\title{
Ein Datenbanksystem (P.A.S.T) zur Verarbeitung und Interpretation von palynologischen Daten aus dem Paläogen Mitteleuropas mit Diversitätsbetrachtungen
}

\author{
Dissertation \\ zur Erlangung des Doktorgrades \\ der Mathematisch-Naturwissenschaftlichen Fakultäten \\ der Georg-August-Universität zu Göttingen
}

\author{
vorgelegt von \\ Thomas Bode \\ aus Göttingen
}

Göttingen 2001 
D7

Referent: Prof. Dr. W. Riegel

Korreferent: Prof. Dr. M. Kappas

Tag der mündlichen Prüfung: 12.12.2001 


\section{Inhaltsverzeichnis}

1. INHALTSVERZEICHNIS..................................................................................................................... 1

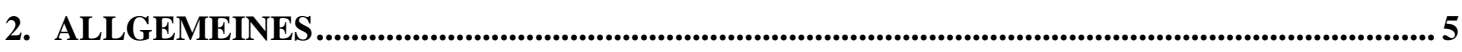

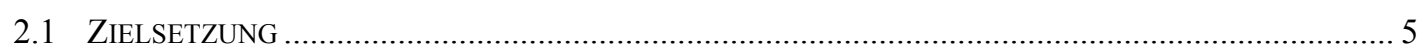

2.2 Historische EnTwicklung Der Palynologie des PALÄOgens MitTeleuropas .................... 6

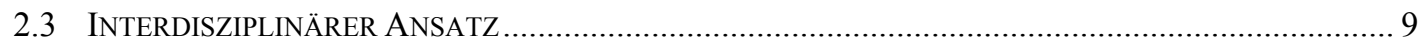

2.4 ELEKTRONISCHE VERARBEITUNGSSYSTEME UND METHODEN IM VERGLEICH ............................... 9

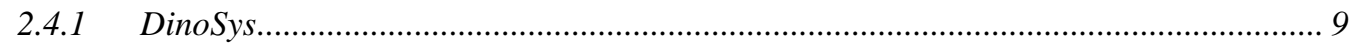

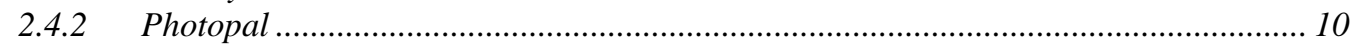

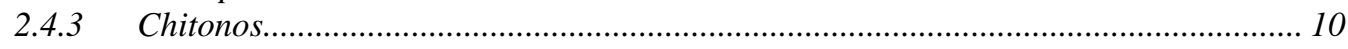

2.4.4 The Plant Fossil Record (PFR2.2) ……………….............................................. 10

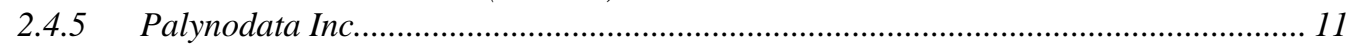

2.4.6 Messeldatenbank …………………………….............................................. 11

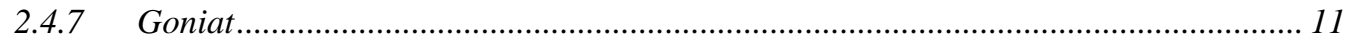

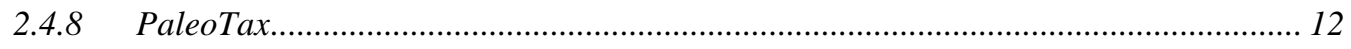

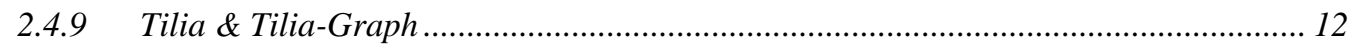

2.4.10 Vergleich der Datenbanken ……………………................................................ 12

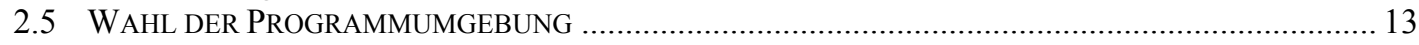

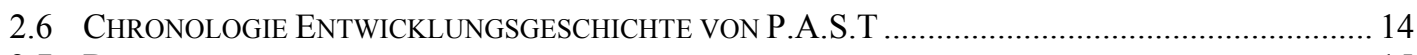

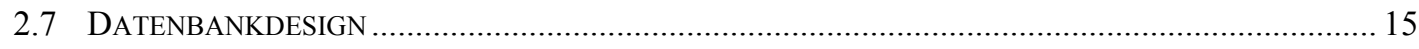

2.7.1 Relationales Datenbankmodell......................................................................... 15

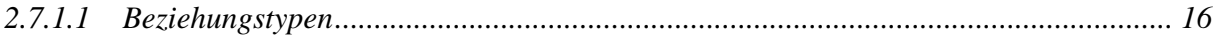

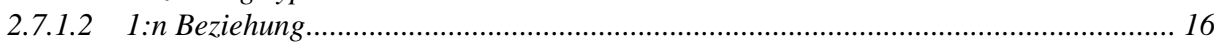

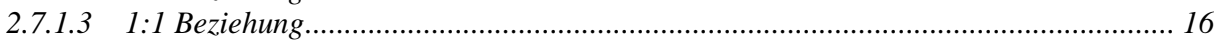

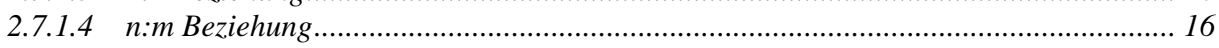

2.8 VORGEHENSWEISE BEI DER ANWENDUNGSENTWICKLUNG ..................................................... 16

2.8.1 Graphische Darstellung der Beziehungen............................................................ 16

2.8.2 Zweck der Normalisierung …………………...................................................... 17

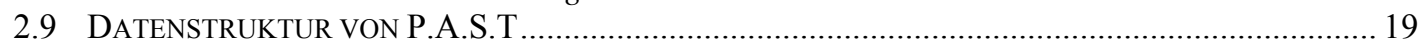

2.10 TABELLEN- UND FORMULARSTRUKTUREN VON P.A.S.T …………........................................ 19

2.11 AllgEMEINE BESCHREIBUNG DER ENTITÄTEN VON P.A.S.T ……………………................... 22

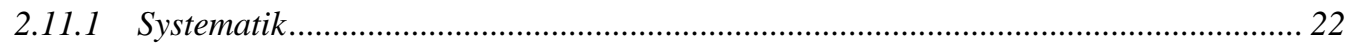

2.11.1.1 Systematische Beschreibung...……………………………………………… 22

2.11.1.2 Morphologische Beschreibungen ……………………………………………… 22

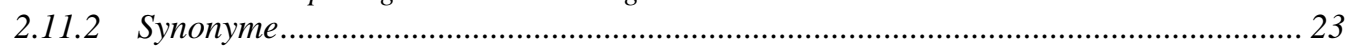

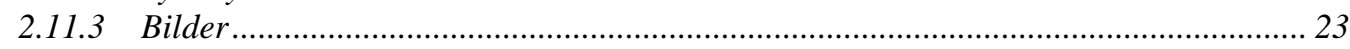

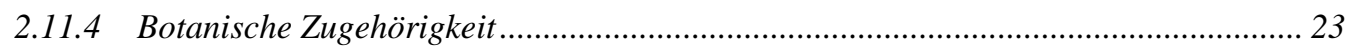

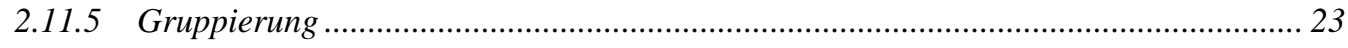

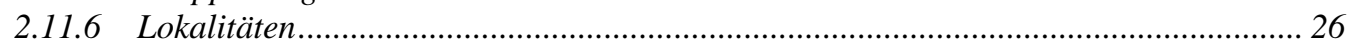

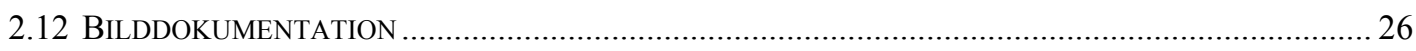

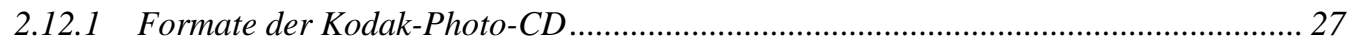

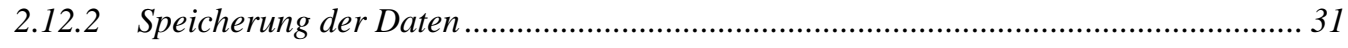

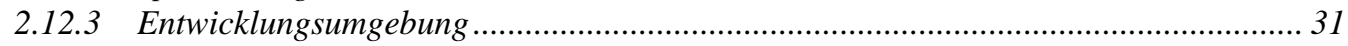

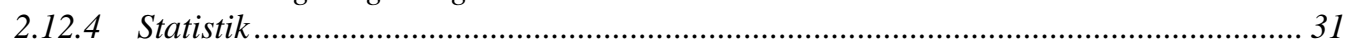

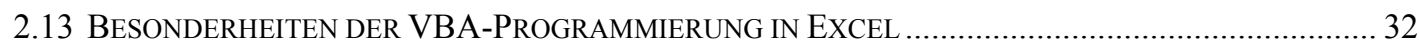

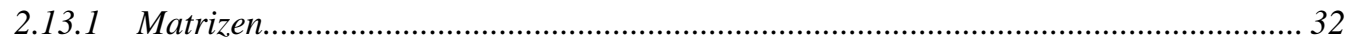

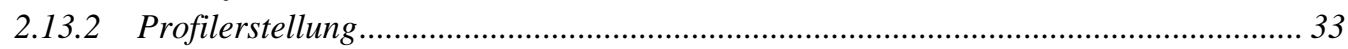

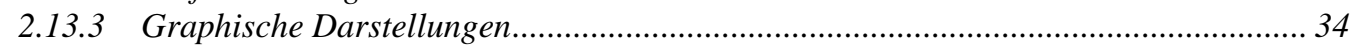

2.13.4 Grenzen der Excelprogrammierung ……………............................................... 35

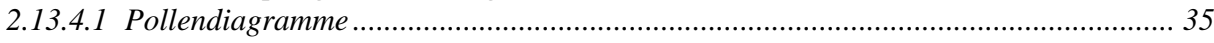

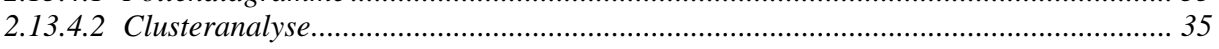

3. BESCHREIBUNG VON P.A.S.T MIT EINEM ANWENDUNGSBEISPIEL...............................36

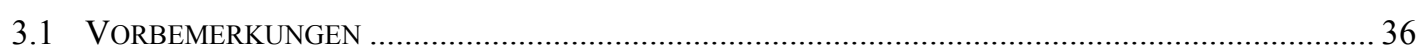

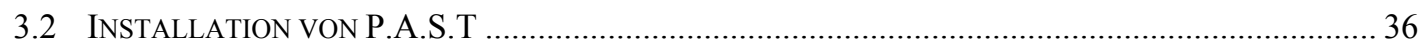

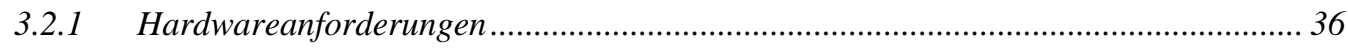


3.2.2 Installation von P.A.S.T von dem Datenträger .................................................. 36

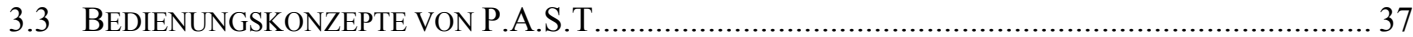

3.3.1 Übersicht (Menüleiste Datei) .......................................................................... 38

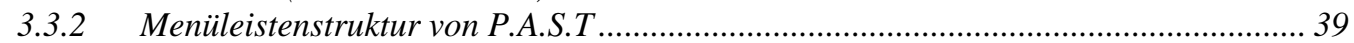

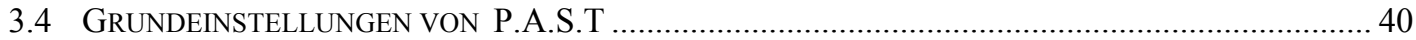

3.5 OBJEKTE EINER DATENBANK MIT BEGRIFFSDEFINITIONEN …….............................................. 40

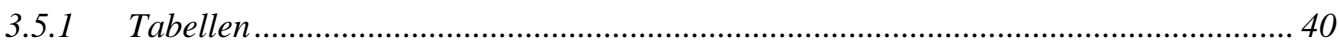

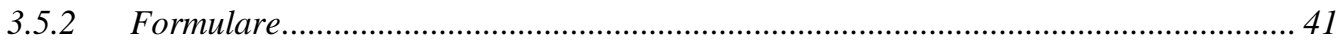

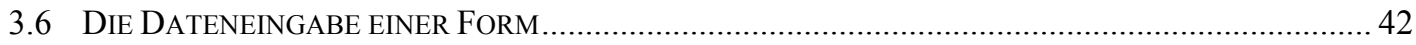

3.6.1 Wechseln und Finden von Datensätzen .................................................................. 44

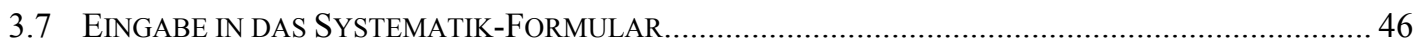

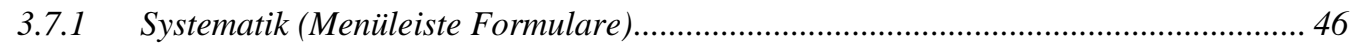

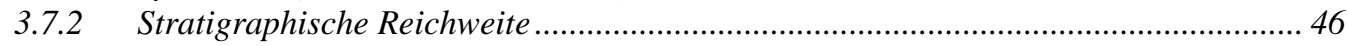

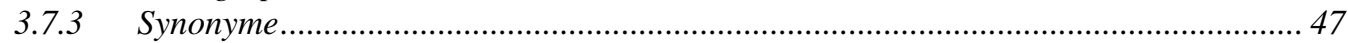

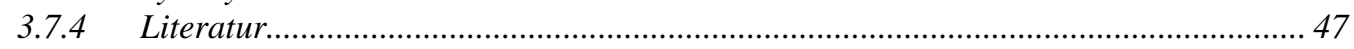

3.7.5 Lokalitäten .................................................................................................. 48

3.7.6 Botanische Zugehörigkeit (nächster lebender Verwandter) .................................... 48

3.7.7 Gruppierung (weiterführende botanische und geographische Informationen) ........... 49

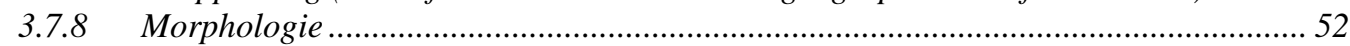

3.7.8.1 Erläuterung der Datenfelder zur Morphologie ...................................................... 55

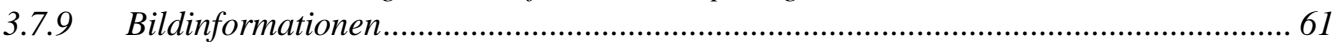

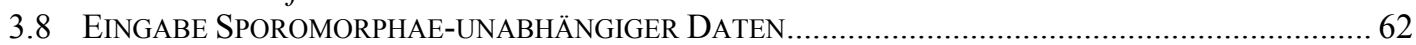

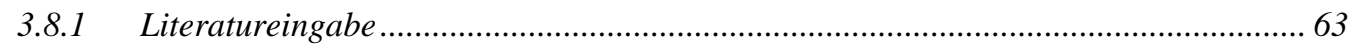

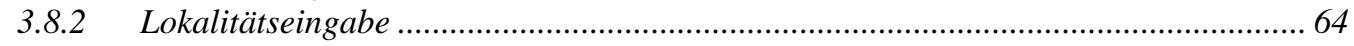

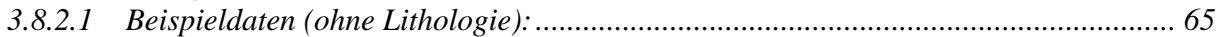

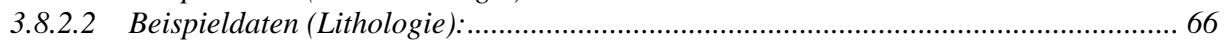

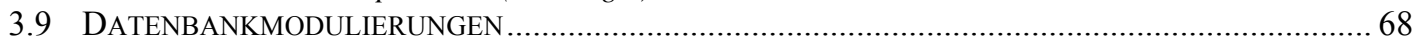

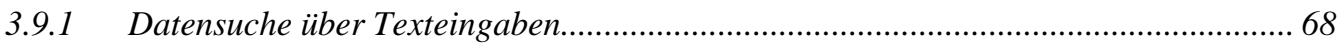

3.9.2 Datensuche über den „Formularbasierten Filter “................................................ 70

3.9.3 Datensuche über den „Auswahlbasierten Filter “ ................................................. 71

3.9.4 Datensuche über stratigraphische Angaben......................................................... 72

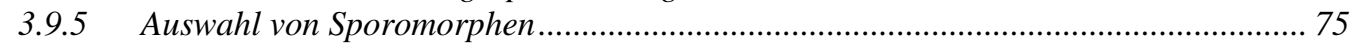

3.10 EXPORTMÖGLICHKEITEN UND ANZEIGEN VON BILDERN MIT P.A.S.T ....................................... 77

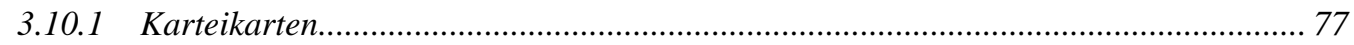

3.10.2 Formularausdruck zur Eingabe der Sporomorphae-Daten ...................................... 79

3.10.3 Abbildungen und Farbtafeln........................................................................... 83

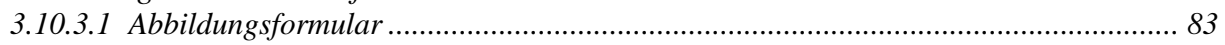

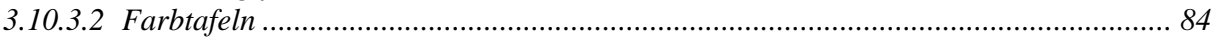

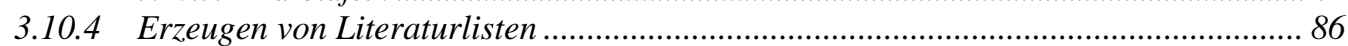

3.10 .5 Erzeugen von Eingabetabellen ......................................................................... 88

3.11 AUSWERTUNG UND GRAPHISCHE DARSTELLUNG DER AUSZÄHLDATEN .................................. 91

3.11.1 Umrechnung der Prozentwerte .......................................................................... 91

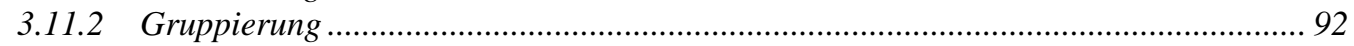

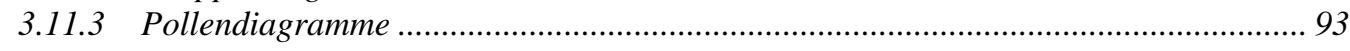

3.11.4 Auswertung der Gruppierungsfunktion .............................................................. 95

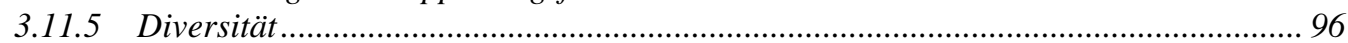

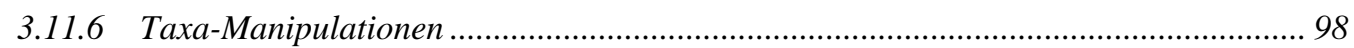

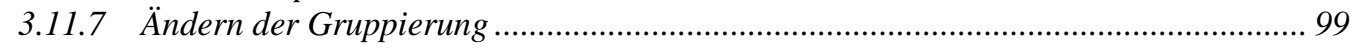

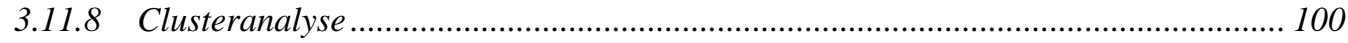

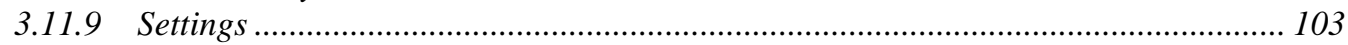

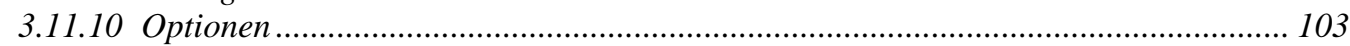

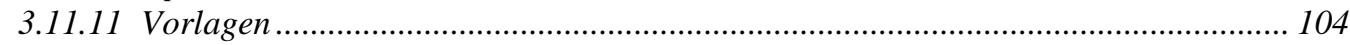

3.11 .12 Kontextmenü .......................................................................................... 105

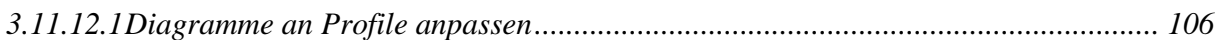

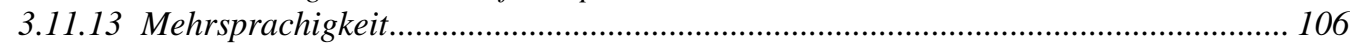

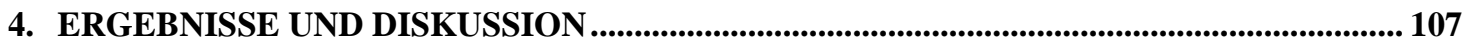

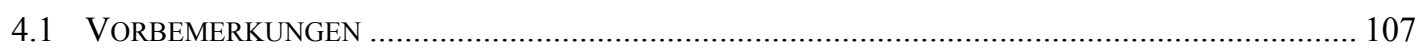

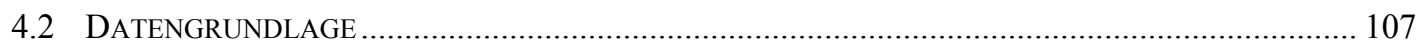

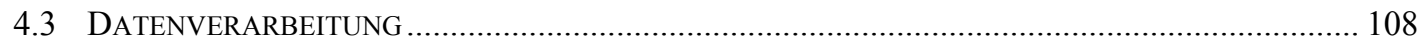

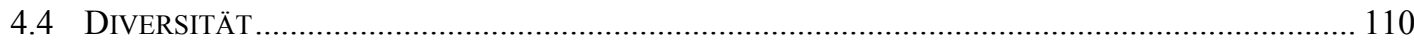


4.4.1 Diversitäts-Berechnungsmethoden ................................................................ 110

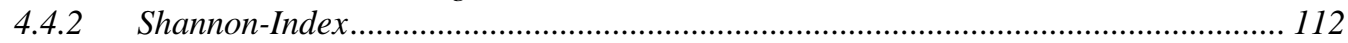

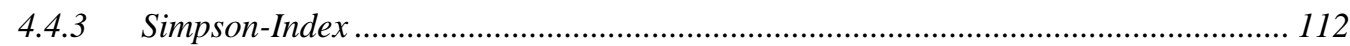

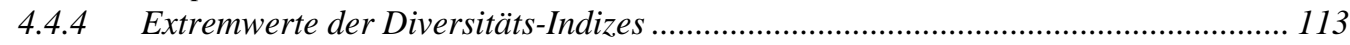

4.4.5 Methoden der Diversitätsberechnung für Lithotypen ............................................ 114

4.4.6 Prozentwerte versus Auszähldaten versus Häufigkeitsklassen ............................... 115

4.4.7 Mittelwertberechnung der Diversitäts-Indizes nach „,Methode 1“ .......................... 115

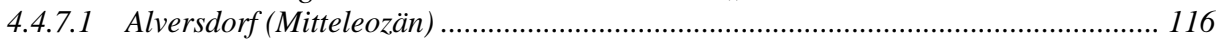

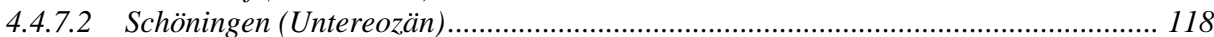

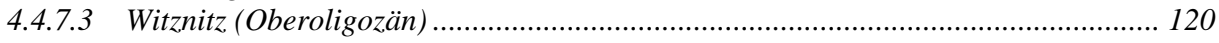

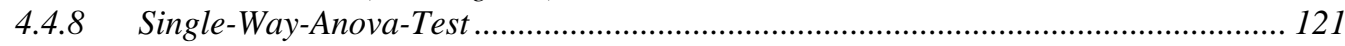

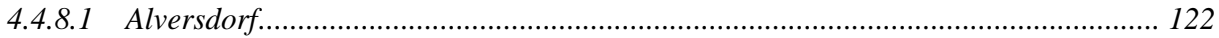

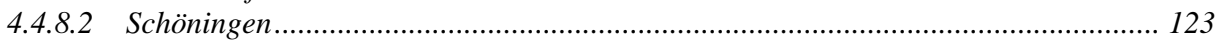

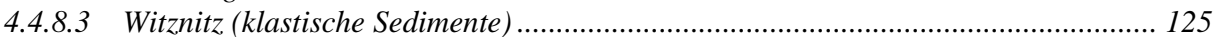

4.4.9 Diskussion der Diversitätsmittelwerte ............................................................ 125

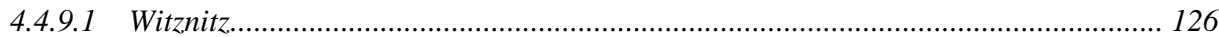

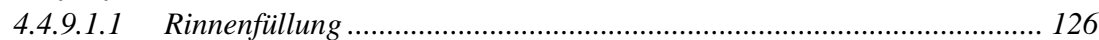

4.4.9.1.2 Paläoboden.............................................................................. 127

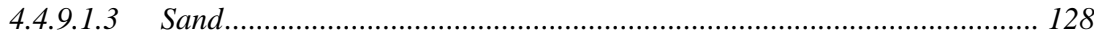

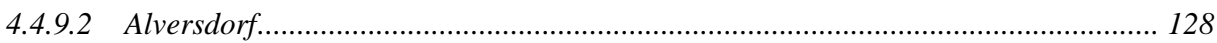

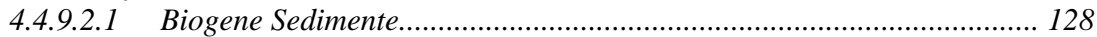

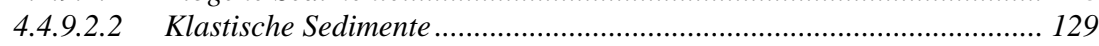

4.4.9.2.3 Biogen-Klastische Sedimente........................................................... 129

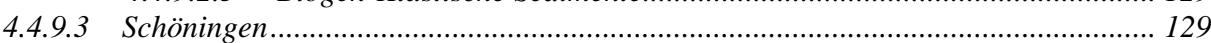

4.4.10 Abhängigkeit der Diversität von der Probengröße …............................................... 129

4.4.10.1 Interpolierende Diversitätsberechnung (Rarefaction)............................................... 130

4.4.10.2 Berechnung der Diversität nach der „,Methode 3“................................................... 131

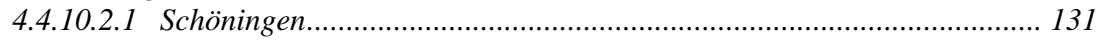

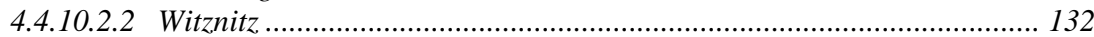

4.4.10.3 Extrapolation der Diversitäten nach Chao-1 .................................................. 133

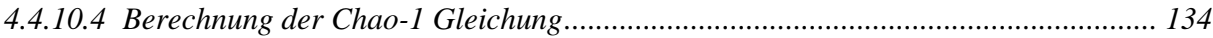

4.4.10.4.1 Schöningen...................................................................... 134

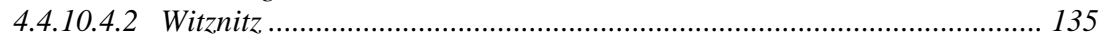

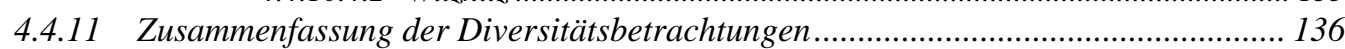

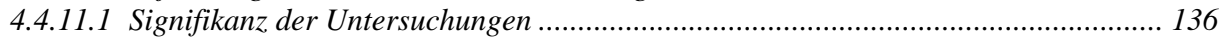

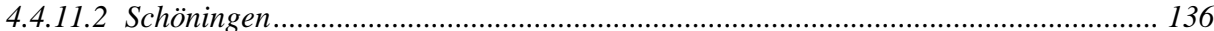

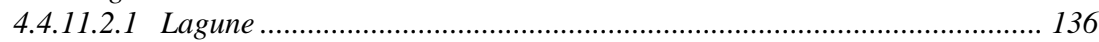

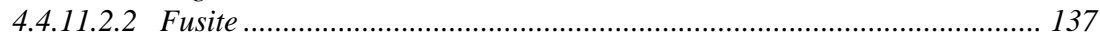

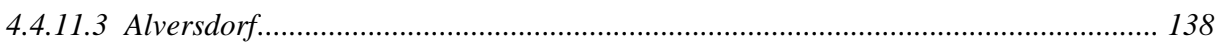

4.4.12 Fazit der Diversitätsbetrachtungen ............................................................. 138

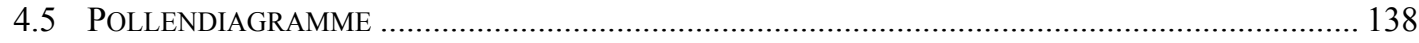

4.5.1 Diskussion der Pollendiagramme im Kontext regionaler und lokaler Aspekte ......... 139

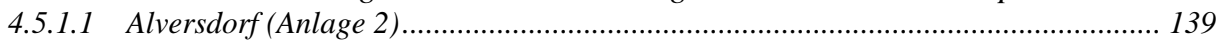

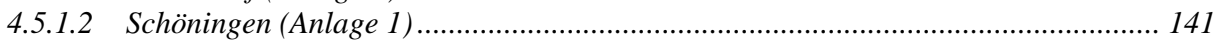

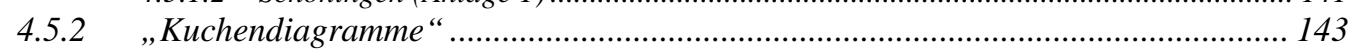

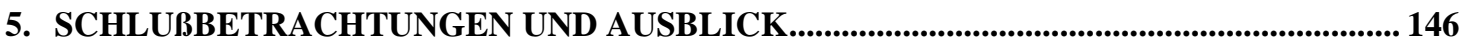

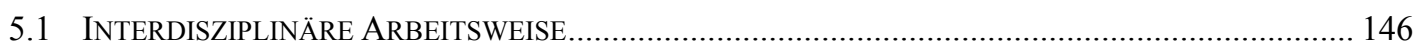

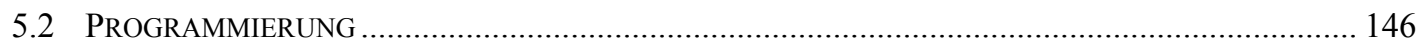

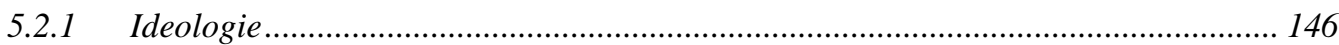

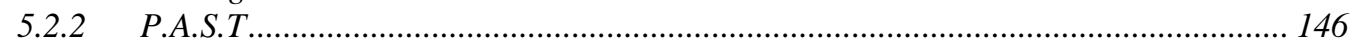

5.2.3 Verknüpfung mit anderen Datenbanken ........................................................... 147

5.2.4 Eingabe weiterer Daten ................................................................................. 147

5.2.4.1 Systematik …............................................................................... 147

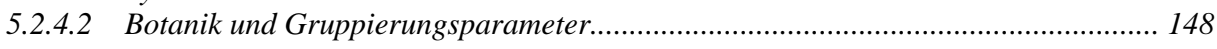

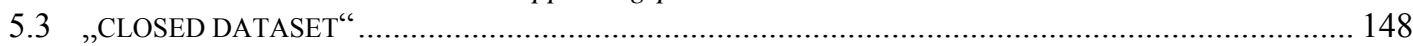

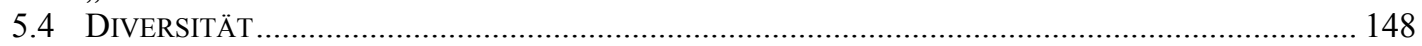

5.4.1 Fehler aufgrund verschiedener Auszähltechniken ............................................ 149

5.4.2 Zukünftige Diversitätsberechnungen .............................................................. 150

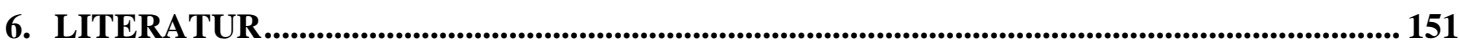

7. ANHANG 


\section{Vorwort}

"There are two ways of constructing a software design: One way is to make it so simple that there are obviously no deficiencies, and the other way is to make it so complicated that there are no obvious deficiencies.

The first method is far more difficult."

C.A.R. Hoare

Diese Arbeit entstand auf Anregung von Prof. Riegel, dem Betreuer dieser Arbeit, dem ich für die Unterstützung bei der Erstellung dieser Arbeit herzlich danke. Die Dissertation entstand unter einem "ungünstigen Stern", da die Gutachter der Deutsche Forschungsgemeinschaft (DFG) die Realisierbarkeit dieses Projektes in Frage stellten, so daß insgesamt zwei Anträge (Pilotprojekt Antrag Ri 154/20-1 und Projekt Ri 154/22-1) mit Argumenten und Vorbehalten gegenüber innovativen Projekten mit etwas höherem Erfolgsrisiko, die z.T. mangelnde Fachkenntnis zeigten, die Dissertation abgelehnt wurde. Aufgrund dieser mißlichen Lage war ich gezwungen, im Rechenzentrum Göttingens (GWDG) und als Netzwerkbeauftrager des Göttinger Zentrums Geowissenschaften GZG, Abteilung Geobiologie (ehemals: Instituts und Museums für Geologie und Paläontologie (IMGP) sowie als Programmierer einer Datenbank über Schwämme (PORIDAT, BOSMAN-PROJEKT, Prof. Reitner) tätig zu sein. Diese Tätigkeiten kamen mir jedoch letztlich wieder bei der Erstellung dieser Arbeit zugute, verlängerten die Arbeiten an der Dissertation jedoch erheblich auf insgesamt fast sechs Jahre.

Außerdem führte der Mangel an Sachmitteln und das Fehlen von Hilfskräften dazu, daß die Eingabe der Daten in die Datenbank nicht wie gewünscht voranschritt. Die in der Einleitung beschriebenen Lokalitäten sind daher bisher nur zum geringen Teil Datenbank-relevant erfaßt. Eine Weiterführung der Eingabe nach Beendigung dieser Arbeit ist daher unerläßlich. Anstrengungen diesbezüglich, das System an geeigneter Stelle zu plazieren, werden von der "Göttinger Arbeitsgruppe" verstärkt unternommen.

Ich möchte insbesondere den Mitarbeitern der "Göttinger Arbeitsgruppe" unter der Leitung meines Betreuers Prof. W. Riegel danken, die mit ihrer steten Diskussionsbereitschaft und Ermunterungen erst die Erstellung dieser Arbeit ermöglichten. $\mathrm{Zu}$ nennen seien hier in alphabetischer Reihenfolge: Herr Dipl.-Geol. Jörg Hammer, Frau Dr. Gudrun Hammer-Schiemann, Herr Dr. Olaf Lenz und Frau Dipl.-Geol. Claudia Natge-Efoghe.

„Last but not least“ danke ich besonders meiner Frau Monika Jedrusik-Bode und unserer gemeinsamen am 26.07.01 geborenen Tochter Anna-Carina. Ohne deren moralische Unterstützung hätten die Arbeiten an dem Programm sicherlich niemals ein Ende gefunden. 


\section{Allgemeines}

\subsection{Zielsetzung}

Das Ziel dieser Arbeit ist es, ein computergestütztes Expertensystem (P.A.S.T = Pollen and Spores of Tertiary) auf der Basis des Datenbanksystems Microsoft Access und der Tabellenkalkulation Microsoft Excel zu entwickeln, um Arbeitsschritte der Palynologie zu automatisieren und zu vereinfachen, die bislang sehr zeitaufwendig, fehleranfällig und häufig redundant durchgeführt werden mußten. Dabei wird berücksichtigt, daß konventionelle Datensammlungen und deren Auswertung und Interpretation in der paläontologischen und biologischen Systematik in absehbarer Zukunft nur dann genutzt werden, wenn sie entsprechend elektronisch aufbereitet und über den PC zugänglich sind.

Dabei geht das entwickelte Programm weit über die Begriffsdefinition einer Datenbank als "Zusammenstellung von Informationen über gleichartige Objekte in Form einer Tabelle" (KULlMANN 1993, S. 398) hinaus. Vielmehr erlaubt das Programm neben den reinen Datenbankfunktionen komplexe interaktiv steuerbare Prozesse zu visualisieren und statistisch auszuwerten.

Insbesondere die Einbeziehung des nächsten lebenden Verwandten ("nearest livingrelatives", z.B. MosBRUGGer, et al. 1997) in Kombination mit palynologischen Auszähl-Daten unter Zuhilfenahme von statistischen Methoden soll neue Möglichkeiten der Interpretation und Rekonstruktion von Pflanzengemeinschaften erschließen, bzw. bestehende palynologische Darstellungsmethoden vereinfachen und erweitern.

Ein weiteres Ziel dieser Arbeit sind Diversitätsbetrachtungen und die Diskussion von Pollendiagrammen aus den Profilen Alversdorf (Mitteleozän) und Schöningen (Untereozän, Helmstedt) sowie Witznitz (Oberoligozän, Leipzig). Die Diversitätsbetrachtungen und die Diskussion der Pollendiagramme sind in erster Linie als Beispiele für die Darstellung der Möglichkeiten von P.A.S.T zu verstehen und deshalb in ihrer wissenschaftlichen Auswertung keineswegs erschöpfend (siehe Kap. 4).

Außerdem soll mit dem System die Möglichkeit geschaffen werden, den hohen bislang erreichten Kenntnisstand in der Palynologie des Paläogens Mitteleuropas zu sichern (siehe Kap. 2.2, S. 6 ff.), die Form und Qualität der Informationen aus den unterschiedlichen Publikationen oder unpublizierten Berichten zu vereinheitlichen und diese Informationen in leicht zugänglicher und vernetzter Form über den PC palynologisch arbeitenden Wissenschaftlern verfügbar zu machen.

Arbeiten aus dem Projekt "Rekonstruktion des Paläogens Mitteleuropas" liefern im Rahmen dieser Arbeit einen Datenpool von ca. 200-300 Formen (die Datenbank wird ständig erweitert) und werden zur ergänzenden Auswertung einiger schon publizierter Arbeiten herangezogen. Neue Ergebnisse werden in Zusammenhang mit Fortsetzungsanträgen (Prof. H. Walther, Dresden, DFG Fortsetzungsantrag zu Wa 757/9-1, Bearbeiter: Dipl.-Geol. J. Hammer) diskutiert. Im Rahmen der Alversorf-Publikation (in Vorbereitung) sind dafür benötigte Programmteile bereits für den angelsächsischen Sprachraum angepaßt worden. Die Übersetzung wird nach der Fertigstellung der Promotion fortgeführt, da der potentielle Nutzerkreis von P.A.S.T damit stark erweitert werden kann. 


\subsection{Historische Entwicklung der Palynologie des Paläogens Mitteleuropas}

Die systematische Bearbeitung fossiler Pollen- und Sporenvergesellschaftungen des Prä-Quartärs und ihre Anwendung auf die Lösung stratigraphischer Probleme hat ihren Anfang mit den frühen Arbeiten von R. PotoniÉ $(1931,1934)$ zur eozänen Braunkohle des Geiseltals genommen. Der Startschuß für die Entwicklung der "Stratigraphischen Palynologie" zu einer eigenständigen und virulenten Disziplin ist somit durch die frühen palynologischen Arbeiten im Paläogen Mitteleuropas gefallen. In den folgenden Jahren weitete sich die Anwendung einerseits auf die neogenen Braunkohlen (z.B. PotoniÉ et al. 1950), andererseits aber auch auf das Mesozoikum und Karbon aus (u.a. PotoniÉ et al. 1954, PotoniÉ et al. 1955). Nach dem zweiten Weltkrieg fand die Palynologie des Prä-Quartärs vor allem durch die Anwendung in der Erdölexploration weltweite Bedeutung. In Deutschland waren es zunächst die Arbeiten von THOMSON und Pflug (Pflug 1952, Thomson \& Pflug, 1953), die die Pionierarbeiten von PotONIÉ und seinen Mitarbeitern auf eine neue systematische Grundlage brachten.

Durch die verstärkte Suche nach Energierohstoffen in der ehemaligen DDR bekam die Tertiär-Palynologie während der sechziger und siebziger Jahre einen neuen Schub durch die Anwendung in der Braunkohlenexploration. Der damals von den ostdeutschen Kollegen erarbeitete Kenntnisstand und die seither vorliegende Datendichte sind für die Palynologie des Paläogens weltweit sicher einmalig und könnten weit über den mitteleuropäischen Raum hinaus Maßstäbe für die systematische und stratigraphische Bearbeitung setzen und als vielseitig verwertbare Quelle für Proxidaten bei paläoökologischen und paläoklimatologischen Rekonstruktionen dienen. Dem steht allerdings im Wege, daß nur ein Teil der Daten veröffentlicht und dann auch nur in weit verstreuten Publikationen zugänglich ist. Der weitaus größte Teil liegt aber wegen der früheren Geheimhaltung nur in verschlüsselter Form in verschiedenen Archiven vor. Die dort gespeicherten Informationen können zur Zeit nur von denjenigen Kollegen gelesen und genutzt werden, die an ihrer Erstellung beteiligt waren und heute noch tätig sind. Mit dem Ausscheiden dieser Generation von Palynologen wird die Kenntnis der nicht veröffentlichten Daten unwiederbringlich verloren gehen. Die aus dieser Bearbeitungsphase stammenden Daten umfassen vor allem Ergebnisse aus dem Weißelster-Becken, dem Geiseltal, dem Bitterfelder Revier, der Egelner Mulde (östliche Fortsetzung der Helmstedter Mulde) und der Oberlausitz.

Mittlerweile hat sich auch in den alten Bundesländern ein umfangreiches Datenmaterial zur Palynologie des Paläogens angesammelt (siehe auch die Zusammenstellung der Lokalitäten auf Seite 8).

Abgeschlossen sind bereits die Arbeiten von THIELE-PFEIFER (1988) über Messel sowie von NiCKEL (1996a) über die Pechelbronner Schichten im nördlichen Oberrheingraben und über das Eckfelder Maar in der Eifel (NICKEL 1996b). SCHULER (1990) bearbeitete mehrere Profile aus dem Paläogen vom Rhonegraben bis in die Hessische Senke palynologisch. Vor und während der Entwicklungsphase dieser Dissertation fand eine ökologisch/palynologisch orientierte Bearbeitung des Helmstedter Paläogens durch die "Göttinger Arbeitsgruppe" im Rahmen von zwei Dissertationen (HAMMER-SCHIEMANN 1998, LENZ 2000) mehrerer Diplomarbeiten und Arbeiten des Betreuers statt. Ergänzend zu diesen Arbeiten kann auch Material von abgeschlossenen Arbeiten aus dem benachbarten Ausland (KRUTZSCH \& VANHOORNE 1977) herangezogen werden. 
Gleiches gilt für Material aus Arbeiten, die seit der Wende in den neuen Bundesländern wieder verstärkt aufgenommen wurden. Hierzu gehören folgende Arbeiten:

\begin{tabular}{|l|l|}
\hline Lokalität & Bearbeiter \\
\hline Egelner Mulde & $\begin{array}{l}\text { KRUTZSCH, Berlin } \\
\text { KIESEL, Freiberg } \\
\text { BLUMENSTENGEL, Halle }\end{array}$ \\
\hline $\begin{array}{l}\text { Region Halle } \\
\text { z.B. Tagebau Merseburg Ost }\end{array}$ & BLUMENSTENGEL \\
\hline Tagebau Schleenhain, Tagebau Witznitz & WALTHER, RIEGEL, HAMMER \\
\hline Tagebau Bockwitz (Thierbachschichten, Oligozän) & GASTALDO et al. \\
\hline Leipziger Bucht (marines Paläogen) & FECHNER \\
\hline
\end{tabular}

Abb. 2.1: Arbeiten aus den neuen Bundesländern.

Die Art der Bearbeitung und der Stand der Veröffentlichungen ist für die genannten Arbeitsgebiete außerordentlich heterogen. Dieser Sachverhalt ergibt sich erwartungsgemäß über einen Zeitraum der einzelnen Bearbeitungen von fast 70 Jahren sowie aus dem Wechsel der Zielsetzungen. Die letzte großregionale Zusammenstellung für Mitteleuropa $\mathrm{zu}$ einem Atlas der tertiären Sporen und Pollen (ThOMSON \& PfLug 1953) liegt inzwischen fast 50 Jahre zurück. Die dort eingesetzte Taxonomie und Nomenklatur wurde von jüngeren Autoren in unterschiedlicher Weise übernommen, abgewandelt oder zum Teil abgelehnt. Es fehlt eine umfassende, moderne Zusammenfassung und Modifikation der Arbeitsmethoden, die als Leitlinie für die taxonomische und nomenklatorische Handhabung dient und gleichzeitig eine autoritative Quelle für stratigraphische, geographische, botanische und ökologische Informationen darstellt.

\begin{tabular}{|c|c|c|c|c|}
\hline Sporenzone & Lokalität & Stratigraphische Einheit & $\begin{array}{l}\text { Bearbeiter, Material- } \\
\text { Ouelle }\end{array}$ & Alter \\
\hline \multirow[t]{6}{*}{$\begin{array}{l}\text { SPP-Zone } 20 \text { [A-I, } \\
\text { II, III] }\end{array}$} & Tgb. Witznitz & \multirow{3}{*}{$\begin{array}{l}\text { Thierbach-Sch. * } \\
\text { Thierbach-Sch. }\end{array}$} & $\begin{array}{l}\text { WALTHER, RIEGEL, } \\
\text { HAMMER }\end{array}$ & \multirow{3}{*}{$\begin{array}{l}\text { Neo-Chatt bis } \\
\text { Basis Miozän }\end{array}$} \\
\hline & Tgb. Bockwitz & & GASTALDO et al. & \\
\hline & Westerwald, Enspel & & KÖHLER & \\
\hline & Oberrhein & Ob. Pechelbronner Sch. & NICKEL & \begin{tabular}{|l|} 
Mittel- bis \\
Ober-Oligozän.
\end{tabular} \\
\hline & $\begin{array}{l}\text { Weißelster-Becken (z.B. } \\
\text { Schleenhain) }\end{array}$ & \multirow[t]{2}{*}{$\begin{array}{l}\text { Haselbach-Tone Bereich } \\
\text { Flöz III-IV }\end{array}$} & $\begin{array}{l}\text { WALTHER, KRUTZSCH et } \\
\text { al., HALFAR et al. }\end{array}$ & \multirow[t]{2}{*}{ Eo-Chatt } \\
\hline & ?Lausitz, Seifhennersdorf & & WALTHER & \\
\hline \multirow[t]{6}{*}{ SPP-Zone 19} & Hessen & & PFLUG & \multirow[t]{6}{*}{$\begin{array}{l}\text { Unter-Oligozän } \\
\text { Latdorf }\end{array}$} \\
\hline & & Melanientone & PFLUG & \\
\hline & Sieblos & & JÄHNICHEN, MARTINI & \\
\hline & Oberrhein & \multirow[t]{3}{*}{ Mittl. Pechelbronner Sch. } & NICKEL, SCHULER & \\
\hline & $\begin{array}{l}\text { Weißelster-Beckenehem. } \\
\text { Tgb. Lochau-Ost }\end{array}$ & & KRUTZSCH et al. & \\
\hline & Lausitz, Kleinsaubernitz & & GOTH & \\
\hline $\begin{array}{l}\text { SPP-Zone } \\
\text { (Zwenkauer } \\
\text { Florenh.) }\end{array}$ & Leipzig, Tgb. Zwenkau & $\begin{array}{l}\text { Zwischenmittel zw. Flöz II } \\
\text { und III ? }\end{array}$ & KRUTZSCH, WALTHER & $\begin{array}{l}\text { Ober-Eozän, } \\
\text { Präbon }\end{array}$ \\
\hline
\end{tabular}




\begin{tabular}{|c|c|c|c|c|}
\hline Sporenzone & Lokalität & Stratigraphische Einheit & \begin{tabular}{|l} 
Bearbeiter, Material- \\
Quelle
\end{tabular} & Alter \\
\hline $\begin{array}{l}\text { SPP-Zone 18 } \\
\text { (,Zeitzer Bild“) }\end{array}$ & $\begin{array}{l}180 \text { Fdpkt. in } \\
\text { Mitteldeutschland, z.B. }\end{array}$ & $\begin{array}{l}\text { Flöze II und III im } \\
\text { Weißelster-Becken }\end{array}$ & KRUTZSCH et al. & $\begin{array}{l}\text { Obereozän, } \\
\text { Präbon }\end{array}$ \\
\hline SPP-Zone 17/18 & Geiseltal & Hangendes d. Geiseltal-S. & KRUTZSCH et al. & $\begin{array}{l}\text { Mittel-Eozän, } \\
\text { Oberstes } \\
\end{array}$ \\
\hline \multirow[t]{2}{*}{ (Zwischenzone) } & Weißelster-Becken & Flöz I und II & KRUTZSCH et al. & \multirow[t]{2}{*}{ Barton } \\
\hline & Egeln-Süd & Hangendes von Flöz I & $\begin{array}{l}\text { KRUTZSCH, } \\
\text { BLUMENSTENGEL. KIESEL }\end{array}$ & \\
\hline \multirow[t]{3}{*}{ SPP-Zone 17} & westl. Halle, Amsdorf & Hauptflöz & \multirow[t]{2}{*}{$\begin{array}{l}\text { KRUTZSCH, } \\
\text { BLUMENSTENGEL }\end{array}$} & \multirow[t]{3}{*}{$\begin{array}{l}\text { Mittel-Eozän, } \\
\text { Oberes }\end{array}$} \\
\hline & $\begin{array}{l}\text { Weißelster-Becken (Tgb. } \\
\text { Profen) }\end{array}$ & & & \\
\hline & Egeln-Süd & Flöz Löderburg & $\begin{array}{l}\text { KRUTZSCH, } \\
\text { BLUMENSTENGEL }\end{array}$ & \\
\hline \multirow{2}{*}{$\begin{array}{l}\text { SPP-Zone 16/17 } \\
\text { (Übergangsphase) }\end{array}$} & Weißelster-Becken & \multirow[t]{2}{*}{ Liegendes von Flöz I } & \multirow[t]{2}{*}{ KRUTZSCH et al. } & $\begin{array}{l}\text { Mittel-Eozän, } \\
\text { Mittleres }\end{array}$ \\
\hline & Egeln Süd & & & Barton \\
\hline \multirow[t]{5}{*}{ SPP-Zone 16} & $\begin{array}{l}\text { Weißelster-B. } \\
\text { (Merseburg Ost) }\end{array}$ & Flöz Merseburg & BLUMENSTENGEL & \multirow{5}{*}{$\begin{array}{l}\text { Mittel-Eozän; } \\
\text { Unteres } \\
\text { Barton }\end{array}$} \\
\hline & Egeln-Süd & Flöz II & $\begin{array}{l}\text { KRUTZSCH, } \\
\text { BLUMENSTENGEL } \\
\end{array}$ & \\
\hline & Subherzyn (Nachterstedt) & Unterflöze & KRUTZSCH & \\
\hline & Helmstedt & Annenberg-Sch. & KRUTZSCH & \\
\hline & Hessen & Borkener Oberflöze & KLEIN-RESINK, RIEGEL & \\
\hline \multirow[t]{2}{*}{$\begin{array}{l}\text { SPP-Zone 15/16 } \\
\text { (Zwischenzone) }\end{array}$} & Geiseltal & $\begin{array}{l}\text { Oberkohle bis } \\
\text { Hangendserie }\end{array}$ & KRUTZSCH et al. & \multirow[t]{2}{*}{$\begin{array}{l}\text { Mittel-Eozän, } \\
\text { Grenzbereich }\end{array}$} \\
\hline & Egeln-Süd & $\begin{array}{l}\text { Äquiv. zu Teilen d. } \\
\text { Annenberg-Sch. }\end{array}$ & KRUTZSCH et al. & \\
\hline \multirow{7}{*}{$\begin{array}{l}\text { SPP-Zone } 15 \\
\text { (Subzonen A-D) }\end{array}$} & Geiseltal & Oberkohle (15D) & KRUTZSCH et al. & \multirow[t]{7}{*}{$\begin{array}{l}\text { Mittel-Eozän, } \\
\text { Lutet }\end{array}$} \\
\hline & & Ob. Mittelkohle (15C) & KRUTZSCH et al. & \\
\hline & & Mittelkohle (15B) & KRUTZSCH et al. & \\
\hline & & Unterkohle (15A) & KRUTZSCH et al. & \\
\hline & Eifel (Eckfeld) & Ölschiefer (=15C) & NICKEL & \\
\hline & Oberrhein (Messel) & Ölschiefer (=15A) & THIELE-PFEIFFER & \\
\hline & Helmstedt* & Oberflöze & \begin{tabular}{|l|} 
LENZ, HAMMER, HAMMER- \\
SCHIEMANN. NATGE-EFOGE \\
\end{tabular} & \\
\hline SPP-Zone $14 / 15$ & Geiseltal & Liegendabschnitt & KRUTZSCH et al. & Grenzbereich \\
\hline (Übergangszone) & Oberrhein (Messel) & \multirow[t]{2}{*}{ Ölschiefer } & THIELE-PFEIFFER, WILDE & $\begin{array}{l}\text { U/M-Eozän } \\
\text { (Ypres/Lutet) } \\
\end{array}$ \\
\hline \multirow[t]{3}{*}{ SPP-Zone 14} & Geiseltal-Südfeld & & \multirow[t]{2}{*}{ KRUTZSCH et al. } & \multirow{3}{*}{$\begin{array}{l}\text { Unter-Eozän } \\
\text { ?Ypres }\end{array}$} \\
\hline & S-Brandenburg & Nedlitzer Schichten & & \\
\hline & Helmstedt & oberer Teil d. Unterflöze & RIEGEL & \\
\hline SPP-Zone 13 & Helmstedt * & \multirow[t]{4}{*}{ Unterflöze } & HAMMER-SCHIEMANN & $\begin{array}{l}\text { Unter-Eozän, } \\
\text { Ypres } \\
\end{array}$ \\
\hline SPP-Zone 12 & Riestedt & & KRUTZSCH & Basis Eozän \\
\hline SPP-Zone 12 & $\begin{array}{l}\text { Belgien, Epinois, } \\
\text { Antweiler }\end{array}$ & & \multirow[t]{2}{*}{$\begin{array}{l}\text { KRUTZSCH \& van HOORNE, } \\
\text { PFLUG, BATTEN }\end{array}$} & \multirow[t]{2}{*}{$\begin{array}{l}\text { Ob. Paläozän } \\
\text { (Landen) }\end{array}$} \\
\hline SPP-Zone 9 & Thüringen, Sangerhausen & & & \\
\hline SPP-Zone 8 & Brandenburg & Nassenheider Sch. & KRUTZSCH & Mittl. Paläozän \\
\hline SPP-Zone 7 & Hannover, Sarstedt & Gründeberger Serie & PFLUG & $\begin{array}{l}\begin{array}{l}\text { Unt. Paläozän } \\
\text { (Mont) }\end{array} \\
\end{array}$ \\
\hline
\end{tabular}

Abb. 2.2: Tabellarische Darstellung der Lokalitäten deren Palynomorphen Datenbank-relevant sind. Bereits mit der Datenbank erfaßte Lokalitätsdaten sind gekennzeichnet $(*)$. 


\subsection{Interdisziplinärer Ansatz}

Neben dem paläobotanischen Ansatz, der die Planung von P.A.S.T sowie die kritischen Beurteilung der Literaturdaten und der Diskussion der Ergebnisse beinhaltet, bestand eine hohe Notwendigkeit Informatik-relevante Arbeitstechniken zu erlernen und zur Schaffung neuer Programmstrukturen anzuwenden. Neben den Regeln der Datenbankentwicklung ist hier in erster Linie die Programmierung zum Teil komplexer Abläufe zu nennen. Hierzu bietet das Microsoft-Office Paket Visual Basic for Applications als relativ gut dokumentiertes, erweiterbares und Programmübergreifendes Werkzeug an. Geschwindigkeitsoptimierte und Windows-nahe Programmteile wurden mit Hilfe der Windows APIs (Windows Application Programming Interface) programmiert. Als unschätzbare Hilfe erwies sich das Internet; insbesondere das Studium umfassender Newsgroups als Diskussionsforum bei Problemen mit Unzulänglichkeiten und Fehlern der Programme führte in der Regel zu Lösungsansätzen.

Die schon im Kapitel 2.1 erwähnte nearest-living-relative-Methode macht ein Auseinandersetzen mit rezenten botanischen Pflanzenfamilien -Gattungen und -Arten nötig. Dabei wurde im Rahmen dieser Arbeit auch enzyklopädische Literatur (insbesondere FUKAREK 1992, 1993, 1994, 1995 und HEYWOOD 1982) eingesetzt, da diese konzentriert auf das Wesentliche beschränkt, vergleichende Informationen liefern kann - ein unschätzbarer Vorteil bei der Verwendung in Datenbanksystemen.

Außerdem fand eine Betrachtung physiogeographischer Sachverhalte statt, die zur Definition der zonalen und z.T. azonalen Vegetationstypen im Gruppierungsteil der Datenbank unerläßlich war.

\subsection{Elektronische Verarbeitungssysteme und Methoden im Vergleich}

Während des Planungsstadiums dieser Arbeit wurde eine Auswahl bestehender paläobotanischer Datenbanksysteme eingehend geprüft, ob und inwieweit eine mögliche Integration oder Anpassung an die hier bearbeitete Fragestellung möglich sei. Im Folgenden werden einige dieser Datenbanken kurz vorgestellt und anschließend miteinander in den Kontext gebracht.

\subsubsection{DinoSys}

A Dinoflagellate Cyst Database

Weegink, J.W., Marks, P.H.

LPP Anzahlation, Laboratory of Palaeobotany and Palynology

Heidelberglaan 2, 3584

Utrecht Netherland

DinoSys ist eine PC-basierende Datenbank über fossile Dinoflagellaten-Zysten veröffentlichter und unveröffentlichter Daten und umfaßte 1998 weltweit ca. 1500 Arten mit 9200 Abbildungen. Die Datenbank dokumentiert die Taxonomie (Beschreibung, Synonyme, Arten und Gattungen mit photographischen Abbildungen), Geographie und Stratigraphie mit Palaeoenvironment-Parametern der fossilen Dinozyste. Die Client-Anwendung (PalSys) ist in Visual Basic als MS-AccessDatenbank programmiert: das Projekt entsteht als Initiative der Laboratory of Palaeobotany and Palynology Anzahlation, Utrecht und wird zum großen Teil von der Erdölindustrie gefördert. 


\subsubsection{Photopal}

Suc, J-P.

Centre de Paléontologie stratigraphique et Paléoécologie

Université Lyon

27-43, boulevard Du 11 Novembre

69622 Villeurbanne Cédex, France

Photopal verarbeitet als interaktive Datenbank rezentes Pollenmaterial mit morphologischen Beschreibungen. Photopal bietet die Möglichkeit 20 Photoabbildungen im Format 756*581 Pixel in Graustufen zu verwalten. Informationen zum pflanzlichen Ursprung, der Ökologie, der Synonyme, der Biologie und der morphologischen Charakteristika werden im Rahmen des kommerziell auf CD-ROM erhältlichen Programmes mitgeliefert.

\subsubsection{Chitonos}

A Personal Image and Data-Acquisition System for the Micropaleontologist van Grootel, G., Hamel, J., Achab, A.

Quebec Geoscience Centre

2700 Rue Einstein

St-Foy, Quebec, Canada

Der Prototyp von Chitonos legt seinen Schwerpunkt auf Chitonozoa - mit der Erweiterungsmöglichkeit auf andere Mikrofossilgruppen.

Chitonos verarbeitet in Echtzeit Bilddaten aus mikroskopischen Aufnahmen über ein Video-Capture-Interface und kann mit elektronischen taxonomischen Katalogen zusammenarbeiten. Das System hat eine Client-Server Architektur, wobei das ClientInterface entweder unter MS-Windows oder IBM-OS/2 betrieben werden kann. Die SQL-Datenbank-Engine wurde mit Gupta's SQL-Windows entwickelt.

\subsubsection{The Plant Fossil Record (PFR2.2)}

Boulter, M.C.

University of East London

Romford Road

London, England

The Plant Fossil Record (PFR) ist ein Projekt, das von der International Organisation of Palaeobotany (eine Sektion der International Union of Biological Science) der Universität London gefördert wird.

PFR stellt eine interaktive, internetbasierende Datenbank mehrerer tausend fossiler Pflanzen dar. Das Taxon kann mit seiner Verbreitung auf paläogeographischen Karten, gegliedert in geologischen Zeitscheiben, auf Gattungsebene dargestellt werden. Über das World-Wide-Web sind Informationen über Arten mit Beschreibungen, Vorkommen und Lokalitäten sowie Verteilungskurven von Pflanzenfamilien und statistische Verfahren zur PCA-Analyse in frei wählbaren Zeitbereichen abrufbar. 


\subsubsection{Palynodata Inc.}

Piel, K.

97 Billings Avenue

Medford, MA 02155 USA

Miller, $\mathrm{M}$.

Amoco Production Company

P.O. Box 3092

Houston, TX 77253 USA

Palynodata wird von dem Geological Survey of Canada (GSC) und der Industrie gefördert. Die Datenbank verfolgt einen ähnlichen Ansatz wie PFR, mit der Speicherung einer großen Anzahl von Taxa unterschiedlicher fossiler Palynomorphengruppen vom Präkambrium bis zum Kanäozoikum.

Palynodata speichert die Daten aus ca. 16000 Publikationen mit Angaben zur Diversität, Herkunft der Formen und Aussterbeereignisse von Palynomorphen (Pollen, Sporen, Dinoflagellaten und Acritarchen). Das System ist allerdings im Gegensatz zu PFR nicht online verfügbar.

\subsubsection{Messeldatenbank}

Senckenbergmuseum

Senckenberganlage

2560325 Frankfurt

Die Senckenbergische Naturforschende Gesellschaft hat sich vertraglich gegenüber dem Land Hessen im Rahmen ihrer Betreiberfunktion zur Dokumentation der Messelfunddaten verpflichtet (PRAHL 1994, S. 197).

Die Datenbank benutzt als Programmplattform Paradox für Windows. Teilweise werden aus Kompatibilitätsgründen dBASE-Komponenten verwendet.

Das Grundkonzept beinhaltet das Speichern sämtlicher in Zukunft stattfindender Grabungen mit der Möglichkeit Bildinformationen einzubinden. Die dBASEGrunddatenbank gibt Informationen über die Grabungsstelle, Geländeansprache, systematische Daten und Hinweise auf Bilddokumente. Die Paradox-Datenmaske unter Windows enthält zusätzlich ein Bildfenster im Format 384 zu 256 Bildpunkten. Die Bilddaten werden direkt in der Datenbank gespeichert.

\subsubsection{Goniat}

Kullmann, J.

Geol.-Paläont. Institut

Sigwartstrasse 10

72076 Tübingen

Bei Goniat wird der Kenntnisstand der paläozoischen Ammonoideen EDV-gerecht zusammengefaßt. Goniat ist als Wissensbasiertes System (WBS), das der raumzeitlichen Diagnose in den Geowissenschaften dienen soll (KULLMANN 1993) konzipiert und basiert auf dBASE.

Es sind sechs unabhängige Hauptdatenbanken beteiligt, die relational miteinander verbunden sind. TAXA enthält systematische Daten der einzelnen TAXA; MORPHA gibt morphologische Informationen $\mathrm{zu}$ den Formen; LIT stellt die Literaturdatenbank dar; LOC enthält die Daten der geographischen Verbreitung; und BOUND die der Zeitebene. Goniat vermittelt teilweise revidierende Angaben und stellt damit nach KULLMANN 1993 eine eigenständige wissenschaftliche Arbeit dar. 


\subsubsection{PaleoTax}

Löser, $\mathrm{H}$.

Postfach 2409

01282 Dresden

PaleoTax verarbeitet taxonomische Daten der mesozoischen Korallen in 20 miteinander verbundenen Datenbanken. Die Datenbasis besteht aus den Komponenten: Zitate in Literatur, Arten und Synonyme, Gattungen, Familien, Unterordnungen, Lokalitäten und stratigraphische Angaben. Die Verwaltung der Daten erfolgt durch ein Programm, das auf eine dBASE-ähnliche Datenbankstruktur aufbaut (LÖSER in KulLmANN 1993), jedoch durch aufwendige Programmierung um weitere Funktionen erweitert wurde. PaleoTax spiegelt objektiv den Stand der Daten wider, der in der Literatur beschrieben wurde, und ist damit weitgehend frei von subjektiven Ansichten des Autors.

\subsubsection{Tilia \& Tilia-Graph}

Grimm, E.

Illinois State Museum

Springfield, Illinois USA

Als einziges der hier vorgestellten Programme verfolgen Tilia \& Tilia-Graph nicht das Ziel Datenbankfunktionen $\mathrm{zu}$ realisieren, sondern wurden entwickelt, um stratigraphische Daten aufzuzeichnen und anzuzeigen. Die DOS-Programme werden kommerziell vertrieben.

Neben den hier vorgestellten Programmen existieren weitere Pollendatenbanken (z.B. European Pollen Database (UPD), World Pollen Database (WPD), die jedoch aufgrund der Beschränkung auf das Quartär nicht weiter diskutiert werden (FISHER 2000).

\subsubsection{Vergleich der Datenbanken}

Die meisten der oben dargestellten Datenbanken basieren auf dBASE. Dieses Datenbanksystem ist zwar relativ weit verbreitet, hat jedoch einige schwerwiegende Nachteile. Da der Ursprung des betagten Systems in der DOS-Zeit der 80er Jahre liegt, ergeben sich zwangsläufig Probleme bei der Integration in ein modernes WindowsSystem. Neben der nur zeichenorientiert arbeitenden dBASE-Variante unter DOS gibt es zwar mittlerweile eine Windows-Version; das "look and feel" kann jedoch nicht unbedingt überzeugen, zumal von DOS auf Windows migrierte Programme in der Regel einiges an veraltetem DOS-Balast mit sich führen. Weiterhin hat der Hersteller Borland mit dem Nachfolgeprodukt Paradox für Windows die führende Marktposition nicht halten können und ist im direkten Vergleich mit z.B. Microsoft ACCESS weniger benutzerfreundlich mit daraus resultierender geringerer Akzeptanz und Verbreitung des Datenbanksystems (s.a. HÜSKES 1996, HÜSKES 1997, GLADIS 1999). Außerdem fehlt die Programm-übergreifende Programmiersprache, die insbesondere bei dem Microsoft Produkt mit Microsoft Visual Basic for Applications ${ }^{\circledR}$ (VBA) durchgehend in der Office-Familie und mit Visual Basic als eigenständiges Produkt vorhanden ist und mittlerweile auch von Fremdherstellern aufgegriffen wird - u.a. mit PERVASIVE SQL (Importmöglichkeit des Pervasive's Active X-Interface in das Visual Basic-System) und COREL DRAW (VBA). Mit dieser Argumentation wird die mögliche Datenbankintegration auf das Access-basierende DINOSYS oder das auf das WorldWideWeb aufsetzende PFR, auf PHOTOPAL und CHITONOS oder auf PALYNODATA eingegrenzt. 
Wie schon KULLMANn 1993 bemerkte, ist PFR (ebenso wie PALYNODATA, Anm. des Autors) im Gegensatz zu GONIAT (oder P.A.S.T, Anm. des Autors) wenig stark "atomisiert" d.h. in kleinste Bestandteile zerlegt und in relational verbundene Datenbanken eingebracht. Erst die detaillierte Zerlegung der einzelnen Merkmale der fossilen Organismen erlaubt eine umfassende wissenschaftliche Bearbeitung, die weit über die stratigraphische Betrachtungsweise hinausgeht. Es besteht nicht der Anspruch, mehrere Fossilgruppen eher oberflächlich $\mathrm{zu}$ betrachten, sondern ganz im Gegenteil eine Möglichkeit zu schaffen, die Palynologie des Paläogens so weit als möglich digital zu erfassen und neue Möglichkeiten der systematischen Bearbeitung und Interpretation palynologischer Ergebnisse zu erschließen.

Insbesondere die Betrachtung der Formen auf Artebene ergibt in P.A.S.T weiterhin Sinn, da damit eine differenzierte Betrachtung nomenklatorischer, taxonomischer und systematischer Probleme möglich ist, die auf der Gattungsebene nur schwer nachvollziehbar sind. Die direkte Internetanbindung über das WorldWideWeb hat in diesem Projekt ebenfalls keine absolute Priorität, da die Daten besser konzentriert mit Abbildungen auf $C D$ verteilt werden.

CHITONOS und PHOTOPAL legen ihren Schwerpunkt auf digitalisiertes Photomaterial. Genau dies ist jedoch bei P.A.S.T zunächst nur von geringer Bedeutung, so daß auch diese Programme zum Zeitpunkt der Planungsphase von P.A.S.T als nicht geeignet für eine Integration erschienen.

Als Darstellungsprogramm numerischer palynologischer Daten kommt TILIA \& TILIA-GRAPH nicht in Frage, da es als DOS-Programm die schon oben erwähnten Nachteile hat und außerdem nicht die Flexibilität einer Tabellenkalkulation zeigt.

Aus dieser Argumentation ergab sich die Notwendigkeit, eine neue Anwendung zu programmieren, da keines der dargestellten Systeme den Anforderungen genügte.

\subsection{Wahl der Programmumgebung}

Als Betriebssystem wurde Microsoft Windows gewählt, da es bei dem zu erwartenden eher Computer-unerfahrenen Benutzer-Clientel sicher die am weitesten verbreitete Plattform im Vergleich zum MacOS oder Unix ist. Die noch 1994 von PRAHL erwähnte geringere Performance von Windows gegenüber Unix ist im Rahmen dieses Projektea relativ unbedeutend und wird daher als Argument vernachlässigt - nicht zuletzt zeigt Windows NT oder Windows 2000 in diesem Bereich erfreuliche Verbesserungen, wenn auch die Absturzfreudigkeit und Fehlerhaftigkeit der Windows-Umgebung gerade auf der Porgrammierebene mehr als lästig ist.

Nach eingehender Prüfung der Marktsituation und Vergleich mehrerer Datenbanksysteme fiel die Wahl auf die relationale Datenbank Microsoft Access, die Tabellenkalkulation Microsoft Excel sowie Microsoft Word und deren gemeinsame Programmiersprache Visual Basic for Applications (VBA). Hiermit ist es leicht möglich, ausgehend von der Datenbank über eine Schnittstelle (Application ObjectProgrammierung) die Tabellenkalkulation und die Textverarbeitung fernzusteuern, was einen dynamischen Datenaustausch zwischen den Anwendungen ermöglicht. Darüber hinaus ist Microsoft Office mit seiner weitestgehenden einheitlichen Programmoberfläche der Einzelprogramme gerade im universitären Bereich weit verbreitet, was die Einarbeitungszeit, Erweiterbarkeit und Installation des auf Access, Excel und Word basierenden P.A.S.T stark vereinfacht. 
Eine mögliche Client/Servervariante - über den SQL-Standard realisierbar - wurde nach eingehender Prüfung wieder verworfen, da zum einen die Programmierung ungleich komplizierter als die der Einplatzvariante ist und zum anderen eine Eingabe der Daten netzunabhängig von ausgewählten Personen erfolgen sollte. Nur so ist es möglich, den hohen Qualitätsstandard der Daten nach eingehender Prüfung durch geeignete Spezialisten zu erzielen. Sollte in Zukunft eine Netzanbindung nötig sein, erlaubt Access 2000 über Datenzugriffsseiten zumindest eine Integration in ein Intranet, so daß nach entsprechender Anpassung des Programmes einer dezentralen Eingabe nichts im Wege steht.

\subsection{Chronologie Entwicklungsgeschichte von P.A.S.T}

1995 Während des Zeitraumes der Antragstellung des Pilotprojektes (Pilotprojekt Antrag Ri 154/20-1) wurde bereits mit der Planung von P.A.S.T begonnen. Die ersten Entwürfe erfolgten mit Asymetrix Toolbook. Dieses Programm verwaltet die Informationen auf sogenannten Seiten, die über entsprechende Befehlsbuttons durchlaufen werden - vergleichbar mit der html-Struktur von Internetseiten. Es stellte sich jedoch schon nach relativ kurzer Zeit heraus, daß dieses System nicht flexibel genug für P.A.S.T sein würde, zumal mit fortschreitender Planung der Gedanke zur Entwicklung einer digitalen Auswertung von palynologischen Auszähldaten entstand - hierzu aber die seitenorientierte Arbeitsweise von Toolbook nicht geeignet erschien. Deshalb fanden die weiteren Entwürfe auf der Grundlage von Microsoft Office und der universell einsetzbaren Programmiersprache VBA statt (s. ausführliche Darstellung auf Seite 13).

1996 Innerhalb der folgenden 6 Monate wurde die grundlegende Struktur der Datenbank festgelegt. Dabei fanden umfangreiche Testläufe mit Beispieldaten statt, die eine wiederholte Überarbeitung der Datenstruktur zur Folge hatte. Im Rahmen dieser Überarbeitungen wurden insbesondere die Datenfelder zur Morphologie in der Göttinger Arbeitsgruppe diskutiert und in P.A.S.T implementiert. Nachdem der systematische Teil der Datenbank weitestgehend vollendet war, wurde der Entwicklungsschwerpunkt auf die Bildbearbeitung gelegt. In dem folgenden sechsmonatigen Zeitraum liegen die photographischen Neuaufnahmen der Alversdorfer Palynomorphen, das Entwickeln der Bildverarbeitungstechniken und die Programmierung der Routinen zur Bildanzeige und Informationen. Im darauffolgenden Jahr wurden Suchroutinen zur Volltextsuche innerhalb der Datenbank entwickelt, und Schritte unternommen, um systematische Daten in die Tabellenkalkulation zu übertragen. Insbesondere die unzureichend und zum Teil fehlerhaft dokumentierten Schnittstellenroutinen von Access nach Excel stellten während dieser Zeit schwer zu überwindende Hürden dar.

1999 Simultan zur Programmierung der nachfolgend benötigten Auswerte- und Darstellungsroutinen der Auszähldaten innerhalb von Excel fand nun die Eingabe der systematischen Palynomorphen-Daten statt, die durch Bilddateneingaben ergänzt wurden. Auch bei der Programmierung der Pollendiagramm-Darstellungen galt es Schwierigkeiten der VBAProgrammierung zu umgehen, die in ungenügend dimensionierten internen Variablen und in Ungenauigkeiten bei Positionierungen graphischer Elemente in Excel zu finden waren. Um die Vorteile der digitalen 
Verarbeitung besser nutzen zu können, wurde der systematische Teil um Gruppierungsfunktionen auf der Grundlage größtenteils rezenter botanischer und geographischer Parameter erweitert, die eine über die klassische Darstellung hinausgehende Verarbeitung der palynologischen Auszähldaten und botanischer Zugehörigkeiten erlauben sollten. Da für die Wahl der Datenfelder umfangreiche Studien fachübergreifender Literatur zur Geographie und Botanik) nötig war, zog sich dieser Arbeitsschritt ebenfalls über etwa 6 Monate hin. Im folgenden Jahr wurde die Dateneingabe weiter vorangetrieben.

2000 Als außerordentlich zeitaufwendig stellte sich die Eingabe der Gruppierungsparameter heraus, da diese Informationen in der Regel nur verstreut und lückenhaft $\mathrm{zu}$ finden sind und nur schwer in ein datenbankrelevantes Schema zu bringen waren. Der nun noch verbliebene Zeitraum wurde zur Fertigstellung der schriftlichen Dissertation benutzt. Zeitgleich fand eine Überarbeitung der Bedienungsoberfläche von P.A.S.T, eine Fehlerbereinigung und eine Programmierung kleinerer Programmroutinen zur Manipulation der Datenmatrix in Excel statt (Diversitätsuntersuchungen, Verfeinerung der Pollendiagramm-Darstellung, programmgesteuerte Möglichkeiten der Korrektur der Datenmatrix).

2001 Eine Verarbeitung der palynologischen Daten aus Alversdorf im Rahmen einer Veröffentlichung sowie einer Erfassung und Darstellung von Daten zum Fortsetzungsantrag (Prof. H. Walther, Dresden, DFG Fortsetzungsantrag zu Wa 757/9-1, Bearbeiter: Dipl.-Geol. J. Hammer) stellen den ersten Praxistest von P.A.S.T dar. Im Rahmen der dazu nötigen Vorbereitungen und in Hinsicht auf die sprachenübergreifende Nutzung des Programms wurde mittlerweile die Bedienungsoberfläche des Auwerteteils von P.A.S.T (Excel) mehrsprachenfähig umprogrammiert.

\subsection{Datenbankdesign}

Vorbemerkungen zu dem Begriff Objekt:

In den nachfolgenden Unterkapiteln wird der Begriff des Objektes mehrfach erwähnt. Ein Objekt ist im weitesten Sinne ein Gegenstand, der durch einen Satz von Eigenschaften und Methoden beschrieben wird, über die auf das Objekt Einfluß genommen werden kann. Über eine Objekteigenschaft kann der Zustand eines Objektes ermittelt oder verändert werden.

Das Taxon kann demnach auch als Objekt bezeichnet werden, da es durch verschiedene Eigenschaften beschrieben werden kann, ebenso die Elemente von Access wie beispielsweise Tabellen, Formulare oder Steuerelemente.

Bei dem Entity-Relationship-Modell stellt ein Objekt nur eine Zeile der Datenbanktabelle dar, wobei die Zeilen durch die Objekteigenschaften/Felder dargestellt sind.

\subsubsection{Relationales Datenbankmodell}

Access gehört zur Klasse der relationalen Datenbanksysteme, das von CoDD 1970 entwickelt wurde.

Beim relationalen Datenmodell stehen als Strukturelemente ausschließlich Relationen, die sich durch verknüpfte Tabellen darstellen lassen, zur Verfügung. Die Datensätze bilden die Zeilen, und die Merkmale des Objekts entsprechen den Spalten einer 
Tabelle. Beziehungen zwischen beliebigen Datensätzen über verschiedene Tabellen werden über gleiche Feldinhalte hergestellt (in diesem Falle entspricht das Objekt z.B. einem Taxon oder einer Literaturstelle). Der Zugriff auf bestimmte Datensätze wird über die Feldinhalte ermöglicht und kann die Durchsuchung sämtlicher Tabellen erforderlich machen. Dementsprechend arbeitet der Benutzer nur mit logischen, mengenorientierten Abfragen, wobei die physische Speicherung und der Datenzugriff für ihn im Hintergrund bleiben.

\subsubsection{Beziehungstypen}

Wie schon erwähnt, werden verschiedene Tabellen über Referenzfelder miteinander verknüpft. Die Art der Verknüpfung wird über sogenannte Komplexitäten definiert. In der Regel handelt es sich bei den Referenzfeldern um sog. Primärschlüssel, die eine eindeutige Kennzeichnung des Datensatzes erlauben, z.B. eine fortlaufende Nummer.

\subsubsection{1:n Beziehung}

Bei P.A.S.T können z.B. jedem Taxon (1) mehrere Bilder (n) über das Referenzfeld Pollenid zugeordnet werden. Dieser Beziehungstyp wird auch als "Eins-zu-Viele" Beziehung bezeichnet.

Neben der 1:n Beziehung werden noch andere Komplexitäten unterschieden.

\subsubsection{1:1 Beziehung}

Bei der 1:1 Beziehung verknüpft die Datenbank einen Datensatz (1) einer Tabelle mit einem anderen Datensatz (1) einer anderen Tabelle. Diese Komplexität wird eingesetzt, um z.B. personenbezogene sicherheitsrelevante Daten von einer Haupttabelle abzutrennen und mit unterschiedlichen Zugriffsrechten zu versehen oder thematisch sehr unterschiedliche Bereiche mit Hilfe von getrennten Tabellen zu strukturieren und/oder an verschiedenen Orten zu speichern.

\subsubsection{4 n:m Beziehung}

Eine weitere Möglichkeit ist eine Komplexität in Form einer n:m Beziehung. Jedes Taxon kann auf mehrere Literaturstellen (m) verweisen. Umgekehrt finden sich zu einer Literaturstelle vielleicht mehrere Taxa (n).

Die ersten beiden Beziehungstypen lassen sich mit Referenzfeldern aus beiden Tabellen definieren. Um die Struktur einer n:m Beziehung zwischen zwei Tabellen zu erzeugen, benötigt man dagegen eine Zwischentabelle (auch Kreuztabelle genannt), in der die Referenzfelder zu den Primärschlüsseln abgelegt werden. Diese Tabelle kann z.B. den Namen Litertaturdetails tragen und beliebige Kombinationen aus Taxa und Literaturstellen speichern. Ein weiterer Vorteil der Zwischentabelle ist das mögliche Hinzufügen von weiteren Informationen. In dem oben genannten Beispiel sind es Hinweise zur Erstbeschreibung und Seitenangaben der angegebenen Literaturstelle.

\subsection{Vorgehensweise bei der Anwendungsentwicklung}

\subsubsection{Graphische Darstellung der Beziehungen}

Für die Darstellung des konzeptuellen Datenschemas gibt es verschiedene Modelle. Neben der von Access angebotenen Darstellung der Beziehungen bietet sich gerade bei größeren Projekten das Entity-Relationship-Modell an, daß 1976 von CHEN entwickelt wurde. Es beinhaltet die Entitäten (Entity=Gegenstand) und die Beziehungen (Relationship) (Abbildung 2.3). 


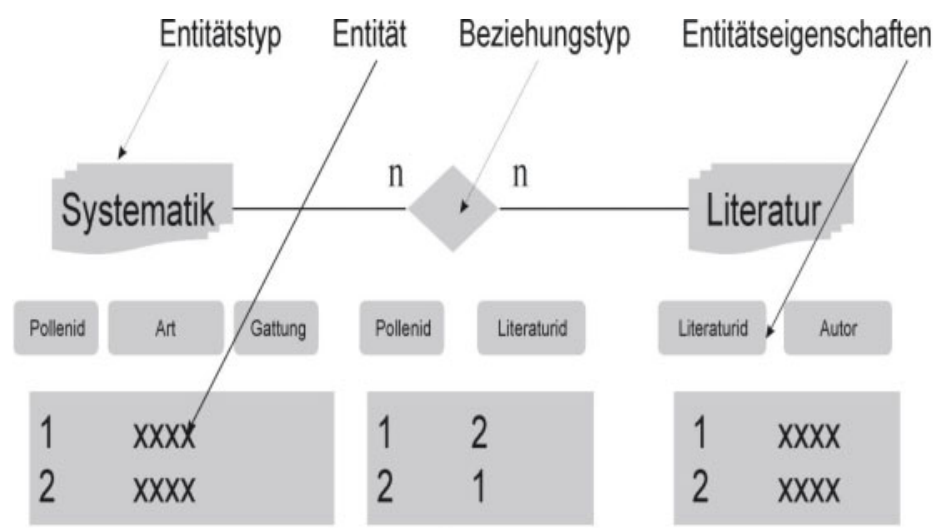

Abb. 2.3: Darstellung der Datenfelder und deren Beziehungen bei der Planung einer Datenbank.

Dieses Verfahren hat sich bei der Entwicklung von P.A.S.T und später bei PORIDAT bewährt und zu relativ kurzen Entwicklungszeiten und weniger Mißverständnissen geführt. Anderseits werden im Rahmen dieser Arbeit die Begriffe Entitäten und Entitätstypen für die Beschreibung von Datenfelder und Tabellen übernommen.

Im nächsten Schritt werden die Objekte des Realitätsausschnittes und ihre Beziehungen unter Berücksichtigung der Normalisierungsregeln definiert. Nach ALPAR 2000 versteht man unter der Normalisierung die Aufspaltung von Relationen in Teilrelationen nach bestimmten Regeln zur Schaffung in sich konsistenter Datenstrukturen.

\subsubsection{Zweck der Normalisierung}

- Vermeidung von Redundanzen, also überflüssige Informationen durch z.B. doppelte Eingaben.

- Vermeidung von Anomalien, die in Zusammenhang mit Datenoperationen auftreten können. Anomalien können bei Lösch- oder Einfüge-Operationen und Modifikationen von Datensätzen auftreten und führen zu Fehlern in der Abbildung der Realität.

- Eindeutiges Modellieren realitätskonformer Sachverhalte.

Die Normalisierung findet in vier Stufen statt, die hier nicht im Einzelnen dargelegt werden. Statt dessen wird auf die Darstellung bei ALPAR 2000 verwiesen. Im Prinzip entsteht bei der Normalisierung der Daten ein Geflecht aus Tabellen, die untereinander in Beziehung stehen und jeweils atomisiert die Eigenschaften eines Objektes beschreiben. Im folgenden wird der Entwicklungszyklus einer Datenbank, exemplarisch für P.A.S.T dargestellt (Abbildung 2.4). 


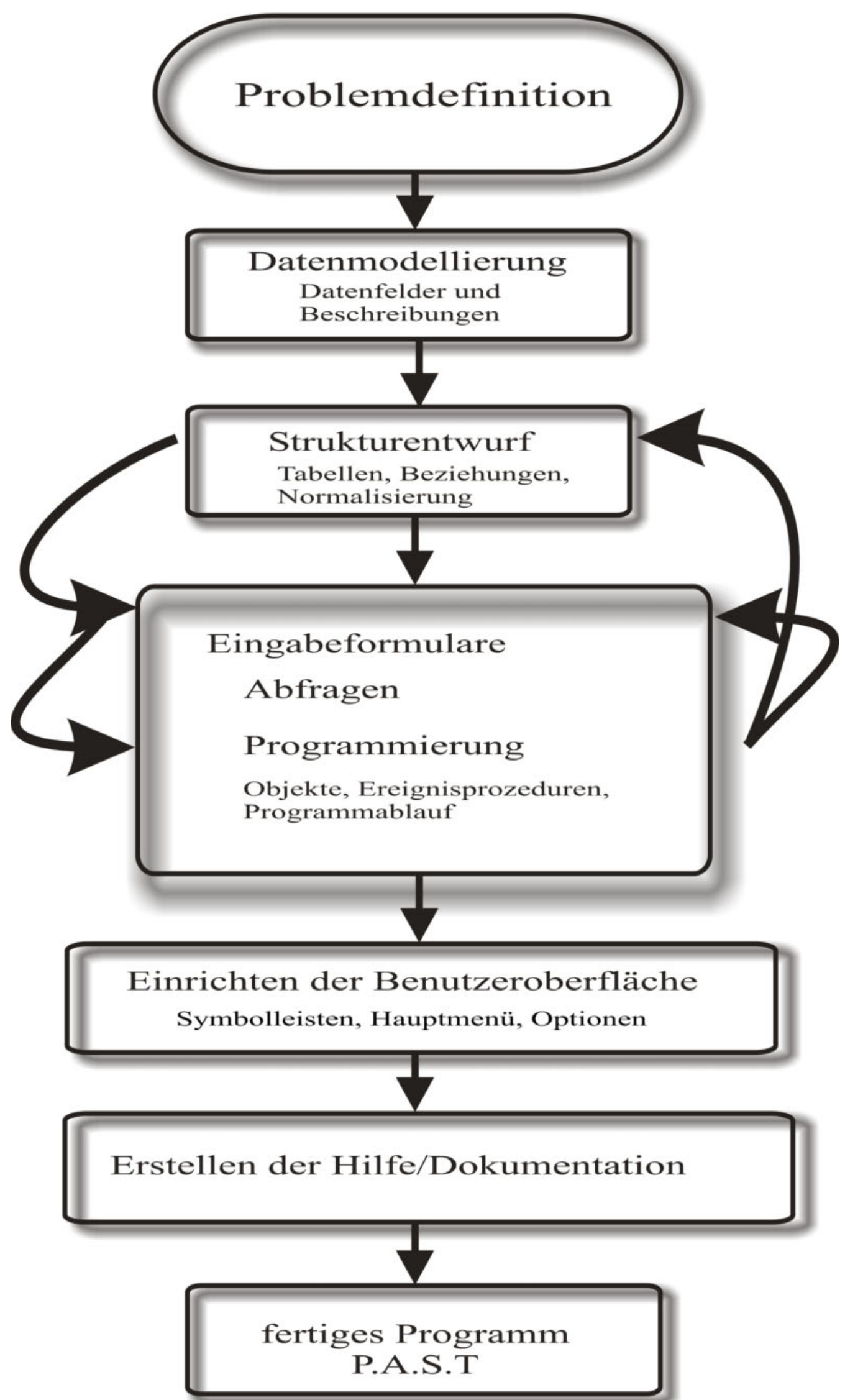

Abb. 2.4: Entwicklung einer Datenbank als Flußdiagramm: Von der Problemdefinition bis zum fertigen Programm. 


\subsection{Datenstruktur von P.A.S.T}

P.A.S.T ist ein Datenbanksystem, das sich aus mehreren Einzeldatenbanken zusammensetzt. Dabei werden die Objekte (hier Formulare, Programmcode etc.) und die Daten in verschiedenen Datenbanken gespeichert. Die Verbindung zwischen der Objektdatenbank und der Datendatenbank entsteht über die eingebundenen Tabellen mit ihren 1:n oder n:m Beziehungen. Mit dieser Aufteilung der Datenbank ist es möglich, die Objektdatenbank zu verändern, ohne den Zugriff auf die Daten zu gefährden. Es ist aus der Objektdatenbank heraus möglich, Verwaltungsoperationen, z.B. Komprimieren von Daten, nur auf die Datendatenbank anzuwenden. Das Trennen von Daten und Objekten bringt noch einen weiteren Vorteil mit sich: Es lassen sich verschiedene Objektdatenbanken, z.B. verschiedener Access-Versionen anlegen, die alle auf den gleichen Datenstamm zugreifen. P.A.S.T verwaltet eine Objektdatenbank und zwei Datendatenbanken. Eine dieser Datendatenbanken speichert nur temporäre speicherintensive Daten, die nach dem Beenden von P.A.S.T wieder freigegeben werden (Abbildung 2.5).

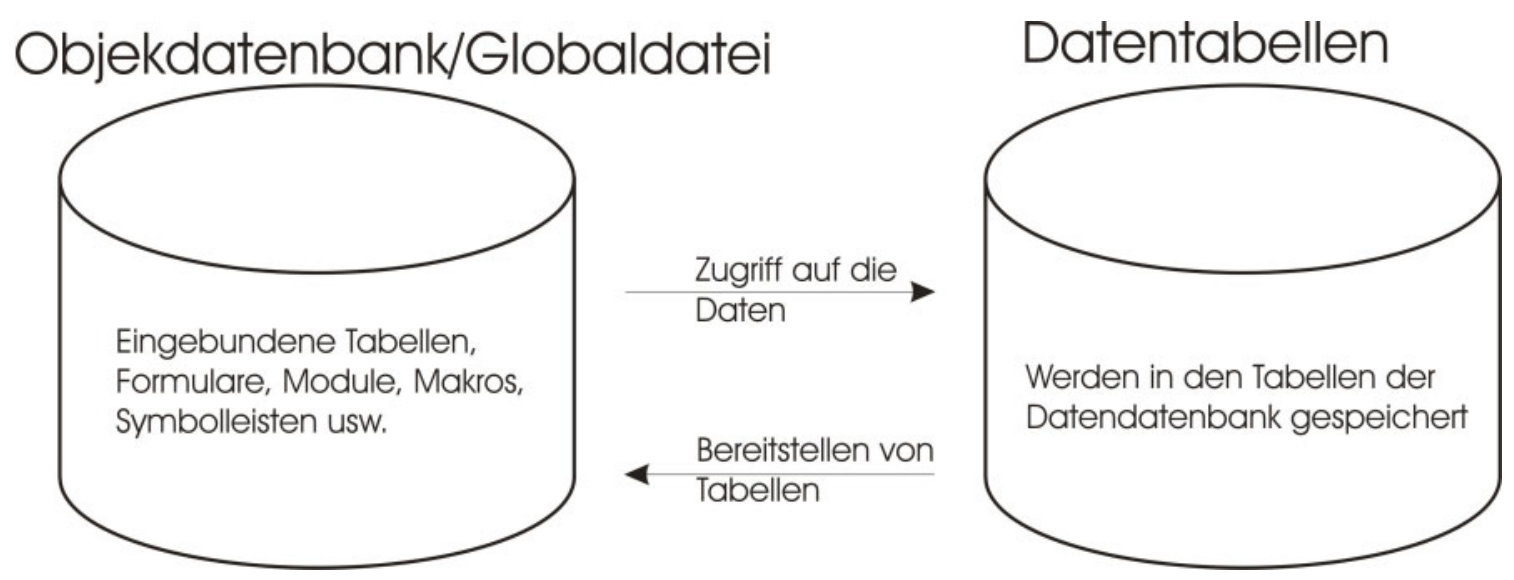

Abb. 2.5: Objekte und Daten werden getrennt verwaltet (verändert nach HoFFBAUER 1999).

\subsection{Tabellen- und Formularstrukturen von P.A.S.T}

Die zentrale Einheit von P.A.S.T stellt die Systematik der Pollen und Sporen dar. Hierarchisch untergeordnet werden weitere Informationen - wie Literatur- oder Bilddaten - dem Taxon zugeordnet. Botanische und geographische Informationen über den nächsten lebenden Verwandten erweitern die Informationsdichte.

In der Systematik werden Lokalitätsdaten benötigt, die Informationen über Fundorte und/oder Typuslokalitäten der Form, den Synonymen oder den Bildern zuordnen. Über diese grundlegenden Informationen geht die Datenstruktur der Lokalitäten über Horizonte und Einheiten in die Tiefe und erreicht letztlich den Bearbeiter eines Profils einer Lokalität. Das Profil wird mit Lithologiedaten makroskopisch beschrieben und anschließend in Excel mit palynologischen Daten erweitert und graphisch ausgegeben.

Die Abbildung 2.6 zeigt schematisch den Zusammenhang der wichtigsten Tabellen und Formulare in P.A.S.T. Detailtabellen, die n:m Beziehungen abbilden und ein Großteil der Tabellen, die Informationen im Rahmen von Kombinationsfeldern liefern, sind nicht dargestellt. 


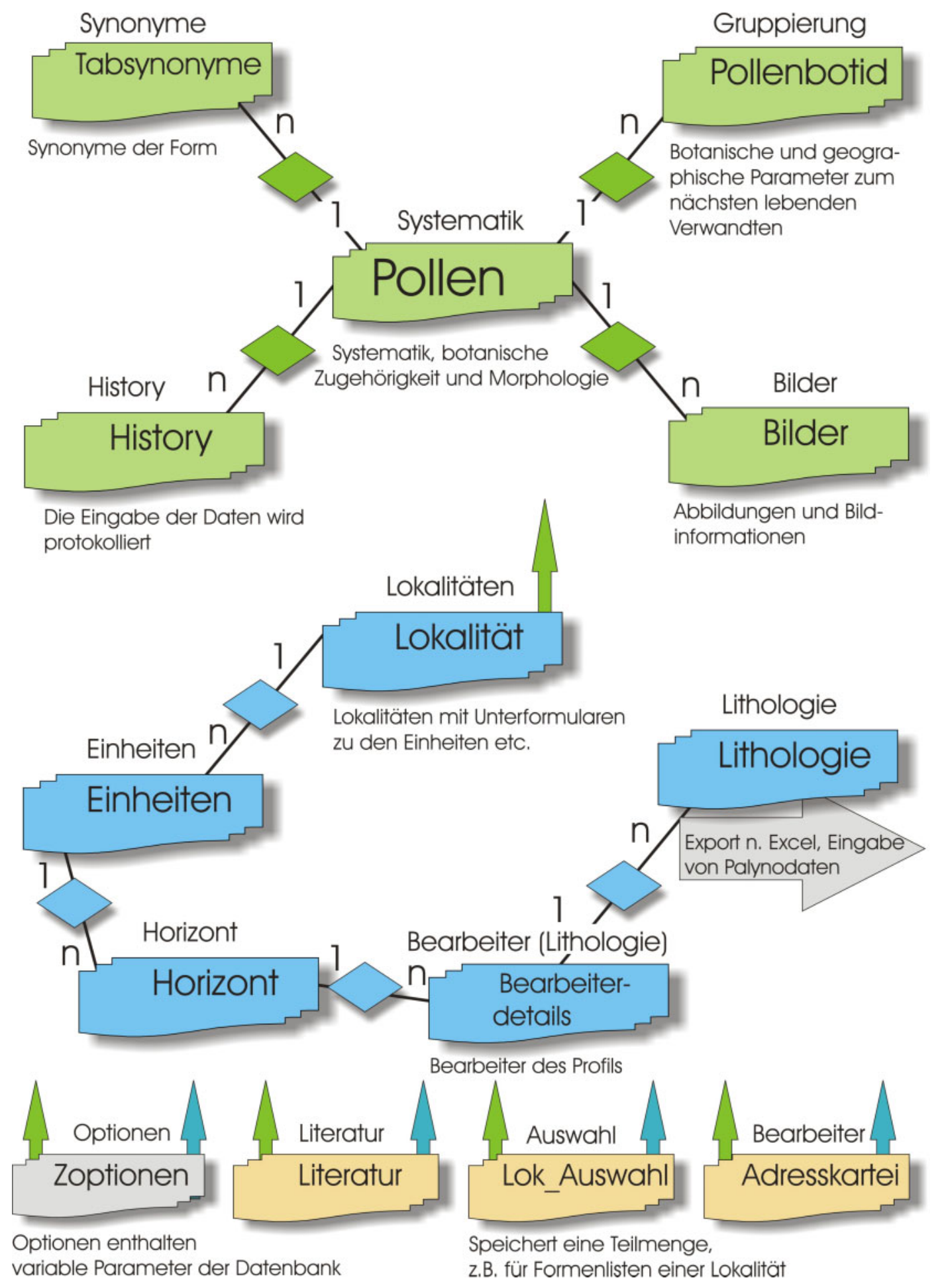

Abb. 2.6: Erkenntlich ist der zentrale Charakter der Systematik mit der Tabelle "Pollen". Über 1:nBeziehungen werden weitere Tabellen mit der Pollentabelle verbunden (über den Tabellensymbolen stehen die Namen der zur Eingabe benötigten Formulare). Lokalitätsdaten speichern lithologische Einheiten, die bis zum Bearbeiter und der Lithologie aufgeschlüsselt werden. Daten, der mit Pfeilen gekennzeichneten Tabellen werden über Kombinationsfelder oder Unterformulare in der Regel in Form von n:m Beziehungen in den gleichfarbigen Formularbereichen abgebildet. So werden z.B. Daten aus der Literaturtabelle im Formular Systematik zur Anzeige des für die stratigraphischen Reichweite zuständigen Autors herangezogen (s. auch "Begriffsdefinitionen" auf Seite 40 und "Eingabe der Systematik" auf Seite 46). Diese Darstellung ist schematisch und zeigt nur einen Bruchteil der in P.A.S.T verwendeten Tabellen und Formulare. 
Im nächsten Schritt wird die Tabellenstruktur von P.A.S.T aufbauend aus der Abbildung 2.6 in einem Beziehungsdiagramm, daß mit Access generiert und erweitert wurde, aufgezeigt. Auch hierbei beschränkt sich die Darstellung auf die wichtigsten Tabellen, die z. T. nur exemplarisch angegeben sind. So sind mit der Systematik (Tabelle „Pollen“) eine ganze Reihe von Tabellen verknüpft, die aus Platzgründen hier nicht dargestellt werden können - und zum Verständnis der Datenstruktur von P.A.S.T nicht zwingend benötigt werden. $\mathrm{Zu}$ den Tabellen sind Datenfelder und Beziehungen zwischen den Tabellen in das Diagramm mit einbezogen (Abbildung 2.7).

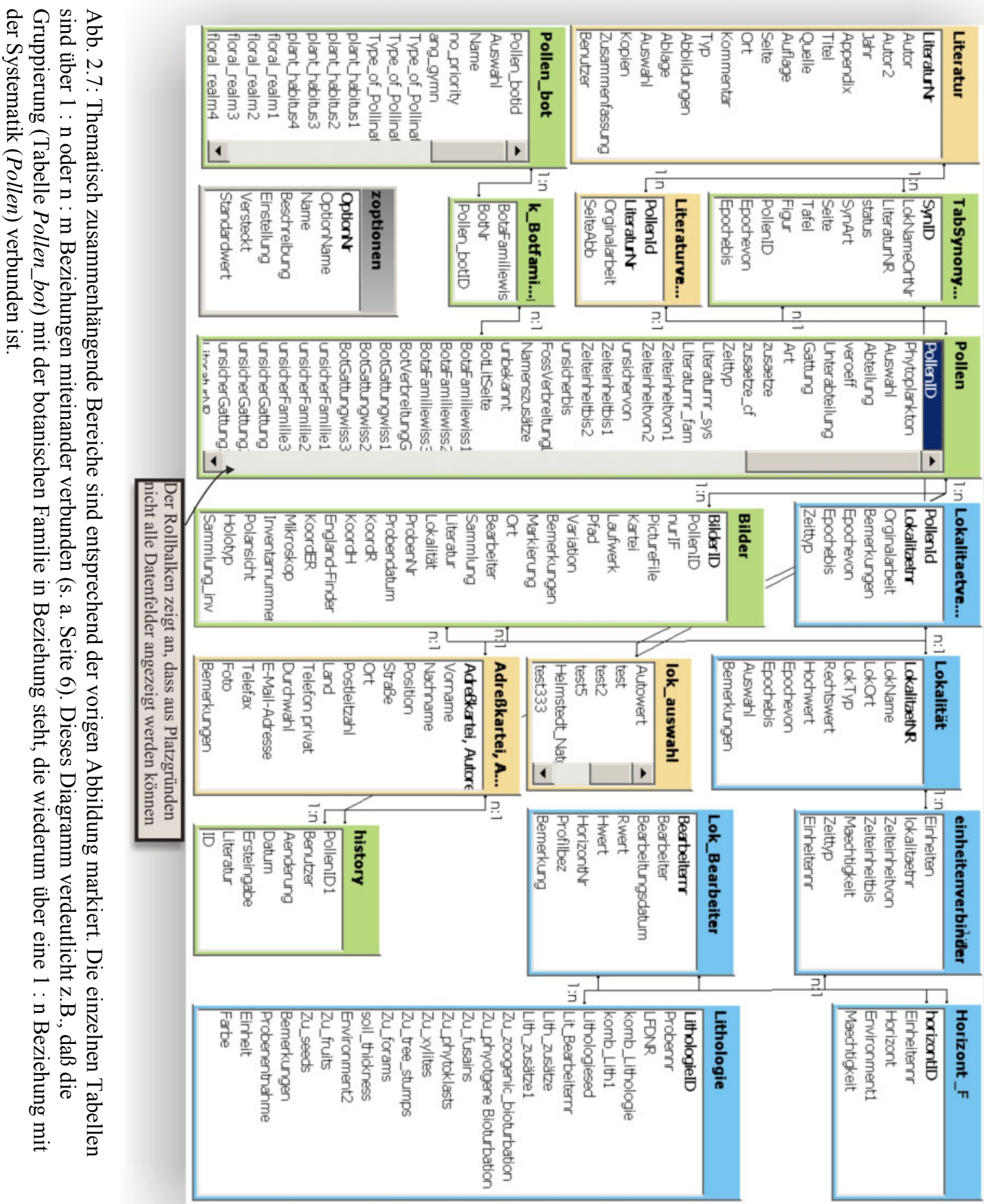




\subsection{Allgemeine Beschreibung der Entitäten von P.A.S.T}

Aufgrund der Komplexität von P.A.S.T ist es nicht möglich, im Rahmen dieser Arbeit sämtliche Datenfelder der Tabellen ausführlich zu beschreiben, da diese Beschreibung ohne Weiteres einen Umfang von ca. 200 Seiten oder mehr erreichen würde (Siehe z.B. Beschreibung der Entitäten der Datenbank ETE (DAMUTH 1993).

Die wesentlichen zur Beschreibung der Realität nötigen Felder und nicht eindeutige Bezeichnungen werden im Rahmen der Eingabe der Daten beschrieben (S. Kap. 3.6, S. 42)

\subsubsection{Systematik}

Die Tabelle „Pollen“ umfaßt die Datenfelder zur Beschreibung der Systematik und der Morphologie mit insgesamt 143 Datenfeldern (Entitäten). Im Folgenden wird die Wahl der Entitäten dieser Tabelle näher erläutert.

\subsubsection{Systematische Beschreibung}

Die systematische Gliederung der Informationen in Art, Gattung, Abteilung, Unterabteilung und stratigraphischer Reichweite wurde den Vorgaben der unten angebenden Literaturstellen entnommen. Die Trennung des Taxonnamens in die Felder Art und Gattung erschien sinnvoll, da häufig auf der Basis dieser Einzelinformationen Filter- und/oder Sortieroperationen durchgeführt werden.

\subsubsection{Morphologische Beschreibungen}

Bei den Entitäten der Morphologie zur Systematik wurde verstärkt die Arbeit von Thomson \& Pflug (1953) über die Pollen und Sporen des mitteleuropäischen Tertiärs herangezogen. Diese Arbeit gibt eine ausführliche Erläuterung der insbesondere in der deutschsprachigen palynologischen Literatur verwandten morphologischen Begriffe. Eine gute Hilfe stellte weiterhin das glossary of pollen and spore (PUNT et al. 2001) dar, das auf das Wesentliche beschränkt, Pollen- und Sporenmorphologische Begriffe definiert (s. auch Kap. 3.7.8.1, S. 52f.)

Um einen Kompromiß zwischen der Genauigkeit der Abbildung der Realität und der praktischen Anwendbarkeit zu erzielen, wurden nachstehende Publikationen auf ihre palynologisch-morphologischen Begriffe durchsucht und die am häufigsten vorkommenden Begriffe nach eingehender Diskussion mit der Göttinger Arbeitsgruppe als Entitäten in P.A.S.T integriert:

ThOMSON \& Pflug (1953); KRUTZSCH (1959, 1962, 1963a,b 1967, 1970, 1971); KRUTZSCH \& VANHOORNE (1977); HAMMER-SCHIEMANN (1998), RIEGEL mündl. Mitteilung.

Daraus entstanden etwa 100 Entitäten, mit denen der größte Teil der vorkommenden Pollen und Sporen morphologisch beschrieben werden kann. Einige Entitäten wurden bewußt allgemeiner gehalten, als dies z.B. mit Hilfe des Glossars von PUNT möglich gewesen wäre. Bei Suchoperationen über eines dieser Felder werden demnach mehrere mögliche Sporomorphae aufgelistet, die das angebende Kriterium erfüllen. Durch das Hinzuziehen weiterer Parameter oder mikrophotographischer Abbildungen läßt sich jedoch weiter eingrenzend vorgehen. Ein Vorteil dieser Methode ist es, auch über ungenaue Angaben zum gewünschten Taxon zu gelangen und Beschreibungs- und Bestimmungs-Ungenauigkeiten in gewissem Rahmen kompensieren zu können. 
Zusätzliche Informationen, die nicht über die vorgegebenen Felder mit P.A.S.T erfaßt werden können, werden über Bemerkungen in Textdarstellung dem Taxon hinzugefügt.

\subsubsection{Synonyme}

(Tabelle "TabSynonyme")

Die Entitäten zur Eingabe der Synonyme entstammen den Vorgaben aus den obigen Literaturstellen. Die interne Datenstruktur von P.A.S.T verwendet die Synonyme zur Generierung vollständiger zitatfähiger Taxonbezeichnungen mit Hilfe der Exportmöglichkeiten von P.A.S.T (S. 77ff).

\subsubsection{Bilder}

(Tabelle "Bilder")

$\mathrm{Zu}$ jeder photographischen Abbildung werden beschreibende Informationen erfaßt, deren Entitäten im Dialog mit Palynologen der Göttinger Arbeitsgruppe entstanden sind. Dazu gehören Daten der mikroskopischen Aufnahme, Sammlungsdaten und Holotyp-Informationen.

\subsubsection{Botanische Zugehörigkeit}

(Tabellen "k_botfamilie", "K_botgattung")

Die Daten des nächsten lebenden Verwandten sind maximal bis auf Gattungsebene aufgeschlüsselt. Es ist jedoch möglich, Artinformationen in einem Bemerkungsfeld anzugeben.

\subsubsection{Gruppierung}

(Tabelle "Pollen_bot")

Die Tabelle der Gruppierung verarbeitet Daten, die sich aus dem nächsten lebenden Verwandten der Sporomorphae ableiten lassen. Dazu zählen sowohl botanische als auch pflanzengeographische Informationen, die letztlich der Rekonstruktion der fossilen Pflanzen-Vergesellschaftung dienen sollen.

Um die Entitäten zur Abbildung der Realität zu erhalten, wurde mit dem Exzerpt der Arbeit von MAI (1995) über die Tertiäre Vegetationsgeschichte Europas begonnen. Diese Arbeit stellt ein umfassendes Werk mit einer Vielzahl von vegetationsrelevanten Daten dar, die es galt, in einen Datenbank-tauglichen Kontext zu bringen. Das nachfolgende Schaubild zeigt das Ergebnis dieser Anstrengungen; dabei sind die Parameter und Entitäten nicht zwingend aus der Arbeit von MAI entnommen worden, sondern wurden durch die letztlich in P.A.S.T verwandten Entitäten ersetzt. Diese Ergänzungen und Veränderungen ergeben sich aus AKIN 1991 und anderen Arbeiten und Mitteilungen (KoEPPEN 1930, TREWARTHA 1968, RitTER 1999 und RIEGEL mündl. Mitteilung). Leider sind in der verfügbaren Literatur nur selten umfassende ökologische Angaben $\mathrm{zu}$ rezenten botanischen Familien und Gattungen $\mathrm{zu}$ finden, deshalb stellte es sich als äußerst schwierig heraus, die Dichte der Datenmatrix auf der einen Seite ausreichend für die zu untersuchenden Fragestellungen zu wählen und andererseits die relevanten Parameter mit Daten füllen zu können.

Ausführliche Daten liegen in erster Linie für europäische und nordamerikanische Familien und Gattungen vor (z.B. ELLENBERG 1979, KRAMER et al. 1990, LINDBACHER 1995, TAKHTAJAN 1997, HEGI 1998). 
Einige Autoren beschränken sich auf die Rekonstruktion der Pflanzen-Assoziationen mit Hilfe klimatischer Daten, die von meteorologischen Stationen innerhalb des Verbreitungsraumes des nächsten lebenden Verwandten quantifiziert werden (z.B. Belz et al. 1994, Mosbrugger 1989, Mosbrugger et al. 1994, 1997). So zeigt MosBrugGer 1993 eine quantitative Rekonstruktion terrestrischer Paläoklimate im Tertiär unter der Annahme, daß tertiäre Pflanzentaxa ähnliche klimatische Bedingungen wie die nächsten lebenden Verwandten benötigten. Die relative Häufigkeit eines Taxons in einer fossilen Flora wird jedoch nicht nur durch paläoklimatische Faktoren und dem Ökosystem bestimmt, sondern auch von zahlreichen taphonomischen Prozessen wie z.B. unterschiedliche Ablagerungsmilieus. Daher wird von MosBRUGGER keine quantitative Auswertung der Häufigkeit eines Taxons durcheführt.

Mit P.A.S.T wird bisher noch keine quantitative Aussage der Klimadaten nach dem Koexistenz-Ansatz von MosBrugGer \& UTESCHER 1993, 1997 durchgeführt, obwohl die Datenmatrix für diese Untersuchungsmethode vorbereitet ist. Kernaussage dieser Methode ist es, mit Hilfe von Klimaparametern ein Koexistenz-Intervall zu ermitteln, in dem die meisten lebenden Verwandten koexistieren können. Diese Implementierung ist in einer weiteren Ausbaustufe problemlos möglich.

\section{Erläuterungen zum Flußdiagramm des nachfolgenden Schaubildes (Abbildung} 2.8)

(Betrachtung von links nach rechts)

Mit P.A.S.T wird nun versucht, weiterführende Informationen über den nächsten lebenden Verwandten zur Verfügung zu stellen. Dazu liefern fossile Taxa Informationen über Verteilungsmuster in Lokalitäten, die als Auszähldaten vorliegen und damit zumindest als relatives Maß für Diversitätsbetrachtungen und taphonomische Untersuchungen herangezogen werden können.

Für die Gattungen und Familien des nächsten lebenden Verwandten wurden über Literaturdaten Merkmale wie Pflanzenhabitus und Pollination erfaßt (aus BENSON 1957, RIEGEL mündl. Mitteilung, HEYWOOD 1992, FUKAREK 1992, 1993, 1994, 1995 , WATSON et al. 1999) die im nächsten Schritt durch Klima, Nährstoffdaten und Substratparameter (aus HEYWOOD 1992, LINDBACHER 1995, RITTER 1999, WATSON et al. 1999 u.a.) erweitert werden können. Sofern bekannt, ergänzen azonale und zonale Vergetationseinheiten die Proxidaten (aus AKIN 1991, TAKHTAJAN 1995, RIEGEL mündl. Mitteilung). Neben den Daten der rezenten botanischen Einheiten, kann für die azonale Vergetationseinheit eine Gliederung von MAI 1995, die auf der Grundlage der Tertiär-Vegetation beruht, ${ }_{2}$ angegeben werden (weitere Literaturangaben: GOTHAN 1954, Hochuli 1978, Thiele-Pfeiffer 1980, Thiele-Pfeiffer 1988, Nickel 1996a, b, diverse Arbeiten von KRUTZSCH u.a.). 


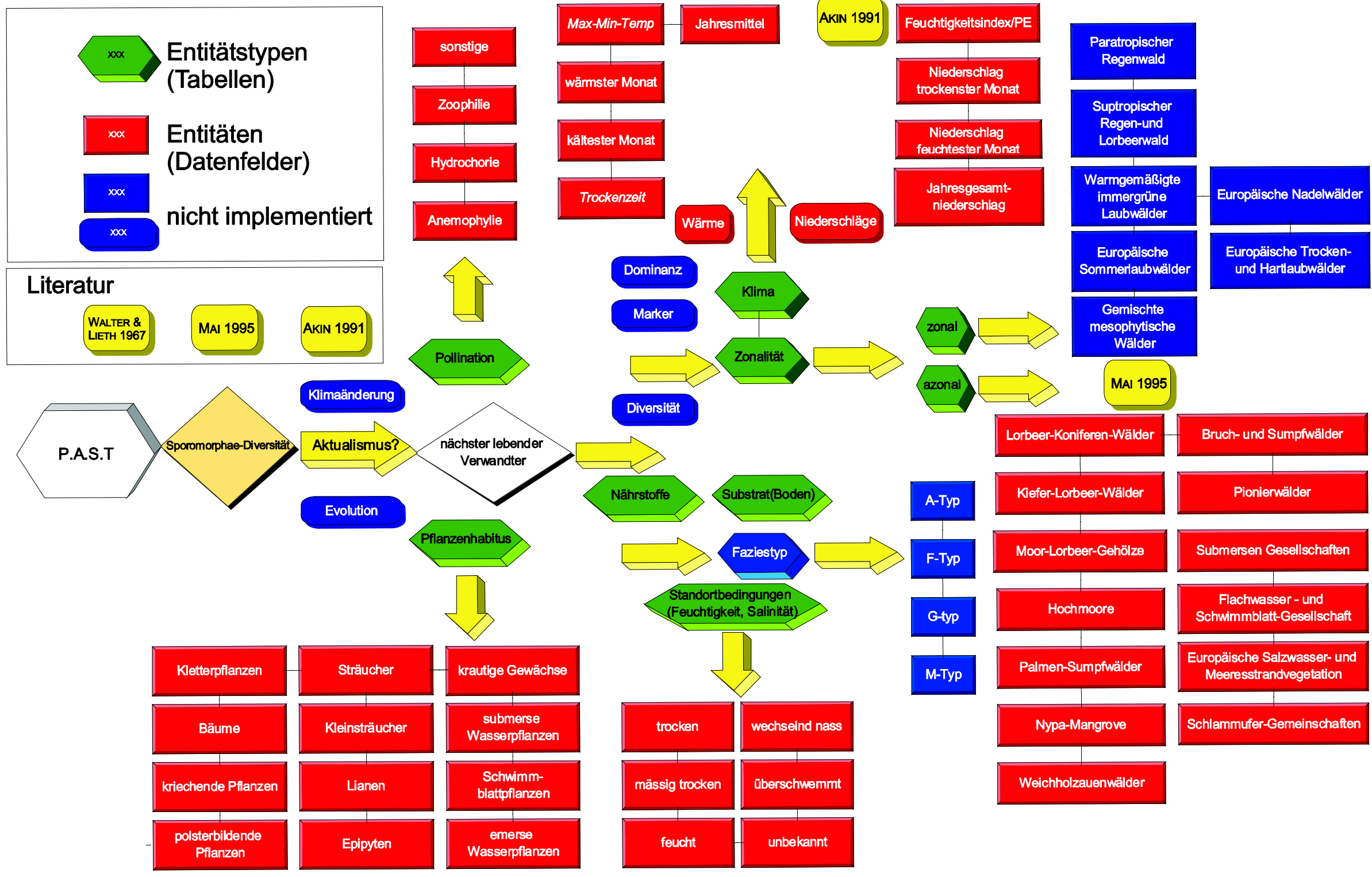

Abb. 2.8: Flußdiaggramm der mit P.A.S.T verarbeiteten Gruppierungsparameter (s. a. Text). Die grünen Felder sind als Entitätstypen (Tabellen) in P.A.S.T integriert. Rot sind die Entitäten angegeben. Eckige blaue Felder (MAI 1995) werden nicht mit P.A.S.T verarbeitet. Abgerundete blaue Felder sind zu Kommentarzwecken eingefügt. Aus Platzgründen ist die rezente azonale und zonale Vegetationseinteilung von P.A.S.T nicht dargestellt. 


\subsubsection{Lokalitäten}

Die grünen Felder sind als Entitätstypen (Tabellen) in P.A.S.T integriert. Rot sind die Entitäten angegeben. Eckige blaue Felder (MAI 1995) werden nicht mit P.A.S.T verarbeitet. Abgerundete blaue Felder sind zu Kommentarzwecken eingefügt. Aus Platzgründen ist die rezente azonale und zonale Vegetationseinteilung von P.A.S.T nicht dargestellt.

Die Entitäten der Lokalitätstabelle umschreiben nur die nötigsten Informationen über Name, Ort und Koordinaten der Lokalität. Erst die Entitäten der Tabellen der Unterformulare "Horizonte", "Einheiten" und "Bearbeiter" eines Profils einer Lokalität lassen Spielraum für Variationen in der Wahl der Entitäten. Um eventuelle spätere Implementierungen weiterführender Auswertungen zu erleichtern, wurden neben dem Namen der Einheiten und Horizonte Angaben wie stratigraphische Reichweite, Mächtigkeit und Informationen über das Ablagerungsmilieu (Horizonte) erfaßt.

Die Entitäten des dem Bearbeiter zugeordneten Profils mit Hilfe der Lithologietabelle umfaßt die makroskopisch erfaßbaren Daten. Die Auswahl der dazu nötigen Entitäten ist einer Reihe von Profilen aus Arbeiten der Göttinger Arbeitsgruppe entnommen und auf die Wesentlichen reduziert in P.A.S.T integriert worden. Nachfolgend eine Übersicht der Anzahl der verwendeten Tabellen, Formulare und Programmzeilen in P.A.S.T (Abbildung 2.9).

\begin{tabular}{|l|l|l|l|l|l|}
\hline Access & $\begin{array}{l}\text { Tabellen } \\
\text { Tabellen } \\
\text { (Formulare) } \\
\text { (Kombinationsfelder } \\
\text { etc.) }\end{array}$ & Formulare & Unterformulare & Abfragen & $\begin{array}{l}\text { Programmzeilen } \\
\text { (ohne } \\
\text { Kommentare) }\end{array}$ \\
\hline 31 & 130 & 46 & 35 & 100 & $\begin{array}{l}\text { ca. 27000 (ca. } 600 \\
\text { Seiten) }\end{array}$ \\
\hline Excel & 1 (Mustertabelle) & 3 & 12 Seiten & - & $\begin{array}{l}\text { Ca. 16000 (ca. } 400 \\
\text { Seiten) }\end{array}$ \\
\hline-
\end{tabular}

Abb. 2.9: Anzahl der Tabellen, Formulare und Programmzeilen von P.A.S.T.

\subsection{Bilddokumentation}

Obwohl die Bilddokumentation bei P.A.S.T nur einen kleinen Baustein bei der Gesamtbeschreibung der abzubildenden Realität bildet, stellt sie aufgrund der hohen Anforderungen an den Speicherbedarf und der Geschwindigkeitsoptimierung bei der Anzeige der Bilder ein gesondert darzustellenden Problem dar.

Der größte Zeitaufwand der Bilddokumentation entsteht bei der Akquisition von optimalen Aufnahmen von Typen oder Typenmaterial. Daher beschränkt sich die Bilddokumentation von P.A.S.T - aufgrund des Mangels an Hilfskräften - bisher auf Formen, deren Bildmaterial leicht zugänglich ist. Aus der umfangreichen Diplomarbeit von NATGE-EFOGHE 1997 wurde das vorliegende Material des Mitteleozäns der Helmstedter Oberflözgruppe photografisch neu verarbeitet.

Die Komplettierung der Bilddokumentation kann allerdings problemlos in einem zweiten Schritt, eventuell in Verbindung mit taxonomischen Revisionsarbeiten im Rahmen der Weiterführung von P.A.S.T erfolgen. 
Die in mehreren Schärfeebenen und wechselnder Kontrasteinstellung neu photographierten Formen wurden anschließend über einen kommerziellen Photo-CDTransfer-Service auf Photo-CD gescannt und gespeichert. Dieser auf dem ersten Blick umständliche Weg gegenüber dem Erfassen der Bilder über einen Haus-eigenen Diascanner ergab zum Zeitpunkt der Bearbeitung (1997) im direkten Vergleich eine deutlich bessere Qualität in der Auflösung und im Detailreichtum. Selbst hochwertige Digitalphoto-Einrichtungen lieferten nicht die gleiche Qualität und Auflösung - die Unterschiede werden jedoch mit jeder Generation der Digitalphotographie geringer, so daß die zukünftige Methode zur Einbindung von Photographien in die Datenbank aufgrund der sofortigen Verfügbarkeit des Bildmaterials und der Vielzahl an Möglichkeiten, z.B. im Bereich der 3-D-Photographie, die Digitalphotographie sein wird.

\subsubsection{Formate der Kodak-Photo-CD}

Die Rohbilder werden im Kodak-eigenen YCC-Farbmodell auf der CD abgelegt (Luminanz, Chrominanz Grün/Rot und Blau/Gelb). Das bislang proprietäre Verfahren zur Komprimierung wurde mittlerweile (seit Dezember 1998) von Kodak im Rahmen eines Toolkits (C-Library) freigegeben. Jedes Bild liegt in fünf unterschiedlichen Auflösungen vor:

Von einer kleinen Vorschau $(128 \times 192$ Pixel $)$ bis zu einem für eine hohe Ausgabequalität vorgesehenen Bild $(2048 \times 3072$ Pixel). Eine Liste aller möglichen Bildgrößen zeigt die Abbildung 2.10:

\begin{tabular}{|l|l|l|l|l|}
\hline Typ & Bezeichnung & Dateigröße & Auflösung & \multirow{2}{*}{ Bemerkung } \\
\hline Base/16 & Thumbnail & $19 \mathrm{kB}$ & $128 \times 192$ & \\
\cline { 1 - 4 } Base/4 & Preview & $74 \mathrm{kB}$ & $256 \times 384$ & \\
\cline { 1 - 4 } Base & VGA & $290 \mathrm{kB}$ & $512 \times 768$ & \\
\hline 4 Base & HDTV & $1.200 \mathrm{kB}$ & $1024 \times 1536$ & Komprimierung \\
\hline 16 Base & Photo & $4.800 \mathrm{kB}$ & $2048 \times 3072$ & Komprimierung \\
\hline
\end{tabular}

Abb. 2.10: Die verschiedenen Typen und Auflösungen der Photo-CD.

Für die Darstellung in P.A.S.T wurde der maximal auflösende Typ 16 Base gewählt und als Ausschnitt von 1200*1000 Punkten - bei übergroßen Sporen bis 1900*1600 Punkten - mit Adobe Photoshop weiterverarbeitet.

Die digitale Bildverarbeitung umfaßte Korrekturen der Farbbalance, Farbton, Sättigung, Lab-Helligkeit, Tonwerte sowie Schärfe und wird in tabellarischer Form für jedes manipulierte Bild festgehalten (Abbildung 2.11). Anschließend wird das Bild im JPG-Format (Qualität "high", Stufe 7) ca. um den Faktor 5-10 komprimiert. Neben dem komprimierten Bild wird ein verlustfrei komprimiertes Tiff-Bild zur eventuellen späteren Weiterverarbeitung auf CD-ROM archiviert. 


\begin{tabular}{|c|c|c|c|c|c|c|c|c|c|c|c|c|c|c|c|c|}
\hline \multicolumn{2}{|c|}{ Film Nr. 1} & \multicolumn{3}{|c|}{ Farbbalance } & \multicolumn{3}{|c|}{ Farbe ersetzen } & \multicolumn{3}{|c|}{ Tonwert } & \multicolumn{2}{|c|}{ Unschärfe } & \multicolumn{2}{|c|}{ Staub/Kratzer } & \multirow{2}{*}{$\begin{array}{l}\text { JPG } \\
\text { Qualität }\end{array}$} & \multirow[t]{2}{*}{ sonstiges } \\
\hline Nr. & Dateiname & $\mathrm{R}$ & $\bar{G}$ & $\mathrm{~B}$ & Farbt & Sättig & Lab & $\mathrm{L}$ & $\bar{M}$ & $\mathrm{R}$ & $\begin{array}{l}\text { Unsc } \\
\mathrm{h}\end{array}$ & $\begin{array}{l}\mathrm{Ra} \\
\mathrm{d}\end{array}$ & $\overline{\operatorname{Rad}}$ & wischen & & \\
\hline \begin{tabular}{|l|}
$1 \_1$ \\
\end{tabular} & $\begin{array}{l}\text { Spinizonocolp. } \\
\text { baculatus }\end{array}$ & & & -30 & & -5 & & & 0,75 & & 150 & 3 & 1 & & hoch & $\begin{array}{l}\text { Kratzer selektiv } \\
\text { entfernt }\end{array}$ \\
\hline 12 & $\begin{array}{l}\text { Spinizonocolp. } \\
\text { baculatus }\end{array}$ & & & -45 & & -5 & & & 0,78 & & 150 & 3 & 1 & & hoch & "" \\
\hline 113 & $\begin{array}{l}\text { Spinizonocolp. } \\
\text { baculatus }\end{array}$ & & & & & & & & 0,93 & & 150 & 3 & 2 & wenig & hoch & \\
\hline
\end{tabular}

Abb. 2.11: Dokumentation der Photoshop-Manipulationen zur Bildverbesserung der Photo-CDRohbilder.

Vergleichende subjektive Tests haben gezeigt, daß die Verlust-behaftete Komprimierung auf die Qualität der Bilder keinen nennenswerten Einfluß hat, da bei der eingesetzten JPG-Komprimierung, insbesondere bei hoher Qualitätseinstellung, in erster Linie nur Veränderungen an den Farbinformationen vorgenommen werden, die für das menschliche Auge kaum wahrnehmbar sind. Durch diese Komprimierung ist es möglich, etwa 2000 - 3000 Bilder auf eine Standard-CD-ROM (ca. 650 MByte) zu speichern.

Um die Bilder innerhalb der Datenbank darzustellen, bieten sich zwei grundsätzlich verschiedene Methoden an. Zum ersten könnten die Bilddaten direkt in die Datenbank eingebunden werden. Wie schon PRAHL 1994 bemerkte, wäre es dabei notwendig, die Farbtiefe und die Auflösung zu reduzieren, damit der Wechsel von einem Datensatz zum nächsten durch die große Datenmenge nicht unnötig verzögert würde. Zudem wäre der Speicherbedarf der Datenbank durch das interne Abspeichern der Bilder sehr hoch.

Die zweite in P.A.S.T verwandte Variante speichert nicht die Bildinformation, sondern nur den Dateinamen des Bildes und baut ein entsprechendes Bildfenster dynamisch auf. Leider unterstützt Access diese Methode nur sehr eingeschränkt. Deshalb wurde die Funktion unter Einsatz des Nedimage-Steuerelementes (Demoversion im Internet unter http://www.frontiernet.net/ ned1/INFO.html) programmiert, das gegenüber dem Picture-Element von Access Vorteile in der Anzeigegeschwindigkeit und Skalierbarkeit zeigt.

Anschließend wird ein verkürzter Teil dieser Programmierung dokumentiert (Abbildung 2.12). 


\section{'Aus den Optionen werden die Pfade der einzelnen Speicherorte extrahiert \\ 'und mit den Dateinamen aus dem Listfeld Picturefile kombiniert \\ 'der gesamte Pfad des Bildes wird dann in das Steuerelement Nedimage eingetragen \\ 'Pfad aus der Optionstabelle über die Funktion getoption ermitteln \\ dirf1 = GetOption("dirft") ' Speicherort auf der Festplatte (Ordner) \\ dircd1 = GetOption("dircdt") ' Speicherort auf der CD (Ordner)}

'Ein SQL-String, der eine Abfrage aus dem Formular Abbildungen darstellt, (Q) wird in ein Recordset, 'das die Datensätze enthält, übertragen und im nächsten Schritt weiterverarbeitet

Set R $=$ Q.OpenRecordset()

'Beginn der Schleife durch die Zeilen - also Datensätze - der Abfrage

Do While R.EOF $=$ False ' Die Schleife wird bis zum Ende abgearbeitet

pict $=$ R.fields! Picturefile 'der Bildname ohne Pfad

'je nach Ordnerangabe im Feld Laufwerk wird der Pfad aus dem Bildname und dem Ordner zusammengesetzt

Select Case R.fields!Laufwerk

Case 1

$$
\text { Pfad }=\text { dirf1 \& "।" \& pict 'setzt den Pfad zusammen }
$$

Case 2

$$
\text { Pfad }=\operatorname{dircd} 1 \& " \mid " \& \text { pict }
$$

End Select

'Die einzelnen Pfade der Bilder werden in ein Array übertragen

$i=i+1$

ReDim Preserve Pictarray(1 To i)

Pictarray $(\mathrm{i})=\mathrm{pfad}$

R.MoveNext 'zum nächsten Datensatz springen

Loop 'Ende der Schleife

$\mathrm{I}=0$

'wird das Array ausgelesen: Die einzelnen Pfade werden den Nedimage-Steuerelementen zugeordnet, 'die in „Meineauflistung“ festgelegt wurden

For Each steuerelement In meineauflistung

steuerelement.Picture $=$ Pictarray (i)

$\mathrm{i}=\mathrm{i}+1$

Next

Abb. 2.12: Stark vereinfachtes Programm (39 Zeilen) der 214 Zeilen umfassenden Subroutinen „thumbsname“ und „thumbsfüllen“ aus dem Modul „Bilder“ von P.A.S.T. Es zeigt die Programmierung des Nedimage-Steuerelementes, das z.B. für die Darstellung der Bilder des Formulars Abbildungen zuständig ist.

Eine besonders schnelle Anzeige einer Übersicht der Abbildungen wurde erreicht, indem zusätzlich zur hochauflösenden Variante die Bilder im verkleinerten Format 250 * 200 Pixel gespeichert wurden. Dieser Vorgang läßt sich mit Photoshop (ab Version 5) unter Benutzung der Automatisierungs- und Stapelverarbeitungs-Funktion ausführen. Zur Reduzierung der Darstellungsverluste bei der Verkleinerung ist in diesem Arbeitsvorgang eine Schärfeverbesserung (Unschärfe maskieren) sinnvoll. Die 
Möglichkeit der Automatisierung kann auch zur Komprimierung einer Vielzahl von Bildern in einem Durchgang und zum Hinzufügen eines Bildmaßstabes genutzt werden (Abbildung 2.13).

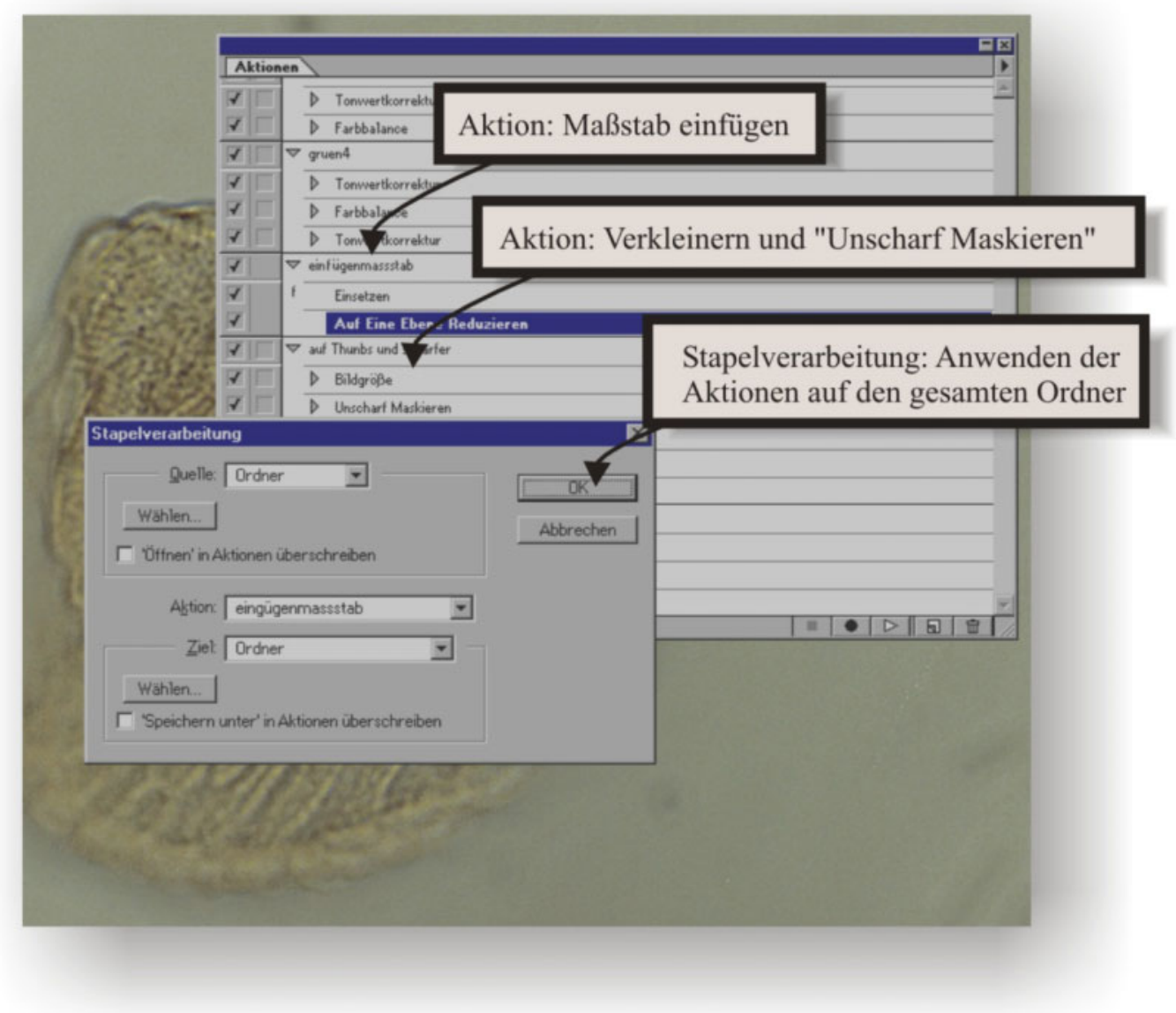

Abb. 2.13: Mit Photoshop lassen sich Operationen automatisieren, wobei Aktionen die Aufgaben definieren. Die Stapelverarbeitung führt Aktionen über mehrere Dateien der Reihe nach aus.

Damit eine Bearbeitung und Ansicht der Bilder auch ohne P.A.S.T möglich ist, werden die Bilder soweit als möglich mit sinnvollen Namen versehen. Folgendes Beispiel soll dies verdeutlichen:

Aceripollenites_microrugulatus_Natge_Hel_Alv_laIF.jpg

Aceripollenites_microrugulatus_Natge_Hel_Alv_lbIF.jpg

Aceripollenites_microrugulatus_Natge_Hel_Alv_2aIF.jpg

Aceripollenites_microrugulatus_Natge_Hel_Alv_2bIF.jpg

Aceripollenites_microrugulatus_Natge_Hel_Alv_la.jpg

Aceripollenites_microrugulatus_Natge_Hel_Alv_lb.jpg

Der Artname wird mit dem Gattungsnamen kombiniert; danach folgt der abgekürzte Bearbeitername (Natge). Anschließend ersetzt ein Kürzel die Lokalitätsbezeichnung (z.B. Hel $=$ Helmstedt, Alv = Alversdorf), das von der Variation (verschiedene Eemplare eines Taxons einer Lokalität), über eine Ziffer angegeben, gefolgt ist (1, 2....). Der danach folgende Buchstabe steht für die Abbildungsebene, wobei maximal vier Ebenen erlaubt sind (a-d). Vor der Dateityp-Bezeichnung (JPG) steht der Kontrastmodus des Mikroskops (normal = keine Angabe, IF für Interferenz-Kontrast). 
Die Bilder der beiden Auflösungsstufen werden in unterschiedlichen Ordnern gespeichert (die Namen sind identisch), deren Pfad in dem Optionsformular angegeben ist (Die einzelnen Namens-Elemente werden über den Unterstrich miteinander verbunden, da Leerzeichen im Namen bei einigen Anzeige- und Konvertierungsprogrammen zu Problemen führen können.

\subsubsection{Speicherung der Daten}

Die verschiedenen Zwischenstadien der Bilder werden in der Regel auf CD-ROM gespeichert. Als Dateisystem kommt nur die Joliet-Erweiterung in Betracht, da sie im Gegensatz zum Plattform-übergreifenden ISO-9660 (Level 1) lange Dateinamen immerhin bis zu einer Länge von 64 Zeichen unterstützt. Da Windows ab Version 95 eine Dateilänge von 255 Zeichen ermöglicht, ist darauf zu achten, daß die Dateinamen 64 Zeichen nicht überschreiten, ansonsten werden beim Archivieren der Daten auf CD die zu langen Dateinamen abgeschnitten.

\subsubsection{Entwicklungsumgebung}

Als Entwicklungsumgebung diente zu Beginn der Arbeiten ein Rechner mit einem Intel-486-Prozessor, der unter Windows95 mit Access 2.0 betrieben wurde. Im Rahmen eines Betriebssystemwechsels auf NT4 und der daraus bedingten Hardwareaufrüstung wurde P.A.S.T auf Access97 umgestellt. Die letzten Programmierschritte fanden auf einem Athlon-700-basierenden Rechner statt, auf dem Office 97 und Office 2000 parallel betrieben wurde, um die Migration auf Access $2000 \mathrm{zu}$ erleichtern. Die unter Windows NT betriebene Workstation ist mit einem NT-Server vernetzt, der zur Spiegelung der Daten über ein Software-basierendes RAID-System verfügt, das von einem nach dem Grandfather-Father-Son (GFS)-arbeitenden Backup unterstützt wird. Damit ist ein ausreichender Schutz gegen Datenverlust durch Hardwareschäden oder Computerviren erreicht.

\subsubsection{Statistik}

Neben der durch P.A.S.T geschaffenen Möglichkeit, die Auszähldaten der Pollen und Sporen programmgesteuert als Diagramm darzustellen sind auch statistische Methoden angewandt bzw. programmiert worden.

Statistische Methoden haben den Vorteil, die zu beurteilende Datenmenge auf ein Minimum zu reduzieren, gleichzeitig aber wichtige Trends hervorzuheben. Durch die Vereinfachung der Daten wird eine Interpretation der palynologischen Daten vereinfacht oder eröffnet neue Ansätze, quantifzierte Aussagen über bestimme Fragestellungen zu treffen.

Folgende statistische Analysen sind mit P.A.S.T realisiert:

- Minimal- und Maximalwerte der Datenmatrix (Kap. 3.11, S. 83)

- Häufigkeitsklassenbildung (Kap. 3.11.8, S. 100)

- Clusteranalyse (Kap. 3.11.8, S. 100)

- Graphische Darstellung der Distanzen nach verschiedenen Verfahren zwischen aufeinanderfolgenden Proben (Kap. 3.11.8, S. 100)

- Diversität (Kap. 3.11.5, S. 96)

Die mathematischen Grundlagen der in P.A.S.T programmierten statistischen Methoden wurden aus den Arbeiten von BACHER 1996, BIRKS 1985, ClAus et al. 1995 und RocK 1988 extrahiert. 


\subsection{Besonderheiten der VBA-Programmierung in Excel}

Die Programmierung der Darstellungs- und Berechnungsroutinen in Excel konnte z.T. nur mit besonderen Methoden durchgeführt werden, da die herkömmlichen Verfahren zum einen nicht die nötige Ablaufgeschwindigkeit der Programmroutinen und zum anderen nicht die graphische Positionierungs-Genauigkeit bei der Darstellung der Pollendiagramme und Profile erzielen. Im Folgenden werden einige der modifizierten Methoden beschrieben.

\subsubsection{Matrizen}

Normalerweise werden Berechnungen innerhalb eines Arbeitsblattes in Excel über sogenannte Ranges durchgeführt. Ein Range stellt definitionsgemäß eine Zelle, eine Zeile, eine Spalte, eine Auswahl von Zellen aus einem oder mehreren zusammenhängenden Zellblöcken oder einen 3D-Bereich dar. Um Berechnungen innerhalb von Ranges durchzuführen, wird in der Exceldokumentation folgender Programmablauf empfohlen.

'Deklaration der Variablen.....

Dim zelle as range

'für jede Zelle im Arbeitsblatt Tabelle1 und dem Bereich von A1 bis D10...

For Each zelle in Worksheets("Tabelle1").Range("A1:A10")

'wenn der Wert der Zelle kleiner als 0,001 ist....

If zelle.Value $<0.001$ Then

'setzte den Wert der Zelle auf $0 . .$.

zelle. Value $=0$

End If

Next zelle

Abb. 2.14: Ein Beispiel für die „Rangemethode“.

Für umfangreiche Berechnungen zur Clusteranalyse ist diese Art der Programmierung jedoch ungeeignet, da die Ablaufgeschwindigkeit sehr niedrig ist. Das nächste Beispiel liefert das selbe Ergebnis - bei umfangreichen Datentabellen jedoch etwa um den Faktor 1000 schneller.

Die hohe Geschwindigkeit wird erreicht, indem ein Range vor der Berechnung in eine Matrize (Array) übertragen wird. Anschließend werden die Arrayelemente manipuliert; das veränderte Array wird anschließend wieder in ein Range übertragen und steht somit wieder für weitere Manipulationen in dem Arbeitsblatt zur Verfügung (Abbildung 2.15).

Da bestimmte Operationen nur auf Rangeelemente anwendbar sind (z.B. Änderungen der Zellenfarbe in Abhängigkeit des Zellenwertes), ist es jedoch nicht immer möglich, 
die Daten in ein Array zu überführen. In diesen Fällen wird weiterhin die „Rangemethode" angewandt.

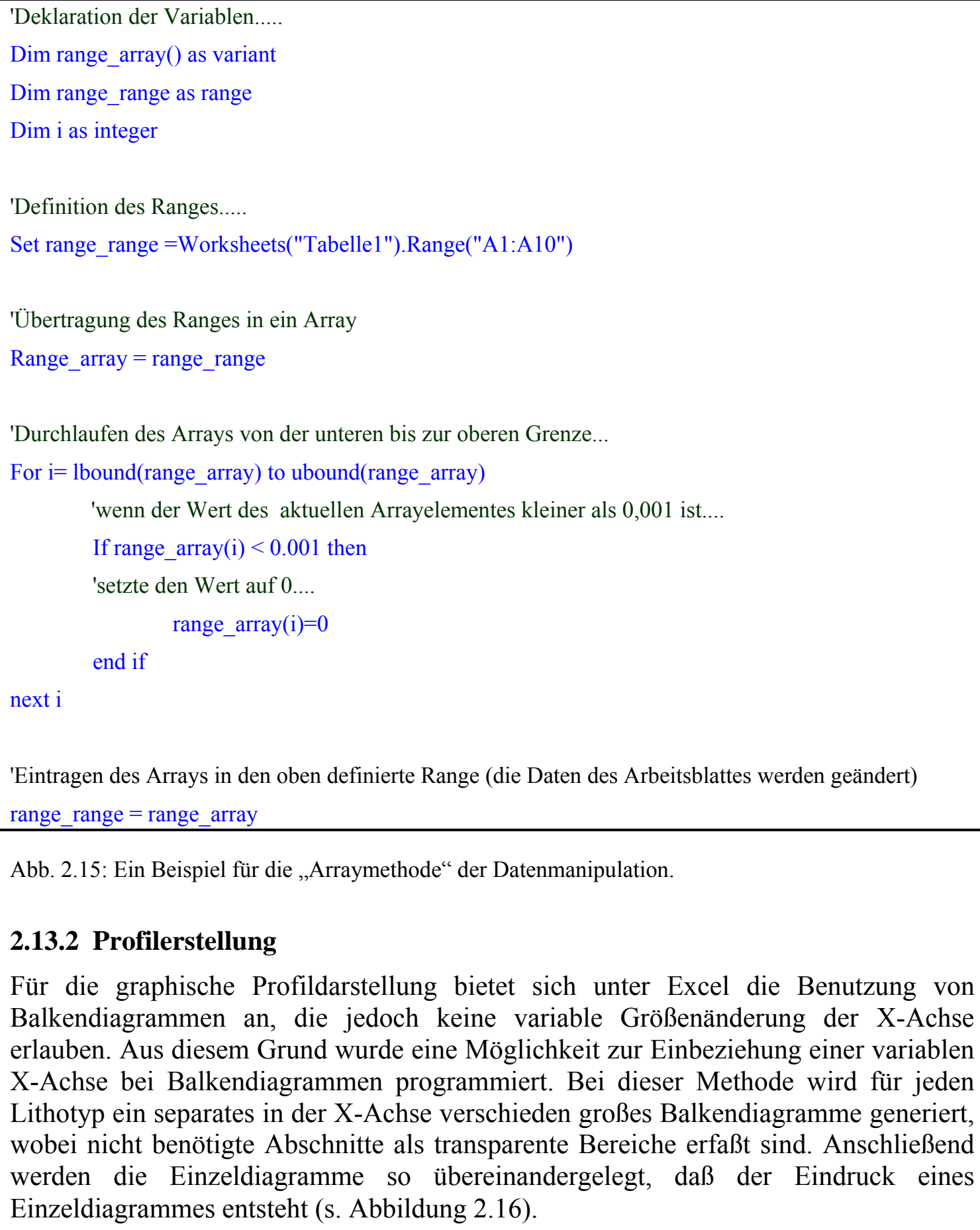

Abb. 2.15: Ein Beispiel für die „Arraymethode“ der Datenmanipulation.

\subsubsection{Profilerstellung}

Für die graphische Profildarstellung bietet sich unter Excel die Benutzung von Balkendiagrammen an, die jedoch keine variable Größenänderung der X-Achse erlauben. Aus diesem Grund wurde eine Möglichkeit zur Einbeziehung einer variablen X-Achse bei Balkendiagrammen programmiert. Bei dieser Methode wird für jeden Lithotyp ein separates in der X-Achse verschieden großes Balkendiagramme generiert, wobei nicht benötigte Abschnitte als transparente Bereiche erfaßt sind. Anschließend werden die Einzeldiagramme so übereinandergelegt, daß der Eindruck eines Einzeldiagrammes entsteht (s. Abbildung 2.16). 


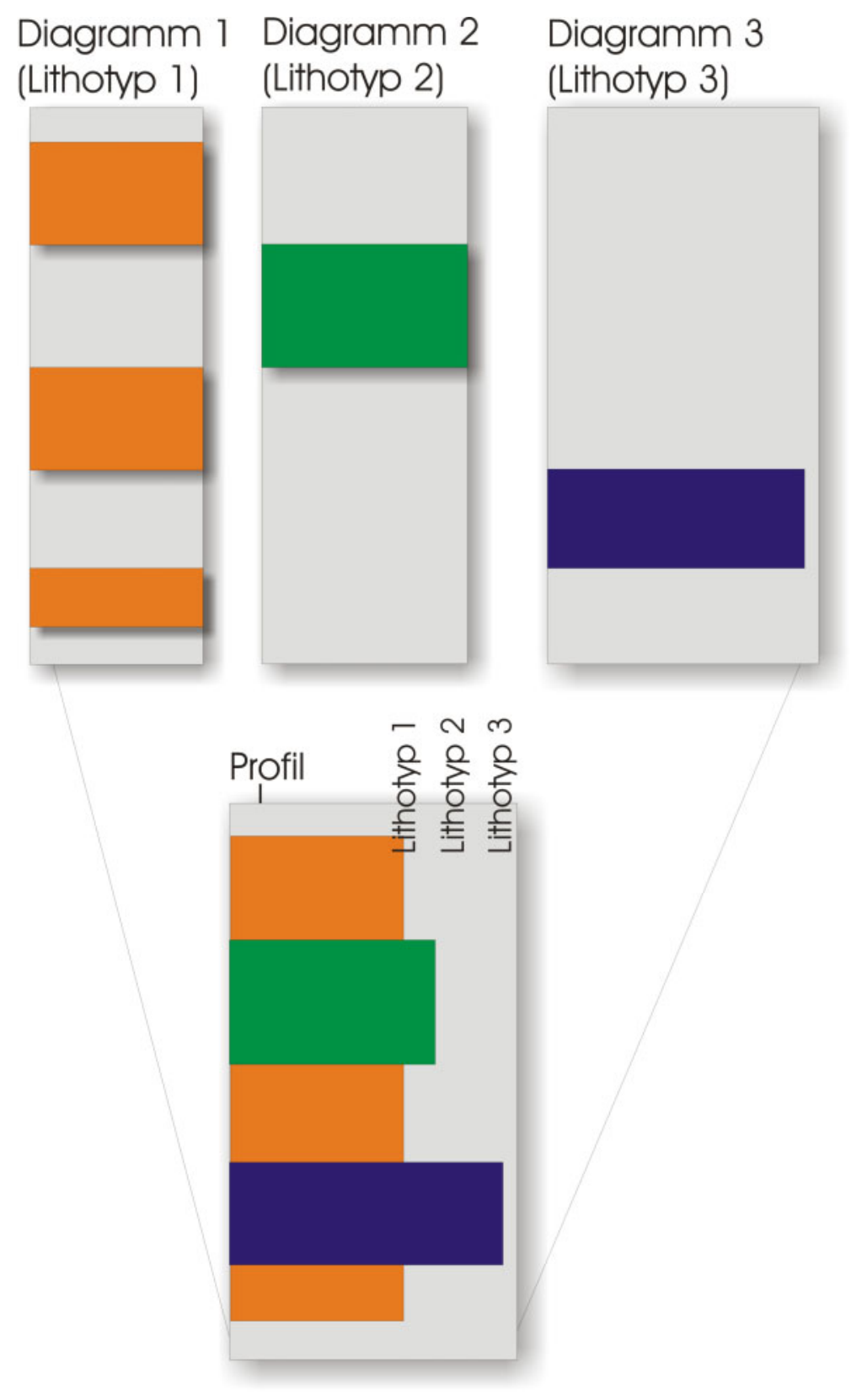

Abb. 2.16: Graphische Darstellung der Profildaten mit Hilfe der Excel-Balkendiagramme.

\subsubsection{Graphische Darstellungen}

Um Pollendiagramme mit Excel zu generieren, wird für jede Form ein X-Y-Diagramm erzeugt und nebeneinander dargestellt. Dabei ist es jedoch unbedingt nötig, daß die Datenbereiche für jedes Diagramm auf einer Positionslinie beginnen und enden. Dies wird durch umfangreiche Neupositionierungen und Größenänderungen erreicht, die über mehrere Stufen angewendet werden.

Die Diagramme werden auf einer Linie angeordnet, dabei wird die Position relativ zur oberen Bildschirmkante vorgegeben.

Im nächsten Schritt werden die Daten für jede Form (Mächtigkeit und Prozentwerte) in das Diagramm übertragen.

Da sich durch eine Datenänderungen die Größe und Position des inneren Diagrammbereiches ändert, ist eine Neupositionierung des Datenbereiches nötig. Dazu dient als 
relative Positionsmarkierung ein Punkt im Datenbereich. Bedingt durch unterschiedlich langen Diagrammtitel verschiebt sich ebenfalls der Diagrammbereich in vertikaler Richtung. Diese Problematik tritt insbesondere bei Verkleinerungen des gesamten Pollendiagrammes auf, um z.B. das Gesamtdiagramm auf unterschiedlichen Papierformaten zu drucken. Deshalb wird der Diagrammtitel ausgelesen und in ein Textfeld innerhalb des Diagrammes positioniert.

Die Gesamtgröße der Diagramme wird im letzten Schritt, nach Beendigung aller Datenmanipulationen, auf eine einheitliche Größe in Relation zur Profildarstellung korrigiert. Leider werden die Positionierungen der Diagramme bei jeder Excelversion unterschiedlich durchgeführt. Für Excel 97 und Excel 2000 werden die Positionierungs-Methoden dynamisch den entsprechenden Versionen angepaßt.

\subsubsection{Grenzen der Excelprogrammierung}

\subsubsection{Pollendiagramme}

Auch wenn mittlerweile Prozessoren und Betriebssysteme mit 64-Bit Architektur und einem 18Gbyte großen Adreßraum auf dem Markt sind, gibt es innerhalb der Anwendungssoftware oft sehr viel engere Grenzen. Insbesondere bei etwas außergewöhnlichen Anwendungsbereichen muß aufwendig ein neuer Weg für die Lösung von Speicherproblemen gefunden werden.

Um eine vollständige Darstellung der Mikrofloren in Form von Pollendiagrammen zu gewährleisten, ist es oft nötig, mehr als 100 Einzeldiagramme gleichzeitig darzustellen. Die VBA-Variable zur Aufnahme der Informationen der Einzeldiagramme innerhalb Excels ist jedoch derart unterdimensioniert, daß nur etwa 20 Diagramme (abhängig von der Komplexität) gleichzeitig in einem Arbeitsblatt angezeigt werden können. Überschreitet man diese Anzahl, kommt es unabhängig vom physikalisch vorhandenem Arbeitsspeicher zu einem Absturz bzw. einer Speicher-Fehlermeldung bei Excel. Weder aufwendige Umstrukturierungen des internen Programmablaufes zur Speicherersparnis noch das Freigeben der Variablenspeicher der Diagrammobjekte brachten hier eine wesentliche Verbesserung der Situation.

Nach umfangreichen Manipulationen bei der Diagrammdarstellung stellte sich heraus, daß der größte Teil des VBA-Variablenspeichers für die Textformatierungen innerhalb der Diagramme verwendet wird. Verhindert man nun die Autoformatierung der Textelemente der Diagramme, erreicht man eine maximale gleichzeitige Darstellung von etwa 130 Diagrammen. Diese Anzahl konnte jedoch nicht weiter gesteigert werden. Sollte dieser Fehler in einer der nächsten Versionen von Office bzw. Excel behoben sein, kann über die Optionseinstellungen bei P.A.S.T (Datenbankteil) die maximal erlaubte Diagrammanzahl verändert werden.

\subsubsection{Clusteranalyse}

Ebenfalls sehr lästig ist die Tatsache, daß die maximale Rangegröße bei Excel (Excel 97 und 2000) nur 65536 Zellen umfassen darf. Insbesondere bei umfangreichen Clusteranalysen bzw. sehr vielen zu vergleichenden Objekten kann dieser Wert jedoch überschritten werden. Die daraus resultierende Fehlermeldung wird von P.A.S.T abgefangen. 


\section{Beschreibung von P.A.S.T mit einem Anwendungsbeispiel}

\subsection{Vorbemerkungen}

Dieses Kaptitel ist als Software-Handbuch für P.A.S.T $\mathrm{zu}$ verstehen. Um den praxisnahen Kontext zu wahren, wird an einem Beispiel die Eingabe eines Taxons Schritt für Schritt beschrieben. Die vorgegebenen Eingabedaten stellen dabei nur eine Empfehlung dar, sofern keine geeigneten Daten zur Verfügung stehen. Daran anschließend werden weiterführende Möglichkeiten der Suche und Ausgabe von Daten aufgezeigt. Um den Einstieg zu erleichtern, werden zu Beginn dieses Kapitels einige grundlegende Datenbank-relevante Begriffe und Bedienungskonzepte erläutert.

Da dieses Kapitel in Form eines Software-Handbuches aufgebaut ist, werden die sprachlichen und stilistischen Empfehlungen von BOEDICKER 1990 berücksichtigt.

Jeder Arbeitsschritt wird mit einer Abbildung des entsprechenden Eingabeformulars verdeutlicht.

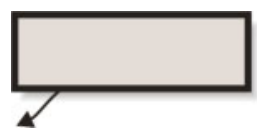

Dabei helfen Anmerkungen in den Abbildungen, die Datenbankfunktionen näher zu erläutern.

1

Für das Anwendungsbeispiel essentielle Anmerkungen sind zusätzlich mit einer Numerierung, die die Reihenfolge der notwendigen Arbeitsschritte angibt, versehen. Weitere nicht gekennzeichnete Anmerkungen geben Informationen, die zum Verständnis der beschriebenen Abläufe beitragen oder weiterführende Möglichkeiten aufzeigen.

\subsection{Installation von P.A.S.T}

\subsubsection{Hardwareanforderungen}

Als Systemanforderung genügt für P.A.S.T im Zuge der rasanten Hardwareentwicklung ein Rechner der mittleren Preisklasse um 2000 DM, der minimal mit einem 17" Bildschirm und einer nominalen Auflösung von 1280*1024 Punkten ausgestattet sein sollte. Um eine zufriedenstellend stabile Arbeitsumgebung zu erhalten, wird Windows NT 4, Windows 2000 oder Windows XP als Betriebssystem empfohlen, die mit einem Arbeitsspeicher von $256 \mathrm{MB}$ als gut ausgestattet gelten.

\subsubsection{Installation von P.A.S.T von dem Datenträger}

Legen Sie die Installations-CD in das CD-Laufwerk Ihres Computers. Rufen Sie nun das Setup-Programm von der CD auf, und befolgen Sie die angezeigten Anweisungen, um das Programm auf Ihrem Rechner zu installieren. 


\subsection{Bedienungskonzepte von P.A.S.T}

Grundsätzlich gibt es drei verschiedene Bedienungskonzepte bei P.A.S.T:

1. Eine Übersicht stellt den Zugriff auf die verschiedenen Formulare hierarchisch dar und ist für Anfänger gut geeignet. Die Übersicht muß jedoch aktiviert bleiben und überdeckt eventuell darunter liegende Formulare. Außerdem sind nicht alle Funktionen über die Übersicht erreichbar. Beim Starten von P.A.S.T wird automatisch die Übersicht geöffnet.

2. Die P.A.S.T eigene Menüstruktur hat etwa die selbe Informationsdichte wie die Übersicht. Sie hat aber den Vorteil, jederzeit zur Verfügung zu stehen und keine Formulare zu überdecken.

3. Als letzte Variante läßt sich P.A.S.T über "Buttons" auf den jeweiligen Formularen steuern. Über diese Variante sind sämtliche Funktionen von P.A.S.T abrufbar, deshalb sollte diese Methode favorisiert werden. Insbesondere Komplexe mehrschichtige Operationen lassen sich über die durch die Formulare im Kontext stehenden Buttons besser durchführen.

Trotzdem kann die Menüstruktur benutzt oder die Übersicht (Menü: Datei) jederzeit aktiviert werden, wenn z.B. ein wichtiges Formular versehentlich geschlossen wurde oder eines der Formulare auf Grund eines Fehlers neu gestartet werden muß. Im Anschluß werden die verschiedenen Bedienungskonzepte kurz erläutert.

Die Beschreibung von P.A.S.T mit einem Anwendungsbeispiel stützt sich auf das dritte Konzept der "Button Methode". Am Anfang jedes Anwendungsbeispiels wird ein Symbol des zum Öffnen des relevanten Formulars/Dialogfeld benötigten Buttons dargestellt. Dahinter steht zusätzlich - sofern vorhanden - der alternativ nutzbare Menüeintrag. 


\subsection{1 Übersicht (Menüleiste Datei)}

Über den Button „Formulare Eingeben, Ansehen“ verzweigt die Übersicht auf die Möglichkeit, die Formulare „Systematik/Botanik/Abbildungen“, „Literatur“, „Lokalitäten“, „Bilder“ und „Benutzerkartei“" zu öffnen (Abbildung 3.1).

Die Hauptübersicht bietet mit dem Button „Exportieren“ die Wahl „Karteikarten“ zu erstellen, Formularmasken der Sporen, der Pollen und der botanischer Zugehörigkeit auszugeben, "Farbtafeln" oder "Karteikarten" zu erstellen oder eine „Eingabetabelle“ zu generieren.

Die Seite "Bearbeiten" ermöglicht die Erstellung einer Auswahl von Sporomorphen, sucht Taxa über stratigraphische Reichweiten oder beliebiger Textfragmente.

Auf der Hauptübersicht kann die Optionsansicht aktiviert werden oder die „Struktur der Übersicht" verändert werden.

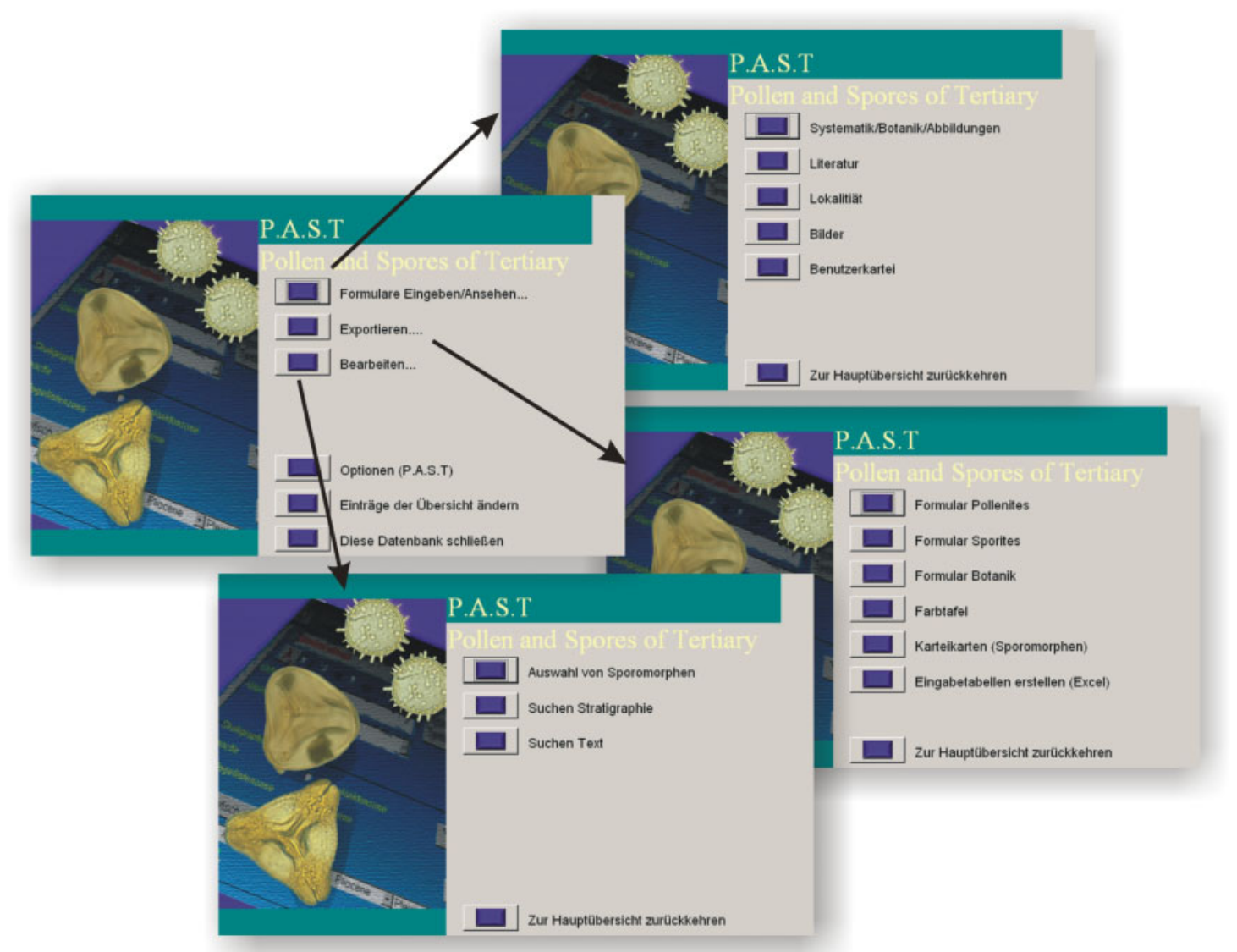

Abb. 3.1: Die Bedienung von P.A.S.T mit Hilfe der Übersicht (Datei: Übersicht). 


\subsubsection{Menüleistenstruktur von P.A.S.T}

\begin{tabular}{|c|c|c|}
\hline Menüeintrag & Beschreibung & Seite \\
\hline Datei & 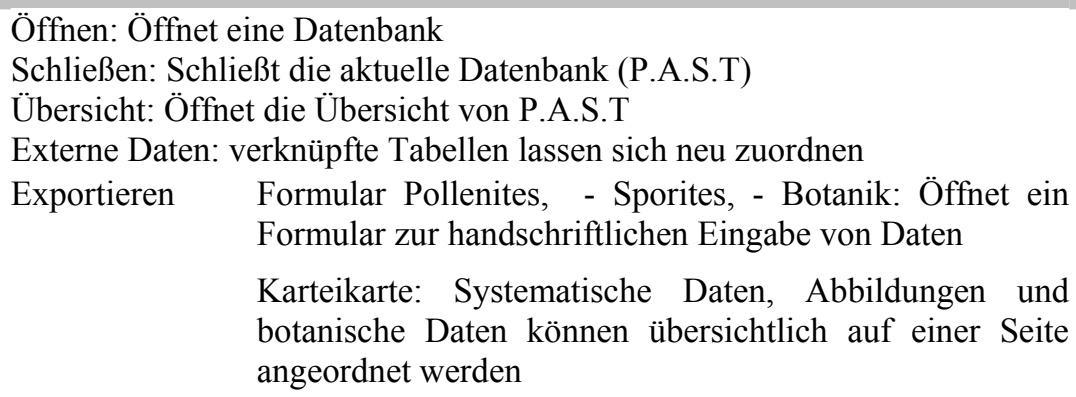 & $77 \mathrm{ff}$ \\
\hline Bearbeiten & $\begin{array}{l}\text { Auswahl von Sporomorphen: Erstellt eine Auswahl aus der Gesamtanzahl } \\
\text { der Taxa (z.B. für Eingabetabellen) } \\
\text { Suchen....Text: Suchen von Taxa durch beliebige Textangaben } \\
\text { Suchen....Stratigraphie: ... nach stratigraphischer Reichweite }\end{array}$ & $\begin{array}{l}75 \mathrm{ff} . \\
72 \mathrm{f} . \\
68 \mathrm{f} .\end{array}$ \\
\hline Formulare & Öffnet die genannten Formulare zur Ansicht oder Eingabe & 46 u.a. \\
\hline Datensätze & $\begin{array}{l}\text { Hierunter werden Wechsel von Datensätzen und Filter zur Einschränkung } \\
\text { oder Suche des Datenpools zusammengefaßt }\end{array}$ & $70 \mathrm{f}$. \\
\hline Extras & $\begin{array}{l}\text { "Datenbank komprimieren" verhindert eine Fragmentierung der Datenbank, } \\
\text { was die Dateigröße verringert und eventuell die Leistung der Datenbank } \\
\text { erhöht } \\
\text { Radiometrische Einstellung verändert die Zuordnung der radiometrischen } \\
\text { Daten zu stratigraphischen Einheiten } \\
\begin{array}{l}\text { Optionen: P.A.S.T relevante Pfade und Einstellungen können hier } \\
\text { eingestellt werden }\end{array}\end{array}$ & $74 \mathrm{f}$. \\
\hline Hilfe & $\begin{array}{l}\text { Definitionen } \\
\begin{array}{l}\text { Zusatzinformationen } \\
\text { abgerufen werden }\end{array}\end{array}$ & 52 u.a. \\
\hline
\end{tabular}

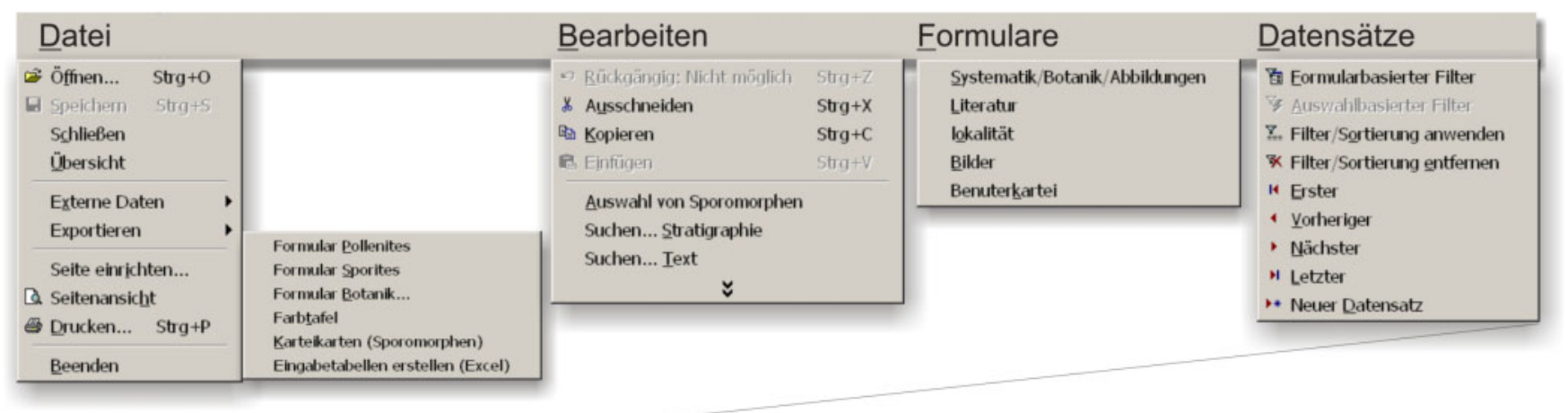

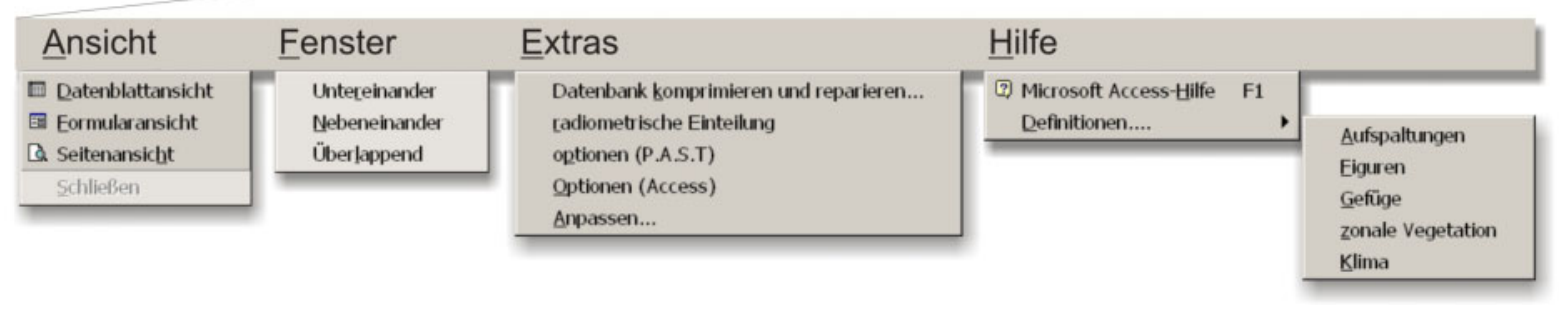

Abb. 3.2: Die Bedienung der Grundfunktionen von P.A.S.T mit Hilfe der Menüleiste: In der Tabelle oben sind die entsprechenden Menüeinträge kurz erläutert und mit einem Verweis auf die Seite im Anwendungsbeispiel ergänzt. 


\subsection{Grundeinstellungen von P.A.S.T}

\section{$\lambda$ Menüleiste Extras}

Nach der Installation von P.A.S.T öffnen Sie das Optionsformular, um einige Einstellungen, die für den Betrieb des Programmes notwendig sind, anzugeben (Abbildung 3.3). In den meisten Fällen können Sie die vorgebenden Einstellungen übernehmen. Eventuell ist nur eine Anpassung der Laufwerksbuchstaben und des Programmnamens des Bildanzeige-Programmes notwendig.

\begin{tabular}{|c|}
\hline $\begin{array}{l}\text { Access kann Bilder aus verschiedenen Ordnern heraus } \\
\text { öffnen. In der Dateiauswahl im Bilderformular stehen die } \\
\text { Begriffe Festplatte, CD und Netzlaufwerk für die hier } \\
\text { angegebenen Pfade. }\end{array}$ \\
\hline $\begin{array}{l}\text { Dieses Programm wird gestartet, wenn im } \\
\text { Abbildungsformular ein vergrößerte Ansicht der Abbildung } \\
\text { aufgerufen wird. Es muss in der Lage sein, als Parameter } \\
\text { einen Dateinamen zu verarbeiten. }\end{array}$ \\
\hline $\begin{array}{l}\text { Hier sind Bilder gespeichert, die zu Definitionen von } \\
\text { Begriffen (z.B. Definition Gruppierungen) benötigt werden. }\end{array}$ \\
\hline $\begin{array}{l}\text { Generierte Eingabetabellen, die in Excel erzeugt werden, } \\
\text { werden hier abgelegt. }\end{array}$ \\
\hline $\begin{array}{l}\text { Excel benötigt eine Datei "pattern.xls", in der Vorlagen und } \\
\text { der Programmcode gespeichert ist. }\end{array}$ \\
\hline $\begin{array}{l}\text { Aufgrund eines Programmfehlers kann Excel nur eine } \\
\text { bestimmte Anzahl von Pollendiagrammen generieren. Wird } \\
\text { diese Zahl über-schritten, muss Excel neugestartet werden. } \\
\text { Dieser Fehler tritt bis Excel } 2000 \text { mit Sicherheit auf. }\end{array}$ \\
\hline $\begin{array}{l}\text { Hier werden die Datendatenbanken (Tabellen) und weitere } \\
\text { temporäre Datenbanken gespeichert. }\end{array}$ \\
\hline
\end{tabular}

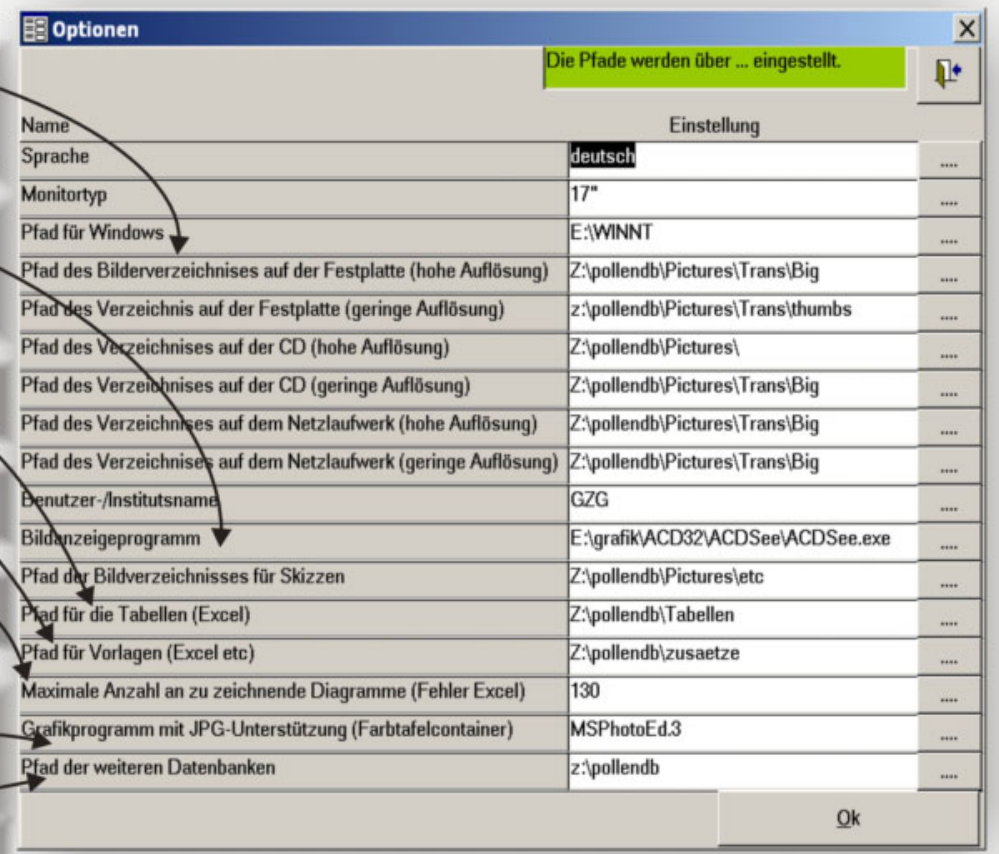

Abb. 3.3: Das Optionsformular zur Einstellung Programm-relevanter Daten.

\subsection{Objekte einer Datenbank mit Begriffsdefinitionen}

\subsubsection{Tabellen}

Synonyme: Dateien (bei dBASE), Tabellenblätter

Sämtliche Informationen werden in tabellarischer Form gespeichert und bilden die Grundlage für Formulare, Berichte und Abfragen.

Tabellen bestehen aus Tabellenfeldern, die durch den Feldnamen, die Länge des Feldes und den Felddatentyp definiert sind. Als wichtigste Felddatentypen seien Autowert-, Text-, Memo-, Zahl- und Ja/Nein-Typen genannt.

Ein Datensatz entspricht einer Reihe innerhalb einer Tabelle. 


\subsubsection{Formulare}

Synonyme: Eingabemaske, Eingabeformular, Popup-Fenster, Dialogbox

Das Popup-Fenster muß als Sonderform eines Formulars geschlossen werden, bevor darunterliegende Fenster/Formulare wieder zugänglich sind.

Formulare stellen die Schnittstelle zum Anwender dar. Auf der Oberfläche der Formulare sind verschiedene Steuerelemente zur Eingabe oder Auswahl von Daten angeordnet (Abbildung 3.4).

$\mathrm{Zu}$ den Steuerelementen gehören Textfelder, die eine freie Eingabe von Informationen einer vorgegebenen Länge erlauben, z.B. die Angabe des Artnamens. Neben Textfeldern gibt es sogenannte Memofelder, die keine Begrenzung der Textmenge vorgeben und für Kommentare oder ähnliches benutzt werden können.

Kombinationsfelder (Synonyme: Auswahlbox, Auswahlfeld, Kombinationsbox, RollupMenü)

Kombinationsfelder geben eine Auswahl von Möglichkeiten vor, z.B. vordefinierte stratigraphische Einheiten, die wiederum in Form einer Tabelle festgehalten sind.

\section{Listenfelder (Synonyme: Auswahlbox, Auswahlfeld)}

Listenfelder ähneln den Kombinationsfeldern, erlauben jedoch im Gegensatz zu diesen eine gleichzeitige Ansicht mehrerer möglicher Auswahlelemente und können zum mehrfachen gleichzeitigen Selektieren befähigt werden.

\section{Befehlsschaltflächen, Umschaltflächen (Synonyme: Schaltflächen, button)}

Neben den unter Auswahlmöglichkeiten wählenden Steuerelementen (mehrere definierte Zuständen) gibt es sogenannte Schaltflächen, die als Befehlsschaltfläche (button) ein einmaliges Ereignis auslösen, z.B. ein anderes Formular öffnen, oder als Umschaltfläche zwischen zwei oder drei Zuständen wechseln. Umschaltflächen werden z.B. im Morphologie-Formular bei der Wahl der Abteilungen Pollenites oder Sporites eingesetzt und sind in der Regel an Ja/Nein-Felder der zugrundeliegenden Tabelle geknüpft.

Eine ähnliche Funktionalität haben Kontrollkästchen, die den Ja/Nein-Zustand jedoch mit Hilfe eines Häkchens symbolisieren.

Befehlsschaltflächen können zu Optionsgruppen zusammengefaßt werden, in der nur eine Auswahl gleichzeitig aktiv sein kann. Eine spezielle Variante der Befehlsschaltfläche stellt das häufig in eine Optionsgruppe gestellte runde Optionsfeld dar.

Innerhalb eines Formulars kann die Komplexität durch ein oder mehrere Unterformulare erhöht werden. Diese verweisen in der Regel auf den "n-ten" Teil einer 1:nBeziehung. Im Systematik-Formular sind z.B. die Literaturstellen in der Ansicht eines Unterformulars abgebildet. 


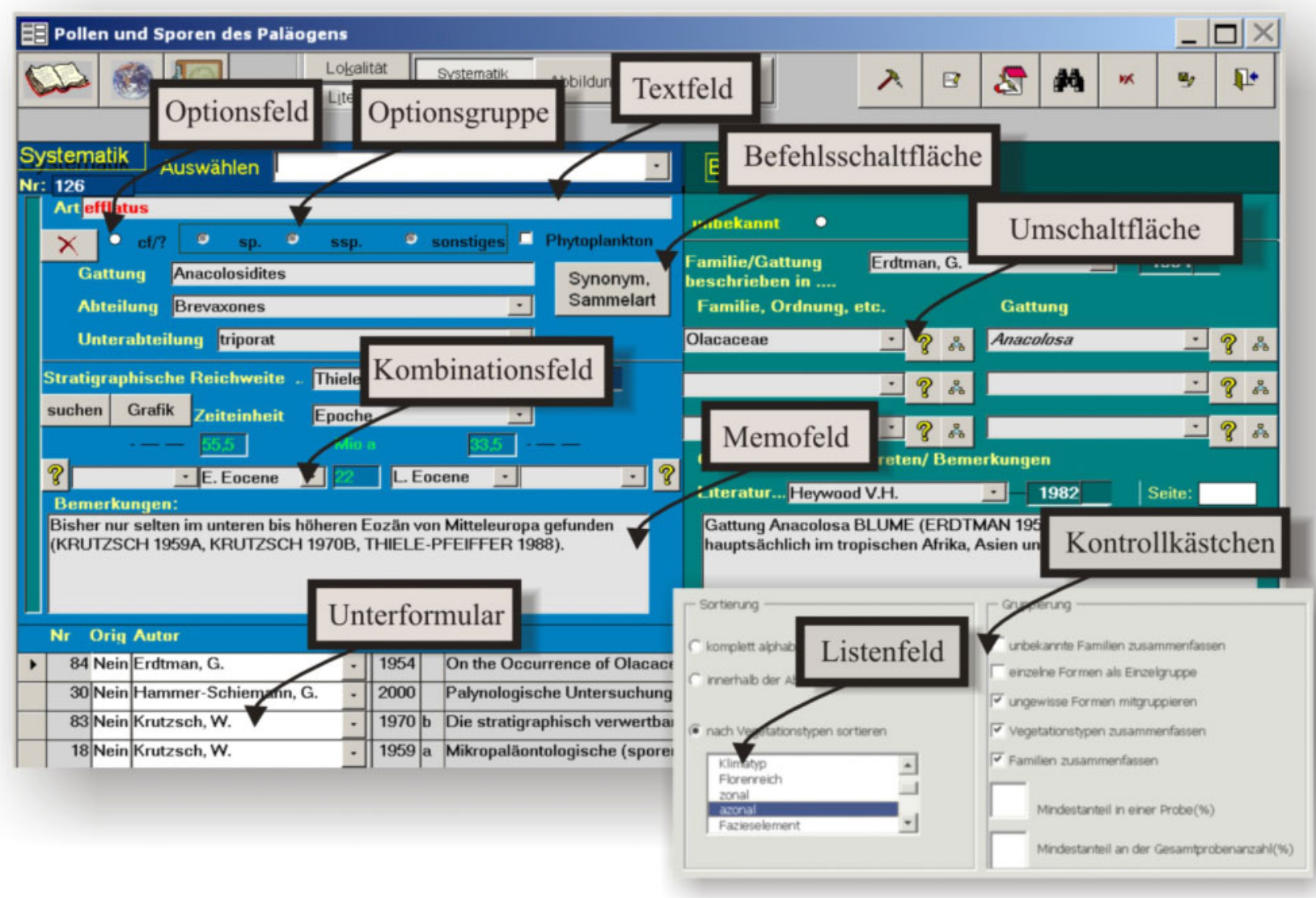

Abb. 3.4: Steuerelemente eines Formulars: Das zugrunde gelegte Beispiel stellt das Eingabeformular der Systematik dar. Für das Beispiel der Listenfelder und Kontrollkästchen dient ein Ausschnitt des ExcelAuswertungs-Formulars.

\subsection{Die Dateneingabe einer Form}

Als Eingabedaten können Sie folgende Informationen verwenden. Dazu gehören Angaben zur Systematik, zur Morphologie der Form, sowie botanische Informationen zum nächsten lebenden Verwandten.

Systematische Daten

Form: Anacolosidites efflatus (POTONIÉ 1931) ERDTMAN 1954

Abteilung: Brevaxones

Unterabteilung: triporat

Stratigraphische Reichweite nach THIELE-PFEIFFER 1988: Eozän

Bemerkungen: Bisher nur selten im unteren bis höheren Eozän von Mitteleuropa gefunden (KRUTZSCH 1959, KRUTZSCH 1970, THIELE-PFEIFFER 1988)

Wichtige Literatur (vollständiger Titel: s. Literaturliste):

ERDTMAN, G. 1954

KRUTZSCH, W. 1959

KRUTZSCH, W. 1970

THIELE-PFEIFFER, H. 1988, S.33

NATGE-EFOGHE, C. 1997, S.75

Wichtige Lokalitäten:

Alversdorf Oberflözgruppe, Mitteleozän

Schöningen Unterflözgruppe, Untereozän 
Synonyme:

1931 Sporites efflatus - PotoniÉ, R., S. 37, Taf. 1, Fig. 17-18 (Cecilie, Geiseltal, M. Eocene)

1954 Anacolosidites efflatus - ERDTMAN, G., S. 804f., Taf. 1, Fig. o. Abbildung

Botanische Daten

Familie Gattung beschrieben in:

ERDTMAN 1954

Familie: Olacaceae

Gattung: Anacolosa

Geographisches Auftreten nach HeYwOOD 1982

Bemerkungen: Gattung Anacolosa Blume (ERDTMAN 1954). Die Familie ist heute hauptsächlich im tropischen Afrika, Asien und Amerika verbreitet.

Morphologische Daten (aus HAMMER-SCHIEMANN 1998)

Äquatorkontur: dreieckig

Polkontur: oval

Figura: Linse

Seitenlinien: stark konkav

Exinenhomogenität: zu Germinalien schmaler

Lage Pore: subäquatorial

Größe Pore: $<4 \mu \mathrm{m}$

Die Form der Pore: rund

Allgemeine Morphologische Beschreibung :

In der Seitenmitte ist eine Einschnürung vorhanden. Des öfteren können plicenartige Falten beobachtet werden, die von Pore zu Pore verlaufen und die Polregion aussparen. An den Ecken erreicht die Exine eine Stärke von $1 \mu \mathrm{m}$ und in der Seitenmitte von ca. 3 $\mu \mathrm{m}$. Bereiche zwischen den Germinalien zeigen eine punctate bis granulate Struktur. Weitere Informationen zur Morphologie:

Bilddaten:

Anacolosidites_efflatus_Natge_Hel_Alv_1aIF.jpg

Beim erstmaligen Öffnen des Systematik-Formulars werden Sie aufgefordert, Ihren Benutzernamen und die zugrunde gelegte Literaturstelle einzugeben (Abbildung 3.5).

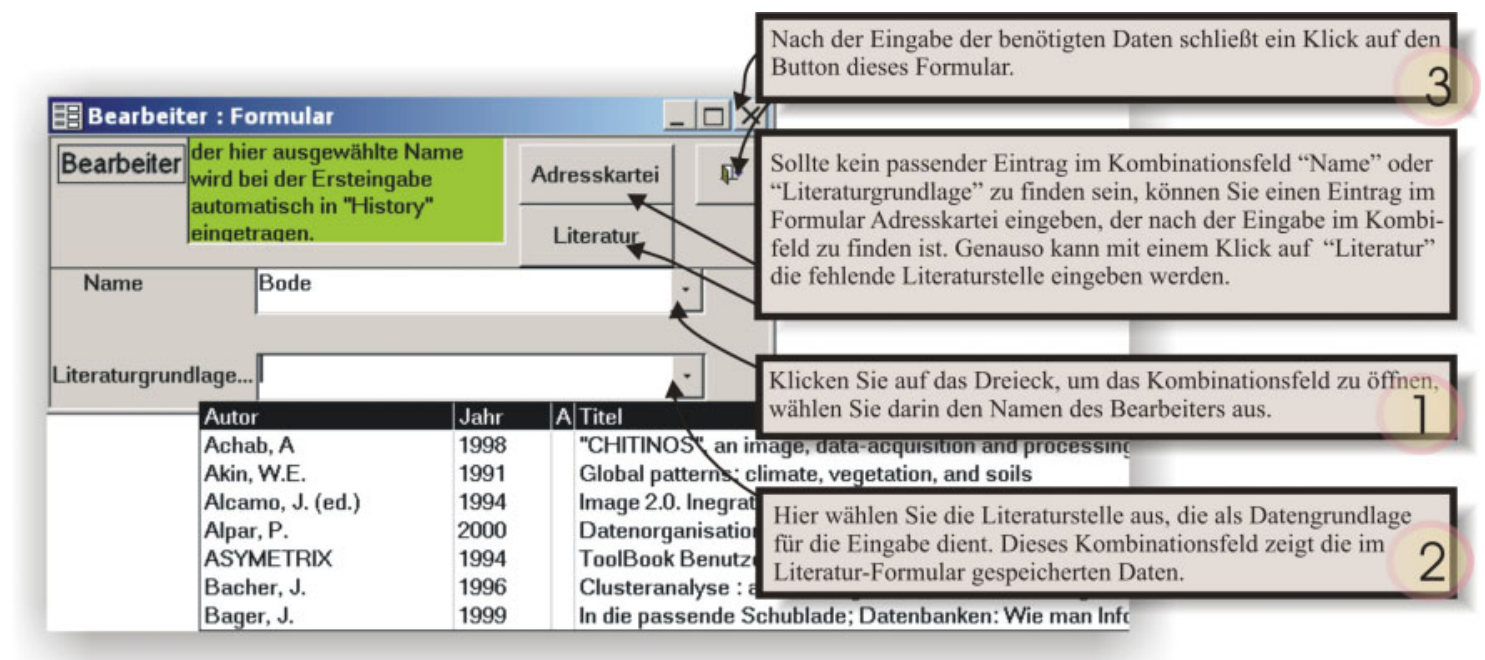

Abb. 3.5: Eingabe des Bearbeiters und der Literaturgrundlage, aus der die Eingabeinformationen entnommen werden. 

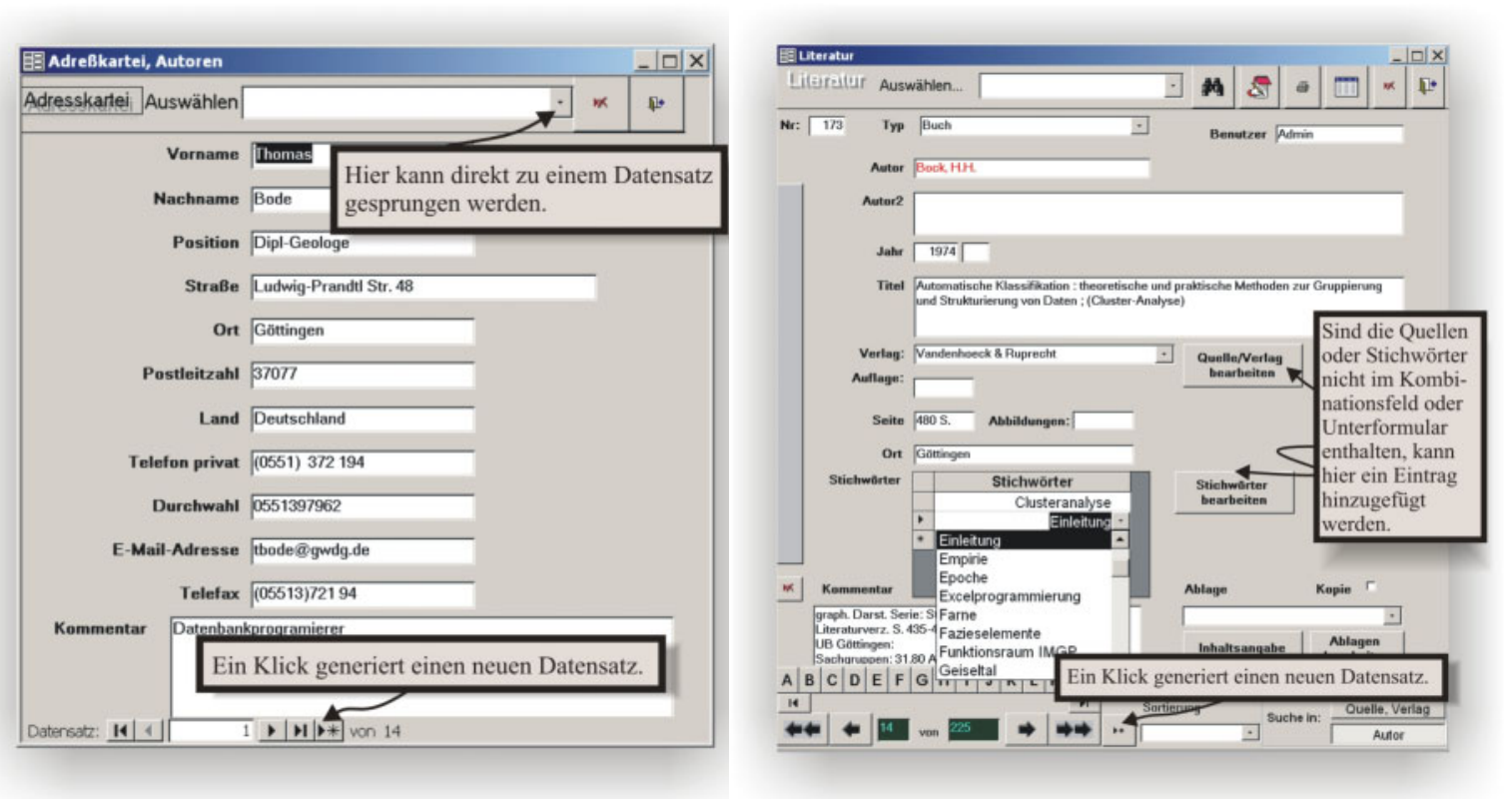

Abb. 3.6: Sind die Angaben des Bearbeiters oder der Literaturgrundlage nicht vorhanden, kann die Eingabe der fehlenden Daten mit den Formularen Adresskartei und Literatureingabe nachgeholt werden. Siehe dazu auch die ausführliche Beschreibung der Literatureingabe auf Seite 62.

\subsubsection{Wechseln und Finden von Datensätzen}

Nachdem Sie die Informationen zum Bearbeiter eingegeben haben, müssen Sie überprüfen, ob der neu einzugebende Datensatz eventuell schon vorhanden ist. Dazu gibt es verschiedene Methoden, die Sie der Reihe nach ausprobieren sollten (Abbildung 3.7).

So können Sie über die Navigations-Schaltflächen zwischen einzelnen Datensätzen wechseln. Dazu sortieren Sie die Datensätze nach „Art“ (Sortierungsfeld in der Fußzeile) und springen mit dem „Schieber“ im blauen Feld der „Direkten Anwahl des Datensatzes" etwa in Richtung der gesuchten Art. Mit dem nun sichtbaren Button „Go" wird die im Textfeld angezeigte Form im Formular aktiviert. Nun können Sie mit den Pfeiltasten „Datensatz vor“ oder „Datensatz zurück“ den gewünschten Datensatz anwählen(Menüleiste Datensätze).

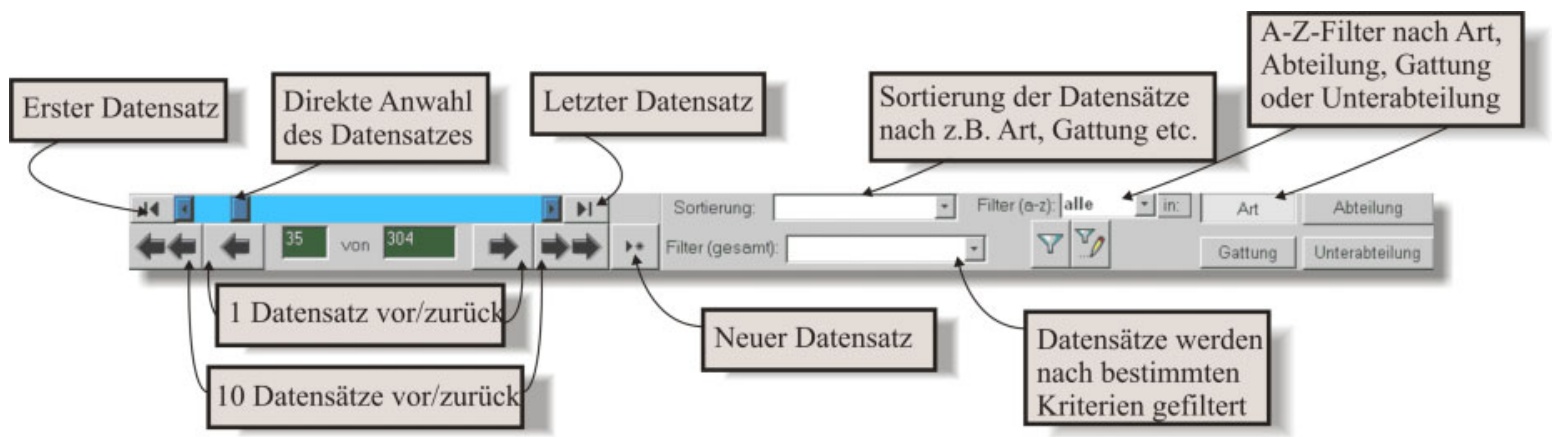

Abb. 3.7: Navigationsschaltflächen in der Fußzeile des Formulars.

Eine weitere Möglichkeit zur direkten Anwahl von Datensätzen bietet die AuswahlKombinationsbox in der Kopfzeile (Abbildung 3.8). Um die Arten anzuzeigen, klicken 
Sie auf den rechten Rand der Kombinationsbox (Rollup-Menü). Zum schnellen Auffinden einer Art geben Sie nur den Anfangsbuchstaben der Form mit der Tastatur ein. Das Kombinationsfeld zeigt daraufhin die erste Form mit diesem Buchstaben in der Auswahl an. Die gewünschte Form wählen Sie nun, indem Sie mit den Pfeiltasten die Kombinationsbox nach unten durchlaufen. Selbstverständlich können Sie auch mit der Maus und dem Positionierungsfeld am rechten Rand der Box eine Auswahl vornehmen - insbesondere bei Feldern mit vielen Einträgen ist diese Suchvariante jedoch etwas mühsam.

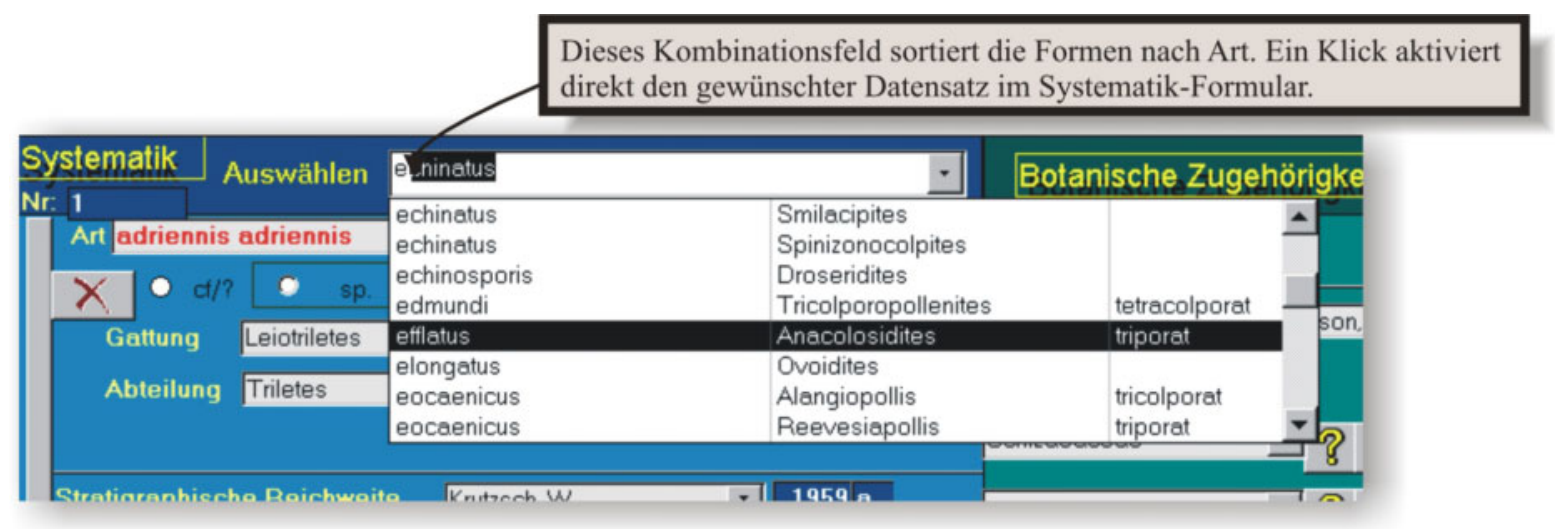

Abb. 3.8: Suchen und aktivieren des Datensatzes über die Auswahlfunktion.

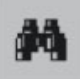

Menüleiste Bearbeiten

Neben der manuellen Datensatzsuche und -navigation bietet Ihnen die P.A.S.T-eigene Suchfunktion eine komfortable Möglichkeit, Datensätze zu finden. Zum Öffnen des Suchfensters klicken Sie im Systematik-Formular auf das Fernrohrsymbol (Abbildung 3.9).

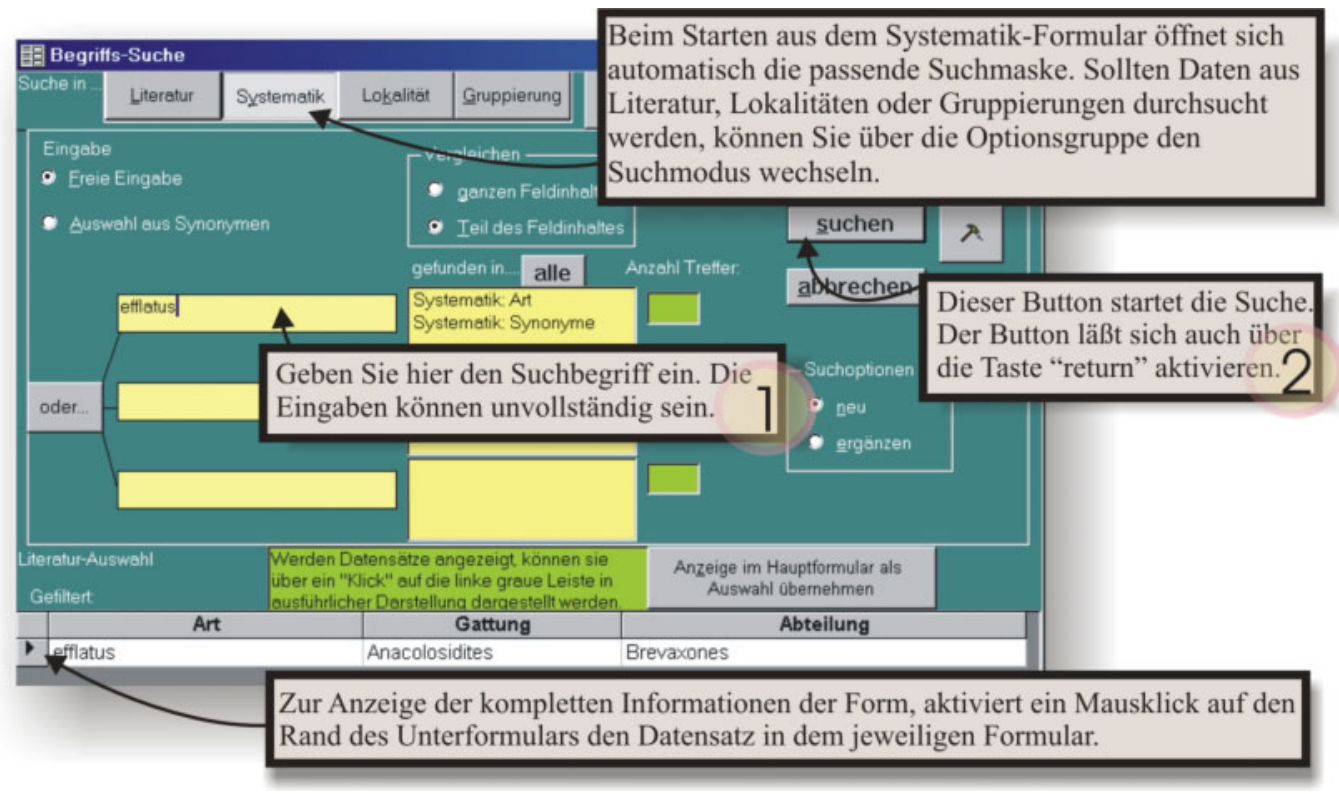

Abb. 3.9: Das Suchen nach Daten in den Formularen. 


\subsection{Eingabe in das Systematik-Formular}

\subsubsection{Systematik (Menüleiste Formulare)}

Sollte die einzugebende Form in der mitgelieferten Datenbank schon vorhanden sein, ist dies im Folgenden kein Problem. Sie können trotzdem die Testeingabe durchführen. Das eventuell erzeugte Duplikat kann später problemlos gelöscht werden.

$\mapsto *$ Menüleiste Datensätze

Die Texteingabe sollte mit einer leeren Datenmaske beginnen, dazu klicken Sie auf den Button „Neuer Datensatz“ in der Fußzeile des Formulars. Geben Sie daraufhin den Artnamen (ohne Gattungsnamen) und den Gattungsnamen in die vorgesehenen Felder ein (Abbildung 3.10).

Den Abteilungs- und den Unterabteilungs-Namen der Form können Sie in den Kombinationsfeldern (Rollup-Menüs) direkt auswählen.

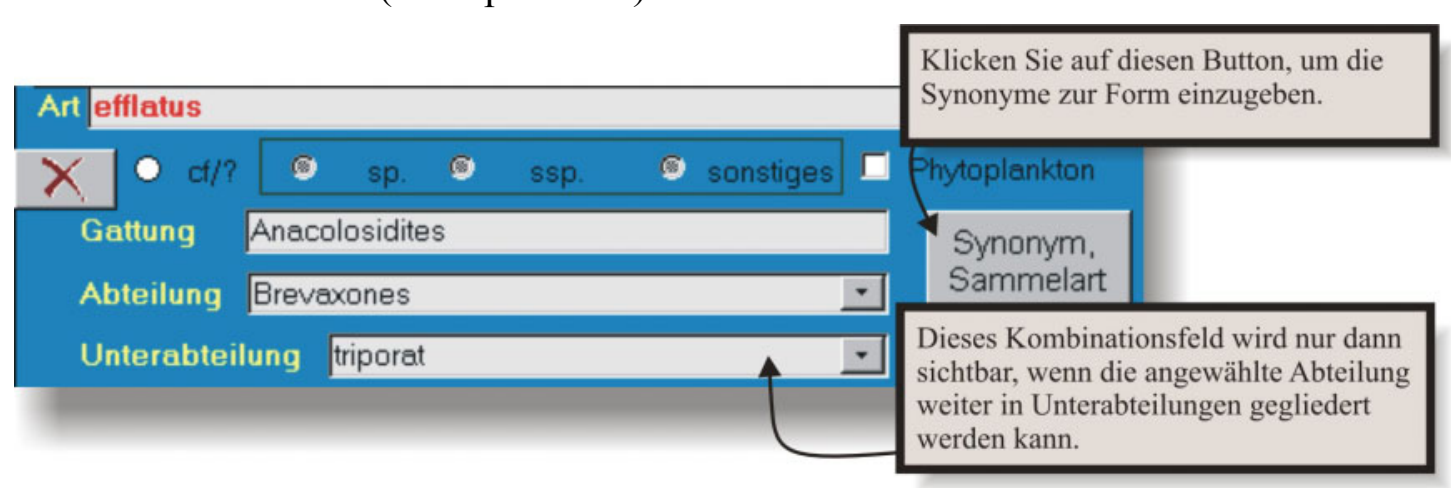

Abb. 3.10: Eingabe in das Systematik-Formular.

\subsubsection{Stratigraphische Reichweite}

Nun folgt der Bereich zur Eingabe der stratigraphischen Reichweite. Das oberste Kombinationsfeld nimmt die relevante Literaturstelle, respektive den verantwortlichen Autor zur Vergabe der Reichweite auf. Weitere für die stratigraphische Eingliederung wichtige Autorennamen fügen Sie im Bemerkungsfeld der Datenbank weiter unten hinzu (Abbildung 3.11).

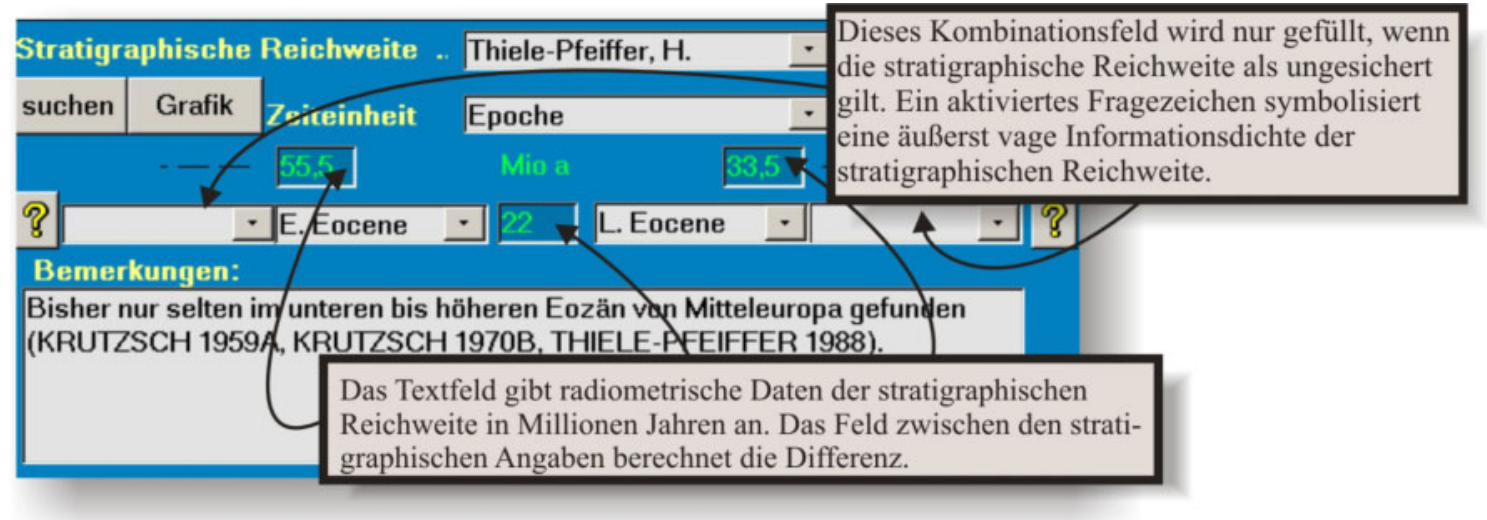

Abb. 3.11: Eingabe in das Systematik-Formular, Stratigraphie. 


\subsubsection{Synonyme}

\section{Synonym,}

Sammelart

Als nächstes sollten Sie die Synonyme eingeben. Hierzu klicken Sie auf den Button neben dem Gattungsfeld. Achten Sie darauf, bei „Art“ den kompletten Artnamen inklusive Gattung einzugeben. Der Autor stellt wieder einen Eintrag aus der Literaturdatenbank dar (Abbildung 3.12).

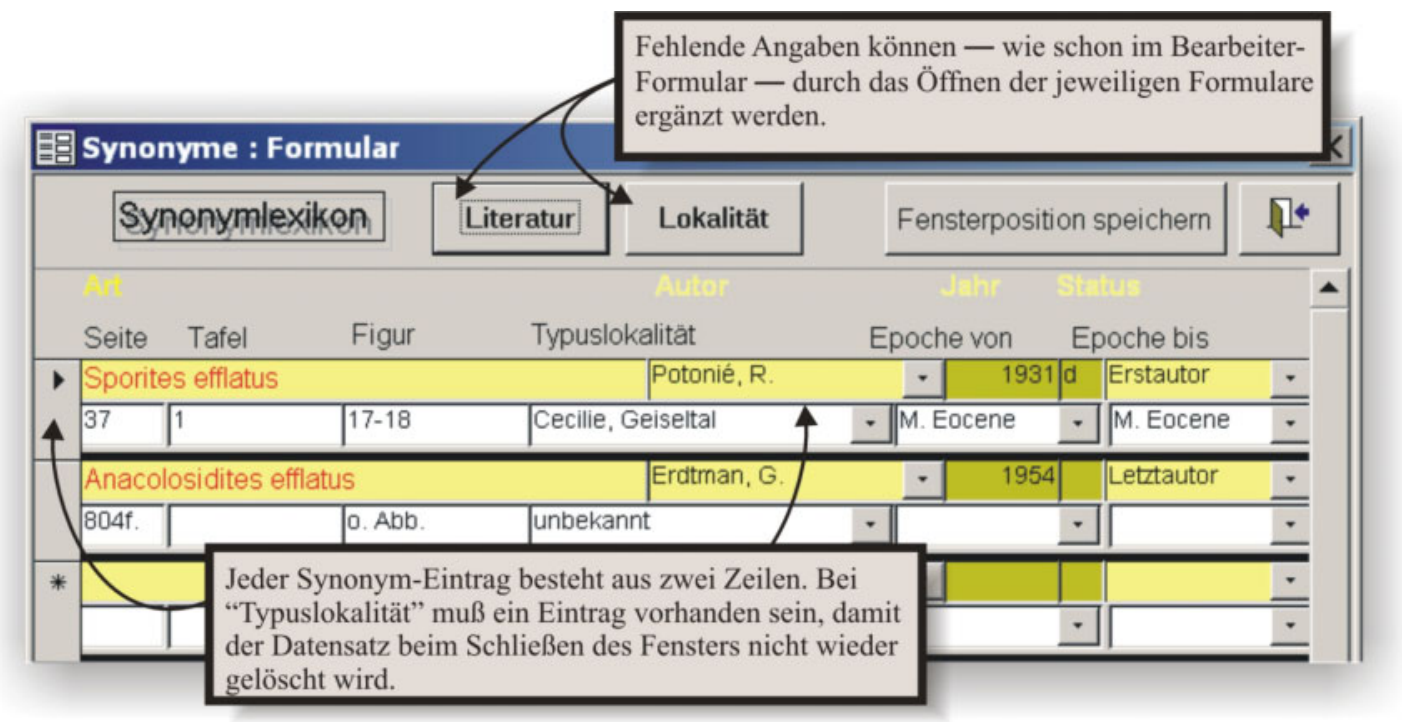

Abb. 3.12: Eingabe in das Systematik-Formular, Synonyme.

Um den Synonym-Datensatz zu vervollständigen, wählen Sie im Kombinationsfeld „Status“ den entsprechenden Eintrag aus. Des Weiteren werden in der nächsten Zeile Angaben zur Seite, Tafel, Figur erwartet. Sollte es nötig sein, einen Datensatz, d.h. einen Synonym-Eintrag zu löschen - z.B. aufgrund einer Fehleingabe - markieren Sie den Datensatz an der linken Seite (horizontaler Pfeil) und drücken anschließend die Taste ,entfernen“.

Anmerkung:

Ist der verantwortliche Autor der Erstbeschreibung innerhalb einer Veröffentlichung eines anderen Autors genannt, wird dieser Zusatz bei Art in Form von "Synonymeintrag (Autor in)" angegeben. Siehe auch nachfolgendes Beispiel des Autors der Erstbeschreibung des Synonyms für das Taxon Triporopollenites rhenanus.

\begin{tabular}{|l|l|l|}
\hline \multicolumn{1}{l}{ Art } & \multicolumn{1}{l}{ Autor } & \multicolumn{1}{l|}{ Jahr } \\
\hline Ostrya? -Poll. granifer rhenanus (THOMSON in:) & POTONIÉ, R. & 1950 \\
\hline
\end{tabular}

\subsubsection{Literatur}

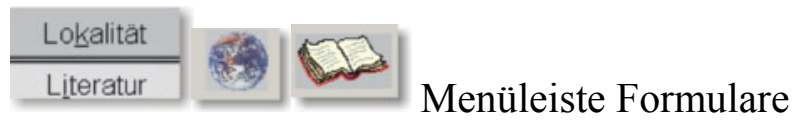

Im unteren Bereich des Systematik-Formulars wird ein Unterformular abgebildet, das je nach Einstellung der Buttons "Lokalität, Literatur" in der Kopfzeile die zugehörigen Literaturstellen oder Lokalitäten anzeigt (Abbildung 3.13). Geben Sie die in dem Beispiel aufgeführten Literaturstellen ein, indem Sie den Autor durch das entsprechende Kombinationsfeld auswählen. 


\begin{tabular}{|c|c|c|c|c|c|c|c|c|c|}
\hline \multicolumn{3}{|c|}{$\begin{array}{l}\text { Wird die Form erstmalig in dieser } \\
\text { Literaturstelle erwähnt, tragen Sie } \\
\text { "ja" bei Originalarbeit ein. }\end{array}$} & \multicolumn{3}{|c|}{$\begin{array}{l}\text { Wählen Sie hier den Autor der einzufügenden Literaturstelle. Fehlt } \\
\text { der Autor, respektive die Literaturstelle, kann über das Buchsymbol } \\
\text { in der Kopfzeile des Systematik-Formulars die Literaturstelle } \\
\text { hinzugefügt werden. }\end{array}$} & & \multicolumn{3}{|c|}{$\begin{array}{l}\text { Hier tragen Sie die relevante } \\
\text { Seitenangabe ein. }\end{array}$} \\
\hline & $\mathrm{Nr}$ Orig futor & & \multicolumn{2}{|r|}{ Titel } & \multirow{2}{*}{$\begin{array}{l}\text { Quelle/Verlag } \\
\text { i| Svensk Bot. Tidskr }\end{array}$} & Aull. $/ \mathrm{He}$ & eft Seiten & i Sente & Jurt \\
\hline - & 84 Nein Erdtman, G. & - & 1954 & On the Occurrence of Olacaceous Pollen Grains is & & 48 & $804-805$ & $\downarrow$ & Jppsala \\
\hline & 30 Nein Hammer-Schiemann, G. & - & 2000 & Palynologische Untersuchungen zur Fazies und d & Dissertation & & 107 & 59 & Universität $\mathrm{G}$ \\
\hline & 83 Nein Krutzsch. W. & $\cdot$ & $1970 \mathrm{~b}$ & b Die stratigraphisch verwertbaren Sporen- und PC. & Jb. Geol. & 3 & $309-379$ & & Berlin \\
\hline & 18 Nein Krutzsch. W. & v & 1959 o & a. Mikropaläontologische (sporenpaläontologische & Geologie & $8(21 / 22)$ & $1-425$ & & Berlin \\
\hline
\end{tabular}

Abb. 3.13: Eingabe in das Systematik-Formular, Literatur.

\subsubsection{Lokalitäten}

\section{Menüleiste Formulare}

Wechseln Sie nun zu den Lokalitäten über den Button in der Kopfzeile; fügen Sie die entsprechenden Datensätze über das Lokalitätsfeld hinzu.

Nicht vorhandene Einträge können über das in der Kopfzeile stehende Symbol Literatur oder die Weltkugel (Lokalitäten) ergänzt werden (siehe auch ausführliche Beschreibung zur Literatureingabe auf Seite $62 \mathrm{ff}$ ).

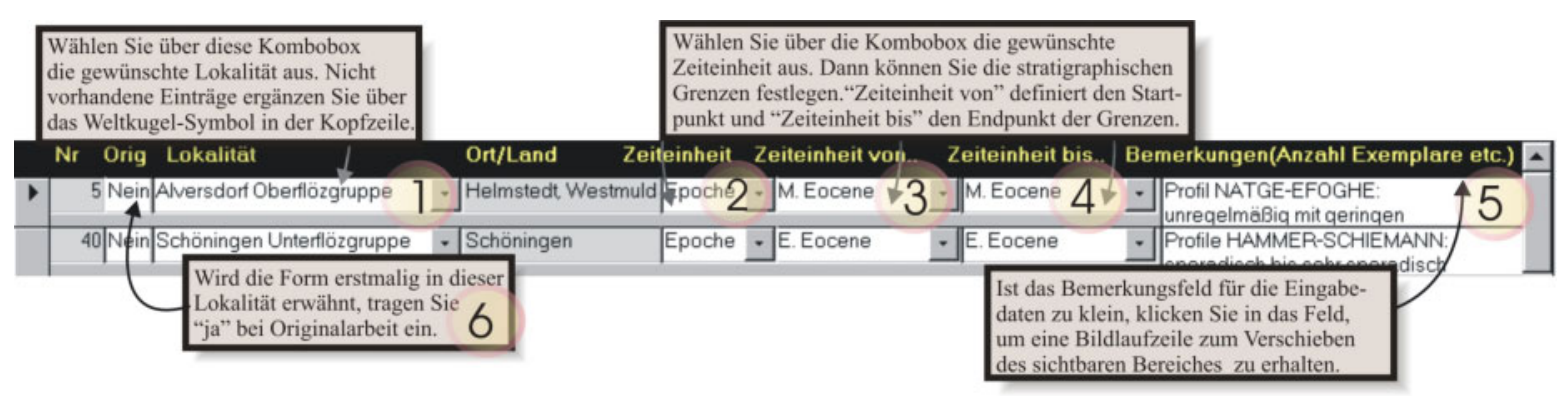

Abb. 3.14: Eingabe in das Systematik-Formular, Lokalitäten.

\subsubsection{Botanische Zugehörigkeit (nächster lebender Verwandter)}

Neben den rein systematischen Daten sind im rechten Bereich des Formulars die botanischen Parameter aufgeführt. Da die botanische Zugehörigkeit bekannt ist, bleibt das Optionsfeld „unbekannt“" unberücksichtigt (Abbildung 3.15).

Das Fragezeichen neben dem Familien-/Ordnungs-Feld wird bei ungewisser botanischer Zuordnung aktiviert. 


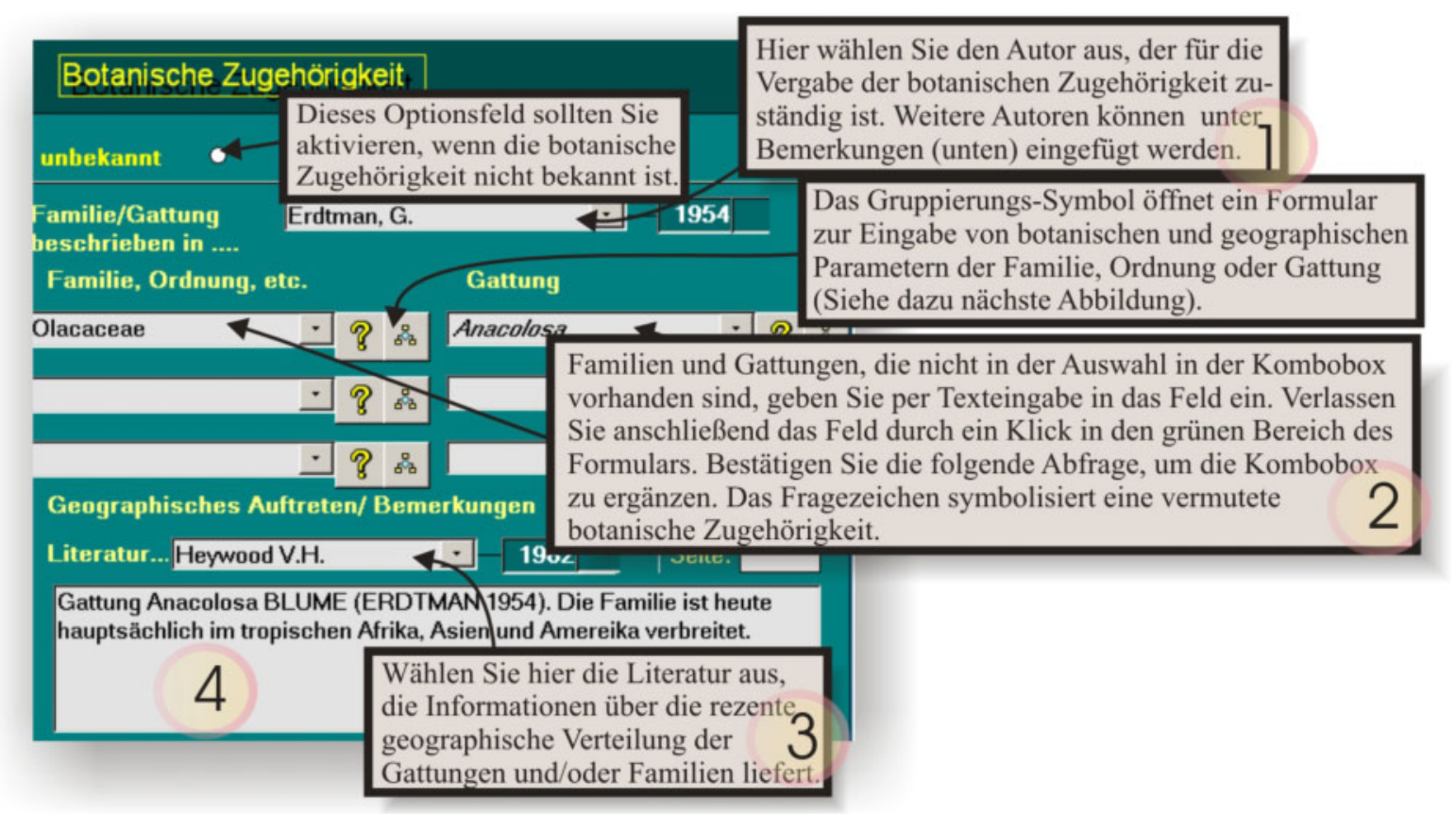

Abb. 3.15: Eingabe in das Systematik-Formular, botanische Zugehörigkeit.

\subsubsection{Gruppierung (weiterführende botanische und geographische Informationen)}

ใ.

Um weiterführende botanische und geographische Informationen einzugeben, wählen Sie das Gruppierungssymbol neben dem botanischen Namen zur jeweiligen Familie/Ordnung oder Gattung aus (im Folgenden als botanische Einheit bezeichnet). Informationen, die Sie im Gruppierungs-Formular eintragen, werden der botanischen Einheit und nicht der Form zugeordnet. Wählen Sie demnach für eine andere Form die selbe botanische Einheit aus, stehen die Gruppierungs-Informationen nach Öffnen des Gruppierungs-Formulars sofort zur Verfügung. Im Eingabebeispiel klicken Sie auf das Gruppierungssymbol neben der Familie Olacaceae. Die botanischen und geographischen Daten sind in der Datenbank gespeichert und müssen nicht neu eingegeben werden (Abbildung 3.16). 


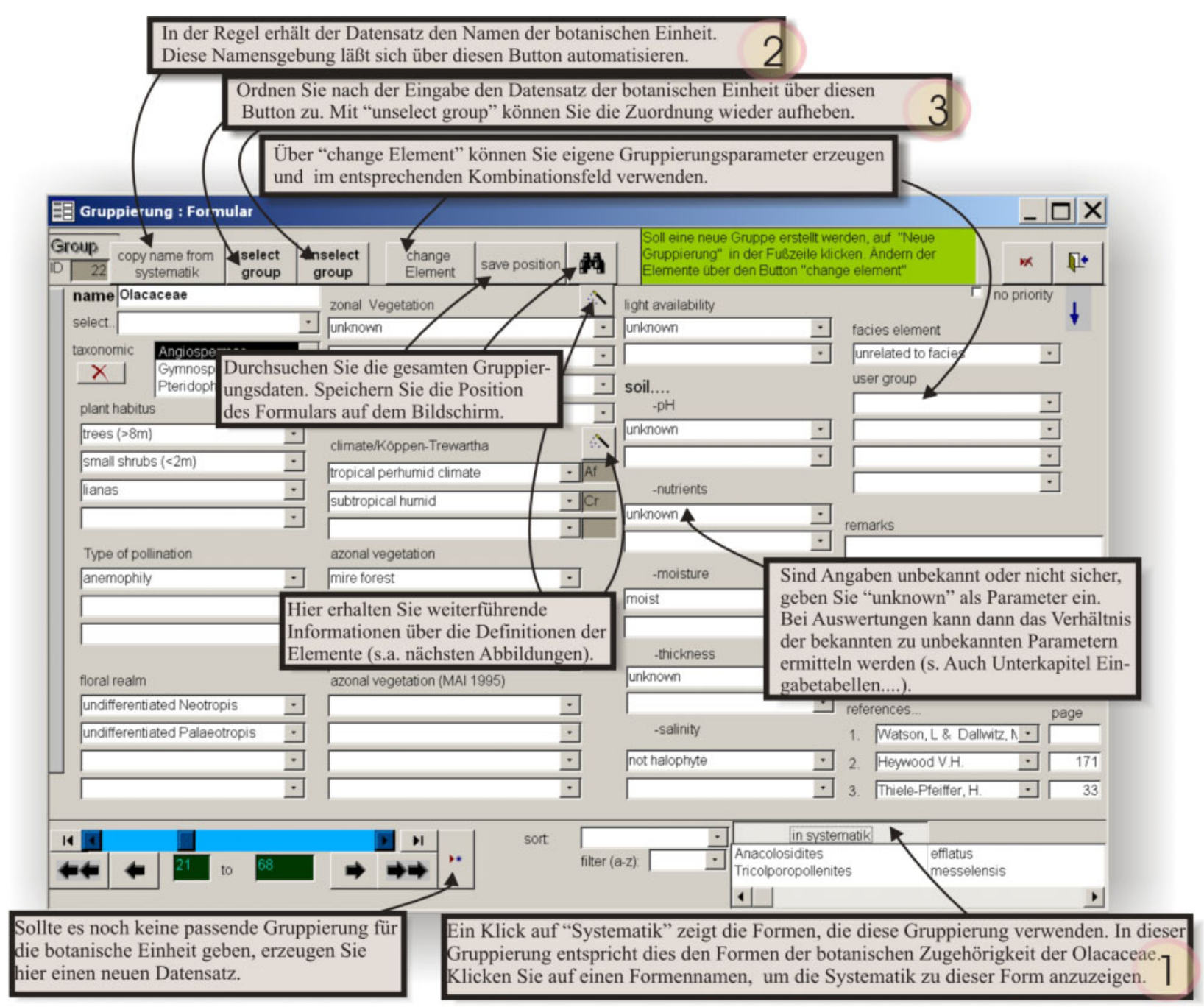

Abb. 3.16: Eingabe der Gruppierungsparameter (botanische und geographische Daten zu den Familien/Ordnungen oder Gattungen).

Sollte noch keine Zuordnung eines Gruppierungs-Datensatzes $\mathrm{zu}$ einer botanischen Einheit vorliegen, werden Sie mit einer Dialogbox darauf hingewiesen. In diesem Fall müssen Sie im Gruppierungs-Formular einen neuen Datensatz generieren und diesen Datensatz über ,select group“ der aktuellen botanischen Einheit zuordnen. Den Namen der Gruppierung setzen Sie über „,copy name from systematik“ der botanischen Einheit gleich. Sie können dies mit einem Klick auf das Gruppierungssymbol neben dem Eintrag Anacolosa überprüfen; für diese Gattung existiert noch kein GruppierungsDatensatz.

Anmerkungen:

Sollten Sie das Gruppierungs-Formular ohne die Zuordnung zu einer botanischen Einheit schließen, können Sie die Zuordnung in dem sich öffnenden Dialogfeld nachholen.

Sie können einen Gruppierungs-Datensatz verschiedenen botanischen Einheiten zuordnen. Unterscheiden sich die Merkmale der Gattung z.B. wenig von denen der Familie, können Sie für beide einen identischen Datensatz verwenden (s. z.B. der Gruppierungs-Datensatz "Selaginellaceae, Selaginella"). 
Für die meisten Parameter gibt es mehrere Eingabemöglichkeiten. Dabei hat die erste (oberste) Eingabe die höchste Priorität. Relevant werden diese Angaben bei der Auswertung der Eingabetabellen (s. auch Kap. 3.10.5, S. 88), die als Sortierungsparameter, z.B. nach azonalen Elementen, die erste Eingabe mit der höchsten Priorität benutzten.

$\nabla$ no priority $\downarrow$

Sie können diese Priorität über „no priority“ im rechten oberen Formularbereich des Gruppierungs-Formulars aufheben; damit werden die Formen in eine eigene Gruppe unter ,undifferenziert" an das Ende der Sortierung der Eingabetabelle gestellt. Sinnvoll ist dies z.B. bei Familien/Gattungen, die an sehr vielen unterschiedlichen, gleichermaßen wichtigen Standorten auftreten können (z.B. Fagacaea, Betulacea, Oleaceae u.a.).

\section{$\therefore$}

Menüleiste Hilfe, Definitionen

Die Abbildungen 3.17 und 3.18 zeigen Formulare zur Definition der zonalen Vegetation und des Klimas, die über den Button auf dem Gruppierungsformular geöffnet werden können (siehe Abbildung 3.16).

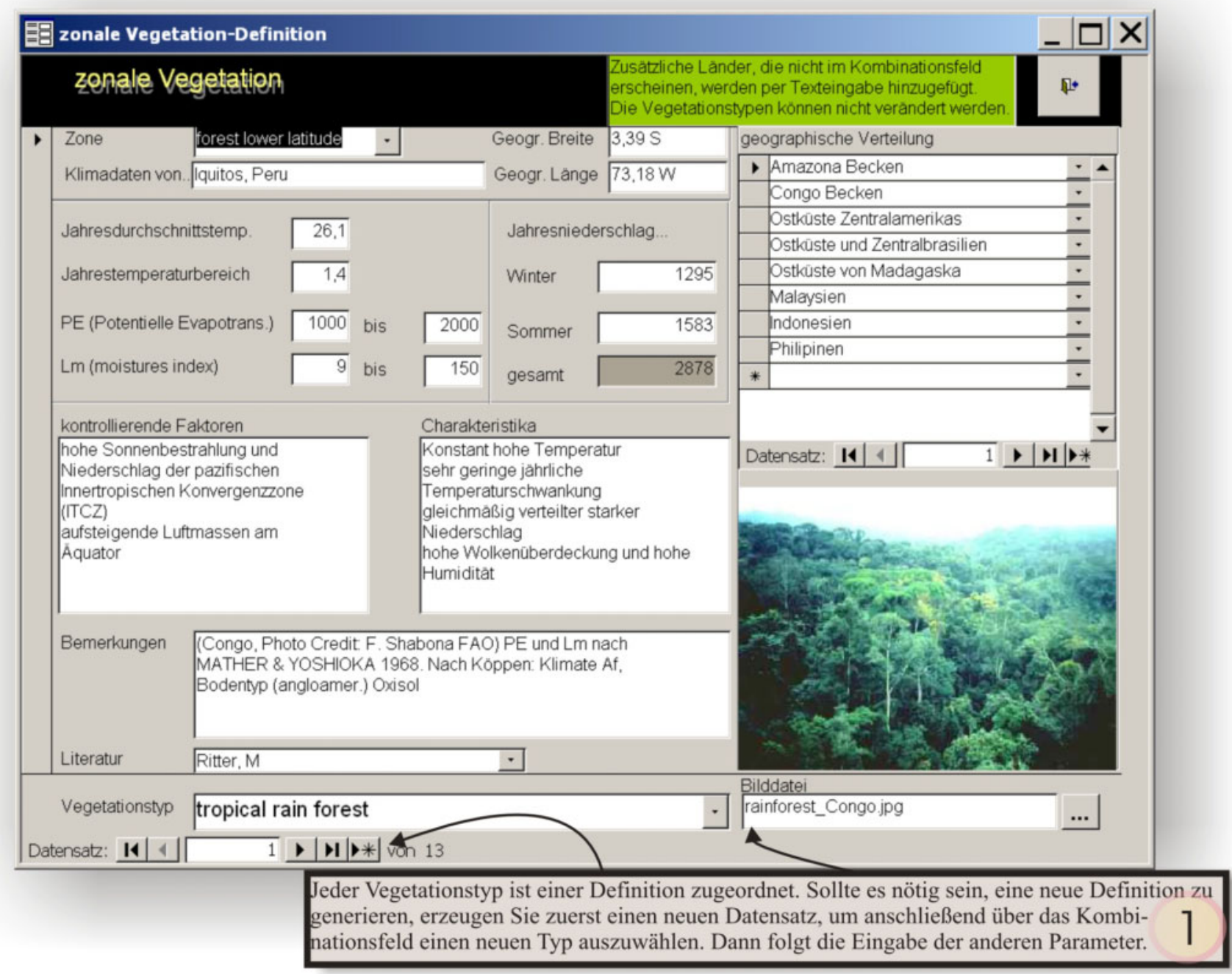

Abb. 3.17: Ansicht der Definition der zonalen Vegetation. Niederschlagsdaten, kontrollierende Faktoren und die geographische Verbreitung wurden mit diesem Formular erfaßt. 


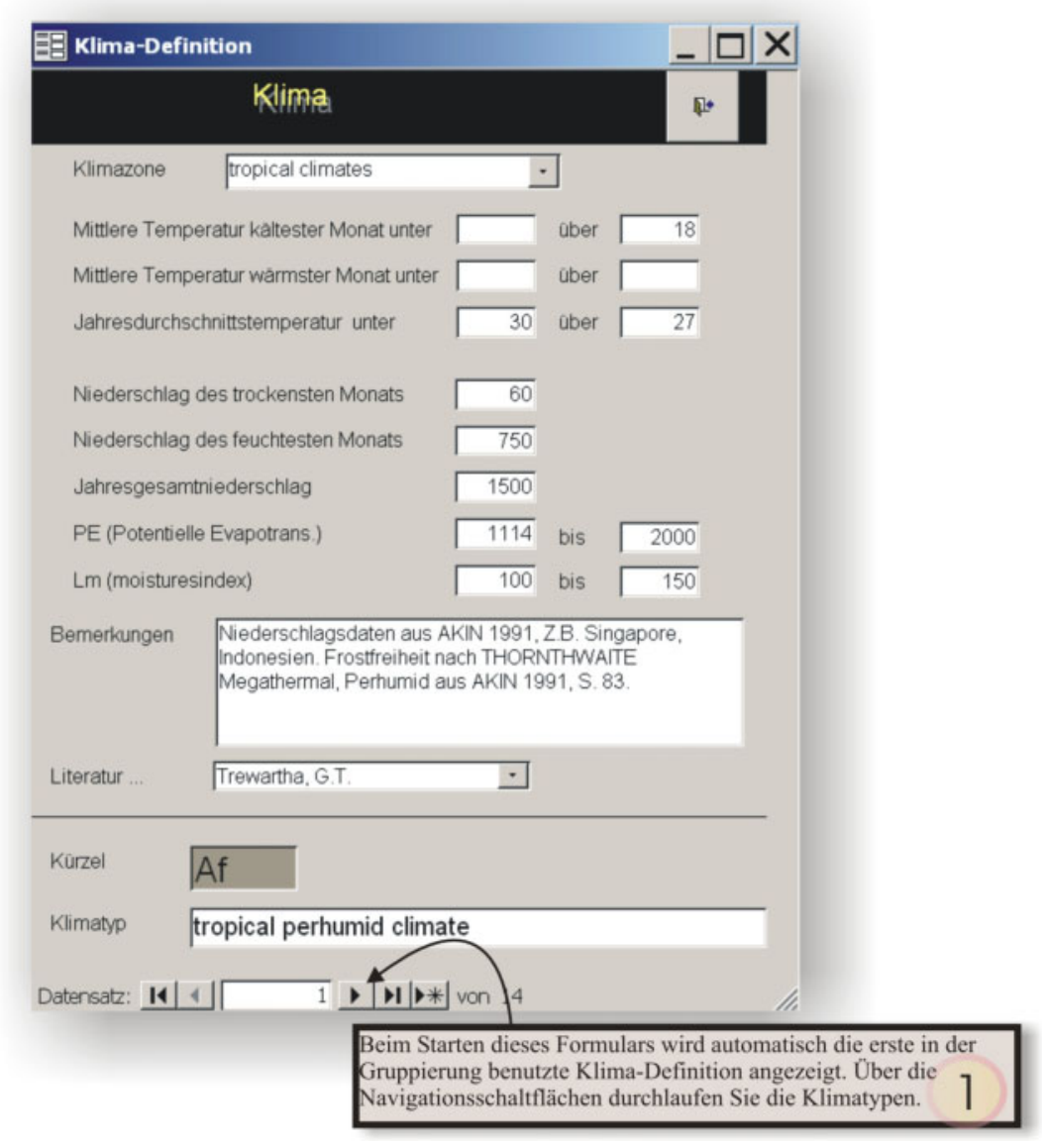

Abb. 3.18: Ansicht der Definition der Klimatypen. Temperaturen, Niederschlagsdaten, die potentielle Evapotranspiration und der Feuchtigkeitsindex wurden mit diesem Formular erfaßt.

\section{Anmerkungen:}

Daten aus den Definitionsformularen werden zum aktuellen Zeitpunkt der P.A.S.TEntwicklung nicht numerisch ausgewertet. Denkbar ist eine Verwendung dieser weiterführenden Daten im Rahmen der Auswertung botanischer und klimatischer Parameter der nächsten lebenden Verwandten über die Eingabetabellen (s.a. Kap. 3.10.5, S. 88). Das Gruppierungs-Formular exportiert durch P.A.S.T bis dato nur die Grunddaten des Klimatyps und des zonalen Vegetationstyps in die Eingabetabellen.

\subsubsection{Morphologie}

Morphologie

Tragen Sie nun die morphologischen Merkmale in das gleichnamige Formular ein, das Sie mit einem Mausklick in der Kopfzeile aktivieren. Die Größenangaben sind bei runden bzw. rundlichen Formen bei „Breite (x)“ einzutragen. Sollte ein Index, also ein durchschnittliches Breiten-/Höhenverhältnis bekannt sein, können Sie diese Angaben direkt neben der Größe eintragen (Abbildung 3.19).

Die Germinalien werden zuerst über die gleichnamige Optionsgruppe ausgewählt, daraufhin sind nur die relevanten Parameter rechts neben der Optionsgruppe sichtbar. Numerische Angaben der Poren- bzw. Colpenanzahl ersetzen dabei Angaben wie triporat, tricolpat etc. Zur Aufnahme der allgemeinen morphologischen Beschreibung ist ein Bemerkungsfeld im rechten oberen Formularbereich vorgesehen. 


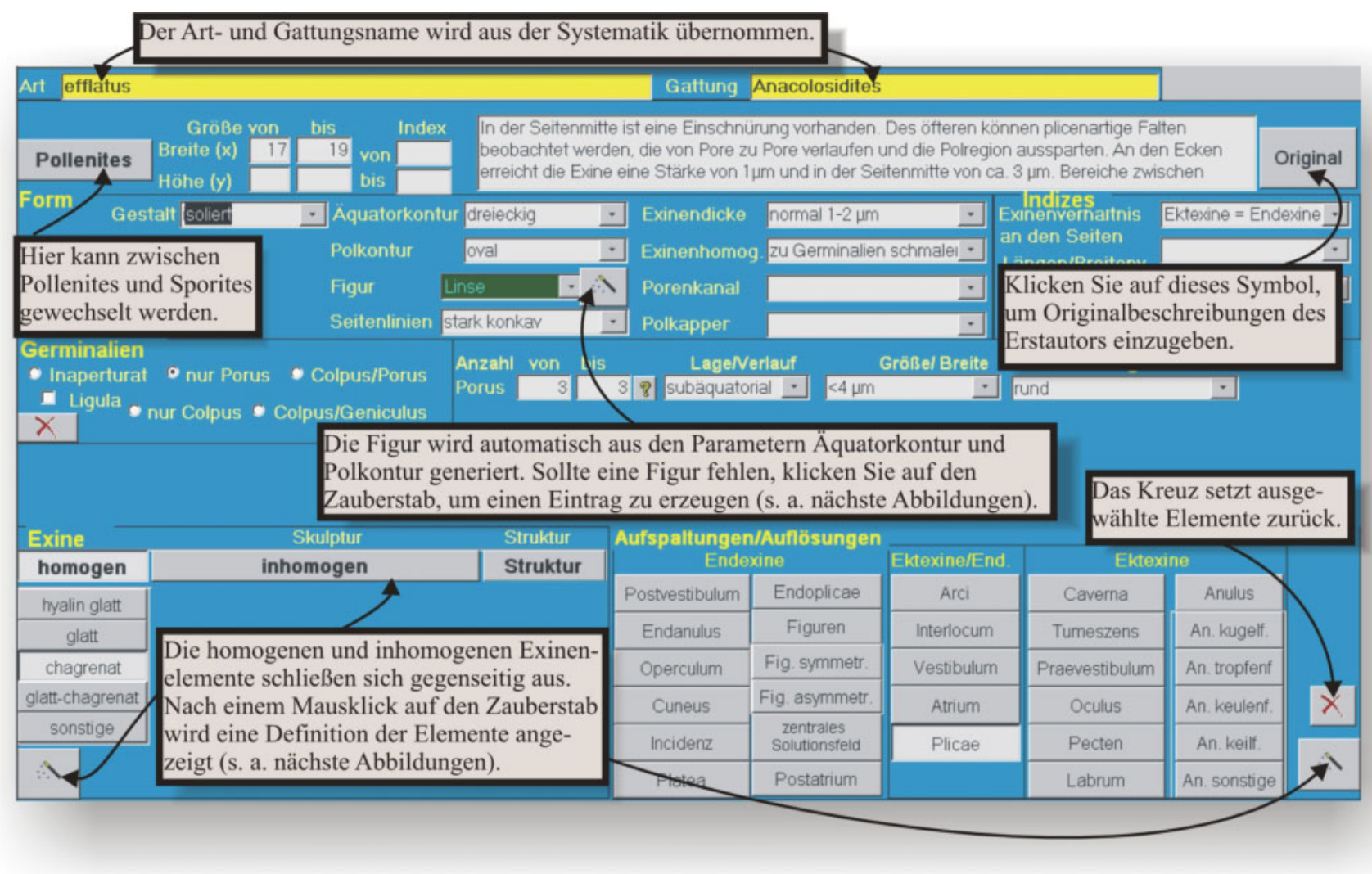

Abb. 3.19: Eingabe der Morphologie.

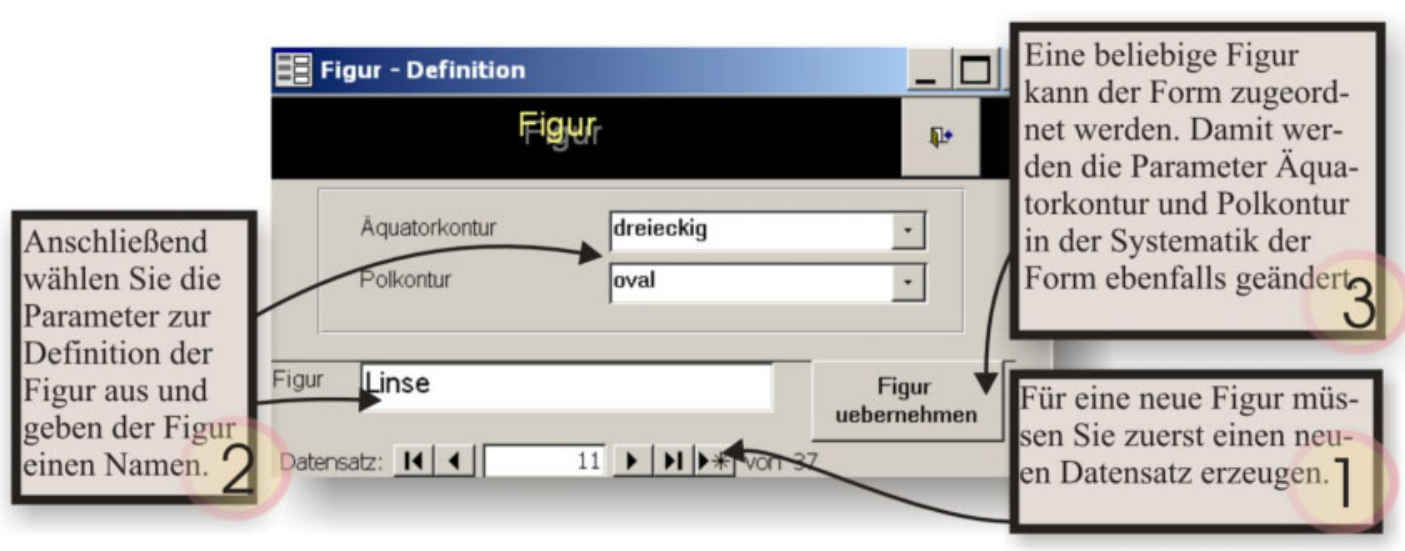

Abb. 3.20: Definition der Figur über die Parameter Äquatorkontur und Polkontur.

\section{Anmerkung:}

In der Literatur werden die Begriffe Äquatorkontur, Polkontur und Figur (oder Figura) nicht einheitlich verwendet. Insbesondere bei dem Begriff "Figur" sind Begriffskombinationen wie "ovale Figura" etc. nicht selten (Abbildung 3.20). 


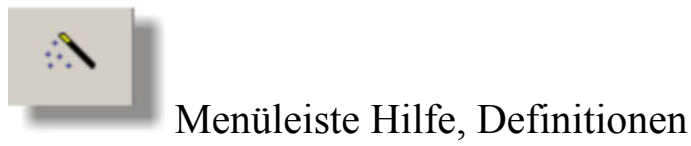

Aus diesem Grund wurde eine Figur-Definition in P.A.S.T integriert, um damit eine Fehlbeschreibung des morphologischen Merkmals zu vermeiden. Bei Textbeschreibungen der morphologischen Merkmale, die als Zitat aus der Literaturgrundlage (siehe auch Seite 43) zu verstehen sind, wurden diese Fehler, mit einem entsprechenden Hinweis, korrigiert.

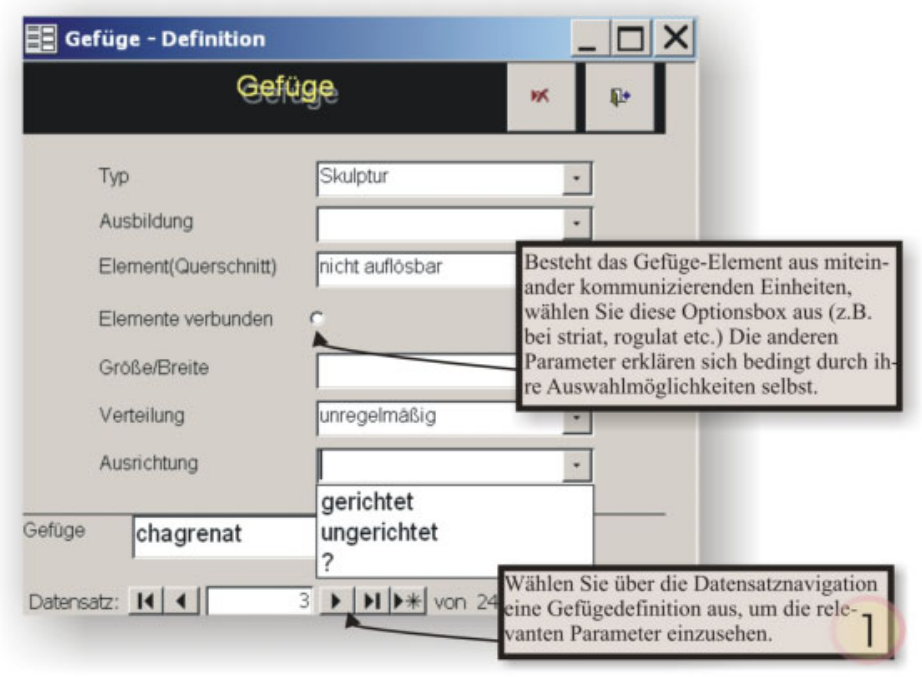

Abb. 3.21: Definition des morphologischen Gefüges über die Parameter Typ, Ausbildung, Elemente, Größe/Breite, Verteilung und Ausrichtung.

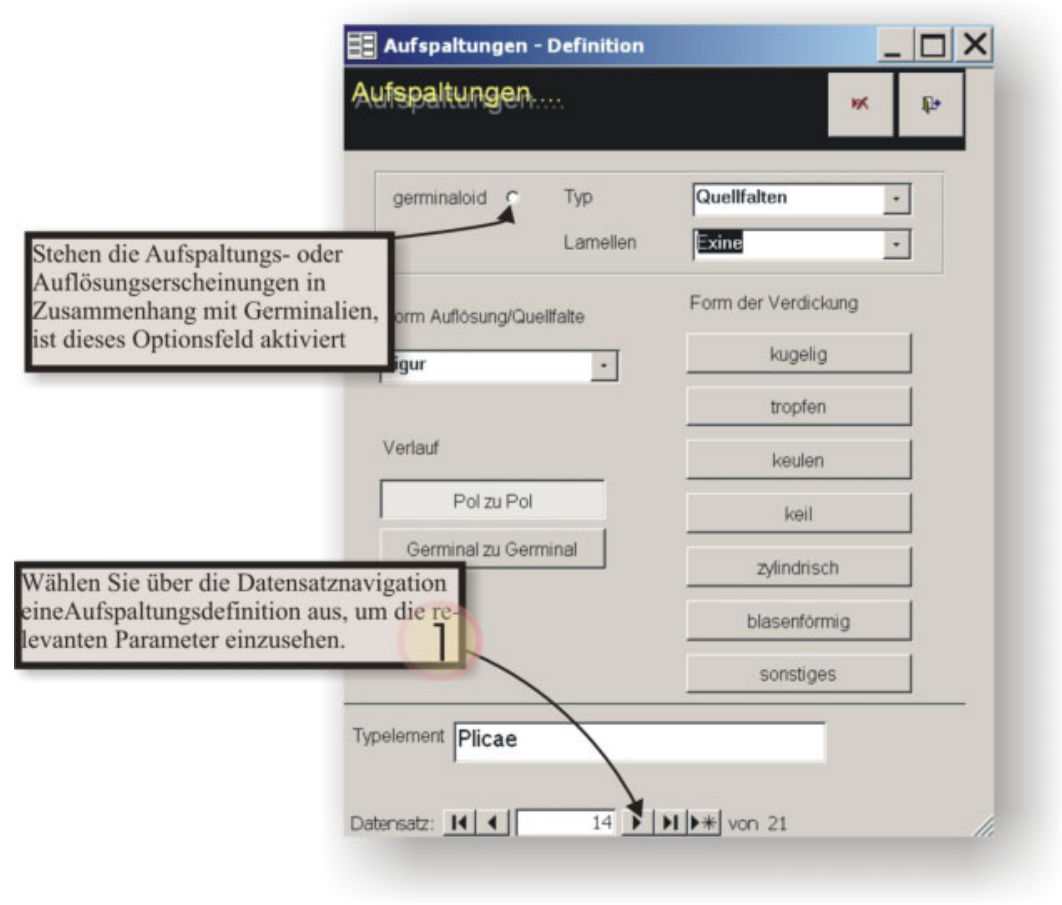

Abb. 3.22: Definition des morphologischen Aufspaltungen/Auflösungen/Quellfalten über die Parameter Typ, Lamellen, Form und Verlauf der Auflösung/Quellfalte sowie Form der Verdickung. 
Anmerkung:

Im Gegensatz zur Figur-Definition werden im Gefüge-Formular und AufspaltungsFormular keine Datensätze hinzugefügt, da die zur Verfügung stehenden Gefüge- und Aufspaltungsvariationen durch die Umschaltflächen auf dem Morphologie-Formular begrenzt sind. Beim Öffnen des Formulars wird automatisch die erste ausgewählte Variation als Datensatz angewählt (Abbildung 3.21 und 3.22).

\subsubsection{Erläuterung der Datenfelder zur Morphologie}

Größe (x und y)

Dieses Textfeld speichert die Größe der Form in $\mu \mathrm{m}$, wobei bei runden bis ovalen Formen die X-Koordinate für den Maximaldurchmesser einschließlich Skulptur angeben wird. X stellt die kurze und Y die lange Achse dar (z.B. bei Longaxonen). Die von-bis-Werte geben die Größenvariationen der Formen aus den Daten der Literaturgrundlage oder der Formen der in der Systematik eingetragenen Lokalitäten an.

\section{Größenindex}

Der Größenindex gibt das Breiten-/Längenverhältnis der Sporomorphae als von-bisWert an.

\section{Gestalt}

Mit Gestalt ist die morphologisch botanische Einheit der Pollen- oder Sporenkörner gemeint. Sie können als Individuum (,,isoliert" oder als Monade), paarweise (Dyade), als Gruppe von vier (Tetrade) oder als unbestimmte Anzahl (Polyade) vorkommen. Weitere Vorgaben dieses Kombinationsfeldes sind "saccate" Formen (Luftsack) oder "Massulae" (ein Verbund von Sporen- oder Pollenkörnern in einer Einheit).

Saccate Sporomorphae können über das bei dieser Einstellung sichtbare Kombinationsfeld in bisaccat (Pollenkorn mit zwei Sacci) und monosaccat (Pollenkorn mit einem einzelnen Saccus) unterteilt werden. Zusätzlich werden die bisaccaten Sporomorphae in Unterhalbkugel, Halbkugel, Überhalbkugel und Vollkugel-Formen unterschieden.

Äquatorkontur, Polkontur

Die Äquatorkontur ist die in der Polansicht sichtbare Umrißlinie; senkrecht dazu verläuft die Polkontur. Über das Kombinationsfeld sind die wichtigsten zweidimensionalen Konturformen ("rund", "oval" etc.) anwählbar. Unter "sonstige" werden Formen, die nicht den Vorgaben entsprechen, zusammengefaßt.

\section{Exinendicke}

Die Dicke der äußersten Wand der Sporomorphen kann in drei Größen, von dünnwandig $(<1 \mu \mathrm{m})$ über normal $(1-2 \mu \mathrm{m})$ bis dickwandig $(>2 \mu \mathrm{m})$, angeben werden. Besonderheiten der Exine sollten im Bemerkungsfeld angegeben werden. 
Exinenhomogenität

Die Exinendicke ist entweder gleichmäßig oder zu den Germinalien/Ecken breiter bzw. schmaler.

\section{Porenkanal}

Der Anulus schließt den Porenkanal schnabelartig ein. Er kann zylindrisch erscheinen oder sich nach außen oder innen erweitern.

\section{Polkappe}

In ca. $3 / 4$ der Höhe der Äquatorebene liegen die Polkappenebenen, die die Polkappen abtrennen. Die Konturen der Polkappen werden bei P.A.S.T in "abgeplattet", "unterhalbkugelig" und "halbkugelig" unterschieden.

\section{Seitenlinie}

Das zwischen den Polkappen gelegene Stück der Meridionalkontur wird als Seitenlinie bezeichnet, die "gerade", "schwach konkav", "stark konkav", "schwach konvex", "stark konvex" und "gebrochen konkav" ausfallen kann. Bei P.A.S.T wird der Begriff der Seitenlinie nicht nur bei Longaxonen (s. THOMSON \& PFLUG 1953) verwandt, so daß bei einer Bestimmungsuntersuchung mit dem Begriff der Seitenlinie z.B. auch triporate Formen und Sporen etc. gefunden werden können.

\section{Eckenverlauf (nur bei Sporites sichtbar)}

Um den charakteristischen Verlauf der Ecken bei Sporen zu erfassen, sind die Parameter "gespitzt", "abgerundet", "gestutzt" und "unterschiedlich" über das Kombinationsfeld wählbar.

\section{Exinenverhältnis an den Seiten}

Mit "Exinenverhältnis an den Seiten" ist das Verhältnis der äußeren Lamellengruppe (Ektexine) und der inneren Lamellengruppe (Endexine) gemeint, beide sind häufig durch einen Zwischenraum getrennt. Auch hier sind sinnvolle Vorgaben wie "unterscheidbar", "nicht unterscheidbar", "Ektexine=Endexine", "Ektexine<Endexine", "Ektexine>Endexine" anwählbar. In der Regel ist die Ektexine mächtiger als die Endexine.

\section{Längen-/Breitenverhältnis}

Das Längen-/Breitenverhältnis bezieht sich auf das Verhältnis der Länge der Pol(1)zur Äquatorachse(b). Es werden 5 Abstufungen mit Hilfe des Kombinationsfeldes unterschieden. 


\section{Porenkanalindex}

Das Verhältnis "Länge des Porenkanals zu Pollendurchmesser" (gemessen in der Achse des Porenkanals) wird als Porenkanalindex bezeichnet. Dieser kann mit größer und kleiner als 1 bzw. als unbekannt ("?") charakterisiert werden.

\section{Germinalien}

Eine Vorauswahl des Germinalapparates in "Inaperturat" (Germinalapparat fehlt), "nur Porus" "Colpus/Porus", "Ligula", "nur Colpus" und "Colpus/Geniculus" zeigt für den gewählten Typ relevante Kombinationsfelder an.

Pollenites

Porus

Anzahl der Poren

Die Anzahl der Poren kann zwischen 1 und einer beliebigen Anzahl angegeben werden.

Lage/Verlauf des Porus

"Äquatorial", "subäquatorial", "äquatorial/subäquatorial", "periporat", "distal/proximal" und ,latiporat" kennzeichnen die Lage des Porus.

Größe des Porus

Hier können die Klassen $<4,4-8,>8(\mu \mathrm{m})$ und "variabel" ausgewählt werden.

Form des Porus

Erlaubt sind die Parameter "rund", "meridional gestreckt", "äquatorial gestreckt", "rund/meridional gestreckt", "rund/äquatorial gestreckt", "rugenartig" und "rund unregelmäßig".

Colpus

Anzahl der Colpen

(s. Porus)

Lage/Verlauf des Colpus

"Parallel", "konvergierend", "divergierend", "percolpat" stehen zur Auswahl.

Größe des Colpus

Die Größe des Colpus bezieht sich auf den Porus und kann mit größer, kleiner, gleich dem Porus festgelegt werden.

Lage der Colpen

Erlaubt sind die Parameter "rund", "meridional gestreckt", "äquatorial gestreckt", "rund/meridional gestreckt", "rund/äquatorial gestreckt", "rugenartig" und "rund unregelmäßig"

\section{Länge der Colpen}

"1/3 der Pollänge", "2/3 der Pollänge", "bis Äquator", "bis $180^{\circ}$ umlaufend", "über $180^{\circ}$ umlaufend", "360 umlaufend" und "bis Pol" geben die Länge der Colpen an.

Sporites

Sporenmarke "von bis" 
Die Sporenmarke wird mit einer Zahl charakterisiert. Die einzeilige colpenähnliche Dehiszenz der monoleten Sporen wird mit 1 gekennzeichnet. Trilete Sporen erhalten die Kennzeichnung 3.

Läsurform der Sporenmarke

Die Differenzierung der Y-Leiste kann mit folgenden Merkmalen charakterisiert werden: "geradlinig", "wellig", "gerade", "geringe Aufspaltung","gerade, deutliche Aufspaltung", "wellig, geringe Aufspaltung","wellig, deutliche Aufspaltung".

Breite der Sporenmarke

Die wählbare Bandbreite reicht von "zart" über "kräftig (Lippen)" bis "kräftig (ohne Lippen)".

Länge der Sporenmarke

Die Längen der Dehiszenzmarke beziehen sich auf den größten meßbaren Radius (r) oder der Länge (L) der Sporomorphae: "1/3r (Trilet)", "2/3r (Trilet)", "3/4r (Trilet)", "4/5r (Trilet)" "1r (Trilet)", "bis Zona (Trilet)", "1/3L (Monolet), "2/3L (Monolet)", "3/4L (Monolet)", "4/5L (Monolet)", "1L (Monolet)".

Tori

Die Tori sind wulstartige Ein- oder Ausstülpungen von Exosporlamellen. Sie verlaufen parallel zur Y-Marke und lassen sich gliedern in: "Bandtori", "Feldtori", "Vollfeldtori", "Faltentori", "Bahnentori", "Pseudotori".

\section{Gefüge}

Mit dem Begriff des Gefüges werden Skulpturelemente (reliefbildende Teile der Exinenoberfläche) und Strukturelemente (Differenzierungen unterhalb der Exinenoberfläche) zusammengefaßt.

\section{Homogene Exine}

Die homogene Exine erscheint unter dem Lichtmikroskop nicht aus Teilchen zusammengesetzt.

Es werden "hyalin glatt" (völlig durchsichtig, stark reflektierend), "glatt", "chagrenat" (undurchsichtig) und Übergänge zwischen glatt und chagrenat unterschieden.

\section{Skulptur}

Inhomogene Exine

Die inhomogene Exine erscheint unter dem Lichtmikroskop aus Teilchen zusammengesetzt.

Punctat: Inhomogentiäten in Form punktförmiger Körper

Baculat: Stäbchen-Skulptur

Verrucat: Warzen-Skulptur

Gemmat: Perlchen-Skulptur

Clavat: Keulchen-Skulptur 
Granulat: Rundliche Körner unter $1 \mu \mathrm{m}$ Durchmesser

Echinat: Stachel-Skulptur

Rugulat: Verlängerte Skulpturelemente unregelmäßig angeordnet

Cicatricos: Parallele Leisten-Skulptur. Die Leisten (Muri) sind breiter als die Zwischenräume oder entsprechen in der Breite etwa den Zwischenräumen.

Hamulat: Ähnlich rugulat; Muri variierender Dicke, die kein Retikulum sondern ein Labyrinth-ähnliches Muster bilden.

Striat: Verlängerte gerichtete Skulpturelemente

Reticulat: Leisten zu Netzen angeordnet

Corrugat: Seitlich verwachsene Warzenzüge wechselnder Scheitelhöhe

Foveolat: Kreisrunde Gruben

Canaliculat: Vertiefungen in Form von parallelen Kanälen, die deutlich schmaler sind als die Zwischenräume zwischen ihnen

Struktur (Gefügeteilchen unterhalb der Exinenoberfläche)

Intrapunktat: Inhomogentiäten in Form punktförmiger Körper

Intrabaculat: Es sind deutlich Stäbchenschichten vorhanden

Intrastriat: Verlängerte Strukturelemente parallel, radial usw. angeordnet

Intrareticulat: Verlängerte Strukturelemente zu Netzen angeordnet

Intrarugulat: rundliche Körner unter $1 \mu \mathrm{m}$ Durchmesser

Abweichende Skulptur-/Strukturelemente (nur bei Sporites sichtbar)

Punktierung: Punktierung des proximalen Bereiches der Spore

Curvatura: Eine Linie bei Trileten Sporen, die sich von den Enden der Laesura erstreckt und die Kontakt-Area abgrenzt

Kontakt-Area (proximal): Bereich der proximalen Seite der Spore, deren Entstehung durch den Kontakt mit anderen Mitgliedern der Tetrade interpretiert wird.

Strukturierung: Strukturierung des distalen Bereiches der Spore.

Als Auflösungen werden die am Germinalapparat lokalisierten Poren und Colpen unterschieden. Großflächige Auflösungszonen, die weit über den Germinalapparat hinausgreifen können, sind das Atrium und der Solutionsmeridian.

Atrium

Ist die Endexine in der Porenregion weitgehend aufgelöst, so daß ein Endoporus entsteht, der mehr als dreimal so groß ist wie der Endoporus, so spricht man von einem Atrium. 
Aufspaltungen in der Ektexine

Der Anulus ist ein „ringförmige Aufblasung“ der Exolamelle $d$ in der Porenregion (Thomson \& Pflug 1953). Es werden verschiedene Formen des Anulus unterschieden: "kugel-", "tropfen-", "keil-" und "keulenförmig" sowie undifferenziert (nur "Anulus").

Das Praevestibulum ist eine interlamellare Aufspaltung der Ektexine, die mit der Pore kommuniziert.

Als Tumeszenz bezeichnet man eine keilförmige Verdickung der Ektexine in Richtung der Porenregion.

Pecten sind Abspaltungen in der Ektexine unter Verlängerung der Stäbchenelemente.

Stülpt sich die unverdickte Ektexine an den Poren "schnauzenartig" aus, spricht man von einem Labrum.

Der Oculus ist eine Verlängerung der Exolamelle in eine ringförmige circumporale Zone und ähnelt dem Anulus, erscheint im Grundriß jedoch umfangreicher als dieser.

Aufspaltung zwischen Ektexine und Endexine

Das Vestibulum ist eine Aufspaltung, die am Germinalapparat lokalisiert ist. Dabei verläuft die Endexine nach der Abspaltung weiter und schließt einen Endoporus ein, der nicht mehr als dreimal größer als der Endoporus ist (s. auch Atrium)

Das Interlocum ist ein Zwischenraum zwischen Ekt- und Endexine, der im ganzen oder nahezu im gesamten Exinenkörper entwickelt ist.

Als Arci werden primäre schlauchförmige Abspaltungen, die benachbarte Vestibula girlandenartig verbinden, bezeichnet.

Bei P.A.S.T werden Endoplicae und Plicae unter "Plicae" zusammengefaßt, da sich eine Unterscheidung nicht immer sicher durchführen läßt. Endoplicae sind schmale durch Quellung entstandene Schläuche zwischen Ekt- und Endexine, die von Atrium zu Atrium verlaufen. Plicae sind Verdopplungen der gesamten Exine.

Eine Caverna ist eine meridionale Aufspaltung.

Aufspaltungen in der Endexine

Der Endanulus ist eine gegen die Pore abgeschlossene "Aufblasung" der Endexine.

Ein Postvestibulum bezeichnet eine Aufspaltung in den Endolamellen, die mit der Endopore kommuniziert.

Das Postatrium entspricht dem Atrium (s. oben), mit dem Unterschied, daß nur die Endexine interlamellar aufgespalten ist. 
Solutionsmeridien

Darunter fallen Auflösungprozesse der Endexine, die unterschiedlich weit in meridionaler Richtung erfolgen.

Als Incidenz wird eine geringe Auflösung, die als Kerbe sichtbar ist, gekennzeichnet.

Der Cuneus bezeichnet eine keilförmige und die Platea eine bahnenförmige Auflösung der Endexine.

\subsubsection{Bildinformationen}

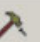

(Menüleiste Formulare: Bilder) Als letztes folgt die Eingabe der Bildinformationen; dazu klicken Sie auf das Bildsymbol in der Kopfzeile. Nach dem Öffnen des Bilder-Formulars wählen Sie das passende Bild über „Bilder eingeben“ und der sich öffnenden Dateiliste aus (Abbildung 3.23).

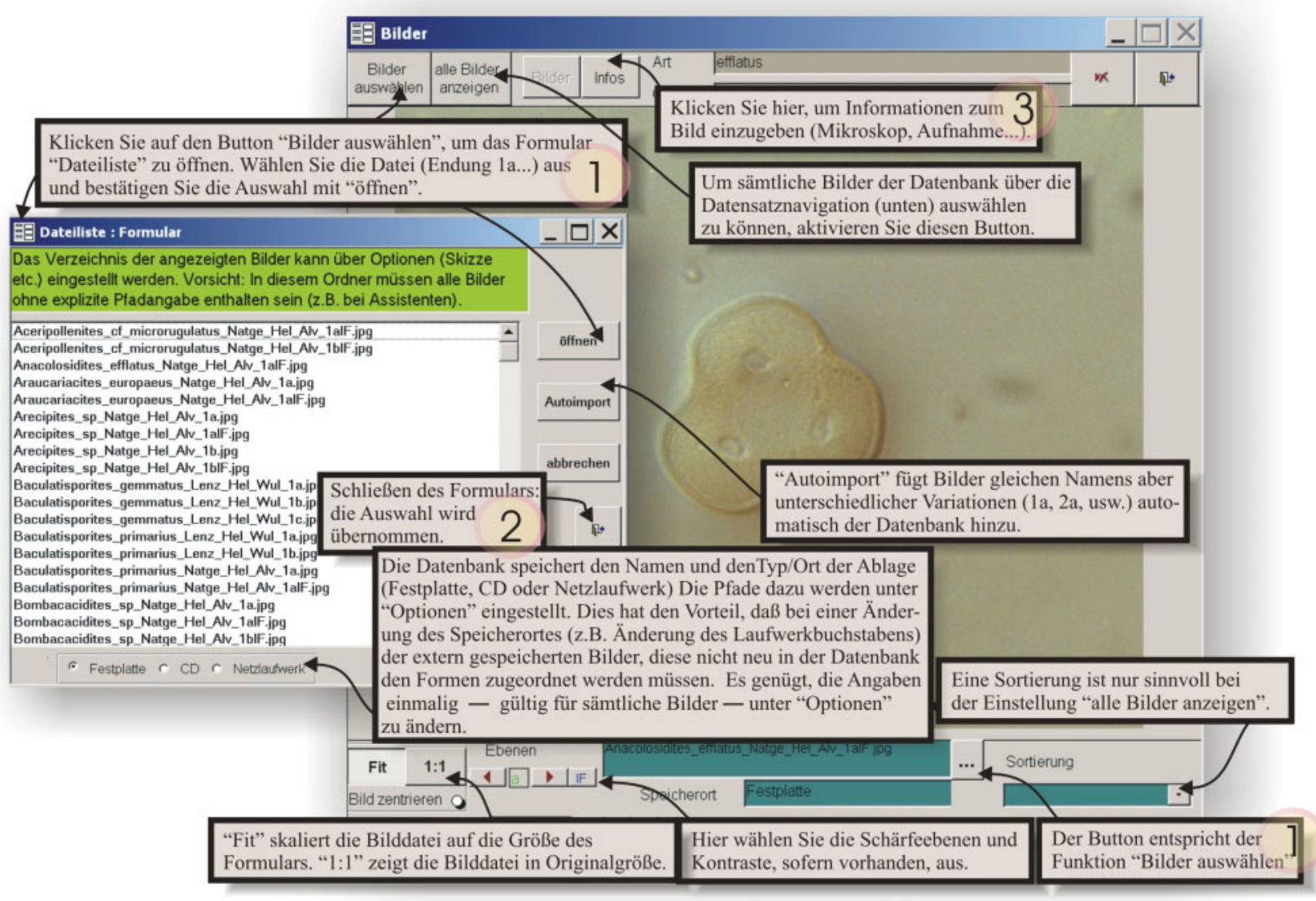

Abb. 3.23: Auswahl der Bilddatei.

Infos

Nachdem Sie die Dateiliste geschlossen haben, können Sie über einen Mausklick auf den „Infobutton“ die Bildbeschreibung eintragen (Abbildung 3.24). Diese Informationen lassen sich im Abbildungsformular gezielt für jedes dargestellte Bild abrufen (s. auch Kap. 3.10.3.1, S. 83 f.). 


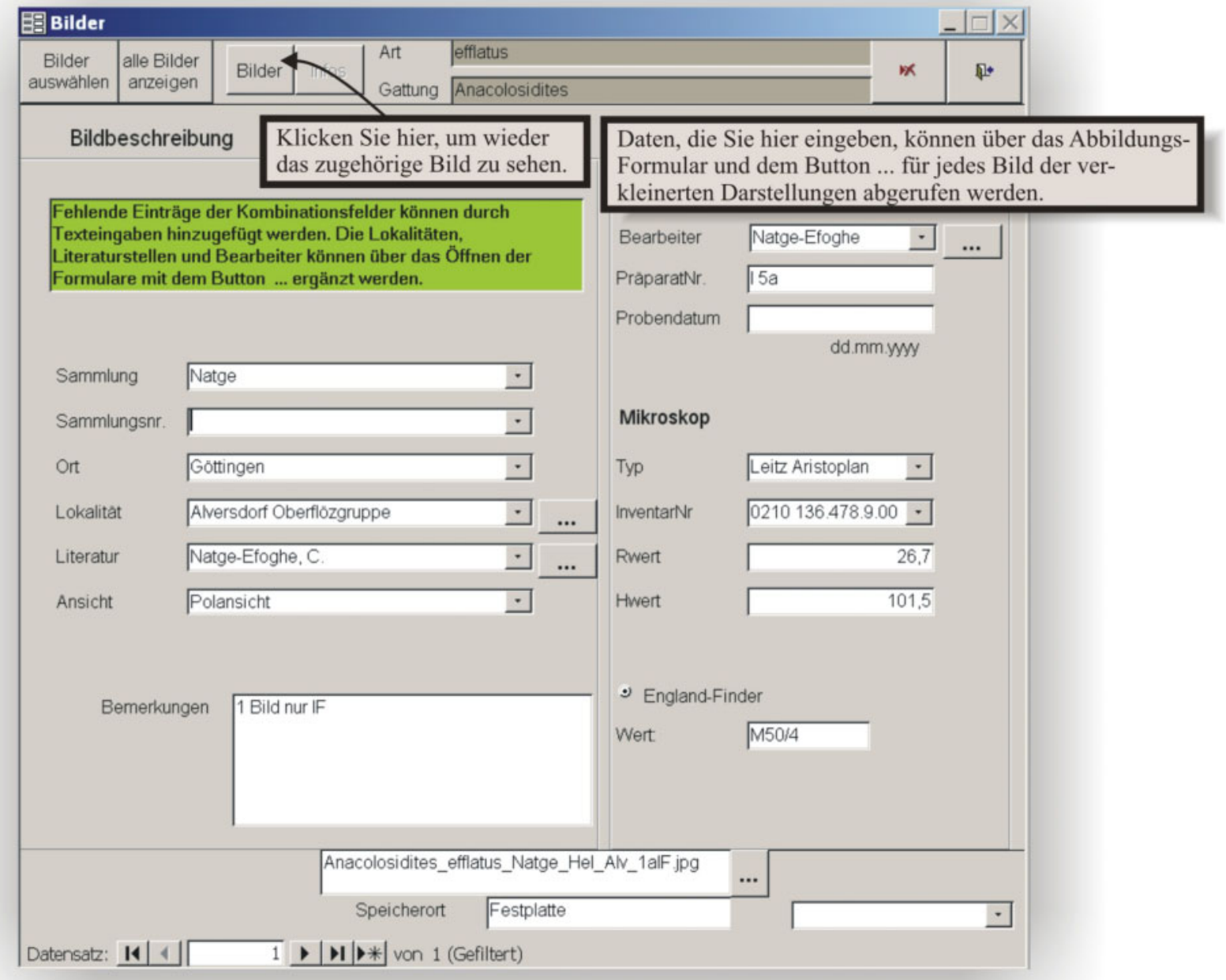

Abb. 3.24: Eingabe der Bildbeschreibung.

\subsection{Eingabe Sporomorphae-unabhängiger Daten}

P.A.S.T verarbeitet neben den Sporomorphae-relevanten Daten auch Informationen über Literaturzitate und Lokalitätsangaben. Im Eingabebeispiel der Sporomorphae fand der Verweis auf diese Tabellen über Kombinationsfelder bereits statt (Kap. 3.6, S. 42). Nachstehend wird die Eingabe einiger Beispieldaten zu der Literatur und den Lokalitäten näher erläutert. 
Menüleiste Formulare: Literatur

Als Eingabedaten zur Literatur benutzten Sie bitte folgende Informationen. Dazu gehören Angaben zur Literatur, zu den Bemerkungen, zur Inhaltsangabe (verkürzt) und zu Stichwörtern.

\subsubsection{Literatureingabe}

\section{Beispieldaten:}

Literaturstelle:

BocK, H.H.(1974): Automatische Klassifikation: theoretische und praktische Methoden zur Gruppierung und Strukturierung von Daten; (Clusteranalyse). - Vandenhoeck \& Ruprecht, 480 S.; Göttingen.

Bemerkungen:

graph. Darst. Serie: Studia mathematica ; 24, Literaturverz. S. 435-467

Inhaltsangabe:

Kapitel 9. Hierarchische Gruppierung. Problem der Festlegung des Schwellenwertes bei der Wahl der Anzahl der Klassen S. 357.

Stichwörter:

Clusteranalyse, Excelprogrammierung

Erzeugen Sie vor der Eingabe einen neuen Datensatz. Auch wenn der einzugebende Datensatz schon vorhanden ist, überprüfen Sie dies über die „Auswählen“Funktion des Literaturformulars (s. auch Abbildung 3.25), fahren Sie trotzdem mit der Eingabe fort (Abbildung 3.25).

Sie können das erzeugte Duplikat nach der Testeingabe über das „Löschen“-Symbol am oberen Formularrand entfernen.

Die Ausgabe von Literaturlisten nach Microsoft Word wird im Kapitel 3.10.4, S. 86 beschrieben. 


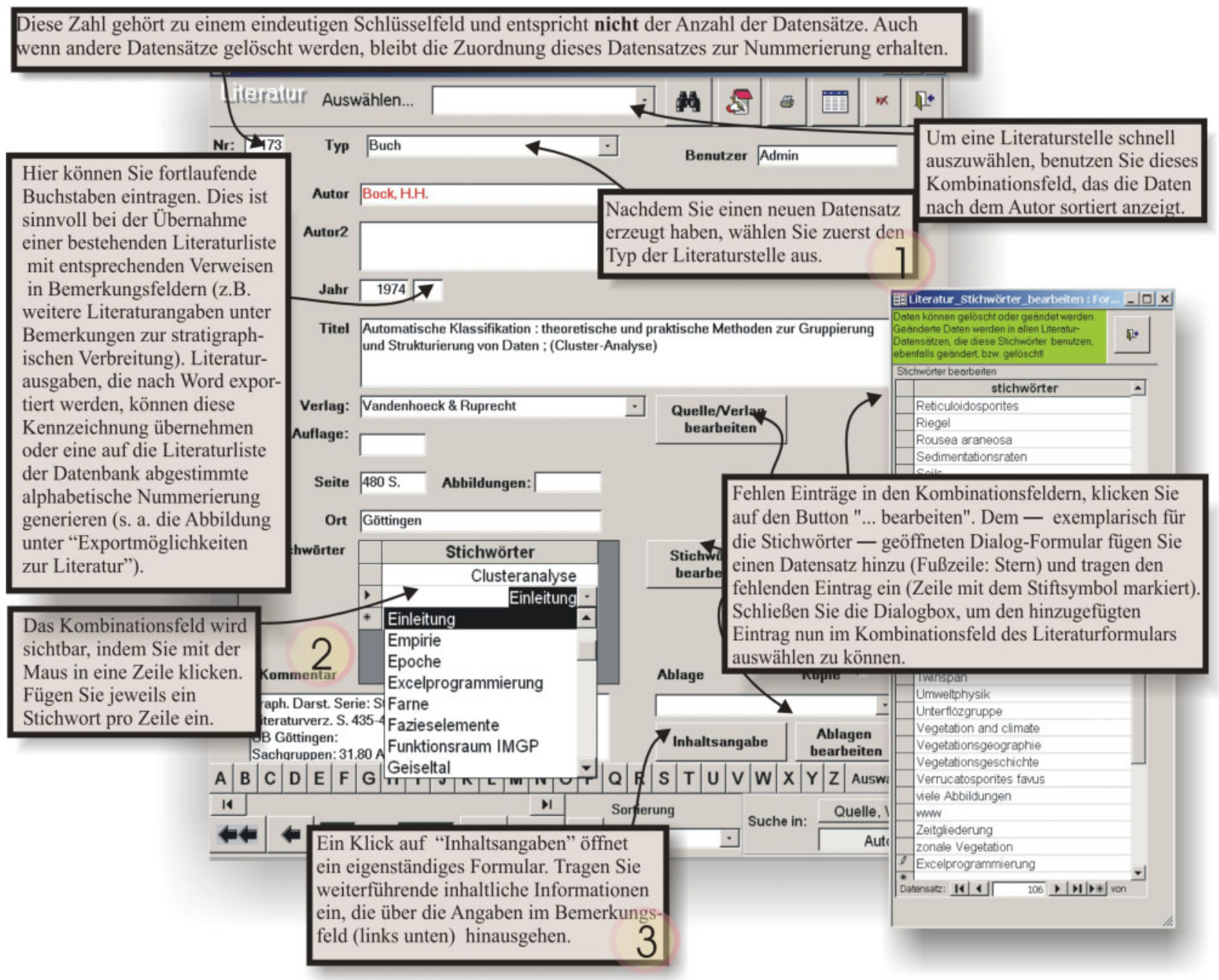

Abb. 3.25: Eingabe der Literatur.

\subsubsection{Lokalitätseingabe}

Menüleiste Formulare: Lokalität

Lokalitätsangaben werden zur Beschreibung der stratigraphischen Reichweite der Sporomorphae und den Typuslokalitäten der Synonyme benötigt. Neben diesen eher rudimentären Angaben, die in erster Linie nur den Namen und die Reichweite der Lokalitäten betreffen, können weiterführende stratigraphische Informationen bis zur detaillierten Beschreibung von Profilen im Formular Lokalität erfaßt werden (Abbildung 3.26). Diese Profilangaben können in Kombination mit SporomorphaeListen nach Excel exportiert und graphisch umgesetzt werden (dazu mehr im Kap. 3.10.5, S. 88).

Als Eingabedaten benutzten Sie Informationen wie: Angaben zum Namen der Lokalität mit weiterführenden Informationen, zur stratigraphischen Reichweite, zu den Einheiten, Horizonten und Bearbeitern mit Profilen (sämtliche Angaben verkürzt).

**

Erzeugen Sie vor der Eingabe einen neuen Datensatz. Auch wenn der einzugebende Datensatz schon vorhanden ist, überprüfen Sie dies über die „Auswählen“- 
Funktion des Lokalitäts-Formulars (s. auch Abbildung 3.26), fahren Sie trotzdem mit der Eingabe fort. Sie können den Test-Datensatz nach der Eingabe über das „LöschSymbol“" problemlos entfernen.

\subsubsection{Beispieldaten (ohne Lithologie):}

Name:

\section{Ort/Land:}

\section{Alversdorf Oberflözgruppe}

Helmstedt, Westmulde

Typ:

Tagebau

Rechtswert:

104432

Hochwert:

525779

Epoche:
M. Eocene

Einheiten:

Victoriaflöz

Reichweite

Mächtigkeiten

Unteres Zwischenmittel

M. Eocene

$1050 \mathrm{~cm}$

$1400 \mathrm{~cm}$

$400 \mathrm{~cm}$

Unterflöz

Horizont des Unterflözes:

\section{",}

Flöz

Bearbeiter des Flözes (UF)

Schiemann

10443230
Environment

limnisch

Rechtswert ungef. Mächtigkeit

400

$\begin{array}{lll}\text { Hochwert } & \text { Profil } & \text { Beprobung } \\ 52577940 & \text { Profil des UF } & 1990(1994)\end{array}$

Dieses Kombinationsfeld bietet eine Einsicht in die gespeicherten Lokalitäten — nach Namen sortiert. Ei Klick auf einen Namen aktiviert die Lokalitätsstelle. Mit der Taste" Escape" wird das Kombinationsfeld ohne Aktivierung der Lokalitätsstelle verlassen.

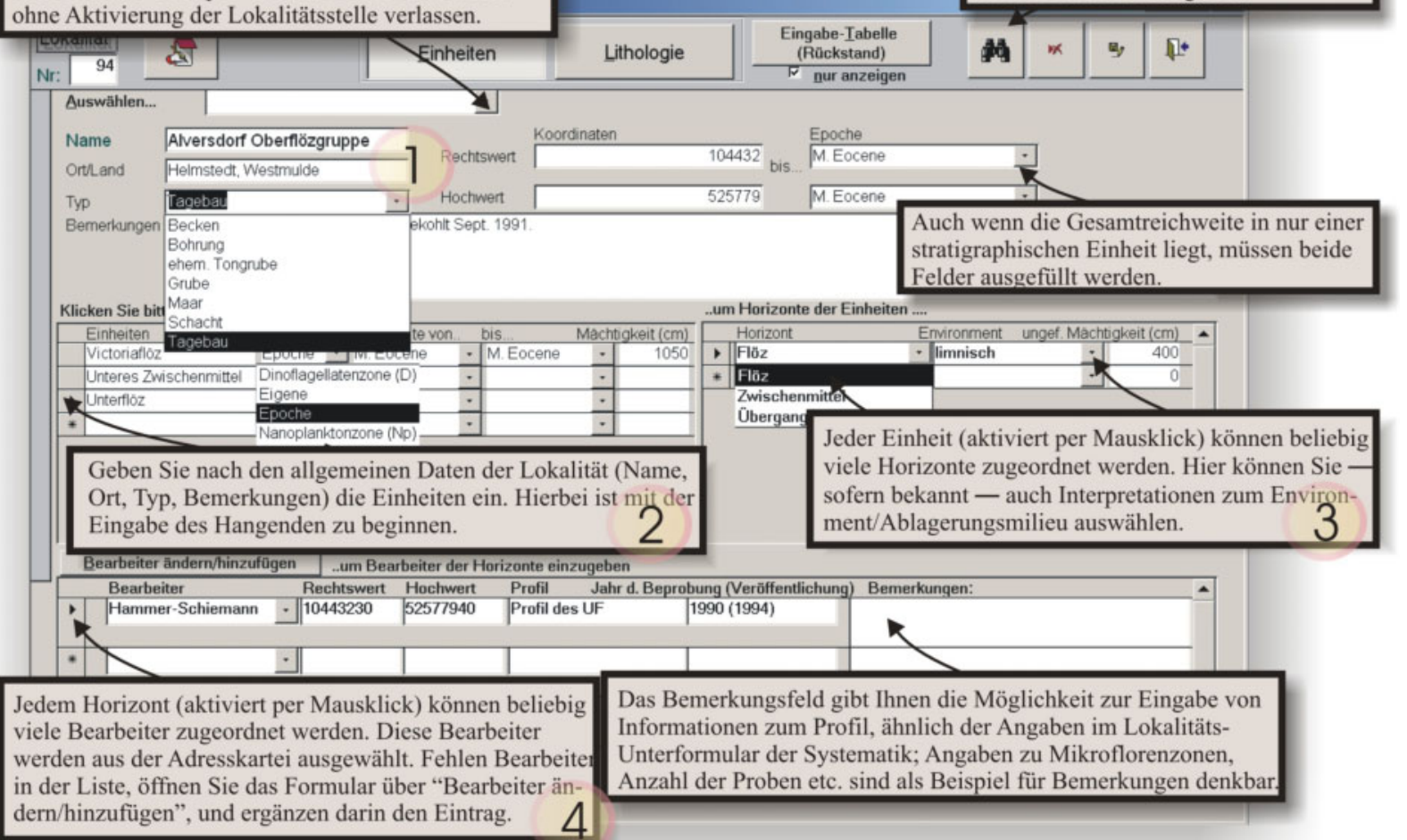

Abb. 3.26: Eingabe der Lokalitätsdaten. 
Anmerkung:

Aufgrund der Komplexität der miteinander verbundenen Unterformulare kann es in seltenen Fällen zu Fehlermeldungen bei der Eingabe kommen. Drücken Sie in diesem Fall die „Escape“ Taste, um die Eingabe rückgängig zu machen. Bei schwerwiegenden Fehlern schließen und öffnen Sie das Lokalitäts-Formular, und wiederholen Sie anschließend die Eingabe (Abbildung 3.27).

\subsubsection{Beispieldaten (Lithologie):}

Einheiten Lithologie

Nach der Eingabe der Beispieldaten zur Lokalität besteht bei aktiviertem Bearbeiter/Profil die Möglichkeit, über den Lithologie-Button (Kopfzeile des Lokalitäts-Formulars) zur Lithologie zu wechseln.

Folgende Daten können als Eingabebeispiel zur Lithologie verwendet werden.

(Mit der Basis beginnend....)

Nr.1 Grundmassenkohle, $70 \mathrm{~cm}$ mächtig, Probenentnahme an der Basis, Farbe dunkelbraun

Nr.2 Sand, $35 \mathrm{~cm}$ mächtig, horizontal geschichtet, feinlameniert, Probenentnahme nach $17 \mathrm{~cm}$, Bemerkungen: Kohlegerölle, Farbe hellbraun

Nr.3 Kohliger Sand 7 cm, keine Schichtung, Farbe schwarz

Nr.4 Sand, $65 \mathrm{~cm}$, keine Schichtung, Probenentnahme nach $30 \mathrm{~cm}$, Bemerkungen: an der Basis Kohlegerölle, Farbe hellbraun, mit zoogener und phytogener Bioturbation

Nr.5 Ton, $18 \mathrm{~cm}$, Farbe grau

Anmerkung:

Access bietet keine Möglichkeit, in einem Endlos-Formular die Dateneingabe von unten nach oben durchzuführen. Andererseits erschien es wenig sinnvoll, bei der Eingabe mit dem Top, und damit entgegen der Gelände-Aufnahmerichtung, zu beginnen. Bei der tabellarischen Ausgabe nach Excel wird diese Darstellung übernommen, erst das graphische Profil in Excel zeigt mit Hilfe der Profilausgabe die richtige Orientierung (siehe auch Kap. 3.10.5, S. 88). 


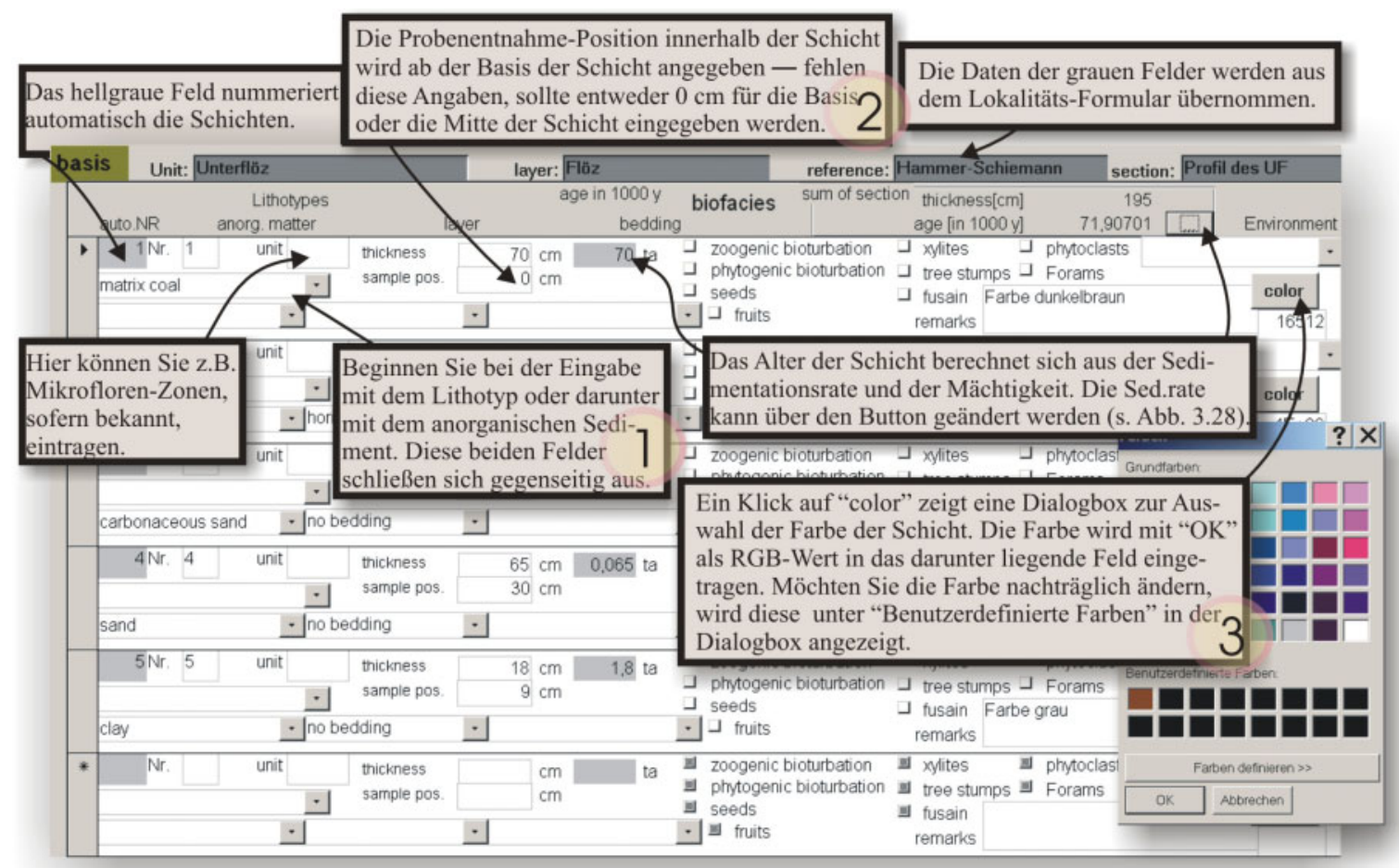

Abb. 3.27: Eingabe der Lithologiedaten im Rahmen des Lokalitäts-Formulars.

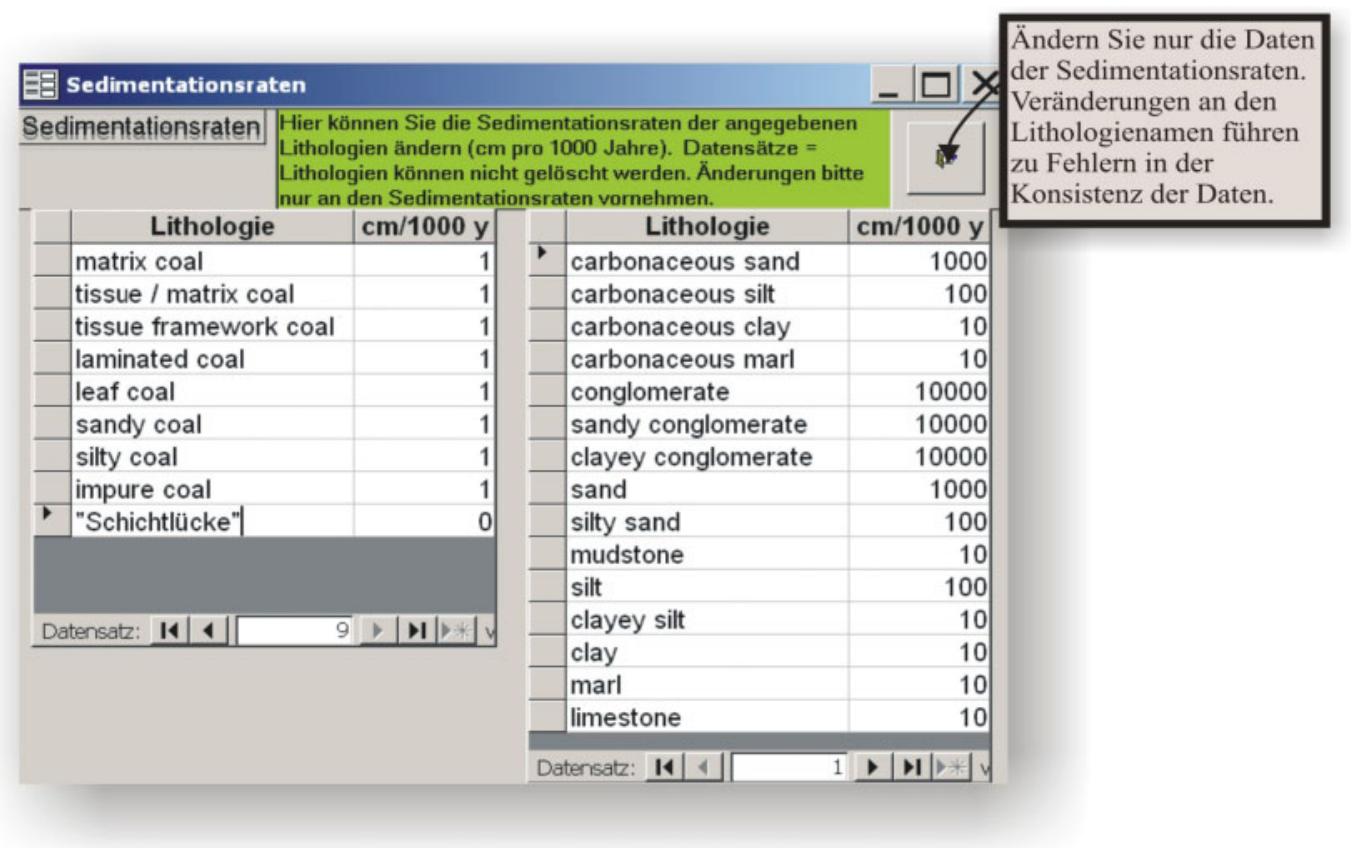

Abb. 3.28: Änderungsmöglichkeit der Sedimentationsraten.

\section{Anmerkung:}

Die angebenden Sedimentationsraten sind nur als Richtwerte $\mathrm{zu}$ verstehen. In Abhängigkeit vom Ablagerungsmilieu sind hier sehr starke Schwankungen möglich.

Um dieser Problematik eine adäquate Möglichkeit der Datenverarbeitung entgegen zu setzen, können für jedes Profil unterschiedliche Alter der Einzelschichten, auf der Basis der aktuell eingestellten Sedimentationsraten, gespeichert werden. Beim 
Schließen des oben beschriebenen Formulars können Sie eine neue Berechnung der Alter des aktuellen Profils durchführen lassen.

Sollten Sie Sedimentationsalter für verschiedene Profile mit unterschiedlichen Sedimentationsraten berechnet haben, können Sie nur die letzte Einstellung der Sedimentationsraten aufrufen, da die Sedimentationsraten im Gegensatz zum Sedimentationsalter nur einmal gespeichert werden.

\subsection{Datenbankmodulierungen}

Wie schon am Anfang dieses Kapitels erwähnt, folgen nun Beispiele einiger weiterführender Möglichkeiten der Datensuche, -Manipulation und -Anzeige. Dazu gehören umfangreiche Möglichkeiten der Sortierung von Daten, dem Filtern von Daten, dem Abspeichern dieser Filter, einer Suche über stratigraphische Reichweiten und dem Bilden von Sporomorphae-Gruppen, die zur weiteren Bearbeitung nach Excel übertragen werden können.

\subsubsection{Datensuche über Texteingaben}

\section{(1)}

Menüleiste Bearbeiten: Suchen....Text

Mit der speziell für P.A.S.T programmierten Suchfunktion können Sie nahezu sämtlicher Datenfelder der Datenbank durchsuchen lassen. Starten Sie die Suchfunktion aus dem Systematik-Formular heraus. Geben Sie, um weiterführende Suchoperationen kennenzulernen, bitte in die Suchfelder die Begriffe ,tripora“ und „tricolpa“ ein (die unvollständige Schreibweise ist durchaus erwünscht) und klicken Sie auf ,Suchen“.

Anmerkung:

Sie können über die Optionsgruppe am oberen Formularrand das zu durchsuchende Formular angeben (Literatur, Lokalitäten, Gruppierung). In diesem Beispiel schränken Sie die Suche nur auf die Systematik und die Morphologie ein.

Anzeige im Hauptormular als
Auswah lobernehmen

Möchten Sie die gefunden Sporomorphae der Reihe nach betrachten, bieten sich zwei Möglichkeiten an. Sie können, wie schon im Kapitel 3.6.1, S. 44 beschrieben, über die graue Leiste am linken Rand die gesuchte Form im SystematikFormular aktivieren. 


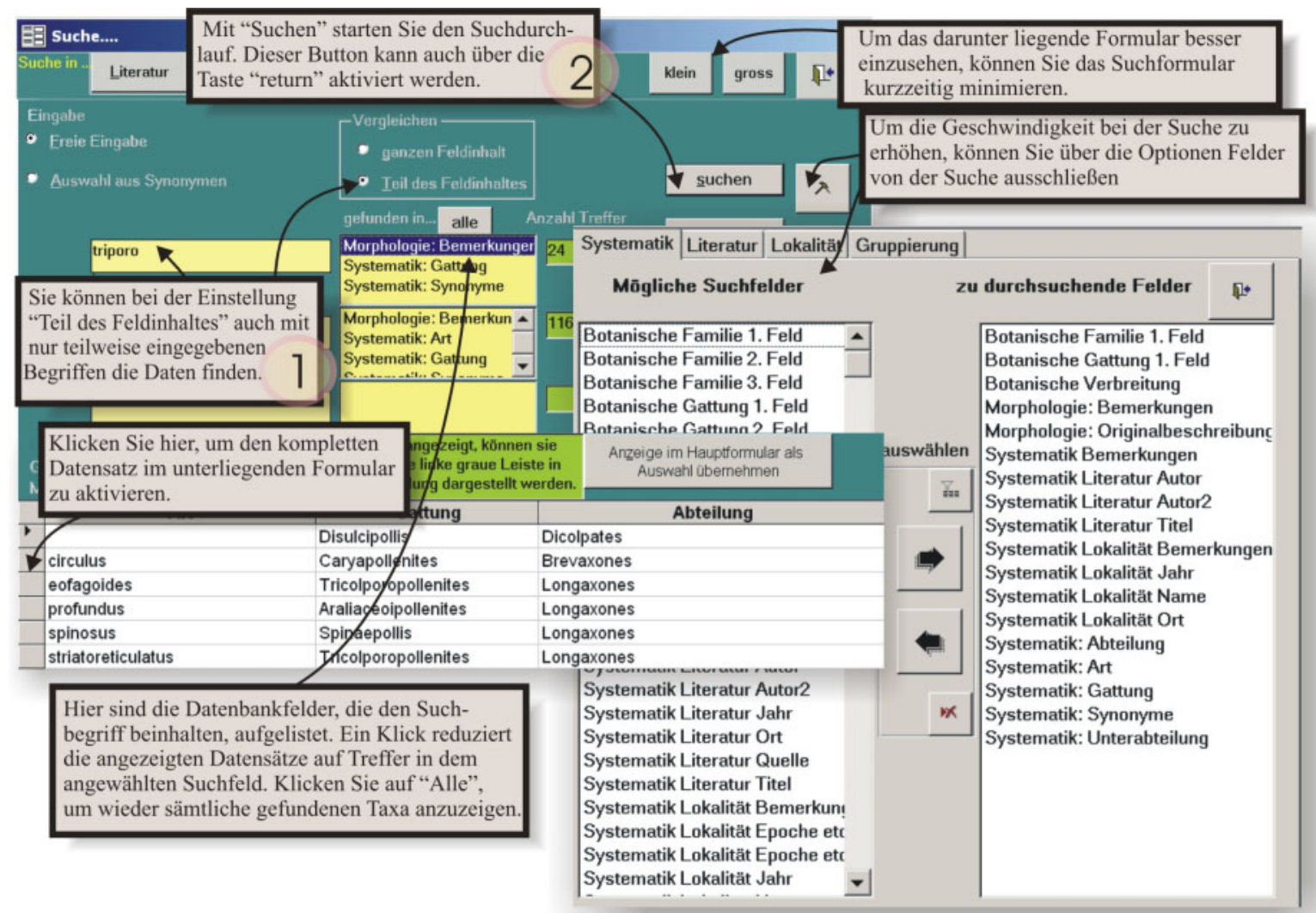

Abb. 3.29: Suchoperationen erleichtern das Finden von Datensätzen.

Weiterhin besteht die Möglichkeit, die gefundenen Sporomorphae komplett als Auswahl in das zugrundeliegende Formular zu übertragen. Dazu klicken Sie auf „Anzeige im Hauptformular als Auswahl übernehmen“.

Über „Filter“ in der Fußzeile des Systematik-Formulars wählen Sie daraufhin diese Auswahl an. Damit wird der Datenpool auf die gefunden Sporomorphae beschränkt. Ein Hinweis für ausgewählte Sporomorphae stellt die grüne Leiste am linken Rand des Formulars dar. Ein Klick auf diese Leiste entfernt Sporomorphae aus der Auswahl oder fügt sie der Auswahl wieder hinzu (s. a. Abbildung 3.30). 


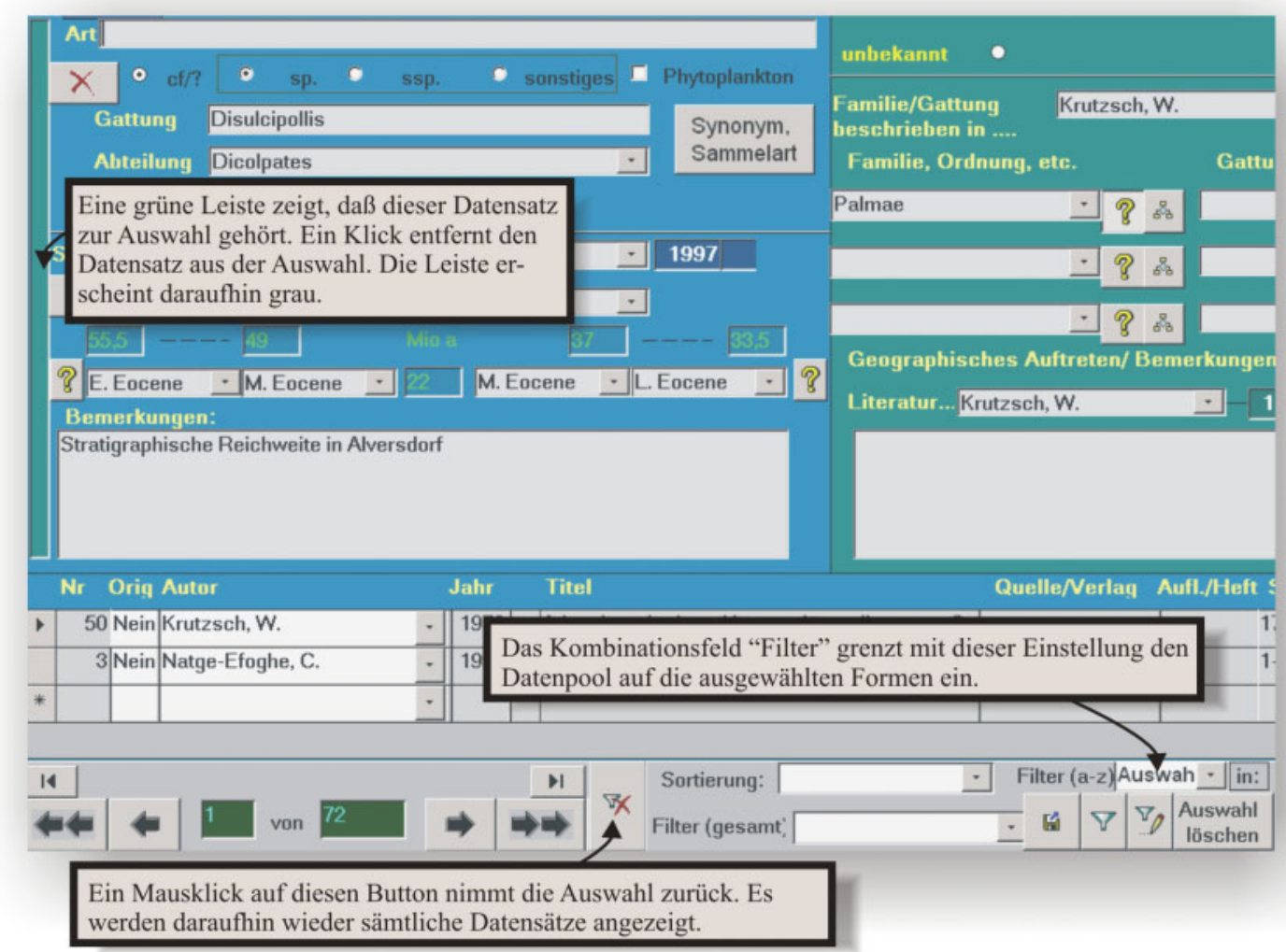

Abb. 3.30: Auswahl der Sporomorphae im Systematik-Formular.

\subsubsection{Datensuche über den ,Formularbasierten Filter“}

(Menüleiste Datensätze: Formularbasierter Filter) Formularbasierte Filter werden standardmäßig allen Access-Datenbanken zur Seite gestellt. Bei der Aktivierung dieses Filters über die rechte Maustaste im Formular oder über die Menüleiste wird Ihnen ein leeres Eingabe-Formular präsentiert, in das Sie einen oder mehrere Datenfelder mit den gesuchten Daten füllen bzw. bei Kombinationsfeldern auswählen können (s. Abbildung 3.31). Bei P.A.S.T können Sie diesen Filter, der dynamisch auf Veränderungen und weitere Eingaben der Datenbank reagiert, abspeichern und nachträglich verändern. So ist es z.B. denkbar, einen Filter zu generieren, der triporate runde Sporomorphae jederzeit aus dem sich verändernden Datenpool anzeigt.

Über die „Bildab“-Taste (Tastatur) können Sie über die Systematik zur Morphologie wechseln und die gewünschten Suchbegriffe eingeben. Achten Sie darauf, daß nur die Suchfelder mit Daten gefüllt sind. Dies können Sie sicherstellen, indem Sie mit der rechten Maustaste in den undefinierten Formularbereich klicken und "Alles löschen" auswählen. 


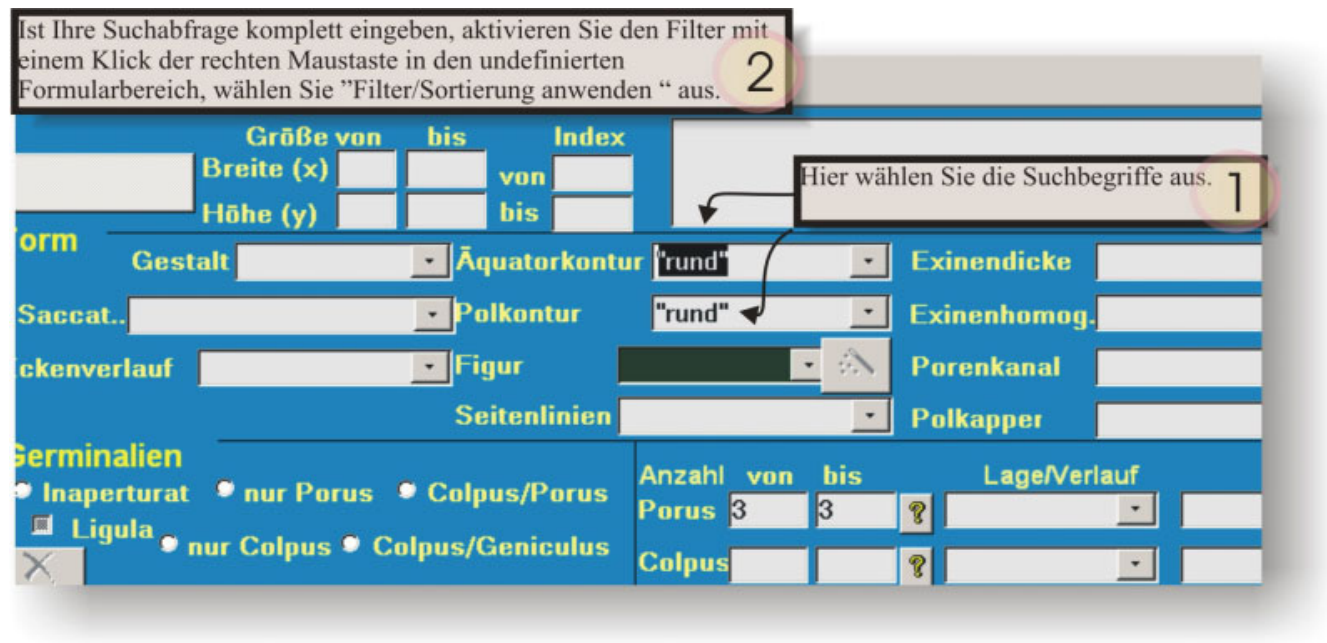

Abb. 3.31: Formularbasierter Filter.

Nachdem Sie den Filter aktiviert haben, können Sie ihn speichern und damit jederzeit abrufen.

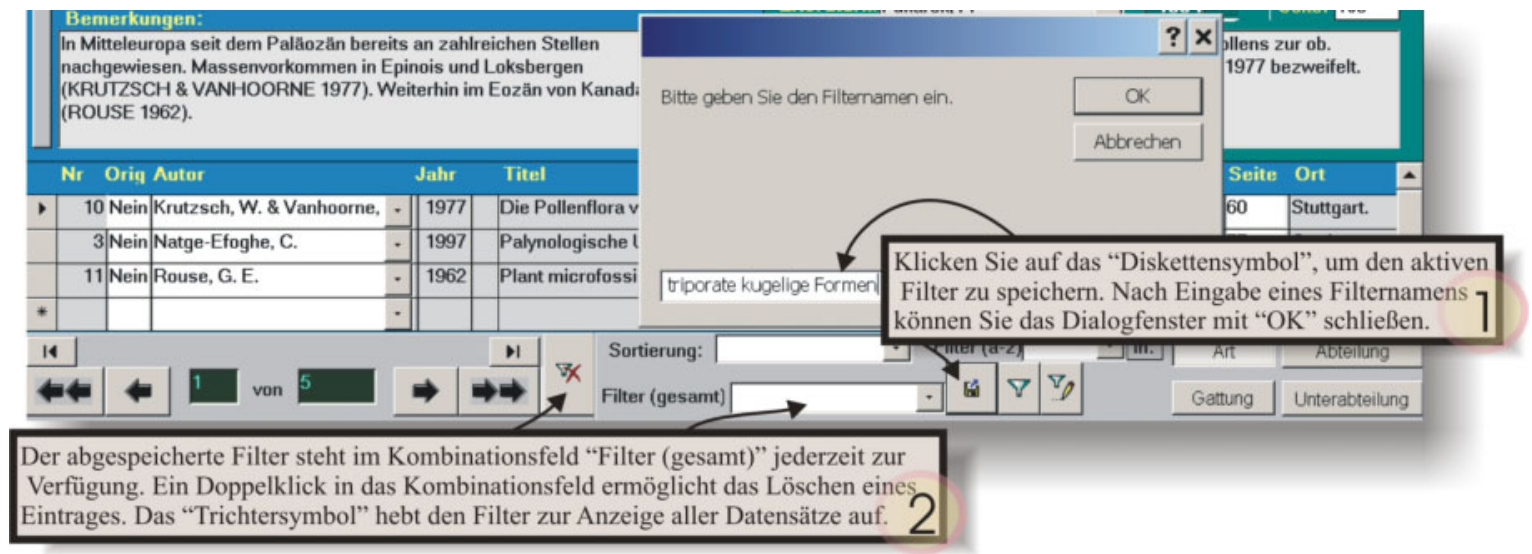

Abb. 3.32: Auswahlbasierenden Filter speichern und abrufen.

\subsubsection{Datensuche über den „Auswahlbasierten Filter“}

(Menüleiste Datensätze: Auswahlbasierter Filter) Neben dem Formularbasierten Filter, der die Angabe von mehreren Suchparametern erlaubt, stellt Ihnen der Auswahlbasierte Filter ein sehr einfaches Werkzeug zum Filtern der Daten zur Verfügung (s. Abb. 3.32). Wählen Sie mit der rechten Maustaste ein mit Daten gefülltes Feld aus und aktivieren Sie die Funktion „Auswahlbasierter Filter“. Die Datenbank zeigt daraufhin nur Datensätze, die das aktivierte Kriterium erfüllen. Auch dieser Filter läßt sich mit der oben beschriebenen Methode abspeichern. Sie können z.B. eine kugelige Form auswählen und sich über den Filtertyp sehr schnell die weiteren in der Datenbank gespeicherten Sporomorphae dieser Morphologie-Eigenschaft anzeigen lassen. Über den am unteren Bildrand aktivierten Karteireiter können Sie mehrere Parameter auch eines Kombinationsfeldes über „oder“ miteinander verknüpfen.

Für die nächste Suchoperation schränken Sie bitte den Datenpool über den Formularbasierten Filter auf Sporomorphae mit runder oder runder-ovaler Äquatorkontur ein; speichern Sie den Filter unter dem Namen „Äquatorkontur rund oder rund-oval“ ab. 


\subsubsection{Datensuche über stratigraphische Angaben}

\section{suchen Grafik}

(Menüleiste Bearbeiten: Suchen...Stratigraphie) Mit der Datensuche über stratigraphische Angaben (linker Button) schränken Sie den Datenpool auf Sporomorphae ein, die in von Ihnen wählbaren stratigraphischen Grenzen auftreten. Achten Sie darauf, für das nachfolgende Beispiel den oben definierten Filter (rundrund-oval) über „Filter-gesamt“" zu aktivieren. Der zu durchsuchende Datenpool wird damit auf ca. 50-60 Sporomorphae eingeschränkt. Geben Sie nun die Suchoptionen entsprechend der Abbildung 3.33 ein.

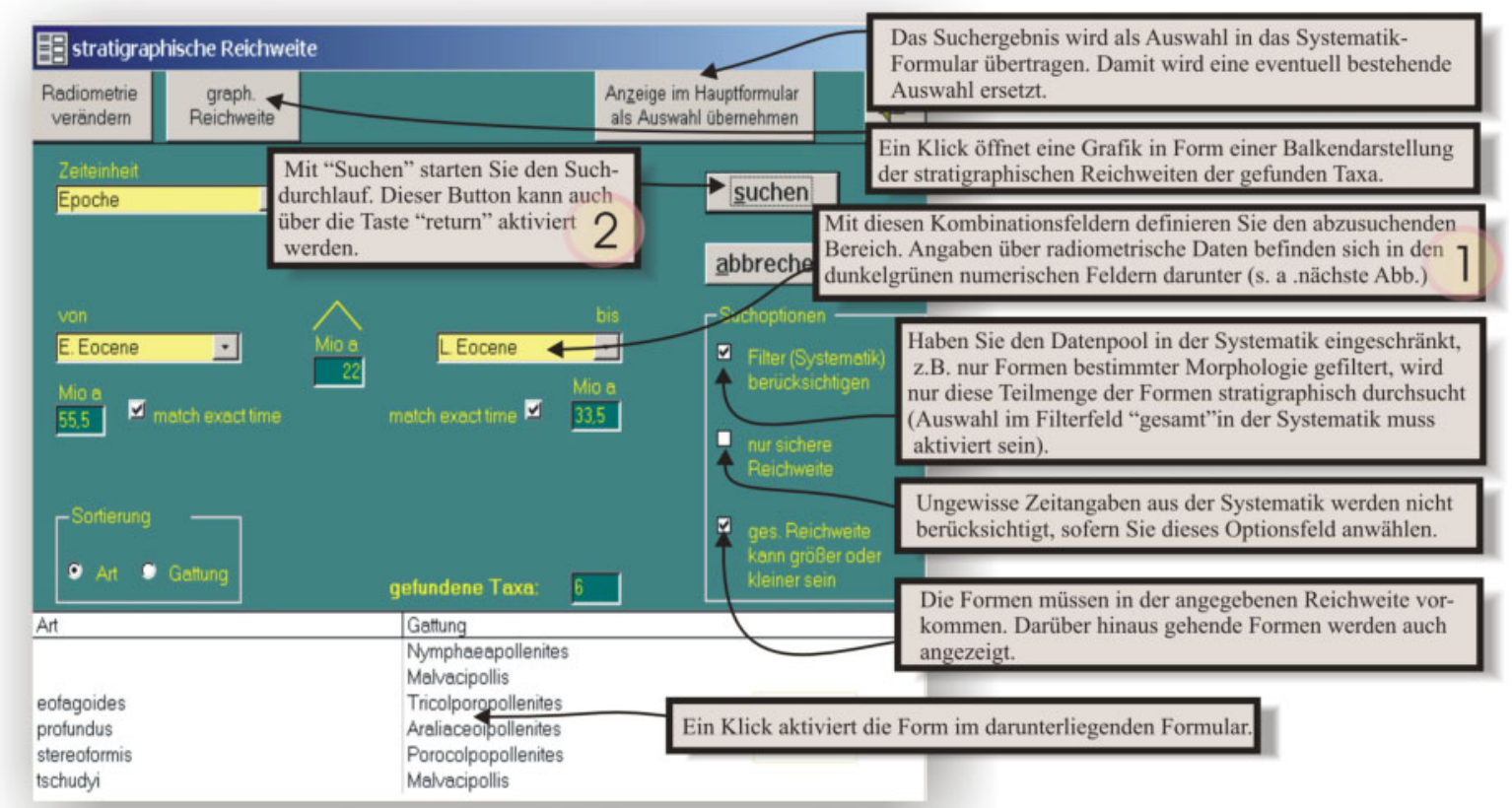

Abb. 3.33: Suche nach Sporomorphae mit Hilfe der stratigraphischen Reichweite.

In den nächsten Darstellungen werden einige Möglichkeiten der stratigraphischen Grenzziehungen anhand des bestehenden Filters aufgezeigt. Wählen Sie dazu die entsprechenden Optionsfelder nacheinander aus, und aktivieren Sie danach den Suchdurchlauf über den Button „,suchen“. Aktivieren Sie die graphische Ausgabe der Reichweite über den Button im Formular (Abbildung 3.33). 

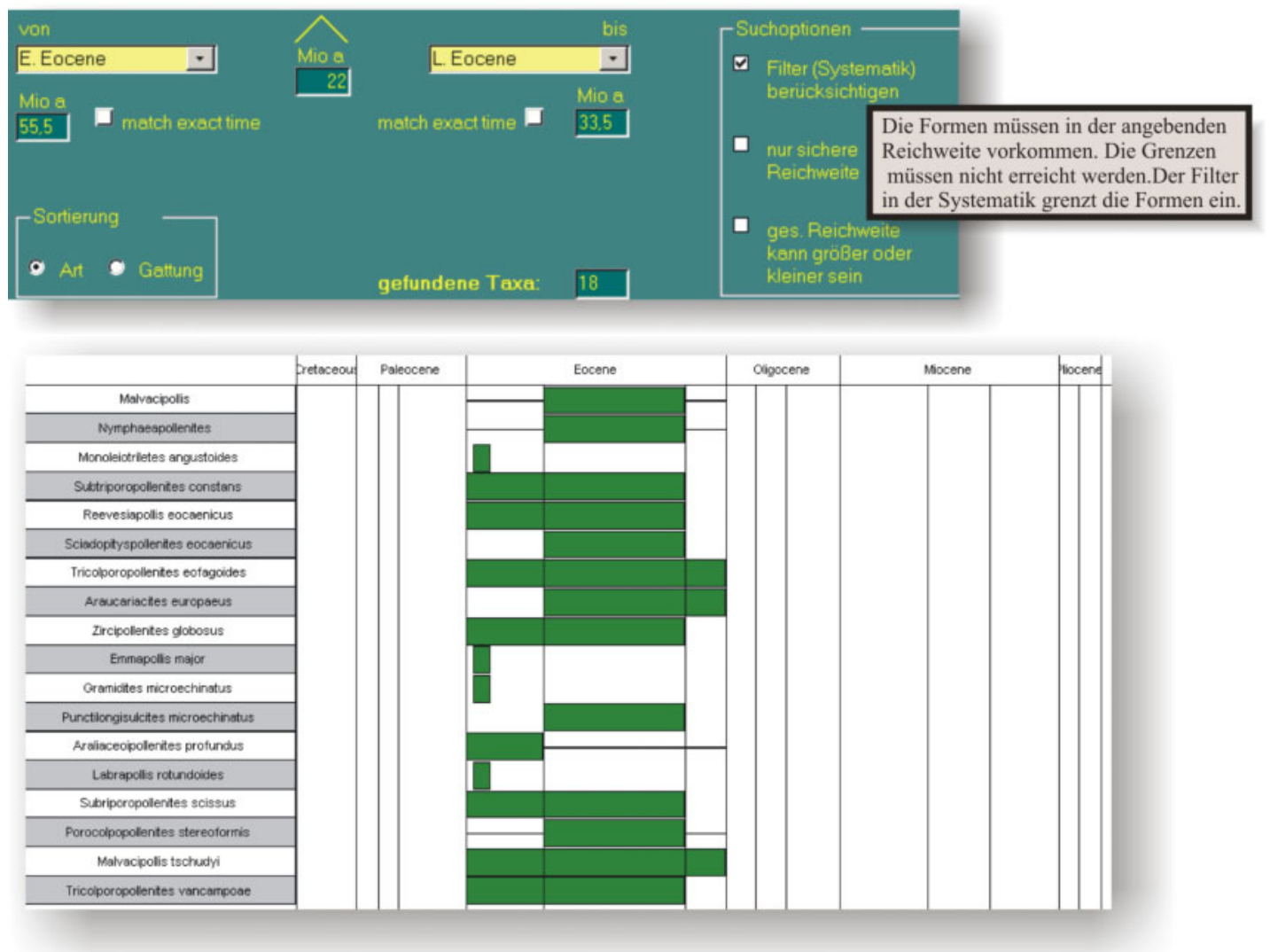

Abb. 3.34: Verschiedene Suchalgorithmen der Stratigraphie (1).
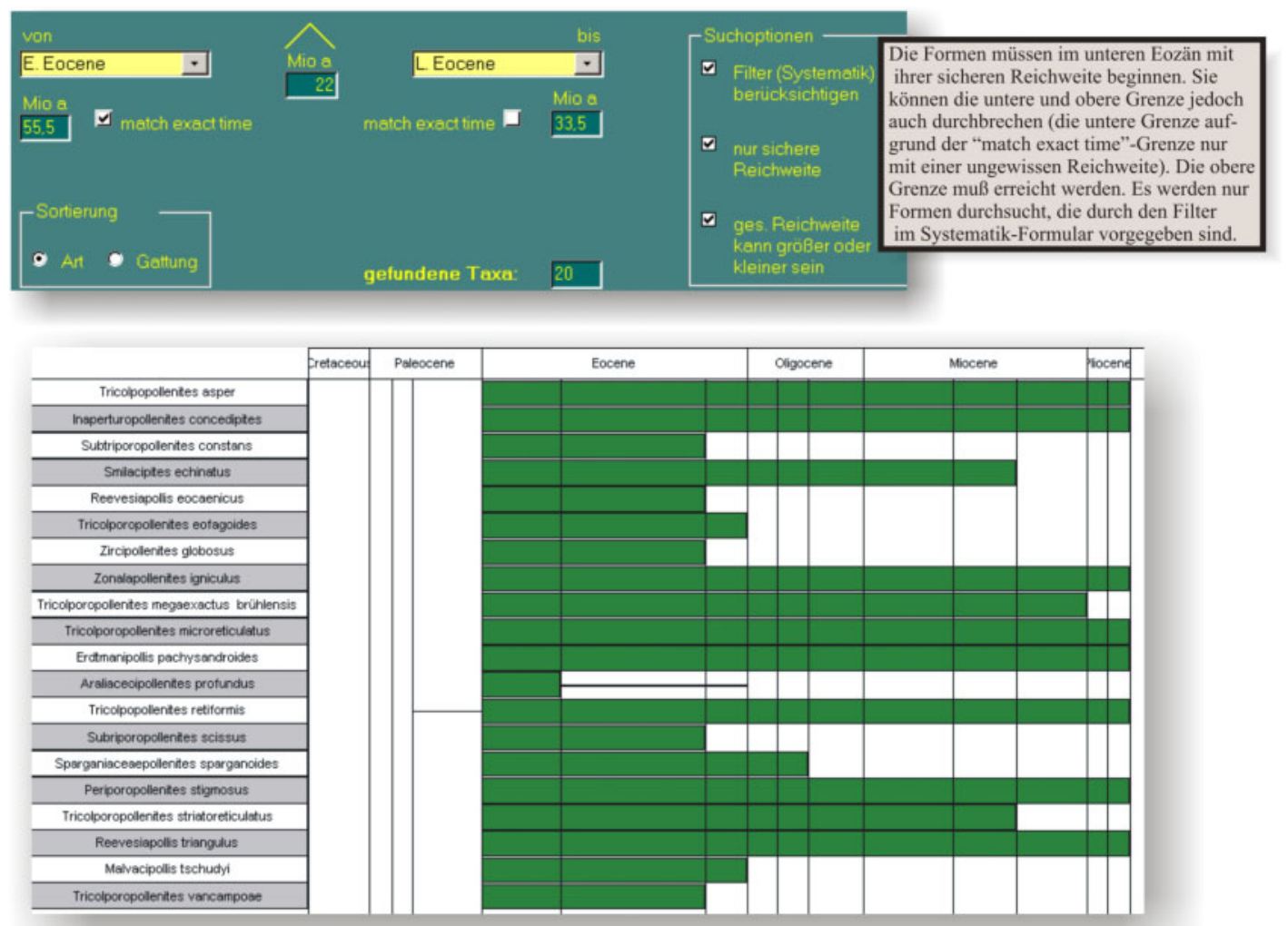

Abb. 3.35: Verschiedene Suchalgorithmen der Stratigraphie (2). 
Anmerkung:

Suchoperationen über stratigraphische Reichweiten erfolgen grundsätzlich über die zugehörigen radiometrischen Alter der Einheiten. Damit wird eine maximale Flexibilität und Genauigkeit auch bei der Suche über verschiedene Zeiteinheiten ermöglicht. Leider ist nicht immer eine sichere Grenzziehung der radiometrischen Daten zu einer Einheit aus der Literatur ersichtlich, da unterschiedliche Bestimmungsmethoden auch unterschiedliche Ergebnisse liefern und die Ergebnisse andererseits mit einer mehr oder weniger großen Ungenauigkeit behaftet sind. Dieser Problematik wurde in P.A.S.T durch eine durch den Benutzer veränderbare Zuordnungstabelle Rechnung getragen.

Radiometrie verändern

(Menüleiste Extras: radiometrische Einteilung) Um die Zuordnungstabelle einsehen bzw. ändern zu können, klicken Sie auf das nebenstehende Symbol aus dem Suchformular 'Stratigraphische Reichweite'.

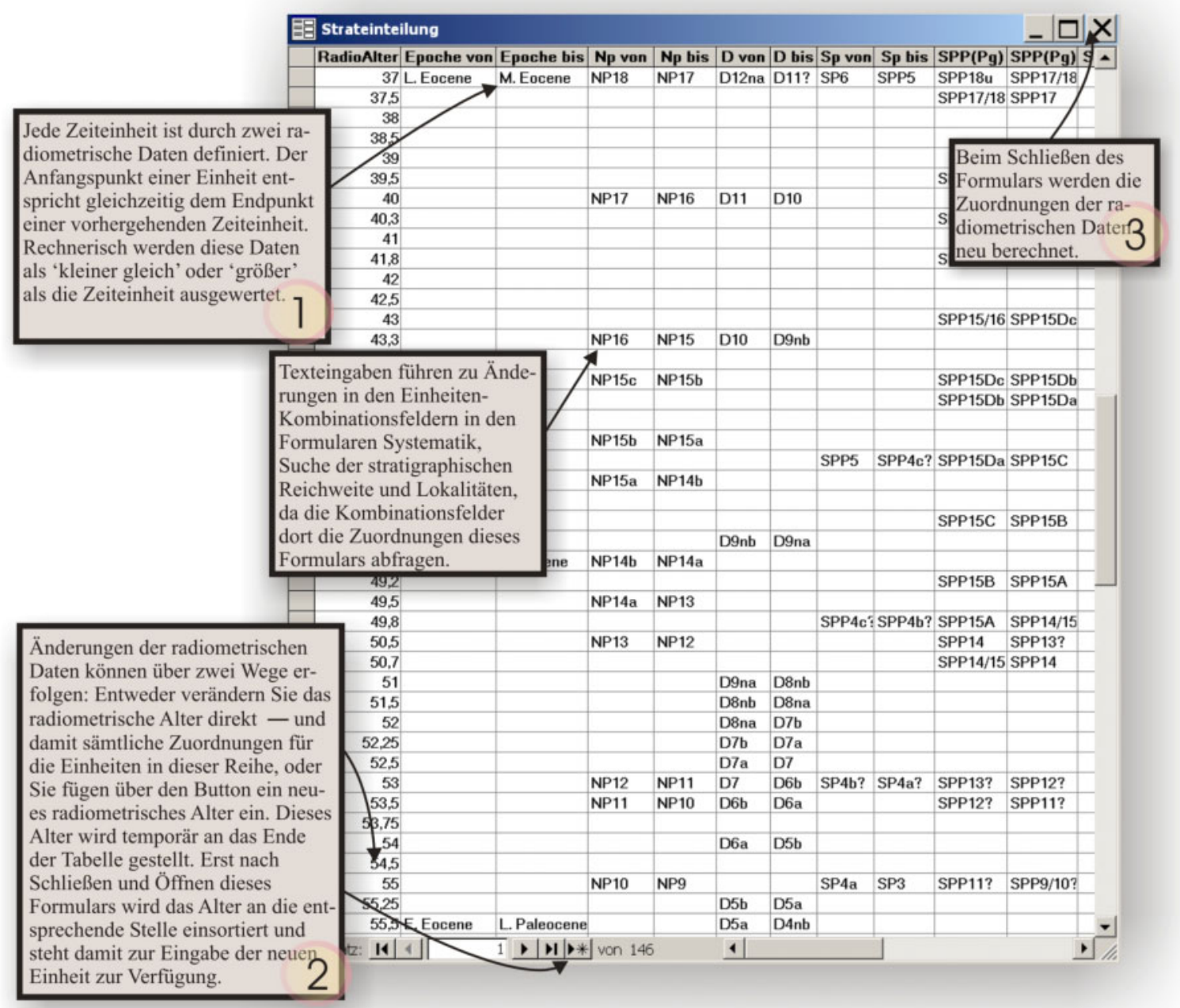

Abb. 3.36: Die Abbildung zeigt die Zordnungstabelle, um radiometrische Daten den stratigraphischen Einheiten zuzuweisen. Änderungen in diesem Formular beeinflussen z.B. die Suche über stratigraphische Reichweiten des Systematik-Formulars. 
Anschließend folgt eine Zusammenfassung der Zeiteinheiten mit Quellenangaben, aus denen die radiometrischen Daten entnommen sind:

\begin{tabular}{|l|l|}
\hline Zeiteinheit & Quelle \\
\hline Epoche & KRUTZSCH 1998 \\
\hline Nanoplanktonzone (NP) & MARTINI 1971 \\
\hline Dinoflagellatenzone (D) & KÖTHE 1990 \\
\hline Sporenzone (SP) & KRUTZSCH 1998 \\
\hline Paläogenzone (SPP, PG) & KRUTZSCH 1998 \\
\hline Säugerzone & LINDSAY, V. 1988 \\
\hline Eigene Zeitgliederung & Der Benutzer kann eine eigene Zeiteinteilung erstellen \\
\hline
\end{tabular}

\subsubsection{Auswahl von Sporomorphen}

\section{Menüleiste Bearbeiten: Auswahl von Sporomorphen}

Das nächste Anwendungsbeispiel behandelt das Zusammenfassen von Sporomorphae in Listen über das Auswahlformular. Diese Formengruppen können abgespeichert und nachträglich verändert werden. Mithilfe der Formengruppen lassen sich z.B. die Sporomorphae einer Lokalität im Zusammenhang bearbeiten. Aus diesem Formular heraus können Sie im Kontext zu den Formenlisten Karteikarten ausgeben, Farbtafeln generieren oder Ausgabetabellen erzeugen, die automatisch zur weiteren Bearbeitung nach Microsoft Excel übertragen werden.

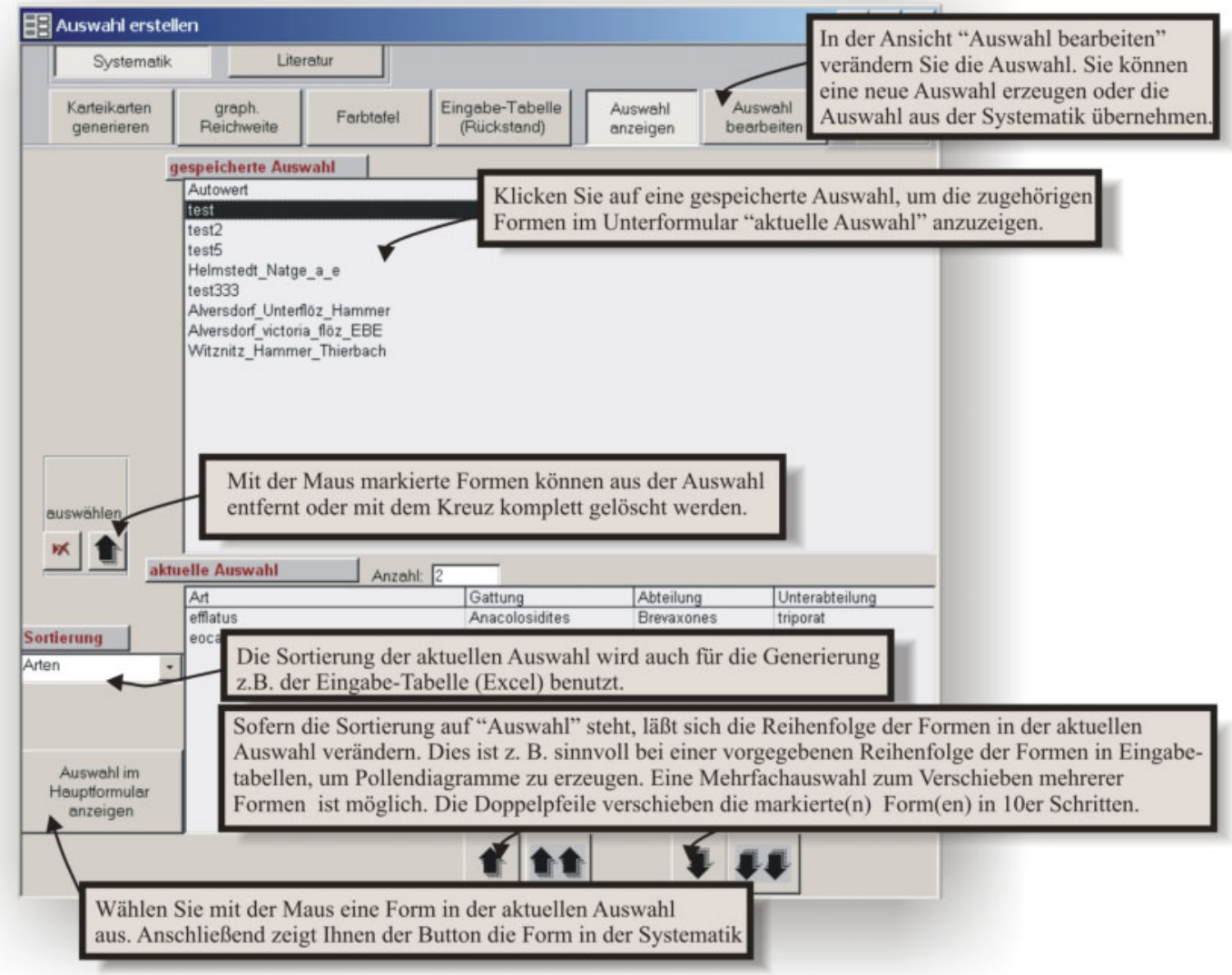

Abb. 3.37: Über "Auswahl erstellen" werden Formengruppen gespeichert. Die Abbildung zeigt den Ansichtsmodus, in der keine Formengruppen verändert werden können. 
Wenn Sie sich mit dem Aufbau der Formularansicht vertraut gemacht haben, wechseln Sie im Formular zur Ansicht „Ausgabe bearbeiten“. Erzeugen Sie eine neue Auswahl (z.B. mit dem Namen: "Test"). Fügen Sie anschließend nachfolgende Sporomorphae über die Pfeile am linken Formularrand dieser neuen Auswahl hinzu.

\section{Stereisporites stereoides}

3. Sciadopityspollenites eocaenicus

5. Plicatopollis plicatus

7. Anacolosidites efflatus

9. Tricolpopollenites liblarensis liblarensis

(Die Reihenfolge der Sporomorphae können Sie über die Pfeiltasten am unteren Formularrand ändern, sofern die Sortierung auf Auswahl steht)

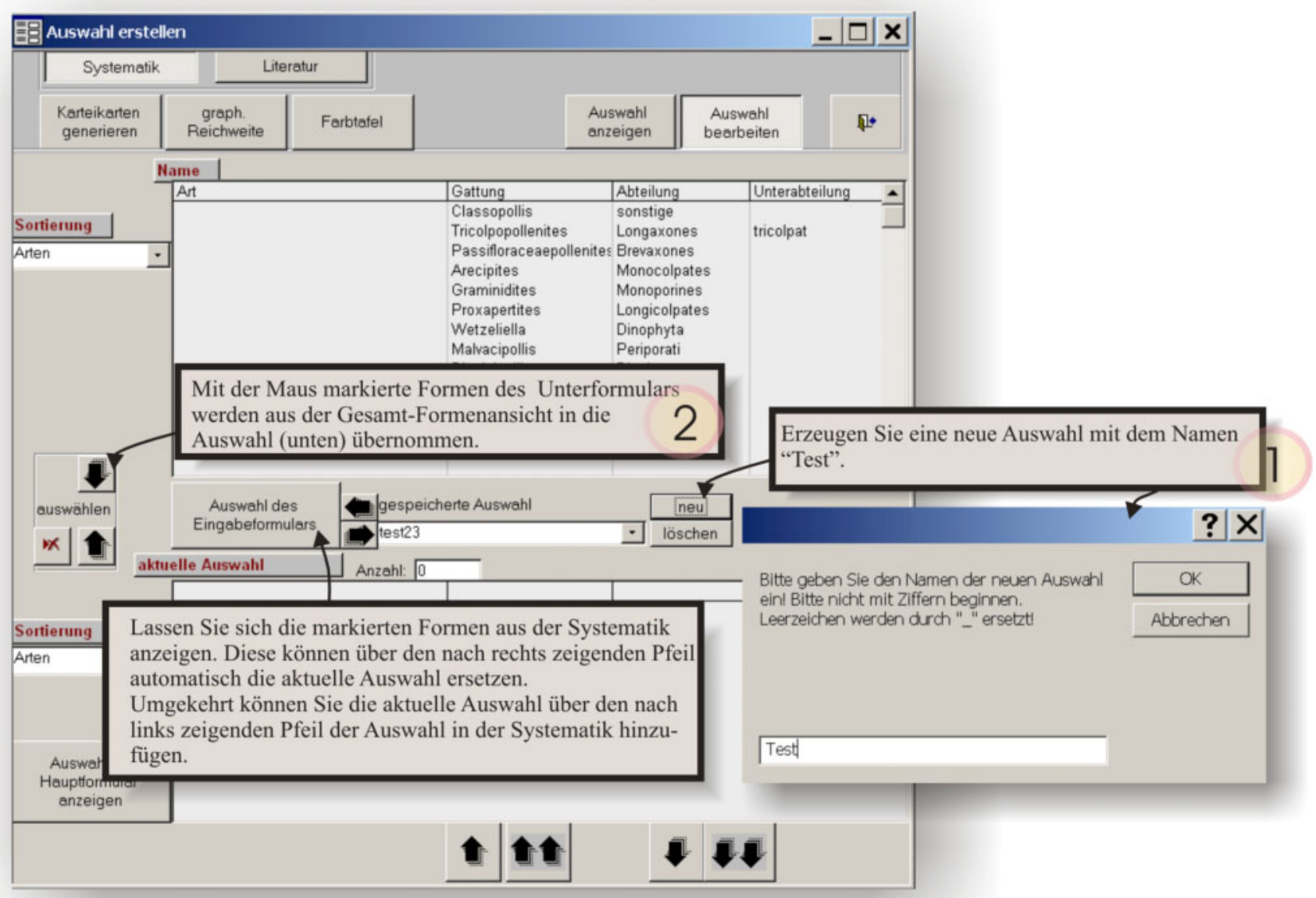

Abb. 3.38: Über "Auswahl bearbeiten" können Formengruppen erzeugt und verändert werden.

Anmerkung:

An dieser Stelle sei noch einmal auf den Unterschied zwischen einem Filter und einer Auswahl hingewiesen: Die Auswahl stellt die Sporomorphae in Form einer Liste zusammen. Der Filter erzeugt dagegen eine dynamische Liste von Sporomorphae mit definierten Kriterien, die sich mit dem Hinzufügen von Sporomorphae in P.A.S.T ändern kann. 


\subsection{Exportmöglichkeiten und Anzeigen von Bildern mit P.A.S.T}

P.A.S.T erlaubt vielfältige Möglichkeiten des Datenexportes und der Visualisierung von Teilmengen der Datenbank. Sie können druckbare Karteikarten, Farbtafeln oder Formenlisten ausgeben. Ebenso ist es möglich, Literaturdaten zitatgerecht aufzuarbeiten und nach Microsoft Word zu übertragen.

2.

Ausgehend von der zuvor erzeugten Auswahl werden Sie im nächsten Schritt die Ausgabe von Karteikarten und Farbtafeln kennenlernen.

\subsubsection{Karteikarten}

Karteikarten können z.B. den Austausch von Informationen unter Palynologen gewährleisten, zu Gelegenheiten, wo die Datenbank nicht zur Verfügung steht. Weiterhin können diese Karten zu Archivierungszwecken ausgedruckt werden.

幽

Dazu öffnen Sie zuerst das Auswahlformular (s. Kap. 3.9.5, S. 75).

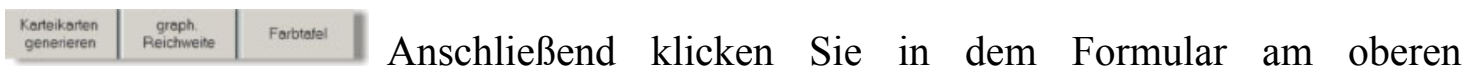
Formularrand auf „Karteikarten generieren“. Bestätigen Sie das Schließen des Systematik-Formulars, das für die Anzeige der Karteikarten nicht benötigt wird. Möchten Sie einen neuen Karteikartensatz auf der Basis einer anderen gespeicherten Auswahl erstellen (z.B. Sporomorphae einer anderen Lokalität), betätigen Sie nach dem Anklicken der entsprechenden Auswahl erneut den Button „Karteikarten generieren".

Drucken Sie über den Dialog Ausgabe/Export den ersten Datensatz, bzw. die erste Karteikarte aus (entweder über ,aktuelle Karteikarte drucken“ oder „Seite von“). Vergleichen Sie den Ausdruck mit der Exportmöglichkeit, die Sie in der DruckDialogbox ebenfalls aktivieren können (Abbildung 3.39). 


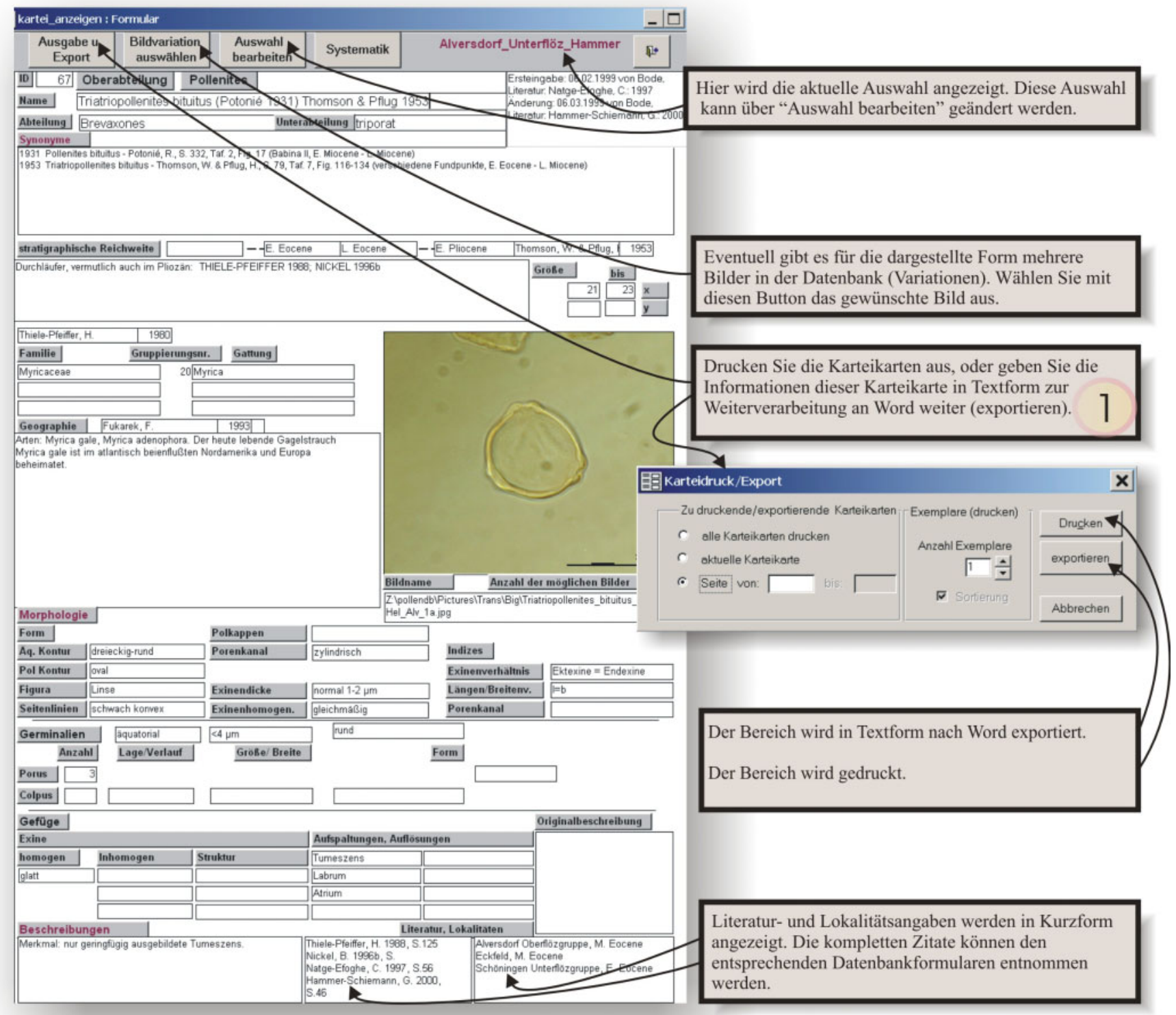

Abb. 3.39: Ausgabe der Auswahl im Rahmen des Karteikarten-Exportes.

Anmerkung:

Der Name des Taxons wird aus dem Art- und Gattungsnamen der Systematik und der Synonymieliste generiert. Dabei wird der Erstautor des Taxons (bzw. des Synonyms) mit Jahreszahl in Klammern gesetzt, gefolgt von dem Letztautor. Dabei muß der vergebene Name des Taxons des Letztautors mit dem Namen, der sich aus der in der Systematik gespeicherten Art und Gattung zusammensetzt, übereinstimmen. Fehlende oder fehlerhafte Einträge in den Synonymielisten führen zu unvollständigen Namen der Taxa auf den Karteikarten und zeigen damit Fehler in der Konsistenz der Synonymieliste an. 


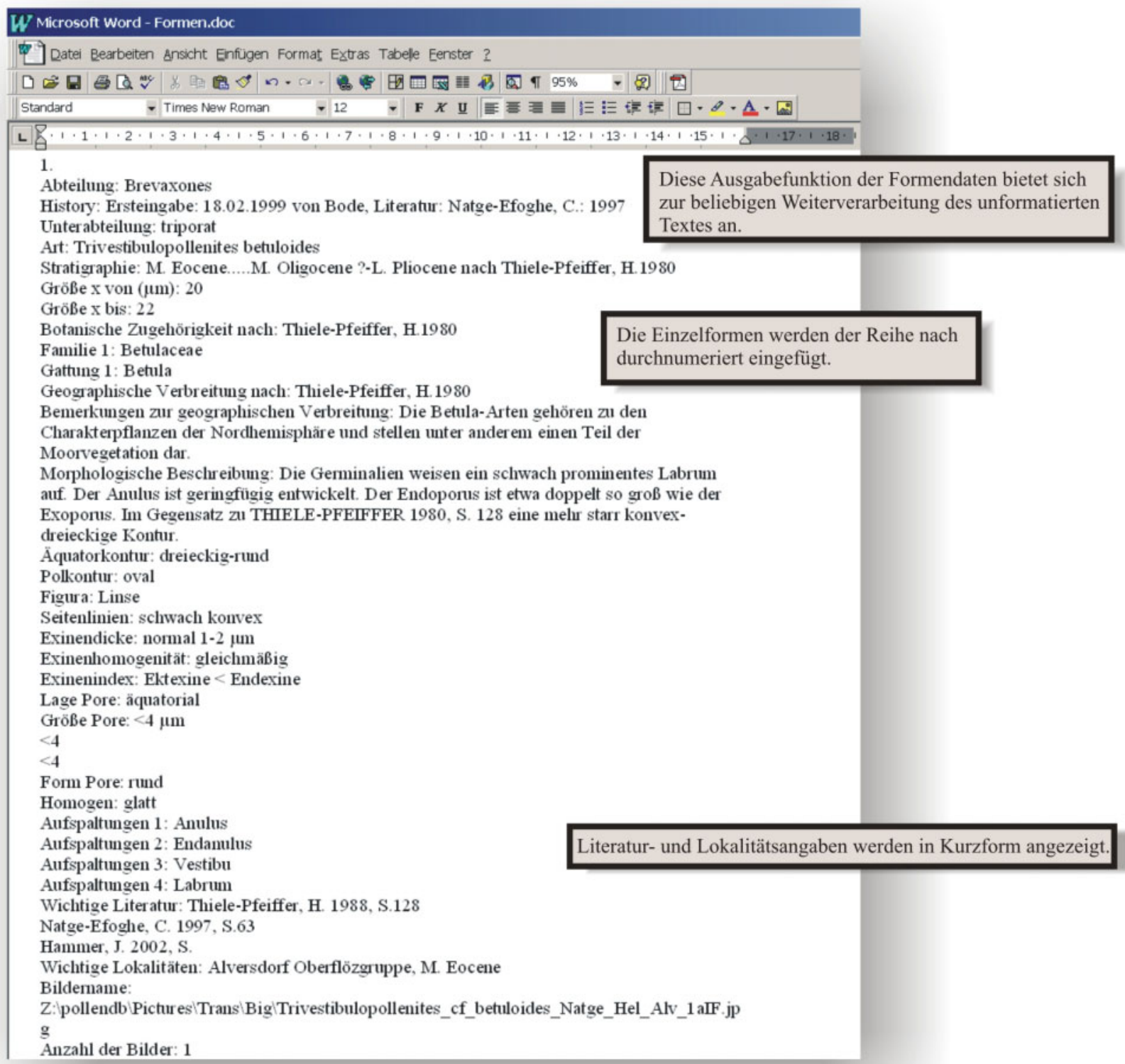

Abb. 3.40: Ausgabe des Karteikartentextes nach Word über "Exportieren" im Druckdialog.

\subsubsection{Formularausdruck zur Eingabe der Sporomorphae-Daten}

Mit P.A.S.T können Eingabeformulare ausgedruckt werden, die es Computerunerfahrenen Anwendern möglich macht, die benötigten Daten für P.A.S.T handschriftlich einzugeben. Diese Daten der Formulare können dann z.B. von palynologisch weniger versierten Hilfskräften P.A.S.T hinzugefügt werden. Diese Funktion erreichen Sie über die Menüleiste, "Datei", "Exportieren", "Formular Pollenites", "Formular Sporites" und "Formular Botanik" (Siehe Abbildungen 3.41 und 3.42). 


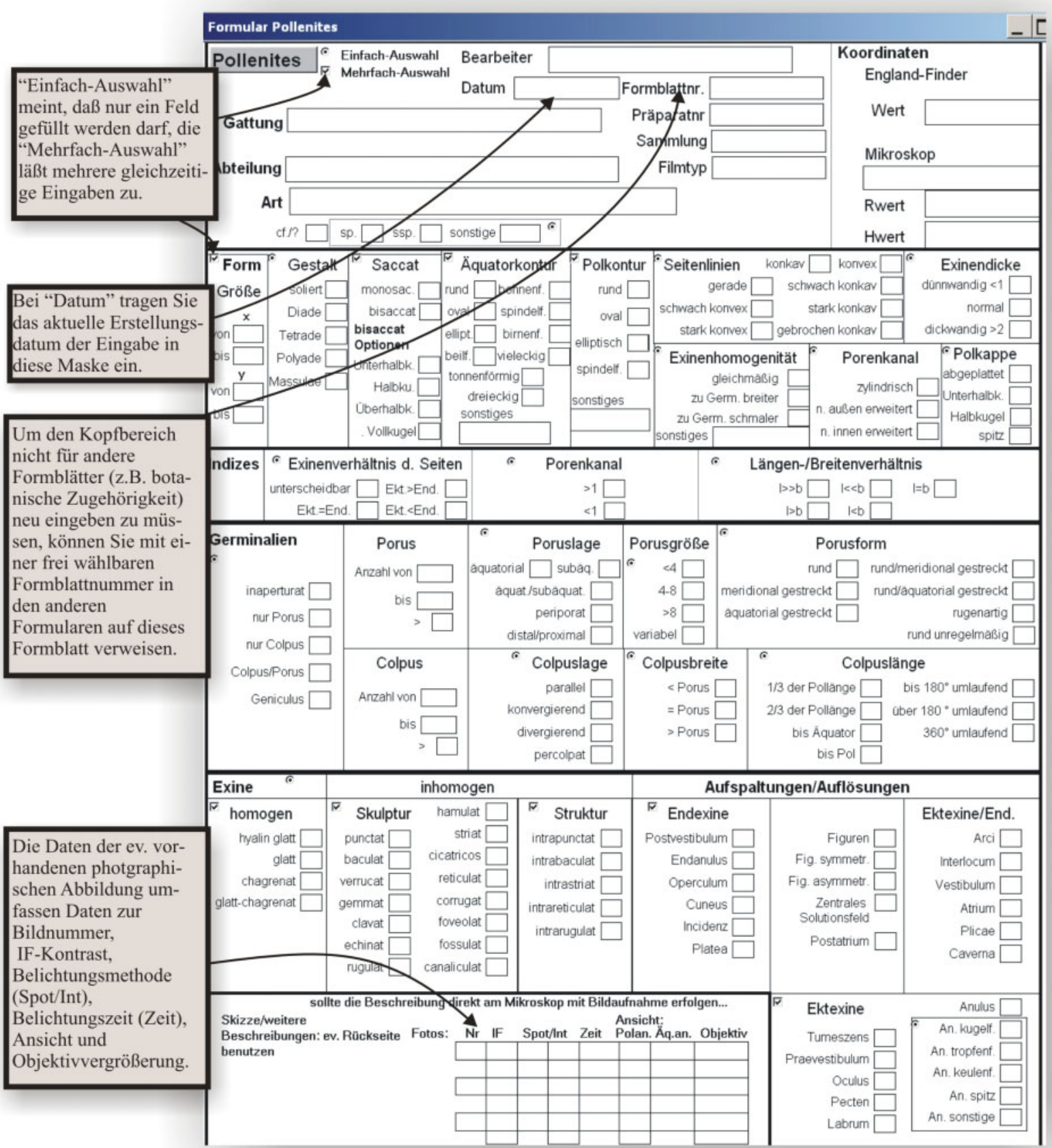

Abb. 3.41: In den Ausdruck dieser Eingabemaske für Pollendaten können handschriftliche Daten eingetragen werden, die im nächsten Schritt in P.A.S.T eingeben werden. 


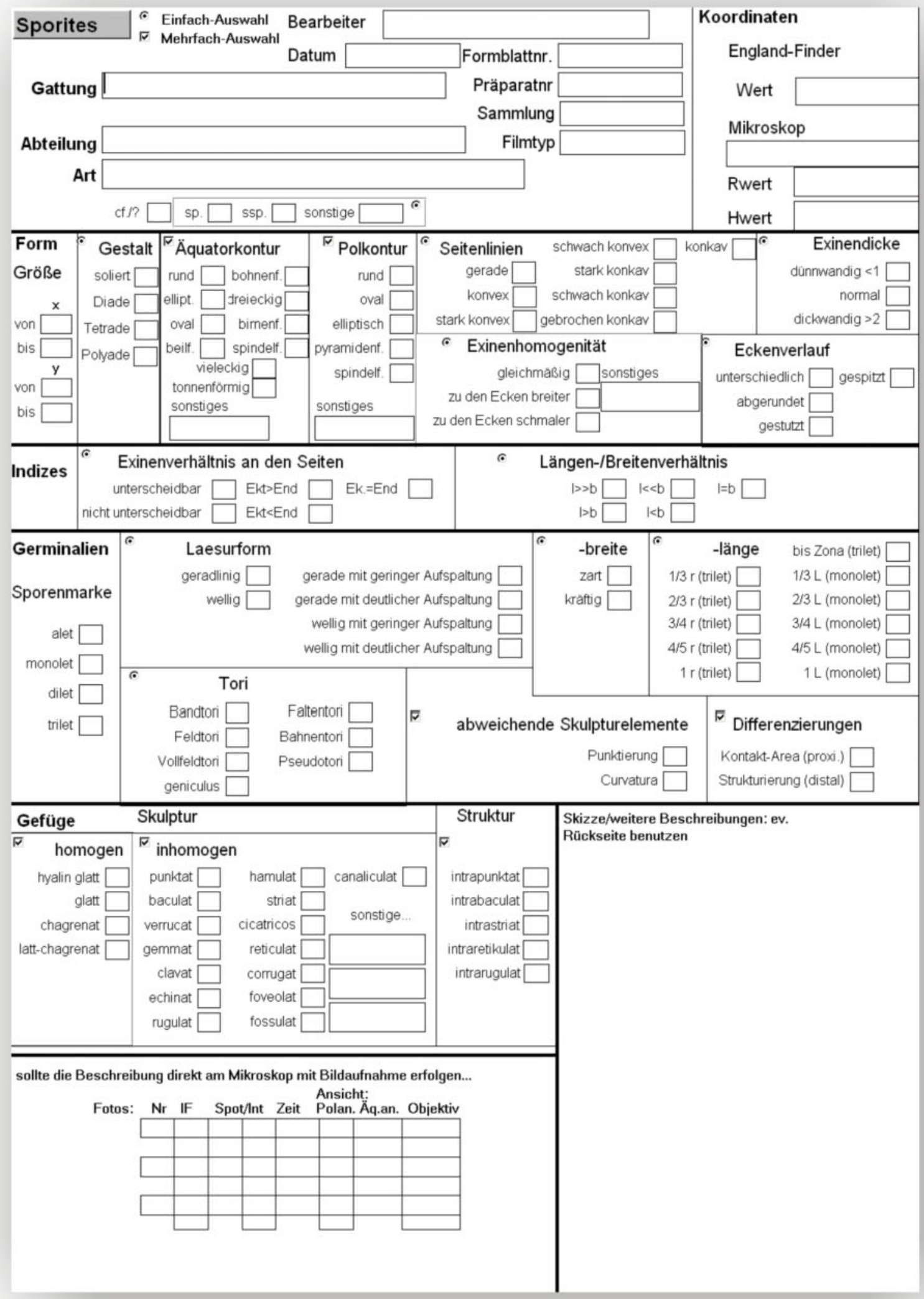

Abb. 3.42:. Eingabemaske für die Daten der Sporen. (Bemerkungen: siehe Abbildung 3.41). 


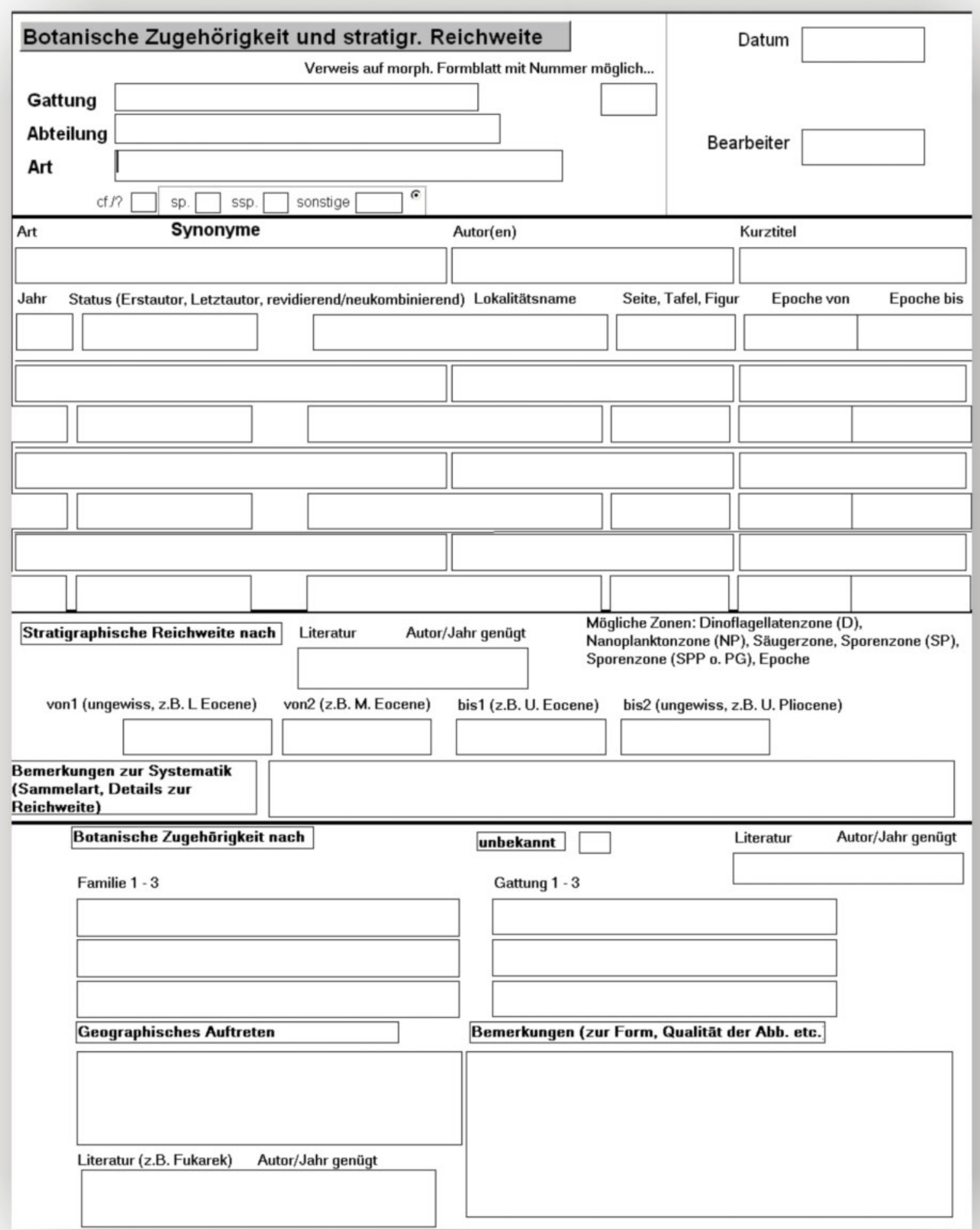

Abb. 3.43: Eingabemaske für die botanischen und stratigraphischen Daten (Bemerkungen: siehe Abbildung 3.41). 


\subsubsection{Abbildungen und Farbtafeln}

Photographische Abbildungen lassen sich über zwei verschiedene Wege mit P.A.S.T anzeigen und verarbeiten. Der naheliegende Schritt führt über die Anzeige des Abbildungsformulars. Sie können Abbildungen aber auch im Rahmen von Farbtafeln flexibel anordnen und/oder ausdrucken lassen.

\subsubsection{Abbildungsformular}

\section{Ausgehend von der Eingabe des Beispieldatensatzes (siehe Kap.} 3.7.9, S. 61) erreichen Sie das Abbildungsformular über einem Mausklick auf den entsprechend benannten Button in der Kopfzeile des Systematik-Formulars. Die Standardeinstellung zeigt daraufhin die verkleinerte(n) Darstellunge(n) der Bilder der Form an. Sie können jedoch davon ausgehend die Anzeige der Bilder über den Filterbutton auf weitere Sporomorphae erweitern oder eingrenzen (mehr dazu in der Abbildung 3.44).

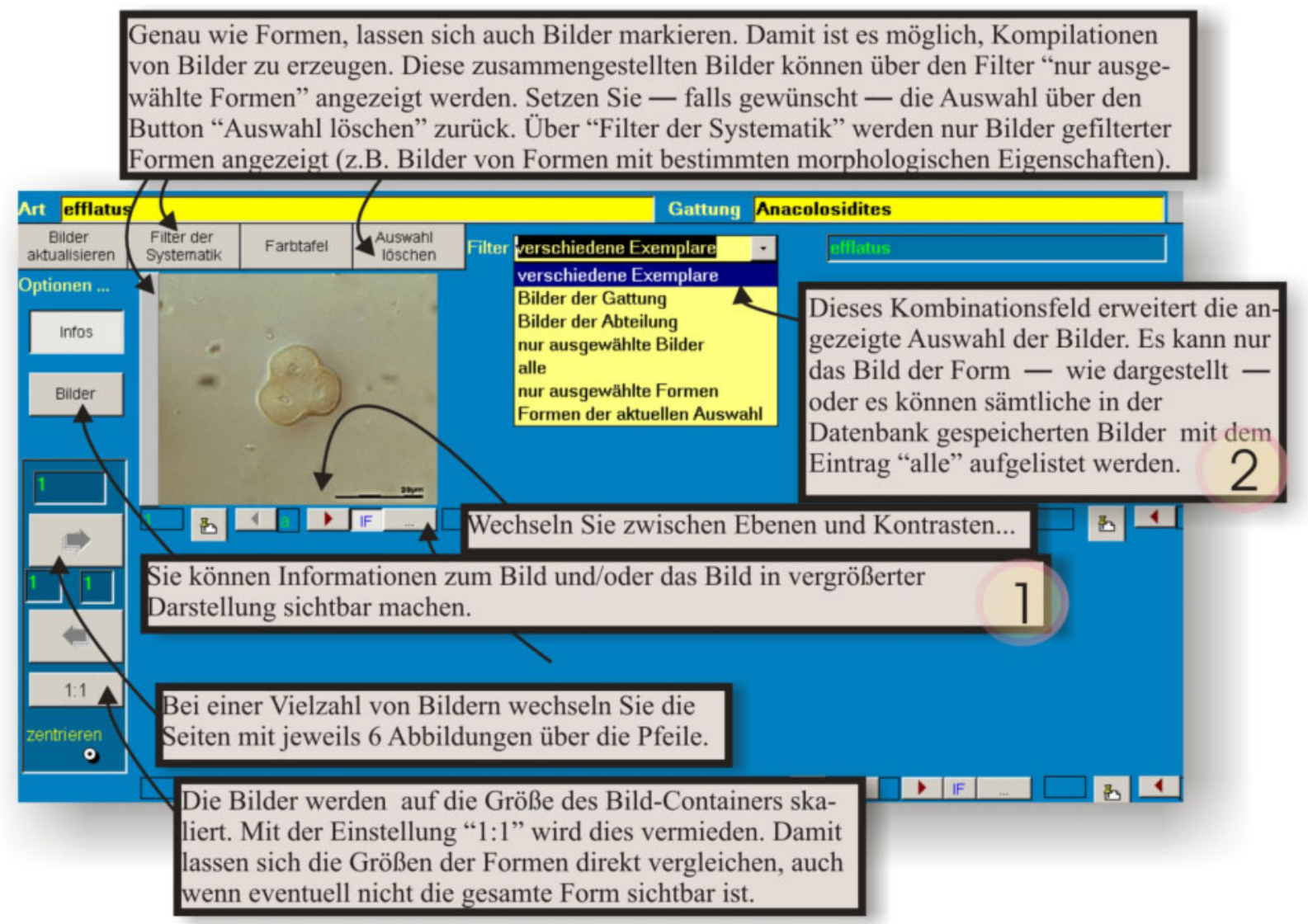

Abb. 3.44: Anzeige der Bilder über das Formular Abbildungen.

Die Abbildung 3.45 zeigt das Formular, das bei der Einstellung "Infos" bei Mausklick auf den Button ... angezeigt wird (s. Tutorial-Schritt 1 der Abbildung 3.44). 


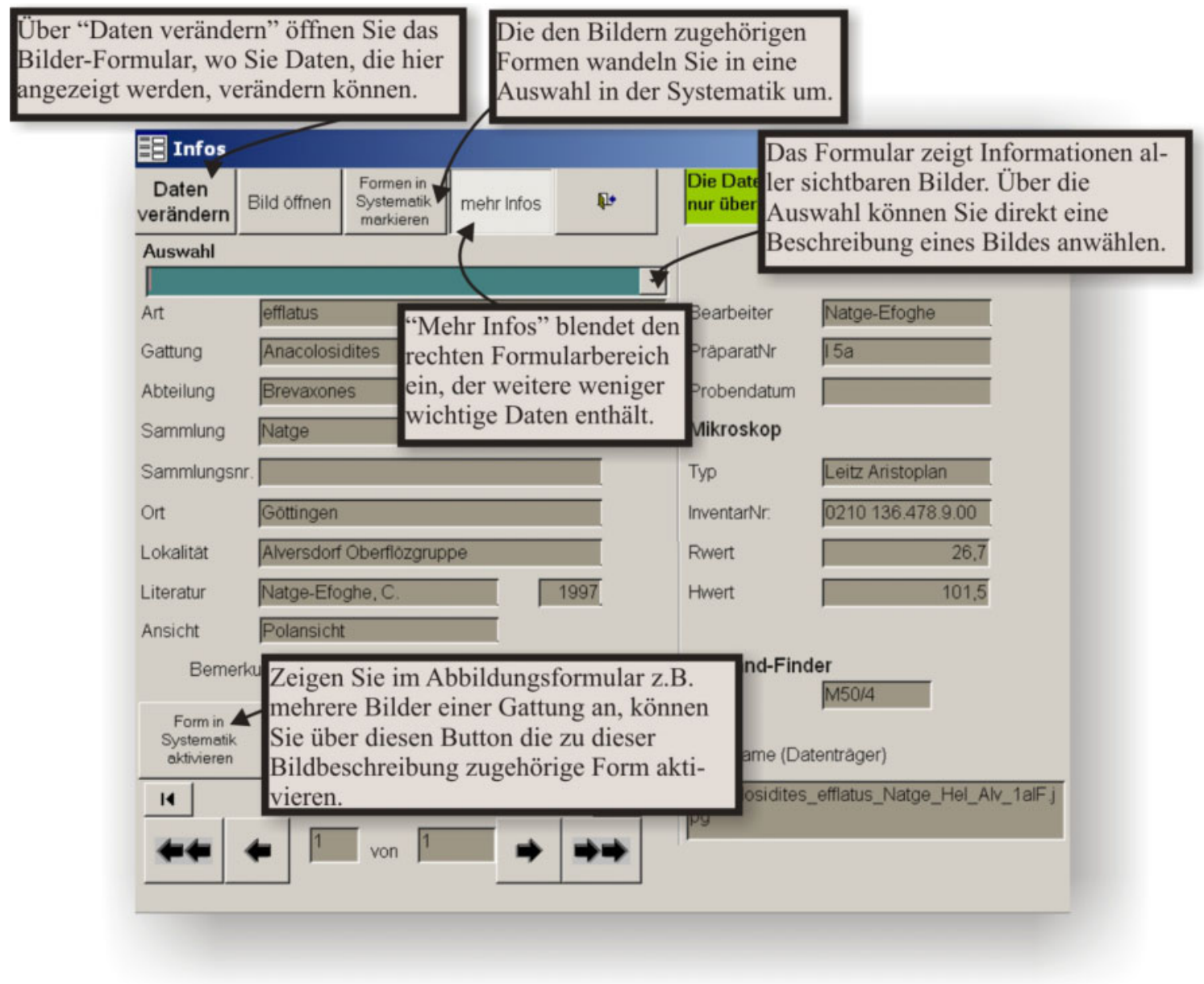

Abb. 3.45: Bildinformationen

\section{Anmerkung:}

Sie können das Bildinformationsformular geöffnet lassen, um die Informationen der angezeigten Bilder schnell abrufen zu können. Ein Klick auf den Info-Button "..." des jeweiligen Bildes aktualisiert den Formularinhalt (Abbildung 3.45, rechtes Teilformular).

Sind mehrere Bilder sichtbar, können Sie über die Navigationsbuttons im Bildinformationsformular zwischen den Daten der im aktuell sichtbaren Bilder wechseln. Dazu gehören auch die Bilder, die nur über die Pfeiltasten des Abbildungsformular erreichbar sind.

\subsubsection{Farbtafeln}

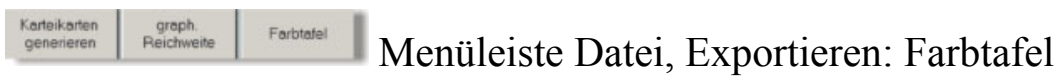

Mit dem Formular „Farbtafel“ ordnen Sie eine Vielzahl von Bildern ausgewählter Sporomorphae übersichtlich auf einer Seite an (Abbildung 3.47). Diese Tafeln können später ausgedruckt oder in andere Dokumente eingebunden werden. Sie erzeugen die Farbtafel über eine gespeicherte Auswahl der Sporomorphae. Zusammengestellt werden die Bilder im Abbildungsformular, das nur die Bilder der aktuellen Auswahl anzeigt (Abbildung 3.46). 


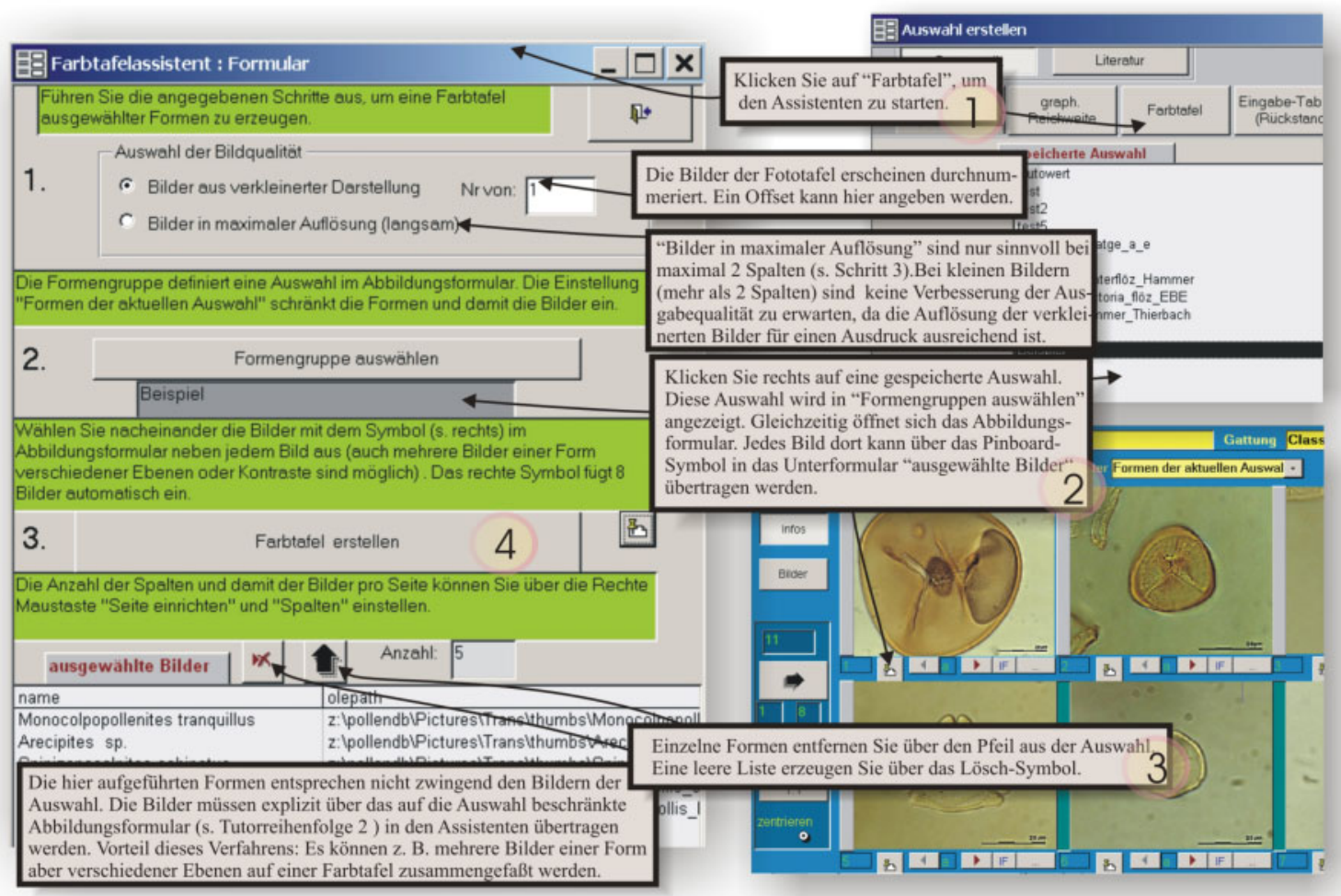

\section{Abb. 3.46: Erstellung einer Farbtafel}

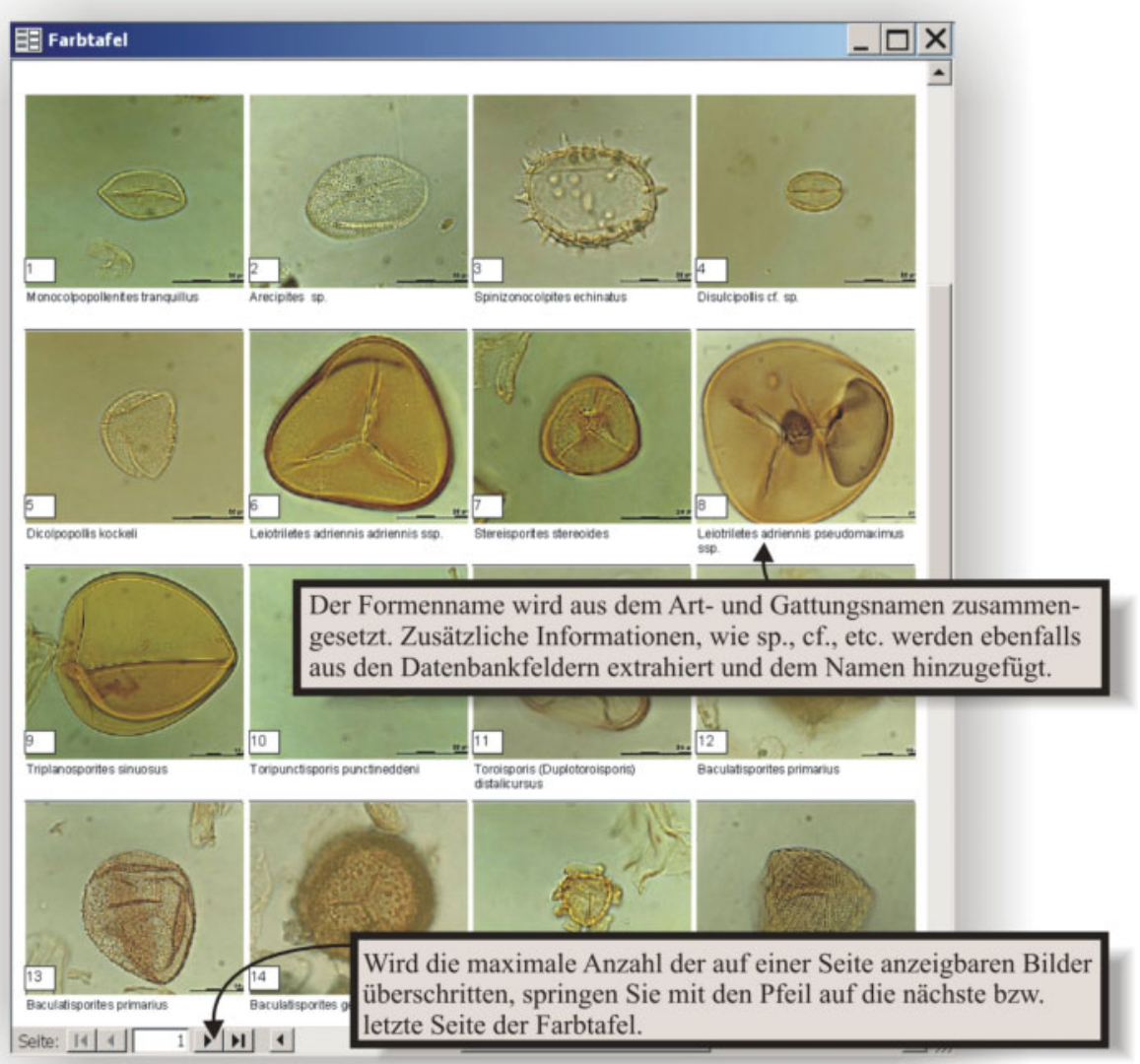

Abb. 3.47: Das Ergebnis des Farbtafel-Assistenten 


\subsubsection{Erzeugen von Literaturlisten}

Über das Eingabeformular Literatur können Literaturlisten nach Word exportiert werden. Dies geschieht über geeignete Formatierungsvorlagen, die frei veränderbar sind. Grundlage der Literaturliste sind die Markierungen im Literaturformular (erkenntlich am „grünen Balken“ des linken unteren Formularrandes, Abbildung 3.48).

\section{Menüleiste Bearbeiten: Auswahl von Sporomorphen}

Sie können Literaturlisten, genau wie im vorhergehenden Beispiel Formenlisten, mit Hilfe des Buttons „Auswahl erstellen“ erzeugen. Starten Sie die Auswahl und erzeugen Sie einige Literaturstellen.

Aktivieren Sie anschließend den Formatierungsdialog.

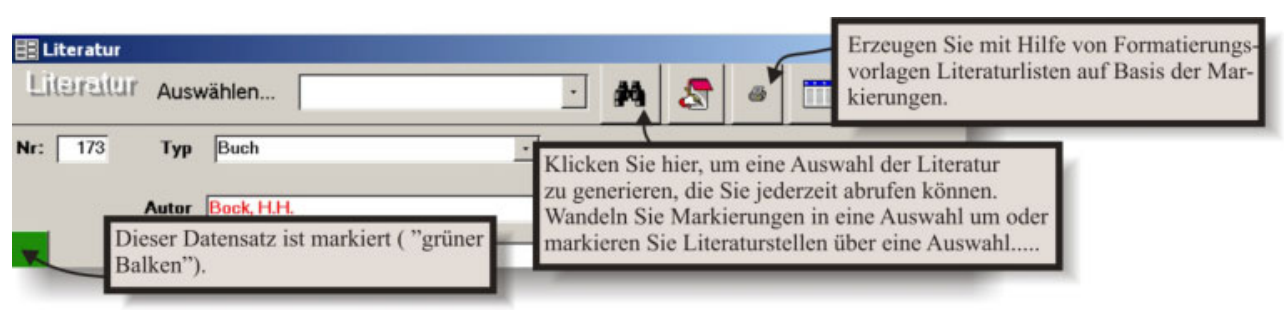

Abb. 3.48: Literaturlisten vorbereiten

$\mathrm{Y}|\mathrm{z}|$ Auswahl|Alle Hinweis: Um nur die markierten Literaturstellen im Formular Literatureingabe anzuzeigen, aktivieren Sie den Auswahlfilter im Eingabeformular.

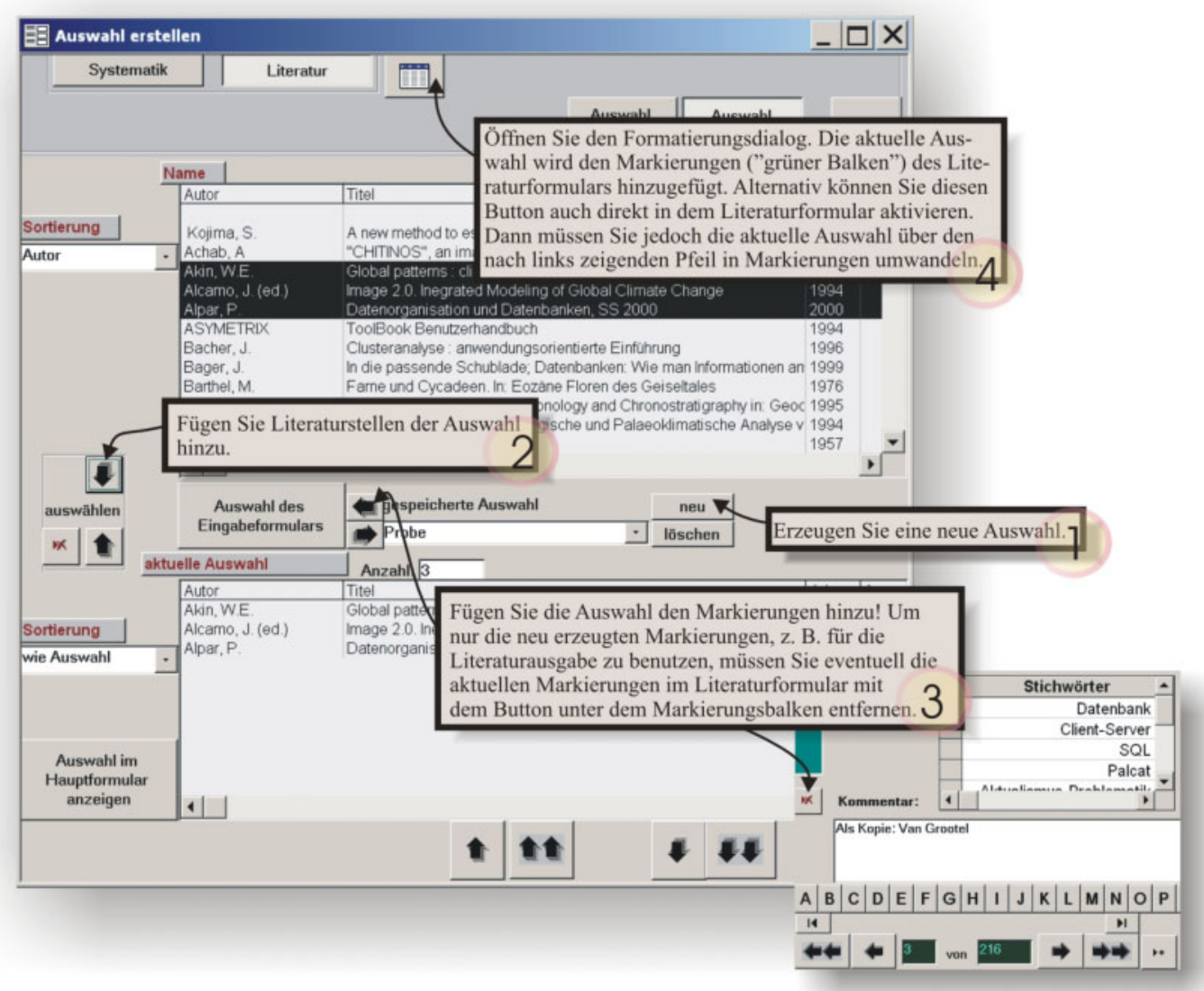

Abb. 3.49: Auswahl der Literatur 


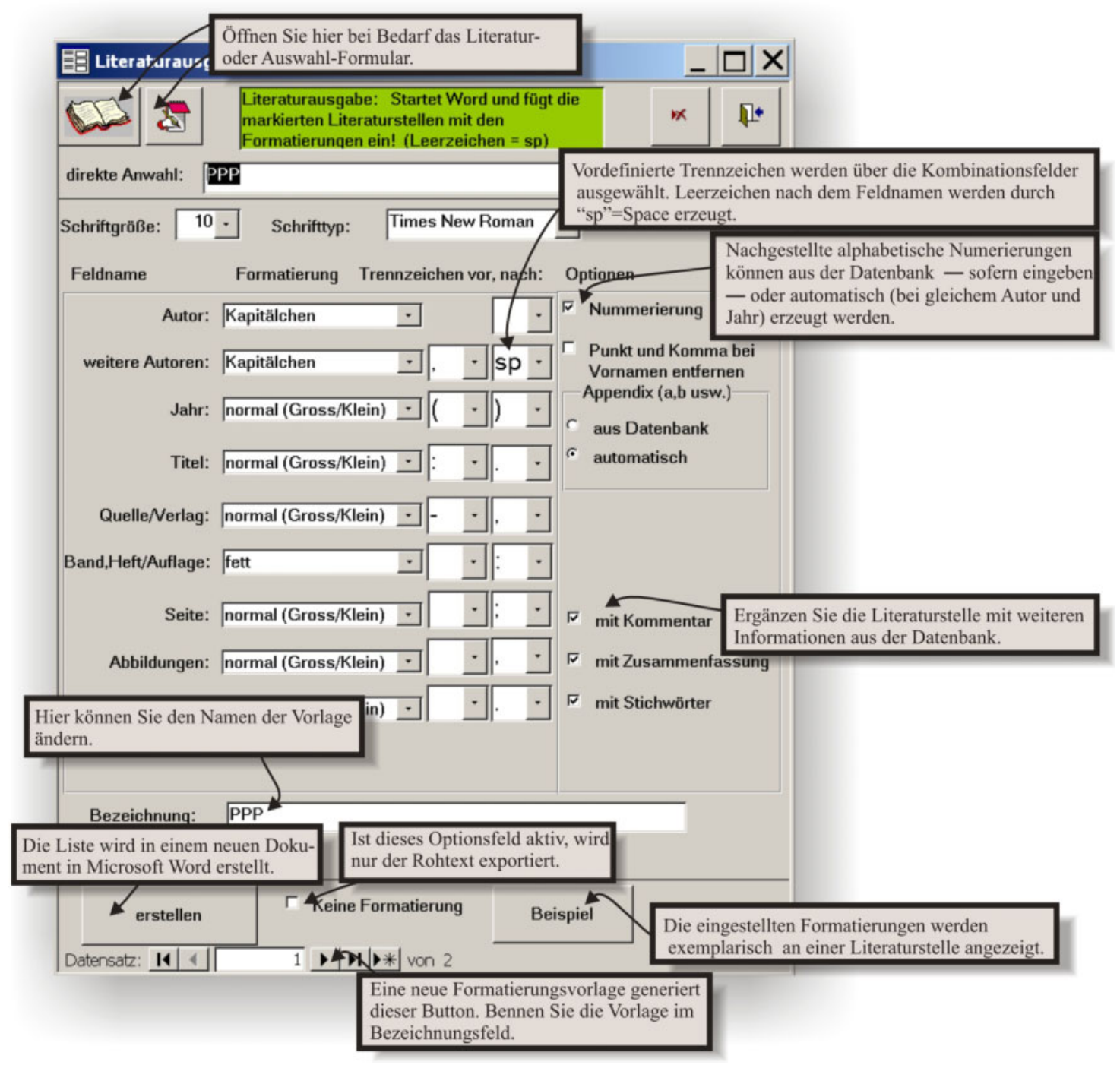

Abb. 3.50: Formatierungsdialog.

Sie können die Absatzstruktur nach belieben verändern. Ein "hängender Einzug" kann z. B über "Alles markieren, Format Absatz: Extra, Hängender Einzug" erzeugt werden.

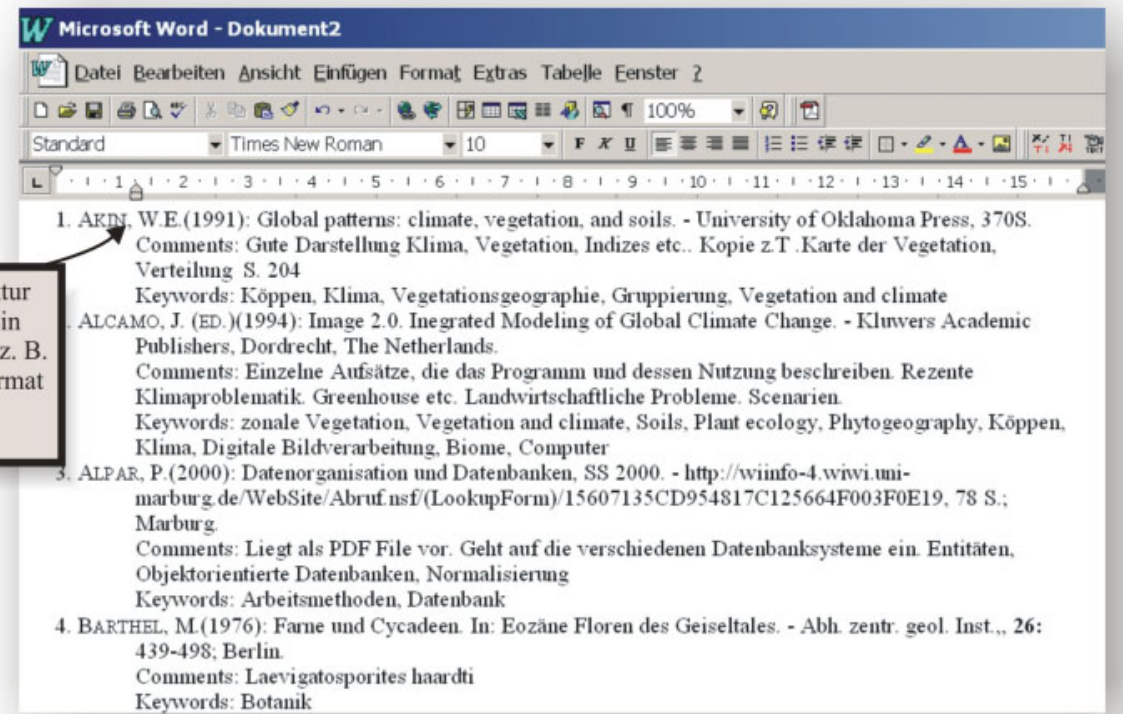

Abb. 3.51: Literaturliste in Word. 


\subsubsection{Erzeugen von Eingabetabellen}

\section{Eingabe-Tabelle}

Eingabe-Tabelle
(Rockstand) (Menüleiste Datei, Exportieren: Eingabetabellen erstellen (Excel)) Um mit P.A.S.T den palynologischen Rückstand in Form von Pollendiagrammen und anderen graphischen Auswertungsmethoden $\mathrm{zu}$ erfassen, ist es möglich, aus Formenlisten Eingabetabellen zu erzeugen.

Diese Tabellen enthalten zusätzlich zur Auflistung der Formennamen Informationen über die nächsten lebenden Verwandten, mit Ihren botanischen und geographischen Parametern (in P.A.S.T: Gruppierungsparameter). Sämtliche Parameter werden den entsprechenden Formularen (i.e.S. Tabellen) der Datenbank entnommen und in die Eingabetabelle, generiert von P.A.S.T, nach Excel übertragen.

Die Eingabetabellen werden in der Regel aus dem Auswahlformular heraus mit Hilfe des Eingabetabellen-Assistenten erzeugt. Sie können die Auswahl, die Sie im Kapitel 3.9.5, S. 75 generiert haben, für die Eingabetabelle benutzten.

Öffnen Sie hierzu das Auswahlformular und klicken Sie auf den Button "Eingabe Tabelle (Rückstand)".

Führen Sie nach Öffnen des Formulars die angegebenen Arbeitsschritte der Reihe nach aus.

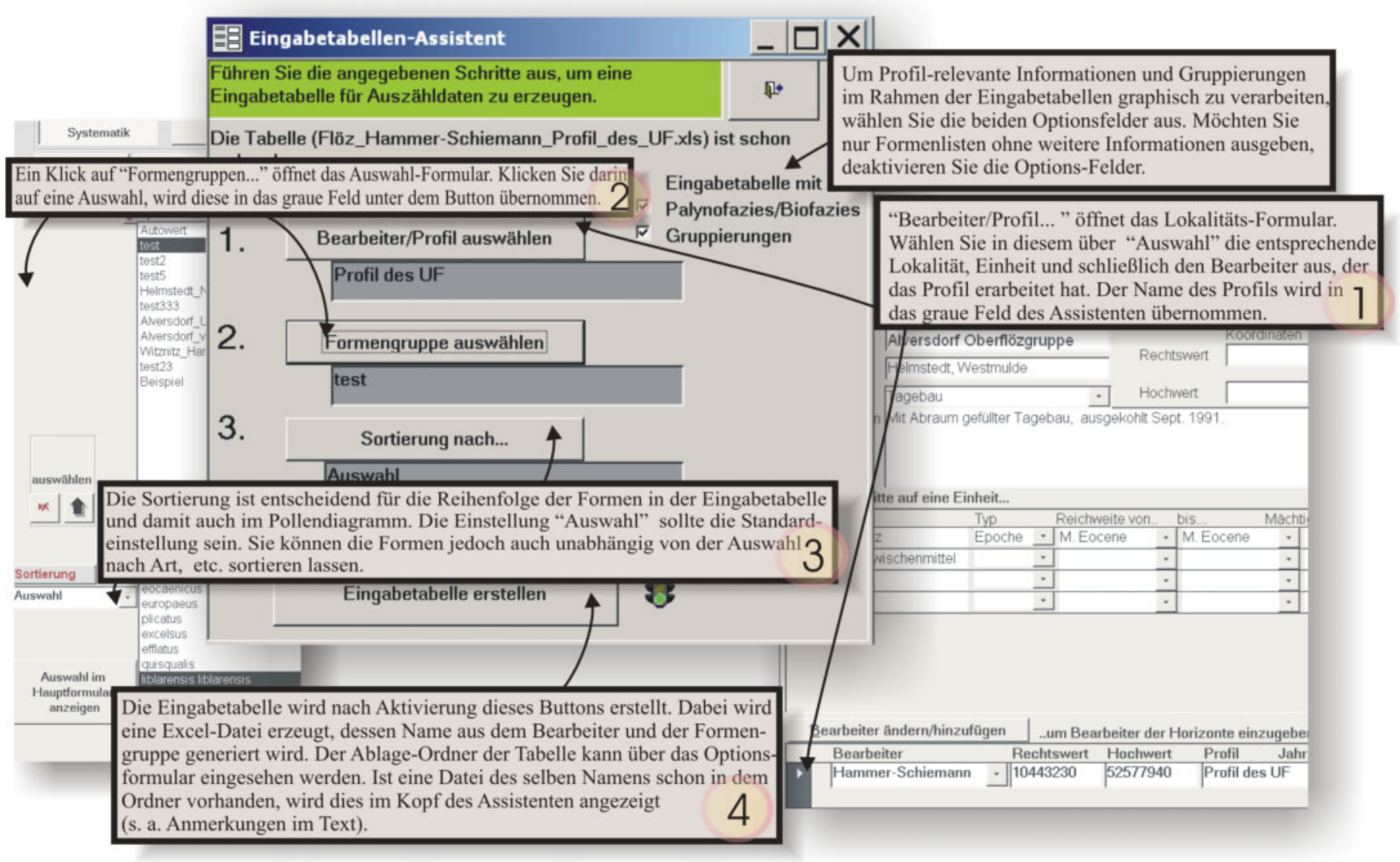

Abb. 3.52: Eingabetabellen-Assistent zur Erzeugung von Profilen, Pollendiagrammen und graphischen Auswertungen. "Bearbeiter/Profil auswählen" öffnet das Lokalitäts-Formular, "Formengruppe auswählen" das Auswahl-Formular. "Sortierung nach..." ist entscheidend für die Reihenfolge der Sporomorphae. 


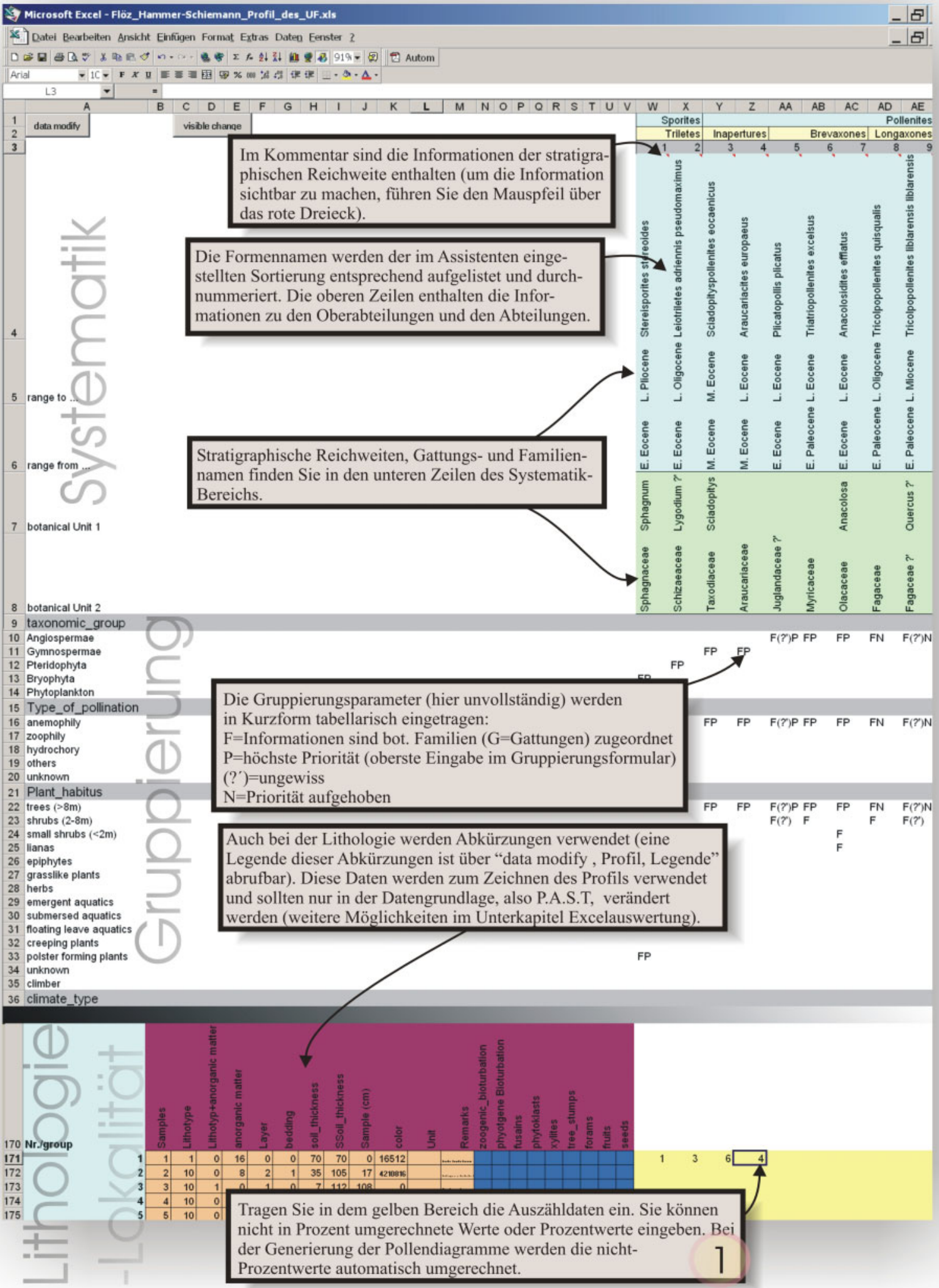

Abb. 3.53: Eingabetabelle für palynologische Auszähldaten. Grautransparent sind die entsprechenden Formulare in P.A.S.T, die zur Eingabe der Daten benötigt werden, abgegrenzt (Systematik, Gruppierung und Lokalität sowie das Unterformular Lithologie). 


\section{Anmerkungen:}

Die Eingabetabellen werden extern gespeichert (Optionsformular: Pfad "für die Tabellen". Siehe auch Kap. 3.4, S. 40). Die Tabellen können auch ohne P.A.S.T geöffnet und bearbeitet werden. In diesem Fall muß jedoch eine weitere Datei, die den Programmroutinen und die Vorlagen für die graphischen Darstellungen enthält, geladen werden. Beim Öffnen der Exceldateien werden Sie auf das Fehlen dieser Datei mit dem Namen "pattern.xls" aufmerksam gemacht. Der Pfad dieser Datei ist dem Optionsformular unter "Pfad für Vorlagen" aus der Datenbank zu entnehmen. Ohne das Laden dieser Datei sind nur die Excel-interen Funktionen abrufbar, jedoch weder das Zeichnen der Pollendiagramme noch das Manipulieren der Daten nach palynologischen Gesichtspunkten ist ohne die Datei "pattern.xls" möglich (siehe auch Kap. 3.11.11, S. 104).

Fehler, die in der Eingabetabelle erkannt werden, sollten in der Datengrundlage - also der Datenbank korrigiert werden. Dies können z.B. fehlerhafte Angaben zu den Gruppierungsparametern, Zuordnungen zu botanischen Familien oder Gattungen sein, die erst beim Zeichnen und Auswerten der Diagramme erkannt werden. Selbstverständlich ist eine Korrektur der Daten - zumindest im eingeschränktem Rahmen auch direkt in der Eingabetabelle möglich (s. Kap. 3.10.5, S. 88 f.). Allerdings sei darauf hingewiesen, daß eine Rückführung der geänderten Daten aus der Eingabetabelle in die Datenbank nicht möglich ist, da sich die Strukturen der Datengrundlage grundlegend von der Excel-Datenmatrix unterscheiden.

Zur Erzeugung der Tabelle sind einige Schritte nötig, die im Einzelnen schon in den vorhergehenden Unterkapiteln erläutert wurden. Anschließend folgt eine Zusammenfassung der Vorgehensweise mit Verweisen auf die relevanten Unterkapitel.

Vorstellbar sind zwei verschiedene Szenarien:

Während der gesamten palynologischen Bearbeitung begleitet P.A.S.T den Palynologen bei Fragen zur Systematik, Morphologie, Botanik, Lithologie bis hin zum Erzeugen der Tabellen zur Planung der palynologischen Auszählung und späteren Darstellung der Ergebnisse.

Die Daten liegen bereits in Form einer Publikation vor und sollen durch P.A.S.T ansprechend und einheitlich dargestellt werden, um sie mit anderen Arbeiten besser vergleichen zu können sowie über die Datenbank anderen Palynologen zugänglich zu machen.

\section{$\mathrm{Zu}$ 1:}

$>\mathrm{Zu}$ Beginn findet die Eingabe der für die Eingabetabelle benötigten Taxa statt, die noch nicht in der Datenbank gespeichert sind (Kap. 3.7, S. 46 ff.).

$>$ Nun folgt die Überprüfung bzw. Eingabe der Gruppierungsparameter des nächsten lebenden Verwandten, sofern eine spätere Auswertung, z.B. das numerische Zusammenfassen von Familien nach azonalen Elementen oder anderen botanisch-geographischen Parametern, erwünscht ist (s. S. 49 ff.)

$>$ Im nächsten Schritt wird Eingabe der Lokalitäts- und Lithologiedaten durchgeführt, falls die Eingabetabelle mit einem lithologischen Profil verbunden werden soll (Kap. 3.8.2, S. 64 ff.).

$>$ Die Generierung der Auswahl der Formen, die für die Eingabetabellen nötig sind (Kap. 3.9.5, S. 75 f.), wird mit der

Erzeugung der Eingabetabelle (Kap. 3.10.5, S. 88) abgeschlossen 
$\mathrm{Zu} 2$ :

$>$ Die ersten drei Schritte entsprechen dem ersten Szenario. Nun erzeugt man eine Auswahl (s. oben) und überträgt diese in die Eingabetabelle (s. oben).

Anschließend fügt man bei Excel temporär in eine neue Zeile die Taxa aus der Archivdatei ein, um eventuelle Fehler bzw. Nichtübereinstimmungen der beiden Listen zu erkennen, bevor die Auszähldaten übertragen werden.

Als nächstes sollte eine Vorsortierung der Formen nach den GruppierungsElementen stattfinden (z.B. azonale Vegetation), um eventuelle fehlerhafte Gruppierungen zu erkennen, die schon in der Datenbank korrigiert werden sollten (Kap. 3.11.2, S. 92).

$>$ Als Letztes sollten die Auszähldaten der Archivdaten in den dafür vorgesehenen Bereich eingefügt werden.

\subsection{Auswertung und graphische Darstellung der Auszähldaten}

Die Auswertung der Auszähldaten findet komplett in Excel statt (Siehe Anmerkungen in Kap. 3.10.5, S. 88 ff.).

Um die Auswertefunktionen zu nutzen, wird ein Grundwissen der Excelbedienung vorausgesetzt, da sich P.A.S.T in die Excel-Programmumgebung einbettet.

\subsubsection{Umrechnung der Prozentwerte}

\section{data modify}

Nach der Eingabe der Daten sollten die Werte in Prozente umgerechnet werden. Dazu klicken Sie in der generierten Excel-Eingabetabelle auf den Button „data modify" am linken oberen Tabellenrand. Alternativ kann dieses Menü auch über einen Klick mit der rechten Maustaste in der Tabelle und dem Menüeintrag „Pollendaten (Formular)“" aufgerufen werden (Abbildung 3.54).

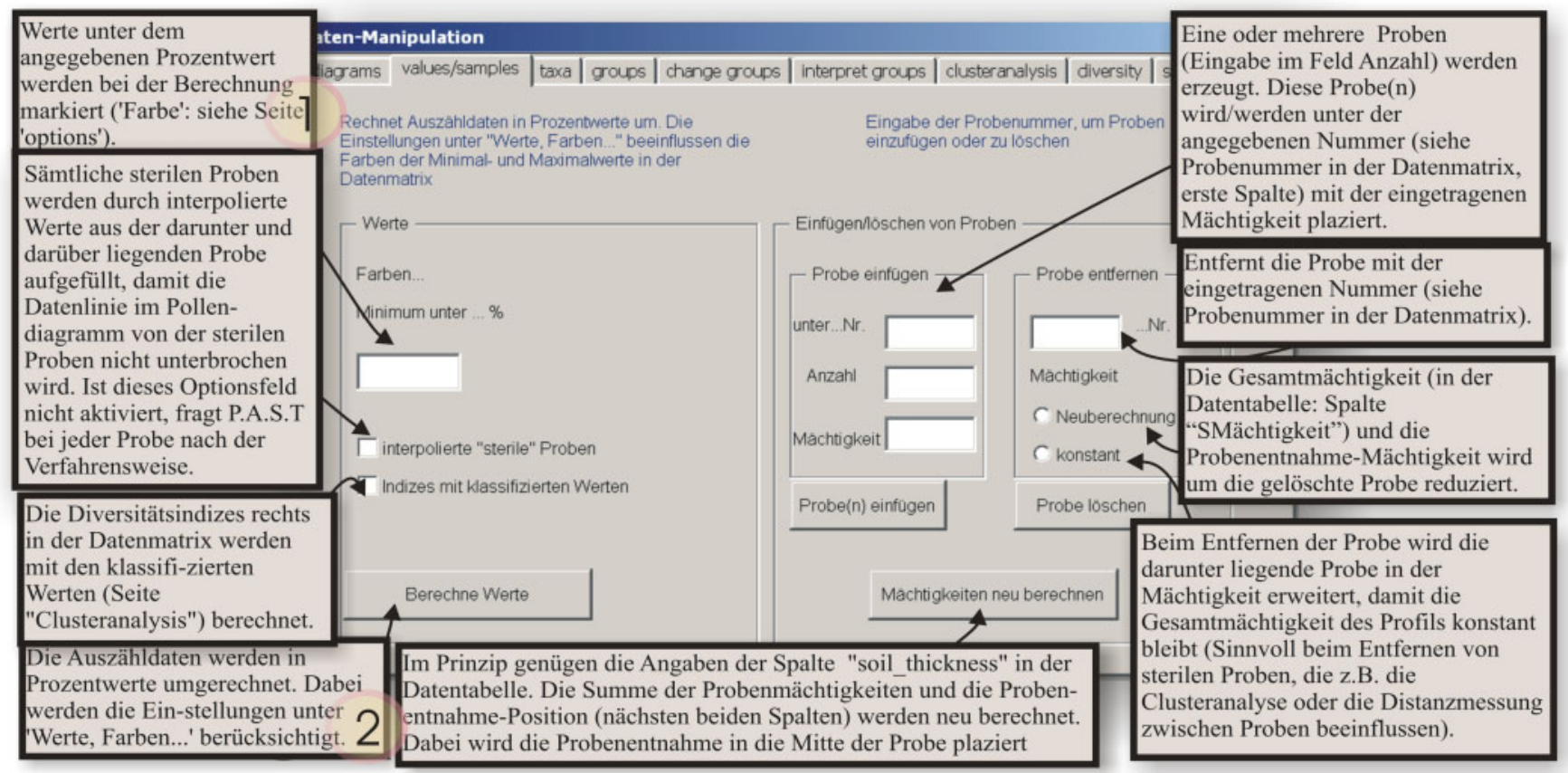

Abb. 3.54: Seite Value/Samples: Umrechnung der Auszähldaten in Prozentwerte sowie „Proben einfügen“ und „löschen“. 
Nachdem Sie die Berechnung aktiviert haben („Berechne Werte“), wird die Berechnungsvariante der sterilen Proben - sofern vorhanden - angemerkt. Eine Interpolation berechnet die sterile Probe als geometrischen Mittelwert (die Mächtigkeit der Proben wird mitberücksichtigt) der umschließenden Proben, so daß bei der Pollendiagrammdarstellung eine Verbindungslinie entsteht. Sinnvoll ist dies bei Proben, die zum Beispiel aus Zeitgründen nicht ausgezählt werden konnten, deren Lithofazies aber im Profil erscheinen soll.

Anmerkung:

Für die Bearbeitung der Auszähldaten ermittelt das Programm den relevanten Datenbereich (z.B. Gruppierungsinformationen, Palynologie, Palynofazies). Dies geschieht über Zellenkoordinaten, die in dafür vorgesehenen Zellen gespeichert sind. Bei sämtlichen Änderungen durch P.A.S.T werden diese Koordinaten neu berechnet; dies kann z.B. das Löschen von Formen, respektive Spalten oder das Löschen von Proben beinhalten (siehe z.B. Kap. 3.11.6, S. 98). Um die Datenkonsistenz zu wahren, sollten deshalb Änderungen der Spalten oder Reihen durch reine Excelfunktionen vermieden werden (Löschen von Datenbereichen etc). Aus diesem Grund sind die Datenbereiche in der Regel wird über „Gültigkeitsbereiche“ geschützt, so daß erst nach einer Neudefinition des Bereiches Eingaben möglich sind (Menü: Daten, Gültigkeit).

Generell wird empfohlen, nach der Eingabe der Auszähldaten eine Sicherheitskopie der Arbeitsmappe anzulegen, da ein versehentliches Neugenerieren der Eingabetabelle aus der Datenbank die bis dato vorgenommenen Änderungen der Eingabetabelle überschreibt.

\subsubsection{Gruppierung}

Im nächsten Schritt sollten die Formen - sofern nötig - gruppiert werden. Dazu rufen Sie die entsprechende Seite auf (Abbildung 3.55).

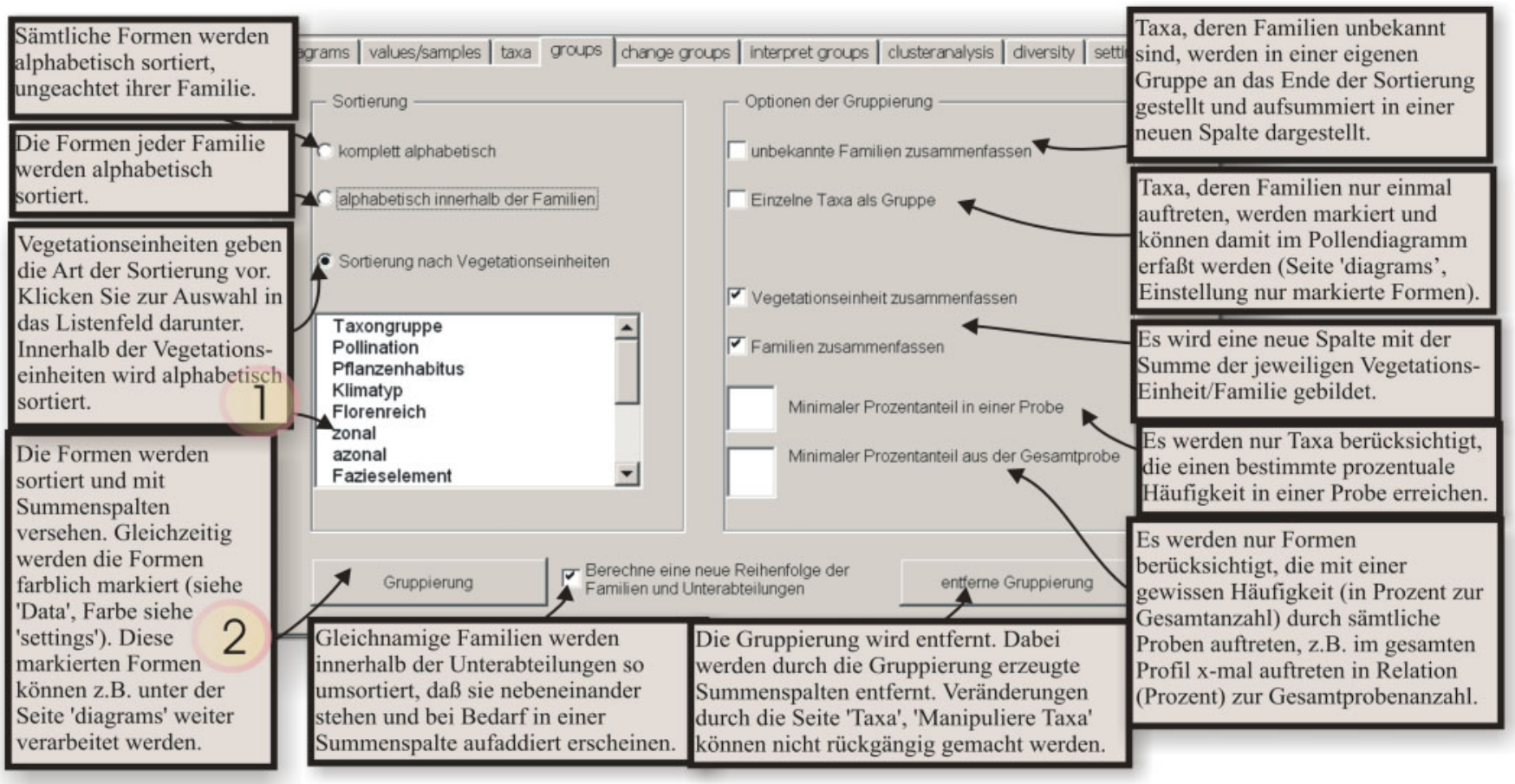

Abb. 3.55: Seite Gruppierung: Zusammenfassen (gruppieren) der Taxa und deren Auszähldaten nach verschiedenen Sortierungsmethoden. 
Das resultierende Arbeitsblatt zeigt die Datenmatrix ergänzt um Summenkurven der jeweiligen Gruppen. Je nach Einstellung der Optionsbuttons (s. Abbildung 3.55) sind die Gruppierungen oder Familien farblich gekennzeichnet (Abbildung 3.56).

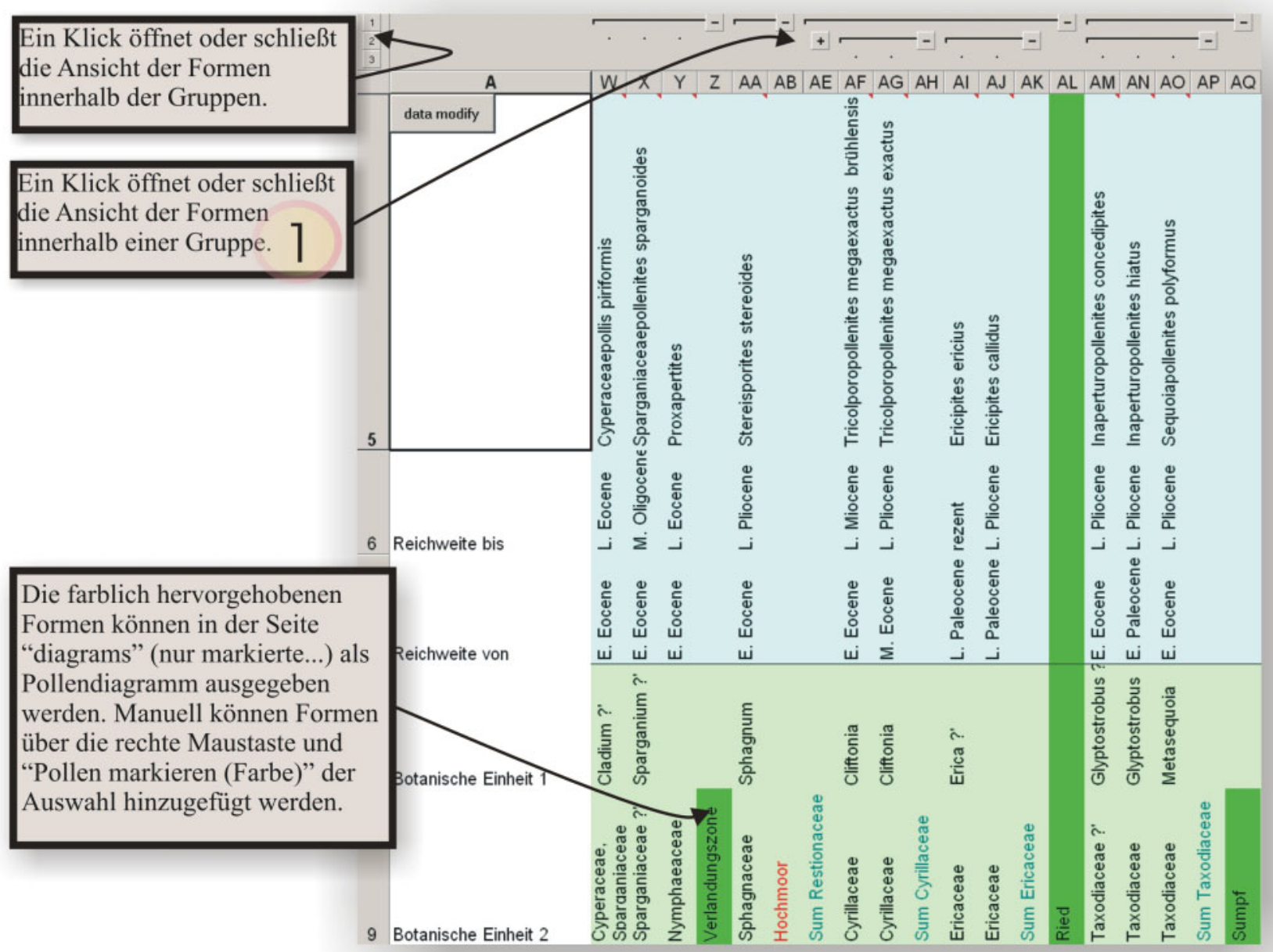

Abb. 3.56: Eine Teilansicht der Ergebnisse der Gruppierungsfunktion von P.A.S.T.

Anmerkung:

Um die Taxa innerhalb einer Gruppe einsehen zu können, muß die Gruppierung über den Tutorial Schritt 1 geöffnet werden. Eventuell muß dazu der „Blattschutz“ aufgehoben werden (s. auch Kap. 3.11.12, S. 105).

\subsubsection{Pollendiagramme}

data modify

Zum Zeichnen der Diagramme klicken Sie auf „data modify“ und wählen die Seite „diagrams“ aus (siehe Abbildung 3.57). Bestimmen Sie das Aussehen der Diagramme über die Optionseinstellungen: Aktivieren Sie das Zeichnen des Profils und der Legende. Bei „Pollendiagramm“ klicken Sie auf "nur markierte...". Anschließend können Sie das Zeichnen der Diagramme aktivieren. 


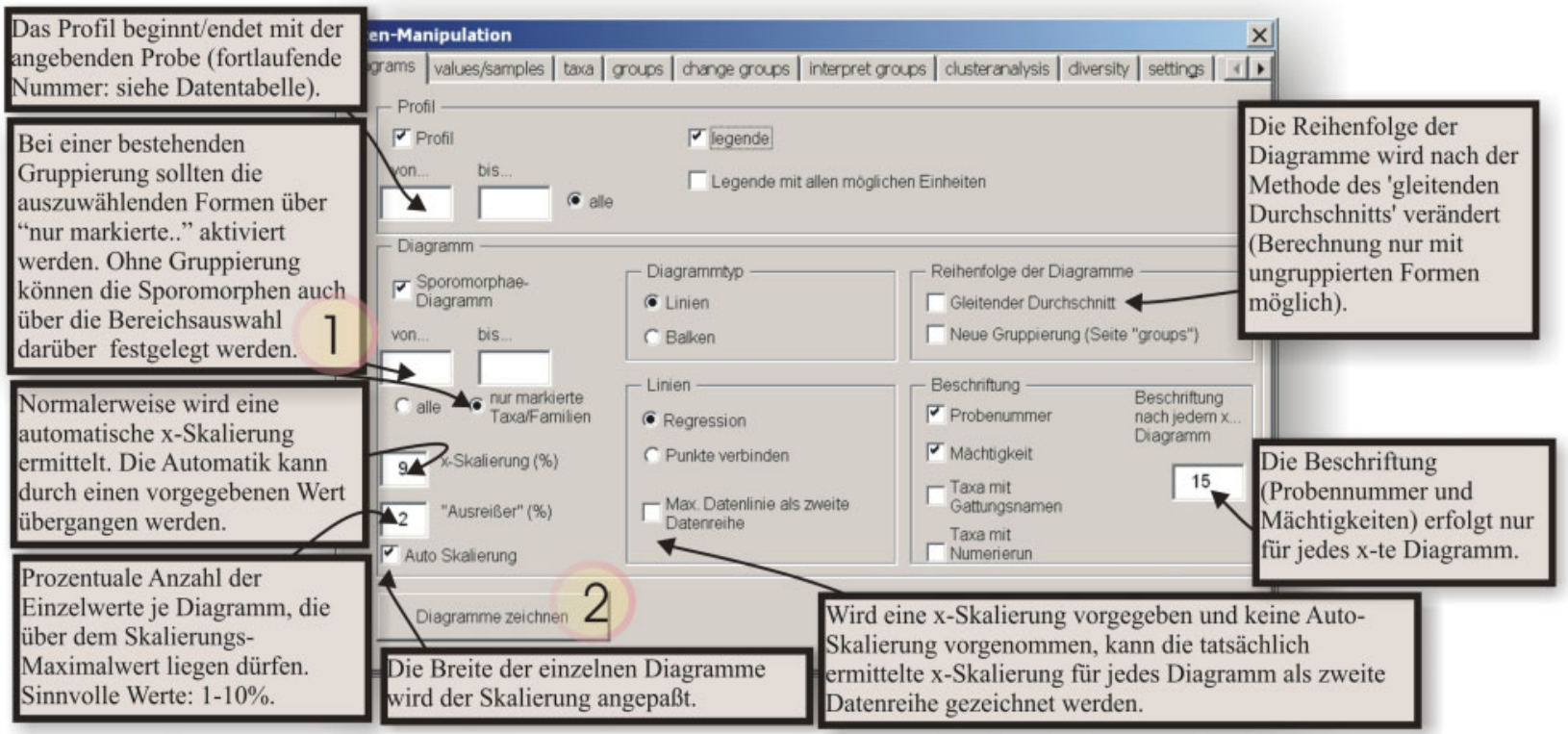

Abb. 3.57: Seite „diagrams“: Hiermit können die Familien und/oder Taxa graphisch in Form von Diagrammen in einem neuen Arbeitsblatt (,Sporomorphae-Diagramm“) gezeichnet werden. Markierte Elemente (z.B. durch die Gruppierungsfunktion) erlauben eine Auswahl der Taxa/Familien oder beliebiger weiterer Gruppierungselemente (Klimakurven etc.).

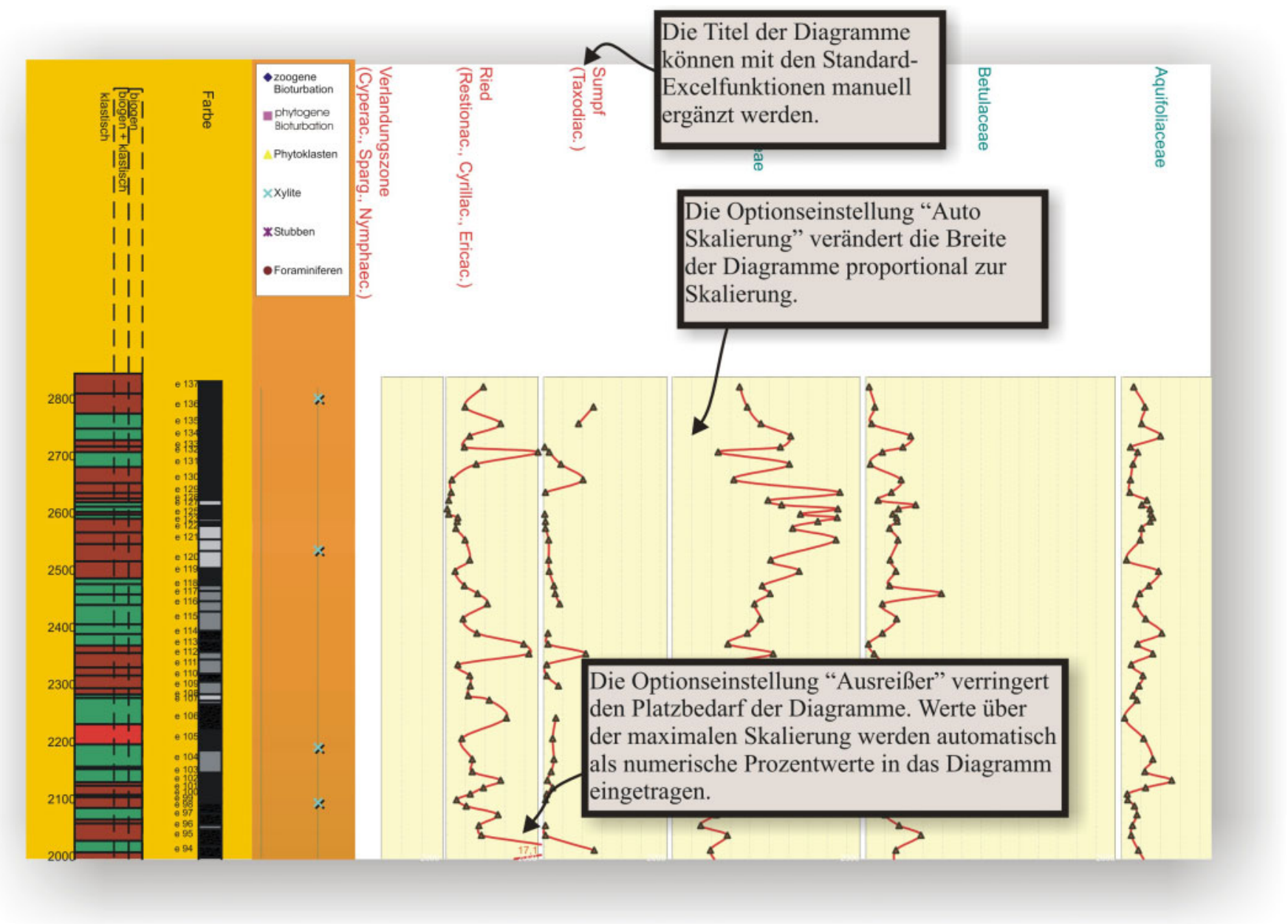

Abb. 3.58: Eine Teilansicht der Diagrammausgabe, generiert in einem neuen Arbeitsblatt (,Sporomorphae-Diagramm"). 


\subsubsection{Auswertung der Gruppierungsfunktion}

Die nachfolgende Funktion von P.A.S.T erlaubt das Zeichnen von „Kuchendiagrammen“ als übersichtliche Darstellung der prozentualen Verhältnisse der Gruppierungsparameter (Abbildung 3.59).

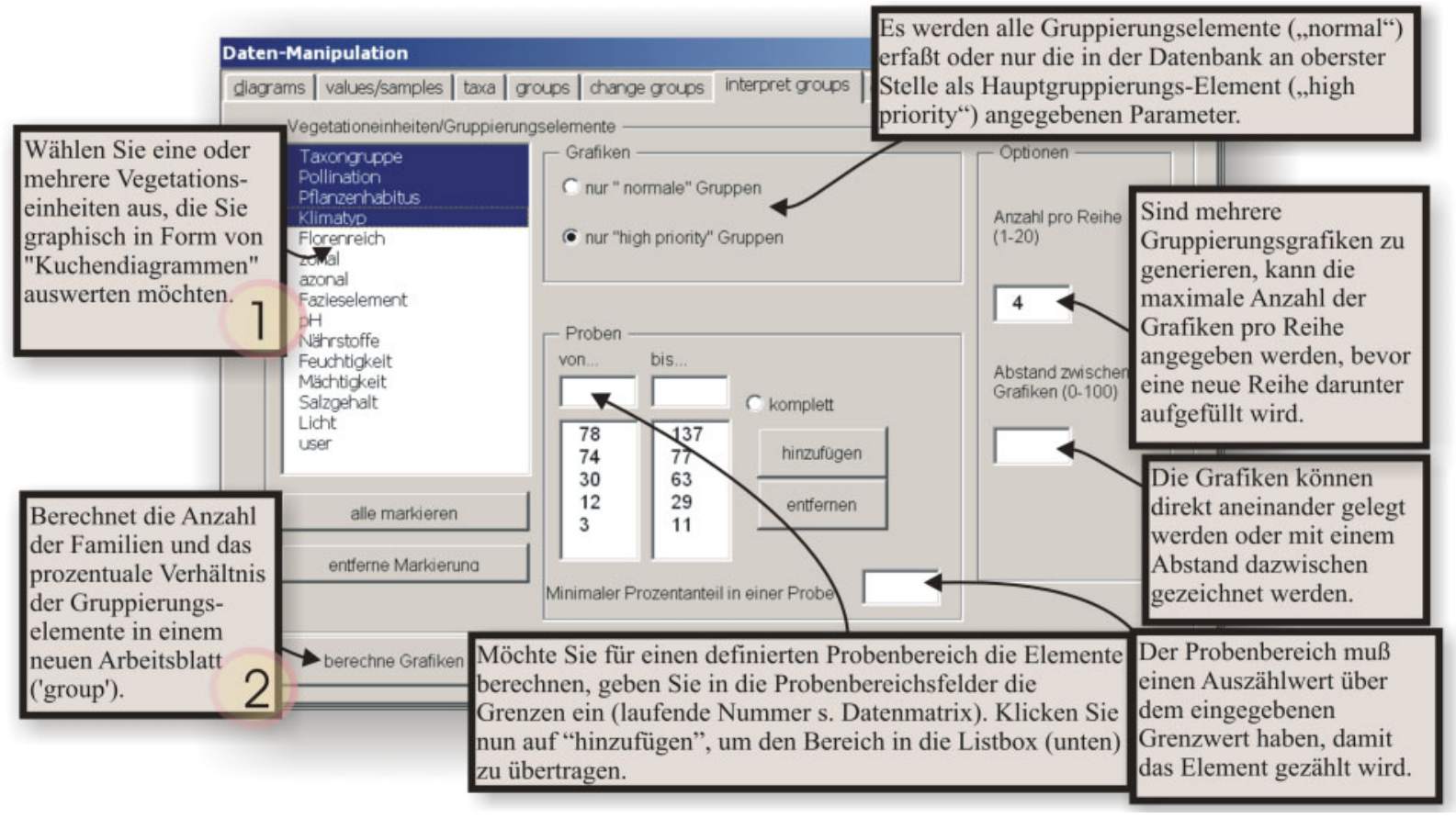

Abb. 3.59: Seite „interpret groups“: Vegetationseinheiten können in Form von „Kuchendiagrammen“ ausgewertet werden.

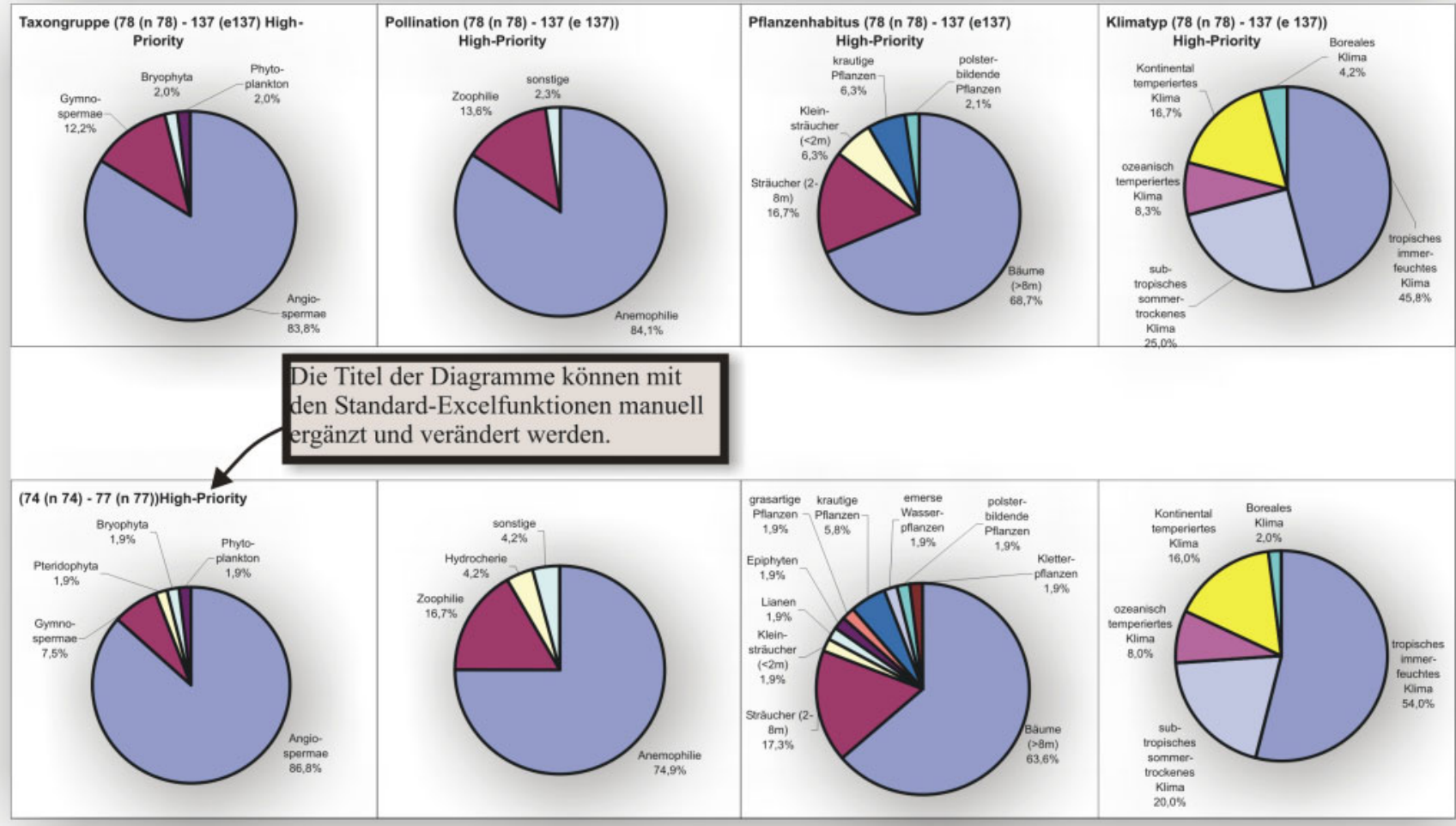

Abb. 3.60: Ein Teilansicht der Diagrammausgabe (Arbeitsblatt ,groups“). 


\subsubsection{Diversität}

Die Berechnung der Diversitäten der in der Datenmatrix vorliegenden Informationen kann mit Hilfe der P.A.S.T-eigenen Statistikroutinen durchgeführt werden (Abbildung $3.61)$.

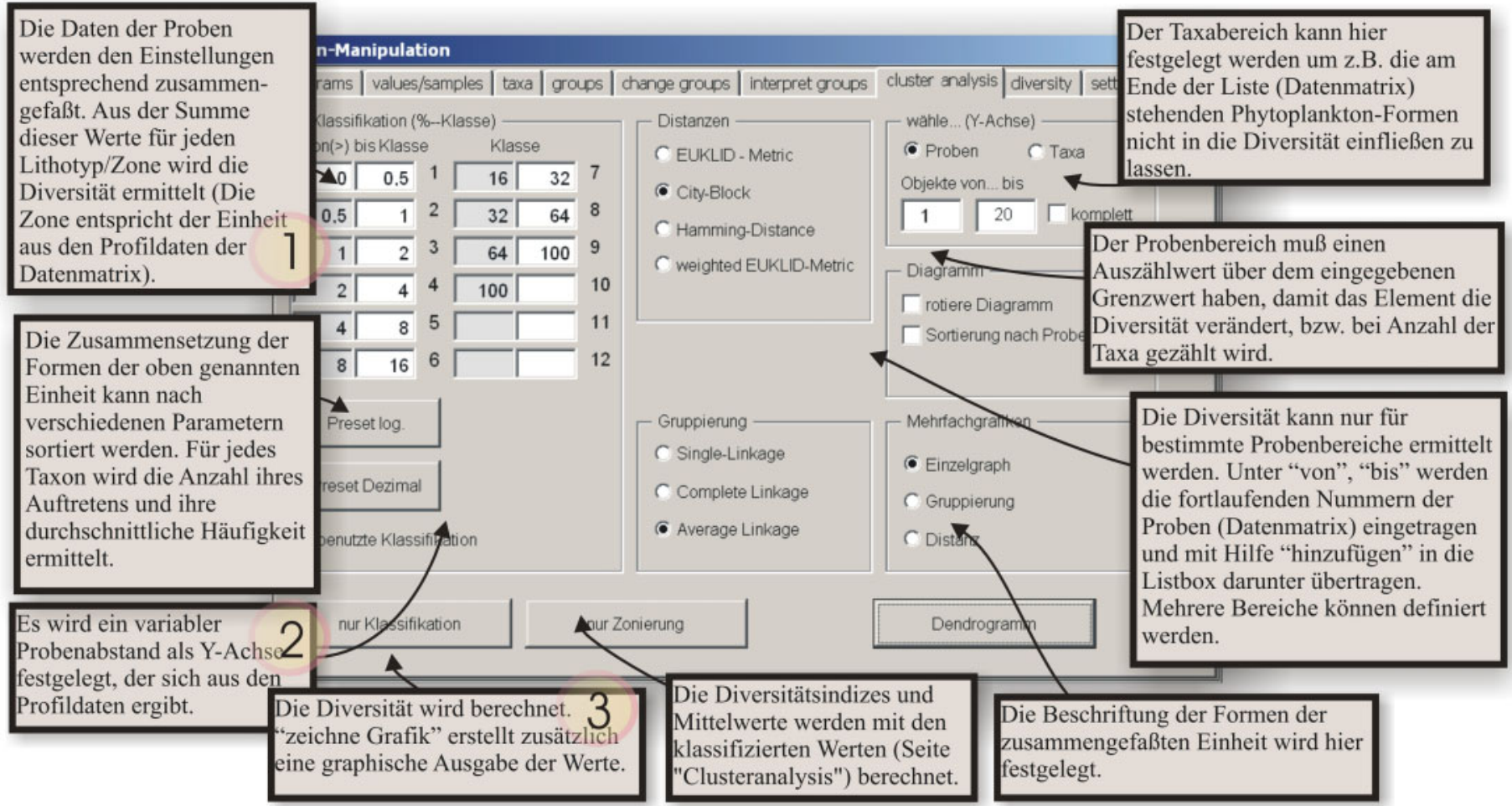

Abb. 3.61: Seite „diversity“: Diversitäten können von Proben, Zonen und Lithotypen ermittelt werden. Eine graphische Ausgabe der Daten in Linien- oder Balkenform ist möglich. Die Zusammensetzung der Taxa mit ihrer Häufigkeit der jeweiligen Zone, der Probe oder des Lithotyps wird ebenfalls ermittelt (s. Abbildung 3.62).

P.A.S.T generiert für die Diversitätsberechnungen ein neues Arbeitsblatt („,diversity“). Darin bieten vielfältige Formate der Ausgabe die Grundlage für weiterführende statistische Untersuchungen. Diversitäten werden für die Summe aller Taxa pro Einheit sowie basierend auf den Diversitätsmittelwerten aller Proben ermittelt. Eine Liste der Diversitäten aller Proben (auch wenn nach Lithotyp oder Zone zusammengefaßt wurde) ergänzt die Daten. Diese Informationen können zum Beispiel für die Erzeugung von Box-Plot-Diagrammen mit geeigneten Programmen benutzt werden (siehe z.B. Kap. 4.4.7.1, S. 116). 
Die Abbildung 3.62 zeigt eine Teilansicht der Ausgabe der numerischen Diversitätsdaten. Aus dieser Darstellungsvariante lassen sich detaillierte Informationen über die Zusammensetzung der Proben, Zonen oder Lithotypen im Kontext zur Diversität ermitteln.

In dem aufgeführten Beispiel kommen demnach in Probe 1 insgesamt 24 Taxa vor, die sortiert nach ihrem durchschnittlichen Auftreten in der untersuchten Einheit (hier Probe) nach rechts aufgelistet werden (in der Abbildung 3.62 aus Platzgründen unvollständig).

Die Diversitäts-Indizes sind links angegeben. Die „Nr (in data)“ kann zum schnellen Auffinden der Form in der Datentabelle benutzt werden.

Unter „Anzahl“ ist die Häufigkeit des Auftretens in der Einheit gemeint. Bei Diversitätsbetrachtungen der Zonen oder Lithotypen variiert dieser Werte entsprechend der Anzahl des jeweiligen Taxa in der zusammengefaßten Einheit (zur Berechnungsmethode der Diversitätsindizes siehe auch Kap. 4.4.1, S. 110).

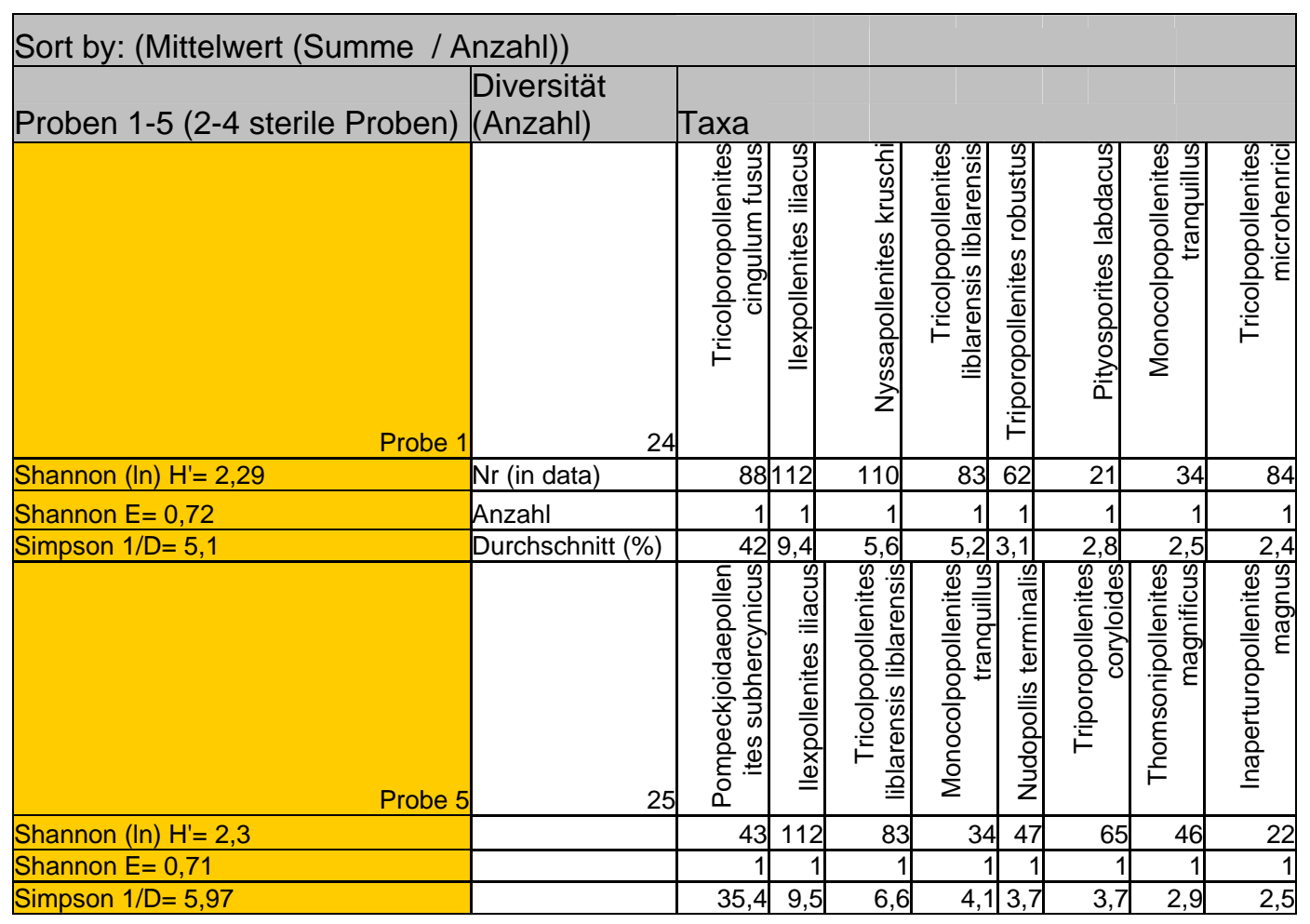

Abb. 3.62: Ein Teilansicht der numerischen Ausgabe der Diversitäten (mit Anpassung des Layouts). 


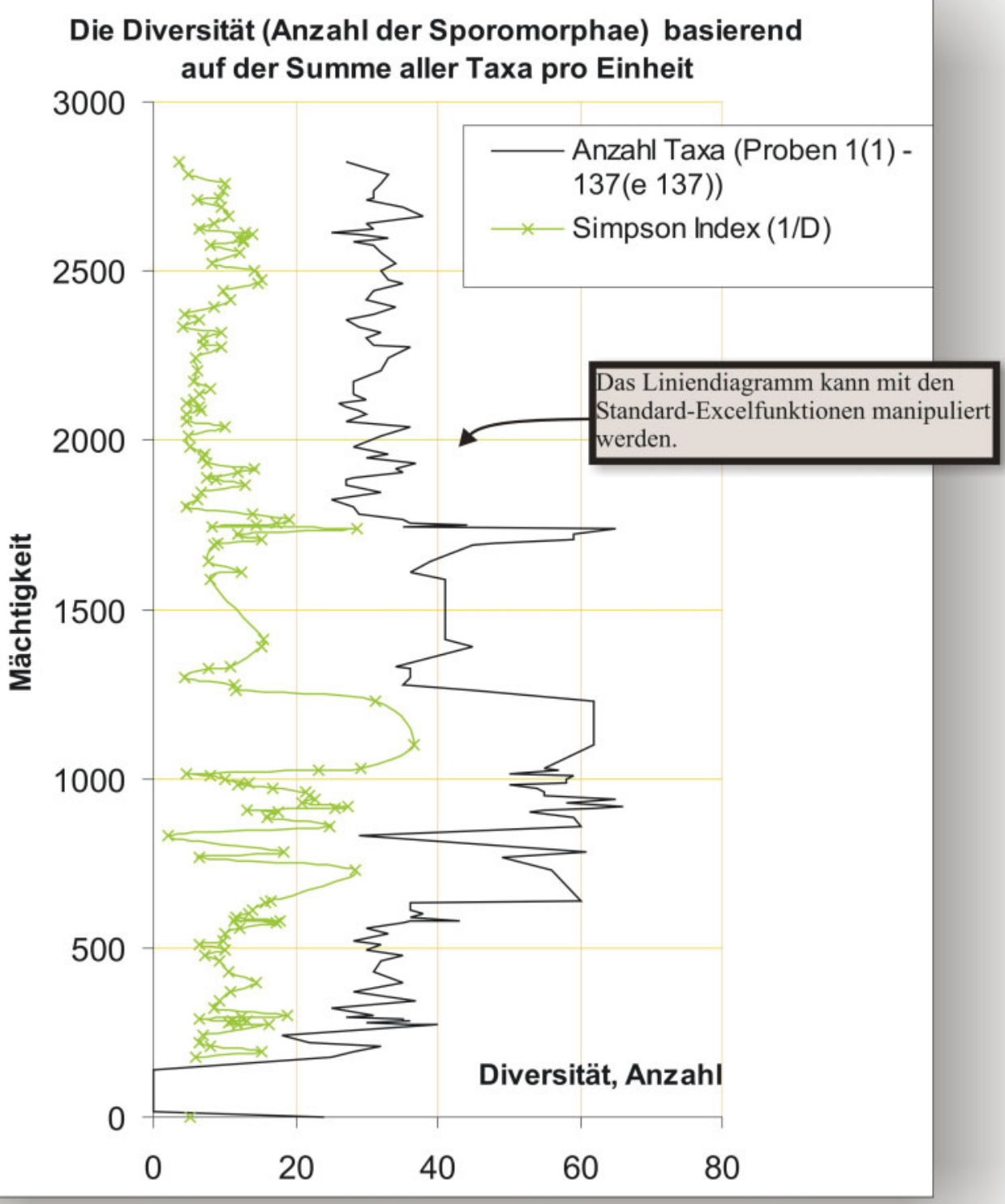

Abb. 3.63: Die Grafik wurde mit der Einstellung der Seite „diversity“ (siehe oben) erzeugt. Mit Hilfe dieser Informationen kann z.B. die Veränderung des Simpson-Index (siehe z.B. Kap. 4.4.1, S. 110 ff.) bei klassifizierten Werten im Gegensatz zu unveränderten Werte im Kontext zur Anzahl der Taxa diskutiert werden.

\subsubsection{Taxa-Manipulationen}

Häufig besteht die Notwendigkeit, Formen zu einer neuen Sammelform zusammenzufassen. Sei es, um die Anzahl der Formen zu reduzieren oder um sehr seltene Formen in eine Sammelform zu übertragen. Dieser Vorgang kann mit P.A.S.T beschleunigt werden. Es können beliebig viele in der Datenmatrix verteilte Formen zu einer neuen Form aufsummiert werden; dabei werden automatisch die Auszähldaten aufsummiert. Dieser Vorgang kann im Gegensatz zur Gruppierungsfunktion nicht rückgängig gemacht werden, deshalb sollte mit einer Sicherheitskopie der Arbeitsmappe gearbeitet werden. 
Auch wenn dieser Vorgang mit den Excel-eigenen Funktionen durchführbar wäre, sollte aus den in den Anmerkungen (Kap.3.11, S. 91 f.) beschriebenen Gründen, die im Folgenden dargelegte Methode benutzt werden (Abbildung 3.64).

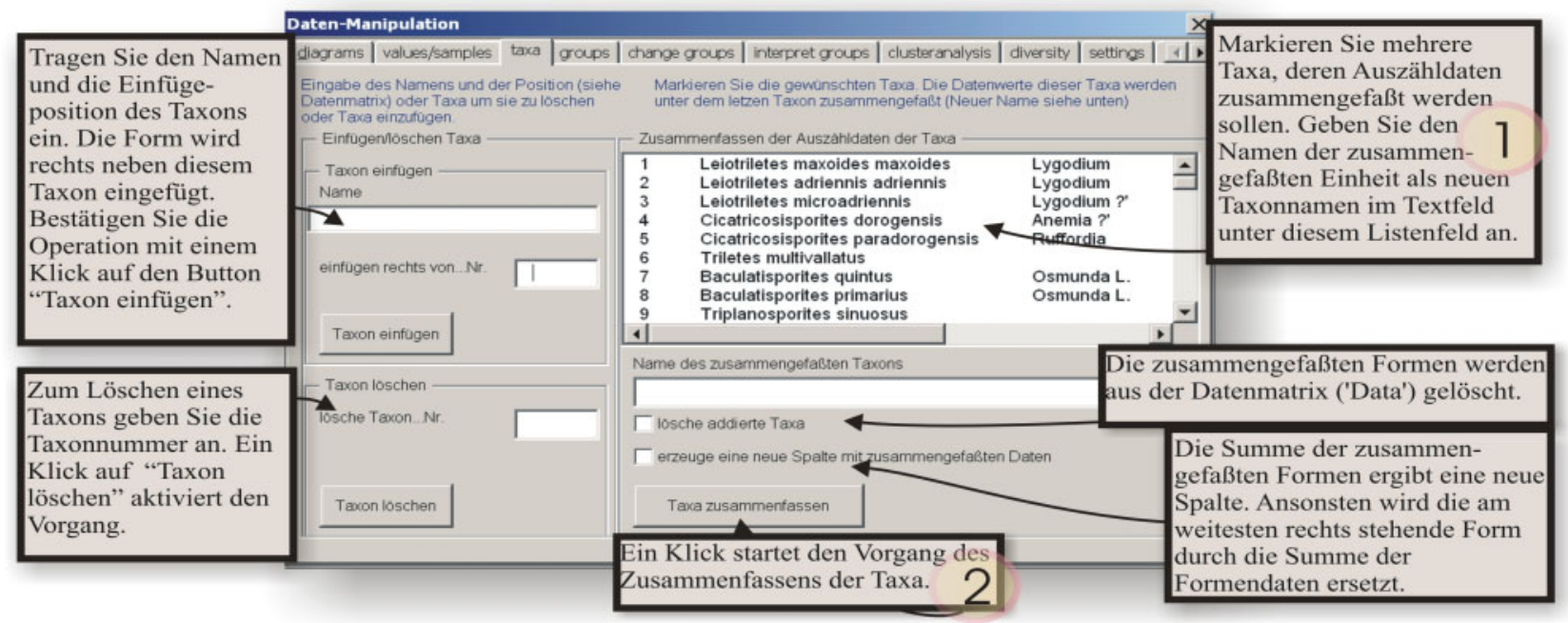

Abb. 3.64: Seite „Taxa“: Mit P.A.S.T können die Daten mehrerer Taxa zu einer neuen Form zusammengefaßt werden. Das Löschen und Hinzufügen von Taxa ist ebenfalls auf dieser Seite möglich.

\subsection{7 Ändern der Gruppierung}

Wie schon mehrfach angemerkt, werden die Gruppierungsinformation aus der Datenbank in das Excel-Diagramm überführt. Kleine Änderungen der Gruppierungszugehörigkeit innerhalb Excels sind möglich, ohne erst umständlich die Eingabetabelle neu generieren zu müssen (siehe Abbildung 3.65). Allerdings kann nur der Hauptparameter („high-priority“), der z.B. für die azonale Sortierung auf der Gruppierungsseite benutzt wird, auf ein anderes Gruppierungselement verschoben werden.

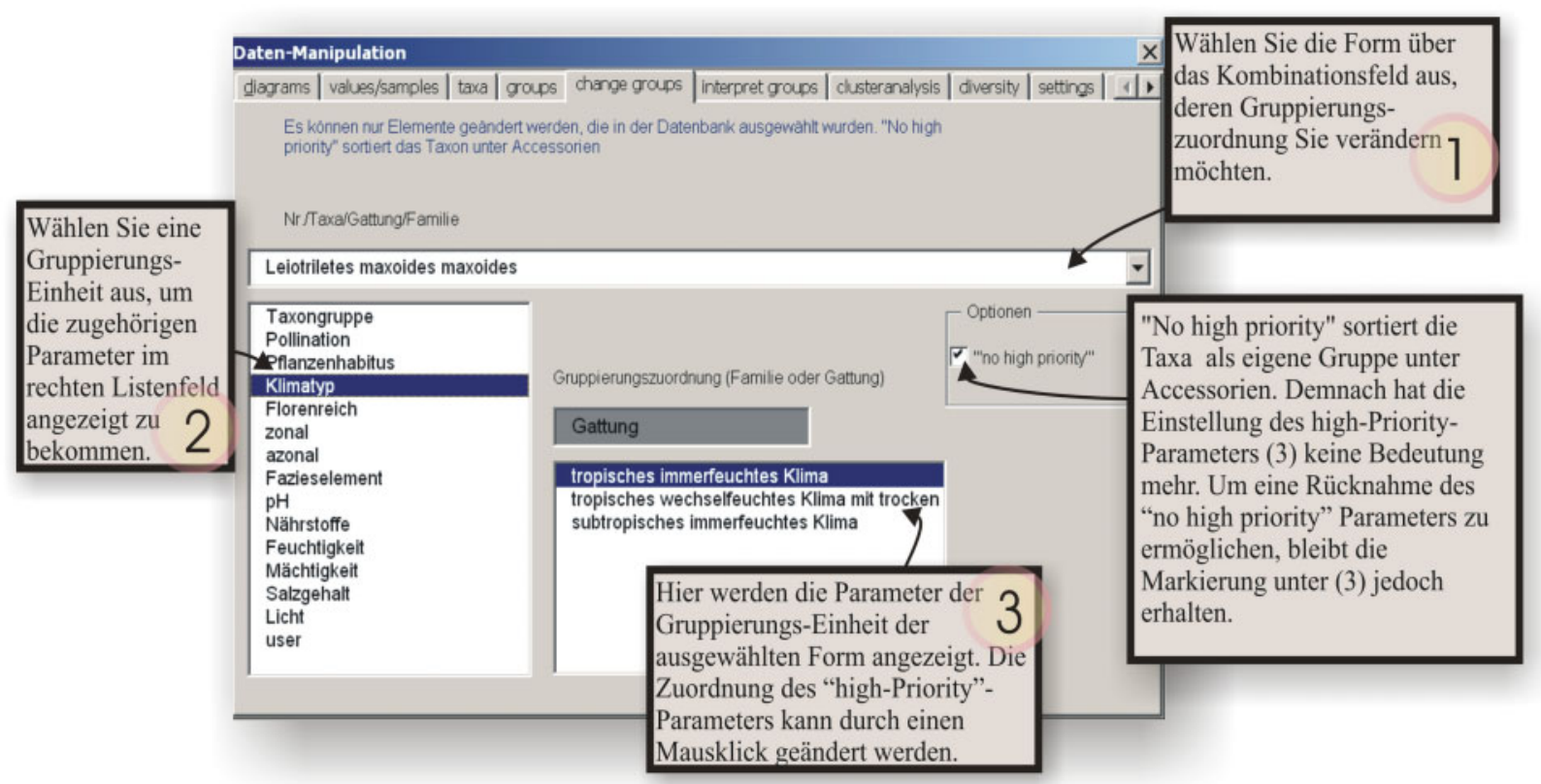

Abb. 3.65: Seite „,change groups“: Änderung des „high priority“ Parameters der Gruppierung. 


\subsubsection{Clusteranalyse}

Die Clusteranalyse-Funktion von P.A.S.T kann in ihrem Funktionsumfang nicht mit Statistikprogrammen, wie z.B. Statistica ${ }^{\circledR}$, konkurrieren. Trotzdem ist insbesondere die Integration in P.A.S.T ein Vorteil bei der schnellen Beurteilung der Clusterbildung von Proben oder Taxa. P.A.S.T stellt nur eine begrenzte Auswahl der Berechnungsmethoden der Clusteranalyse bereit, deshalb kann die Datenmatrix - auch als Matrix der Häufigkeitsklassen - isoliert in einem separaten Arbeitsblatt ausgegeben werden, so daß ein Import der Daten in andere Programme vereinfacht wird.

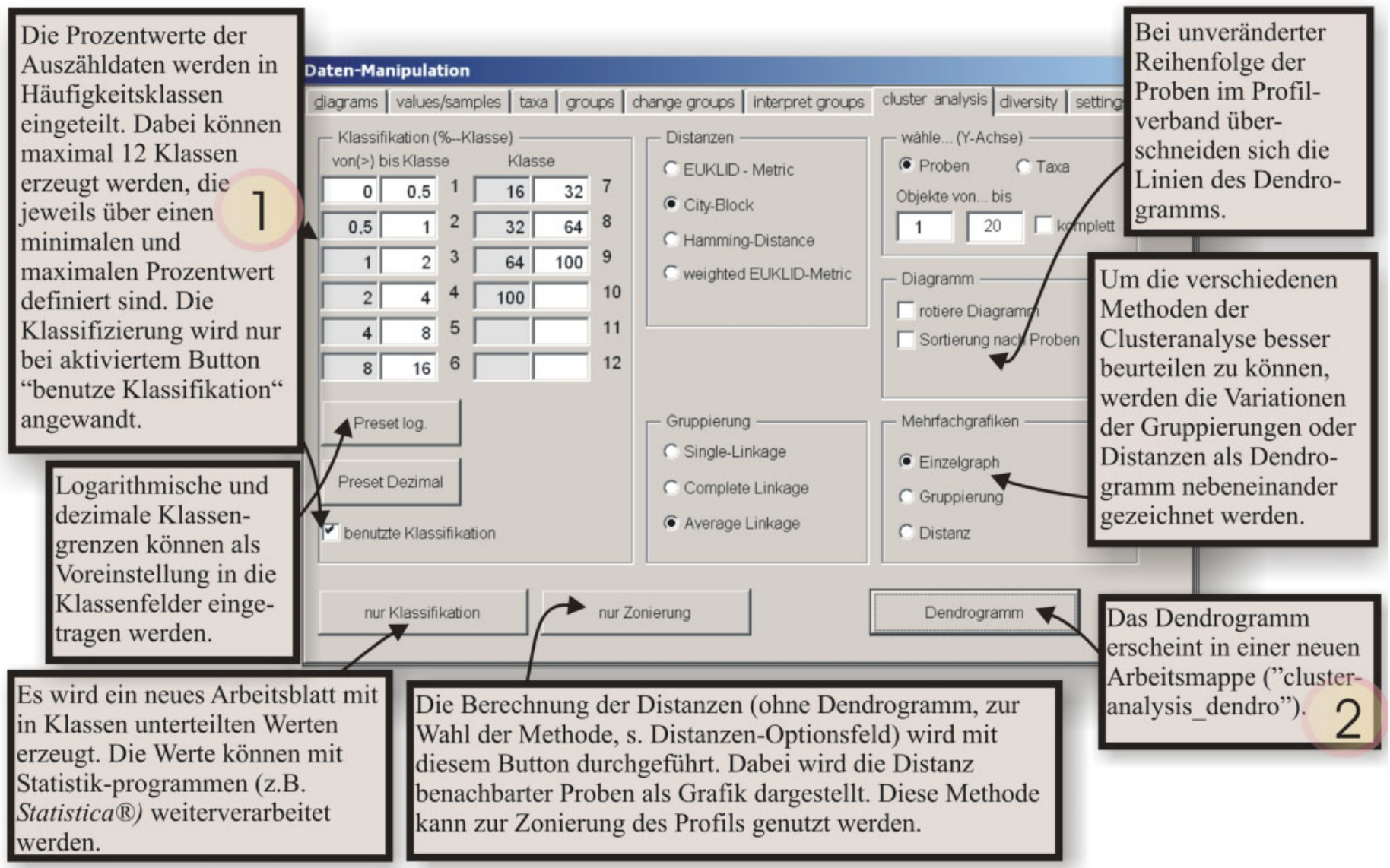

Abb. 3.66: Seite „cluster analysis“: Berechnung der Clusterdendrogramme und der HäufigkeitsklassenMatrix. 


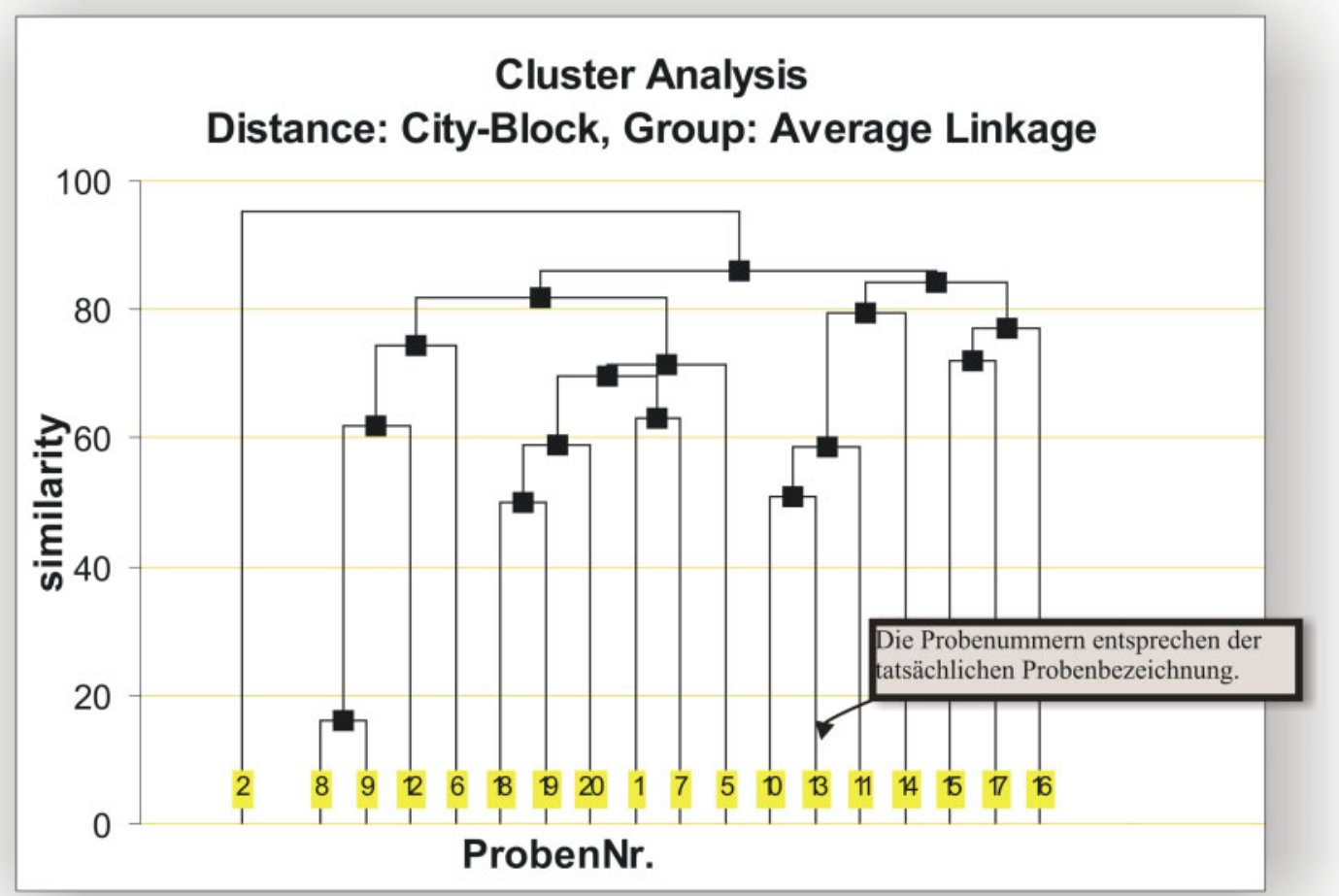

Abb. 3.67: Clusteranalyse der Proben (Beispiel: Profil Alversdorf, s. Anlage).

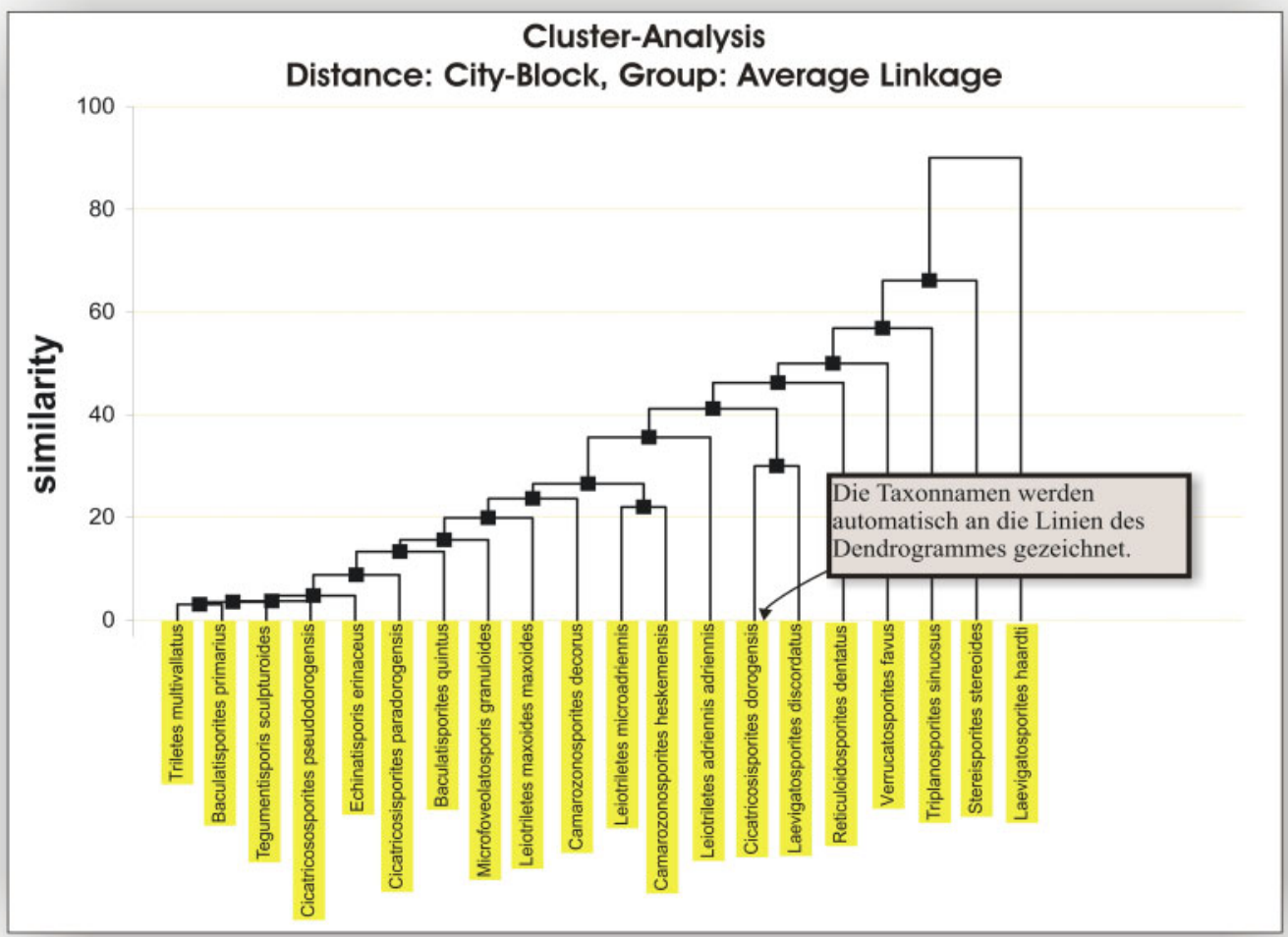

Abb. 3.68: Clusteranalyse der Taxa (Beispiel: s. Abbildung 3.67). 
Einen eher experimentellen Charakter hat die Funktion „Sortierung nach Proben“, da bei dieser Darstellung die entstehende Grafik bei einer größeren Anzahl von Proben unübersichtlich wird (s. Abbildung 3.69). Der Gedanke, die Unterschiede der Proben zueinander im Verbund des Profils zu betrachten, wurde bei der Funktion „Autozonierung“ weiter verfolgt. Hierbei werden aus der Matrix aller Distanzen zueinander nur die Distanzen benachbarter Proben extrahiert und in ein Diagramm übertragen (Anwendungsbeispiel: Kap. 4.4.9.1, S. 126 f.).

\section{Cluster-Analysis Distance: City-Block, Group: Average Linkage}

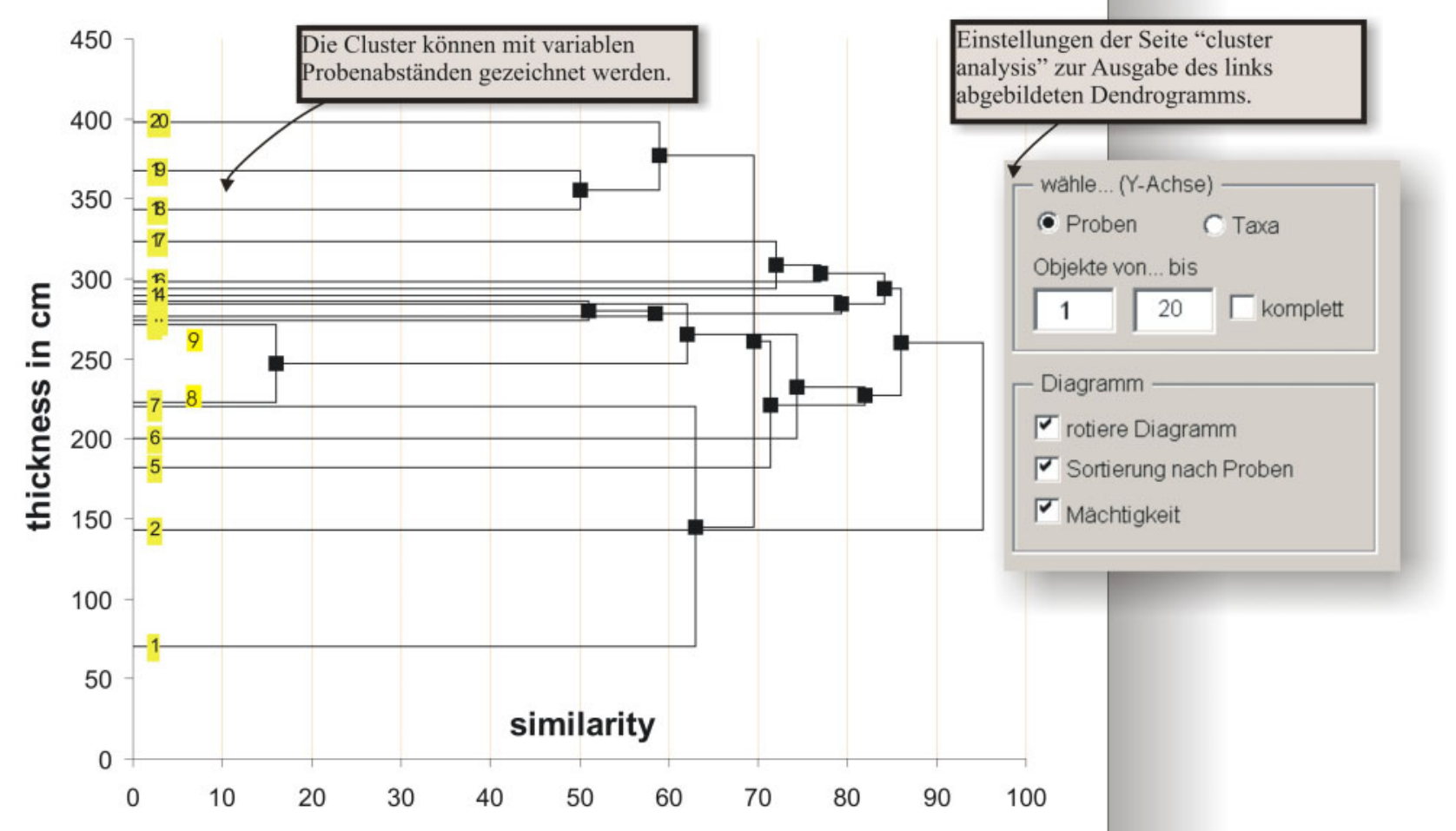

Abb. 3.69: Clusteranalyse der Proben (Proben in der Reihenfolge des Profils). Erkenntlich ist ein geringer Unterschied der Proben 8 und 9. Der Komplex 18, 19, 20 hebt sich von den sehr heterogenen Proben darunter ab. Die Anzahl der gleichzeitig zu betrachtenden Proben sollte nicht zu groß gewählt werden, da sonst die Überschneidungen der Linien die Grafik sehr unübersichtlich erscheinen lassen. 


\subsubsection{Settings}

Die Größe der Diagramme und die Farbeinstellungen der Datenmatrix werden über die folgende Seite geändert (Abbildung 3.70). Außerdem können die Vorlagen, die für das Aussehen der Diagramme zuständig sind, in Vorlagensets gespeichert werden (s. dazu Kap. 3.11.11, S.104).

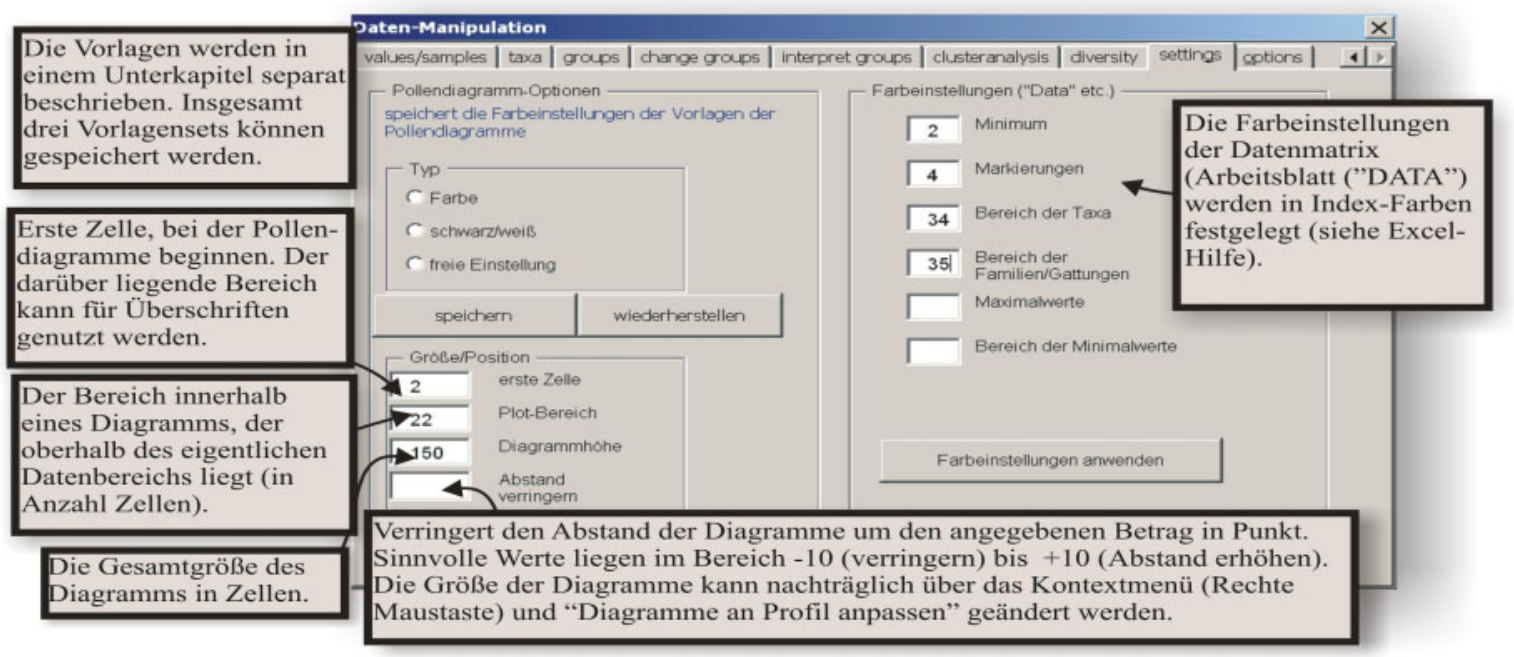

Abb. 3.70: Seite ,settings“: Einstellungen der Vorlagen, Farben und Größe der Diagramme.

\subsubsection{Optionen}

Unter Optionen sind das Zusammenfügen von Arbeitsblättern und die DatenansichtsFunktionen erreichbar (Abbildung 3.71).

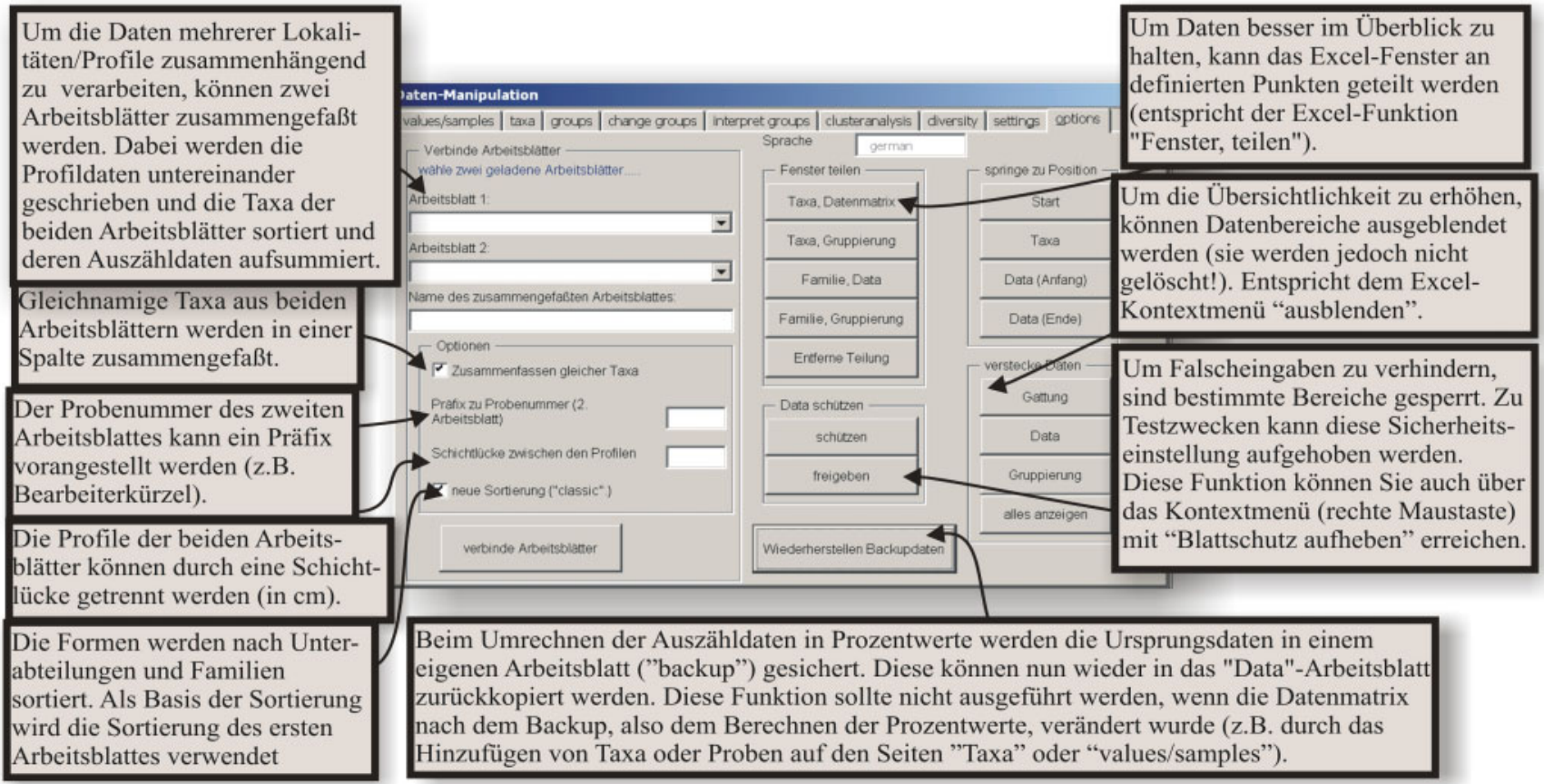

Abb. 3.71: Seite „options“: Arbeitsblätter zusammenfügen und die Datenansicht manipulieren. 


\subsubsection{Vorlagen}

\section{visible change}

Das Aussehen der Diagramme kann über Vorlagen an zentraler Stelle eingestellt werden. Dazu gehören Informationen zur Farbwahl, Schriftarten etc. Diese Einstellungen werden in der Datei ,pattern.xls“ gespeichert (siehe auch Anmerkungen zum Kap. 3.10.5, S. 88 ff.). Veränderungen an der Vorlage implizieren ein Neugenerieren der Diagramme über die entsprechenden Seiten.

Zum Öffnen der Vorlageneinstellungen klicken Sie auf den Button „visible change“ am linken oberen Rand des Arbeitsblatts „Data“. Sind die Änderungen durchgeführt, kann mit dem Button ,save“ oder einem Wechsel der Arbeitsmappe zur Datenmatrix zurückgekehrt werden (Abbildung 3.72 und 3.73).

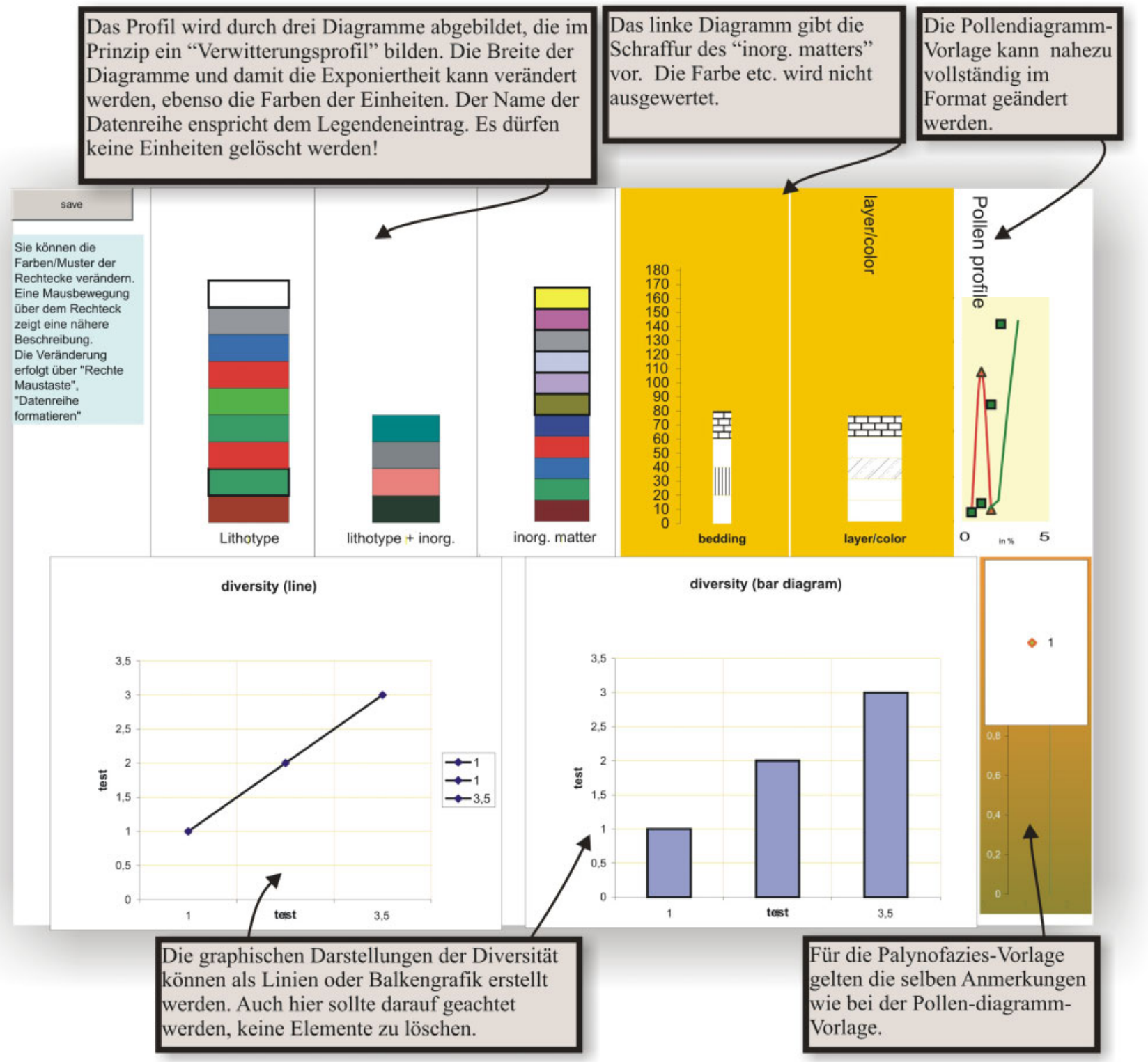

Abb. 3.72: Vorlagen der Diagrammdarstellungen von P.A.S.T (erste Seite). 


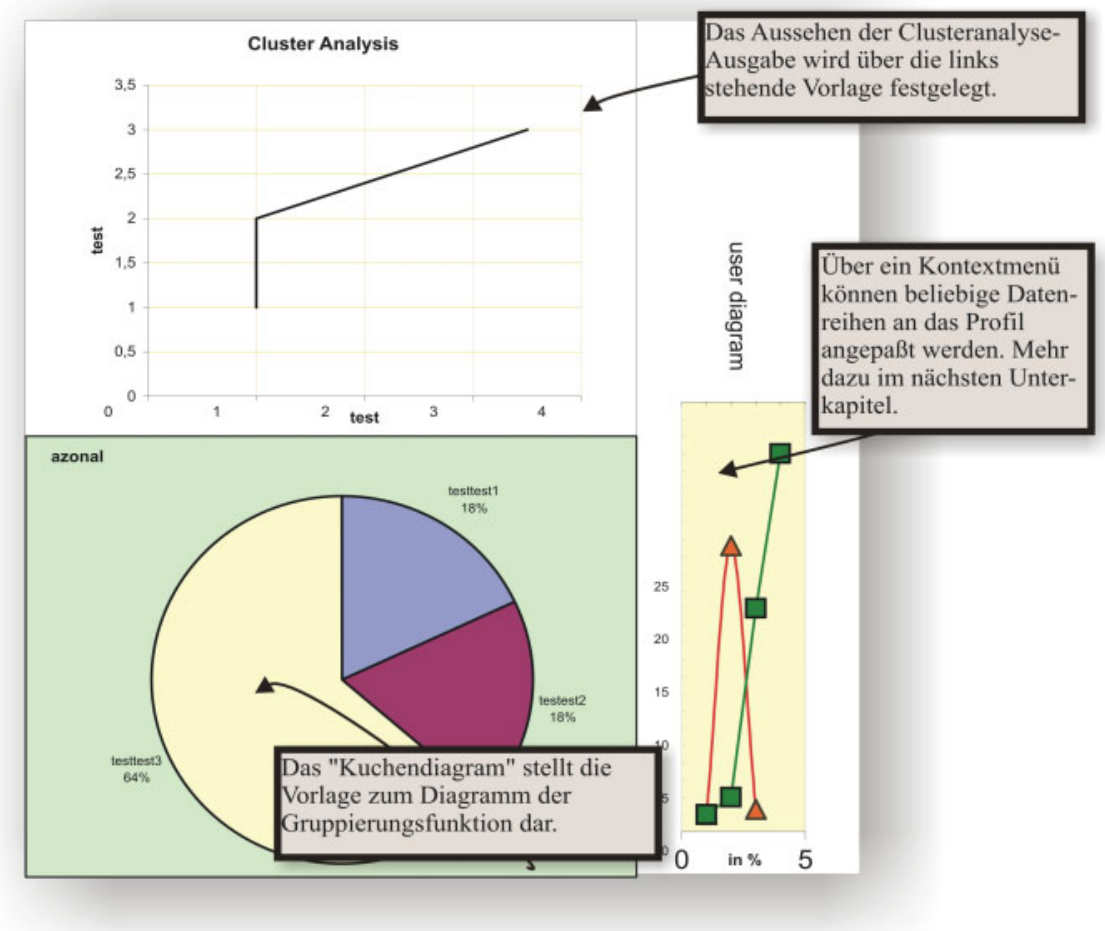

Abb. 3.73: Vorlagen der Diagrammdarstellungen von P.A.S.T (zweite Seite).

\section{Anmerkungen:}

Die Vorlagen werden, genau wie die Programmroutinen des Excel-Teils von P.A.S.T, in der Datei „pattern.xls“ in einem Tabellenblatt gespeichert. Leider ist es mit Exceleigenen Funktionen nicht möglich, das Löschen von Objekten in der Vorlagentabelle zu verhindern, deshalb sollten Veränderungen an den Diagrammen in der Vorlage nur mit entsprechender Vorsicht vorgenommen werden.

\subsubsection{Kontextmenü}

Das Kontextmenü wird über die rechte Maustaste aufgerufen. Die speziellen P.A.S.TFunktionen werden anschließend beschrieben (Abbildung 3.74).

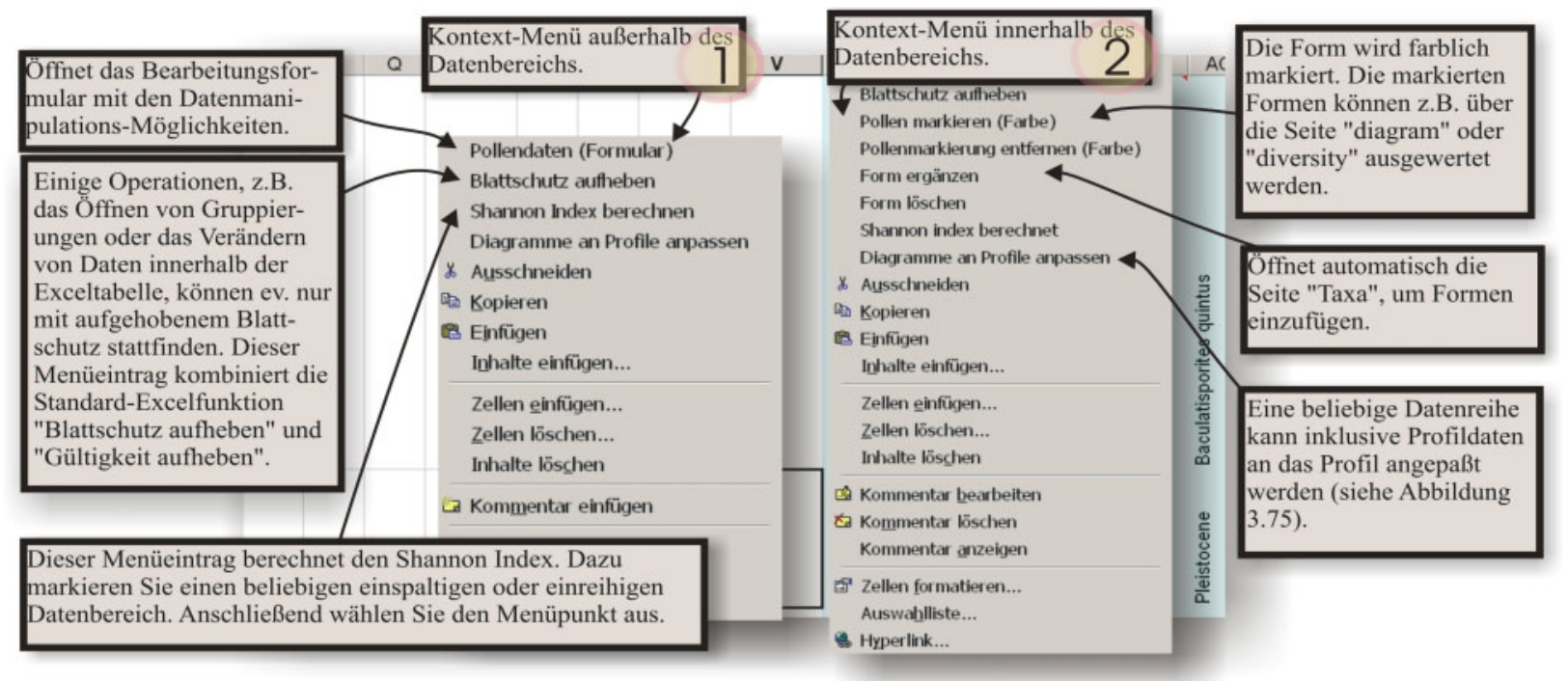

Abb. 3.74: Kontext-Menü von P.A.S.T . 


\subsubsection{Diagramme an Profile anpassen}

Die über das Kontext-Menü aufzurufene Funktion der Diagrammanpassung an Profile erfüllt zwei Aufgaben:

1. Bei Veränderungen an Pollendiagrammen verschiebt sich häufig die Ausrichtung der Diagramme zueinander. Deshalb können Sie die Diagramme über einen Aufruf dieses Menüeintrages neu ausrichten. Die Größen der Diagramme werden der Seite ,settings“ des „Data-modify“-Formulars entnommen (Abbildung 3.70). Eine Größenänderung ist jedoch auch in diesem Formular über das Optionsfeld „Größe/Position“ möglich (s. Abbildung 3.75).

2. Ein- oder mehrspaltige Datenreihen können einem Profil angepaßt werden. Mehrspaltige Datenreihen müssen in der ersten Spalte die Mächtigkeitsangaben zu den Probeentnahmepunkten enthalten.

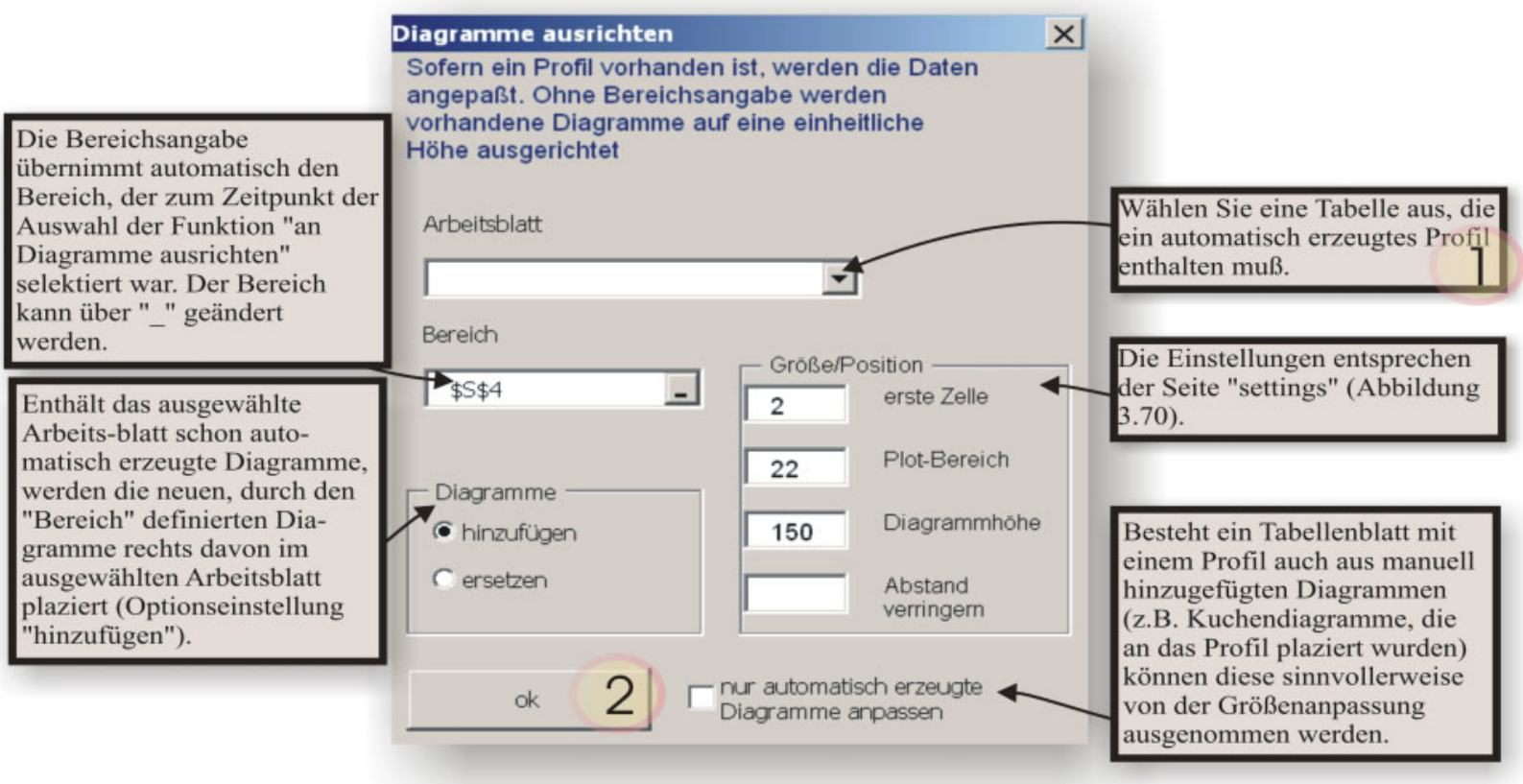

Abb. 3.75: „Diagramme ausrichten“-Formular des Kontext-Menüs von P.A.S.T.

\subsubsection{Mehrsprachigkeit}

language

Klicken Sie auf den Button „language“ am linken oberen Rand des „Data“-Arbeitsblattes, um die Spracheneinstellungen zu ändern (Abbildung 3.76).

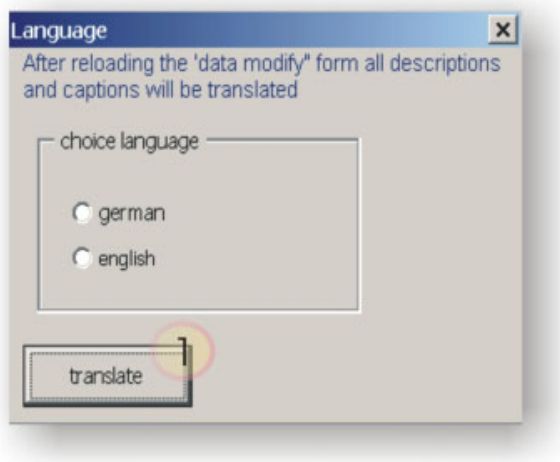

Abb. 3.76: Formular „language“ 


\section{Ergebnisse und Diskussion}

\subsection{Vorbemerkungen}

Die flexible Arbeitsweise von P.A.S.T erlaubt es, die palynologischen Auszähldaten nach verschiedenen Kriterien $\mathrm{zu}$ sortieren, zusammenzufassen, auszuwerten und darzustellen. Diese Vorgänge sind nahezu vollständig automatisiert, so daß wenige „Mausklicks“ ausreichen, numerische und graphische Darstellungen der Ergebnisse zu erhalten.

Um die Vorteile dieser neuen Arbeitstechniken aufzuzeigen, war es nicht nötig, für diese methodisch orientierte Arbeit primäre Daten im Rahmen einer palynologischen Arbeit zu erzeugen, sondern es konnte auf z.T. unveröffentlichte und veröffentliche Daten aus der Göttinger Arbeitsgruppe zurückgegriffen werden. Dies ermöglicht eine Gegenüberstellung der „konservativen“ Methodik gegenüber P.A.S.T sowie eine Vereinfachung und Erweiterung der Interpretation der vorliegenden Darstellungen.

Da die Daten dieser Arbeiten primär unter ökologischen und systematischen Fragestellungen entstandenen sind, beruhen sämtliche sedimentologischen Angaben beruhen ausschließlich auf Geländebeobachtungen und -aufnahmen. Eine ergänzende Laboruntersuchung z.B. zu Korngrößenverteilungen konnte im gesetzten Zeitrahmen dieser Arbeit nicht durchgeführt werden.

\subsection{Datengrundlage}

Um die Diskrepanzen aufgrund unterschiedlicher Aufbereitungs- und Auszählmethoden bei einer breiten Palette von Bearbeitern zu minimieren, wurde hier auf Arbeiten zurückgegriffen, die in der Arbeitsgruppe Prof. Riegel im Zeitraum von 1992 bis 2001entstanden sind und somit unter vergleichsweise einheitlichen Voraussetzungen erarbeitet wurden.

Dazu gehören mehrere Diplomarbeiten und eine Dissertation, die sich im Rahmen des Gemeinschaftsprojektes „Rekonstruktion terrestrischer Ökosysteme im Paläogen Mitteleuropas" mit der eozänen Kohle der Tertiärmulden des Helmstedter Braunkohlenreviers im nördlichen Harzvorland bei Helmstedt befaßten.

Außerdem wurden Daten aus einer Dissertation (HAMMER 2002 in Vorbereitung) über die Sedimentologie und Palynofazies der fluviatilen Ablagerungen des Oberoligozäns in der Leipziger Bucht erfaßt (Tagebau Witznitz, Profil 11, Mächtigkeit ca. 3,5m).

Schiemann 1994, NAtge-Efoghe 1997 und EBe 1992 untersuchten die Palynologie der Hangenden Flözgruppe der Westmulde bei Helmstedt (Alversdorf), die das etwa 3 bis $4 \mathrm{~m}$ mächtige Unterflöz, das „Untere Zwischenmittel“ und das ca. $10 \mathrm{~m}$ mächtige Victoriaflöz umfaßt. Das darüber folgende Treueflöz mit einer Mächtigkeit von ca. $20 \mathrm{~m}$ war zum Zeitpunkt der Bearbeitung nicht mehr zugänglich. Im Folgenden werden die stratigraphisch zusammengesetzten Profile als Einzelprofil behandelt.

HAMMER-SCHIEMANN 1998 untersuchte die Palynologie im unteren Abschnitt der Unterflözgruppe der Tagebaue Schöningen-Nordfeld und -Südfeld (s. a. Abbildung 4.1) in zwei miteinander korrelierten Profilen (Nordfeld und Südfeld), deren Aufnahmepunkte etwa $1,5 \mathrm{~km}$ auseinanderliegen. Sie bestehen aus einem etwa $10 \mathrm{~m}$ mächtigen Hauptflöz (HF) und einem darüberliegenden ca. $5 \mathrm{~m}$ bis $7 \mathrm{~m}$ mächtigen Zwischenmittel (ZM1). Anschließend folgt ein etwa 3-4 m mächtiges Flöz (FL1), das 
von einem 3 bis 4,5 m mächtigen Zwischenmittel (ZM2) abgelöst wird. Den Abschluß des bearbeiteten Profils bildet das Flöz 2 (FL2), welches etwa 2,5 bis 3,5 m mächtig ist. Die Palynologie der Zwischenmittel des Nordfeld-Profils liegt nicht vor.

Eine detaillierte Beschreibung des geologischen Rahmens der Helmstedter Schichtenfolge befindet sich bei SCHIEMANN (1998).

\subsection{Datenverarbeitung}

Sämtliche Profildaten wurden aus der oben genannten Literatur extrahiert und in das P.A.S.T-konforme Format übertragen. Dabei wurde die Systematik von P.A.S.T um die jeweiligen Formenlisten der oben genannten Arbeiten erweitert. Gruppierungsparameter (z.B. botanische Zugehörigkeit, azonale Vegetation, Klima usw.), Literatur-, Morphologie- und Lokalitätsdaten ergänzten im Rahmen dieser Eingabe die auf Abruf zur Verfügung stehende Informationsdichte von P.A.S.T.

Insbesondere die Verarbeitung der Daten aus den ,älteren“ Diplomarbeiten erwies sich als schwierig, da die Auszähldaten nur in gedruckter Form vorlagen und daher ,von Hand" in die Datenbank eingeben werden mußten.

Insgesamt konnten somit etwa 280 Formen in die Datenbankstruktur überführt werden. Die lithologischen Informationen und Auszähldaten von ca. 300 Proben mit ca. 30000 Einzelwerten ergeben den augenblicklichen numerischen Datenbestand von P.A.S.T. 


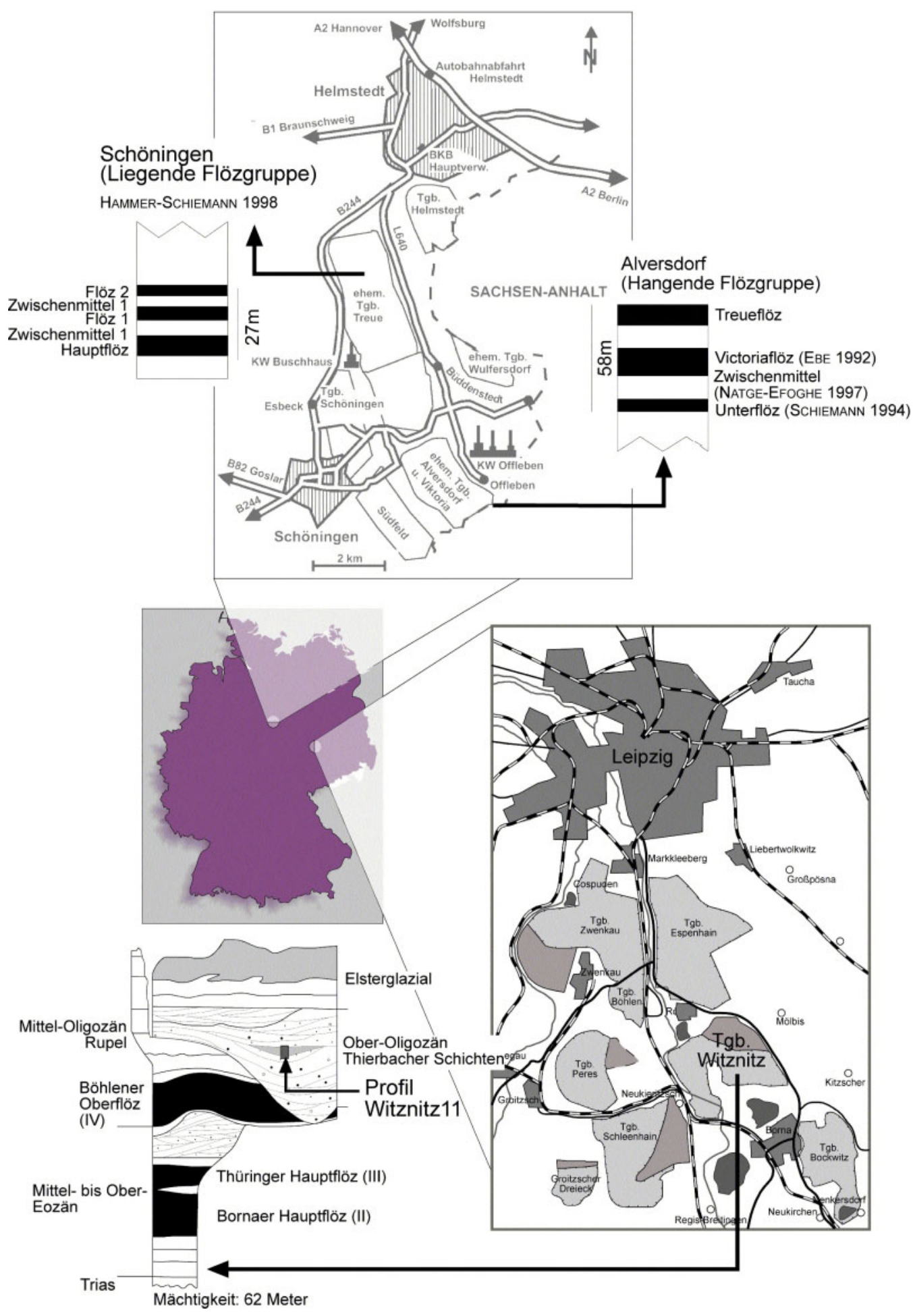

Abb. 4.1: Das Helmstedter Braunkohlenrevier (verändert nach LOOK 1984 und LENZ 2000) mit der Lage der Profile und Bearbeiter. Dargestellt ist weiterhin die Lage des Profils Witznitz 11 (Leipzig). 


\subsection{Diversität}

Neben der Clusteranalyse und der Möglichkeit die Daten für weitere statistische Methoden in externen Statistikprogrammen aufzubereiten und $\mathrm{zu}$ exportieren, sind Diversitätsberechnungen direkt mit P.A.S.T realisierbar.

Insbesondere die Tatsache, daß fossile Pollen und Sporen in sehr unterschiedlichen sedimentären Faziesbereichen $\mathrm{zu}$ finden sind, legt die Notwendigkeit nahe, eine Abhängigkeit der Diversität zum sedimentären Ablagerungsraum der Palynomorphen zu untersuchen.

Es steht außer Frage, daß eine Sortierung von Pollen und Sporen innerhalb eines fluviatilen Systems oder in Deltabereichen möglich ist - dies belegen eine Vielzahl von Autoren, die sich in erster Linie mit rezenten Ökosystemen befaßt haben (z.B. DimbleBY 1985, FAll 1987, Holmes 1990, TRAVERSE 1994, Ziegler 1988 u.a.). Sie haben Ökosysteme betrachtet, bei denen andere beeinflussende Faktoren von dem taphonomischen Prozeß der Sortierung und Anreicherung deutlich zu trennen bzw. aufgrund von simulierten Laboruntersuchungen (z.B. HOLMES 1990) nicht vorhanden sind.

Im Gegensatz dazu stellen die in dieser Dissertation bearbeiten Ökosysteme äußerst heterogene Systeme dar, die in ihrer zeitlichen Entwicklung starken faziellen und ökologischen Wechseln unterworfen waren, so daß ein ganze Reihe weiterer Faktoren die Diversität beeinflußt haben muß.

Es stellt sich nun die Frage, ob eine nach statistischen Methoden durchgeführte Untersuchung eine Isolierung des Faktors Sortierung ermöglicht und wie deutlich dieser von anderen palynofaziellen und lithofaziellen Prozessen zu trennen ist.

$\mathrm{Zu}$ Beginn díeses Kapitels werden die verschiedenen Methoden der Diversitätsberechnung erläutert. Anschließend folgen die Ergebnisse der Anwendung dieser Methoden zu den Profilen Alversdorf (Mitteleozän), Schöningen (Untereozän) und Witznitz (Ober-Oligozän). Da die verschiedenen Methoden zum Teil sehr unterschiedliche Ergebnisse liefern, wird die Diskussion zusammenhängend durchgeführt (Kap. 4.4.9, S. 125 und eine Zusammenfassung im Kapitel 4.4.11.1, S. 136 ff.)

\subsubsection{Diversitäts-Berechnungsmethoden}

Bei der Wahl der Methode zur Diversitätsberechnung sind einige Überlegungen nötig, da es eine Vielzahl von Möglichkeiten gibt, die Diversität in Form eines Index numerisch abzubilden. Einige Autoren (z.B. ASHRAF 1995, ALROY 2001) benutzen die Diversität als Synonym für die Anzahl der Taxa, wobei jedoch die Häufigkeitsverteilung der Taxa unberücksichtigt bleibt. Dieses Vorgehen läßt sich aus der Tatsache begründen, daß die unterschiedliche Pollenproduktivität der Mutterpflanze sowie taphonomische Prozesse die Häufigkeitsverteilung der Palynomorphen unterschiedlich stark beeinflussen.

Weiterhin ist $\mathrm{zu}$ beachten, daß die Verteilung der Palynomorphen als Organe von Pflanzen nur ein relatives Maß für die Verteilung der Mutterpflanzen darstellen.

Diversitäts-Indizes ermitteln den Parameter Heterogenität innerhalb einer Stichprobe und den Parameter Artenreichtum gleichermaßen (MAGURRAN 1988). Grundsätzlich unterscheiden sich die nichtparametrischen Indizes darin, welche der oben genannten Parameter stärker in die Berechnung einfließen. Insbesondere die Bedingung, Indizes auch mit Verteilungsdaten zu berechnen, die nur als Prozentwerte vorliegen, schränkt 
die Wahl der Indizes auf solche ein, die bei proportionalen Änderungen konstant bleiben. Bei der Annahme, daß in verschiedenen Proben in der Regel etwa die gleiche Anzahl an Punkten ausgezählt wird, verändert die Umrechnung der Rohdaten in Prozentwerte den Index demnach nur unbedeutend (Abbildung 4.2).

Folgendes Beispiel soll dies anhand zweier Indizes verdeutlichen:

\begin{tabular}{|l|l|l|}
\hline Index & Probe 1 & Probe 2 \\
\hline & 10 & 5 \\
& 10 & 5 \\
& 10 & 5 \\
& 10 & 5 \\
& 10 & 5 \\
& 10 & 5 \\
& 10 & 5 \\
& 10 & 5 \\
& 10 & 5 \\
& 10 & 5 \\
\hline Shannon $\mathrm{H}^{6}$ & 2,30 & 2,30 \\
Brillouin HB & 2,13 & 2,01 \\
\hline
\end{tabular}

Abb. 4.2: Der Shannon-Index wird nicht durch proportionale Änderungen des Datensatzes beeinflußt.

Der Shannon- sowie der Simpson-Index erfüllen diese Forderung.

Als zweite Bedingung seien solche Indizes favorisiert, bei denen der Artenreichtum den stärksten Einfluß ausübt und damit die unterschiedlichen Häufigkeitsverteilungen, bedingt durch die unterschiedliche Pollenproduktion, den Index nicht übermäßig stark verfälschen. Diese Bedingung wird am ehesten durch den Shannon-Index erfüllt. Trotzdem wird bei P.A.S.T ergänzend der Simpson-Index berechnet, der als zusätzlicher Indikator Schwankungen in der Palynomorphenverteilung überproportional anzeigt (s. auch Abbildung 4.3).

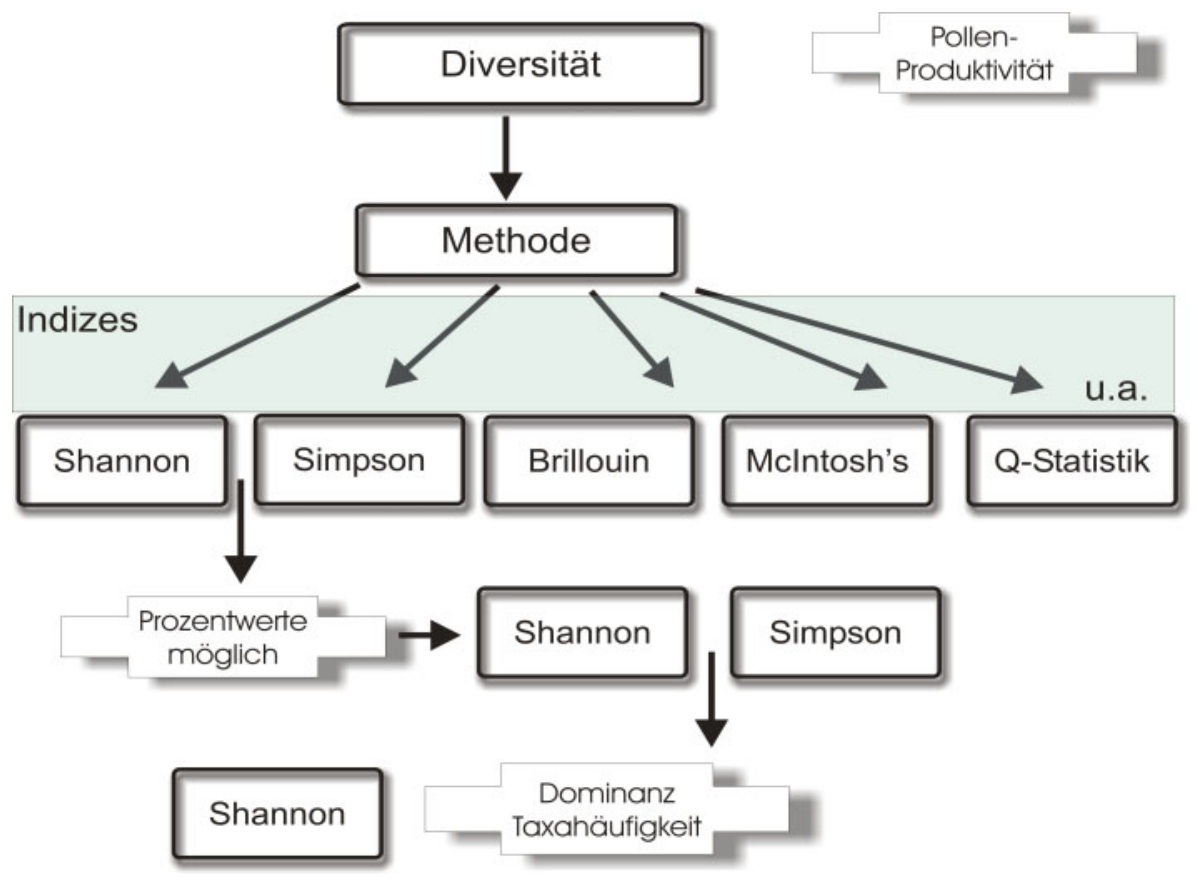

Abb. 4.3: Wahl der Diversitäts-Indizes: Die Berechnung des Indizes sollte auch mit Prozentwerten möglich sowie durch die Taxa-Häufigkeit dominiert sein. 


\subsubsection{Shannon-Index}

Diese Form der Indexberechnung geht aus der Informationstheorie hervor (SHANNON 1949) und mißt die Ordnung (bzw. Unordnung) innerhalb eines definierten Systems. Diese Ordnung wird in ökologischen Studien durch die Anzahl und Verteilung der Individuen für jede Art charakterisiert (FREDERICK 2000).

Eine Messung der Diversität - unabhängig von der Probengröße - wird durch folgende Formel ausgedrückt:

$\mathrm{H}^{\prime}=$ Diversität pro Individuum (für große Vergesellschaftungen)

$\mathrm{Pi}=$ Wahrscheinlichkeit, mit der ein zufällig ausgewähltes Individuum zu einer Klasse gehört

$\mathrm{S}=$ Anzahl unterschiedlicher Spezies/Palynomorphen

$(\log )=$ natürlicher Logarithmus

$H^{\prime}=-\sum_{i} p i \log p i$

Der Shannon-Diversitätsindex liegt gewöhnlich zwischen 1,5 und 4.

Mithilfe des Artenreichtums (N) und des Shannon-Index ist es möglich die „Evenness“

- also die Ähnlichkeit der Häufigkeiten der verschiedenen Spezies zu ermitteln.

$$
E=\frac{H}{\log (N)}
$$

Mit P.A.S.T können E und $\mathrm{H}^{`}$ für beliebige Datenpools berechnet werden (Proben, Lithotypen oder Zonen).

\subsubsection{Simpson-Index}

Dieser Index wurde von SIMPSON 1949 entwickelt und kann auf unterschiedliche Art berechnet werden. Allen Berechnungsarten gemeinsam ist die Ermittlung von $\mathrm{P}_{\mathrm{i}}$, als der Quotient aus der Anzahl der gegebenen Arten (n) und der Summe aller untersuchten Arten (N).

1) Simpson-Index (infinite Gemeinschaft)

$D=\sum P_{i}^{2}$

(finite Gemeinschaft)

$D=\sum\left(\frac{n(n-1)}{N(N-1)}\right.$

2) Simpson-Index der Diversität:

$=1-D$

Dieser Term ermittelt die Wahrscheinlichkeit, daß zwei zufällig selektierte Individuen in einer Gemeinschaft zu unterschiedlichen Kategorien (z.B. Spezies) gehören. 
3) Reziproker Simpson-Index

$$
=\frac{1}{D}
$$

Der reziproke Simpson-Index ermittelt die Anzahl der gleichen Kategorien (z.B. Arten), die der Simpson-Index produziert.

P.A.S.T berechnet den reziproken Simpson-Index.

\subsubsection{Extremwerte der Diversitäts-Indizes}

Die möglichen Minimal- und Maximalwerte des Shannon- und des Simpson-Index verdeutlichen folgende Tabellen:

Identische Häufigkeitswerte

\begin{tabular}{|l|l|l|l|l|l|l|l|} 
Artname & \# Anzahl $\mathrm{P}_{\mathrm{i}}$ & $\mathrm{P}_{\mathrm{i}}{ }^{2}$ & $\mathrm{P}_{\mathrm{i}} \ln \left[\mathrm{P}_{\mathrm{i}}\right]$ & \multicolumn{2}{|l|}{ Messung } & Wert \\
\hline Spezies 1 & 40 & 0.200 & 0.040 & -0.322 & $\mathrm{~S}$ & 5 \\
\hline Spezies 2 & 40 & 0.200 & 0.040 & -0.322 & $\mathrm{D}$ & 0.200 \\
\hline Spezies 3 & 40 & 0.200 & 0.040 & -0.322 & $1-\mathrm{D}$ & 0.800 \\
\hline Spezies 4 & 40 & 0.200 & 0.040 & -0.322 & $1 / \mathrm{D}$ & 5.000 \\
\hline Spezies 5 & 40 & 0.200 & 0.040 & -0.322 & $\mathrm{H}$ & 1.609 \\
\hline Summe & 200 & 1.000 & & & & $\mathrm{E}$ & 1.000 \\
\hline
\end{tabular}

Eine dominante Spezies

\begin{tabular}{|l|l|l|l|l|l|l|} 
Artname & \# Anzahl $\mathrm{P}_{\mathrm{i}}$ & $\mathrm{P}_{\mathrm{i}}{ }^{2}$ & $\mathrm{P}_{\mathrm{i}} \ln \left[\mathrm{P}_{\mathrm{i}}\right]$ & \multicolumn{2}{|l|}{ Messung } & Wert \\
\hline Spezies 1 & 1 & 0.005 & 0.000 & -0.026 & $\mathrm{~S}$ & 5 \\
\hline Spezies 2 & 1 & 0.005 & 0.000 & -0.026 & $\mathrm{D}$ & 0.960 \\
\hline Spezies 3 & 196 & 0.980 & 0.961 & -0.020 & $1-\mathrm{D}$ & 0.040 \\
\hline Spezies 4 & 1 & 0.005 & 0.000 & -0.026 & $1 / \mathrm{D}$ & 1.041 \\
\hline Spezies 5 & 1 & 0.005 & 0.000 & -0.026 \\
\hline Summe & 200 & 1.000 & & & $\mathrm{H}$ & 0.126 \\
\hline
\end{tabular}

Nur eine Spezies

\begin{tabular}{|l|l|l|l|l|l|l|} 
Artname & \# Anzahl $\mathrm{P}_{\mathrm{i}}$ & $\mathrm{P}_{\mathrm{i}}{ }^{2}$ & $\mathrm{P}_{\mathrm{i}} \ln \left[\mathrm{P}_{\mathrm{i}}\right]$ & \multicolumn{2}{|l|}{ Messung } & Wert \\
\hline Spezies 1 & 0 & 0.000 & 0.000 & 0.000 & $\mathrm{~S}$ & 1 \\
\hline Spezies 2 & 0 & 0.000 & 0.000 & 0.000 & $\mathrm{D}$ & 1.000 \\
\hline Spezies 3 & 200 & 1.000 & 1.000 & 0.000 \\
\hline Spezies 4 & 0 & 0.000 & 0.000 & 0.000 \\
\hline Spezies 5 & 0 & 0.000 & 0.000 & 0.000 & 1 - D & 0.000 \\
\hline Summe & 200 & 1.000 & & & $\mathrm{H}$ & 1.000 \\
\hline
\end{tabular}

Abb. 4.4: Maximal- und Minimalwerte der Indizes: Demnach kann E = Evenness minimal 0 (nur eine Spezies) und maximal 1 sein (identische Spezies). Die möglichen Werte für 1/D = reziproker SimpsonIndex betragen minimal 1 und maximal die Anzahl der Spezies. 


\subsubsection{Methoden der Diversitätsberechnung für Lithotypen}

Die Berechnung der Diversitäts-Indizes kann sehr unterschiedlich erfolgen. Im Folgenden werden die in dieser Arbeit angewandten Methoden vorgestellt:

1) Man berechnet für jede Probe des gleichen Lithotyps den Diversitätsindex und ermittelt daraus einen Mittelwert der Diversität.

2) Man stellt ein Inventar aller Formen auf, die insgesamt in allen Proben dieses Lithotyps vorkommen. Anschließend wird die durchschnittliche Individuenzahl für jedes Taxon aus allen Proben ermittelt. Aus dieser Liste kann anschließend die Diversität errechnet werden.

3) Man ermittelt die Diversität einer zufällig ausgewählten Probe. Anschließend wählt man eine weitere Probe wiederum zufällig aus und berechnet die Diversität des nun entstandenen Datenpools der beiden Proben neu. So fährt man fort, bis sämtliche Proben in die Diversitätsberechnung eingegangen sind.

Die Methoden 1 und 2 sind direkt mit P.A.S.T realisiert. Bei der Methode 3 wurde auf das Programm EstimateS (COLWELL 2001) zurückgegriffen. Die Methode 1 und 3 zeigen die plausibelsten Ergebnisse bei Lithotypen-Diversitäten und wurden deshalb für die Berechnungen eingesetzt.

Die Methode 2 hat sich in Bezug auf Lithotypen-Diversitäten als nicht sinnvoll erwiesen, da die Probenanzahl des jeweiligen Lithotyps die Diversität sehr stark beeinflußt. Die Diversität nähert sich asymptotisch mit Zunahme der Probenzahl an die Gesamtanzahl der Taxa an. Diese Methode ist jedoch sinnvoll bei der Diversitätsbestimmung von Probenbereichen oder Zonen, deren Proben als zusammenhängender Komplex betrachtet werden können - so als hätte man den Probenahmebereich erhöht. Da die Anwendung dieser Methode für die Klärung der im Kapitel 4.4 angesprochene Fragestellung keine zusätzlichen Informationen liefert, wird sie im Rahmen dieser Arbeit nicht näher diskutiert. Bei zukünftigen andersartigen Untersuchungen steht sie jedoch auf „Mausklick“ in P.A.S.T zur Verfügung.

Weiterhin ist es möglich, die Diversität von zuvor in Häufigkeitsklassen transformierten Prozentwerten zu ermitteln. Damit wird eine gewisse Homogenisierung der Auszähldaten erreicht, so daß z.B. zoophile Taxa mit geringer Pollenproduktion weniger stark unterrepräsentiert werden, da der maximale Unterschied zwischen den Extremen aufgrund der Klassifizierung nur die Summe der Gesamt-Klassenzahl betragen kann.

Bei P.A.S.T sind die Grenzen der Häufigkeitsklassen frei wählbar. Für die Berechnung der Indizes wurde die Einteilung der Prozentwerte in Häufigkeitsklassen nach dem „logarithmic abundance class scheme“ von GAUCH (1982) benutzt. Hierbei wird die Reichweite der Häufigkeitsklasse zur vorhergehenden Klasse jeweils verdoppelt.

\begin{tabular}{|l|l|}
\hline Klasse & Reichweite \\
\hline 0 & $0 \%$ \\
\hline 1 & $>0 \%-0,5 \%$ \\
\hline 2 & $>0,5 \%-1 \%$ \\
\hline
\end{tabular}




\begin{tabular}{|l|l|}
\hline 3 & $>1 \%-2 \%$ \\
\hline 4 & $>2 \%-4 \%$ \\
\hline 5 & $>4 \%-8 \%$ \\
\hline 6 & $>8 \%-16 \%$ \\
\hline 7 & $>16 \%-32 \%$ \\
\hline 8 & $>32 \%-64 \%$ \\
\hline 9 & $>64 \%$ \\
\hline
\end{tabular}

Abb. 4.5: Die von GAUCH (1982) vorgeschlagene Methode zur Einteilung der Prozentwerte in das „logarithmic abundance class scheme“.

\subsubsection{Prozentwerte versus Auszähldaten versus Häufigkeitsklassen}

Die Einteilung der Prozentwerte in Häufigkeitsklassen, bewirkt eine deutliche Erhöhung des Shannon-Index gegenüber den unklassifizierten Werten, da der Shannon-Index bei homogeneren Verteilungen generell zunimmt (siehe auch Kap. 4.4.4, S. 113). Die Berechnung des Index auf der Grundlage der Prozentwerte zeigt dagegen nur eine geringe Abweichung zu den Werten der Rohdaten, sofern die Probengröße relativ konstant ist (siehe auch Kap. 4.4.1, S. 110). Der Ton zeigt hier eine etwas höhere Diversität bei den Prozentwerten gegenüber den Rohdaten auf, da die Probengröße, zumindest im Profil Witznitz, durchschnittlich signifikant kleiner ist, was reziprok den relativen Anteil des Tones erhöht (Abbildung 4.6) . Die Problematik der variablen Probengröße wird im Kapitel 4.4.10, S. 129 näher erläutert.

\section{Diversitätsindex der Lithotypen (Witznitz, Profil 11)}

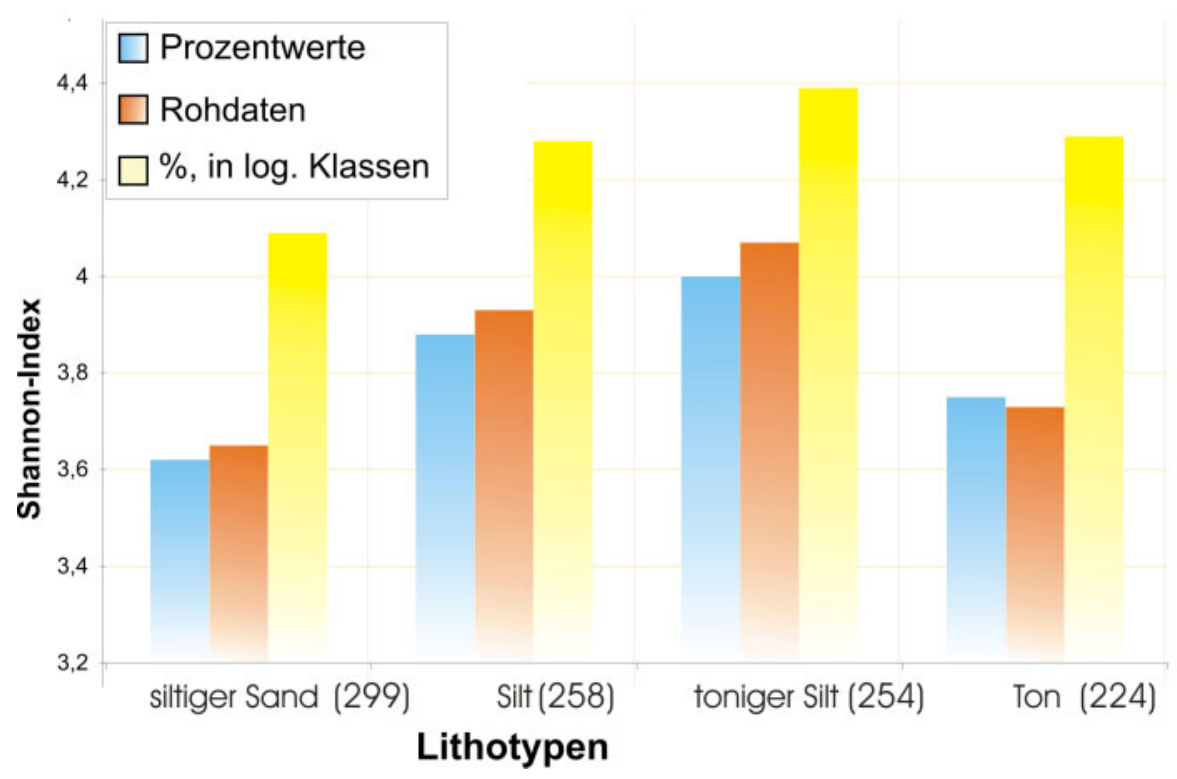

Abb. 4.6: Klassifizierte Werte erhöhen den Diversitätsindex signifikant, da eine dadurch bedingte homogenere Verteilung einen höheren Shannon-Index impliziert. In Klammern: die Anzahl der Auszählpunkte als Durchschnittswert über sämtliche Proben des jeweiligen Lithotyps.

\subsubsection{Mittelwertberechnung der Diversitäts-Indizes nach ,Methode 1“6}

Die Abbildung 4.7 zeigt das Histogramm der Shannon-Indizes sämtlicher Proben für Alversdorf, beruhend auf in Häufigkeitsklassen umgerechnete Prozentwerte. Die 
Häufigkeitsklassen-Anzahl wurde nach PANOFSKY und BRIER 1958 nach folgender Formel ermittelt:

$K=5 \log n$

Dabei sei log der dekadische Logarithmus und $\mathbf{n}$ der Stichprobenumfang

\subsubsection{Alversdorf (Mitteleozän)}

Das Histogramm in der Abbildung 4.7 zeigt deutlich eine mehrgipflige Verteilung. Es sind zwei unterscheidbare Häufigkeitsmaxima zu beobachten. Orange sind kohlige Proben (biogene Sedimente) und grün Proben des Zwischenmittels (klastische und klastisch-biogene Sedimente) dargestellt.

Die Proben aus der Kohle weisen generell einen niedrigeren Index gegenüber den Zwischenmittelproben auf - auch wenn einige kohlige Proben einen höheren Index besitzen und in den Bereich des Zwischenmittels hineinreichen. Umgekehrt gibt es eine geringe Anzahl von Proben des Zwischenmittels mit signifikant niedrigeren Diversitäten.

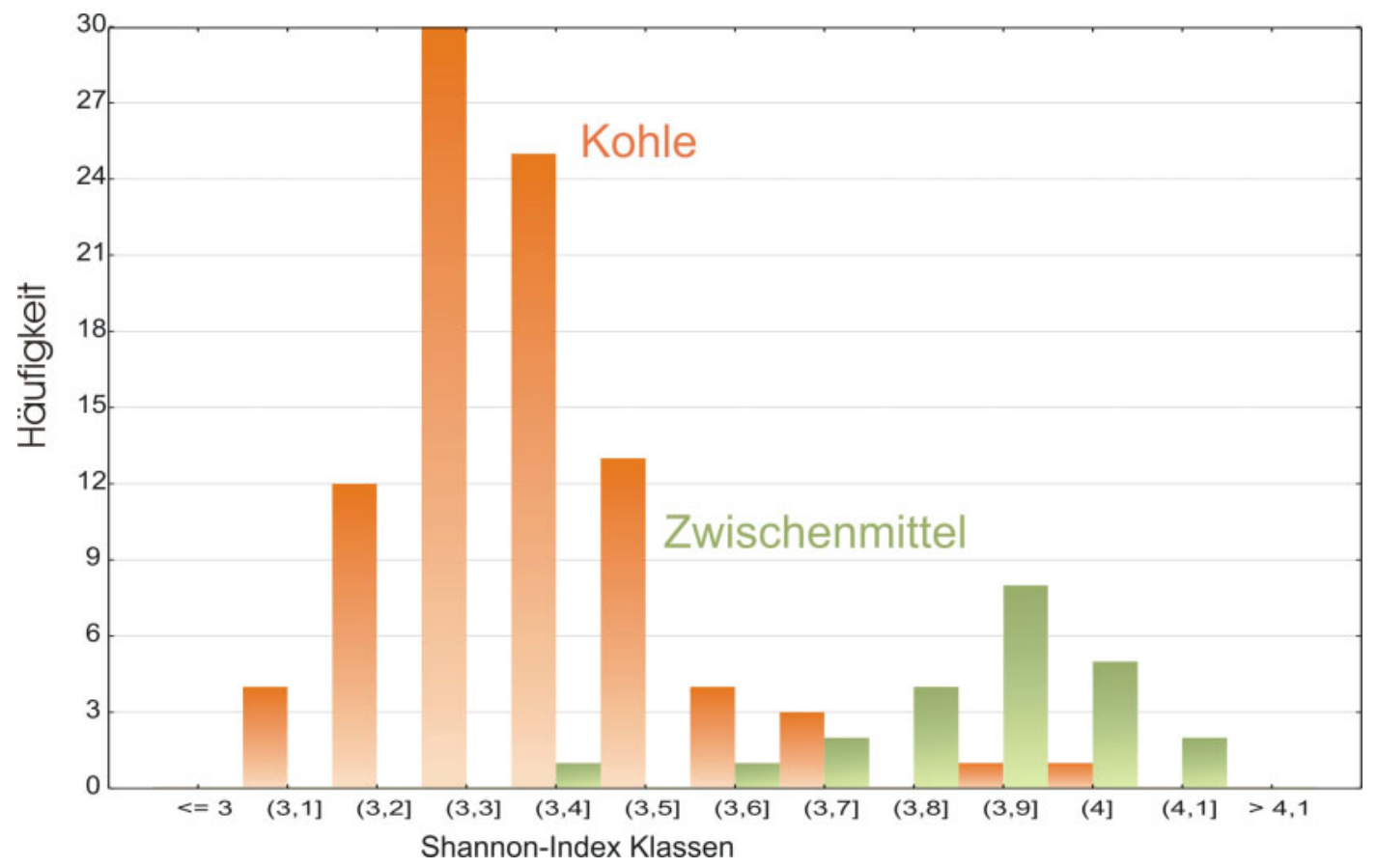

Abb. 4.7: Histogramm der Diversitäten sämtlicher Proben aus Alversdorf (Die Angabe der Klasse bezieht sich jeweils auf die obere Grenze (zur Erläuterung der „Ausreißer“, siehe Text). Die Klasse [4] beinhaltet beispielsweise Einzelwerte zwischen 3,9 - <4).

Eine detaillierte Auflistung der Mittelwerte der Diversitäten nach der „Methode 1“ (siehe Kap. 4.4.5) ist nachfolgend in Form eines Box-Plot-Diagramms abgebildet (Abbildung 4.8). Bei dieser Darstellungsform kann die Standardabweichung vom arithmetischen Mittelwert (als \pm Werte) visualisiert werden. Der Mittelwert läuft demnach jeweils durch die Mitte der Balken, wobei die Standardabweichung proportional mit der Mächtigkeit der Balken zunimmt. Neben dem Shannon-Index (gelb) zeigt der Simpson-Index (orange) verstärkt die Diversität in Abhängigkeit der Häufigkeitsverteilung an. Theoretisch kann der Simpson-Index maximal die Anzahl der Taxa erreichen. 
Der Mittelwert des Shannon-Diversitätsindex der Kohle in Alversdorf liegt bei ca. 3,3, wobei die tonige Kohle mit einem Durchschnittswert von 3,7 das Maximum darstellt (,Ausreißer"). Im Histogramm (Abbildung 4.7) bilden zwei der drei Proben der tonigen Kohle die sog. Ausreißer.

Im Zwischenmittel markiert der kohlige Sand mit einem Durchschnittswert von 3,3 die untere Grenze und bildet die Ausreißerproben nach unten im Histogramm. Der Silt erreicht mit einen Shannon-Index von 3,9 das Maximum, gefolgt vom Sand, von kohligem Ton und Ton. Dieser zeigt eine auffällig hohe Standardabweichung - genau wie der kohlige Sand, der mit einem durchschnittlichen Index von 3,3 eher den Kohleproben zuzuordnen ist.

Der Simpson-Index bestätigt diese Trends, visualisiert jedoch verstärkt die hohe Variation der Einzeldiversitäten der Lithotypproben. Die Evenness des Shannon-Index liegt relativ konstant bei 0,95-0,96. Auffällig ist jedoch die relativ hohe Varianz der Evenness im Bereich des kohligen Sandes (Diskussion der Ergebnisse, siehe Kap. 4.4.9.2, S. 128).

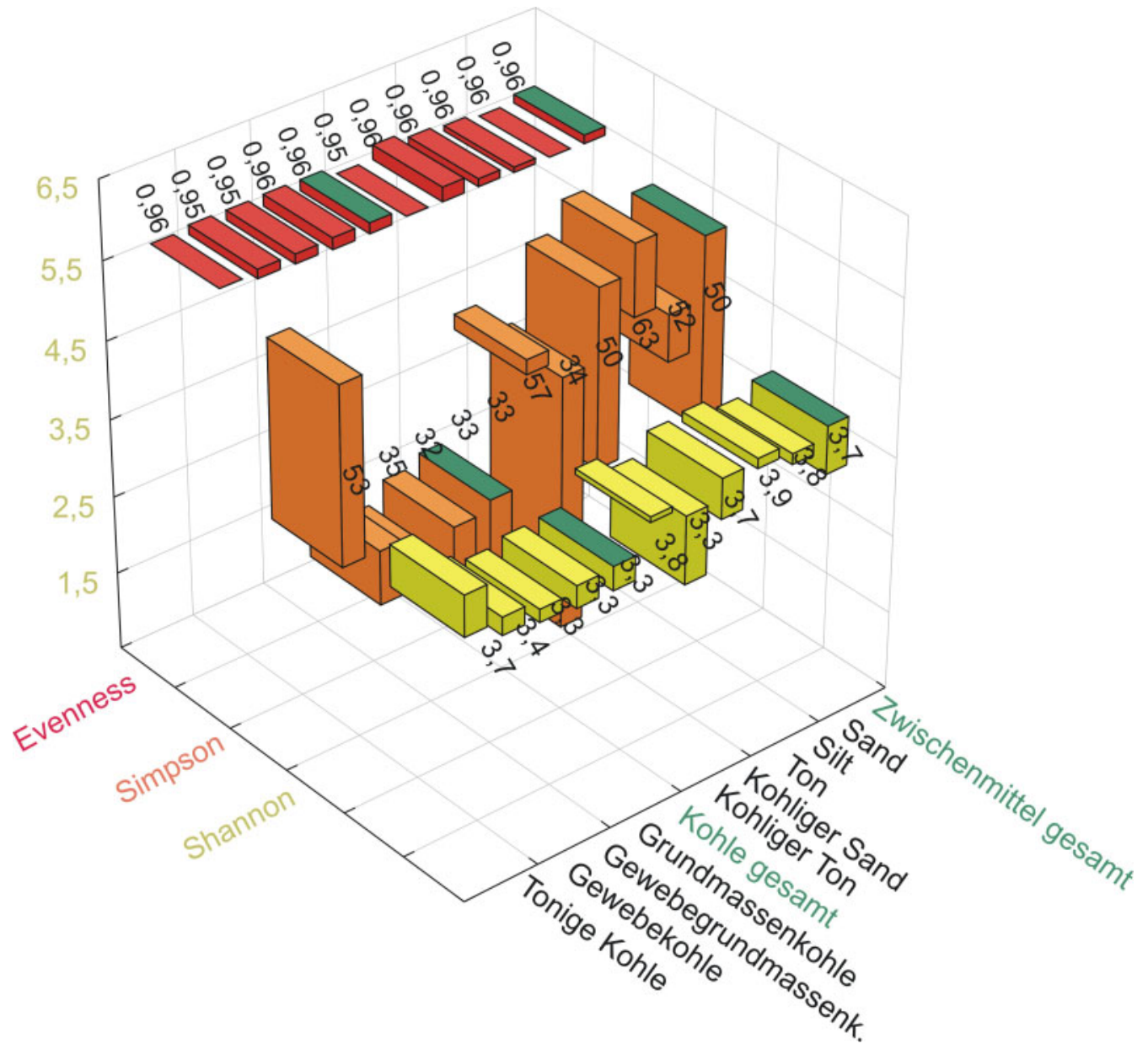

Abb. 4.8: Box-Plot-Diagramm der Proben aus Alversdorf: Dargestellt sind die arithmetischen Mittelwerte der Diversitäts-Indizes mit ihren Standardabweichungen. Der Mittelwert ist zusätzlich in numerischer Form angeben. Die Y-Skala ist nur für den Shannon-Index relevant. 


\subsubsection{Schöningen (Untereozän)}

Das Histogramm der Proben aus dem Tagebau Schöningen (s. Abbildung 4.9) läßt im Gegensatz zu den Proben aus Alversdorf keine signifikante multimodale Verteilung erkennen. Die Anpassungskurve (Normalverteilung) zeigt auch für die Mittelwerte keine signifikanten Unterschiede zwischen der Kohle und dem Zwischenmittel. Auffällig häufig ist bei den Kohlen die Klasse 3,1-3,2 vertreten. Die Zwischenmittelproben haben ihre häufigste Klasse bei 3,3-3,4 und liegt damit in ihrer Diversität etwas höher als die häufigste Klasse der Kohleproben.

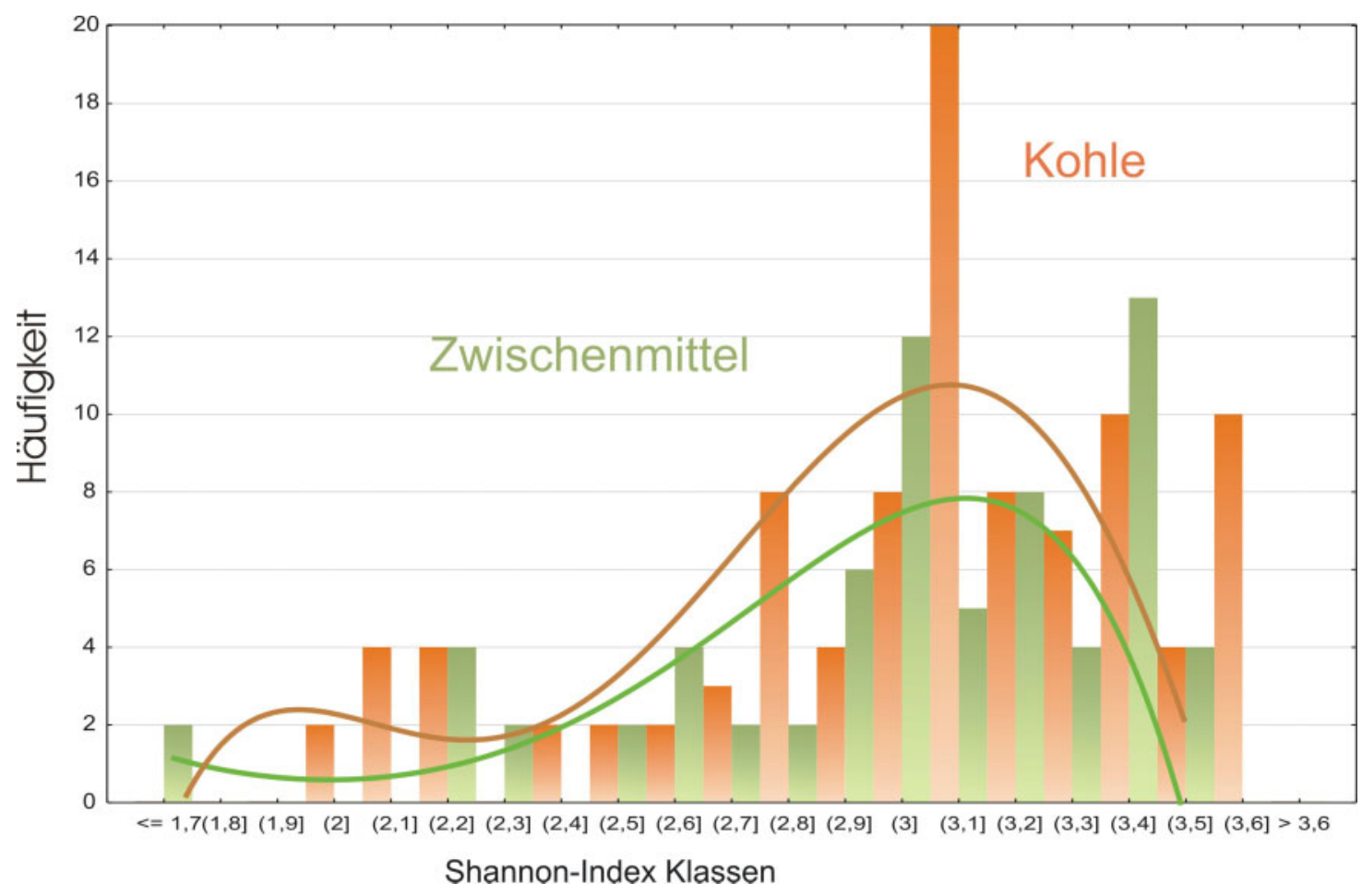

Abb. 4.9: Histogramm der Diversitäten sämtlicher Proben aus Schöningen. Die Angabe der Klasse bezieht sich jeweils auf die obere Grenze. Die Anpassungskurve berechnet sich aus einer Normalverteilung.

Das Box-Plot-Diagramm der Diversitäts-Mittelwerte (s. Abbildung 4.10) bestätigt die hohe Varianz der Diversitäten. Es läßt sich - zumindest mit dieser Methode - keine Trennung zwischen den Kohleproben und den Zwischenmittelproben feststellen. 
Die erhöhte Heterogenität der Häufigkeitsverteilungen, im Gegensatz zu den Proben aus Alversdorf, zeigt die Evenness des Shannon-Index an: Sie liegt im Bereich von 0,7 bis 0,8 . Auffällig ist im direkten Vergleich mit Alversdorf die hohe Standardabweichung der Proben insgesamt und im Speziellen bei den Kohleproben und dem tonigen Silt. (Diskussion der Ergebnisse, siehe Kap. 4.4.9.3, S. 129).

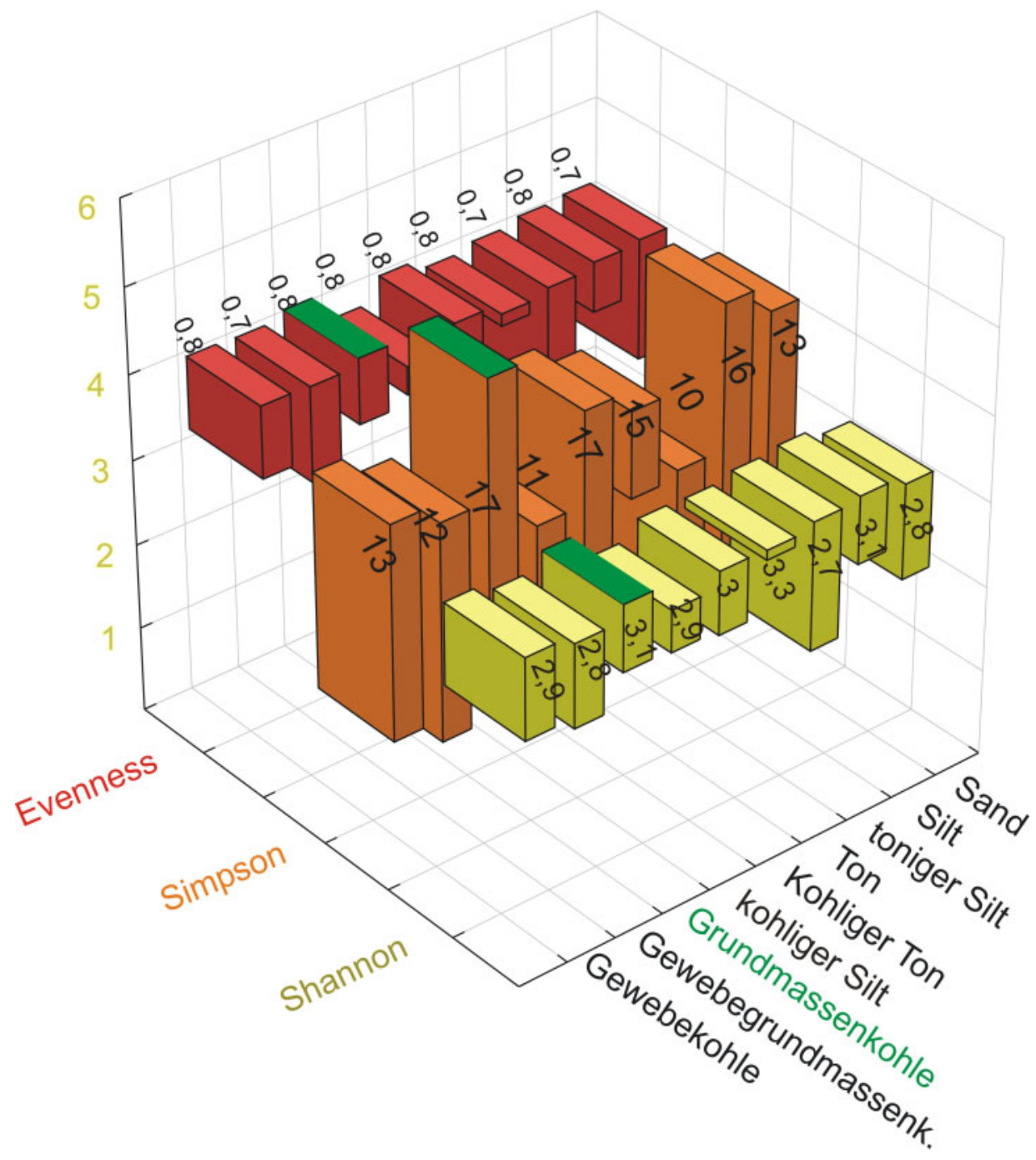

Abb. 4.10: Box-Plot-Diagramm der Proben aus Schöningen: Dargestellt sind die arithmetischen Mittelwerte der Diversitätsindizes mit ihren Standardabweichungen. Der Mittelwert ist in numerischer Form angeben. Die Y-Skala ist nur für den Shannon-Index relevant. Die Grenze zwischen der Kohleund der Zwischenmittel-Diversität ist andersfarbig markiert. Auf Grund der hohen Varianz der Mittelwerte ergibt die Errechnung des Gesamtdiversitäts-Mittelwerts der Kohle und des Zwischenmittels keinen Sinn, deshalb wurde auf die Darstellung dieser Werte verzichtet. 


\subsubsection{Witznitz (Oberoligozän)}

Aufgrund der geringen Probenzahl der Witznitzproben des Profils 11 wurde auf das Histogramm verzichtet, da keine sinnvolle Klassenzahl gebildet werden konnte.

Das Box-Plot-Diagramm (s. Abbildung 4.11) zeigt die selben Trends wie in Alversdorf. Im Silt ist die höchste Diversität mit 4 festzustellen, gefolgt von dem tonigen Silt mit 3,9 und dem Sand mit 3,8. Die geringste Diversität weisen die Proben des Tons auf. Hier liegt die Diversität um 3,6. Die Evenness ist gleichbleibend hoch bei 0,98 und weist eine geringe Standardabweichung auf. (Diskussion der Ergebnisse, siehe Kap. 4.4.9.1, S. 126).

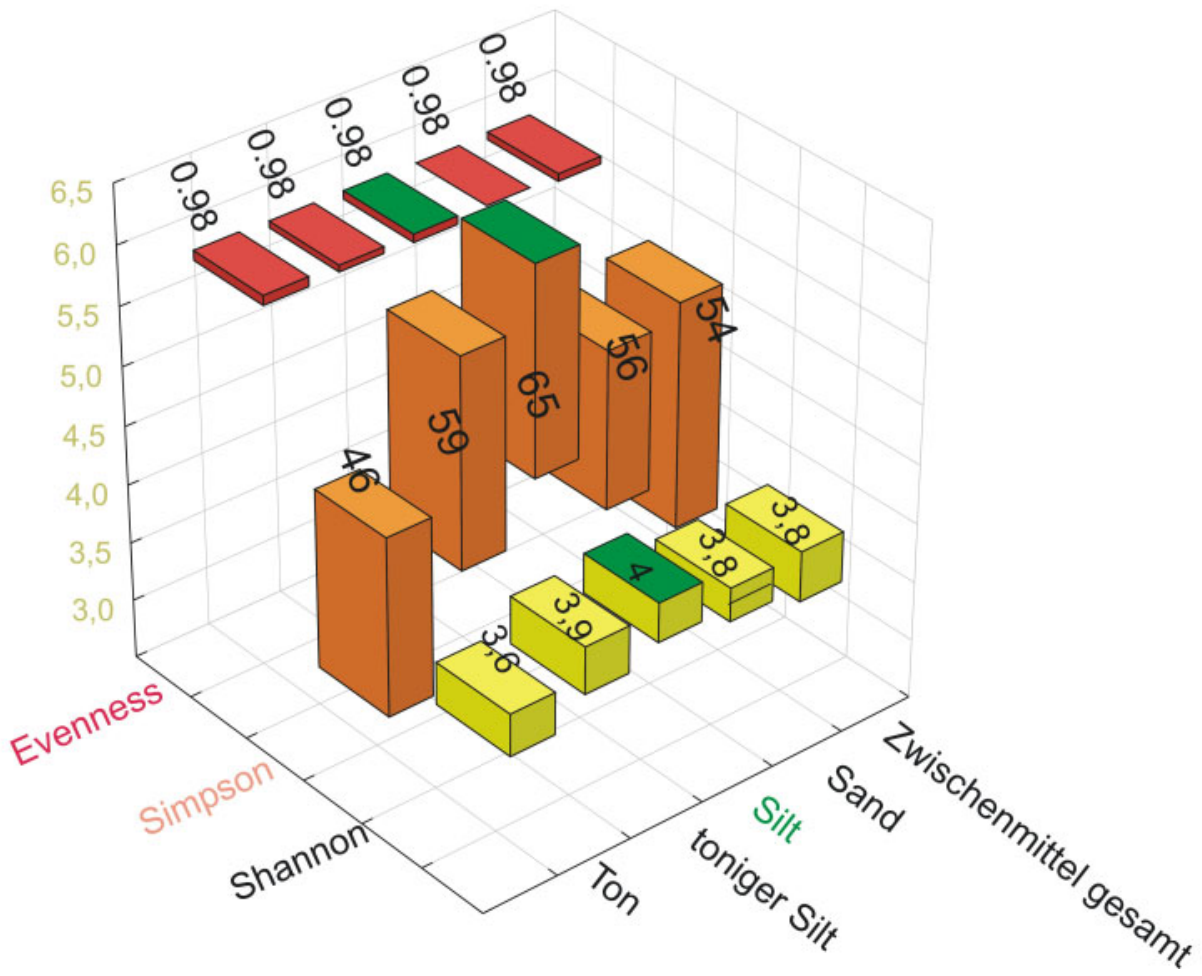

Abb. 4.11: Box-Plot-Diagramm der Proben aus Witznitz (Profil 11): Dargestellt sind die arithmetischen Mittelwerte der Diversitäts-Indizes mit ihren Standardabweichungen. Der Mittelwert ist in numerischer Form angeben. Die Y-Skala ist nur für den Shannon-Index relevant. Die höchste Diversität weist der Silt mit einem Shannon-Index 4.0 und dem reziproken Simpson-Index mit 65 auf (grün markiert). 


\subsubsection{Single-Way-Anova-Test}

In nächsten Schritt muß geklärt werden, in welchem Masse Sortierungsprozesse die Diversität in der Taphozönose beeinflußt haben. Hierzu wurde der Anova-Test angewandt, der die Varianzen vom Mittelwert der Probendiversitäten (Shannon-Index) miteinander vergleicht und deren Signifikanz ermittelt. Die Mittelwerte berechnen sich nach der Diversitäts-Berechnungsmethode 1 (s. Kap. 4.4.5, S. 114).

Die Anova-Tabelle zeigt eine Zerlegung der Varianzen der Daten in zwei Komponenten: eine zwischen den einzelnen Proben (Intragruppen-Varianz) der jeweiligen Lithotypen und eine weitere zwischen den Gruppen der Lithotypen insgesamt (Intergruppen-Varianz).

Der F-Quotient der Gruppen zeigt das Verhältnis der Varianzen zwischen den Gruppen zu den Varianzen innerhalb der Gruppe an. Sobald der P-Wert des F-Testes größer oder gleich 0,05 ist, kann nicht mehr von einer statistisch signifikanten Differenz zwischen den Mittelwerten der verschiedenen Gruppen gesprochen werden (bei einer 95-prozentigen Wahrscheinlichkeit). D.F. gibt die Anzahl der Freiheitsgrade an, die mit $D F=n-1$ berechnet werden. Der Grenzwert, von dem an ein Kontrast in der Multiple-Range-Tabelle signifikant ist, ergibt sich aus der Anzahl der Freiheitsgrade und dem Alpha-Wert (5\%) und ist als Tabellenwert festgelegt.

Innerhalb der Multiple-Range-Tabelle werden homogene Gruppen durch die Anzahl $X$ dargestellt, in denen es jeweils keine signifikante statistische Differenz gibt. Um die Reichweite der Mittelwerte zu ermittelt, wurde die Fisher's last significant differenceMethode (LSD) angewandt, bei der ein fünfprozentiges Risiko besteht, daß eine NullDifferenz als eine signifikante Differenz bezeichnet wird. 


\subsubsection{Alversdorf}

Die nachfolgenden Tabellen zeigen, daß sich die Proben des Alversdorf-Profils innerhalb der Lithotypen signifikant $(\mathrm{P}=0,0033)$ mit einem Verhältnis von 4,79 von den Varianzen zwischen den Lithotypen insgesamt unterscheiden. Die Auswertung der Multiple-Range-Tabelle ergibt eine signifikante Differenz der Diversitäten zwischen dem kohligen Ton und dem kohligen Sand, dem Ton und dem kohligen Sand sowie dem kohligen Sand und dem Silt. Im Gegensatz dazu gibt es nur sehr geringe Unterschiede der Mittelwertvarianzen zwischen dem kohligen Ton mit Silt oder Sand und der Fraktion des Tones mit dem Sand (Zur Diskussion der Ergebnisse siehe auch Kap. 4.4.9.2, S. 128 und Kap. 4.4.11.3, S. 138).

\begin{tabular}{|c|c|c|c|c|}
\hline \multicolumn{2}{|l|}{ Anova Tabelle } & & & \\
\hline Summe der Quadrate & D.F. & $\begin{array}{l}\text { Mean } \\
\text { Quadrate }\end{array}$ & F-Ratio & P-Wert \\
\hline $\begin{array}{ll}\text { Intragruppen-Var. } & 1,308 \\
\text { Intergruppen-Varianz } & 5,50588\end{array}$ & $\begin{array}{l}4 \\
36\end{array}$ & $\begin{array}{l}0,327001 \\
0,0682161\end{array}$ & 4,79 & 0,0033 \\
\hline Gesamt (korr,) & 40 & & & \\
\hline
\end{tabular}

\section{Multiple Range Tests}

Methode: 95,0 Prozent LSD

\begin{tabular}{|l|l|l|l|}
\hline Material & Anzahl & Mean & Homogenität \\
\hline Kohliger Sand & 5 & 3,264 & $\mathrm{X}$ \\
\hline Ton & 26 & 3,68231 & $\mathrm{X}$ \\
\hline Sand & 3 & 3,76667 & $\mathrm{X}$ \\
\hline Kohliger Ton & 2 & 3,845 & $\mathrm{X}$ \\
\hline Silt & 5 & 3,918 & $\mathrm{X}$ \\
\hline \multicolumn{3}{|l|}{} \\
\hline Kontrast & Differenz & $+/-\mathrm{Bereich}$ \\
\hline Kohliger Ton - Kohliger Sand & $*_{0} 0,601$ & 0,443181 \\
\hline Kohliger Ton - Ton & 0,162692 & 0,388696 \\
\hline Kohliger Ton - Silt & $-0,0730001$ & 0,443181 \\
\hline Kohliger Ton - Sand & 0,0783333 & 0,48355 \\
\hline Kohliger Sand - Ton & $*_{-}-0,438308$ & 0,258667 \\
\hline Kohliger Sand - Silt & $*_{-}-0,674$ & 0,335013 \\
\hline Ton - Silt & $-0,235692$ & 0,258667 \\
\hline Ton - Sand & $-0,0843589$ & 0,322986 \\
\hline Silt - Sand & 0,151333 & 0,38684 \\
\hline
\end{tabular}

*signifikante Differenz zwischen den Lithotypen 


\subsubsection{Schöningen}

Im Vergleich zu Alversdorf kann in Schöningen nicht von einer signifikanten Trennung zwischen und innerhalb der Gruppen gesprochen werden. Insbesondere wenn die biogen-klastischen Sedimente mit in die Berechnung einfließen, fällt der FWert von 1,46 auf 0,55. Dies bedeutet, daß die Varianz innerhalb der Gruppen einen größeren Wert als zwischen den Gruppen erreicht.

Für den Multiple Range Test ergeben sich maximale Kontraste der Fraktionen Silt zu Sand und dem tonigen Silt zum Sand, die jedoch nicht signifikant sind. Äußerst geringe Kontraste zeigen die kohligen Tone zum kohligen Silt, zum tonigen Silt und zum Silt sowie der tonige Silt zum Silt (Zur Diskussion der Ergebnisse siehe auch Kap. 4.4.9.3, S. 129 und Kap. 4.4.11.2, S. 136).

(biogene Sedimente)

\begin{tabular}{|c|c|c|c|c|}
\hline \multicolumn{2}{|l|}{ Anova Tabelle } & & & \\
\hline Summe der Quadrate & D.F. & $\begin{array}{l}\text { Mean } \\
\text { Quadrate }\end{array}$ & F-Ratio & P-Wert \\
\hline $\begin{array}{ll}\text { Intragruppen-Var. } & 0,459854 \\
\text { Intergruppen-Varianz } & 5,50588\end{array}$ & $\begin{array}{l}2 \\
35\end{array}$ & $\begin{array}{l}0,229927 \\
0,157311\end{array}$ & 1,46 & 0,2457 \\
\hline Gesamt (korr.) $\quad 5,96575$ & 37 & & & \\
\hline
\end{tabular}

(biogene und biogen-klastische Sedimente)

\begin{tabular}{|c|c|c|c|c|c|}
\hline \multicolumn{3}{|l|}{ Anova Tabelle } & & & \\
\hline \multicolumn{2}{|c|}{ Summe der Quadrate } & \multirow{2}{*}{$\begin{array}{l}\text { D.F. } \\
4 \\
30\end{array}$} & \multirow{2}{*}{$\begin{array}{l}\text { Mean } \\
\text { Quadrate } \\
0,0760994 \\
0,137515\end{array}$} & \multirow{2}{*}{$\frac{F \text {-Ratio }}{0,55}$} & \multirow{2}{*}{$\frac{\text { P-Wer }}{0,6981}$} \\
\hline $\begin{array}{l}\text { Intragruppen-Var } \\
\text { Intergruppen-Var }\end{array}$ & $\begin{array}{l}0,304354 \\
4,12546\end{array}$ & & & & \\
\hline Gesamt (korr.) & 4,42892 & 34 & & & \\
\hline
\end{tabular}




\section{Multiple Range Tests}

Methode: 95,0 Prozent LSD

\begin{tabular}{|l|l|l|l|}
\hline Material & Anzahl & Mean & Homogenität \\
\hline Sand & 4 & 2,7825 & $\mathrm{X}$ \\
\hline toniger Silt & 5 & 3,052 & $\mathrm{X}$ \\
\hline Kohliger Ton & 7 & 2,91743 & $\mathrm{X}$ \\
\hline Kohliger Silt & 8 & 2,9075 & $\mathrm{X}$ \\
\hline Silt & 11 & 3,0525 & $\mathrm{X}$ \\
\hline
\end{tabular}

\begin{tabular}{|l|l|l|}
\hline Kontrast & Differenz & + -Bereich \\
\hline Kohliger Ton - kohliger Silt & 0,0639286 & 0,39196 \\
\hline Kohliger Ton - toniger Silt & $-0,0805714$ & 0,443452 \\
\hline Kohliger Ton - Silt & $-0,0940259$ & 0,366169 \\
\hline Kohliger Ton - Sand & 0,188928 & 0,474687 \\
\hline Kohliger Silt - toniger Silt & $-0,1445$ & 0,43175 \\
\hline Kohliger Silt - Silt & $-0,157955$ & 0,35905 \\
\hline Toniger Silt - Silt & $-4,99964 \mathrm{E}-4$ & 0,31185 \\
\hline Toniger Silt - Sand & 0,2695 & 0,381936 \\
\hline Silt - Sand & 0,27 & 0,336836 \\
\hline
\end{tabular}

signifikante Differenz zwischen den Lithotypen 


\subsubsection{Witznitz (klastische Sedimente)}

Die Sedimente aus Profil Witznitz 11 zeigen im Vergleich mit den anderen Profilen den zweithöchsten F-Wert von 4,21. Signifikante Kontraste konnten zwischen dem Ton und dem tonigen Silt, bzw. dem Ton und dem Silt ermittelt werden. Der geringste Kontrast besteht zwischen dem tonigen Silt und dem Sand (Zur Diskussion der Ergebnisse siehe auch Kap. 4.4.9.1, S. 126 und Kap. 4.4.11.1, S. 136).

\begin{tabular}{|l|l|lll|}
\hline \multicolumn{2}{|l|}{ Anova Tabelle } \\
\begin{tabular}{|llllll|}
\hline \multicolumn{2}{|l|}{ Summe der Quadrate } & D.F. & $\begin{array}{l}\text { Mean } \\
\text { Quadrate }\end{array}$ & F-Ratio & P-Wert \\
\hline Intragruppen-Var. & 0,408891 & 3 & 0,136297 & $\mathbf{4 , 2 1}$ & 0,0176 \\
Intergruppen-Var. & 0,679285 & 21 & 0,0323469 & & \\
\hline Gesamt (korr.) & 1,08818 & 24 & & & \\
\hline
\end{tabular}
\end{tabular}

\section{Multiple Range Tests}

Methode: 95,0 Prozent LSD

\begin{tabular}{|l|l|l|l|}
\hline Material & Anzahl & Mean & Homogenität \\
\hline Ton & 10 & 3,637 & $\mathrm{X}$ \\
\hline Sand & 4 & 3,8375 & $\mathrm{XX}$ \\
\hline toniger Silt & 8 & 3,875 & $\mathrm{X}$ \\
\hline Silt & 2 & 3,98 & $\mathrm{X}$ \\
\hline \multicolumn{3}{|l|}{} \\
\hline Kontrast & Differenz & $+/$-Bereich \\
\hline Ton - toniger Silt & $*_{-} 0,238$ & 0,177415 \\
\hline Ton - Silt & $*_{-} 0,343$ & 0,246213 \\
\hline Ton - Sand & $-0,2005$ & 0,221276 \\
\hline toniger Silt - Silt & $-0,105$ & 0,253216 \\
\hline toniger Silt - Sand & 0,0375 & 0,229042 \\
\hline Silt - Sand & 0,1425 & 0,285666 \\
\hline
\end{tabular}

* signifikante Differenz zwischen den Lithotypen

\subsubsection{Diskussion der Diversitätsmittelwerte}

Die Diversitäten der Palynomorphen aus den Proben der Profile Alversdorf und Witznitz 11, die mit Hilfe des Shannon-Index ermittelt wurden, zeigen eine mehr oder weniger starke Variabilität in Abhängigkeit der Korngröße der klastischen und klastisch-biogenen Sedimente. Die Ursache dieser Abhängigkeit kann zumindest auf den ersten Blick den Sortierungseffekten zugeordnet werden, da Sporomorphen als Teil der Suspensionsfracht in einem Wasserkörper betrachtet werden können.

So konnte HoLMES 1990 in einer Laborstudie in Verbindung mit einem Feldversuch (Silwood Lake, Berkshire, England) eine Abhängigkeit der Palynomorphen-Sortierung 
von der Korngröße feststellen. Die größten Sporomorphen-Taxa (z.B. Farnsporen, einige Gräserpollen) wurden primär im Bereich des Deltas, mittelgroße Pollen (einige Gräserpollen, Corylus) im See und kleine Taxa (Urticaceae) größtenteils in Sedimenten distal zum Delta abgelagert. Eine weitere Untersuchung des Verhaltens von Sporomorphen als sedimentäre Partikel wurden von BRUSH \& BRUSH 1972 durchgeführt. Sie stellten eine deutliche Anreicherung der Palynomorphen in feinkörnigen Sedimenten fest.

Muller 1959, Cross et al. 1966 und CHEN 1987 u.a. konnten an rezenten Studien ebenfalls nachweisen, daß der Transport von Sporomorphen in einem Wasserkörper normalerweise mit der Feinsilt/Ton-Fraktion der Mineralkörner assoziiert ist. Diese Mineralkörner liegen damit eine Korngrößenklasse unter der Sporomorphengröße, was mit der geringeren Dichte der Palynomorphen zu erklären ist.

Im Profil Alversdorf ergeben die Diversitätsberechnungen für den Silt die höchste Diversität, gefolgt von dem kohligen Ton, dem Sand und dem Ton. Im Profil 11 von Witznitz verläuft die Reihenfolge von Silt mit der höchsten Diversität über den tonigen Silt, zu Sand und Ton generell ähnlich.

Auffällig ist die Tatsache, daß der Ton im Gegensatz zu den Ergebnissen aus dem Vergleich mit rezenten Ökosystemen eine relativ geringe Diversität aufweist. Außerdem zeigt der Sand eine hohe Diversität, die in Anbetracht des großen Korngrößen-Unterschiedes zu den Palynomorphen erstaunlich ist (Interpretation siehe auch Kap. 4.4.9.1.3).

\subsubsection{Witznitz}

\subsection{Rinnenfüllung}

Zumindest für Witznitz kann eine mögliche Ursache für die geringe Diversität des Tones ein Fazies- und Vegetationswechsel sein. Eine Zusammenfassung der ökologischen Interpretation dieses Profils im Kontext zur Diversität soll dies veranschaulichen (Pollendiagramme s.a. Fortsetzungsantrag $\mathrm{zu}$ Wa 757/9-1 und HAMMER 2002 in Vorbereitung).

Deutlich ist eine Diversitätsabnahme von der Basis des Profils zum Top zu erkennen. Eine erstmalige deutliche Abnahme der Diversität findet im schräggeschichten Sand der Probe 11 statt. Zum Top setzt sich die Abnahme der Diversität mit dem Einsetzen der Tone in den „Overbank fines“ fort. 

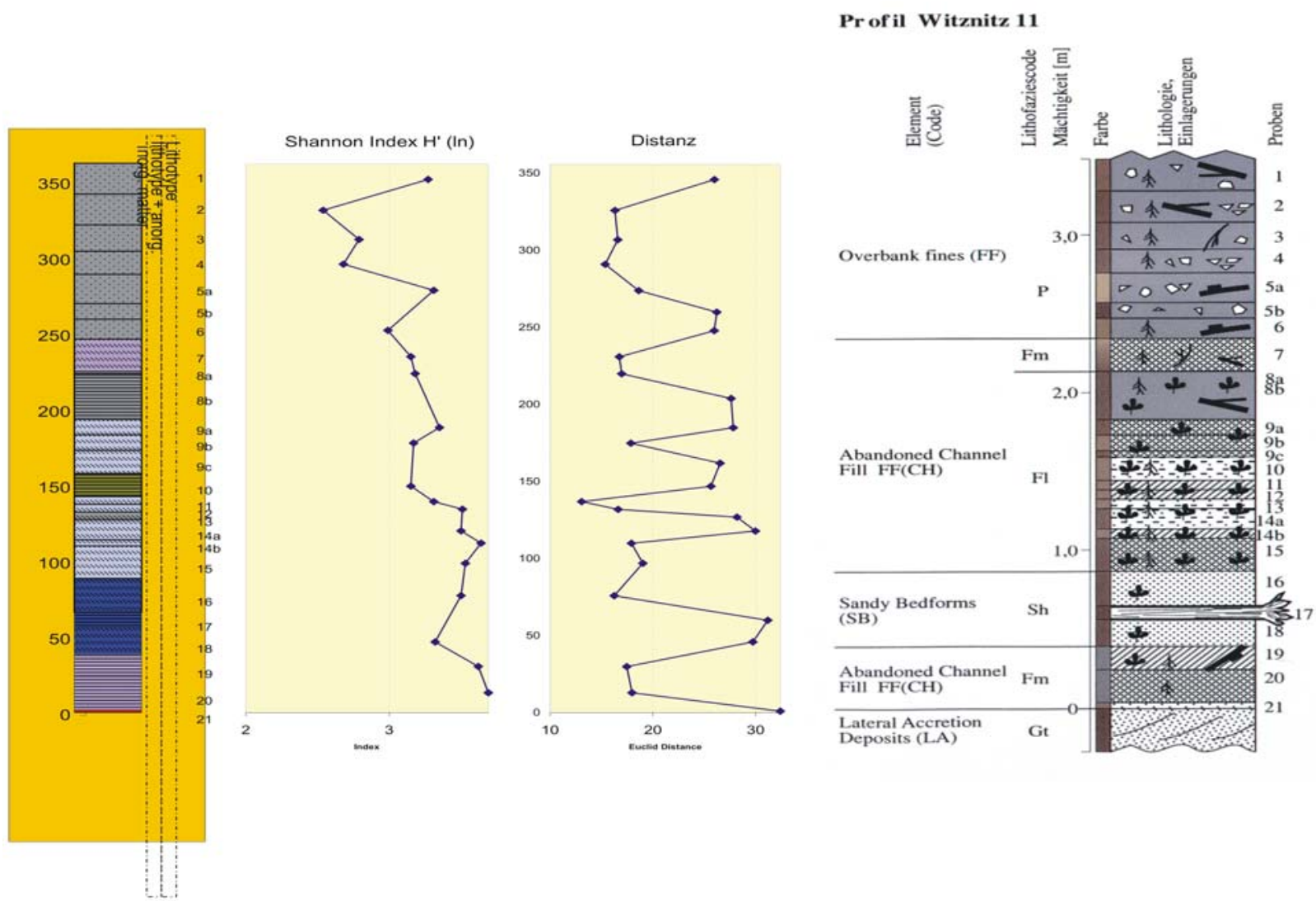

Abb. 4.12: Profil Witznitz 11: Das linke Profil und die Diagramme sind mit P.A.S.T generiert (Legende siehe Anlage), rechts nach HAMMER 2002 (in Vorbereitung). Es ist eine deutliche Diversitätsabnahme zum Top zu erkennen. Das Diagramm „Distanz“ stellt die Unterschiede der Probe zur nächsten Probe dar, ermittelt nach der Euklid-Distance-Methode. Eine geringe Distanz bedeutet demnach, daß sich die zusammenhängenden Proben stark ähneln; eine große Distanz spricht für einen Wechsel in der Vegetation, bzw. eine Unähnlichkeit der Proben. Daran ist deutlich die einheitliche Fazies in den „Overbank-fines“ zu erkennen.

Die Interpretation für das Profil Witznitz 11 ergibt eine Sukzession von einem flußbegleitenden Auewald zur Vegetation eines mesophytischen Waldes (Tone im Top des Profils).

Die Auewaldvegetation konnte makrobotanisch z.B. durch Liquidambar, Alnus, Populus und palynologisch sowie carpologisch durch Taxodium u.a. nachgewiesen werden (HAMMER mündl. Mitteilung).

\subsection{Paläoboden}

Die als fahlgraue Tone mit Wurzelstrukturen angesprochenen Tone am Top des Profils wurden von HAMMER (mündl. Mitteilung) als Paläoboden gedeutet, dessen primäre Sedimente unter dem Einfluß eines verlandenden Altwasserarmes standen. In den Tonen konnte ein deutlicher Wechsel der Vegetation mit einem Auftreten von Onagraceen und Salvinia ermittelt werden. Salvinia ist ein Indikator für stehendes Gewässer im Endstadium einer Altwasserrinne, in die im Gegensatz zur aktiven Rinne ein geringer Eintrag von regionalen Pollen erfolgt. Dies kann die Abnahme der Diversität im oberen Bereich des Profils und damit im Ton erklären. 
Die Entstehung eines Paläobodens führte in einem sekundären Prozeß mit hoher Wahrscheinlichkeit zu einer weiteren Abnahme der Diversität, da die Bodenbildung die Zersetzung von Pollen und Sporen dramatisch verstärkt. Im Gegensatz dazu sind mechanische Zerstörungen im Wasserkörper unbedeutend. ZIEGLER 1988 konnte nachweisen, daß Sedimentpartikel kleiner als $750 \mu \mathrm{m}$ in einem Flußsystem mit normaler Fließgeschwindigkeit nicht gerundet werden können. Die Korngröße der Palynomorphen liegt jedoch in der Regel weit unter diesem Grenzwert - daher ist eine mechanische Zerstörung der Palynomorphen in einem Fließgewässer mit niedrigen Fließgeschwindigkeiten äußerst unwahrscheinlich.

Im Gegensatz dazu findet in terrestrischen Böden eine erhebliche Zersetzung der Pollen und Poren statt. Nach DimbleBY 1985 ist der pH-Wert in diesen Böden der wichtigste Faktor bei der Sporomorphen-Erhaltung und Zersetzung. So wird die Erhaltungsfähigkeit von Pollen mit steigendem pH-Wert stark reduziert. DIMBLEBY nimmt weiterhin an, daß unter bestimmen Umständen der $\mathrm{pH}$-Wert einen größeren Einfluß auf die Erhaltungsfähigkeit haben kann als oxidierende oder reduzierende Bedingungen. TsCHUDY (1969) stellt dagegen den Eh-Wert als den wichtigsten Faktor der Zersetzung von Palynomorphen heraus. Ein niedriger Eh-Wert führt zu reduzierenden anaeroben Bedingungen mit einer guten Erhaltungsfähigkeit des Sporopollenins bzw. der Zellulose. Ebenso beschleunigen Bakterien und Pilze die Pollendegradation in einem Bodenhorizont (HOLLOWAY 1981).

\subsection{Sand}

Die relativ hohe Diversität der Palynomorphen in der Kornfraktion des Sandes kann verschiedene Ursachen haben. BRUSH \& BRUSH (1972) und FALL 1987 fanden ebenfalls Sporomorphen in Sandlagen. Folgende Erklärungsmodelle sind denkbar:

$>$ Holmes 1994 vermutet, daß bei höheren Strömungsgeschwindigkeiten Turbulenzen in der Wassersäule die Palynomorphen relativ schnell zu Boden tragen. Dort werden sie von turbulenzartigen Wirbeln, verursacht durch prominente Sandkörner, in die Porenräume der Sandlagen transportiert und dort fixiert, was zu einer Anreicherung von Palynomorphen führen kann.

Ebenso ist es denkbar, daß der Polleneintrag aus der lokalen bis extra-lokalen Flora in Phasen geringerer Fließgeschwindigkeiten eingetragen und abgelagert wird. Dies würde auch die feinere Fraktion des klastischen Sediments erklären und kann z.B. saisonal bedingt sein.

\subsubsection{Alversdorf}

Gegenüber den Diversitäten in den Kohlen kann in Alversdorf ein deutlicher Anstieg der Diversitäten in den Zwischenmitteln festgestellt werden. Dieser Anstieg ist mit großer Wahrscheinlichkeit auf den erhöhten Polleneintrag aus der umgebenden regionalen Flora in Lagunen über das fluviatile Milieu zurückzuführen. Im Gegensatz dazu bildet die Kohle in erster Linie die lokale Flora ab.

\subsection{Biogene Sedimente}

In den Grundmassenkohlen, Gewebegrundmassenkohlen und Gewebekohlen konnte keine Abhängigkeit der Diversität nachgewiesen werden, da die sedentäre Bildung des Torfs mit großer Wahrscheinlichkeit keinem Sortierungs- bzw. Anreicherungseffekt unterliegt.

Eine Ausnahme bildet die tonige Kohle, die eine höhere Diversität als die anderen biogenen Lithotypen aufweist und eventuell eher den sedimentären Bildungen zuzuordnen ist. 


\subsection{Klastische Sedimente}

Dagegen kann ein Sortierungseffekt in den klastischen Sediment-Proben aus dem Profil Alversdorf mehr oder weniger deutlich mit Hilfe des Single-Way-Anova-Tests nachgewiesen werden.

Ungeachtet der durch die deutlich multimodale Verteilung im Histogramm suggerierten Trennung der Diversitäten der Kohleproben von den Zwischenmittelproben, ist die Signifikanz der Kontraste der klastischen Sedimente untereinander uneinheitlich und z.T. nur schwach ausgeprägt. Auffällig ist z.B. der geringe Kontrast zwischen dem Ton und dem Sand - wie schon im Profil Witznitz festgestellt. Die im Kapitel 4.4.9.1.3, S. 128 aufgeführten Überlegungen zur Erklärung dieses Phänomens könnten demnach auch in Alversdorf ihre Gültigkeit haben.

\subsection{Biogen-Klastische Sedimente}

Zudem weisen einige zu dem Zwischenmittel gestellten biogen-klastischen Sedimente (z.B. der kohlige Sand) eine relativ geringe Diversität und eine hohe Varianz auf. So liegt der Schluß nahe, daß die Palynomorphen in diesen Proben größtenteils aus den dispers verteilten aufgearbeiteten Kohlebruchstücken stammen und damit die geringere Diversität der Kohle wiedergeben.

\subsubsection{Schöningen}

Das Histogramm sowie der Anova-Test ergeben keine signifikante Unterscheidung der Lithotypen hinsichtlich der Diversität. Eine mögliche Erklärung ist die unterschiedliche Fazies der Zwischenmittelproben gegenüber den fluviatilen Sedimenten von Witznitz und Alversdorf.

Die Zwischenmittel im Schöningen-Profil sind durch Transgressionshorizonte charakterisiert, die durch das häufige Auftreten von Dinoflagellaten dokumentiert sind. Insgesamt können die Zwischenmittel Z1 und Z2 als Brackwasser-Lagunen gedeutet werden (HAMmER-SchiEMANN 1998, Lietzow 1991). Dies ließe den Schluß zu, daß im marinen Milieu die Diversität der Palynomorphen nicht durch Sortierungseffekte beeinflußt ist, beziehungsweise andere taphonomische Prozesse die Diversität beeinträchtigen. Dem widersprechen jedoch Untersuchungen anderer Autoren, die auch im marinen Milieu Sortierungs- und Anreicherungseffekte feststellen konnten (HOLMES 1990 u.a.). Ob dieser Effekt unter Berücksichtigung einer anderen Untersuchungsmethode auch in Schöningen nachgewiesen werden kann oder ob die lagunären Bildungsbedingungen keinen Sortierungseffekt herbeiführen, beantworten die nächsten Kapitel.

\subsubsection{Abhängigkeit der Diversität von der Probengröße}

Leider ist die geforderte Gleichmäßigkeit der Probengröße in den untersuchten Profilen nicht immer gewährleistet. Aufgrund der ökologischen Fragestellungen der zu Grunde liegenden Arbeiten ist zum Beispiel in Schöningen das Phytoplankton der marin beeinflußten Proben nur als Gruppe erfaßt worden, was das Phytoplankton als Diversitätsindikator ausschließt. Eine Subtraktion der Phytoplankton-Daten unter Neuberechnung der Prozentwerte brachte keine Verbesserung bei der Erfassung der vollständigen Diversität, da die resultierenden Probengrößen zu klein sind.

Um die Problematik der dadurch veränderten Diversität darzustellen, werden zu Beginn dieses Unterkapitels interpolierende Verfahren angewandt, die jedoch keine Diversitäten in Form von Indizes, sondern nur die Anzahl der Taxa berücksichtigen können. 
Daran anschließend zeigen extrapolierende Maßnahmen die zu erwartende maximale Diversität für jeden Lithotyp.

\subsubsection{Interpolierende Diversitätsberechnung (Rarefaction)}

In dieser Arbeit wurde die Rarefaction-Methode benutzt, da sie in der Literatur weit verbreitet ist (z.B. ASHRAF 1995, WENJUN 1998, u.a.). Zur Berechnung der Rarefaction wurde auf die Programme Past (HAMMER 2001) und Rarefaction Calculator (BRZUSTOWSKI 2001) zurückgegriffen. Bei den folgenden Berechnungen wurden ausschließlich Rohdaten (keine Prozentwerte!) als Datenbasis verwendet.

Die statistische Rarefaction-Methode dient dazu, die Anzahl der Taxa einer Unterprobe von Individuen aus einer definierten Probe zu interpolieren. Mithilfe dieser Methode ist es möglich, die Qualität einer kleineren Probe in Bezug auf deren Diversität zu ermitteln. Sie eignet sich jedoch nur bedingt dazu, die Diversität einer größeren Probe aus einer kleinen Probe zu extrapolieren (s. ALROY 2001).

Die Rarefaction-Methode produziert eine Hyperbelkurve, die die vermutete Anzahl von Arten gegenüber der Probengröße darstellt. Eine umfangreiche Darstellung dieser Methode gibt KREBS 1989.

Folgende Gesichtspunkte sind bei der Benutzung der Rarefaction-Methode zu beachten:

\section{\begin{tabular}{|l|l|l}
\hline Methode & Bedingung & Datenbasis \\
\hline
\end{tabular}}

Vergleich taxonomischer Diversität von Proben unterschiedlicher kleinerer Größen

\section{Die Proben müssen} taxonomisch ähnlich sein, bzw. aus dem selben Habitat entnommen sein

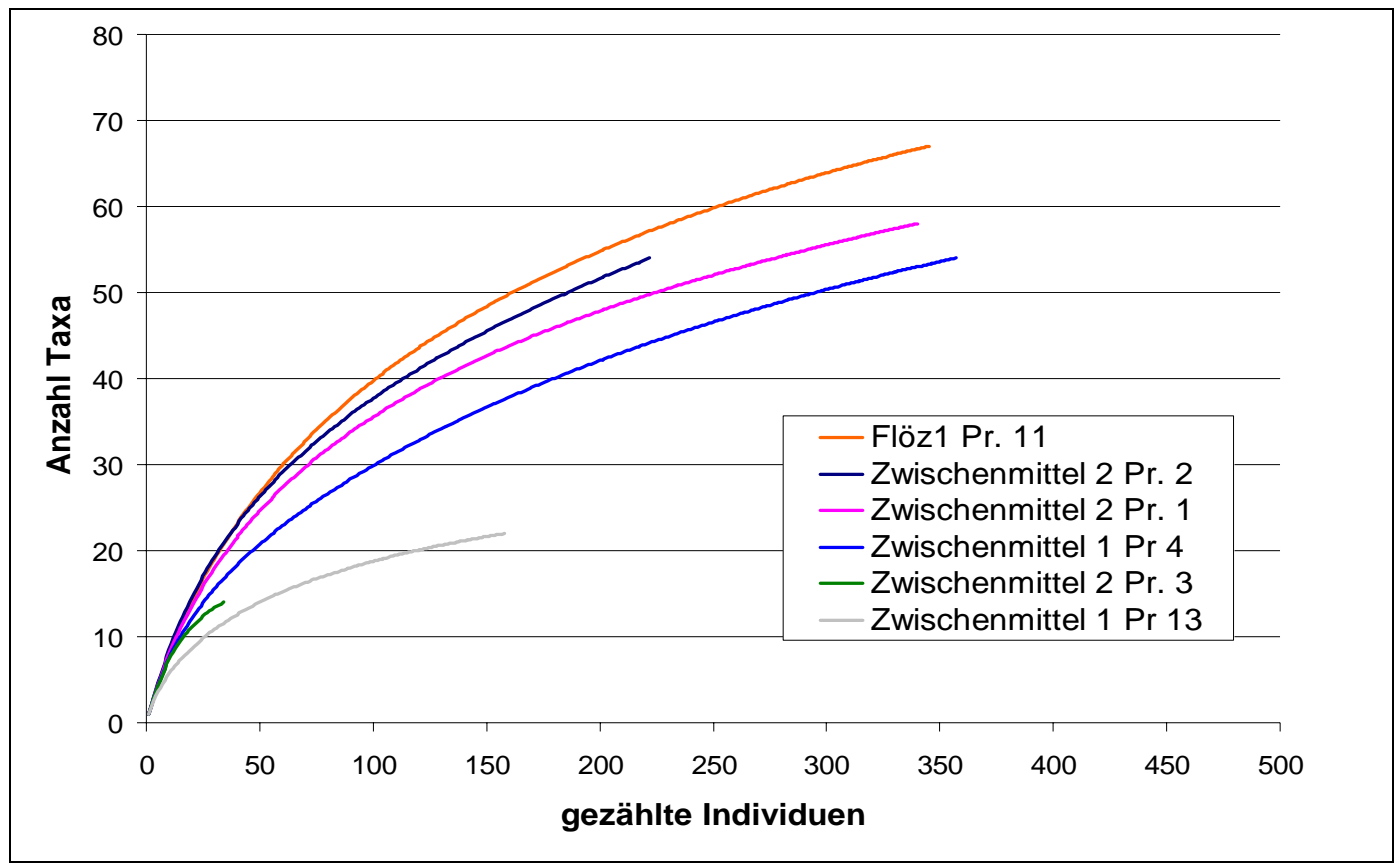

Abb. 4.13: Rarefaction-Kurven einiger ausgewählter Proben aus dem Schöningen Profil (Südfeld). Deutlich ist die geringe Anzahl der Individuen in einigen Proben zu erkennen, so daß nicht die tatsächliche Diversität - erkenntlich an der großen Steigung der Kurven - erfaßt werden konnte. 
Insbesondere Proben aus dem Zwischenmittel weisen eine auffällig geringe Individuenzahl auf, was eine mögliche Ursache der geringen Diversität gegenüber den Kohlenproben sein könnte.

\subsubsection{Berechnung der Diversität nach der, ,Methode 3“}

Die Anwendung der „Methode 3“ (s. Kap. 4.4.5) der Diversitätsberechnung zeigt, daß die Diversität mit dem Hinzuziehen weiterer Proben stetig zunimmt und einen maximalen Wert erreicht, der sich je nach Qualität der Probennahme asymptotisch einer Geraden annähern sollte. Der größte Vorteil dieser Methode gegenüber der „Methode 1“ liegt darin, daß Proben mit einer geringen Individuenzahl die Gesamtdiversität nicht so stark negativ beeinflussen, da die Gesamtheit aller Proben den Index bei der „Methode 3“ sukzessiv erhöht.

\subsection{Schöningen}

Die Abbildung 4.14 belegt für die Diversität im Zwischenmittel aufgrund der geringen Individuenzahl eine große Ungenauigkeit, so daß eine Aussage zur Sortierung äußerst schwierig ist. Allerdings könnte die geringe Diversität des Sandes auf einen Sortierungseffekt hindeuten. Aussagen über Absolutwerte können aufgrund der für die Fragestellung der Diversitätsberechnung unzureichenden Datengrundlage nicht sicher getroffen werden - hierzu wäre eine Neuauszählung der Proben nötig, was im Rahmen dieser Promotion nicht durchführbar ist (mehr dazu im Kapitel 5.4.2, S. 150).

Vergleicht man die Steigung der Kurven der Abbildung 4.14 und 4.15 wird deutlich, daß im Zwischenmittel aufgrund der geringen Individuenzahl nicht die tatsächliche Diversität ermittelt werden konnte.

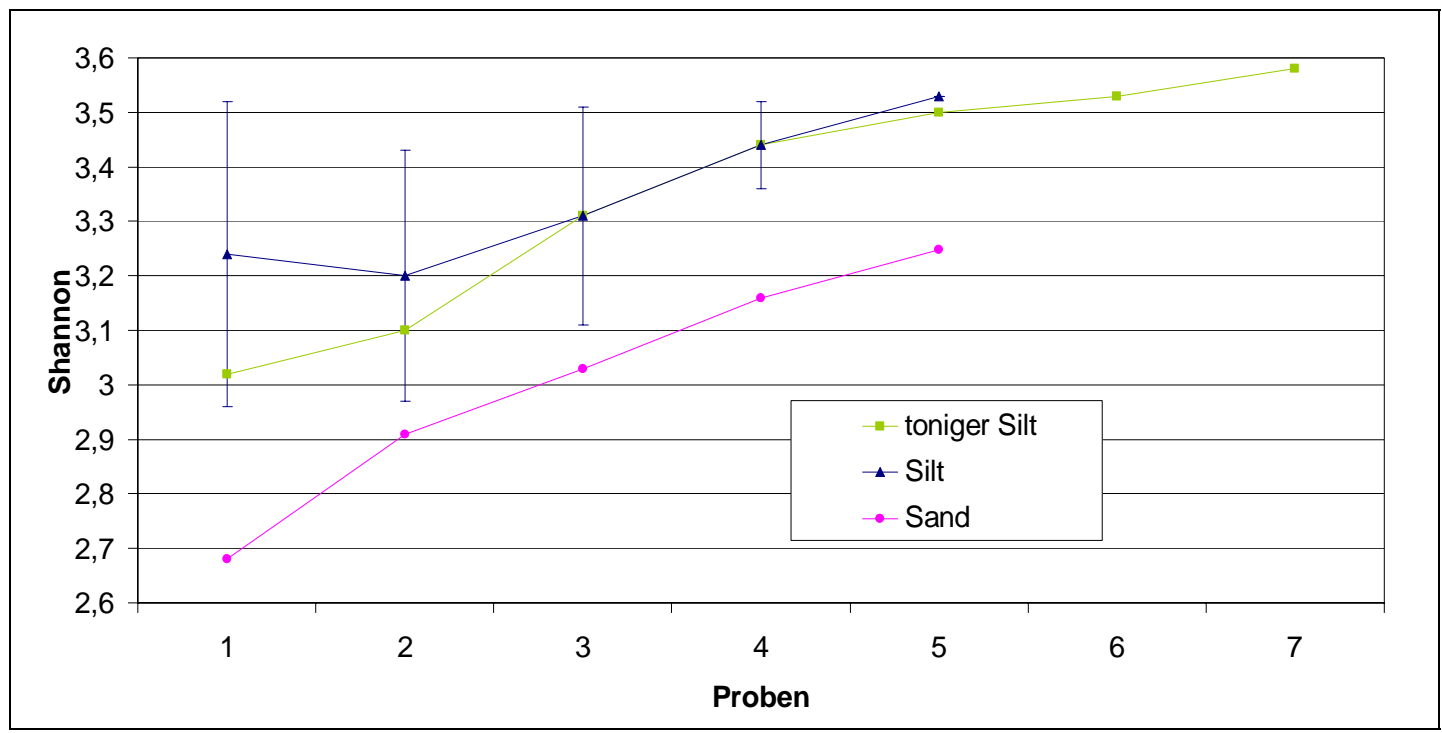

Abb. 4.14: Darstellung der Diversitäten nach der „Methode 3“, Zwischenmittel. Die Diversität der Proben aus dem Zwischenmittel ist nicht komplett erfaßt worden. Die Standardabweichungen des tonigen Siltes und des Sandes sind aus Gründen der Übersichtlichkeit nicht dargestellt - sie bewegen sich in Grenzen, die dem Silt ähneln.

Die Diversitäten der Proben aus der Kohle nähern sich asymptotisch einer Geraden und damit einem Maximalwert an (Abbildung 4.15) . Es ist davon auszugehen, daß auch bei einer höheren Individuenzahl keine signifikante Zunahme der Diversität zu 
erwarten wäre. Die Schwankungen am Anfang der Kurven sind aufgrund der hohen Standardabweichung nicht signifikant.

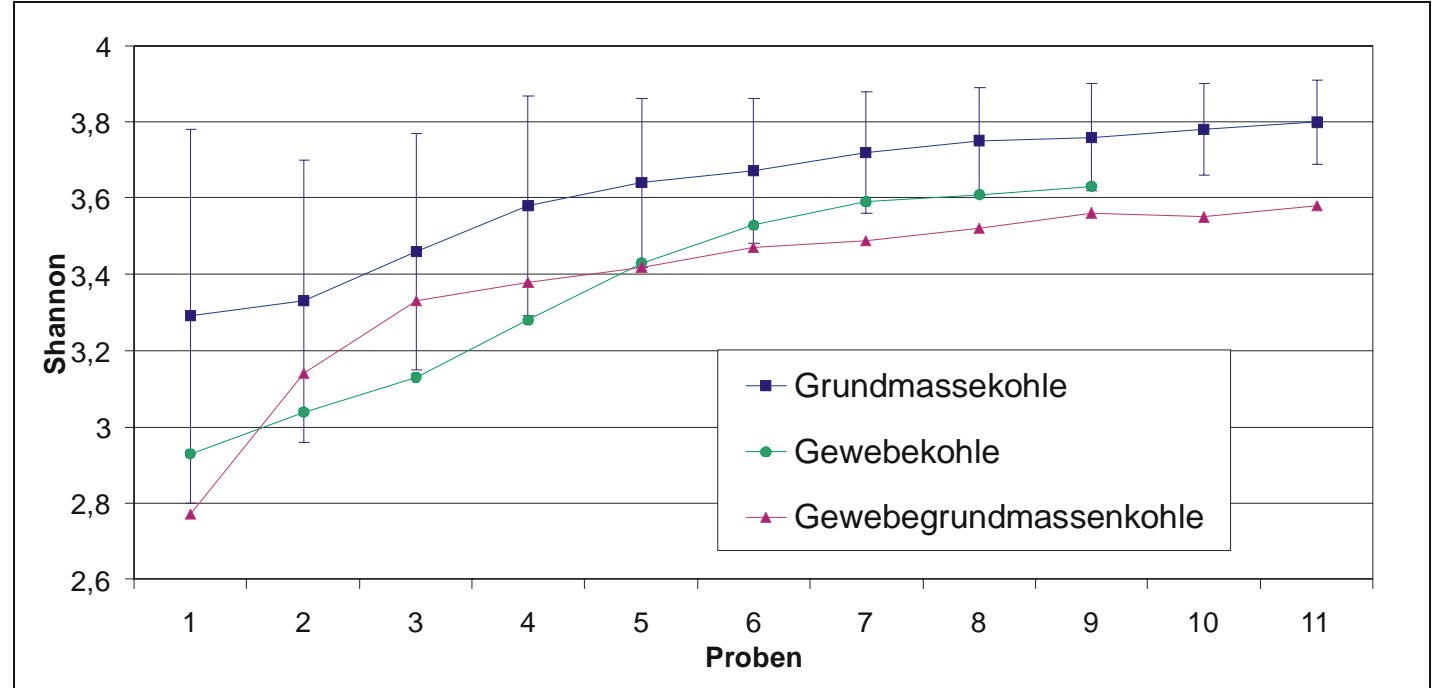

Abb. 4.15: Extrapolation der Diversitäten nach „Methode 3“, Kohle. Die Kurven nähern sich asymptotisch einem Maximalwert an. Die Diversität ist mit hoher Wahrscheinlichkeit komplett erfaßt worden.

\subsection{Witznitz}

Auch wenn die Probenanzahl des Witznitz-Profils 11 im Vergleich zu dem Schöningen-Profil gering ist, spiegelt die ermittelte Diversität mit größerer Wahrscheinlichkeit die tatsächlichen Verhältnisse eher wieder. Die Kurven nähern sich asymptotisch einem Maximalwert an. Insbesondere die geringe Diversität der Tone des Paläobodens kann mit Hilfe der „Methode 3“ signifikant bestätigt werden. Auffällig ist die geringe Standardabweichung der Shannon-Indizes gegenüber den Schöningen-Indizes.

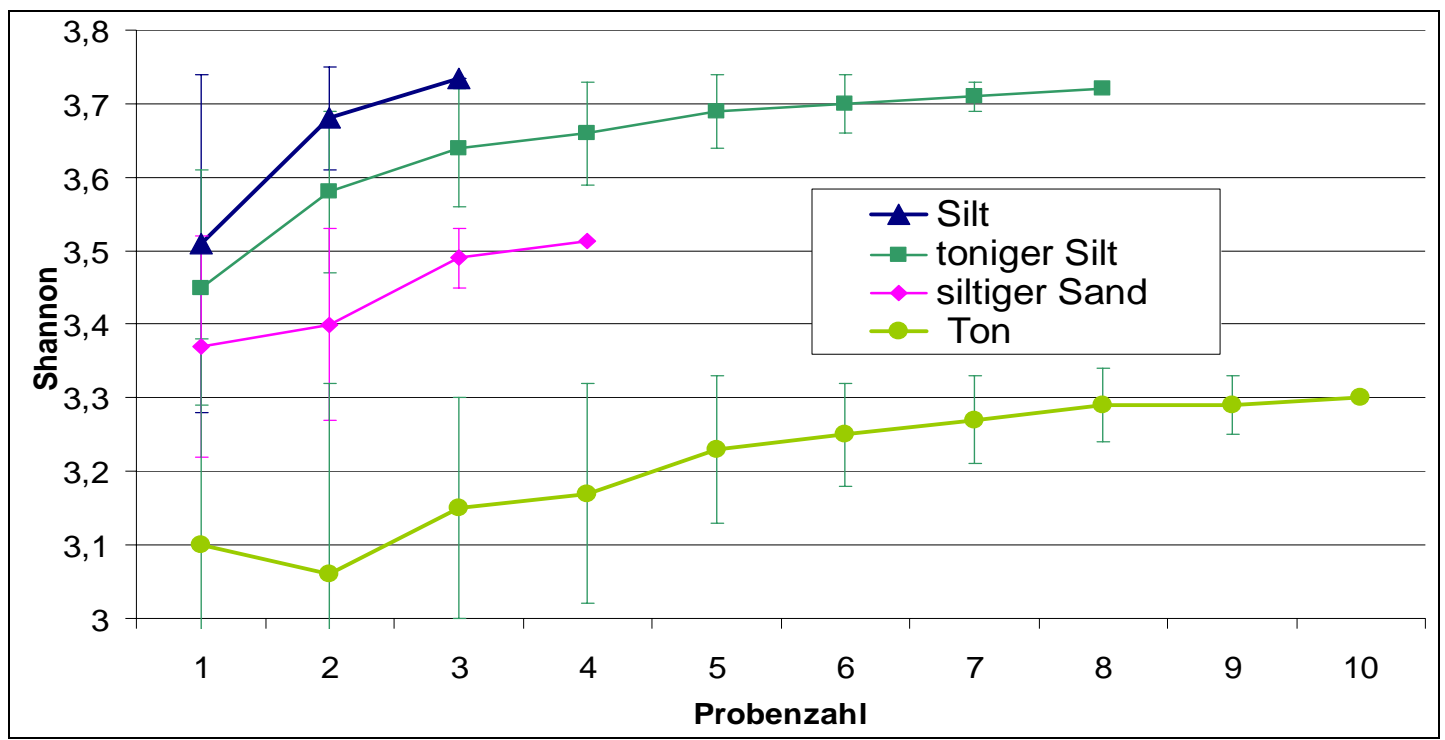

Abb. 4.16: Extrapolation der Diversitäten nach der „Methode 3“, Witznitz, klastische Sedimente. Die Kurven nähern sich asymptotisch einem Maximalwert an. Die Diversität ist mit hoher Wahrscheinlichkeit nahezu komplett erfaßt worden. Auch mit dieser Methode ist ein Sortierungseffekt sehr deutlich. 


\subsubsection{Extrapolation der Diversitäten nach Chao-1}

Neben der Interpolation durch die Rarefaction-Methode existiert eine Vielzahl weiterer Methoden um die Diversität zu extrapolieren (ALROY 2001). Der grundsätzliche Gedanke bei der Extrapolation liegt darin, daß die Gesamtdiversität als eine Funktion der relativen Anzahl der Arten in jeder Probenhäufigkeitsklasse zu verstehen ist. Diese Berechnung enthält gegenüber den vorherigen Methoden einen spekulativen Charakter, da hier auf der Basis von Häufigkeitsdaten eine unter infiniten Probengrößen zu erhaltende Diversität ermittelt wird. Außerdem ist es nicht möglich, über eine Extrapolation einen Index zu berechnen, deshalb ist unter Diversität in diesem Kapitel die Anzahl der Taxa zu verstehen. Folgende Argumente sprechen im Vergleich mit anderen Extrapolierungsmethoden für den Chao-1 Test:

1. Die Berechnung des Chao-1-Tests benötigt eine große Zahl von Singletons und Doubletons (s. nächstes Unterkapitel). Ermittelt man das prozentuale Auftreten der Minimalwerte in den verschiedenen Profilen, wird ersichtlich, daß dieser Sachverhalt in den bearbeitenden Profilen gegeben ist (Abbildung 4.17).

\begin{tabular}{|l|l|}
\hline Profil & $<=1 \%$ \\
\hline Schöningen Nordfeld & $58 \%$ \\
\hline Schöningen Südfeld & $62 \%$ \\
\hline Alversdorf (komplett) & $50 \%$ \\
\hline Witznitz & $33 \%$ \\
\hline
\end{tabular}

Abb. 4.17: Der prozentuale Anteil der Auszähldaten, die unter oder gleich 1\% liegen

2. Aufgrund der Rarefaction-Kurven ist bekannt, daß in Schöningen die Diversität in den Zwischenmitteln mit Sicherheit unvollständig ermittelt wurde. Ein Vergleich der verschiedenen Methoden der Extrapolation für den Lithotyp Silt in Schöningen zeigt die Abbildung 4.18. Da man von einer deutlich geringeren ermittelten Diversität (in diesem Fall die Anzahl der Taxa) ausgehen kann, kommen nur die Methoden Jack-2 und Chao-1 in Betracht. Aufgrund der besser geeigneten Datenberechnung (s. Argument 1) wurde die Chao-1Berechnung gewählt.

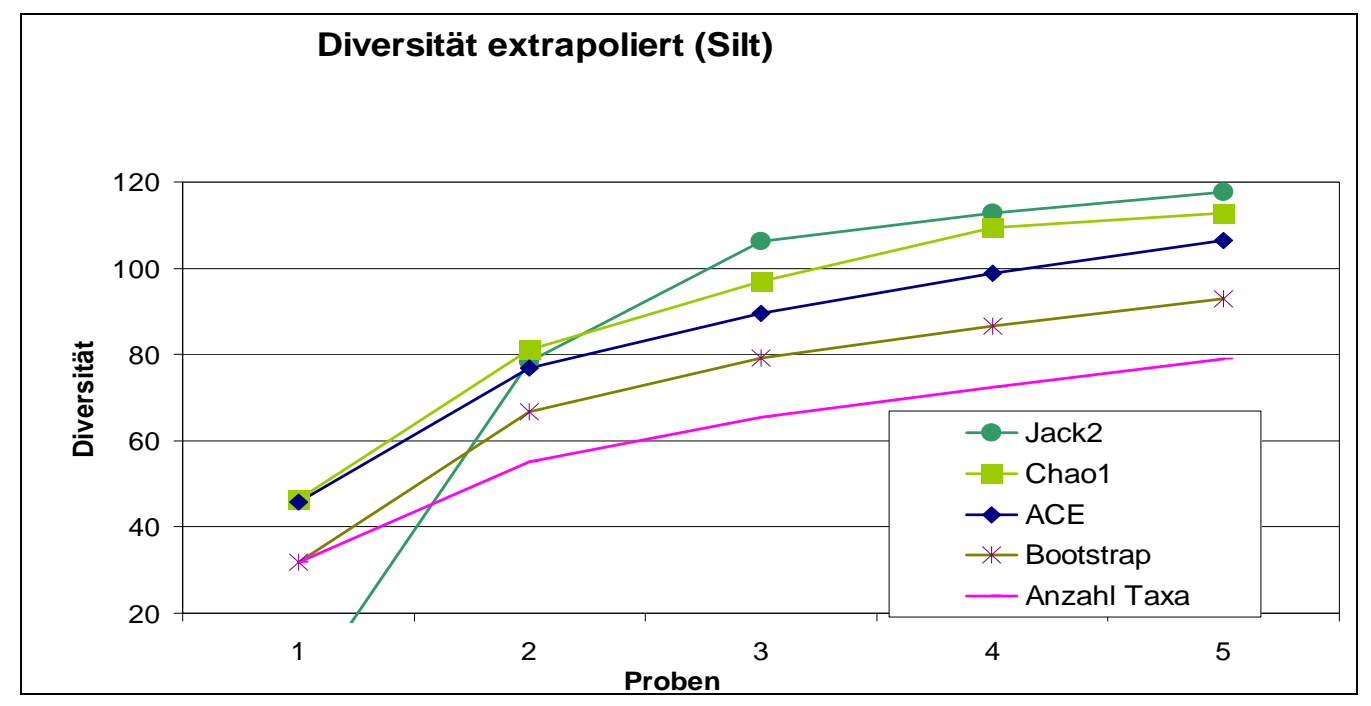

Abb. 4.18: Extrapolationsmethoden für den Lithotyp Silt aus dem Proben des Profils Schöningen. 


\subsubsection{Berechnung der Chao-1 Gleichung}

Die Chao-1-Gleichung (nach CHAO 1987) berechnet sich wie folgt:

$D_{C H}=D_{S}+\left(\frac{N_{S F 1}^{2}}{2 \mathrm{~N}_{\mathrm{SF} 2}}\right)$

Wobei $\mathrm{D}_{\mathrm{CH}}$ die geschätzte Diversität, bzw. die Speziesanzahl ergibt, $\mathrm{D}_{\mathrm{S}}$ umfaßt die Anzahl der beprobten Arten innerhalb eines Intervalls, $\mathrm{N}_{\mathrm{SF} 1}$ ist die Anzahl der Taxa, die genau einmal auftreten (sog. ,Singletons“) und $\mathrm{N}_{\mathrm{SF} 2}$ die Anzahl der Taxa, die mit genau zwei Exemplaren (,Doubletons“) auftreten.

\subsection{Schöningen}

Die Abbildung 4.19 gibt bei der Benutzung der Chao-1-Gleichung für alle Lithotypen des Zwischenmittels einen nahezu identischen Verlauf der Diversitäten bei dem sukzessiven Hinzuziehen sämtlicher Proben. Dies spricht dafür, daß in den Zwischenmittel-Sedimenten keine Sortierung erfaßt ist. Im Vergleich mit der Abbildung 4.20 ist insbesondere die geringe Diversität des unkorrigierten Siltes aufgrund der zu geringen Probengrößen erkennbar. Die extrapolierte Diversität liegt etwa 20 bis $25 \%$ über den unkorrigierten Werten. Die niedrige Diversität des Sandes, berechnet mit der „Methode 3“, kann hier nicht bestätigt werden.

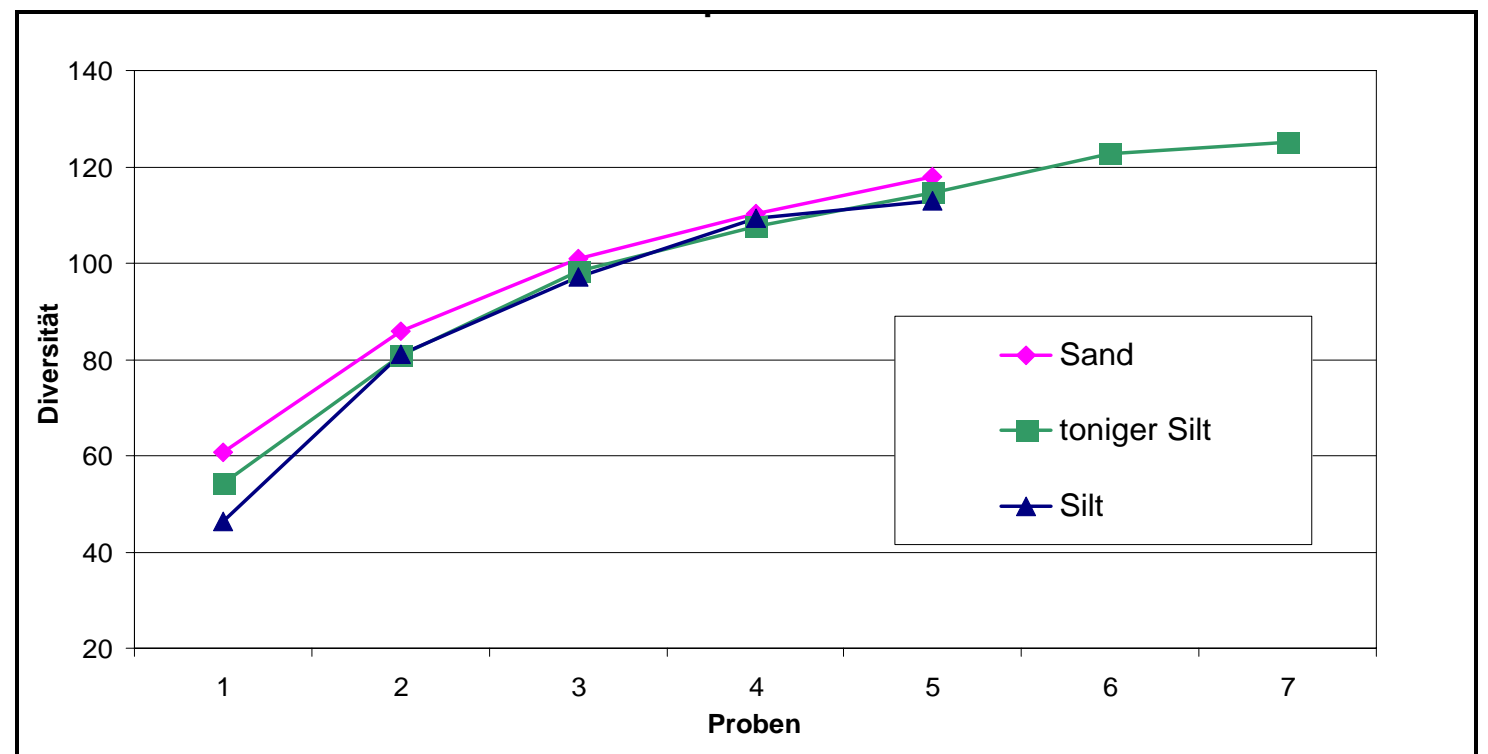

Abb. 4.19: Extrapolation der Anzahl der Taxa in Schöningen nach der Chao-1-Methode. Es ist kein signifikanter Unterschied der Diversitäten der verschiedenen Lithotypen zu erkennen. 


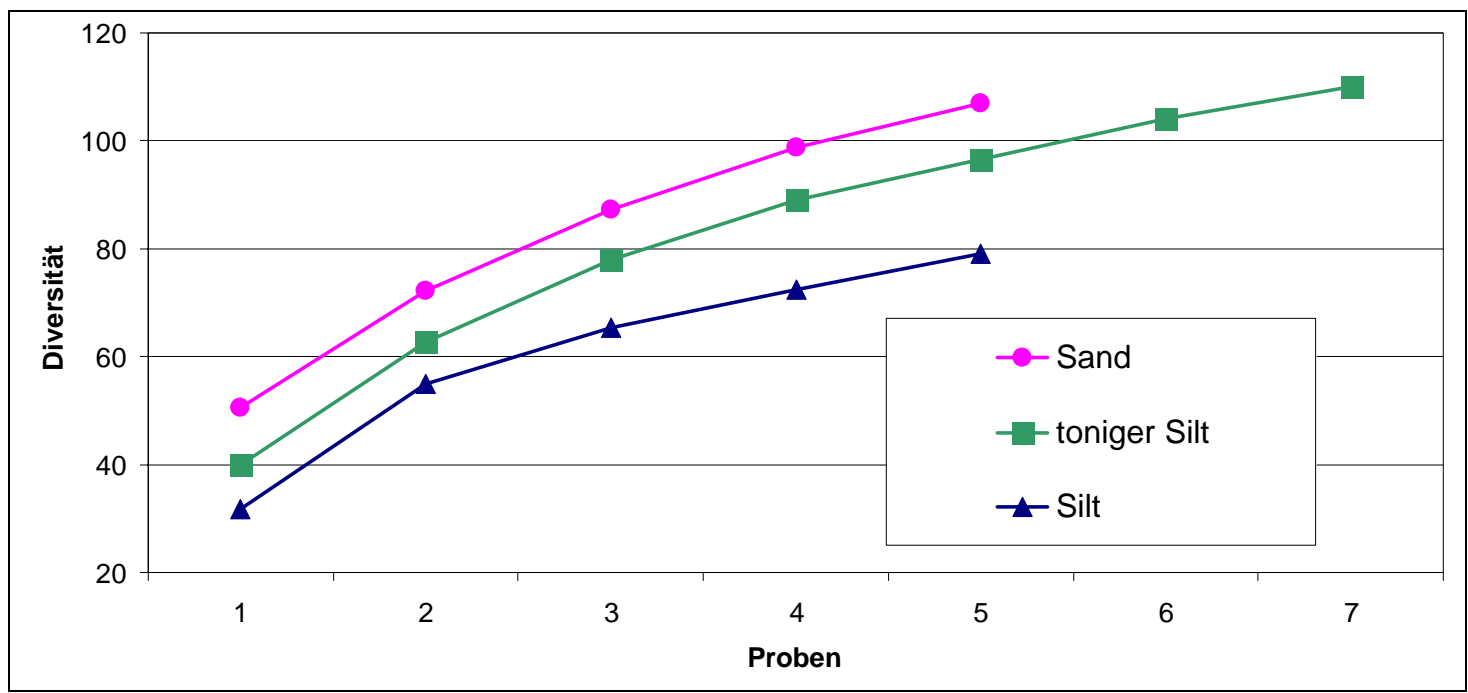

Abb. 4.20: Anzahl der Taxa der Proben in Schöningen.

\subsection{Witznitz}

Im Gegensatz dazu zeigt das Profil Witznitz 11 (Oberoligozän) eine deutliche Trennung der Diversitäten nach dem Lithotyp - wie auch schon aufgrund der vorherigen Methoden vermutet (siehe Abbildung 4.21). Die extrapolierte Diversität liegt etwa 10 bis $15 \%$ über den unkorrigierten Werten. Die geringere Diversität des Siltes gegenüber dem Ton ist nicht signifikant, da aufgrund der geringen Probenzahl eine hohe Standardabweichung vorliegt.

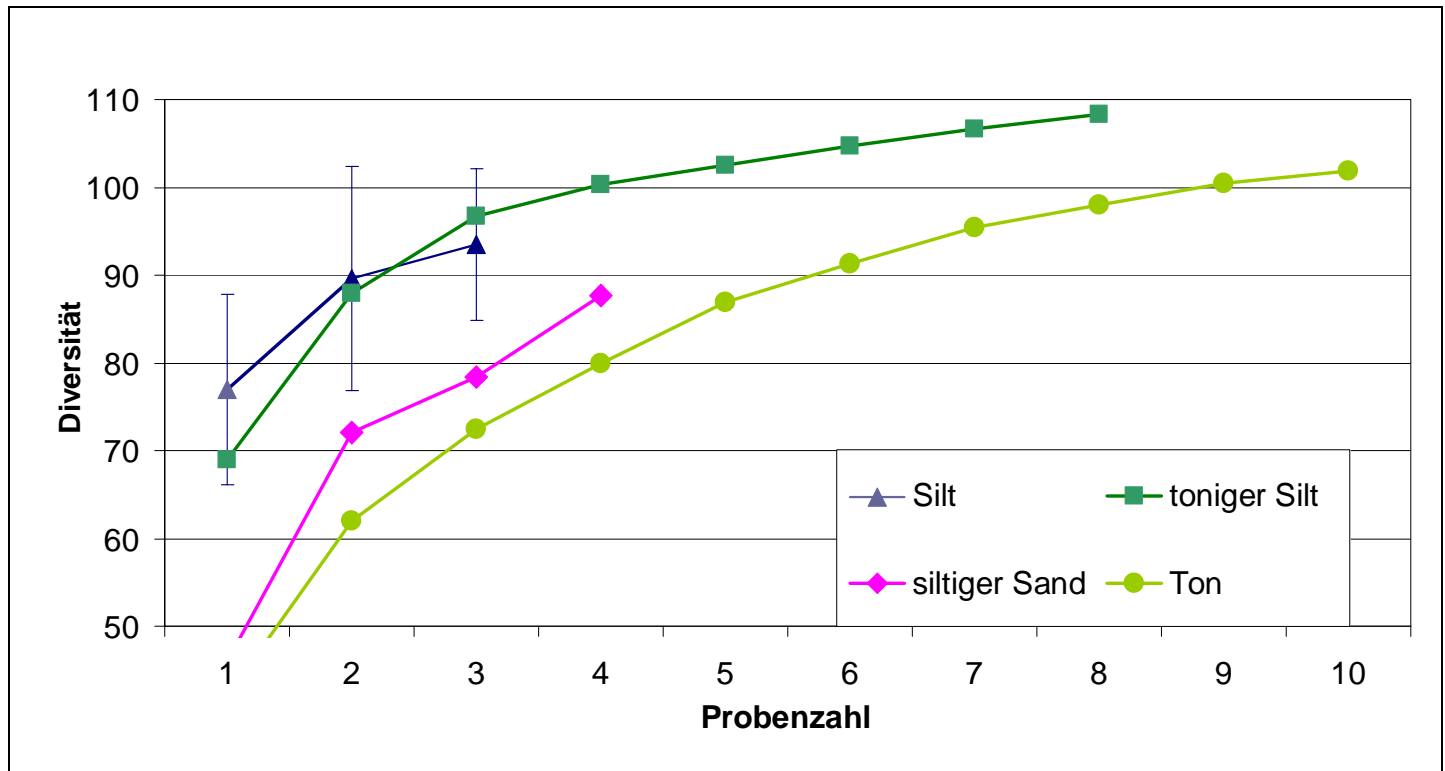

Abb. 4.21: Extrapolation der Anzahl der Taxa nach der Chao-1-Methode. Es ist ein signifikanter Unterschied der Diversitäten der verschiedenen Lithotypen zu erkennen. Aus Gründen der Übersichtlichkeit ist die Standardabweichung ausschließlich für den Silt dargestellt. Die Standardabweichungen der anderen Lithotypen liegen in ähnlichen Größenordnungen. 


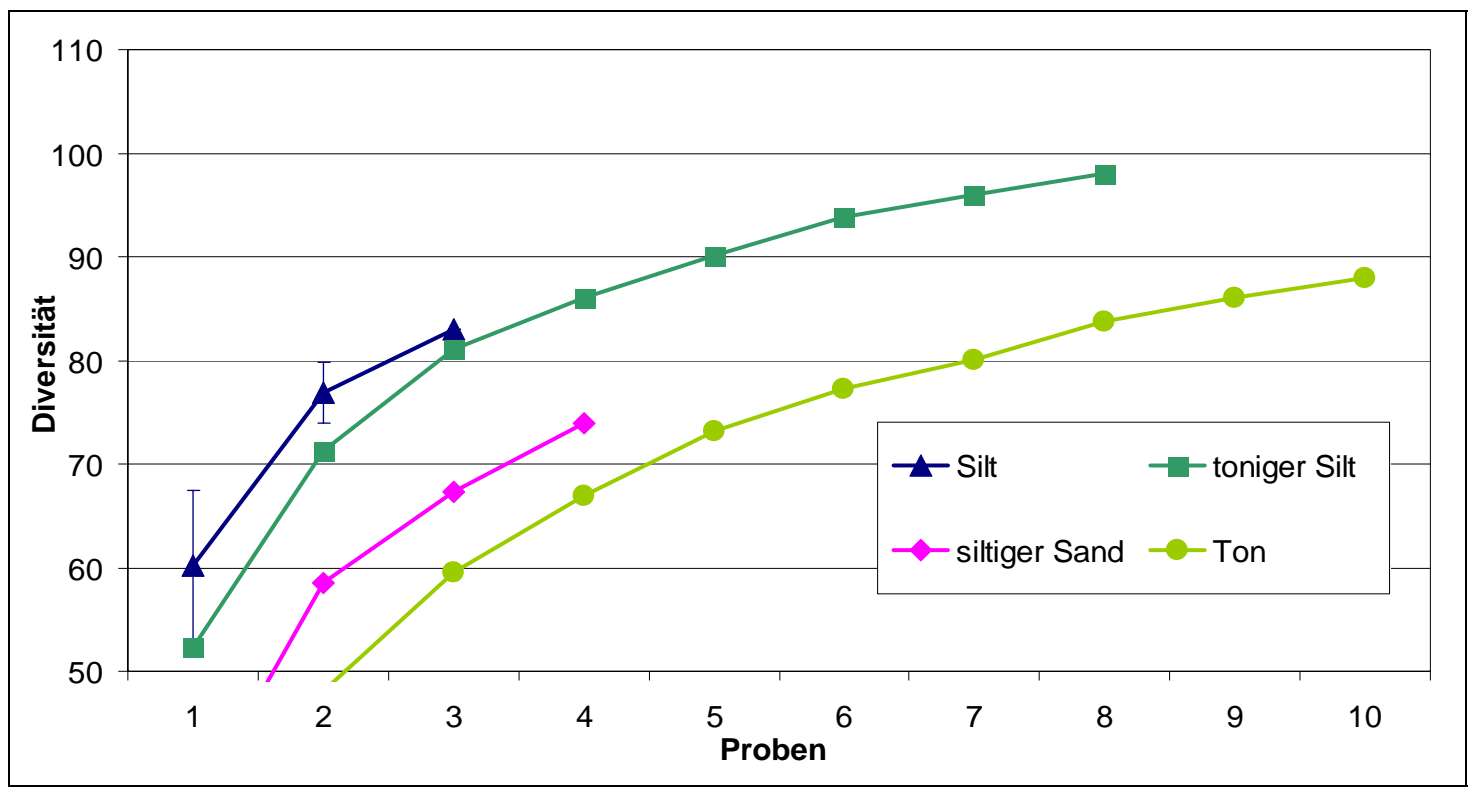

Abb. 4.22: Die Anzahl der Taxa nach der „Methode 3“. Bemerkung zu der Standardabweichung: s. Abbildung 4.21.

\subsubsection{Zusammenfassung der Diversitätsbetrachtungen}

\subsubsection{Signifikanz der Untersuchungen}

Vergleicht man die Ergebnisse der verschiedenen Methoden der Diversitätsberechnungen, zeigt nur das Profil Witznitz (Oberoligozän) in allen Tests einen signifikanten Unterschied der Diversitäten innerhalb der klastischen Sedimente. Dies kann verschiedene Ursachen haben: Es kann ein Sortierungseffekt innerhalb der relativ einheitlichen Fazies eines fluviatilen Flußsystem angenommen werden. Des weiteren verstärkt die geringe Diversität des Paläobodens die Unterscheidung der klastischen Lithotypen untereinander, da sekundäre Prozesse die Diversität im Paläoboden erheblich reduzieren.

\subsubsection{Schöningen}

Im Schöningen-Profil (Untereozän) besteht das Hauptproblem in der zum Teil zu geringen Probengröße, was die Diversitäten auf der Basis der Mittelwertberechnungen stark verfälscht, d.h. reduziert. Mit der „Methode 3“ und insbesondere der Extrapolation mit Hilfe des Chao-1-Tests kann ein Teil dieses dadurch verursachten Fehlers korrigiert werden. Inwieweit die Extrapolation dabei „des Guten zuviel“" korrigiert, kann nicht sicher belegt werden. Hier wären erneute Auszählungen und zusätzliche Laboruntersuchungen nötig. Nach der Korrekturrechnung zeigen die klastischen Sedimente eine mehr oder weniger einheitliche Diversität. Es scheint also, daß es in dem Ökosystem, soweit es durch die Proben des Schöningen-Profils abgebildet ist, keinen oder einen nur sehr geringen, nicht signifikant nachweisbaren Sortierungseffekt gegeben hat.

\subsection{Lagune}

Die Interpretation des Ökosystems als eine lagunäre Bildung (HAMMER-SCHIEMANN 1998, LIETZOW 1991) kann eventuell als Lösungsansatz für die fehlende Sortierung der Palynomorphen dienen. 
So ist eine Küsten-Lagune nach KJERFVE 1994 durch eine ganze Reihe von Faktoren beeinflußt, wie Flußeintrag, Wind, Tidaleffekte, Verdunstung und Oberflächentemperatur-Gleichgewicht. In dem relativ kleinräumigen Ökosystem spielen Sortierungseffekte nach Korngröße eine nur untergeordnete Rolle. Nach BIRD 1994 und PRYOR 1975 ist eine Lagune geprägt durch Zyklen der Erosion, des Transportes und der Sediment-Akkumulation, da Strömungen, verursacht durch Wind und Tiden, in dem flachen Wasserkörper verstärkt ihre Wirkung entfalten können. Außerdem ist Brackwasser ein Elektrolyt, der feinkörnige Sedimente - also auch Palynomorphen in Form von „Flocken“ bindet und ausfällt. Diese feinen Partikel in Aggregaten tendieren dazu, schneller zu Boden zu sinken (NiCHOLS \& BOON 1994, PEJRUP 1986a, b).

Der Ablagerungsort der unterschiedlichen Kornfraktionen innerhalb der Lagune ist von der Menge des Sedimenteintrages von den Rändern sowie der Tiefe der Lagune abhängig. So tritt beispielsweise „Mud“ im Zentralbecken auf, während Sande an den Randbereichen häufig in tiefen, abgeschnürten oder restriktiven Mikrotidal-Lagunen, die einen hohen Eintrag von feinen Sedimenten durch Flüsse aufweisen, abgelagert werden (RYAN \& GODELL 1972). Andererseits werden Sande in zentralen Teilen und „Mud“ entlang der Ränder abgelagert, wenn es sich um eine flache abgeschnürte oder restriktive Mikrotidal-Lagune handelt, in der der Eintrag von Feinsedimenten gering ist (BRETT 1963).

Es ist vorstellbar, daß die gröber klastischen Sedimente mit dem Zufluß von Salzwasser als Bodenfracht in die Lagune eingeschwemmt werden, wenn die Strömungsgeschwindigkeit ein gewisses Niveau überschritten hat (s.a. DRONKERS 1986a, 1986b). Dabei wurden jedoch nur wenige Pollen und Sporen aus dem terrestrischen Umfeld, aber eine signifikante Menge von Phytoplankton mitgeführt. Dies könnte durch die geringe Diversität des Sandes in dem Profil Schöningen nach der „Methode 3“ belegt sein.

Die abgelagerten Palynomorphen stammen vermutlich aus der lokalen bis regionalen Flora und werden als konstante Pollensedimentation abgelagert, oder sie werden als Schwebefracht von einem Fluß in die Lagune transportiert, in der durch die oben genannten Prozesse der Aufarbeitung und Resuspension keine Sortierung festgestellt werden kann.

Mangrovenelemente, die mit vereinzelten Pollen der Gattung Avicennia und der Palmengattung Nypa in dem Zwischenmittel nachgewiesen werden konnten (z.B. Südfeld Zone I = Proben 5 - 14 im Zwischenmittel 1) sprechen für eine weniger geschlossene Lagune, da Mangroven bevorzugt im Intertidalbereich auftreten, was einen dauerhaften Kontakt der Lagune zum Meer impliziert (BIRD 1994); diese Lagune - als Übergang zu einem Ästuar - könnte demnach folgerichtig als ästuarine Lagune bezeichnet werden (BIRD 1994).

\subsection{Fusite}

Auffällig ist weiterhin das Auftreten von Fusiten in den kohligen Proben (s. Anlagen Pollendiagramme), deren Ursache vermutlich Brände sind, die die Flora des Umlandes und damit die Diversität auch in den klastischen Sedimenten beeinflussen. Die Bildung von Pioniergesellschaften nach diesen Bränden löst eine stabile etablierte Vegetation ab. Diese Fluktuationen führen zu einer eher heterogenen Abbildung in der Palynofazies, was als eine Ursache der hohen Anzahl von Auszähldaten unter oder gleich $1 \%$ in dem untersuchten Profilen (Nord- und Südfeld) angesehen werden kann. 


\subsubsection{Alversdorf}

Auf die Proben aus Alversdorf (Mitteleozän), das ein vorwiegend terrestrisches, z.T. fluviatiles Ökosystem repräsentiert, konnte nur die „Methode 1 “ angewandt werden, da für die anderen Methoden eine Rohdatenmatrix nötig gewesen wäre, die jedoch nicht zur Verfügung stand. Außerdem sind die Tone gegenüber den anderen Kornfraktionen stark überrepräsentiert, was eine Extrapolation z.B. nach der Chao-1-Methode verbietet (s. Darstellung bei ALROY 2001).

Gegenüber den Kohlen weisen klastische Sedimente in Alversdorf nach der „Methode $1^{\text {" }}$ und dem anschließenden Anova-Test eine relativ deutliche Erhöhung der Diversität durch Anreicherungseffekte auf. Es kann daher angenommen werden, daß in terrestrischen Milieus, anders als im randmarinen Milieu wie in Schöningen, in klastischen Sedimenten Anreicherungseffekte auftreten. Eine Trennung innerhalb dieser klastischen Sedimente aufgrund von Sortierungseffekten kann ebenfalls angenommen werden.

\subsubsection{Fazit der Diversitätsbetrachtungen}

Ein generelles Problem besteht in der statistischen Trennung der Palynofazies von der Lithofazies in der Taphozönose. Verzahnen sich die Effekte, kann eine Trennung nur sehr schwer und nur unter extrem spekulativen Interpretationsversuchen stattfinden. So ist es völlig ungeklärt, wie stark zum Beispiel der Paläoboden die Diversität reduziert oder andererseits die Sukzession innerhalb des Profils Witznitz die Diversität beeinflußt.

Diese Probleme werden durch die z.T. gering signifikanten Unterschiede der Zwischen- und Inngruppenvarianzen der klastischen Sedimente ausgedrückt (s. Kap. 4.4.8, S. 83).

\subsection{Pollendiagramme}

Um die Möglichkeiten der Pollendiagramm-Darstellungen von P.A.S.T aufzuzeigen und andererseits eine eventuelle Abhängigkeit der Pflanzen-Vergesellschaftungen von der Lithofazies zu visualisieren, wurden zwei Varianten der Darstellung generiert (s. Anlagen). Diese Varianten stellen nur einen Bruchteil der Möglichkeiten dar, die mit P.A.S.T unter minimalem Zeitaufwand erzielbar sind. Da für das Profil Witznitz (HAMMER, in Vorbereitung) bisher keine vollständigen Gruppierungs-Informationen (z.B. azonale Parameter und Klimabedingungen des botanischen Verwandten) vorliegen, erschien es nicht sinnvoll, dazu ein Pollendiagramm in dieser Arbeit abzubilden (siehe jedoch Profildarstellung mit Diversitäten in Kap. 4.4.9.1, S. 126 f.).

Entgegeben den bisherigen Darstellungen in der Literatur werden die Prozentwerte nicht als Balkendiagramm sondern als Liniendiagramm gezeichnet. Ein Vorteil dieser Methode liegt in der besseren Wahrnehmung von isolierten Werten, da diese durch eine Unterbrechung der Verbindungslinie im Diagramm deutlich sind. Im Gegensatz dazu werden „Durchläufer“ mit einer verbundenen Linie über das gesamte Profil schnell sichtbar. Alternativ können auch Balkendiagramme mit P.A.S.T gezeichnet werden, die Programmbeschränkung von Excel erlaubt bei dieser Methode allerdings keine Profildarstellung mit variablen Probenabständen.

1. Die Variante 1 zeigt eine „klassische“ Darstellung der Pollendiagramme als Familiendiagramm, ergänzt um weitere Informationen, die nicht aus den zitierten Arbeiten exzerpiert, sondern ausschließlich mit P.A.S.T generiert und in dieser Dissertation erarbeitet wurden. 
Das Diagramm zeigt die Summenkurven der azonalen Gruppierungsparameter (z.B. Auewald-, Riedelemente etc.). In Einzelfällen werden auch Familien abgebildet, ebenso wie bestimmte Fazieselemente (z.B. Tricolporopollenites microreticulatus, Plicapollis pseudoexcelsus u.a.). Die Formen einer Familie und deren Gruppierungsparameter lassen sich innerhalb von P.A.S.T mit den entsprechenden Auszähldaten ein- oder ausblenden, so daß jederzeit die komplette Datenmatrix einsehbar ist und das Pollendiagramm dynamisch geändert werden kann (s. Kap. 3.11.2, S. 92 f.).

Botanische Familien, die gleichwertig in mehreren azonalen Bereichen vorkommen können, werden unter der Rubrik ,azonal undifferenziert“ eingeordnet. Das Diagramm zeigt weiterhin die Diversitätsveränderung über das Profil (Anzahl der Arten, Shannon-Index), das prozentuale Verhältnis der tropischen und subtropischen Formen des nächsten lebenden Verwandten, sowie die Summen-Prozentwerte der primär anemophilen und zoophilen Formen. Eine Auswahl des Pflanzenhabitus der nächsten lebenden Verwandten ergänzt die Darstellung. Diese Informationen stammen direkt aus der Datenbank und wurden über die Gruppierungsfunktion in Excel automatisch erzeugt.

Die „Kuchendiagramme“ geben Informationen über das prozentuale Verhältnis der Gruppierungsparameter für alle Taxa bzw. deren botanische Familienzugehörigkeit innerhalb eines zusammenhängenden definierten Probenbereichs. Bei dieser Darstellungsform wird nur das Auftreten der Familien aber nicht deren prozentuales Verhältnis ihrer Formen berücksichtigt. Bei der hier gewählten Darstellung wurde nur der erste dominante Parameter („High priority“-Parameter) ausgewertet (zur Datenbankeingabe dieser Daten siehe auch Kap. 3.7.7, S. 51).

Die Distanzkurve gibt den Unterschied zwischen benachbarten Proben nach der Euklid-Distance-Methode an. Hohe Werte signalisieren große numerische Unterschiede der Proben. Kleine aufeinanderfolgende Werte zeigen dagegen eher homogene Verhältnisse an. Die Wahl der Distanzberechnungsmethode kann mit P.A.S.T variiert werden.

2. Eine erstmals in dieser Arbeit vorgeschlagene Variante kann die Bedeutung der Lithofazies aufzeigen. Dabei werden die Proben entsprechend ihres Lithotyps umsortiert und mit einheitlichen Mächtigkeiten als Profil aufgetragen, so daß Abhängigkeiten der Palynofazies von der Lithofazies sowie die Relation der lokalen und der regionalen Vegetation leichter erkannt werden können (s. Anlagen). Innerhalb des Lithotyps bleibt die Reihenfolge der Probe von der Basis zum Top erhalten.

Bis auf die in diesem Kontext nicht sinnvolle Distanzkurve entsprechen die zusätzlichen Informationen im Diagramm der Darstellungsvariante 1.

\subsubsection{Diskussion der Pollendiagramme im Kontext regionaler und lokaler Aspekte}

\subsubsection{Alversdorf (Anlage 2)}

Das Profil wurde aus folgenden Arbeiten stratigraphisch zusammengesetzt. Dabei sortiert P.A.S.T die Taxa automatisch nach palynologisch-botanischen Gesichtspunkten. Gleiche Taxa werden in einer Spalte zusammengefaßt. 


\begin{tabular}{|l|l|}
\hline $\begin{array}{l}\text { Präfix der Probe- } \\
\text { nummerierung }\end{array}$ & $\begin{array}{l}\text { Bearbeiter der Einzelprofile (von der } \\
\text { Basis zum Top) }\end{array}$ \\
\hline & SCHIEMANN 1994 \\
\hline, $\mathrm{n}^{“ 6}$ & NATGE-EFOGHE 1997 \\
\hline, $\mathrm{e}^{6}$ & EBE 1992 \\
\hline
\end{tabular}

Das sortierte Pollendiagramm zeigt deutlich die Trennung der Kohleproben von den klastischen Zwischenmittelproben. Der relativ konstante Shannon-Index in den Kohlen steigt stetig mit dem Beginn der Zwischenmittelproben an.

Eine deutliche Zunahme der Sumpf- und Aueelemente kann in den Zwischenmittelproben festgestellt werden. Der Anteil der Auevegetation kann nach FLORES 1983 und POCKNALL \& FLORES 1987 als eher lokaler Pollenniederschlag in „Crevasse splays“ oder „overbank-fines“" gedeutet werden.

Im Gegensatz dazu nehmen die Bruchwald-Elemente im Zwischenmittel ab, obwohl ein gewisser Ausgleich durch die Juglandaceen und Betulaceen stattfindet, die sich reziprok zueinander verhalten, wohl auch durch den relativen Rückgang der sehr dominanten Fagaceen. Aufgrund ihres schnellen Wachstums kann davon ausgegangen werden, daß Betulaceen Standorte sehr schnell besiedeln können und damit zumindest Anfangs einen gewissen Konkurrenzdruck gegenüber den anspruchsvolleren Juglandaceen ausüben können.

Ebenso ist im Zwischenmittel der Anteil der Fagaceen-Formen reduziert, der als „azonal undifferenziert" eingeordnet ist. Sofern die Fagaceen der lokalen Flora zugeordnet werden, kann diese Abnahme durch die Verringerung des Einflußes der lokalen Flora erklärt werden.

Nach NichOls und PockNALl 1984 wird das Verhältnis der lokalen zur regionalen Vegetation im starken Masse von der Höhe der Vegetation beeinflußt. Eine offene niedrige Vegetation fängt sowohl lokalen als auch regionalen Pollenniederschlag ein, was zu einer Überdeckung der lokalen Vegetation führen kann. Eine hochstehende Vegetation tendiert dazu, die regionale Vegetation herauszufiltern, insbesondere wenn eine geschlossene Baumschicht vorliegt. Dies führt dann zu einer Dominanz der lokalen Flora. Die relative oder absolute Zunahme der Betulaceen-Pollen in dem Alversdorfer Zwischenmittel kann somit durch den abnehmenden Filtereffekt der Fagaceen erklärt werden.

Auffällig ist die zunehmende bzw. gleichbleibende Diversität in den marin beeinflußten Proben, die durch hohe Anteile von Dinoflagellaten gekennzeichnet sind. Im Gegensatz zu der Interpretation von NATGE-EFOGHE 1997, die die ZwischenmittelSedimente einem lagunären Milieu zuordnet, spricht dies eher für eine Delta- bzw. Ästuar-Situation. MULLER 1959 konnte am Beispiel des rezenten Orinoco-Deltas eine deutliche Anreicherung von Palynomorphen im „Offshore“-Bereich des Deltas nachweisen.

Die Normapolles-Elemente sind an bestimmte Faziesbereiche gebunden und treten gehäuft an der Basis der Kohlen und in einzelnen Lagen der Zwischenmittel auf. Sie können deshalb als ökologisch zusammengehörige Gruppe als Teil eines Ökotons betrachtet werden. 
Pinaceen weisen am Top der Kohle und in den Zwischenmitteln hohe Werte auf. Zumindest Letzteres kann auf die gute Schwebfähigkeit ihrer saccaten Pollenkörner zurückzuführen sein (PECK 1974a, b). Nach COHEN et al. 1984 und COWARIN et al. 1979, die rezente Habitate untersucht haben (Okefenokee swamp, scrub-shrubwetland), weisen Pinaceen-Häufigkeiten von 10-20\% auf substantielle lokale Bestände auf levees hin. Eine Überschreitung dieser Grenzwerte im Profil Alversdorf wird jedoch nur in sehr wenigen Einzelproben erreicht, insbesondere punktuell in den Flözen (Gewebekohle). Wegen der relativ geringen anzunehmenden Streuung im Bruchwald sprechen diese Werte deutlich dafür, daß die Pinaceen der Unterflöze von Alversdorf ein Element der Lokalflora sind.

Bemerkenswert ist die Tatsache, daß sich in den kohligen Basisschichten der Unterflöze von Alversdorf (Profil SchIEMANN) die oben dargestellten Trends fortsetzen. In diesen Bereichen zeichnet sich verstärkt eine regionale bis extra-lokale Beeinflussung der Flora ab. Windblütige Formen, wie Betulaceen werden angereichert. Außerdem treten Anzeiger von offenen Wasserflächen, wie Nyssaceen und Botryococcus stärker hervor, während die Vertreter einer höheren Baumschicht, die als natürliche Barriere für windtransportierte Pollen und Sporen wirken könnte, proportional abnehmen (NiCHOLS und POCKNALL 1984). Bestätigt wird diese Aussage von SCHIEMANN 1994, die für die Bildung des Unterflözes größtenteils eine offene Wasserfläche, bzw. eine Nyssa-Taxodium-Vergesellschaftung in der Nähe von offenen Wasserflächen annimmt. In diesem Bereich kann also ein verstärkter Einfluß der extralokalen bis regionalen Vegetation angenommen werden, der sicherlich erheblich über dem von POCKNALL und FLORES (1987) angenommen Gesamtanteil von 3-5\% innerhalb einer lokal-dominierten Vegetation liegt.

\subsubsection{Schöningen (Anlage 1)}

Die graphische Darstellung des Diversitätsverlaufes zeigt keine Zunahme der Diversität im Zwischenmittel, was mit den statistischen Untersuchungen in den Kapiteln 4.4.7.2, S. 118 und 4.4.10.4.1, S. 134 korreliert.

Auffällig ist der hohe, allerdings stark schwankende Anteil der Betulaceen, die als Pioniergewächse rasch freie Flächen besiedeln können. Betulaceen-Pollen sind besonders stark in den oberen Teilen der Flöze vertreten, wo Fusitlagen besonders häufig und markant sind. Diese Fusitlagen entstehen als Folge von Moorbränden, durch die freie Flächen für die Besiedelung durch Betulaceen geschaffen wurden. Die Betulaceen treten zusammen mit den Taxodiaceen auf und bilden Vergesellschaftungen, die auch als „Hammocks“ gedeutet werden können (LENZ 2001). Wo sie in Analogie zu den rezenten Florida-Everglades innerhalb der Süßwassermarsch auf erhöhten Standorten oder kleinen Senken gebildet werden (RIEGEL 1965). Allerdings erreichen die Taxodiaceen-Pollen aus den „cypress hammocks" der Everglades Anteile von 40 - 80\% (RIEGEL 1965), im Profil Schöningen jedoch nur Werte bis maximal 27\% (innerhalb der Sumpfelemente: Taxodiaceen und Schizaeaceen). Dies deutet darauf hin, daß im Profil keine „,cypress hammocks" unmittelbar angetroffen wurden. Ob die Taxodiaceen in Schöningen typische mit den Everglades vergleichbare Hammocks gebildet haben oder vielmehr assoziierte Elemente eines Bruchwaldes waren, bleibt daher fraglich.

Im Pollendiagramm (siehe Anlage) stellen die Taxodiaceen den überwiegenden Anteil des azonalen Elementes Sumpf dar, deshalb wurde auf eine getrennte Darstellung als Einzeldiagramm verzichtet. 
In der Ostmulde der Wulfersdorfer Flöze der Oberen Flözgruppe konnte LENZ 2001 mit Hilfe von Isopollenkarten deutliche Hinweise auf das Vorhandensein von Hammocks finden, die allerdings nicht durch Taxodiaceen, sondern vor allem durch Palmen gekennzeichnet sind.

Die Palmen treten in Schöningen zwar primär in den Bereichen auf, in denen auch Taxodiaceen vorhanden sind, zeigen aber in Detail gegenläufige Trends. Sie sind also eventuell ebenfalls auf Hammocks angesiedelt, bevorzugen aber eher trockenere Standorte (s. a. LENZ 2001).

Die Entwicklung einer Farnflur wird durch das verstärkte Auftreten von Polypodiaceen in der Kohle angezeigt. Diese Farnflur kann z.B. in Randbereichen von Bruchwäldern oder am Rand von Flußrinnen auftreten. In einigen Teilen des Flözes erreichen sie hohe Werte und sind damit vermutlich die Hauptelemente des Bodenbewuchses, da sie aufgrund ihrer speziellen „Fortpflanzungsmethode“ und ihres niedrigen Habitus gegenüber den windblütigen Formen im regionalen Pollenregen unterrepräsentiert sind.

Die Auevegetation nimmt im Gegensatz zu dem Profil Alversdorf in den Zwischenmitteln ab. Dieser Effekt kann auf die nicht ausreichende Probengröße (s. Kap. 4.4.10.1, S.130) bzw. den relativen Anstieg der Dinoflagellaten in den marin beeinflußten Proben zurückzuführen sein.

Die Betulaceen zeigen in den Zwischenmitteln als allochthone regionale Elemente eines entfernten Bruchwaldes ebenfalls geringe Werte.

Sehr geringe Prozentwerte erzielen die windblütigen Pinaceen, die wohl ausschließlich als regionale Vegetation des Hinterlandes gedeutet werden können.

Die prozentuale Abnahme der ,,azonal undifferenziert“" einsortierten Formen sowie der Fagaceen ist in den marinen Dinoflagellaten-Proben sehr deutlich. Andererseits ist nach der Rarefaction-Methode (Kap. 4.4.10.1, S. 130) belegt, daß diese Proben nicht die vollständig mögliche Diversität abbilden.

Im Gegensatz zu dem insgesamt gesehen palynologisch recht homogen zusammengesetzten Profil von Alversdorf zeigt das Profil von Schöningen deutlichere Veränderungen in den Pollenspektren sowohl innerhalb der Flözabfolge als auch zwischen Flöz und Zwischenmittel. Dies ist auch durch die hohe Varianz (s. Kap. 4.4.7.2, S. 118f.) in den Proben belegt. 


\subsection{2 ,Kuchendiagramme“6}

P.A.S.T erlaubt die Auswertung der nach Excel exportierten Gruppierungsparameter des zugehörigen botanischen Verwandten.

Dabei können folgende Daten graphisch in Form eines „Kuchendiagramms“ dargestellt werden:

\begin{tabular}{|c|c|}
\hline Taxongruppe & Fazieselement \\
\hline Pollination & $\mathrm{pH}$ \\
\hline Pflanzenhabitus & Nährstoffe \\
\hline Klimatyp & Feuchtigkeit \\
\hline Florenreich & Mächtigkeit \\
\hline zonal & Salzgehalt \\
\hline azonal (rezent) & Licht \\
\hline $\begin{array}{l}\text { azonal sensu } \\
\text { (Tertiär) }\end{array}$ & Benutzerdefiniert \\
\hline
\end{tabular}

Bei der Auswertung dieser Informationen ist stets die Problematik des Aktualismus zu beachten, die davon ausgeht, daß die Klimaansprüche der fossilen Arten durch die klimatischen Ansprüche der rezenten Vergleichstaxa approximiert und dadurch die paläoklimatischen Verhältnisse für eine Flora rekonstruiert werden können (BELZ \& Mosbrugger 1994). Eine umfassende Diskussion dieser Problematik findet sich bei LENZ 2001.

Da die klimatischen und ökologischen Ansprüche der fossilen Formen und der rezenten Vergleichstaxa nicht notwendigerweise übereinstimmen, ist es möglich, eine benutzerdefinierte Gruppierung fossiler Pflanzen einzugeben. So kann z.B. neben der Zuordnung zur azonalen Vegetation auf der Basis des nächsten lebenden Verwandten auch eine Gliederung von MAI 1995 benutzt werden (siehe auch Kap. 3.7.7, S. 49), die auf der Zuordnung innerhalb der Tertiär-Vegetation beruht (s. auch Abbildung 2.8).

Die Eingliederung der Pollen und Sporen zu den jeweiligen Gruppierungen wird hauptsächlich auf der botanischen Familien- und seltener auf Gattungszugehörigkeit durchgeführt. Es ist jedoch jederzeit möglich, mit Hilfe der Datenbank genauere Informationen einzutragen, um damit die Interpretationsmöglichkeiten zu erweitern.

Mit dem momentanen Stand der Eingabe sind jedoch schon Interpretationen möglich, die weit über die bisher in der Literatur gebräuchliche Zuordnung der Pollen und Sporen in Kategorien von Klimaelemente wie Wärme liebende Elemente, Gemäßigte Elemente, Intermediäre Elemente und Fazieselemente hinausgehen (KRUTZSCH \& MAJEWSKI 1967, HAMMER-SCHIEMANN 1998).

Für den exemplarisch herausgegriffenen Parameter Klima geben EBE 1992, SCHIEMANN 1994, NATGE-EFOGHE 1997 und HAMMER-SCHIEMANN 1998 für die Profile Alversdorf und Schöningen eine klare Dominanz der wärmeliebenden Elemente an. Tropische oder subtropische Klimabedingungen sind allerdings durch diese Gruppierung in eine gemeinsame Gruppe thermophiler Elemente nicht als solche $\mathrm{zu}$ erkennen. Die Aufstellung nach den Angaben der Datenbank in Form der Kuchendiagramme (s. Anlagen und Abbildung 3.60, S.52) schlüsselt die klimatischen Parameter dagegen sehr viel differenzierter auf. 
Eine klare Dominanz der warmen Elemente gegenüber den gemäßigten Elementen ergibt sich daraus für das Profil Schöningen (Untereozän) mit einem Anteil von etwa 50\% an tropischen Elementen und die subtropischen Elementen ca. 20\% an. Der Rest teilt sich unter die temperierten und borealen (arktotertiären) Klimate auf. Im Profil Alversdorf (Mitteleozän) sind die tropischen mit ca. 60\% und die subtropischen Elemente mit 20-25\% vertreten. Dem entspricht eine relative Abnahme temperierter und borealer Klimate (Angaben zu den weiteren Klimabereichen, siehe Anlage).

Die Vorstellungen über das Klima im Mitteleozän sind recht gegensätzlich. So hält MAI (1995) für das Eozän tropische Temperaturen und Jahresmittel über $20^{\circ} \mathrm{C}$ in Europa für unrealistisch, da seiner Meinung nach lediglich das azonale Mangrovenelement Nypa als tropische Form angesehen werden kann. Alle anderen als Indikatoren für tropisches Klima herangezogenen Formen wie z. B. die Palmen können auch bis in die gemäßigte Zone vordringen und sogar in Gebieten wie dem Himalaja vorkommen. Anzunehmen ist für das Eozän eine Wintertrockenheit, deren Dauer zwei bis vier Monate betragen konnte.

Es wird demnach ein subtropisches Wechselklima angenommen bzw. für das Mitteleozän (insbesondere im Geiseltal) subtropisches wintertrockenes Klima.

KRUTZSCH et al. 1992 gehen von einer insgesamt höheren Durchschnittstemperatur mit eher tropischem Klima aus.

Im Vergleich zu MAI 1987 kann nach LENZ 2001 für die marine Randlage der Wulfersdorfer Flözgruppe eine etwas höhere Temperatur als im Geiseltal und ein Klima mit subtropisch/tropischem bis tropischem Charakter angenommen werden, das durch die Wassertemperaturen in der „Nordsee“ bestimmt wurde. Nach unabhängigen Isotopen-Daten waren die Wassertemperaturen dort mit Abstand die höchsten im gesamten Tertiär (Abbildung 4.24).

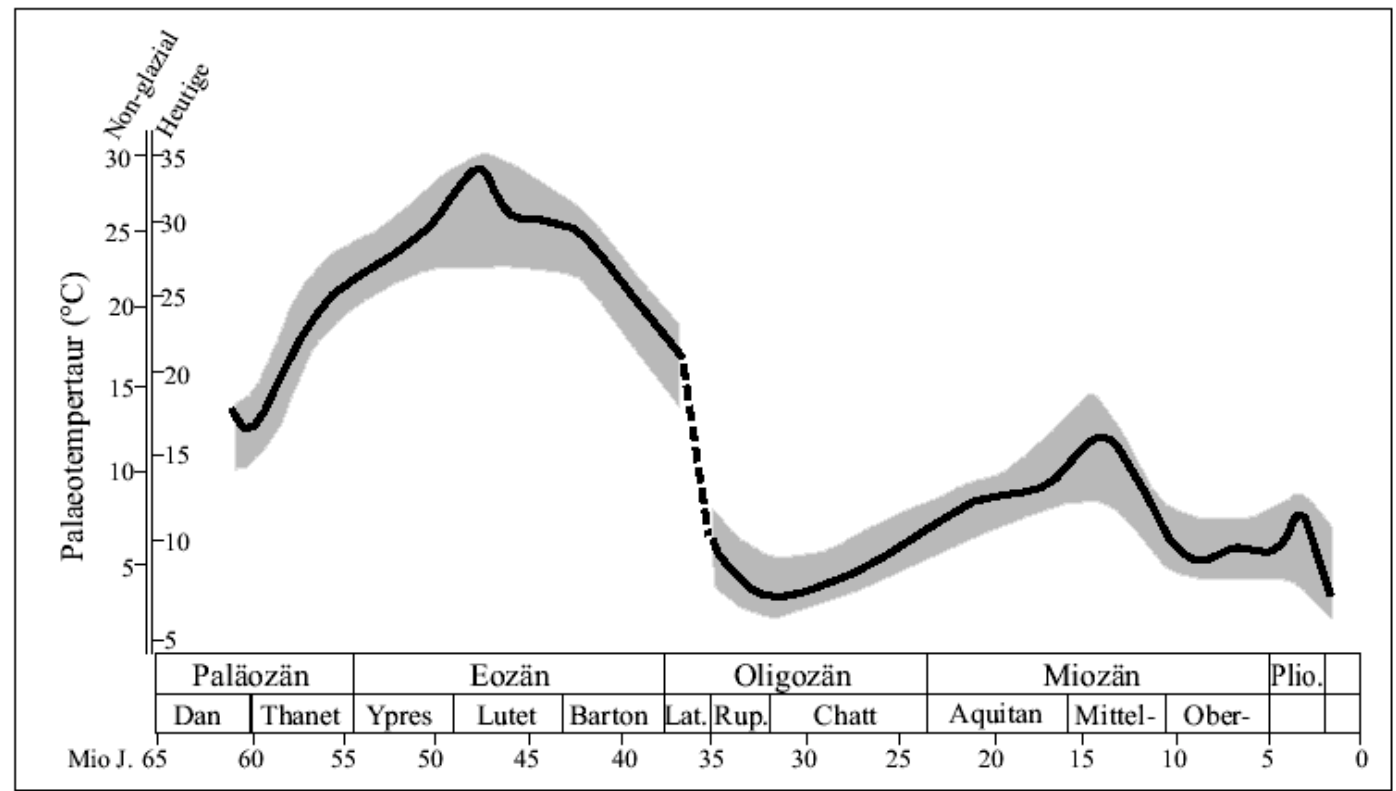

Abb. 4.24: Isotopen-Temperatur-Kurve der tertiären Nordsee (nach ${ }^{18} \mathrm{O}$-Nachweisen in Karbonaten von Muschelschalen aus der Nordsee zusammengestellt); die wahren Wasser-Temperaturen liegen innerhalb der grauen Fläche (nach BUCHARDT 1978). Der Unterschied zwischen den nonglazialen und gegenwärtigen Temperaturskalen wird durch die Veränderung der Sauerstoff-Isotopen-Komposition des Ozeanwassers verursacht, eine Folge der Akkumulation von Gletschereis in der Antarktis. 
Da das Palynomorphen-Inventar in den Profilen der Oberflözgruppe in der Ostmulde und der Westmulde (LENZ 2001) nahezu identisch ist, können auch für die Flöze im Tagebau Alversdorfer (Mitteleozän) ähnliche klimatische Verhältnisse angenommen werden, da die Alversdorfer Flöze nach der paläogeographischen Rekonstruktion nur geringfügig weiter landeinwärts gelegen haben müssen (nach ZIEGLER 1990).

Im Profil Schöningen (Untereozän) ist die Anzahl der tropischen Formen geringer, obwohl die subtropischen Formen nicht in dem selben Maße zunehmen. Vielmehr erhöht sich die Anzahl der temperierten und borealen Elemente.

HAMMER-SCHIEMANN 1998 schließt aus der hohen Zahl an thermophilen Elementen, in Verbindung mit dem Vorkommen der intermediären Elemente auf ein subtropisches Klima, das jedoch auch kühlere Elemente zuläßt.

Ein weiterer Aspekt, der bei der Rekonstruktion des Klimas mit berücksichtigt werden muß, sind die zahlreichen Fusitlagen in den Flözen, die auf kurzzeitige Austrocknung der Moore mit Trockenperioden hindeuten (SCHIEMANN 1998).

RIEGEL et al. (1999) weisen auf die markanten Gegensätze in der petrographischen Konstitution zwischen den Kohlen der mitteleozänen Oberflözgruppe und der untereozänen Unterflözgruppe hin und erklären das Fehlen von Fusitlagen und die starke Vorherrschaft fein-detritischer Kohlelithotypen (Grundmassenkohlen) in der Oberflözgruppe mit intensiver aerober Zersetzung bei relativer Bodentrockenheit und mangelnder Brennbarkeit bei extremer Luftfeuchtigkeit in einem ausgeglichenen perhumiden Klima. Ein solches Klima könnte auch ohne nennenswerte Temperaturerhöhung gegenüber dem Untereozän eine Ausbreitung der tropischen Elemente im Mitteleozän begünstigt haben. 


\section{Schlußbetrachtungen und Ausblick}

\subsection{Interdisziplinäre Arbeitsweise}

Die vorliegende Arbeit ist methodisch-orientiert und hat ihren Schwerpunkt in der Kombination der Arbeitsfelder Informatik und Palynologie in Verbindung mit den Fachgebieten der Botanik, Geographie und Sedimentologie. Erst eine kritische Bewertung und Eingabe palynologischer Daten schafft in Verbindung mit statistischen Programmen und den verschiedenen Kombinationen Möglichkeiten für eine wissenschaftliche Auswertung großer Datenmengen, die bisher nicht oder nur sehr zeitaufwendig realisierbar war.

Die Diversitätsbetrachtungen und die Diskussion der Pollendiagramme sind in erster Linie als Beispiele für die Darstellung der Möglichkeiten von P.A.S.T zu verstehen und deshalb in ihrer wissenschaftlichen Auswertung keineswegs erschöpfend.

Die in der vorliegenden Arbeit vorgenommene verstärkte Einbeziehung des nächsten lebenden Verwandten zur Interpretation paläogener Ökosysteme mit Hilfe der Gruppierungsinformation aus der Datenbank (Kap. 4.5.2, S. 143 und Kap. 3.11.4, S. 95) stellt ein vorsichtiges „Herantasten“ zur Verbesserung bisheriger palynologischer Ergebnisse dar.

Inwieweit die Genauigkeit der Ökosystem-Rekonstruktion zu erhöhen ist, hängt im großen Maße von der vollständigen Eingabe und kritischen Beurteilung der Gruppierungsparameter zu den nächsten lebenden Verwandten ab. Dies sollte bei der Neueingabe bzw. der Revision der bisher eingegebenen Daten in die Datenbank stets berücksichtigt werden.

\subsection{Programmierung}

\subsubsection{Ideologie}

Bei der Programmierung von P.A.S.T wurde auf die Benutzung von Standardsoftware mit Microsoft Office $\mathbb{C}$ wertgelegt, deren Programmierschnittstellen mittlerweile einen Standard bilden. Damit steht die Programmbasis mit großer Wahrscheinlichkeit auch in Zukunft zur Verfügung, was einer Insellösung der darauf aufbauenden Programmumgebung P.A.S.T entgegenwirkt. Eine stetige Weiterentwicklung und Anpassung an die neuesten Standards ist damit gewährleistet.

\subsubsection{P.A.S.T}

In P.A.S.T können systematische mit botanischen Daten in Echtzeit korreliert werden. Dabei können Synonymlisten überprüft, Karteikarten für den praktisch arbeitenden Palynologen angelegt, Literatur- Lokalitäts- und Bilddaten verwaltet werden. Auf diesem Wege sind systematische Revisionen möglich, die ohne P.A.S.T um ein Vielfaches aufwendiger und fehleranfälliger wären.

Stratigraphische Daten in verschiedenen Zeitskalen erlauben den Vergleich der Reichweiten der Palynomorphen, z.B. mit Pflanzen-Makrofossilien, und bilden damit eine Schnittstelle zu anderen Datenbanken.

Gruppierungsinformationen, wie Klima, azonale und zonale Vegetation etc., zu den nächsten lebenden Verwandten stehen auf Abruf bereit. Der sekundenschnelle Export von numerischen Profildaten und Gruppierungsinformationen nach Excel schafft nach 
der Eingabe oder dem Import von Auszähldaten Möglichkeiten der statistischen und graphischen Auswertung innerhalb der Programmumgebung.

Pollendiagramme können auf der Ebene von Formtaxa oder Zugehörigkeit zu botanischen Familien nach verschiedenen Auswahlkriterien ausgegeben werden. Dabei ist es möglich, die Formen nach beliebigen Gruppierungsparametern zu sortieren und deren Summenkurven zu ermitteln. P.A.S.T ist so flexibel programmiert, daß beliebige auch „per Hand“ eingegebene numerische Daten mit den Profilen korreliert werden können. Nicht zuletzt kann P.A.S.T die Daten für externe Statistikprogramme aufbereiten, z.B. in frei wählbare Häufigkeitsklassen einteilen, wenn die zur Verfügung stehenden Funktionen in P.A.S.T den Anforderungen nicht genügen oder weitere Aufgabenfelder erschlossen werden müssen.

Diversitäten (Anzahl der Taxa, Shannon-Index, Simpson-Index) werden mit P.A.S.T für Einzelproben, beliebige Zonen und Lithotypen errechnet. Die Daten stehen in vielfältigen Formatierungen zur Verfügung und sind damit für weitere Berechnungen (Varianzen, Box-Plot-Diagramme, Anova-Test, etc.) in anderen Programmen wie z.B. Statistika $\bigcirc$ gerüstet. Der Excelteil ist bereits multilingual programmiert, so daß das System nach entsprechenden Eingaben der Übersetzungen länderübergreifend genutzt werden kann.

\subsubsection{Verknüpfung mit anderen Datenbanken}

Aufgrund der Programmierung von P.A.S.T mit Hilfe von Standardsoftware ist es relativ problemlos möglich, die Daten von P.A.S.T mit anderen systematischen Datenbanken zu verbinden. So kann z.B. ein rezent-botanisches Datenbanksystem mit der botanischen Familie innerhalb der Systematik von P.A.S.T relational verknüpft werden.

\subsubsection{Eingabe weiterer Daten}

Da die für die Eingabe der Daten eingeplanten wissenschaftlichen Hilfskräfte nicht bewilligt wurden (siehe Einleitung) ist die Eingabe der Daten bisher noch recht begrenzt und muß nach Beendigung dieser Arbeit weitergeführt werden, um ein vollständiges Bild der Flora und Vegetation sowie des Klimas und der Ökologie des terrestrischen Paläogens in Mitteleuropa zu erhalten. Als weitere Datengrundlage können dazu folgende Arbeiten dienen (in alphabetischer Reihenfolge):

\subsubsection{Systematik}

HAMMER-SCHIEMANN 1998 (Schöningen, Untereozän, bereits eingegeben)

KRUTZSCH 1962a, b, 1963a, b, 1967a, 1970a, b, 1971, 1976 (Paläogen, Geiseltal, Mitteldeutschland)

KRUTZSCH \& VANHOORNE 1977 (Belgien)

LENZ 2001 (Helmstedt, Oberflözgruppe, Mitteleozän)

NICKEL 1996 a, b (Oberrhein, Unteroligozän; Eckfeld, Mitteleozän)

THIELE-PFEIFFER 1988 (Messel, Mitteleozän)

THOMSON \& PfLUG 1953 (diverse Lokalitäten in Mitteleuropa)

sowie weitere laufende Arbeiten in Helmstedt/Schöningen, bei Egeln, im Gebiet um Halle, im Geiseltal und im Weißelster-Becken (Obereozän bis Oberoligozän) 


\subsubsection{Botanik und Gruppierungsparameter}

ELLENBERG 1979, 1991

FUKAREK 1992, 1993, 1994

HEGI 1998

HEYWOOD 1982

LINDBACHER 1995

MAI 1995

TAKHTAJAN 1997

WATSON \& DALLWITZ 1999

\section{3 ,closed dataset ${ }^{66}$}

Die Problematik des „closed data set“ - also die Abhängigkeit der Häufigkeitsverteilungen untereinander - wird nach Meinung des Autors durch die Schaffung von Häufigkeitsklassen nicht aufgehoben (im Gegensatz zu LENZ 2001), da die Abhängigkeit der Daten auch nach der Häufigkeitsklassenbildung weiter besteht. Bestätigt wird diese Aussage z.B. von PrAuss 2000, ROCK 1988 und anderen, die ebenfalls keine Verbesserung des Datensatzes in Bezug auf ein offenes oder teilweise offenes System mit Hilfe der Häufigkeitsklassen erreichen konnten.

Man muß sich vielmehr fragen, ob ein offenes System für die Untersuchung der Diversität überhaupt nötig ist, da das ökologische Verhalten von Pflanzengemeinschaften im Prinzip auch als ein ,closed data set“ beschrieben werden kann. Insbesondere in der lokalen Flora verändern Konkurrenzbedingungen auf kleinstem Raum das Verhalten einer Pflanzensippe, was eine gegenseitige Abhängigkeit dahingehend impliziert, daß Pflanzen, die sich unter günstigen Wettbewerbsbedingungen durchaus ausbreiten könnten, durch andere, besser an die Standortbedingungen angepaßte Pflanzen verdrängt bzw. in der Ausdehnung reduziert werden.

Die Standortbedingungen der Pflanzen in Mitteleuropa, unter Berücksichtigung der Konkurrenzbedingungen der Pflanzen untereinander, sind von ELLENBERG 1979, 1991, LINDBACHER 1995 als ökologische Zeigerwerte erfaßt worden.

Die Bildung von Häufigkeitsklassen ist jedoch durchaus sinnvoll, um starke Schwankungen in den Auszähldaten aufgrund der unterschiedlichen Pollenproduktion etwas auszugleichen.

Um daraus die tatsächliche Zusammensetzung der Vegetation abzuschätzen, wäre zusätzlich ein Faktor hilfreich, der die Unterschiede der Pollenproduktion zwischen den verschiedenen Taxa korrigierte, z.B. Windblütige versus Insektenblütige. Da bisherige Untersuchungen, z.B. von TAUBER 1965 u.a. nur für eine nicht ausreichende Anzahl von Taxa vorliegen, konnten im Rahmen dieser Arbeit diese Daten nicht ausgewertet werden, da für eine Datenbank eine nahezu lückenlose Informationsdichte nötig ist.

\subsection{Diversität}

Zusammenfassend kann gesagt werden, daß es mit geeigneten statistischen Methoden durchaus möglich ist, Diversitätsbetrachtungen mit Daten, die gänzlich unter anderen Aufgabenstellungen erzeugt wurden, durchzuführen, auch wenn primär eine einheitliche Probennahme, Aufbereitung, taxonomische Behandlung und einheitliche Auszähltechniken den Interpretationsspielraum der Ergebnisse erhöhen würden. 


\subsubsection{Fehler aufgrund verschiedener Auszähltechniken}

Der tatsächliche Wert der Entropie und damit der Diversität ist sehr stark davon abhängig, auf welcher taxonomischer Ebene die Pollen und Sporen klassifiziert wurden. Wurde die Klassifizierung auf Art-Ebene durchgeführt, erhält man eine deutlich höhere Diversität als bei einer Klassifikation auf Gattungsebene. Man sollte also bei der Gegenüberstellung von Diversitäten zweier Zeitscheiben darauf achten, daß die Klassifizierung auf gleichem Niveau durchgeführt wurde.

Insbesondere bei Palynomorphen ist es üblich, auf Grund der Vielzahl von verschiedenen Taxa von Pollen und Sporen eine Vereinfachung des Datensatzes durchzuführen und die Pollen und Sporen so zu gruppieren, daß sie ähnliche Ablagerungs- und Standortbedingungen reflektieren (z.B. LENZ 2001). Dies wird jedoch die Diversität mit hoher Wahrscheinlichkeit reduzieren, so daß bei Diversitäts-Betrachtungen stärkere Gruppierungen vermieden werden sollten.

Bei Diversitäts-Untersuchungen an fossilen Palynomorphen durchzuführen, sollten folgende Gesichtspunkte beachtet werden:

Wie schon bei ASHRAF 1995 beschrieben, sollte die Individuenzahl dynamisch nach der Rarefaction-Methode angepaßt werden.

Die Approximation der Probengröße mit Hilfe der Rarefaction-Kurve erlaubt jedoch keine Aussage über den tatsächlich vorhandenen Fehler bei der Auszählung. Insbesondere die Tatsache, daß ein sehr großer Teil der Auszählwerte unter einem Prozent liegt (siehe Kap. 4.4.10.3, S. 133), impliziert eine hohe Fehlerquote bei diesen Einzelwerten.

Nach THOMPSON 1992 kann die benötigte Probengröße in Abhängigkeit der zu erwartenden Genauigkeit nach folgender Formel ermittelt werden:

$n=\frac{Z^{2} \alpha^{*} p(1-p)}{E^{2}}$

Umstellung der Gleichung nach E:

$E=\sqrt{\frac{Z^{2} \alpha^{*} p(1-p)}{n}}$

$\mathrm{n}=$ benötigte Probengröße

$Z=$ Tabellenwert (bei Konfidenzintervall: $95 \%, Z=1,96$ )

$\mathrm{p}=$ Prozentanteil des Taxons

$\mathrm{E}=$ Konfidenzintervall der maximal erlaubten Abweichung zum Prozentanteil

Nimmt man eine durchschnittliche Probengröße von 300 Individuen an, so ergibt sich für Formen, deren Häufigkeit unter bzw. gleich einem Prozent liegen, ein Fehler von ca. $100 \%$.

Dieser Fehler kann durch das Zusammenfassen von Formen, wie z.B. bei LENZ 2001 beschrieben, wieder relativiert werden.

Der Widerspruch zu der Forderung, die Diversitäts-Berechnung nur mit einzelnen Taxa durchzuführen, kann mit P.A.S.T entschärft werden, da je nach Fragestellung in Minutenschnelle die Datenmatrix entsprechend umgerechnet werden kann. 


\subsubsection{Zukünftige Diversitätsberechnungen}

In der vorliegenden Untersuchung wurde darauf verzichtet, die Pollenkorngröße in Abhängigkeit des Sedimentes zu ermitteln, da die Größe der Palynomorphen bei der Auszählung nicht für jedes Individuum angegeben wird, auf der anderen Seite ein durchschnittlicher Datenbankwert der dort gespeicherten Palynomorphengröße für diese Art der Untersuchung zu ungenau erschien und bei Testrechnungen kein signifikantes Ergebnis lieferte.

Nach verschiedenen, rezente Ökosysteme untersuchende Autoren ist es nicht nur möglich, eine generelle Anreicherung von Sporomorphen in der Feinsilt/Ton-Fraktion eines fluviatilen Sedimentes zu ermitteln, sondern zuätzlich eine Anreicherung bestimmter Pollenformen und -größen, je nach Mineralkorngröße des assoziierten Sediments, festzustellen.

So werden kleine Formen sowie gut schwimmende Formen (z.B. die eher großen bisaccaten Pollenkörner) in der Regel erheblich länger in Suspension gehalten (MULLER 1959) und sind deshalb stärker in der Feinsilt/Ton-Fraktion des assoziierten Sediments vertreten. Aufgrund der Auswertung dieser Informationen wäre ein zusätzlicher Parameter, neben der Diversität, zur Erkennung von Sortierungseffekten gegeben (s. a. PECK 1974).

Außerdem müßte die Abhängigkeit der Diversität vom Sedimenttyp anhand der totalen Menge in Gramm des Sedimentes bestimmt werden. Bei der hier benutzten Auszählmethode wird ein Raster über eine zweidimensionale Fläche gelegt und so lange ausgezählt, bis die gewünschte Individuenzahl erreicht ist; damit ist die absolute prozentuale Menge der Palynomorphen und der organische Anteil insgesamt im Sediment nicht bekannt. 


\section{Literatur}

AKIN, W. E. (1991): Global patterns: climate, vegetation, and soils. - University of Oklahoma Press, $370 \mathrm{~S}$.

AlPAR, P. (2000): Datenorganisation und Datenbanken, SS 2000. - http://wiinfo4.wiwi.un-marburg.de/WebSite/Abruf.nsf/(LookupForm)/ 15607135CD954817C125664F003F0E19; Marburg.

Alroy, J. (2001): Methods for removing sampling biases from diversity curves. http://www.nceas.ucsb.edu/ alroy/Alroy.sampling.html, Santa Barbara.

Ashraf, A. R., Mosbrugger, V (1995): Palynologie und Palynostratigraphie des Neogens der Niederrheinischen Bucht. Teil 1 Sporen. - Palaeontographica, 235: 61-173; Stuttgart.

BACHER, J. (1996): Clusteranalyse: anwendungsorientierte Einführung. - Oldenbourg, 2.: 424 S.; Muenchen.

Battarbee, R. W. \& Flower, R. J. (1984): The inwash of catchment diatoms as a source of error in the sediment-based reconstruction of $\mathrm{pH}$ in an acid lake. Limnol. Oceanogr., 29: 1325-1329.

Belz, G., Mosbrugger, V. (1994): Systematisch-paläoökologische und paläoklimatische Analyse von Blattfloren im Mio-/Pliozän der Niederrheinischen Bucht (NW-Deutschland). - Palaeontographica, Abt. B, 233: 19-156; 60 Abb., 12 Tab., 12 Taf., Stuttgart.

Benson, J. (1957): Plant Classification. - D.C. Heath and Company, 688 S.; Boston.

BIRD E. C. F. (1994): Physical Setting and Geomorphology of Coastal Lagoons. In: Costal Lagoon Processes (KJERFVE, B., ed.). - Elsevier, 9-39; Amsterdam, New York, Tokyo.

BIRKS, H. J. \& GORDON, A. D. (1985): Numerical methods in quaternary pollen analysis. - Acad. Pr., 317 S.; London.

Bock, H. H. (1974): Automatische Klassifikation: theoretische und praktische Methoden zur Gruppierung und Strukturierung von Daten; (Cluster-Analyse). Vandenhoeck \& Ruprecht, 480 S.; Göttingen.

BOEDICKER, D. (1990): Handbuch-Knigge, Software-Handbücher schreiben und beurteilen. - Wissenschaftsverlag, 284 S.; Manheim.

BRETT, C. E. (1963): Relationships between marine invertebrate infauna distribution and sediment type distribution in Bogue Sound, North Carolina. - Unveröffentlichte Dissertation. 202 S.; North Carolina.

BRUSH, G. S. \& BRUSH, L. M. (1972): Transport of pollen in a sediment laden channel: a laboratory study. - American Journal of Science, 272: 359-81.

BRZUstowsKi, J. (2001): Rarefaction Calculator. - http://www.biology.ualberta.ca/ jbrzusto/rarefact.php, Alberta.

BUCHARDT, B. (1978): Oxygen isotope palaeotemperatures from the Tertiary period in the North Sea area. - Nature, 275: 121-123; London. 
CHAO, A. (1987): Estimating the population size for capture-recapture data with unequal catchability. - Biometrics, 43: 783-791.

CHEN, P. (1976): The Entity-Relationship Model - Toward a Unified View of Data. ACM TODS, 1: 9-36.

CHEN, Y. (1987): Pollen and sediment distribution in a small crater lake in Northeast Queensland, Australia. - Pollen et Spores, 29: 98-110.

Claus, G., Finze, F. R., Partzsch, L. (1995): Statistik für Soziologen, Pädagogen, Psychologen und Mediziner. - Verlag Harri Deutsch, 2.: 476S.; Frankfurt am Main.

CodD, E. F. (1970): A Relational Model of Data for Large Shared Data Banks. Communications of the ACM, 6: 337-387.

ColWELL R. K. (2001): EstimateS. - http://viceroy.eeb.uconn.edu/EstimateS.

Cross, A. T., Thompson, G. G., \& Zaitzeff, J. B. (1966): Source and distribution of palynomorphs in botton sediments, southern part of Gulf of California. Marine Geology, 4: 467-524.

DAmuth, J. (1993): ETE Database Manual. - Evolution of Terrestrial Ecosystems Consortium, Departement of Paleobiology, 195 S.; Washington.

DIMBLEBY, G. W. (1985): The palynology of Archaeological sites. - Acad. Pr., 176 S.; London.

DRONKERS, J. (1986a): Tidal asymmetry and estuarine morphology. - Netherlands Journal of Sea Research, 20: 117-131.

DRONKERS, J. (1986b): Tide-induced residual transport of fine sediment. In Lecture Notes on Coastal and Estuarine Studies, Volume 16, Physics of Shallow Estuaries and Bays (van de KREEK, J., ed.). - Springer Verlag, 228-241; New York.

EBE, A. (1992): Palynologische Untersuchungen am Flöz Victoria im Tagebau Alversdorf (Eozäne Braunkohle von Helmstedt). - unveröffentlichte Diplomarbeit, 48 S.; Göttingen.

EllenBerG, H. (1979): Zeigerwerte der Gefäßpflanzen Mitteleuropas. - Goltze, 2. Aufl.: 122 S.; Göttingen.

EllenberG, H. (1991): Zeigerwerte von Pflanzen in Mitteleuropa. - Goltze, 3.: 248 S.; Göttingen.

ERdtMAn, G. (1954): On the Occurrence of Olacaceous Pollen Grains in eocene Deposits in Germany. - Svensk. Bot. Tidskr., 48: 804-805; Uppsala.

FALL, P. L. (1987): Pollen Taphonomy in a Canyon Stream. - Quaternary Research, 28: $393-406$.

FISHER, H. (2000): QUATERNARY DATA. - http://ibs.uel.ac.uk/ibs/other/ env/quat.htm.

FloRES, R. M. (1983): Basin facies analysis of coal-rich Tertiary fluvial deposits, nothern Powder River Basin, Montana and Wyoming. In Modern and Ancient Fluvial Systems (COLLINSON, J. D. \& LEWIN J, ed.). - International Association of Sedimentology Special Publications, 6: 501-515. 
FREDERICK, J. A. (2000): Biofilm \& Biodiversity. - http://www.mdsg.umd.edu/ Education/biofilm/diverse.htm.

FREDERIKSEN, N. O. (1973): New mid-Tertiary spores and pollen grains from Mississippi and Alabama. - Tulane studies in geology and palaeontology, 10 (2): 65-86.

FREDERIKSEN, N. O. (1980): Sporomorphs from the Jackson Group (Upper Eocene) and Adjacent Strata of Mississippi and Western Alabama. - Geol. Survey Prof. Paper, 1084: 1-175; Washington.

Frederiksen, N. O., Wiggins, V. D., Ferguson, I. K., Dransfield, John \& Ager, C. M. (1985): Distribution, Paleoecology, Paleoclimatology, and botanical affinity of the Eocene Pollen Genus Diporoconia n. gen. - Palynology, 9: 3760 .

FuKAreK, F. (1992): Urania Pflanzenreich; Moose, Farne, Nacktsamer. - Urania Verlag, Bd II: 390 S.; Leipzig, Jena, Berlin.

FuKAREK, F. (1995): Urania Pflanzenreich; Vegetation. - Urania Verlag, Bd V: 1-420; Leipzig, Jena, Berlin.

FukareK, F., Schultze-Motel, J.; Siegel, M. (1993): Urania Pflanzenreich; Blütenpflanzen 1. - Urania Verlag, Bd III: 590 S; Leipzip, Jena, Berlin.

FukareK, F., Schultze-Motel, J.; Siegel, M. (1994): Urania Pflanzenreich; Blütenpflanzen 2. - Urania Verlag, Bd IV: 609 S.; Leipzig, Jena, Berlin.

Gauch, H. G. (1982): Multivariate Analysis in Community Ecology. - Cambridge Univ. Press, New York.

GLADIS, R. (1999): Ordnungskräfte; Relationale Desktop-Datenbanken für Windows und MacOS Prüfstand, Desktop-Datenbanken, Access, Approach, 4D, FileMaker, dBase, Paradox, FoxPro, Desktop-Datenbanken. - CT-Magazin, 4: 134; Hannover.

Gothan, W., Weyland, H. (1954): Lehrbuch der Paläobotanik. - Acad. Verlag Berlin, 521 S.; Berlin.

Hammer, J.: Sedimentologie und Palynofazies der fluviatilen Ablagerungen des Oberoligozäns in der Leipziger Bucht (Tagebau Witznitz). - Dissertation (in Vorbereitung), Göttingen.

Hammer,Ø, Harper, D. A. T. RyAn, P. D. (2001): PAST - PAlaeontological STatistics. - http://folk.uio.no/ohammer/past/.

HAMmer-SchiemanN, G. (1998): Palynologische Untersuchungen zur Fazies und Ökologie der Unterflözgruppe im Tagebau Schönigen (Untereozän, Helmstedt, Bez. Braunschweig). - Dissertation, 107 S.; Universität Göttingen.

Hegi, G., Conert, H. J., JÄger, E. J., Kadereit, J. W., Schultze-Motel, W., Wagenitz, G., Weber, H. E. (Hrsg.) (1998): Illustrierte Flora von Mitteleuropa. - Parey Buchverlag, 3. Aufl.: Berlin.

HeYwood V. H. (1982): Blütenpflanzen der Welt. - Jb. Preuß. Geol. L.-Amt, 335 S; Basel, Boston, Stuttgart.

Hochuli, P. A. (1978): Palynologische Untersuchungen im Oligozän und Untermiozän der zentralen und westlichen Paratethys. - Beitr. Paläonz. Österr., 4: 1-123; Wien. 
Hoffbauer, M., Spielmann, C. (1999): Access 2000: Programmierung, das Buch, Spezialwissen für Experten. - Sybex, 1. Aufl.: 765 S.; Düsseldorf.

Holloway, R. G. (1981): Preservation and experimental diagenesis of the pollen exine. - Dissertation, Texas, A \& M University.

Holmes, P. L. (1990): Differential transport of spores and pollen: A laboratory study. Rev. Palaeobotan. Palynol., 64: 289-296.

Holmes, P. L. (1994): The sorting of spores and pollen by water: experimental and field evidence. In: Sedimentation of Organic Particles, (TRAVERSE, A., ed.). - Cambridge Univ. Press, 9-32, Cambridge.

HÜSKES, R. (1996): Heger und Sammler; Endanwender-Datenbanken für Windows und Mac Prüfstand, Desktop-Datenbanken, Access, dBASE, Paradox, F\&A, 4D First, Approach, Omnis (Mac), DataEase, Wordperfect InfoCentral. - CTMagazin, 4: 296; Hannover.

HÜSKES, R. (1997): Ordnungshüter; Die Datenbanken Access 97, Approach 97, Paradox 7 und StarBase Prüfstand, Office. - CT-Magazin, 6: 146; Hannover.

KJerfve, B. (ed.) (1994): Costal Lagoon Processes. - Elsevier, Amsterdam, London, New York, Tokyo.

Koeppen, W. (1930): Handbuch der Klimatologie: in fünf Bänden. - Bornträger, Berlin.

Köthe, A. (1990): Paleogene Dinoflagellates from Northwest Germany. - Geol. Jb. A, 118: 111 S.; Hannover.

Kramer, K. U. \& Green, P. S., Kubitzki, K (ed.) (1990): The Families and Genera of Vascular Plants. I Pteridophytes and Gymnosperms. - Springer Verlag, I: 404 S.; Berlin, Heidelberg.

KREBS, C. J. (1989): Ecological Methodology. - Harper \& Row, New York.

KRUTZSCH, W. (1959): Mikropaläontologische (sporenpaläontologische) Untersuchungen in der Braunkohle des Geiseltales. Teil I. - Geologie, 8 (21/22): 1425; Berlin.

KRUTZSCH, W. (1962a): Atlas der mittel- und jungtertiären dispersen Sporen und Pollen, sowie der Mikroplanktonformen des nördlichen Mitteleuropas. Lieferg., I: Laevigate und toriate trilete Sporenformen: 1-108; Berlin.

KRUTZSCH, W. (1962b): Stratigraphisch bzw. botanisch wichtige neue Sporen- und Pollenformen aus dem deutschen Tertiär.- Geologie, 11 (3): 265-307; Berlin.

KRUTZSCH, W. (1963a): Atlas der mittel- und jungtertiären dispersen Sporen- und Pollen sowie der Mikroplanktonformen des nördlichen Mitteleuropas.- Lieferg. II. Die Sporen der Anthocerotaceae und der Lycopodiaceae.1-141; Berlin.

KRUTZSCH, W. (1963b): Atlas der mittel- und jungtertiären dispersen Sporen und Pollen sowie der Mikroplanktonformen des nördlichen Mitteleuropas. Lieferg., III: 128; Berlin.

KRUTZSCH, W. (1967): Atlas der mittel- und jungtertiären dispersen Sporen und Pollen sowie der Mikroplanktonformen des nördlichen Mitteleuropas. Lieferg. IV und $\mathbf{V}$ : weitere azonotrilete (apiculate, muronate), zonotrilete, monolete und alete Sporenformen. 1-232; Berlin. 
KRUTZSCH, W. (1970a): Atlas der mittel- und jungtertiären dispersen Sporen und Pollen sowie der Mikroplanktonformen des nördlichen Mitteleuropas. Lieferg. VII: Monoporate, monocolpate, longicolpate, dicolpate und ephedroide (polyplicate) Pollenformen.1-175; Berlin.

KRUTZSCH, W. (1970b): Die stratigraphisch verwertbaren Sporen- und Pollenformen des mitteleuropäischen Altteriär. - Jb. Geol., 3: 309-379; Berlin.

KRUTZSCH, W. (1971): Atlas der mittel- und jungtertiären dispersen Sporen und Pollen sowie der Mikroplanktonformen des nördlichen Mitteleuropas. Lieferg. VI: Coniferenpollen (Saccites und "Inaperturates").1-234; Berlin.

KRUTZSCH, W. (1976): Die Mikroflora der Braunkohle des Geiseltales. Teil IV: Die stratigraphische Stellung des Geiseltalprofils im Eozän und die sporenstratigraphische Untergliederung des mittleren Eozäns. In: Eozäne Floren des Geiseltales. - Abh. zentr. geol. Inst.,, 26: 47-92; Berlin.

KRUTZSCH, W. (1998): „Handout“. - Probleme der Palynologie tertiärer Sedimente in Mittel- und Norddeutschland, 3: Hannover.

Krutzsch, W., Blumenstengel, H., Kiesel, Y. ， RÜfFle, L. (1992): Paläobotanische Klimagliederung des Alttertiärs (Mitteleozän bis Oberoligozän) in Mitteldeutschland und das Problem der Verknüpfung mariner und kontinentaler Gliederungen. - N. Jb. Geol., Abh., 186, H. 1/2: 137 - 253; Stuttgart.

KRUTZSCH, W. \& MAJEWSKI, J. (1967): Zur Methodik der pollenstratigraphischen Zonengliederung im Jungtertiär Mitteleuropas. - Abh. zentr. geol. Inst., 10: 8398; Berlin.

Krutzsch, W. \& VANHOORne, R. (1977): Die Pollenflora von Epinois und Loksbergen. - Jb. Preuß. Geol. L.-Amt, Abt. B (163): 1-110; Stuttgart.

Kullmann, J., LöSER, H. (1993): Die Datenbanken PaleoTax und Goniat- Vorstufen eines paläontologischen Informationssystems. - Paläont. Z., 67 (3/4): 397-405; Stuttgart.

LENZ, O. (2000): Paläoökologie eines Küstenmoores aus dem Eozän Mitteleuropas am Beispiel der Wulfersdorfer Flöze und deren Begleitschichten (Helmstedter Oberflözgruppe, Tagebau Helmstedt). - http://webdoc.gwdg.de/diss/2000/ lenz/index.htm, 228 S.; Göttingen.

Lietzow, A. (1991): Das Paläogen des Tagebaus Schöningen, Baufeld Esbeck bei Helmstedt (östliches Niedersachsen). - unveröffentlichte Diplomarbeit, 104 S.; Göttingen.

LiNDBACHER, R. (1995): Phanart, Datenbank der Gefäßpflanzen Mitteleuropas. Erklärung der Kennzahlen, Aufbau und Inhalt. - Veröffentlichungen des Geobotanischen Institutes der eidg. Tech. Hochschule, Stiftung Rübel, 125: 436 S.; Zürich.

Lindsay, E. H., Fahlbusch, V., Mein, P. (1988): A contribution to the Neogene terrestric Biostratigraphy of Europe based on Rodents. - Proceedings of a NATO Advanced Research workshop on European Neogene Mammal Chronology. - Plenum Press, 211-236; New York. 
LOOK, E. R. (1984): Geologie und Bergbau im Braunschweiger Land. Dokumentation zur geol. Wanderkarte Braunschweiger Land. - Ber. naturhist. Ges. Hannover, 127: 1-467; Hannover

MAGURRAN, A. E. (1988): Ecological diversity and its measurement. - Princeton Univ. Press, 179 S.; Princeton, NJ.

MAI, D. H. (1995): Tertiäre Vegetationsgeschichte Europas. - Gustav Fischer Verlag, $691 \mathrm{~S}$; Suttgart, New York.

MARTINI, E. (1971): Standard Tertiary and Quaternary calcareous nannoplankton zonation. In Proc. 2nd Int. Conf. Planktonic Microfossils Roma (FARINACCI, A., ed.), 2: 739-785; Rom.

Mosbrugger, V. (1989): Zur Gliederung und Benennung von Taphozönosen. - Cour. Forsch. Inst. Senckenberg, 109: 17-28; Franfurt a. M..

Mosbrugger, V., Gee, C. T., Belz, G., Ashraf, A.R. (1994): Three-dimensional reconstruction of an in-situ Miocene peat forest from the Lower Rhine Embayment, northwestern Germany; new methods in palaeovegetation analysis. - Palaeogeogr., Palaeoclimatol., Palaeoecol., 110; 3-4: 295-317; Amsterdam.

Mosbrugger, V. \& UTESCHER, T. (1993): Quantitative terrestrial paleoclimate reconstructions in the Tertiary - possibilities and limitations of a new approach. - Terra Nova, Abstr. Suppl., 5: 727; Oxford.

Mosbrugger, V. \& Utescher, T. (1997): The coexistence approach; a method for quantitative reconstructions of Tertiary terrestrial palaeoclimate data using plant fossils. - Palaeogeogr., Palaeoclimatol., Palaeoecol., 134: 61-86; Amsterdam.

Muller, J. (1959): Palynology of recent Orinoco delta and shelf sediments: report of the Orinoco Shelf expedition. - Micropalaeontology, 5 (1): 1-32.

NATGE-EFoGHE， C. (1997): Palynologische Untersuchungen am unteren Zwischenmittel im Tagebau Alversdorf, Mitteleozän, Helmstedter Oberflözgruppe. - unveröffentlichte Diplomarbeit, 182 S.; Göttingen.

Nichols, M, M. \& Boon, J. D. (1994): Sediment Transport Processes in Costal Lagoons. In: Costal Lagoon Processes (KJERFVE, B., ed.). - Elsevier, 157220; Amsterdam, London, New York, Tokyo.

Nichols, D. J. \& PockNALl D. T. (1984): Relationship of palynofacies to coaldeposition environments in the upper Paleocene of the Gulf Coast Basin, Texas, and the Powder River Basin, Montana and Wyoming. In Sedimentation of Organic Particles (TRAVERSE, A., ed.). - Cambridge Univ. Press, 217-237.

Nickel, B. (1996a): Palynofazies und Palynostratigraphie der Pechelbronn Schichten im nördlichen Oberrheintalgraben. - Jb. Preuß. Geol. L.-Amt, Abt B (240): 1151; Stuttgart.

NiCKEL, B. (1996b): Die mitteleozäne Mikroflora von Eckfeld bei Manderscheid/Eifel. - Mainzer Naturwissenschaftliches Archiv, 56 (Beiheft 16): 2-121; Mainz.

PANOFsKy, H. A. \& Brier, G. W. (1958): Some Applications of Statistics to Meteorology. - Pennsylvania State University, 224 S.; University Park.

PECK, R. (1974): Studies of pollen distribution in the Oakdale catchment. Dissertation, Cambridge University. 
PEJRUP, M. (1986a): Parameters affection fine-grained suspended sediment concentrations in a shallow micro-tidal estuary. - Estuarine, Costal and Shelf Science, 22: 241-254; Ho Bugt, Dänemark.

PEJRUP, M. (1986b): Flocculated suspended sediment in a micro-tidal environment. Sedimentary Geology, 57: 249-256.

Pflug, H. (1952): Palynologie und Stratigraphie der eozänen Braunkohlen von Helmstedt. - Paläont. Z., 26: 112-137; Stuttgart.

Pocknall, D. T. \& Flores, R. M. (1987): Palynomorph biozones for the Fort Union and Wasatch Formations (upper Paleocene-lower Eocene), Powder River Basin, Wyoming and Montana. - Palaios, 2: 133-145.

PotoniÉ, R. (1931): Zur Mikroskopie der Braunkohlen. Tertiäre Blütenstaubformen. Jb. Preuß. Geol. L.-Amt, 30 (16): 325-333; Halle.

Potonié, R. (1934): Zur Mikrobotanik des eozänen Humodils des Geiseltales. - Arb. Inst. Paläobot. u. Petrogr. Brennsteine, 4: 25-125; Berlin.

PotoniÉ, R., KREMP, G. (1954): Die Gattungen der paläozoischen Sporae dispersae und ihre Stratigraphie. - Geol. Jb., 69: 111-194; 5, Hannover.

POTONIÉ, R., KREMP, G. (1955): Die Sporae dispersae des Ruhrkarbons. Ihre Morphographie und Stratigraphie mit Ausblicken auf Arten anderer Gebiete und Zeitabschnitte, Teil II. - Palaeontographica, B, 98: 1-136; Stuttgart.

POtOnié, R., Thomson, P.W. \& Thiergart, F. (1950): Zur Nomenklatur und Klassifikation der neogenen Sporomorphae (Pollen und Sporen). - Geol. Jb., 65: 35-69; Hannover.

PRAUSS, M. (2000): The oceanographic and climatic interpretation of marine palynomorph phytoplankton distribution from Mesozoic, Cenozoic and Recent sections. - GAGP, Selbstverlag der Geologischen Institute der Georg-AugustUniversität 76: 118 S., Göttingen.

PRAHL, K. (1994): Datenbankkonzept für die Messel-Dokumentation. - CFS, 170: 197208; Frankfurt a.M.

PRYOR, w. A. (1975): Biogenic sedimentation and alteration of argillaceous sediments in shallow marine environments. - Geological Society of American Bulletin, 86: $1244-1254$.

PUNT, W., BLACKMORE, S. , NILSSON, S. , THOMAS A. L. E. (2001): glossary of pollen and spore terminology. - http://www.bio.uu.nl/ palaeo/glossary/glos-txt.htm \#Pattern, Utrecht.

RIEGEL, W. (1965): Palynology of Environments of Peat Formation in Southwestern Florida. - Dissertation, 189 S.; Pennsylvania, State Univ., State Park.

Riegel, W., Bode, T., Hammer, J., Hammer-Schiemann, G., Lenz, O. \& Wilde, V. (1999): The Paleoecology of the Lower and Middle Eocene at Helmstedt, Northern Germany-Study in Contrasts. - Acta Palaeobotanica.

RitTER, M (1999): The Physical Environment. - http://www.uwsp.edu/acaddept/ geog/faculty/ritter/geog101/modules/biosphere/biomes_toc.html, Wisconsin.

Rock, N. M. S. (1988): Numerical geology: a source guide, glossary, and selective bibliography to geological uses of computers and statistic. - Springer Verlag, XI: 427 S; Berlin. 
RYAN, J, J,. \& GODELL, H. G. (1972): Marine geology and estuarine history of Mobile Bay, Alabama. In: Environmental Framework of Coastal Plain Estuaries, NELSON , B.W. (ed.). - Geological Society of American Memoir, 133: 517554.

SchiEmanN, G. (1994): Palynologische Untersuchungen am Unterflöz der Helmstedter Oberflözgruppe (Mitteres Eozän) im Tagebau Alversdorf. - unveröffentlichte Diplomarbeit, 74 S.; Göttingen.

SchUler, M. (1990): Palynologie et biostratigraphie de l' Eocene et de l' Oligocene inferieur dans les fosses rhenan, rhodanien et de Hesse. - Jb. Preuß. Geol. L.Amt, 190: 1-503; Orléans.

SHANNON, C. E., WeAVER, W. (1949): The mathematical theory of communication. Urbana IL: University of Illinois Press, Illinois.

SiMPSON, E. H., Measurement of diversity (1949) - Nature, 163: S. 688.

TAKHTAJAN, A. L. (1997): Diversity and classification of flowering plants. - Columbia Univ. Pres, X: 643 S.; New York.

TAUBER, H. (1965): Differential pollen dispersion and the interpretation of pollen diagrams. - Danmarks Geologiske. Undersølgelse., Række 2, Number 89, Dänemark.

Thiele-Pfeiffer, H. (1980): Die miozäne Mikroflora aus dem Braunkohletagebau Oder bei Wackersdorf/ Oberpfalz. - Jb. Preuß. Geol. L.-Amt, Abt. B (174): 95224; Stuttgart.

Thiele-Pfeiffer, H. (1988): Die Mikroflora aus dem mitteleozänen Ölschiefer von Messel bei Darmstadt.- Palaeontographica. - Jb. Preuß. Geol. L.-Amt, Abt. B (211): 1-86; Stuttgart.

Thompson, S. K. (1992): Sampling. - Wiley, New York.

Thomson, W. \& Pflug, H. (1953): Pollen und Sporen des mitteleuropäischen Tertiärs. - Palaeontographica, Abt. B (94): 1-138; Stuttgart.

TRAVERSE, A. (1994): Sedimentation of Organic Particles. - Cambridge Univ. Press, $544 \mathrm{~S}$.

Trewartha, G. T. (1968): An introduction to weather and climate. - McGrawHill, 4. Aufl.: 408 S.; New York.

TsCHUDY, R. H. (1969): Relationship of palynomorphs to sedimentation. - Aspects of Palynology, 76-96; New York.

Tschudy, R. H. (1973): Stratigraphic Distribution of significant Eocene Palynomorphs of the Mississippi Embayment.- Geol. Survey Prof. Paper, 743, B: Washington.

Watson, L \& Dallwitz, M. J. (1999): The Families of Flowering Plants. http:/www.rrz.uni-hamburg.de/biologie/b_online/ibc99/delta/angio/.

WenJun, Z. (1998): Species Richness Estimation. - http://mathsoft.stormpages.com/ ecotools/index_biodiversity_species.htm.

ZIEGLER, C. K., LICK, W. (1988): The Transport of Fine-grained Sediments in Shallow Waters. - Environmental Geology and Water Sciences, 11: 123-132.

ZIEGLER, P. A. (1990): Geological atlas of western and central Europe. - Shell Internationale Petroleum Maatschapping B. V, 239 S.; Den Haag. 


\section{Anhang}

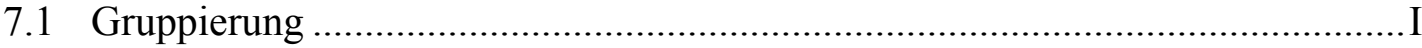

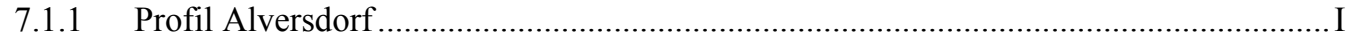

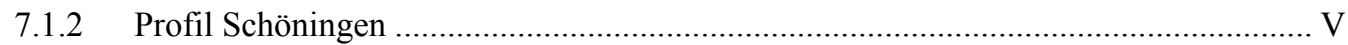

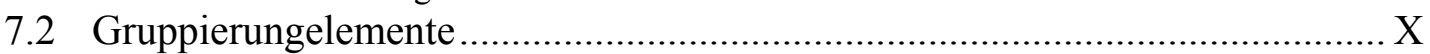

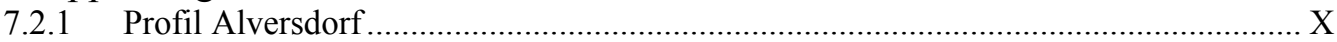

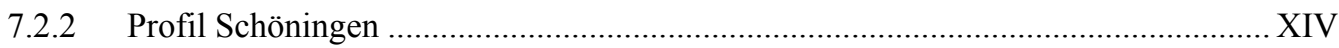

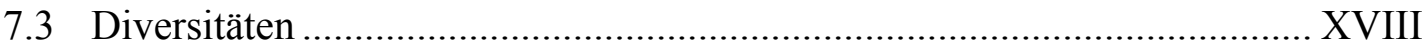

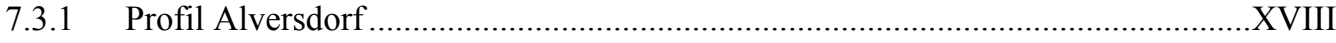

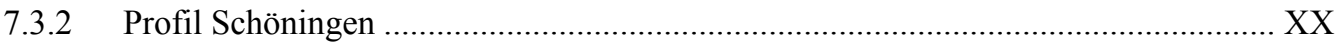

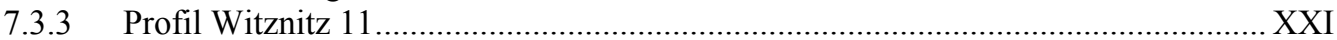

\subsection{Gruppierung}

Da es sich bei den Auszähldaten um Literaturdaten handelt, wurde auf die Darstellung dieser Informationen im Rahmen dieser Dissertation verzichtet. Die folgenden Tabellen zeigen die Taxa und deren botanische Zugehörigkeit sowie die Einordnung der Familien in die Gruppierungselemente (hier azonale Vegetation). Sämtliche der folgenden Tabellen wurden direkt mit P.A.S.T aus der Datenbank erzeugt - mit entsprechenden Anpassungen des Layouts (Rotation, Farbanpassungen und Selektion der relevanten Elemente). Grün sind die im Pollendiagramm abgebildeten Familien/Gruppierungselemente gekennzeichnet. Die abgebildeten Familien/Gruppierungselemente in den Profilen „sortiert nach Lithotyp“ bilden eine Teilmenge dieser markierten Elemente. Die Mehrsprachigkeit ist durchaus beabsichtigt und beruht auf den in verschiedenen Entwicklungsstufen von P.A.S.T entstandenen Auswertungen und Darstellungen.

\subsubsection{Profil Alversdorf}

\begin{tabular}{|lrr|}
\hline Statistik: Botanische Zugehörigkeit & Gattungen & Familien \\
\hline unbekannt (Anzahl) & 75 & 13 \\
\hline fraglich (Anzahl) & 41 & 31 \\
\hline bekannt (Anzahl) & 96 & 106 \\
\hline$\%$ (bekannt(incl. fraglich)/unbekannt) & $70,1 \%$ & $77,4 \%$ \\
\hline$\%$ (bekannt(excl. fraglich)/unbekannt) & $40,1 \%$ & $54,7 \%$ \\
\hline
\end{tabular}

\begin{tabular}{|c|c|c|c|c|}
\hline Taxa & \multicolumn{2}{|c|}{ stratigr. Reichweite } & \multirow{2}{*}{\multicolumn{2}{|c|}{$\begin{array}{l}\text { Familie/ } \\
\text { Gruppierungselement } \\
\text { Cyperaceae, Sparganiaceae, } \\
\text { Nymphaeaceae }\end{array}$}} \\
\hline Cyperaceaepollis piriformis & L. Eocene & E. Eocene & & \\
\hline $\begin{array}{l}\text { Sparganiaceaepollenites } \\
\text { sparganoides }\end{array}$ & M. Oligocene? & E. Eocene & Sparganium? & Sparganiaceae? \\
\hline \multirow[t]{2}{*}{ Proxapertites } & L. Eocene & E. Eocene & & Nymphaeaceae (Monocolpates) \\
\hline & & & & Verlandungszone \\
\hline \multirow[t]{2}{*}{ Stereisporites stereoides } & L. Pliocene & E. Eocene & Sphagnum & Sphagnaceae \\
\hline & & & & Hochmoor \\
\hline Milfordia minima & E. Miocene & L. Paleocene & Lyginia. & Restionaceae \\
\hline \multirow[t]{2}{*}{ Milfordia incerta } & M. Miocene & E. Paleocene & Leptocarpus? & Restionaceae \\
\hline & & & & Sum Restionaceae \\
\hline $\begin{array}{l}\text { Tricolporopollenites } \\
\text { megaexactus brühlensis }\end{array}$ & L. Miocene & E. Eocene & Cliftonia & Cyrillaceae \\
\hline $\begin{array}{l}\text { Tricolporopollenites } \\
\text { megaexactus exactus }\end{array}$ & L. Pliocene & M. Eocene & Cliftonia & Cyrillaceae \\
\hline & & & & Sum Cyrillaceae \\
\hline
\end{tabular}




\begin{tabular}{|c|c|c|c|c|}
\hline Taxa & \multicolumn{2}{|c|}{ stratigr. Reichweite } & Gattung & $\begin{array}{l}\text { Familie/ } \\
\text { Gruppierungselement }\end{array}$ \\
\hline Ericipites ericius & rezent & L. Paleocene & Erica? & Ericaceae \\
\hline \multirow[t]{3}{*}{ Ericipites callidus } & L. Pliocene & L. Paleocene & & Ericaceae \\
\hline & & & & Sum Ericaceae \\
\hline & & & & Ried \\
\hline $\begin{array}{l}\text { Inaperturopollenites } \\
\text { concedipites }\end{array}$ & L. Pliocene & E. Eocene & Glyptostrobus? & Taxodiaceae? \\
\hline Inaperturopollenites hiatus & L. Pliocene & E. Paleocene & Glyptostrobus & Taxodiaceae \\
\hline \multirow[t]{3}{*}{ Sequoiapollenites polyformus } & L. Pliocene & E. Eocene & Metasequoia & Taxodiaceae \\
\hline & & & & Sum Taxodiaceae \\
\hline & & & & Sumpf \\
\hline Smilacipites echinatus & M. Miocene & E. Eocene & Smilax & Smilacaceae \\
\hline Araucariacites europaeus & L. Eocene & M. Eocene & & Araucariaceae \\
\hline Emmapollis pseudoemmaensis & L. Pliocene & Cretaceous & Ascarinopsis? & Chloranthaceae? \\
\hline Magnolipollis graciliexinus & M. Miocene & M. Eocene & Magnolia & Magnoliaceae \\
\hline Nudopollis terminalis & M. Eocene & L. Paleocene & & Myricaceae? \\
\hline Triatriopollenites rurensis & L. Pliocene & E. Eocene & Myrica & Myricaceae \\
\hline \multirow[t]{2}{*}{ Triatriopollenites bituitus } & E. Pliocene & E. Eocene & Myrica & Myricaceae \\
\hline & & & & Sum Myricaceae \\
\hline Plicatopollis plicatus & L. Eocene & E. Eocene & & Juglandaceae? \\
\hline Momipites punctatus & L. Pliocene & E. Paleocene & Engelhardtia? & Juglandaceae \\
\hline Momipites quietus & M. Oligocene? & M. Paleocene? & Engelhardtia & Juglandaceae \\
\hline Caryapollenites triangulus & L. Eocene & L. Paleocene & Carya & Juglandaceae \\
\hline Caryapollenites circulus & L. Eocene & E. Paleocene & Carya & Juglandaceae \\
\hline Caryapollenites simplex & L. Pliocene & E. Eocene & Carya & Juglandaceae \\
\hline Subtriporopollenites constans & M. Eocene & E. Eocene & & Juglandaceae? \\
\hline Subtriporopollenites anulatus & L. Eocene & L. Paleocene & Carya? & Juglandaceae \\
\hline $\begin{array}{l}\text { Platycaryapollenites } \\
\text { platycaryoides }\end{array}$ & L. Eocene & M. Paleocene? & Platycarya & Juglandaceae \\
\hline $\begin{array}{l}\text { Platycaryapollenites } \\
\text { miocaenicus }\end{array}$ & L. Miocene & E. Eocene & Platycarya & Juglandaceae \\
\hline \multirow[t]{2}{*}{ Platycaryapollenites semicyclus } & L. Eocene & E. Eocene & Platycarya? & Juglandaceae \\
\hline & & & & Juglandaceae \\
\hline Pterocaryapollenites stellatus & Pleistocene & M. Eocene & Platycarya? & Juglandaceae(Brevaxones) \\
\hline Triporopollenites robustus & L. Oligocene & E. Paleocene & & Betulaceae? \\
\hline Triporopollenites rhenanus & L. Pliocene & E. Eocene & Ostrya & Betulaceae \\
\hline Triporopollenites megagranifer & L. Oligocene & E. Paleocene & & Betulaceae? \\
\hline Triporopollenites coryloides & L. Pliocene & E. Eocene & Corylus & Betulaceae \\
\hline Trivestibulopollenites betuloides & L. Pliocene & M. Eocene & Betula & Betulaceae \\
\hline Polyvestibulopollenites verus & rezent & E. Eocene & Alnus & Betulaceae \\
\hline \multirow[t]{2}{*}{ Carpinuspollenites carpinoides } & rezent & M. Eocene & Carpinus & Betulaceae \\
\hline & & & & Betulaceae \\
\hline Porocolpopollenites vestibulum & L. Pliocene & E. Eocene & Symplocos? & Symplocaceae? \\
\hline $\begin{array}{l}\text { Porocolpopollenites } \\
\text { rarobaculatus }\end{array}$ & L. Miocene & E. Eocene & Symplocos & Symplocaceae \\
\hline $\begin{array}{l}\text { Porocolpopollenites } \\
\text { stereoformis }\end{array}$ & L. Eocene & E. Eocene & & Symplocaceae? \\
\hline \multirow[t]{2}{*}{ Symplocospollenites orbis } & L. Miocene & E. Paleocene & & Symplocaceae? \\
\hline & & & & Sum Symplocaceae \\
\hline Polyporopollenites undulosus & rezent & L. Paleocene & Ulmus? & Ulmaceae \\
\hline Polyporopollenites eoulmoides & M. Eocene & E. Paleocene & & Ulmaceae \\
\hline Celtipollenites intrastructurus & M. Miocene & L. Paleocene & Celtis & Ulmaceae \\
\hline \multirow[t]{2}{*}{ Celtipollenites laevigatus } & M. Eocene & M. Eocene & Celtis? & Ulmaceae? \\
\hline & & & & Sum Ulmaceae \\
\hline Intratriporopollenites instructus & L. Miocene & E. Paleocene & Tilia & Tiliaceae \\
\hline Anacolosidites efflatus & L. Eocene & E. Eocene & Anacolosa & Olacaceae \\
\hline $\begin{array}{l}\text { Tricolporopollenites } \\
\text { pseudocingulum }\end{array}$ & L. Miocene & E. Eocene & & Anacardiaceae? \\
\hline $\begin{array}{l}\text { Tricolporopollenites solé-de- } \\
\text { portai }\end{array}$ & E. Miocene & M. Paleocene? & & Anacardiaceae? \\
\hline
\end{tabular}




\begin{tabular}{|c|c|c|c|c|}
\hline Taxa & \multicolumn{2}{|c|}{ stratigr. Reichweite } & \multirow{2}{*}{\multicolumn{2}{|c|}{$\begin{array}{l}\text { Familie/ } \\
\text { Gruppierungselement } \\
\text { Sum Anacardiaceae }\end{array}$}} \\
\hline & & & & \\
\hline $\begin{array}{l}\text { Tricolporopollenites } \\
\text { marcodurensis }\end{array}$ & L. Pliocene & E. Eocene & Cissus? & Vitaceae \\
\hline Tricolporopollenites edmundi & L. Pliocene & E. Eocene & Mastixia? & Cornaceae? \\
\hline $\begin{array}{l}\text { Tricolporopollenites } \\
\text { vancampoae }\end{array}$ & M. Eocene & E. Eocene & Monotes? & Dipterocarpaceae? \\
\hline Spinaepollis spinosus & L. Miocene & E. Paleocene & & Euphorbiaceae? \\
\hline Ilexpollenites iliacus & rezent & E. Eocene & Ilex & Aquifoliaceae \\
\hline \multirow[t]{2}{*}{ Ilexpollenites margaritatus } & L. Pliocene & E. Eocene & Ilex? & Aquifoliaceae \\
\hline & & & & Aquifoliaceae \\
\hline $\begin{array}{l}\text { Tetracolporopollenites } \\
\text { manifestus }\end{array}$ & M. Miocene & E. Paleocene & Planchonella? & Sapotaceae \\
\hline $\begin{array}{l}\text { Tetracolporopollenites } \\
\text { sapotoides }\end{array}$ & M. Miocene & E. Eocene & Planchonella? & Sapotaceae \\
\hline Tetracolporopollenites obscurus & M. Miocene & E. Paleocene & Palaquium? & Sapotaceae \\
\hline $\begin{array}{l}\text { Tetracolporopollenites } \\
\text { kirchheimeri }\end{array}$ & L. Miocene & E. Paleocene & Pouteria? & Sapotaceae \\
\hline \multirow[t]{2}{*}{ Tetracolporopollenites abditus } & M. Eocene & M. Eocene & & Sapotaceae \\
\hline & & & & Sum Sapotaceae \\
\hline Aceripollenites microrugulatus & L. Pliocene & M. Eocene & Acer & Aceraceae \\
\hline Spinulaepollis arceuthobioides & L. Pliocene & M. Eocene & Arceuthobium & Loranthaceae \\
\hline Araliaceoipollenites reticuloides & L. Pliocene & E. Eocene & Hedera & Araliaceae \\
\hline \multirow[t]{2}{*}{ Droseridites echinosporis } & L. Eocene & M. Eocene & Nepenthes & Nepenthaceae \\
\hline & & & & Bruchwald \\
\hline Echinatisporis erinaceus & E. Eocene & E. Paleocene & Selaginella & Moose \\
\hline \multirow[t]{2}{*}{ Tegumentisporis sculpturoides } & M. Eocene & M. Eocene & Selaginella & Moose \\
\hline & & & & Moose \\
\hline $\begin{array}{l}\text { Punctilongisulcites } \\
\text { microechinatus }\end{array}$ & M. Eocene & M. Eocene & & Hydrocharitaceae \\
\hline Tricolpopollenites retiformis & L. Pliocene & L. Paleocene & Salix & Salicaceae \\
\hline \multirow[t]{2}{*}{$\begin{array}{l}\text { Tricolporopollenites } \\
\text { staresedloensis }\end{array}$} & L. Oligocene & E. Eocene & Salix? & Salicaceae? \\
\hline & & & & Sum Salicaceae \\
\hline Nyssapollenites kruschi & L. Pliocene & E. Eocene & Nyssa & Nyssaceae \\
\hline \multirow[t]{3}{*}{ Rhoipites angustus } & E. Oligocene & L. Paleocene & Nyssa? & Nyssaceae? \\
\hline & & & & Nyssaceae \\
\hline & & & & Auewald/Galeriewald \\
\hline Pityosporites labdacus & L. Pliocene & E. Eocene & Pinus & Pinaceae \\
\hline \multirow[t]{3}{*}{ Inaperturopollenites magnus } & L. Pliocene & E. Paleocene & Pseudotsuga? & Pinaceae \\
\hline & & & & Sum Pinaceae \\
\hline & & & & Sum Bergwald \\
\hline \multirow[t]{2}{*}{ Tricolpopollenites parmularius } & L. Pliocene & E. Paleocene & Eucommia? & Eucommiaceae? \\
\hline & & & & Sum Hochwald \\
\hline Spinizonocolpites echinatus & E. Oligocene & Cretaceous & Nypa & Palmae \\
\hline $\begin{array}{l}\text { Tricolporopollenites } \\
\text { mansfeldensis }\end{array}$ & M. Eocene & E. Paleocene & Rhizophora? & Rhizophoraceae? \\
\hline Diporoconia iszkaszentgyöergyi & L. Eocene & E. Eocene & Lyxia? & Apocynaceae? \\
\hline \multirow[t]{2}{*}{ Malvacipollis } & L. Eocene & E. Eocene & & Malvaceae? \\
\hline & & & & Mangrove \\
\hline Reevesiapollis eocaenicus & M. Eocene & E. Eocene & Reevesia & Sterculiaceae \\
\hline $\begin{array}{l}\text { Tricolporopollenites } \\
\text { pseudointergranulatus }\end{array}$ & L. Miocene & M. Eocene & Paracyclea? & Menispermaceae? \\
\hline \multirow[t]{2}{*}{ Thymelipollis retisculpturius } & L. Miocene & M. Eocene & Phaleria & Thymeliaceae \\
\hline & & & & Sum unbekannt \\
\hline Leiotriletes maxoides maxoides & L. Pliocene & E. Eocene & Lygodium & Farne \\
\hline Leiotriletes adriennis adriennis & M. Oligocene ? & E. Paleocene & Lygodium & Farne \\
\hline Leiotriletes microadriennis & M. Eocene & E. Eocene & Lygodium? & Farne \\
\hline Cicatricosisporites dorogensis & M. Oligocene ? & M. Paleocene? & Anemia? & Farne \\
\hline $\begin{array}{l}\text { Cicatricosisporites } \\
\text { paradorogensis }\end{array}$ & L. Oligocene & L. Paleocene & Ruffordia & Farne \\
\hline
\end{tabular}




\begin{tabular}{|c|c|c|c|c|}
\hline Taxa & \multicolumn{2}{|c|}{ stratigr. Reichweite } & \multirow[t]{2}{*}{ Gattung } & \multirow{2}{*}{$\begin{array}{l}\text { Familie/ } \\
\text { Gruppierungselement } \\
\text { Farne }\end{array}$} \\
\hline Triletes multivallatus & E. Pliocene & E. Eocene & & \\
\hline Baculatisporites quintus & Pleistocene & M. Eocene & Osmunda & Farne \\
\hline Baculatisporites primarius & L. Pliocene & E. Eocene & Osmunda & Farne \\
\hline Triplanosporites sinuosus & M. Miocene & L. Paleocene & & Farne \\
\hline $\begin{array}{l}\text { Microfoveolatosporis } \\
\text { granuloides }\end{array}$ & L. Eocene & M. Eocene & Schizaea & Farne \\
\hline $\begin{array}{l}\text { Cicatricososporites } \\
\text { pseudodorogensis }\end{array}$ & E. Oligocene & E. Eocene & Schizaea & Farne \\
\hline Laevigatosporites haardti & L. Miocene & E. Paleocene & Pronephrium? & Farne \\
\hline Laevigatosporites discordatus & M. Oligocene? & E. Paleocene & & Farne \\
\hline Verrucatosporites favus & L. Pliocene & E. Eocene & Polypodium ? & Farne \\
\hline Reticuloidosporites dentatus & M. Eocene & M. Eocene & & Farne \\
\hline Camarozonosporites decorus & L. Pliocene & M. Eocene & $\begin{array}{l}\text { Lycopodium } \\
\text { cernuum }\end{array}$ & Farne \\
\hline \multirow[t]{2}{*}{$\begin{array}{l}\text { Camarozonosporites } \\
\text { heskemensis }\end{array}$} & L. Oligocene & E. Paleocene & $\begin{array}{l}\text { Lycopodium } \\
\text { cernuum }\end{array}$ & Farne \\
\hline & & & & Farne \\
\hline Monocolpopollenites tranquillus & E. Miocene & L. Paleocene & Phoenix & Palmae \\
\hline Monocolpopollenites magnus & M. Eocene & M. Eocene & & Palmae \\
\hline Arecipites & L. Eocene & E. Eocene & Sabal? & Palmae \\
\hline \multirow{2}{*}{ Dicolpopollis kockeli } & E. Miocene & E. Eocene & Calamus? & Palmae \\
\hline & & & & Palmae \\
\hline $\begin{array}{l}\text { Compositoipollenites } \\
\text { rhizophorus }\end{array}$ & L. Eocene & L. Paleocene & Polyporandra & Icacinaceae \\
\hline Pistillipollenites mcgregorii & L. Eocene & M. Paleocene? & Rusbyantes? & Gentianaceae? \\
\hline $\begin{array}{l}\text { Tricolpopollenites liblarensis } \\
\text { liblarensis }\end{array}$ & L. Miocene & E. Paleocene & Quercus? & Fagaceae? \\
\hline Tricolpopollenites microhenrici & L. Pliocene & E. Paleocene & Quercus? & Fagaceae? \\
\hline Tricolpopollenites quisqualis & L. Oligocene & E. Paleocene & & Fagaceae \\
\hline Tricolpopollenites henrici & L. Miocene & L. Eocene & Quercus? & Fagaceae? \\
\hline Tricolpopollenites asper & L. Pliocene & E. Eocene & Quercus & Fagaceae \\
\hline $\begin{array}{l}\text { Tricolporopollenites cingulum } \\
\text { fusus }\end{array}$ & E. Miocene & E. Paleocene & Castanopsis? & Fagaceae \\
\hline $\begin{array}{l}\text { Tricolporopollenites cingulum } \\
\text { pusillus }\end{array}$ & L. Pliocene & E. Eocene & Castanopsis & Fagaceae \\
\hline \multirow[t]{2}{*}{$\begin{array}{l}\text { Tricolporopollenites cingulum } \\
\text { oviformis }\end{array}$} & L. Pliocene & L. Paleocene & Castanopsis & Fagaceae \\
\hline & & & & Fagaceae \\
\hline Tricolpopollenites vegetus & Pleistocene & L. Paleocene & & Hamamelidaceae? \\
\hline \multirow[t]{2}{*}{ Tricolporopollenites microporitus } & L. Eocene & E. Paleocene & Corylopsis & Hamamelidaceae \\
\hline & & & & Sum Hamamelidaceae \\
\hline $\begin{array}{l}\text { Tricolporopollenites } \\
\text { microreticulatus }\end{array}$ & L. Pliocene & E. Eocene & Fraxinus? & Oleaceae? \\
\hline \multirow[t]{2}{*}{ Cupressacites insulipapillatus } & L. Miocene & M. Eocene & Thujopsis? & Cupressaceae \\
\hline & & & & azonal undifferenziert \\
\hline Cycadopites lusaticus & M. Miocene & M. Eocene & & $\begin{array}{l}\text { Cycas-Ginkgo-Encephalitus- } \\
\text { Zamia-Formen? }\end{array}$ \\
\hline $\begin{array}{l}\text { Pompeckjoidaepollenites } \\
\text { subhercynicus }\end{array}$ & L. Eocene & L. Paleocene & & Normapolles \\
\hline Plicapollis pseudoexcelsus & L. Eocene & E. Paleocene & & Normapolles \\
\hline Subriporopollenites scissus & M. Eocene & E. Eocene & & Normapolles \\
\hline \multirow[t]{2}{*}{ Thomsonipollenites magnificus } & M. Eocene & E. Paleocene & & Normapolles \\
\hline & & & & Normapolles \\
\hline Labrapollis labraferus & L. Eocene & E. Paleocene & & \\
\hline Tricolporopollenites laevigatus & M. Eocene & E. Paleocene & & \\
\hline Tricolporopollenites cognitus & M. Oligocene? & E. Eocene & & \\
\hline Tricolporopollenites baculatus & M. Eocene & E. Paleocene & & \\
\hline Polycolpites micropunctatus & M. Eocene & E. Eocene & & \\
\hline Polycolpites helmstedtensis & L. Eocene & E. Eocene & & \\
\hline Reticulataepollis intergranulatus & L. Eocene & E. Eocene & & \\
\hline Phytoplankton indet. & & & & \\
\hline
\end{tabular}




\begin{tabular}{|llll|}
\hline Taxa & stratigr. Reichweite & Gattung & Familie/ \\
\hline Dinoflagellaten & & \\
\hline Planctonites & & \\
\hline Prasinophyceae & & \\
\hline Acritarcha & & & \\
\hline varia & & & Botryococcaceae \\
\hline Botryococcus braunii & rezent & Cretaceous & Botryococcus \\
\hline Ovoidites elongatus & rezent & Cretaceous & Zygnemataceae \\
\hline & & & Sum Accessorien \\
\hline
\end{tabular}

\subsubsection{Profil Schöningen}

\begin{tabular}{|lll|}
\hline Statistik: Botanische Zugehörigkeit & Gattungen & Familien \\
\hline unbekannt (Anzahl) & 108 & 74 \\
\hline fraglich (Anzahl) & 38 & 46 \\
\hline bekannt (Anzahl) & 153 & 145 \\
\hline$\%$ (bekannt(incl. fraglich)/unbekannt) & $80,1 \%$ & $75,9 \%$ \\
\hline$\%$ (bekannt(excl. fraglich)/unbekannt) & $60,2 \%$ & $51,8 \%$ \\
\hline
\end{tabular}

\begin{tabular}{|c|c|c|c|c|}
\hline Taxa & \multicolumn{2}{|c|}{ stratigr. Reichweite } & Gattung & $\begin{array}{l}\text { Familie/ } \\
\text { Gruppierungselement }\end{array}$ \\
\hline $\begin{array}{l}\text { Sparganiaceaepollenites } \\
\text { sparganoides }\end{array}$ & M. Oligocene? & E. Eocene & Sparganium? & Sparganiaceae? \\
\hline Cyperaceaepollis piriformis & L. Eocene & E. Eocene & Cladium? & Cyperaceae \\
\hline \multirow{2}{*}{ Nympheapollenites } & L. Eocene & E. Eocene & Nymphea & Nymphaeaceae \\
\hline & & & & Sum lake succession \\
\hline Stereisporites verrugranulus & L. Pliocene & Cretaceous & Sphagnum & Sphagnaceae \\
\hline Stereisporites eovalidus & SPP12? & SPP11? & Sphagnum & Sphagnaceae \\
\hline \multirow[t]{3}{*}{ Stereisporites breviancoris } & L. Pliocene & E. Eocene & Sphagnum & Sphagnaceae \\
\hline & & & & Sum Sphagnaceae \\
\hline & & & & Sum bog \\
\hline Milfordia incerta & M. Miocene & E. Paleocene & Leptocarpus? & Restionaceae \\
\hline $\begin{array}{l}\text { Tricolporopollenites } \\
\text { megaexactus brühlensis }\end{array}$ & L. Miocene & E. Eocene & Cliftonia & Cyrillaceae \\
\hline \multirow{4}{*}{ Ericipites callidus } & rezent & L. Paleocene & Erica? & Ericaceae \\
\hline & L. Pliocene & L. Paleocene & & Ericaceae \\
\hline & & & & Sum Ericaceae \\
\hline & & & & Sum reed \\
\hline Leiotriletes maxoides maxoides & L. Pliocene & E. Eocene & Lygodium & Schizaeaceae \\
\hline Leiotriletes microadriennis & M. Eocene & E. Eocene & Lygodium? & Schizaeaceae? \\
\hline Triletes multivallatus & E. Pliocene & E. Eocene & & Schizaeaceae? \\
\hline Ischyossporites asolidus & L. Pliocene & L. Paleocene & Lygodium? & Schizaeaceae? \\
\hline \multirow[t]{2}{*}{ Cicatricosisporites dorogensis } & M. Oligocene? & M. Paleocene? & Anemia? & Schizaeaceae? \\
\hline & & & & Sum Schizaeaceae \\
\hline Baculatisporites gemmatus & L. Eocene & E. Paleocene & & Osmundaceae? \\
\hline \multirow[t]{2}{*}{ Baculatisporites primarius } & L. Pliocene & E. Eocene & Osmunda & Osmundaceae \\
\hline & & & & Sum Osmundaceae \\
\hline Retitriletes rueterbergensis & rezent & E. Paleocene & Lycopodium & Lycopodiaceae \\
\hline \multirow[t]{2}{*}{$\begin{array}{l}\text { Camarozonosporites } \\
\text { heskemensis }\end{array}$} & L. Oligocene & E. Paleocene & $\begin{array}{l}\text { Lycopodium } \\
\text { cernuum }\end{array}$ & Lycopodiaceae \\
\hline & & & & Sum Lycopodiaceae \\
\hline $\begin{array}{l}\text { Sciadopityspollenites } \\
\text { eocaenicus }\end{array}$ & M. Eocene & M. Eocene & Sciadopitys & Taxodiaceae \\
\hline $\begin{array}{l}\text { Inaperturopollenites } \\
\text { concedipites }\end{array}$ & L. Pliocene & E. Eocene & Glyptostrobus? & Taxodiaceae? \\
\hline Inaperturopollenites hiatus & L. Pliocene & E. Paleocene & Glyptostrobus & Taxodiaceae \\
\hline Sequoiapollenites polyformus & L. Pliocene & E. Eocene & Metasequoia & Taxodiaceae \\
\hline & & & & Sum Taxodiaceae \\
\hline
\end{tabular}




\begin{tabular}{|c|c|c|c|c|}
\hline Taxa & stratigr. $\mathrm{Re}$ & iweite & Gattung & $\begin{array}{l}\text { Familie/ } \\
\text { Gruppierungselement }\end{array}$ \\
\hline \multirow[t]{2}{*}{ Arecipites butomoides } & L. Pliocene & E. Pliocene & Butomus? & Butomaceae \\
\hline & & & & Sum swamp \\
\hline Smilacipites echinatus & M. Miocene & E. Eocene & Smilax & Smilacaceae \\
\hline Emmapollis pseudoemmaensis & L. Pliocene & Cretaceous & Ascarinopsis? & Chloranthaceae? \\
\hline Magnolipollis megafiguratus & L. Pliocene & E. Eocene & Magnolia? & Magnoliaceae \\
\hline Nudopollis terminalis & M. Eocene & L. Paleocene & & Myricaceae? \\
\hline Plicapollis pseudoexelsus & L. Eocene & E. Paleocene & & Myricaceae? \\
\hline Triatriopollenites rurensis & L. Pliocene & E. Eocene & Myrica & Myricaceae \\
\hline Triatriopollenites bituitus & E. Pliocene & E. Eocene & Myrica & Myricaceae \\
\hline \multirow[t]{2}{*}{$\begin{array}{l}\text { Triporopollenites } \\
\text { paleobetuloides }\end{array}$} & SPP11? & SPP11? & Myrica & Myricaceae \\
\hline & & & & Sum Myricaceae \\
\hline Triporopollenites coryloides & L. Pliocene & E. Eocene & Corylus & Betulaceae \\
\hline Plicatopollis plicatus & L. Eocene & E. Eocene & & Juglandaceae? \\
\hline Momipites punctatus & L. Pliocene & E. Paleocene & Engelhardtia? & Juglandaceae \\
\hline Momipites quietus & M. Oligocene? & M. Paleocene? & Engelhardtia & Juglandaceae \\
\hline $\begin{array}{l}\text { Platycaryapollenites } \\
\text { platycaryoides }\end{array}$ & L. Eocene & M. Paleocene? & Platycarya & Juglandaceae \\
\hline $\begin{array}{l}\text { Platycaryapollenites } \\
\text { miocaenicus }\end{array}$ & L. Miocene & E. Eocene & Platycarya & Juglandaceae \\
\hline Caryapollenites circulus & L. Eocene & E. Paleocene & Carya & Juglandaceae \\
\hline Caryapollenites triangulus & L. Eocene & L. Paleocene & Carya & Juglandaceae \\
\hline Pterocaryapollenites stellatus & Pleistocene & M. Eocene & Platycarya? & Juglandaceae \\
\hline Subtriporopollenites subporatus & E. Eocene & M. Paleocene? & & Juglandaceae? \\
\hline $\begin{array}{l}\text { Subtriporopollenites } \\
\text { magnoporatus }\end{array}$ & L. Paleocene & E. Paleocene & & Juglandaceae? \\
\hline \multirow[t]{2}{*}{ Subtriporopollenites constans } & M. Eocene & E. Eocene & & Juglandaceae? \\
\hline & & & & Sum Juglandaceae \\
\hline Celtipollenites intrastructurus & M. Miocene & L. Paleocene & Celtis & Ulmaceae \\
\hline Intratriporopollenites instructus & L. Miocene & E. Paleocene & Tilia & Tiliaceae \\
\hline Anacolosidites efflatus & L. Eocene & E. Eocene & Anacolosa & Olacaceae \\
\hline $\begin{array}{l}\text { Porocolpopollenites } \\
\text { rarobaculatus }\end{array}$ & L. Miocene & E. Eocene & Symplocos & Symplocaceae \\
\hline \multirow[t]{2}{*}{ Porocolpopollenites vestibulum } & L. Pliocene & E. Eocene & Symplocos? & Symplocaceae? \\
\hline & & & & Sum Symplocaceae \\
\hline Periporopollenites stigmosus & L. Pliocene & E. Eocene & Liquidambar? & Hamamelidaceae? \\
\hline \multirow[t]{2}{*}{ Tricolpopollenites vegetus } & Pleistocene & L. Paleocene & & Hamamelidaceae? \\
\hline & & & & Sum Hamamelidaceae \\
\hline $\begin{array}{l}\text { Tricolporopollenites } \\
\text { pseudocingulum }\end{array}$ & L. Miocene & E. Eocene & & Anacardiaceae? \\
\hline \multirow[t]{2}{*}{$\begin{array}{l}\text { Tricolporopollenites solé-de- } \\
\text { portai }\end{array}$} & E. Miocene & M. Paleocene? & & Anacardiaceae? \\
\hline & & & & Sum Anacardiaceae \\
\hline $\begin{array}{l}\text { Tricolporopollenites } \\
\text { satzveyensis }\end{array}$ & L. Pliocene & E. Paleocene & Mastixia? & Cornaceae? \\
\hline \multirow[t]{2}{*}{ Tricolporopollenites edmundi } & L. Pliocene & E. Eocene & Mastixia? & Cornaceae? \\
\hline & & & & Sum Cornaceae \\
\hline $\begin{array}{l}\text { Tricolporopollenites } \\
\text { marcodurensis }\end{array}$ & L. Pliocene & E. Eocene & Cissus? & Vitaceae \\
\hline Tricolporopollenites messelensis & L. Eocene & E. Eocene & Ligustrum? & Olacaceae? \\
\hline $\begin{array}{l}\text { Tricolporopollenites } \\
\text { vancampoae }\end{array}$ & M. Eocene & E. Eocene & Monotes? & Dipterocarpaceae? \\
\hline Spinaepollis spinosus & L. Miocene & E. Paleocene & & Euphorbiaceae? \\
\hline Araliaceoipollenites profundus & L. Eocene & E. Eocene & Euphorbia? & Euphorbiaceae? \\
\hline & & & & Sum Euphorbiaceae \\
\hline Spinulaepollis arceuthobioides & L. Pliocene & M. Eocene & Arceuthobium & Loranthaceae \\
\hline Ilexpollenites iliacus & rezent & E. Eocene & Ilex & Aquifoliaceae \\
\hline Ilexpollenites margaritatus & L. Pliocene & E. Eocene & Ilex? & Aquifoliaceae \\
\hline & & & & Sum Aquifoliaceae \\
\hline Tetracolporopollenites & M. Miocene & E. Eocene & Planchonella? & Sapotaceae \\
\hline
\end{tabular}




\begin{tabular}{|c|c|c|c|c|}
\hline Taxa & \multicolumn{2}{|c|}{ stratigr. Reichweite } & Gattung & $\begin{array}{l}\text { Familie/ } \\
\text { Gruppierungselement }\end{array}$ \\
\hline \multicolumn{5}{|l|}{ sapotoides } \\
\hline \multicolumn{5}{|l|}{$\begin{array}{l}\text { Tetracolporopollenites } \\
\text { manifestus }\end{array}$} \\
\hline \multirow[t]{3}{*}{$\begin{array}{l}\text { Tetracolporopollenites } \\
\text { kirchheimeri }\end{array}$} & L. Miocene & E. Paleocene & Pouteria? & Sapotaceae \\
\hline & & & & Sum Sapotaceae \\
\hline & & & & Sum mire forest \\
\hline Neogenisporis pseudoneddeni & L. Pliocene & E. Eocene & & Polypodiaceae? \\
\hline Laevigatosporites haardti & L. Miocene & E. Paleocene & Pronephrium? & Polypodiaceae? \\
\hline Laevigatosporites discordatus & M. Oligocene? & E. Paleocene & & Polypodiaceae \\
\hline Laevigatosporites nutidus & L. Pliocene & E. Eocene & & Polypodiaceae \\
\hline \multirow[t]{2}{*}{ Verrucatosporites favus } & L. Pliocene & E. Eocene & Polypodium? & Polypodiaceae \\
\hline & & & & Sum Polypodiaceae \\
\hline Tricolpopollenites retiformis & L. Pliocene & L. Paleocene & Salix & Salicaceae \\
\hline Nyssapollenites kruschi & L. Pliocene & E. Eocene & Nyssa & Nyssaceae \\
\hline \multirow[t]{2}{*}{ Rhoipites angustus } & E. Oligocene & L. Paleocene & Nyssa? & Nyssaceae? \\
\hline & & & & Sum Nyssaceae \\
\hline Pityosporites labdacus & L. Pliocene & E. Eocene & Pinus & Pinaceae \\
\hline Zonalapollenites igniculus & L. Pliocene & E. Eocene & Tsuga & Pinaceae \\
\hline \multirow[t]{3}{*}{ Inaperturopollenites magnus } & L. Pliocene & E. Paleocene & Pseudotsuga? & Pinaceae \\
\hline & & & & Sum Pinaceae \\
\hline & & & & Sum montane forest \\
\hline Tricolpopollenites parmularius & L. Pliocene & E. Paleocene & Eucommia? & Eucommiaceae? \\
\hline \multirow[t]{2}{*}{ Rousea araneosa } & L. Eocene & E. Eocene & & Bignoniaceae? \\
\hline & & & & Sum high canopy forest \\
\hline Malvacipollis tschudyi & L. Eocene & E. Eocene & & Malvaceae \\
\hline \multirow[t]{2}{*}{$\begin{array}{l}\text { Tricolporopollenites } \\
\text { mansfeldensis }\end{array}$} & M. Eocene & E. Paleocene & Rhizophora? & Rhizophoraceae? \\
\hline & & & & Sum mangrove \\
\hline \multirow[t]{2}{*}{ Reevesiapollis triangulus } & L. Pliocene & E. Eocene & Reevesia & Sterculiaceae \\
\hline & & & & Sum unknown \\
\hline Toroisporis neddeni & L. Eocene & E. Eocene & & Gleicheniaceae? \\
\hline \multirow[t]{2}{*}{ Concavisporites rugulatus } & E. Eocene & E. Paleocene & & Gleicheniaceae? \\
\hline & & & & Sum Gleicheniaceae \\
\hline Ephedrepites treplinensis & E. Miocene & L. Oligocene & Ephedra & Ephedraceae \\
\hline Monocolpopollenites tranquillus & E. Miocene & L. Paleocene & Phoenix & Palmae \\
\hline \multirow[t]{2}{*}{ Dicolpopollis kockeli } & E. Miocene & E. Eocene & Calamus? & Palmae \\
\hline & & & & Sum Palmae \\
\hline $\begin{array}{l}\text { Compositoipollenites } \\
\text { rhizophorus }\end{array}$ & L. Eocene & L. Paleocene & Polyporandra & Icacinaceae \\
\hline Pistillipollenites mcgregorii & L. Eocene & M. Paleocene? & Rusbyantes? & Gentianaceae? \\
\hline Triporopollenites rhenanus & L. Pliocene & E. Eocene & Ostrya & Betulaceae \\
\hline Triporopollenites robustus & L. Oligocene & E. Paleocene & & Betulaceae? \\
\hline Triporopollenites megagranifer & L. Oligocene & E. Paleocene & & Betulaceae? \\
\hline \multirow[t]{2}{*}{ Polyvestibulopollenites verus } & rezent & E. Eocene & Alnus & Betulaceae \\
\hline & & & & Sum Betulaceae \\
\hline $\begin{array}{l}\text { Tricolpopollenites liblarensis } \\
\text { liblarensis }\end{array}$ & L. Miocene & E. Paleocene & Quercus? & Fagaceae? \\
\hline Tricolpopollenites microhenrici & L. Pliocene & E. Paleocene & Quercus? & Fagaceae? \\
\hline Tricolpopollenites asper & L. Pliocene & E. Eocene & Quercus & Fagaceae \\
\hline $\begin{array}{l}\text { Tricolporopollenites cingulum } \\
\text { fusus }\end{array}$ & E. Miocene & E. Paleocene & Castanopsis? & Fagaceae \\
\hline $\begin{array}{l}\text { Tricolporopollenites cingulum } \\
\text { pusillus }\end{array}$ & L. Pliocene & E. Eocene & Castanopsis & Fagaceae \\
\hline & & & & Sum Fagaceae \\
\hline $\begin{array}{l}\text { Tricolporopollenites } \\
\text { microreticulatus }\end{array}$ & L. Pliocene & E. Eocene & Fraxinus? & Oleaceae? \\
\hline Cupressacites cuspidataeformis & L. Pliocene & M. Eocene & Juniperus & Cupressaceae \\
\hline Cupressacites bockwitzensis & L. Pliocene & M. Miocene & Juniperus? & Cupressaceae \\
\hline
\end{tabular}




\begin{tabular}{|c|c|c|c|c|}
\hline \multirow[t]{3}{*}{ Taxa } & \multicolumn{2}{|c|}{ stratigr. Reichweite } & Gattung & $\begin{array}{l}\text { Familie/ } \\
\text { Gruppierungselement }\end{array}$ \\
\hline & & & & Sum Cupressaceae \\
\hline & & & & Sum azonal undifferenziert \\
\hline Monoleiotriletes gracilis & M. Oligocene? & Cretaceous & & \\
\hline Triplanosporites sinuosus & M. Miocene & L. Paleocene & & \\
\hline $\begin{array}{l}\text { Punctatisporites ?Crassimaximu } \\
\text { s }\end{array}$ & E. Miocene & E. Eocene & & \\
\hline Stereisporites rueterbergensis & L. Pliocene & E. Eocene & & \\
\hline Stereisporites cingulatus & L. Pliocene & E. Eocene & & \\
\hline \multicolumn{5}{|l|}{ Stereisporites sp.1 } \\
\hline \multicolumn{5}{|l|}{ Toroisporis sp.1 } \\
\hline \multicolumn{5}{|l|}{ Toroisporis sp.2 } \\
\hline \multicolumn{5}{|l|}{ Trilete Spore sp.1 } \\
\hline Foveasporis agathoecus & M. Eocene & E. Paleocene & & \\
\hline \multicolumn{5}{|l|}{ Foveotriletes sp. } \\
\hline \multicolumn{5}{|l|}{ Trilete Sporen } \\
\hline Laevigatosporites ?bisulcatoides & L. Miocene & E. Eocene & & \\
\hline \multicolumn{5}{|c|}{ Laevigatosporites sp.1 } \\
\hline \multicolumn{5}{|l|}{ Laevigatosporites sp.2 } \\
\hline \multicolumn{5}{|l|}{ Verrucatosporites favus1 } \\
\hline Extrapunctatosporis maximus & L. Pliocene & E. Eocene & & \\
\hline \multicolumn{5}{|l|}{ Verrucate varia } \\
\hline Classopollis & Cretaceous & Cretaceous & & Cheirolepidaceae \\
\hline \multicolumn{5}{|l|}{ Pollenkorn mit Saum } \\
\hline \multicolumn{5}{|l|}{ Inaperturpoll. sp.A } \\
\hline \multicolumn{5}{|l|}{ Inaperturate Pollenformen } \\
\hline \multicolumn{5}{|l|}{$\begin{array}{l}\text { Ephedrepites sp. senso } \\
\text { Krutzsch }\end{array}$} \\
\hline Graminidites & rezent & Cretaceous & & Gramineae \\
\hline Zircipollenites globosus & M. Eocene & E. Eocene & & \\
\hline \multicolumn{5}{|l|}{ Cycadopites 1} \\
\hline \multicolumn{5}{|l|}{ Cycadopites gracilis } \\
\hline Arecipites convexus & M. Miocene & E. Eocene & Sabal & Arecaceae \\
\hline \multicolumn{5}{|l|}{ Magnoliaformen1 } \\
\hline \multicolumn{5}{|l|}{ Magnoliaformen } \\
\hline Basopollis basalis & M. Eocene & E. Paleocene & & Juglandales? \\
\hline Basopollis atumescens & M. Eocene & E. Paleocene & & Juglandales? \\
\hline & & & & Sum Juglandales \\
\hline Triatriopollenites hannoverensis & E. Eocene & E. Paleocene & & Myricales? \\
\hline Vacuopollis concavus & E. Eocene & E. Paleocene & & Myricales? \\
\hline Vacuopollis microconcavus & E. Eocene & E. Paleocene & & Myricales? \\
\hline & & & & Sum Myricales \\
\hline $\begin{array}{l}\text { Pompeckjoidaepollenites } \\
\text { subhercynicus }\end{array}$ & L. Eocene & L. Paleocene & & Sum Juglandales \\
\hline Triatriopoll. quietus & & & & Sum Myricales \\
\hline Triatriopoll. sp. & & & & \\
\hline Subriporopollenites scissus & M. Eocene & E. Eocene & & \\
\hline Subriporopoll. sp. & & & & \\
\hline Triporopoll. sp.1 & & & & \\
\hline Trivestibulopollenites veloides & L. Miocene & M. Paleocene? & & \\
\hline Thomsonipollenites magnificus & M. Eocene & E. Paleocene & & \\
\hline Interpollis microsupplingensis & M. Eocene & E. Paleocene & & \\
\hline Bombacacidites & L. Eocene & E. Eocene & Bombax & Bombacaceae \\
\hline Labrapollis rotundoides & SPP11? & SPP11? & & \\
\hline Labrapollis labraferus & L. Eocene & E. Paleocene & & \\
\hline Porocolpopollenites & & & & \\
\hline
\end{tabular}




\begin{tabular}{|c|c|c|}
\hline Taxa & stratigr. Reichweite & $\begin{array}{l}\text { Familie/ } \\
\text { Gruppierungselement }\end{array}$ \\
\hline \multicolumn{3}{|l|}{ hemicolpites } \\
\hline \multicolumn{3}{|l|}{ Porocolpopollenites sp. } \\
\hline \multicolumn{3}{|l|}{ Symplocospollenites rotundus } \\
\hline Pentapollenites pentangulus & M. Oligocene? & \\
\hline \multicolumn{3}{|l|}{ Periporopoll. echinatus } \\
\hline Erdtmanipollis pachysandroides & L. Pliocene & Pachysandra \\
\hline Persicarioipollis persicarioides & E. Paleocene & Loganiaceae? \\
\hline Duplopollis golzowense & E. Paleocene & Sapindaceae? \\
\hline Tricolporopollenites belgicus & L. Paleocene & \\
\hline Tricolporopollenites eofagoides & E. Eocene & Rubiaceae? \\
\hline $\begin{array}{l}\text { Tricolporopollenites } \\
\text { striatoreticulatus }\end{array}$ & M. Miocene & \\
\hline Tricolporopollenites ornatus & E. Eocene & \\
\hline \multicolumn{3}{|l|}{$\begin{array}{l}\text { Tricolpopoll. sp. } 2 \text { senso B. } \\
\text { Nickel }\end{array}$} \\
\hline \multicolumn{3}{|l|}{ Tricolpopoll. sp.1 } \\
\hline \multicolumn{3}{|l|}{ Aceripollenites cf. striatus } \\
\hline Retitricolpites pflugii & L. Paleocene & \\
\hline Polycolpites helmstedtensis & E. Eocene & \\
\hline \multicolumn{3}{|l|}{ Tricolporopoll. oleoides } \\
\hline \multicolumn{3}{|l|}{ Tricolporopoll. cf. megaporatus } \\
\hline \multicolumn{3}{|l|}{ Tricolporopoll. striatopunctatus } \\
\hline \multicolumn{3}{|l|}{ Tricolporopoll. sp.2 } \\
\hline \multicolumn{3}{|l|}{ Tricolporopoll. sp.5 } \\
\hline \multicolumn{3}{|l|}{ Tricolporopoll. sp.6 } \\
\hline \multicolumn{3}{|l|}{ Tricolporopoll. sp.9 } \\
\hline \multicolumn{3}{|l|}{ Tricolporopoll. sp.12 } \\
\hline \multicolumn{3}{|l|}{ Tricolporopoll. sp.13 } \\
\hline \multicolumn{3}{|l|}{ Tricolporopoll. sp.15 } \\
\hline \multicolumn{3}{|l|}{ Tricolporopoll. sp.19 } \\
\hline \multicolumn{3}{|l|}{ Tricolporopoll. sp.28 } \\
\hline \multicolumn{3}{|l|}{ Tricolporopoll. sp.30 } \\
\hline \multicolumn{3}{|l|}{ Tricolporopoll. sp.31 } \\
\hline \multicolumn{3}{|l|}{ Tricolporopoll. sp.32 } \\
\hline \multicolumn{3}{|l|}{ Tricolporopoll. sp.35 } \\
\hline \multicolumn{3}{|l|}{$\begin{array}{l}\text { Nuxpollenites sp. senso } \\
\text { Frederiksen }\end{array}$} \\
\hline Verrutricolporites cruciatus & & \\
\hline Botryococcus braunii & Cretaceous & Botryococcaceae \\
\hline Planctonites & & \\
\hline Phytoplankton indet. & & \\
\hline Ovoidites elongatus & & \\
\hline Dinoflagellaten & & \\
\hline Acritarcha & & \\
\hline Prasinophyceae & & \\
\hline varia & & \\
\hline Plankton & & \\
\hline & & Sum Accessorien \\
\hline
\end{tabular}




\subsection{Gruppierungelemente}

Nachfolgend werden die Gruppierungselemente der Familien dargestellt.

Erläuterung der Abkürzungen:

F: Informationen sind botanischen Familien zugeordnet

P: höchste Priorität, erste Eingabe im Gruppierungsformular

?: Familienzugehörigkeit ungewiß

\subsubsection{Profil Alversdorf}

\begin{tabular}{|c|c|c|c|c|c|c|c|c|c|c|c|c|c|c|c|c|c|c|c|c|c|c|c|c|c|}
\hline & 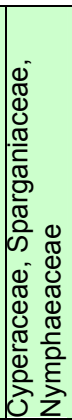 & 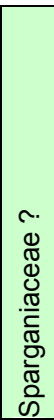 & 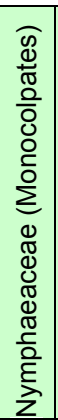 & 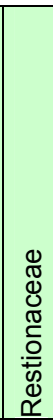 & 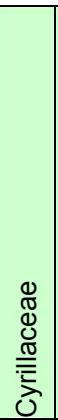 & 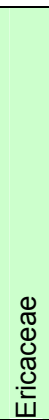 & 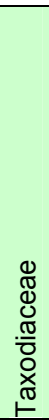 & 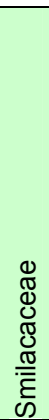 & 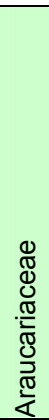 & 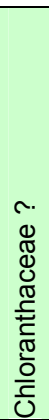 & 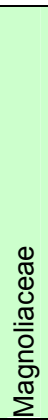 & 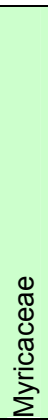 & 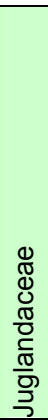 & 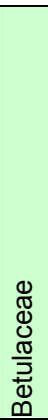 & 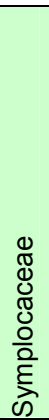 & 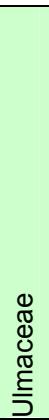 & 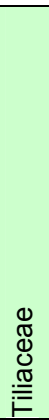 & 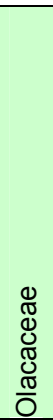 & 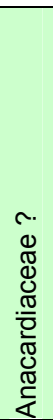 & $\begin{array}{l}0 \\
\mathbb{D} \\
\mathbb{8} \\
\mathbb{\pi} \\
\stackrel{ \pm}{ \pm}\end{array}$ & 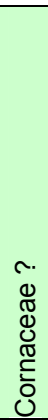 & 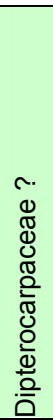 & 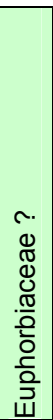 & 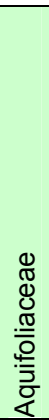 & $\begin{array}{l}0 \\
\mathbb{8} \\
8 \\
0 \\
\pi \\
\mathbb{0} \\
\frac{8}{\pi} \\
\mathbb{0}\end{array}$ \\
\hline Taxongruppe & & & & & & & & & & & & & & & & & & & & & & & & & \\
\hline Angiospermae & FP & FP & FP & FP & FP & FP & & FP & & FP & FP & FP & FP & FN & FP & FP & FP & FP & FP & FP & FP & FP & FP & FP & FP \\
\hline Gymnospermae & & & & & & & FP & & FP & & & & & & & & & & & & & & & & \\
\hline Pollination & & & & & & & & & & & & & & & & & & & & & & & & & \\
\hline Anemophilie & & & & FP & & FP & FP & & FP & FP & & FP & FP & $\mathrm{FN}$ & $\mathrm{FP}$ & $\mathrm{FP}$ & FP & FP & & FP & & & & & FP \\
\hline Zoophilie & FP & FP & FP & & & & & FP & & $\mathrm{F}$ & FP & & & & & & & & & & & FP & FP & FP & \\
\hline Hydrocherie & $F$ & & & & & & & & & & & & & & & & & & & & & & & & \\
\hline sonstige & $F$ & & & & & & & & & & & & & & & & & & & & & & & & \\
\hline Pflanzenhabitus & & & & & & & & & & & & & & & & & & & & & & & & & \\
\hline Bäume (>8m) & & & & & & $\mathrm{F}$ & $\mathrm{FP}$ & & $\mathrm{FP}$ & $\mathrm{F}$ & $\mathrm{FP}$ & FP & FP & $\mathrm{F}$ & & FP & FP & FP & $\mathrm{F}$ & & FP & FP & $F$ & FP & FP \\
\hline Sträucher (2-8m) & & & & & FP & $\mathrm{F}$ & & & & FP & $F$ & $F$ & $\mathrm{~F}$ & FN & FP & $\mathrm{F}$ & $\mathrm{F}$ & & FP & $\mathrm{F}$ & $\mathrm{F}$ & & $\mathrm{F}$ & $\mathrm{F}$ & $\mathrm{F}$ \\
\hline Kleinsträucher $(<2 \mathrm{~m})$ & $\mathrm{F}$ & & & & & FP & & FP & & & & & & & & & & $\mathrm{F}$ & & & $\mathrm{F}$ & & & & \\
\hline Lianen & & & & & & & & $\mathrm{F}$ & & & & & & & $\mathrm{F}$ & & & $\mathrm{F}$ & $\mathrm{F}$ & & & & & & \\
\hline grasartige Pflanzen & $\mathrm{FP}$ & FP & & & & & & & & & & & & & & & & & & & & & & & \\
\hline krautige Pflanzen & & & & FP & & & & & & $\mathrm{F}$ & & & & & & & $\mathrm{F}$ & & & & $\mathrm{F}$ & & FP & & \\
\hline $\begin{array}{l}\text { emerse } \\
\text { Wasserpflanzen }\end{array}$ & & & FP & & & & & & & & & & & & & & & & & & & & & & \\
\hline Kletterpflanzen & & & & & & & & & & & & & & & & & & & & $\mathrm{FP}$ & & & & & \\
\hline Klimatyp & & & & & & & & & & & & & & & & & & & & & & & & & \\
\hline $\begin{array}{l}\text { tropisches } \\
\text { immerfeuchtes Klima }\end{array}$ & & & FP & FP & FP & & $\mathrm{FP}$ & FP & & FP & FP & FP & & & FP & & & FP & FP & $\mathrm{FP}$ & & FP & \multicolumn{2}{|l|}{ FP } & FP \\
\hline $\begin{array}{l}\text { tropisches } \\
\text { wechselfeuchtes } \\
\text { Klima mit extremer } \\
\text { Feuchtperiode im } \\
\text { Sommer }\end{array}$ & & $\mathrm{F}$ & & & & & & & & & & & & & & & $F$ & & & & & $F$ & & & \\
\hline $\begin{array}{l}\text { tropisches } \\
\text { wechselfeuchtes } \\
\text { Klima mit trockener } \\
\text { Periode im Winter }\end{array}$ & & & & & & $\mathrm{F}$ & & & & & & & & & & $\mathrm{F}$ & & & & & & $\mathrm{F}$ & & & \\
\hline $\begin{array}{l}\text { subtropisches } \\
\text { sommertrockenes } \\
\text { Klima }\end{array}$ & & & $\mathrm{F}$ & & & $\mathrm{F}$ & & & $\mathrm{FP}$ & $\mathrm{F}$ & $\mathrm{F}$ & & $\mathrm{FP}$ & $\mathrm{F}$ & & & & & & $\mathrm{F}$ & & & & FP & \\
\hline $\begin{array}{l}\text { subtropisches } \\
\text { immerfeuchtes Klima }\end{array}$ & & & & & $F$ & & G & $\mathrm{F}$ & & & & & & & $\mathrm{F}$ & & & $\mathrm{F}$ & $\mathrm{F}$ & $\mathrm{F}$ & $\mathrm{F}$ & & & & $\mathrm{F}$ \\
\hline $\begin{array}{l}\text { Ozeanisch } \\
\text { temperiertes Klima }\end{array}$ & & FP & & $\mathrm{F}$ & & & & $\mathrm{F}$ & & & $\mathrm{F}$ & $\mathrm{F}$ & $\mathrm{F}$ & & & $\mathrm{F}$ & $\mathrm{F}$ & & & & $\mathrm{FP}$ & & $\mathrm{F}$ & $\mathrm{F}$ & \\
\hline $\begin{array}{l}\text { Kontinental } \\
\text { temperiertes Klima }\end{array}$ & $\mathrm{FP}$ & & $\mathrm{F}$ & & & & G & & & & & & $F$ & & & FP & $\mathrm{FP}$ & & & & $\mathrm{F}$ & & $\mathrm{F}$ & $\mathrm{F}$ & \\
\hline Boreales Klima & & & & & & FP & & & & & & & & FN & & & & & & & & & & & \\
\hline Tundrenklima & $\mathrm{F}$ & $\mathrm{F}$ & & & & & & & & & & & & $\mathrm{F}$ & & & & & & & & & & & \\
\hline Florenreich & & & & & & & & & & & & & & & & & & & & & & & & & \\
\hline $\begin{array}{l}\text { Antarctis Neu } \\
\text { Seeland }\end{array}$ & & & & & & & & & & & & & & & & & & & & & & & FP & & \\
\hline $\begin{array}{l}\text { Australis trop. Nord- } \\
\text { und Ost-Australien }\end{array}$ & & & & & & & & & & & & & & & & & & & FP & & & & & & $\mathrm{F}$ \\
\hline Australis undiff. & & & & $\mathrm{F}$ & & & & & FP & & & & & & & & & & & & & & $\mathrm{F}$ & & \\
\hline Capensis undiff. & & & & FP & & & & $\mathrm{F}$ & & & & $\mathrm{F}$ & & & & & & & & & & & & & \\
\hline Holarktis undiff & & FP & & & & FP & FP & & & & FP & $\mathrm{F}$ & FP & & & $\mathrm{F}$ & & & & & FP & & & & \\
\hline $\begin{array}{l}\text { Neotropis } \\
\text { amazonisch }\end{array}$ & & & & & $F$ & & & & & & & & & & & & & & & & & & & & \\
\hline Neotropis karibisch & & & & & FP & & & & & & & & & & & & & & & & & & & & \\
\hline Neotropis undiff. & & & & & & $\mathrm{F}$ & $G$ & FP & $\mathrm{F}$ & FP & $\mathrm{F}$ & FP & $\mathrm{F}$ & & $\mathrm{F}$ & FP & & FP & $\mathrm{F}$ & FP & $\mathrm{F}$ & & $\mathrm{F}$ & $\mathrm{F}$ & FP \\
\hline Paleotropis Afrika & & & & & & & & & & & & & & & & & & & $\mathrm{F}$ & & & & & FP & \\
\hline Paleotropis undiff. & & & & $\mathrm{F}$ & & $\mathrm{F}$ & & $\mathrm{F}$ & $F$ & $\mathrm{~F}$ & $\mathrm{~F}$ & $\mathrm{~F}$ & $\mathrm{~F}$ & & FP & $\mathrm{F}$ & & $\mathrm{F}$ & & $\mathrm{F}$ & $\mathrm{F}$ & FP & $\mathrm{F}$ & & $\mathrm{F}$ \\
\hline
\end{tabular}




\begin{tabular}{|c|c|c|c|c|c|c|c|c|c|c|c|c|c|c|c|c|c|c|c|c|c|c|c|c|c|}
\hline & 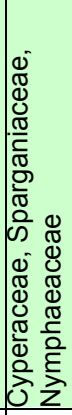 & 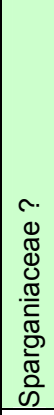 & 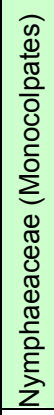 & 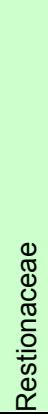 & 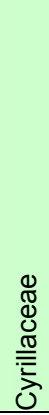 & 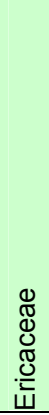 & 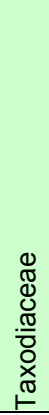 & 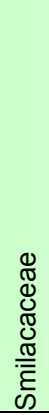 & 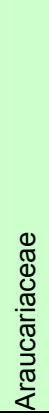 & 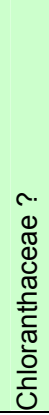 & 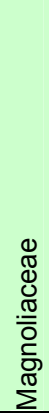 & 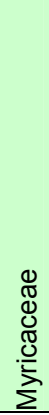 & $\begin{array}{l}0 \\
\mathbb{0} \\
0 \\
0 \\
\frac{\pi}{0} \\
\frac{1}{\pi} \\
\frac{\pi}{0} \\
\end{array}$ & 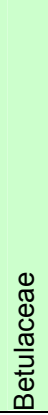 & 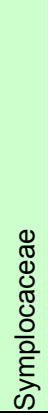 & 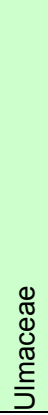 & 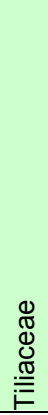 & $\begin{array}{l}\mathbb{\Phi} \\
\mathbb{D} \\
\mathbb{0} \\
\mathbb{ల} \\
\mathbb{ত} \\
0\end{array}$ & 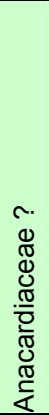 & 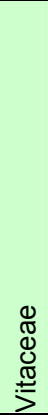 & 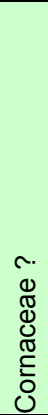 & 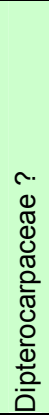 & 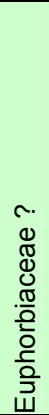 & $\begin{array}{l}\mathbb{1} \\
\mathbb{8} \\
\mathbb{8} \\
0 \\
\frac{\pi}{0} \\
\frac{.0}{5} \\
\frac{0}{0} \\
\frac{0}{4}\end{array}$ & $\begin{array}{l}0 \\
\mathbb{8} \\
\mathbb{8} \\
0 \\
\mathbb{\pi} \\
0 \\
0 \\
\frac{8}{0} \\
0\end{array}$ \\
\hline kosmopolitisch & $\mathrm{FP}$ & & FP & & & & & & & & & & & $\mathrm{FN}$ & & & FP & & & & & & & & \\
\hline zonal & & & & & & & & & & & & & & & & & & & & & & & & & \\
\hline $\begin{array}{l}\text { Tropischer } \\
\text { Regenwald }\end{array}$ & & & $\mathrm{F}$ & & & & $\mathrm{F}$ & $\mathrm{F}$ & & $\mathrm{F}$ & $\mathrm{F}$ & & & & $\mathrm{F}$ & & $\mathrm{F}$ & & & $\mathrm{F}$ & $\mathrm{F}$ & $\mathrm{F}$ & & & $\mathrm{F}$ \\
\hline $\begin{array}{l}\text { Tropischer Monsun } \\
\text { Regenwald }\end{array}$ & & & & & & & FP & $\mathrm{F}$ & & FP & FP & & & & FP & $\mathrm{F}$ & & & & $\mathrm{FP}$ & & FP & & & \\
\hline $\begin{array}{l}\text { Mediterranes } \\
\text { Sclerophylles } \\
\text { "Waldland" } \\
\end{array}$ & & & FP & & & & & $\mathrm{FP}$ & $\mathrm{F}$ & & $\mathrm{F}$ & & FP & & & FP & FP & & & & $\mathrm{F}$ & & & & \\
\hline $\begin{array}{l}\text { Laub- und Mischwald } \\
\text { mittlerer Breite } \\
\text { (immergrün) }\end{array}$ & & & & & & & $\mathrm{F}$ & & & & $\mathrm{F}$ & & & & & & & & & & $\mathrm{F}$ & & & & \\
\hline $\begin{array}{l}\text { Laub- und Mischwald } \\
\text { mittlerer Breite } \\
\text { (sommergrün) }\end{array}$ & $\mathrm{F}$ & & $\mathrm{F}$ & & & & $\mathrm{F}$ & & & & & & $\mathrm{F}$ & & & $\mathrm{F}$ & $\mathrm{F}$ & & & & $\mathrm{FP}$ & & & & \\
\hline $\begin{array}{l}\text { Nördlicher } \\
\text { Koniferenwald }\end{array}$ & $\mathrm{FP}$ & & & & & & & & & & & & & & & & & & & & & & & & \\
\hline arktische Tundra & $\mathrm{F}$ & & & & & & & & & & & & & & & & & & & & & & & & \\
\hline kosmopolitisch & & $\mathrm{F}$ & & & & $\mathrm{F}$ & & & & & & & & & & & & & & & & & & & \\
\hline azonal & & & & & & & & & & & & & & & & & & & & & & & & & \\
\hline $\begin{array}{l}\text { Verlandungszone- } \\
\text { Schwimmblatt- } \\
\text { pflanzengürtel }\end{array}$ & & & $\mathrm{F}$ & & & & & & & & & & & & & & & & & & & & & & \\
\hline $\begin{array}{l}\text { Verlandungszone } \\
\text { undifferenziert }\end{array}$ & $\mathrm{FP}$ & FP & FP & $\mathrm{F}$ & & & & & & & & & & & & & & & & & & & & & \\
\hline Niedermoor & & & & & & $\mathrm{F}$ & & & & & & $\mathrm{F}$ & & & & & & & & & & & & & \\
\hline Hochmoor & & & & & & & & & & & & & & & & & & & & & & & & & \\
\hline Ried & $\mathrm{F}$ & $\mathrm{F}$ & & $\mathrm{FP}$ & $\mathrm{FP}$ & $\mathrm{FP}$ & & & & & & & & $\mathrm{F}$ & & & & & & & & & & & \\
\hline Marsch & & & & & & & & & & & & & & & & & & & & & & & & & \\
\hline Sumpf & $\mathrm{F}$ & & & & & $\mathrm{F}$ & $\mathrm{FP}$ & & & & & & & & & & & & & & & & & & \\
\hline Bruchwald & & & & & $\mathrm{F}$ & & & FP & FP & FP & FP & $\mathrm{FP}$ & $\mathrm{FP}$ & FP & FP & FP & FP & FP & FP & $\mathrm{FP}$ & FP & FP & FP & FP & $\mathrm{FP}$ \\
\hline $\begin{array}{l}\text { Auewald/Galeriewald } \\
\text { undifferenziert }\end{array}$ & & & & & & & $\mathrm{F}$ & & & & & $\mathrm{F}$ & $\mathrm{F}$ & $\mathrm{F}$ & & $\mathrm{F}$ & $\mathrm{F}$ & & & & $\mathrm{F}$ & & & & $\mathrm{F}$ \\
\hline Nebelwald & & & & & & & & & & & & & & & & & & & & & & & & & $\mathrm{F}$ \\
\hline Bergwald & & & & & & $\mathrm{F}$ & & & & & & & & & $\mathrm{F}$ & & & & & & & & & & \\
\hline Hochwald & & & & & & & & & & & & & & $\mathrm{F}$ & & & & & & & & & & & \\
\hline Fazieselement & & & & & & & & & & & & & & & & & & & & & & & & & \\
\hline Fazieselement & FP & FP & FP & FP & & FP & $\mathrm{FP}$ & & & & & & & & & & & & & & & & & FP & \\
\hline $\begin{array}{l}\text { faziesunabhängiges } \\
\text { Element }\end{array}$ & & & & & $\mathrm{FP}$ & & & $\mathrm{FP}$ & $\mathrm{FP}$ & FP & $\mathrm{FP}$ & $\mathrm{FP}$ & $\mathrm{FP}$ & $\mathrm{FN}$ & $\mathrm{FP}$ & $\mathrm{FP}$ & $\mathrm{FP}$ & $\mathrm{FP}$ & $\mathrm{FP}$ & $\mathrm{FP}$ & $\mathrm{FP}$ & $\mathrm{FP}$ & $\mathrm{FP}$ & & $\mathrm{FP}$ \\
\hline $\mathrm{pH}$ & & & & & & & & & & & & & & & & & & & & & & & & & \\
\hline sauer & $\mathrm{FP}$ & & & $\mathrm{FP}$ & & & & & & & & & & & & & & & & & & & & & \\
\hline schwach sauer & & & & & & FP & & & & & & & & & & & & & & & & & & & \\
\hline unbekannt & & FP & FP & & $\mathrm{FP}$ & & $\mathrm{FP}$ & $\mathrm{FP}$ & $\mathrm{FP}$ & $\mathrm{FP}$ & $\mathrm{FP}$ & $\mathrm{FP}$ & $\mathrm{FP}$ & $\mathrm{FN}$ & $\mathrm{FP}$ & $\mathrm{FP}$ & $\mathrm{FP}$ & $\mathrm{FP}$ & $\mathrm{FP}$ & $\mathrm{FP}$ & $\mathrm{FP}$ & $\mathrm{FP}$ & $\mathrm{FP}$ & $\mathrm{FP}$ & $\mathrm{FP}$ \\
\hline Nährstoffe & & & & & & & & & & & & & & & & & & & & & & & & & \\
\hline nährstoffarm & & & & FP & & FP & & & & & & & & & & & & & & & & & & & \\
\hline $\begin{array}{l}\text { mässig } \\
\text { nährstoffreich }\end{array}$ & & & & & & & & & & & & & $\mathrm{FP}$ & & & & & & & & & & & & \\
\hline nährstoffreich & & $\mathrm{FP}$ & & & & & & & & & & & & & & & & & & & & & & & $\mathrm{FP}$ \\
\hline unbekannt & FP & & FP & & FP & & & FP & FP & FP & FP & FP & & $\mathrm{FN}$ & & FP & FP & FP & FP & FP & FP & FP & FP & FP & \\
\hline Feuchtigkeit & & & & & & & & & & & & & & & & & & & & & & & & & \\
\hline trocken & & & & FP & & $\mathrm{F}$ & & & & & & & & & & & & & & & & & & & \\
\hline mässig trocken & & & & & & & & & FP & & & & & & & $\mathrm{F}$ & $\mathrm{F}$ & & & & & & & & \\
\hline feucht & & & & & FP & FP & $\mathrm{F}$ & FP & & FP & & FP & & $\mathrm{FN}$ & FP & FP & FP & FP & FP & & FP & FP & FP & FP & $\mathrm{FP}$ \\
\hline nass & & & & $\mathrm{F}$ & & & & & & & & & & & & & & & & & & $\mathrm{F}$ & & & \\
\hline wechselnd nass & FP & $\mathrm{F}$ & & & & & FP & & & & & & & & & & & & & & & & & & \\
\hline überschwemmt & & FP & FP & & & & & & & & & & & & & & & & & & & & & & \\
\hline unbekannt & & & & & & & & & & & FP & & FP & & & & & & & FP & & & & & \\
\hline Mächtigkeit & & & & & & & & & & & & & & & & & & & & & & & & & \\
\hline unbekannt & FP & FP & FP & FP & $\mathrm{FP}$ & $\mathrm{FP}$ & $\mathrm{FP}$ & $\mathrm{FP}$ & $\mathrm{FP}$ & FP & FP & $\mathrm{FP}$ & FP & $\mathrm{FN}$ & FP & FP & FP & FP & FP & FP & FP & $\mathrm{FP}$ & FP & $\mathrm{FP}$ & $\mathrm{FP}$ \\
\hline Salzgehalt & & & & & & & & & & & & & & & & & & & & & & & & & \\
\hline nicht Halophyt & FP & FP & FP & FP & FP & FP & FP & FP & FP & FP & FP & FP & FP & $\mathrm{FN}$ & FP & FP & FP & FP & FP & FP & FP & FP & FP & FP & $\mathrm{FP}$ \\
\hline Licht & & & & & & & & & & & & & & & & & & & & & & & & & \\
\hline Schattenpflanzen & & & & & & & & FP & & & & & & & & & & & & FP & & & & & \\
\hline $\begin{array}{l}\text { Halbschatten- } \\
\text { pflanzen }\end{array}$ & $\mathrm{FP}$ & & & & & & & $\mathrm{F}$ & & & & & & & $\mathrm{FP}$ & & & & & $\mathrm{F}$ & & & & & \\
\hline Vollichtpflanzen & & & FP & & & & $\mathrm{FP}$ & & FP & & & & & & & & & & & & & FP & & & \\
\hline unbekannt & & FP & & FP & FP & FP & & & & FP & FP & FP & FP & $\mathrm{FN}$ & & FP & FP & FP & FP & & FP & & FP & FP & FP \\
\hline
\end{tabular}




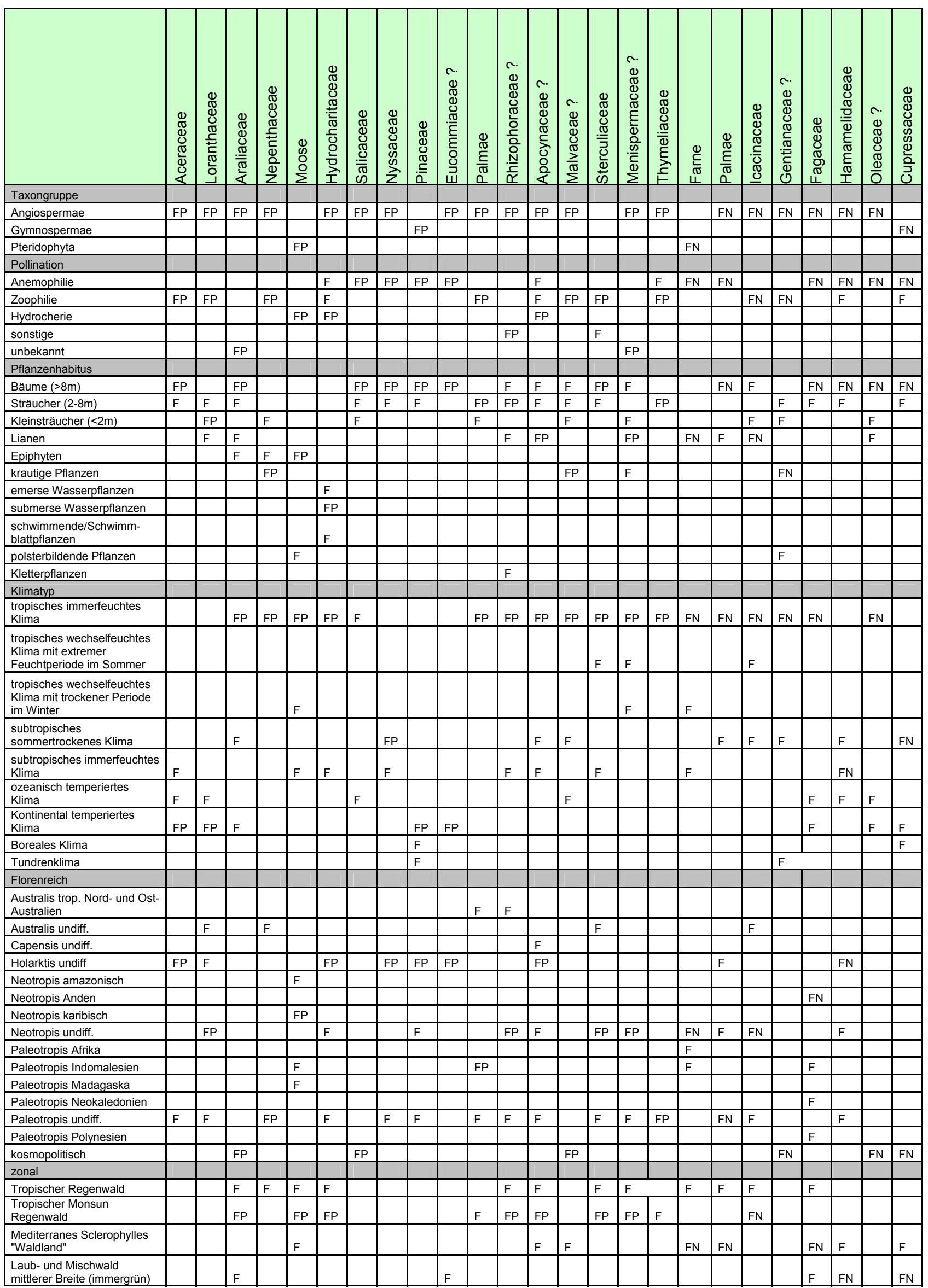




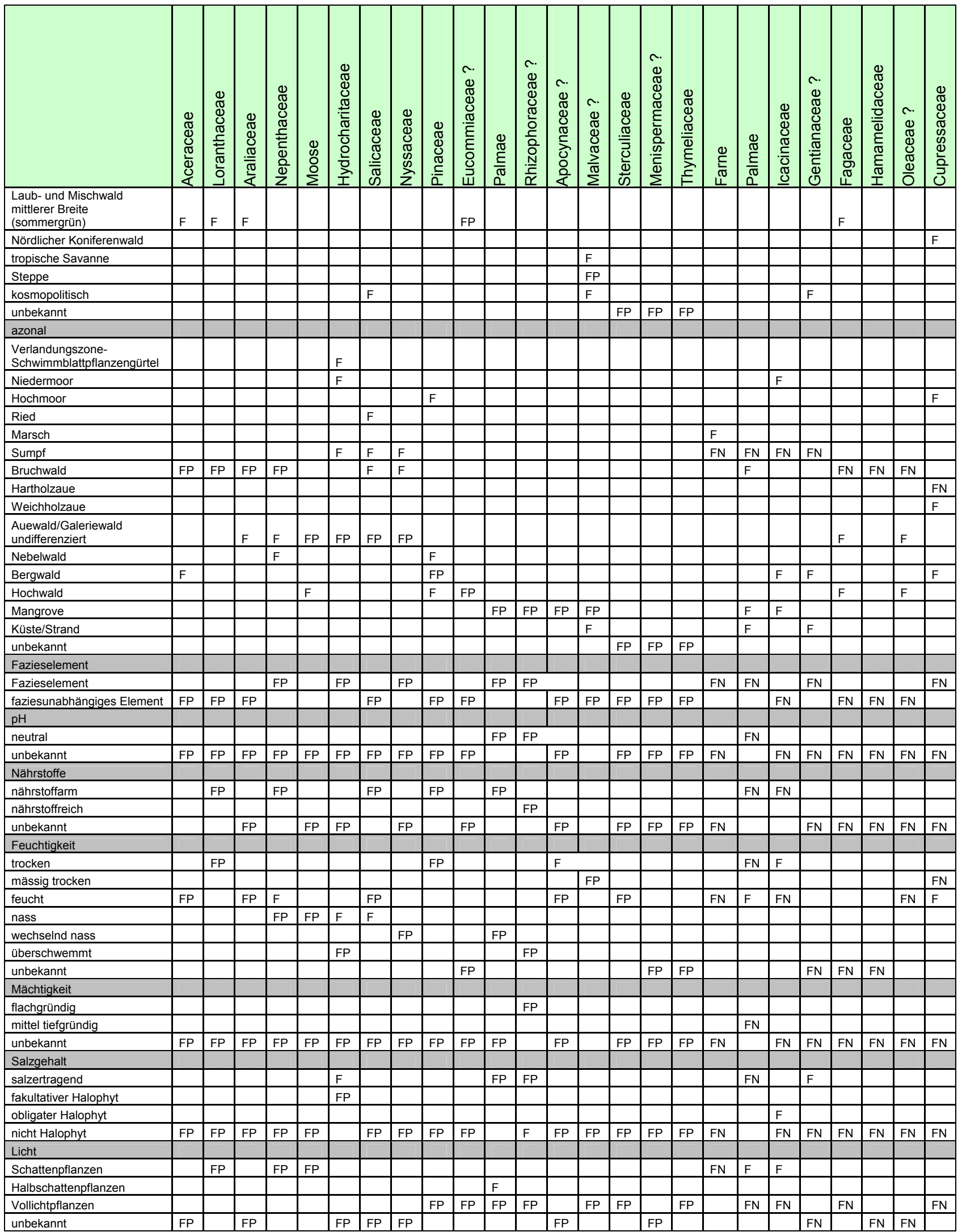




\subsubsection{Profil Schöningen}

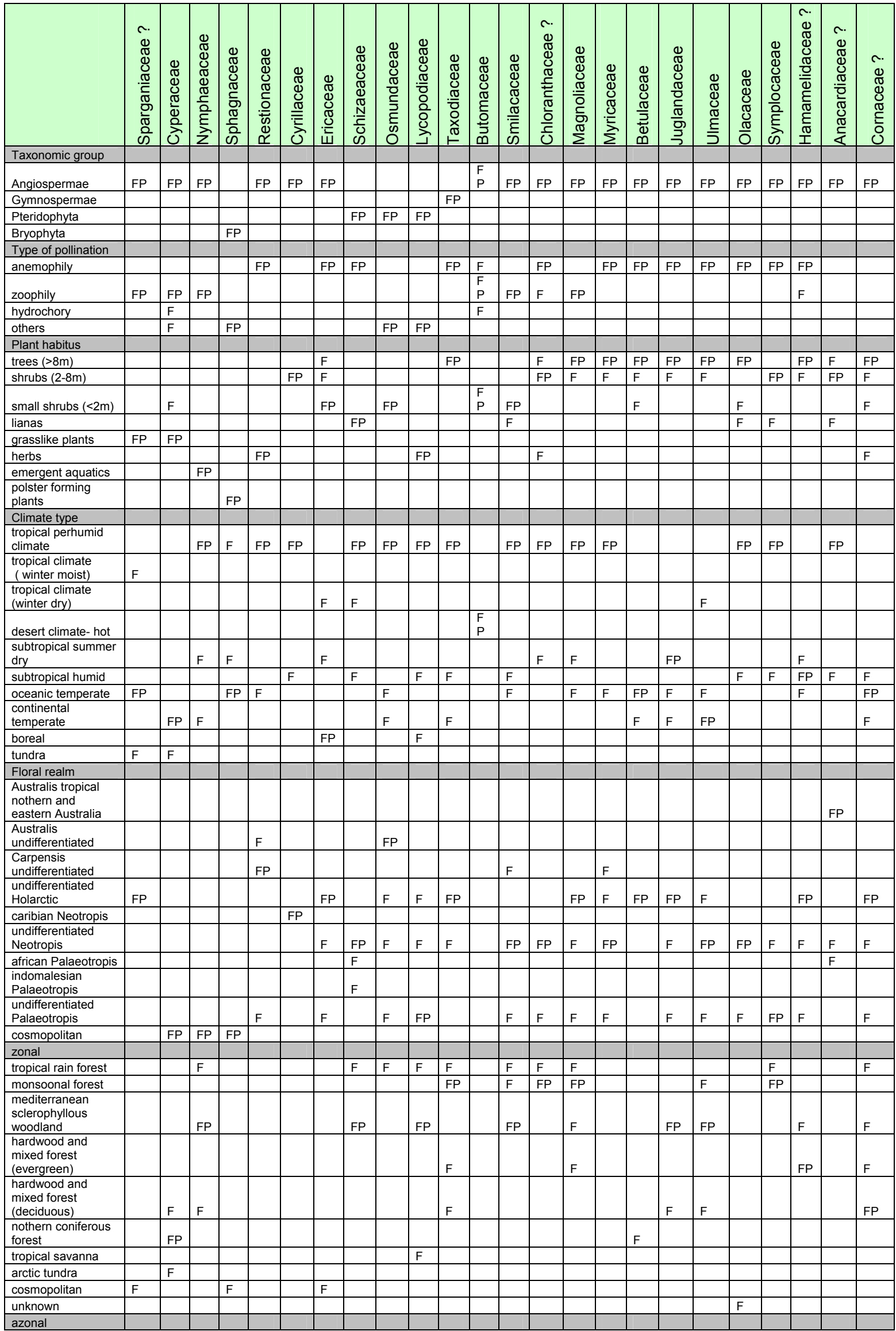




\begin{tabular}{|c|c|c|c|c|c|c|c|c|c|c|c|c|c|c|c|c|c|c|c|c|c|c|c|c|}
\hline & 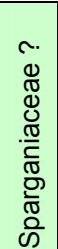 & 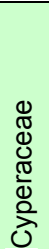 & 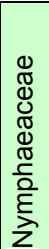 & 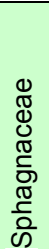 & 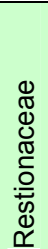 & 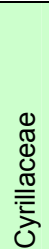 & 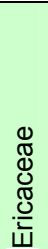 & 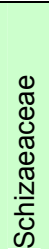 & 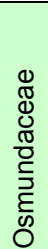 & 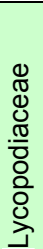 & 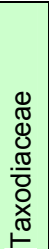 & 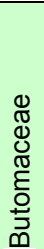 & 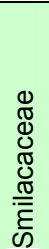 & 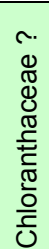 & 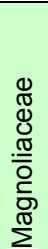 & $\begin{array}{l}0 \\
\mathbb{D} \\
\mathbb{D} \\
0 \\
\mathbb{J} \\
.0 \\
\sum \\
\sum\end{array}$ & 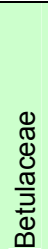 & $\begin{array}{l}0 \\
\mathbb{D} \\
\mathbb{0} \\
\mathbb{J} \\
\frac{0}{0} \\
\frac{\pi}{0} \\
\frac{\pi}{J} \\
\end{array}$ & 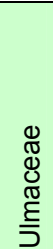 & 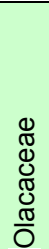 & 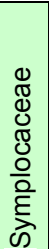 & 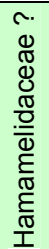 & 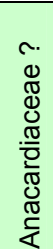 & 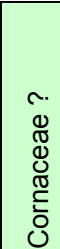 \\
\hline $\begin{array}{l}\text { lake succession - } \\
\text { zone of submersed } \\
\text { aquatics }\end{array}$ & & & & & & & & & & & & $\mathrm{F}$ & & & & & & & & & & & & \\
\hline $\begin{array}{l}\text { lake succession - } \\
\text { zone of floating leaf } \\
\text { aquatics }\end{array}$ & & & $\mathrm{F}$ & & & & & & & & & & & & & & & & & & & & & \\
\hline $\begin{array}{l}\text { lake succession - } \\
\text { reed/sedge zone }\end{array}$ & & & & & & & & & & & & $\mathrm{F}$ & & & & & & & & & & & & \\
\hline lake succession & $\mathrm{FP}$ & $\mathrm{FP}$ & FP & & $\mathrm{F}$ & & & & & & & & & & & & & & & & & & & \\
\hline mire & & & & $\mathrm{F}$ & & & $\mathrm{F}$ & & $\mathrm{F}$ & $\mathrm{F}$ & & & & & & $\mathrm{F}$ & & & & & & & & \\
\hline bog & & & & FP & & & & & & & & & & & & & & & & & & & & \\
\hline reed & $\mathrm{F}$ & $\mathrm{F}$ & & & FP & FP & FP & & $\mathrm{F}$ & $\mathrm{F}$ & & & & & & & & & & & & & & \\
\hline marsh & & & & & & & & $\mathrm{F}$ & & & & & & & & & & & & & & & & \\
\hline swamp & & $\mathrm{F}$ & & & & & $\mathrm{F}$ & FP & FP & $\mathrm{FP}$ & $\mathrm{FP}$ & $\begin{array}{l}F \\
P\end{array}$ & & & & & & & & & & & & \\
\hline mire forest & & & & & & $\mathrm{F}$ & & & $\mathrm{F}$ & & & & $\mathrm{FP}$ & FP & $\mathrm{FP}$ & $\mathrm{FP}$ & $\mathrm{FP}$ & $\mathrm{FP}$ & $\mathrm{FP}$ & $\mathrm{FP}$ & $\mathrm{FP}$ & $\mathrm{FP}$ & $\mathrm{FP}$ & FP \\
\hline $\begin{array}{l}\text { riparian/gallery } \\
\text { forest }\end{array}$ & & & & & & & & & & & $\mathrm{F}$ & & & & & $\mathrm{F}$ & & $\mathrm{F}$ & $\mathrm{F}$ & & & & & $\mathrm{F}$ \\
\hline montane forest & & & & & & & $\mathrm{F}$ & & & & & & & & & & & & & & $\mathrm{F}$ & & & \\
\hline Facies element & & & & & & & & & & & & & & & & & & & & & & & & \\
\hline facies related & $\mathrm{FP}$ & $\mathrm{FP}$ & FP & $\mathrm{FP}$ & $\mathrm{FP}$ & & $\mathrm{FP}$ & FP & $\mathrm{FP}$ & $\mathrm{FP}$ & $\mathrm{FP}$ & & & & & & & & & & & & & \\
\hline unrelated to facies & & & & & & FP & & & & & & & FP & FP & FP & FP & FP & FP & $\mathrm{FP}$ & FP & $\mathrm{FP}$ & FP & $\mathrm{FP}$ & FP \\
\hline unknown & & & & & & & & & & & & $\begin{array}{l}\mathrm{F} \\
\mathrm{P} \\
\end{array}$ & & & & & & & & & & & & \\
\hline $\mathrm{pH}$ & & & & & & & & & & & & & & & & & & & & & & & & \\
\hline strongly alkaline & & & & & & & & & & $\mathrm{FP}$ & & $\begin{array}{l}\mathrm{F} \\
\mathrm{P}\end{array}$ & & & & & & & & & & & & \\
\hline acid & & FP & & FP & FP & & & & $\mathrm{FP}$ & & & & & & & & & & & & & & & \\
\hline weakly acid & & & & & & & FP & & & & & & & & & & & & & & & & & \\
\hline unknown & $\mathrm{FP}$ & & FP & & & FP & & FP & & & $\mathrm{FP}$ & & FP & FP & $\mathrm{FP}$ & FP & $\mathrm{FP}$ & FP & $\mathrm{FP}$ & FP & $\mathrm{FP}$ & FP & $\mathrm{FP}$ & FP \\
\hline nutrients & & & & & & & & & & & & & & & & & & & & & & & & \\
\hline oligotrophic & & & & FP & FP & & FP & & FP & & & & & & & & & & & & & & & \\
\hline mesotrophic & & & & & & & & & & & & & & & & & & $\mathrm{FP}$ & & & & & & \\
\hline eutrophic & $\mathrm{FP}$ & & & & & & & & & & & & & & & & & & & & & & & \\
\hline unknown & & FP & FP & & & $\mathrm{FP}$ & & $\mathrm{FP}$ & & $\mathrm{FP}$ & & $\begin{array}{l}\mathrm{F} \\
\mathrm{P}\end{array}$ & $\mathrm{FP}$ & $\mathrm{FP}$ & $\mathrm{FP}$ & $\mathrm{FP}$ & $\mathrm{FP}$ & & $\mathrm{FP}$ & $\mathrm{FP}$ & & FP & $\mathrm{FP}$ & FP \\
\hline moisture & & & & & & & & & & & & & & & & & & & & & & & & \\
\hline dry & & & & & $\mathrm{FP}$ & & $\mathrm{F}$ & & & & & & & & & & & & & & & & & \\
\hline moderate dry & & & & & & & & & & & & & & & & & & & $\mathrm{F}$ & & & & & \\
\hline moist & & & & & & FP & $\mathrm{FP}$ & FP & & $\mathrm{FP}$ & $\mathrm{F}$ & & $\mathrm{FP}$ & FP & & FP & & & $\mathrm{FP}$ & $\mathrm{FP}$ & $\mathrm{FP}$ & & $\mathrm{FP}$ & $\mathrm{FP}$ \\
\hline wet & & & & FP & $\mathrm{F}$ & & & & FP & $\mathrm{F}$ & & & & & & & & & & & & & & \\
\hline alternate wet/dry & $\mathrm{F}$ & FP & & & & & & & $\mathrm{F}$ & & $\mathrm{FP}$ & & & & & & & & & & & & & \\
\hline flooded & $\mathrm{FP}$ & & FP & & & & & & & & & & & & & & & & & & & & & \\
\hline unknown & & & & & & & & & & & & $\begin{array}{l}\mathrm{F} \\
\mathrm{P}\end{array}$ & & & $\mathrm{FP}$ & & $\mathrm{FP}$ & FP & & & & FP & & \\
\hline Soil thickness & & & & & & & & & & & & & & & & & & & & & & & & \\
\hline shallow & & & & $\mathrm{FP}$ & & & & & & & & & & & & & & & & & & & & \\
\hline unknown & $\mathrm{FP}$ & FP & FP & & FP & FP & FP & FP & $\mathrm{FP}$ & $\mathrm{FP}$ & FP & $\begin{array}{l}F \\
P\end{array}$ & FP & FP & FP & FP & FP & FP & $\mathrm{FP}$ & FP & FP & FP & FP & FP \\
\hline salinity & & & & & & & & & & & & & & & & & & & & & & & & \\
\hline not halophyte & FP & $\mathrm{FP}$ & FP & $\mathrm{FP}$ & $\mathrm{FP}$ & $\mathrm{FP}$ & FP & $\mathrm{FP}$ & $\mathrm{FP}$ & $\mathrm{FP}$ & $\mathrm{FP}$ & $\begin{array}{l}\mathrm{F} \\
\mathrm{P}\end{array}$ & FP & $\mathrm{FP}$ & $\mathrm{FP}$ & $\mathrm{FP}$ & $\mathrm{FP}$ & FP & FP & $\mathrm{FP}$ & $\mathrm{FP}$ & FP & $\mathrm{FP}$ & FP \\
\hline Light availability & & & & & & & & & & & & & & & & & & & & & & & & \\
\hline shade plants & & & & $\mathrm{FP}$ & & & & $\mathrm{FP}$ & FP & & & & $\mathrm{FP}$ & & & & & & & & & & & \\
\hline partly shade plants & & $\mathrm{FP}$ & & & & & & & & & & & $\mathrm{F}$ & & & & & & & & $\mathrm{FP}$ & & & \\
\hline fully exposed plants & & & FP & & & & & & & & $\mathrm{FP}$ & & & & & & & & & & & & & \\
\hline unknown & $\mathrm{FP}$ & & & & $\mathrm{FP}$ & $\mathrm{FP}$ & FP & & & $\mathrm{FP}$ & & $\begin{array}{l}\mathrm{F} \\
\mathrm{P}\end{array}$ & & $\mathrm{FP}$ & $\mathrm{FP}$ & $\mathrm{FP}$ & $\mathrm{FP}$ & FP & FP & $\mathrm{FP}$ & & FP & $\mathrm{FP}$ & FP \\
\hline
\end{tabular}




\begin{tabular}{|c|c|c|c|c|c|c|c|c|c|c|c|c|c|c|c|c|c|c|c|c|c|c|c|c|c|}
\hline & 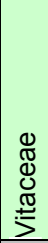 & 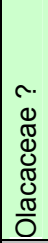 & 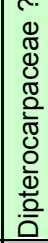 & 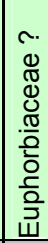 & 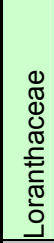 & 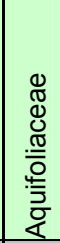 & $\begin{array}{l}0 \\
\mathbb{\pi} \\
0 \\
0 \\
\pi \\
0 \\
0 \\
\mathbb{0} \\
0 \\
0\end{array}$ & 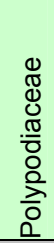 & 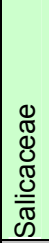 & 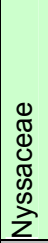 & 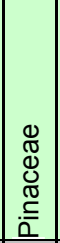 & 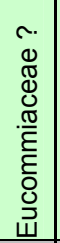 & 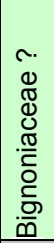 & 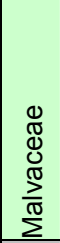 & 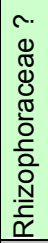 & 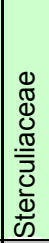 & 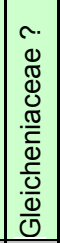 & 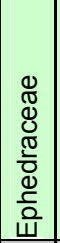 & \begin{tabular}{|l}
$\mathbb{\Xi}$ \\
$\tilde{\sigma}$ \\
$\frac{E}{\tilde{\sigma}}$ \\
$\alpha$
\end{tabular} & 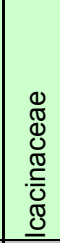 & 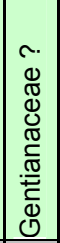 & 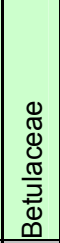 & 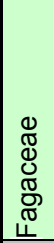 & 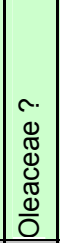 & $\begin{array}{l}0 \\
\mathbb{8} \\
\mathbb{1} \\
0 \\
\mathbb{0} \\
0 \\
0 \\
0 \\
0 \\
0 \\
0 \\
0\end{array}$ \\
\hline Taxonomic group & & & & & & & & & & & & & & & & & & & & & & & & & \\
\hline Angiospermae & FP & FP & FP & FP & FP & FP & \begin{tabular}{|l|}
$F P$ \\
\end{tabular} & & FP & FP & & FP & FP & FP & FP & & & & $\mathrm{FN}$ & \begin{tabular}{|l|}
$\mathrm{FN}$ \\
\end{tabular} & $\mathrm{FN}$ & \begin{tabular}{|l|}
$\mathrm{FN}$ \\
\end{tabular} & $\mathrm{FN}$ & $\mathrm{FN}$ & \\
\hline Gymnospermae & & & & & & & & & & & \begin{tabular}{|l|}
$F P$ \\
\end{tabular} & & & & & & & $\mathrm{FN}$ & & & & & & & $\mathrm{FN}$ \\
\hline Pteridophyta & & & & & & & & FP & & & & & & & & & $\mathrm{FN}$ & & & & & & & & \\
\hline Type of pollination & & & & & & & & & & & & & & & & & & & & & & & & & \\
\hline anemophily & FP & FP & & & & & FP & & FP & FP & FP & FP & & & & & & \begin{tabular}{|l|}
$\mathrm{FN}$ \\
\end{tabular} & FN & & & \begin{tabular}{|l|}
$\mathrm{FN}$ \\
\end{tabular} & $\mathrm{FN}$ & $\mathrm{FN}$ & $\mathrm{FN}$ \\
\hline zoophily & & & FP & FP & FP & FP & & & & & & & FP & FP & & FP & & & & $\mathrm{FN}$ & FN & & & & $\mathrm{F}$ \\
\hline others & & & & 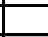 & & & & FP & & & & & & & FP & $\mathrm{F}$ & $\mathrm{FN}$ & & & 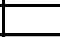 & 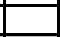 & & 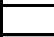 & 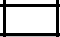 & \\
\hline Plant habitus & & & & & & & & & & & & & & & & & & & & & & & & & \\
\hline trees $(>8 \mathrm{~m})$ & & FP & FP & $\mathrm{F}$ & & FP & FP & & FP & FP & FP & FP & FP & $\mathrm{F}$ & $\mathrm{F}$ & FP & & & FN & $\mathrm{F}$ & & $\mathrm{F}$ & FN & FN & FN \\
\hline shrubs $(2-8 \mathrm{~m})$ & $\mathrm{F}$ & & & $\mathrm{F}$ & $\mathrm{F}$ & $\mathrm{F}$ & $\mathrm{F}$ & & $\mathrm{F}$ & $\mathrm{F}$ & $\mathrm{F}$ & & $\mathrm{F}$ & $\mathrm{F}$ & FP & $\mathrm{F}$ & & \begin{tabular}{|l|}
$\mathrm{FN}$ \\
\end{tabular} & & & $\mathrm{F}$ & FN & $\mathrm{F}$ & & $\mathrm{F}$ \\
\hline small shrubs $(<2 \mathrm{~m})$ & & $\mathrm{F}$ & & & FP & & & & $\mathrm{F}$ & & & & & $\mathrm{F}$ & & & & & & $\mathrm{F}$ & $\mathrm{F}$ & & & $\mathrm{F}$ & \\
\hline lianas & & $\mathrm{F}$ & & & $F$ & & & & & & & & $\mathrm{~F}$ & & $\mathrm{~F}$ & & & & $\mathrm{~F}$ & FN & & & & $\mathrm{F}$ & \\
\hline epiphytes & & & & & & & & FP & & & & & & & & & & & & & & & & & \\
\hline herbs & & & & FP & & & & $\mathrm{F}$ & & & & & & FP & & & $\mathrm{FN}$ & & & & $\mathrm{FN}$ & & & & \\
\hline $\begin{array}{l}\text { polster forming } \\
\text { plants }\end{array}$ & & & & & & & & & & & & & & & & & & & & & $\mathrm{F}$ & & & & \\
\hline climber & FP & & & & & & & & & & & & & & $\mathrm{F}$ & & & & & & & & & & \\
\hline Climate type & & & & & & & & & & & & & & & & & & & & & & & & & \\
\hline $\begin{array}{l}\text { tropical perhumid } \\
\text { climate }\end{array}$ & FP & FP & FP & FP & & & FP & FP & $\mathrm{F}$ & & & & FP & FP & FP & FP & $\mathrm{FN}$ & & $\mathrm{FN}$ & $\mathrm{FN}$ & $\mathrm{FN}$ & & $\mathrm{FN}$ & $\mathrm{FN}$ & \\
\hline $\begin{array}{r}\text { tropical climate } \\
\text { ( winter moist) }\end{array}$ & & & $\mathrm{F}$ & & & & & $\mathrm{F}$ & & & & & & & & $\mathrm{F}$ & & $\mathrm{FN}$ & & $\mathrm{F}$ & & & & & \\
\hline $\begin{array}{l}\text { tropical climate } \\
\text { (winter dry) }\end{array}$ & & & $\mathrm{F}$ & & & & & & & & & & $\mathrm{F}$ & & & & $\mathrm{F}$ & & & & & & & & \\
\hline steppe climate - hot & & & & & & & & & & & & & & & & & & $\mathrm{F}$ & & & & & & & \\
\hline $\begin{array}{l}\text { desert climate - } \\
\text { cold }\end{array}$ & & & & & & & & & & & & & & & & & & $\mathrm{F}$ & & & & & & & \\
\hline $\begin{array}{l}\text { subtropical summer } \\
\text { dry }\end{array}$ & $\mathrm{F}$ & & & & & $\mathrm{FP}$ & & & & FP & & & & $\mathrm{F}$ & & & & & $\mathrm{F}$ & $\mathrm{F}$ & $\mathrm{F}$ & $\mathrm{F}$ & & & FN \\
\hline subtropical humid & $\mathrm{F}$ & $\mathrm{F}$ & & & & & $\mathrm{F}$ & & & $\mathrm{F}$ & & & $\mathrm{F}$ & & $\mathrm{F}$ & $\mathrm{F}$ & $\mathrm{F}$ & & & & & & & & \\
\hline oceanic temperate & & & & $\mathrm{F}$ & $\mathrm{F}$ & $\mathrm{F}$ & & $\mathrm{F}$ & $\mathrm{F}$ & & & & & $\mathrm{F}$ & & & & & & & & & $\mathrm{F}$ & $\mathrm{F}$ & \\
\hline $\begin{array}{l}\text { continental } \\
\text { temperate }\end{array}$ & & & & $\mathrm{F}$ & FP & $\mathrm{F}$ & & & & & $\mathrm{FP}$ & FP & & & & & & & & & & & $\mathrm{F}$ & $\mathrm{F}$ & $\mathrm{F}$ \\
\hline boreal & & & & & & & & & & & $\mathrm{F}$ & & & & & & & & & & & \begin{tabular}{|l|}
$\mathrm{FN}$ \\
\end{tabular} & & & $\mathrm{F}$ \\
\hline tundra & & & & & & & & & & & $\mathrm{F}$ & & & & & & & & & & $\mathrm{F}$ & $\mathrm{F}$ & & & \\
\hline Floral realm & & & & & & & & & & & & & & & & & & & & & & & & & \\
\hline $\begin{array}{l}\text { Antarctic New } \\
\text { Zealand }\end{array}$ & & & & FP & & & & & & & & & & & & & & & & & & & & & \\
\hline $\begin{array}{l}\text { Australis tropical } \\
\text { nothern and } \\
\text { eastern Australia }\end{array}$ & & & & & & & $\mathrm{F}$ & & & & & & & & $\mathrm{F}$ & & & & & & & & & & \\
\hline $\begin{array}{l}\text { Australis } \\
\text { undifferentiated }\end{array}$ & & & & $\mathrm{F}$ & $\mathrm{F}$ & & & & & & & & $\mathrm{F}$ & & & $\mathrm{F}$ & & & & $\mathrm{F}$ & & & & & \\
\hline $\begin{array}{l}\text { Carpensis } \\
\text { undifferentiated }\end{array}$ & & & & & & & & & & & & & & & & & $\mathrm{F}$ & & & & & & & & \\
\hline $\begin{array}{l}\text { undifferentiated } \\
\text { Holarctic }\end{array}$ & & & & & $\mathrm{F}$ & & & & & FP & FP & FP & $\mathrm{F}$ & & & & & & $\mathrm{F}$ & & & & & & \\
\hline andean Neotropis & & & & & & & & & & & & & & & & & & & & & & & $\mathrm{FN}$ & & \\
\hline $\begin{array}{l}\text { undifferentiated } \\
\text { Neotropis }\end{array}$ & FP & FP & & $\mathrm{F}$ & FP & $\mathrm{F}$ & FP & & & & $\mathrm{F}$ & & FP & & FP & FP & $\mathrm{F}$ & & $\mathrm{F}$ & $\mathrm{FN}$ & & & & & \\
\hline african Palaeotropis & & & & & & FP & & & & & & & & & & & & $\mathrm{F}$ & & & & & & & \\
\hline $\begin{array}{l}\text { indomalesian } \\
\text { Palaeotropis }\end{array}$ & & & & & & & & & & & & & & & & & & & & & & & $\mathrm{F}$ & & \\
\hline $\begin{array}{l}\text { new caledonian } \\
\text { Palaeotropis }\end{array}$ & & & & & & & & & & & & & & & & & & & & & & & $\mathrm{F}$ & & \\
\hline $\begin{array}{l}\text { undifferentiated } \\
\text { Palaeotropis }\end{array}$ & $\mathrm{F}$ & $\mathrm{F}$ & FP & $\mathrm{F}$ & $\mathrm{F}$ & & $\mathrm{F}$ & & & $\mathrm{F}$ & $\mathrm{F}$ & & $\mathrm{F}$ & & $\mathrm{F}$ & $\mathrm{F}$ & $\mathrm{FN}$ & $\mathrm{FN}$ & $\mathrm{FN}$ & $\mathrm{F}$ & & & & & \\
\hline $\begin{array}{l}\text { polymesian } \\
\text { Palaeotropis }\end{array}$ & & & & & & & & & & & & & & & & & & & & & & & $\mathrm{F}$ & & \\
\hline cosmopolitan & & & & & & & & FP & FP & & & & & FP & & & & & & & $\mathrm{FN}$ & $\mathrm{FN}$ & & FN & FN \\
\hline zonal & & & & & & & & & & & & & & & & & & & & & & & & & \\
\hline tropical rain forest & $\mathrm{F}$ & & $\mathrm{F}$ & & & & $\mathrm{F}$ & $\mathrm{F}$ & & & & & $\mathrm{F}$ & & $\mathrm{F}$ & $\mathrm{F}$ & $\mathrm{F}$ & & $\mathrm{F}$ & $\mathrm{F}$ & & & $\mathrm{F}$ & & \\
\hline monsoonal forest & FP & & FP & & & & & FP & & & & & FP & & FP & FP & $\mathrm{FN}$ & & & $\mathrm{FN}$ & & & & & \\
\hline $\begin{array}{l}\text { mediterranean } \\
\text { sclerophyllous } \\
\text { woodland }\end{array}$ & & & & & & & & $\mathrm{F}$ & & & & & $\mathrm{F}$ & $\mathrm{F}$ & & & $\mathrm{F}$ & & $\mathrm{FN}$ & & & & $\mathrm{FN}$ & & $\mathrm{F}$ \\
\hline $\begin{array}{l}\text { hardwood and } \\
\text { mixed forest } \\
\text { (evergreen) }\end{array}$ & & & & & & & & & & & & $\mathrm{F}$ & $\mathrm{F}$ & & & & & & & & & & $\mathrm{F}$ & & $\mathrm{FN}$ \\
\hline $\begin{array}{l}\text { hardwood and } \\
\text { mixed forest } \\
\text { (deciduous) }\end{array}$ & & & & & $\mathrm{F}$ & & & $\mathrm{F}$ & & & & FP & & & & & & & & & & & $\mathrm{F}$ & & \\
\hline $\begin{array}{l}\text { nothern coniferous } \\
\text { forest }\end{array}$ & & & & & & & & & & & & & & & & & & & & & & & & & $\mathrm{F}$ \\
\hline tropical savanna & & & & & & & & & & & & & & $F$ & & & & & & & & & & & \\
\hline prairie & & & & & & & & & & & & & & & & & & $\mathrm{F}$ & & & & & & & \\
\hline
\end{tabular}




\begin{tabular}{|c|c|c|c|c|c|c|c|c|c|c|c|c|c|c|c|c|c|c|c|c|c|c|c|c|c|}
\hline & 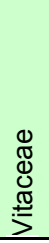 & 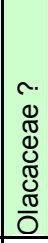 & 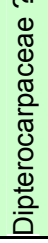 & 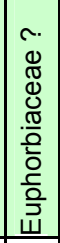 & 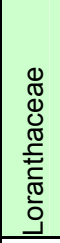 & 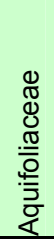 & 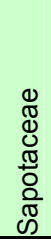 & 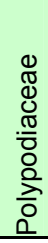 & 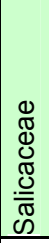 & 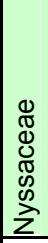 & 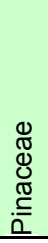 & 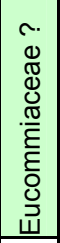 & $\begin{array}{c}. \\
0 \\
\mathbb{\pi} \\
0 \\
0 \\
. \frac{\pi}{ \pm} \\
0 \\
\frac{0}{0} \\
.0 \\
0\end{array}$ & $\begin{array}{l}\mathbb{\Xi} \\
\mathbb{J} \\
\mathbb{U} \\
\mathbb{\pi} \\
\frac{2}{\pi} \\
\Sigma\end{array}$ & 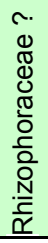 & 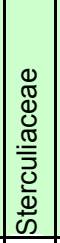 & 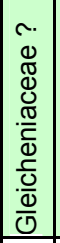 & 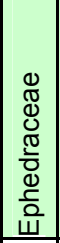 & \begin{tabular}{|l|}
$\mathbb{\Xi}$ \\
$\tilde{E}$ \\
$\frac{\tilde{E}}{\widetilde{\sigma}}$ \\
$\tilde{\Omega}$
\end{tabular} & 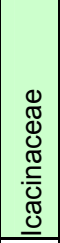 & 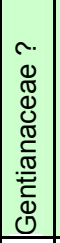 & 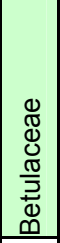 & 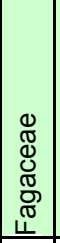 & \begin{tabular}{|c|}
0 \\
0 \\
$\mathbb{D}$ \\
0 \\
0 \\
$\mathbb{E}$ \\
0 \\
0 \\
\end{tabular} & 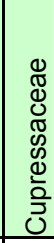 \\
\hline steppe & & & & & & & & & & & & & & FP & & & & $\mathrm{FN}$ & & & & & & & \\
\hline semidesert & & & & & & & & & & & & & & & & & & $\mathrm{F}$ & & & & & & & - \\
\hline cosmopolitan & & & & & & & & & $\mathrm{F}$ & & & & & $\mathrm{F}$ & & & & 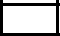 & & & $\mathrm{F}$ & & & & 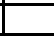 \\
\hline unknown & & $\mathrm{F}$ & & & & & & & & & & & & & & FP & & & & & & & & & \\
\hline azonal & & & & & & & & & & & & & & & & & & & & & & & & & 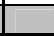 \\
\hline \multicolumn{26}{|l|}{$\begin{array}{l}\text { lake succession - } \\
\text { zone of floating leaf } \\
\text { aquatics }\end{array}$} \\
\hline mire & & & & & & & & $\mathrm{F}$ & & & & & & & & & & & & $F$ & & & & & \\
\hline bog & & & & & & & & $\mathrm{F}$ & & & $\mathrm{F}$ & & & & & & \begin{tabular}{|l|}
$\mathrm{FN}$ \\
\end{tabular} & \begin{tabular}{|l|}
$\mathrm{FN}$ \\
\end{tabular} & & & & & & & $\mathrm{F}$ \\
\hline reed & & & & & & & & & $\mathrm{F}$ & & & & & & & & & & & & & $F$ & & & 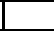 \\
\hline swamp & & & & & & & & $\mathrm{F}$ & $\mathrm{F}$ & $F$ & & & & & & & $F$ & 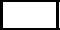 & FN & $\mathrm{FN}$ & FN & & & & \\
\hline mire forest & FP & FP & FP & FP & FP & FP & FP & & $\mathrm{F}$ & $F$ & & & & & & & & 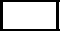 & $\mathrm{F}$ & & & $\mathrm{FN}$ & FN & FN & \\
\hline upper flood plane & & & & & & & & & & & & & & & & & & & & & & & & & $\mathrm{FN}$ \\
\hline lower flood plane & & & & & & & & & & & & & & & & & & 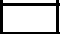 & & & & & & & $\mathrm{F}$ \\
\hline $\begin{array}{l}\text { riparian/gallery } \\
\text { forest }\end{array}$ & & & & & & & $\mathrm{F}$ & FP & FP & FP & & & $\mathrm{F}$ & & & & $\mathrm{F}$ & $\mathrm{F}$ & & & & $\mathrm{F}$ & $\mathrm{F}$ & $\mathrm{F}$ & \\
\hline fog forest & & & & & & & $\mathrm{F}$ & & & & $\mathrm{F}$ & & & & & & & 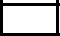 & & & & & & & \\
\hline montane forest & & & & & & & & & & & FP & & $F$ & & & & $F$ & 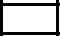 & 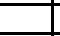 & $\mathrm{F}$ & $\mathrm{F}$ & & - & 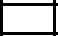 & $\mathrm{F}$ \\
\hline high canopy forest & & & & & & & & & & & $\mathrm{F}$ & \begin{tabular}{|l|}
$F P$ \\
\end{tabular} & FP & & & & & & & & & $\mathrm{F}$ & $\mathrm{F}$ & $\mathrm{F}$ & \\
\hline mangrove & & & & & & & & & & & & & & FP & FP & & & - & $\mathrm{F}$ & $\mathrm{F}$ & & & & & \\
\hline coast/beach & & & & & & & & & & & & & & $\mathrm{F}$ & & & & 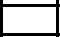 & $\mathrm{F}$ & & $\mathrm{F}$ & & & & 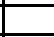 \\
\hline unknown & & & & & & & & & & & & & & & & FP & & & & & & & & & \\
\hline \multicolumn{26}{|l|}{ Facies element } \\
\hline facies related & & & & & & FP & & FP & & FP & & & & & FP & & FN & & \begin{tabular}{|l|}
$\mathrm{FN}$ \\
\end{tabular} & & FN & & & & $\mathrm{FN}$ \\
\hline unrelated to facies & FP & FP & FP & FP & \begin{tabular}{|l|} 
FP \\
\end{tabular} & & FP & & FP & & FP & FP & FP & FP & & FP & & & & \begin{tabular}{|l|}
$\mathrm{FN}$ \\
\end{tabular} & & \begin{tabular}{|l|}
$\mathrm{FN}$ \\
\end{tabular} & \begin{tabular}{|l|}
$\mathrm{FN}$ \\
\end{tabular} & $\mathrm{FN}$ & \\
\hline unknown & & & & & & & & & & & & & & & & & & \begin{tabular}{|l|}
$\mathrm{FN}$ \\
\end{tabular} & & & & & & & \\
\hline \multicolumn{26}{|l|}{$\mathrm{pH}$} \\
\hline weakly alkaline & & & & & & & & & & & & & & & & & & $\mathrm{FN}$ & & & & & & & \\
\hline neutral & & & & & & & & & & & & & & & $\mathrm{FP}$ & & & & \begin{tabular}{|l|}
$\mathrm{FN}$ \\
\end{tabular} & & & & & & \\
\hline acid & & & & & & & & FP & & & & & & & & & & & & & & & & & \\
\hline unknown & FP & FP & FP & FP & \begin{tabular}{|l|l|}
$F P$ \\
\end{tabular} & FP & FP & & FP & FP & FP & FP & \begin{tabular}{|l|} 
FP \\
\end{tabular} & & & $\mathrm{FP}$ & $\mathrm{FN}$ & & & \begin{tabular}{|l|}
$\mathrm{FN}$ \\
\end{tabular} & FN & \begin{tabular}{|l|}
$\mathrm{FN}$ \\
\end{tabular} & FN & $\mathrm{FN}$ & $\mathrm{FN}$ \\
\hline \multicolumn{26}{|l|}{ nutrients } \\
\hline oligotrophic & & & & & \begin{tabular}{|l|}
$F P$ \\
\end{tabular} & & & FP & FP & & FP & & & & & & & & \begin{tabular}{|l|}
$\mathrm{FN}$ \\
\end{tabular} & \begin{tabular}{|l|}
$\mathrm{FN}$ \\
\end{tabular} & & & & & \\
\hline mesotrophic & & & & & & & & & & & & & & & & & & $\mathrm{FN}$ & & & & & & & \\
\hline eutrophic & & & & & & & FP & & & & & & & & FP & & & & & & & & & & \\
\hline unknown & FP & FP & FP & FP & & FP & & & & FP & & FP & FP & & & FP & FN & & & & FN & FN & FN & $\mathrm{FN}$ & $\mathrm{FN}$ \\
\hline \multicolumn{26}{|l|}{ moisture } \\
\hline dry & & & & & FP & & & & & & $\mathrm{FP}$ & & & & & & & & $\mathrm{FN}$ & $\mathrm{F}$ & & & & & \\
\hline moderate dry & & & & & & & & & & & & & FP & FP & & & & $\mathrm{FN}$ & & & & & & & $\mathrm{FN}$ \\
\hline moist & & FP & FP & FP & & FP & FP & FP & FP & & & & $\mathrm{F}$ & & & \begin{tabular}{|l|}
$F P$ \\
\end{tabular} & FN & & $\mathrm{F}$ & $\mathrm{FN}$ & & $\mathrm{FN}$ & & $\mathrm{FN}$ & $\mathrm{F}$ \\
\hline wet & & & $\mathrm{F}$ & & & & & & $\mathrm{F}$ & & & & & & & & $F$ & & & & & & & & \\
\hline alternate wet/dry & & & & & & & & & & FP & & & & & & & & & & & & & & & \\
\hline flooded & & & & & & & & & & & & & & & FP & & & & & & & & & & \\
\hline unknown & FP & & & & & & & & & & & FP & & & & & & & & & \begin{tabular}{|l|}
$\mathrm{FN}$ \\
\end{tabular} & & \begin{tabular}{|l|}
$\mathrm{FN}$ \\
\end{tabular} & & \\
\hline \multicolumn{26}{|l|}{ Soil thickness } \\
\hline shallow & & & & & & & & & & & & & & & FP & & & & & & & & & & \\
\hline moderate thick & & & & & & & & & & & & & & & & & & & \begin{tabular}{|l|}
$\mathrm{FN}$ \\
\end{tabular} & & & & & & \\
\hline thick & & & & & & & & & & & & & & & & & & $\mathrm{FN}$ & & & & & & & \\
\hline unknown & FP & FP & FP & FP & \begin{tabular}{|l|}
$F P$ \\
\end{tabular} & FP & FP & FP & FP & FP & FP & 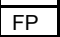 & \begin{tabular}{|l|} 
FP \\
\end{tabular} & & & FP & \begin{tabular}{|l|l|}
$\mathrm{FN}$ \\
\end{tabular} & & & \begin{tabular}{|l|}
$\mathrm{FN}$ \\
\end{tabular} & \begin{tabular}{|l|l|}
$\mathrm{FN}$ \\
\end{tabular} & \begin{tabular}{|l|}
$\mathrm{FN}$ \\
\end{tabular} & \begin{tabular}{|l|l|}
$\mathrm{FN}$ \\
\end{tabular} & $\mathrm{FN}$ & $\mathrm{FN}$ \\
\hline \multicolumn{26}{|l|}{ salinity } \\
\hline salt tolerant & & & & & & & & & & & & & & & FP & & & & \begin{tabular}{|l|l|}
$\mathrm{FN}$ \\
\end{tabular} & & $\mathrm{F}$ & & & & \\
\hline obligate halophyt & & & & & & & & & & & & & & & & & & & & $\mathrm{F}$ & & & & & \\
\hline not halophyte & FP & FP & FP & FP & FP & FP & FP & FP & FP & FP & FP & FP & FP & FP & $\mathrm{F}$ & FP & \begin{tabular}{|l|}
$\mathrm{FN}$ \\
\end{tabular} & $\mathrm{FN}$ & & \begin{tabular}{|l|}
$\mathrm{FN}$ \\
\end{tabular} & FN & FN & \begin{tabular}{|l|}
$\mathrm{FN}$ \\
\end{tabular} & $\mathrm{FN}$ & $\mathrm{FN}$ \\
\hline Light availability & & & & & & & & & & & & & & & & & & & & & & & & & \\
\hline shade plants & $\mathrm{FP}$ & & & & \begin{tabular}{|l|}
$F P$ \\
\end{tabular} & & & FP & & & & & & & & & $\mathrm{FN}$ & & $\mathrm{F}$ & $\mathrm{F}$ & & & & & \\
\hline partly shade plants & $\mathrm{F}$ & & & & & & & & & & & & & & & & & & & & & & & & \\
\hline fully exposed plants & & & \begin{tabular}{|l|} 
FP \\
\end{tabular} & & & & & & & & FP & FP & FP & FP & FP & \begin{tabular}{|l|} 
FP \\
\end{tabular} & & & \begin{tabular}{|l|}
$\mathrm{FN}$ \\
\end{tabular} & \begin{tabular}{|l|}
$\mathrm{FN}$ \\
\end{tabular} & & & \begin{tabular}{|l|}
$\mathrm{FN}$ \\
\end{tabular} & & $\mathrm{FN}$ \\
\hline unknown & & FP & & FP & & FP & FP & & FP & FP & & & & & & & & $\mathrm{FN}$ & & & \begin{tabular}{|l|}
$\mathrm{FN}$ \\
\end{tabular} & \begin{tabular}{|l|}
$\mathrm{FN}$ \\
\end{tabular} & & $\mathrm{FN}$ & \\
\hline & & & & & & & & & & & & & & & & & & & & & & & & & \\
\hline
\end{tabular}




\subsection{Diversitäten}

\subsubsection{Profil Alversdorf}

\begin{tabular}{|c|c|c|c|c|c|c|c|c|c|}
\hline $\begin{array}{l}\dot{\Sigma} \\
0 \\
\frac{0}{0} \\
\frac{c}{0} \\
\stackrel{J}{J} \\
\stackrel{J}{J}\end{array}$ & 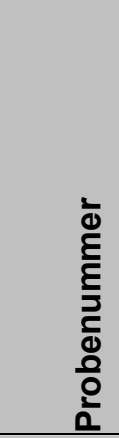 & 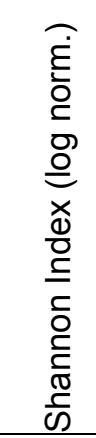 & 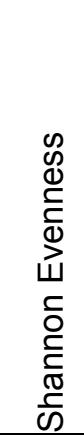 & 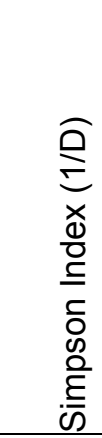 & 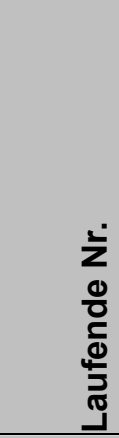 & 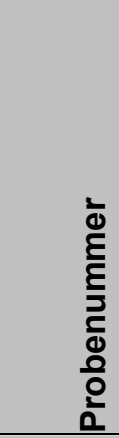 & 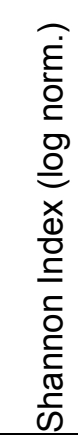 & 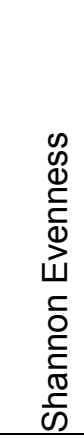 & 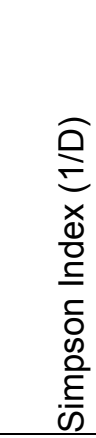 \\
\hline 1 & 1 & 3,05 & 0,96 & 24,48 & 69 & n 69 & 3,52 & 0,95 & 39,85 \\
\hline 2 & 2 & & & & 70 & n 70 & 3,37 & 0,94 & 33,00 \\
\hline 3 & 3 & & & & 71 & n 71 & 3,48 & 0,95 & 38,02 \\
\hline 4 & 4 & & & & 72 & n 72 & 3,62 & 0,95 & 43,82 \\
\hline 5 & 5 & 3,04 & 0,94 & 23,52 & 73 & n 73 & 3,69 & 0,95 & 47,50 \\
\hline 6 & 6 & 3,28 & 0,98 & 32,86 & 74 & n 74 & 3,91 & 0,96 & 62,11 \\
\hline 7 & 7 & 3,34 & 0,96 & 33,71 & 75 & n 75 & 3,88 & 0,95 & 58,64 \\
\hline 8 & 8 & 2,99 & 0,97 & 23,14 & 76 & n 76 & 3,99 & 0,96 & 67,15 \\
\hline 9 & 9 & 2,84 & 0,98 & 20,53 & 77 & n 77 & 3,34 & 0,94 & 31,50 \\
\hline 10 & 10 & 3,52 & 0,95 & 39,97 & 78 & n 78 & 3,57 & 0,94 & 40,46 \\
\hline 11 & 11 & 3,42 & 0,95 & 36,14 & 79 & e 79 & 3,46 & 0,97 & 39,09 \\
\hline 12 & 12 & 3,25 & 0,96 & 30,04 & 80 & e 80 & 3,41 & 0,96 & 36,34 \\
\hline 13 & 13 & 3,42 & 0,95 & 36,34 & 81 & e 81 & 3,26 & 0,97 & 31,30 \\
\hline 14 & 14 & 3,47 & 0,98 & 40,39 & 82 & e 82 & 3,21 & 0,96 & 29,26 \\
\hline 15 & 15 & 3,38 & 0,95 & 34,03 & 83 & e 83 & 3,14 & 0,98 & 27,86 \\
\hline 16 & 16 & 3,12 & 0,95 & 25,98 & 84 & e 84 & 3,35 & 0,97 & 34,57 \\
\hline 17 & 17 & 3,37 & 0,98 & 36,61 & 85 & e 85 & 3,20 & 0,97 & 29,40 \\
\hline 18 & 18 & 3,04 & 0,94 & 23,60 & 86 & e 86 & 3,22 & 0,98 & 31,01 \\
\hline 19 & 19 & 3,39 & 0,94 & 33,02 & 87 & e 87 & 3,15 & 0,95 & 27,45 \\
\hline 20 & 20 & 3,17 & 0,95 & 27,25 & 88 & e 88 & 3,43 & 0,96 & 37,47 \\
\hline 21 & 21 & 3,37 & 0,95 & 33,67 & 89 & e 89 & 3,46 & 0,98 & 39,70 \\
\hline 22 & 22 & 3,22 & 0,94 & 27,86 & 90 & e 90 & 3,52 & 0,97 & 42,02 \\
\hline 23 & 23 & 3,37 & 0,97 & 35,87 & 91 & e 91 & 3,21 & 0,94 & 28,44 \\
\hline 24 & 24 & 3,34 & 0,94 & 32,24 & 92 & e 92 & 3,34 & 0,96 & 33,57 \\
\hline 25 & 25 & 3,24 & 0,95 & 29,37 & 93 & e 93 & 3,25 & 0,98 & 31,46 \\
\hline 26 & 26 & 3,32 & 0,96 & 33,02 & 94 & e 94 & 3,30 & 0,95 & 31,70 \\
\hline 27 & 27 & 3,14 & 0,94 & 26,53 & 95 & e 95 & 3,43 & 0,96 & 37,14 \\
\hline 28 & 28 & 3,34 & 0,96 & 33,44 & 96 & e 96 & 3,10 & 0,94 & 25,05 \\
\hline 29 & 29 & 3,28 & 0,96 & 32,11 & 97 & e 97 & 3,26 & 0,96 & 30,83 \\
\hline 30 & 30 & 3,44 & 0,97 & 37,90 & 98 & e 98 & 3,28 & 0,97 & 32,66 \\
\hline 31 & 31 & 3,43 & 0,96 & 36,73 & 99 & e 99 & 3,13 & 0,95 & 27,00 \\
\hline 32 & 32 & 3,62 & 0,96 & 46,36 & 100 & e 100 & 3,10 & 0,95 & 26,15 \\
\hline 33 & 33 & 3,39 & 0,95 & 34,34 & 101 & e 101 & 3,27 & 0,96 & 31,67 \\
\hline 34 & 34 & 3,50 & 0,96 & 40,51 & 102 & e 102 & 3,26 & 0,98 & 31,48 \\
\hline 35 & 35 & 3,43 & 0,96 & 36,55 & 103 & e 103 & 3,22 & 0,97 & 30,34 \\
\hline
\end{tabular}




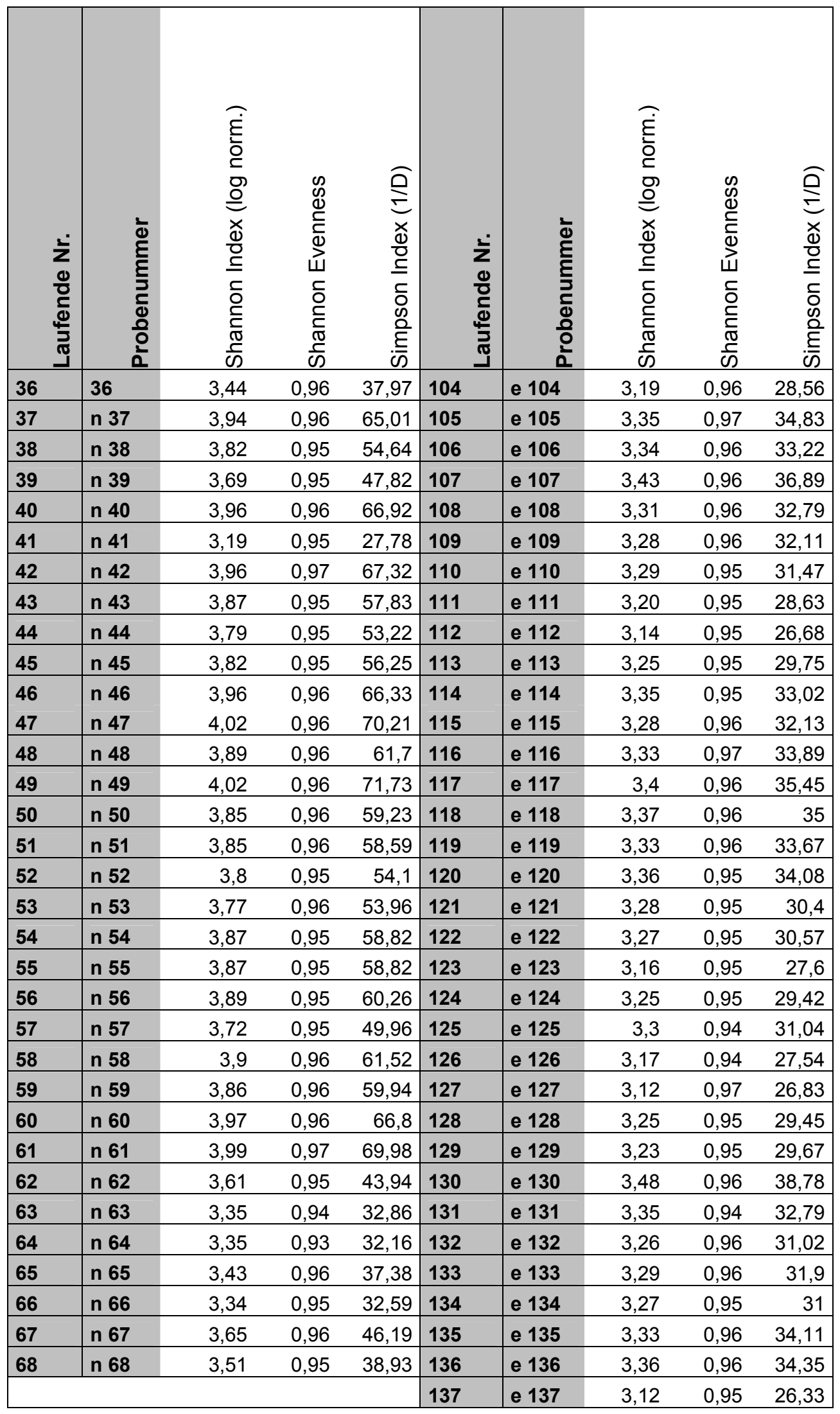




\subsubsection{Profil Schöningen}

\begin{tabular}{|c|c|c|c|c|c|c|c|c|c|}
\hline $\begin{array}{l}\stackrel{\Sigma}{2} \\
\frac{1}{0} \\
\frac{0}{0} \\
\stackrel{0}{2} \\
\frac{\pi}{3}\end{array}$ & 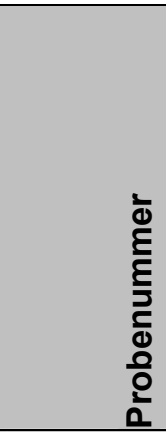 & 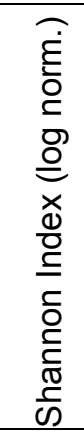 & 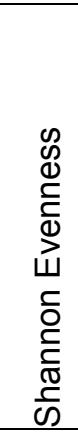 & 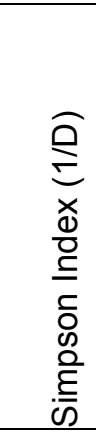 & 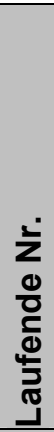 & 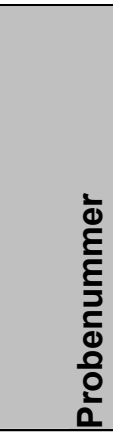 & 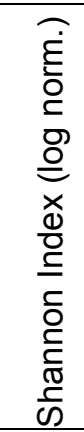 & 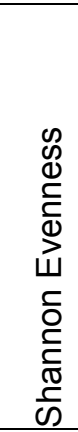 & 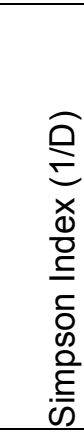 \\
\hline 1 & $35 \mathrm{H}$ & 3,05 & 0,80 & 14,76 & $\overline{47}$ & 8ZM1 & 2,64 & 0,69 & 5,81 \\
\hline 2 & $34 \mathrm{H}$ & 3,11 & 0,80 & 14,28 & 48 & 7ZM1 & & & \\
\hline 3 & $33 \mathrm{H}$ & & & & 49 & 6ZM1 & 3,48 & 0,86 & 28,51 \\
\hline 4 & $32 \mathrm{H}$ & 3,39 & 0,87 & 25,20 & 50 & 5ZM1 & 3,37 & 0,87 & 24,80 \\
\hline 5 & $31 \mathrm{H}$ & 3,02 & 0,80 & 13,71 & 51 & 4ZM1 & 3,11 & 0,78 & 14,67 \\
\hline 6 & $30 \mathrm{H}$ & 3,54 & 0,85 & 26,30 & 52 & 3ZM1 & 3,37 & 0,83 & 21,57 \\
\hline 7 & $29 \mathrm{H}$ & 3,35 & 0,84 & 23,91 & 53 & 2ZM1 & 3,31 & 0,82 & 18,89 \\
\hline 8 & $28 \mathrm{H}$ & 3,31 & 0,82 & 18,93 & 54 & 1ZM1 & 3,14 & 0,79 & 15,92 \\
\hline 9 & $27 \mathrm{H}$ & 3,04 & 0,78 & 12,20 & 55 & $12 \mathrm{~F} 1$ & 3,04 & 0,77 & 11,59 \\
\hline 10 & $26 \mathrm{H}$ & 3,44 & 0,85 & 26,13 & 56 & 11F1 & 3,53 & 0,84 & 21,16 \\
\hline 11 & $25 \mathrm{H}$ & 3,60 & 0,88 & 35,19 & 57 & 10F1 & 3,04 & 0,79 & 13,06 \\
\hline 12 & $24 \mathrm{H}$ & 3,37 & 0,87 & 28,31 & 58 & 9F1 & 2,70 & 0,72 & 8,45 \\
\hline 13 & $23 \mathrm{H}$ & & & & 59 & $8 \mathrm{~F} 1$ & & & \\
\hline 14 & $22 \mathrm{H}$ & 3,37 & 0,83 & 24,03 & 60 & 7F1 & 2,32 & 0,68 & 6,70 \\
\hline 15 & $21 \mathrm{H}$ & 3,16 & 0,80 & 14,42 & 61 & 6F1 & 3,08 & 0,78 & 12,44 \\
\hline 16 & $20 \mathrm{H}$ & 2,94 & 0,80 & 12,38 & 62 & $5 F 1$ & 2,50 & 0,72 & 8,21 \\
\hline 17 & $19 \mathrm{H}$ & 2,91 & 0,76 & 11,16 & 63 & 4F1 & 2,95 & 0,78 & 13,08 \\
\hline 18 & $18 \mathrm{H}$ & 3,06 & 0,79 & 13,89 & 64 & 3F1 & 2,10 & 0,59 & 3,86 \\
\hline 19 & $17 \mathrm{H}$ & 3,23 & 0,81 & 18,20 & 65 & $2 \mathrm{~F} 1$ & 2,74 & 0,74 & 9,88 \\
\hline 20 & $16 \mathrm{H}$ & 3,10 & 0,82 & 17,75 & 66 & 1F1 & 2,95 & 0,75 & 10,27 \\
\hline 21 & $15 \mathrm{H}$ & 3,54 & 0,84 & 26,56 & 67 & 1ZM2 & 3,36 & 0,83 & 18,80 \\
\hline 22 & $14 \mathrm{H}$ & 3,34 & 0,84 & 23,54 & 68 & 2ZM2 & 2,96 & 0,74 & 8,26 \\
\hline 23 & $13 \mathrm{H}$ & 3,09 & 0,80 & 14,38 & 69 & 3ZM2 & 1,25 & 0,47 & 1,91 \\
\hline 24 & $12 \mathrm{H}$ & 3,12 & 0,81 & 18,56 & 70 & 4ZM2 & 2,87 & 0,73 & 8,68 \\
\hline 25 & $11 \mathrm{H}$ & 3,17 & 0,80 & 16,95 & 71 & 5ZM2 & & & \\
\hline 26 & $10 \mathrm{H}$ & 2,58 & 0,74 & 7,95 & 72 & 6ZM2 & 2,86 & 0,78 & 10,55 \\
\hline 27 & $9 \mathrm{H}$ & 2,89 & 0,75 & 11,54 & 73 & 7ZM2 & 2,59 & 0,74 & 6,38 \\
\hline 28 & $8 \mathrm{H}$ & 2,18 & 0,60 & 3,85 & 74 & $8 Z M 2$ & 2,56 & 0,73 & 6,21 \\
\hline 29 & $7 \mathrm{H}$ & & & & 75 & 9ZM2 & 2,91 & 0,74 & 9,55 \\
\hline 30 & $6 \mathrm{H}$ & 2,18 & 0,57 & 3,70 & 76 & 10ZM2 & 2,97 & 0,76 & 10,34 \\
\hline 31 & $5 \mathrm{H}$ & & 0,55 & 3,67 & 77 & 17F2 & 3,34 & 0,85 & 21,20 \\
\hline 32 & $4 \mathrm{H}$ & 2 & 0,54 & 3,51 & 78 & 16F2 & 3,16 & 0,80 & 16,55 \\
\hline 33 & $3 \mathrm{H}$ & & 0,55 & 3,70 & 79 & 15F2 & 2,98 & 0,76 & 10,14 \\
\hline 34 & $2 \mathrm{H}$ & 2,42 & 0,62 & 4,71 & 80 & 14F2 & 2,95 & 0,77 & 11,53 \\
\hline 35 & $1 \mathrm{H}$ & 2,13 & 0,56 & 3,71 & 81 & 13F2 & 2,69 & 0,74 & 8,65 \\
\hline 36 & 19ZM1 & 3,18 & 0,86 & 20,53 & 82 & $12 \mathrm{~F} 2$ & & & \\
\hline 37 & 18ZM1 & 3,34 & 0,85 & 19,75 & 83 & 11F2 & 3,29 & 0,82 & 15,97 \\
\hline 38 & 17ZM1 & & & & 84 & 10F2 & 3,54 & 0,85 & 30,47 \\
\hline 39 & 16ZM1 & 3,05 & 0,75 & 11,24 & 85 & 9F2 & & & \\
\hline 40 & 15ZM1 & & & & 86 & $8 \mathrm{~F} 2$ & 3,30 & 0,80 & 14,41 \\
\hline 41 & 14ZM1 & 2,73 & 0,80 & 9,50 & 87 & 7F2 & 3,07 & 0,78 & 13,65 \\
\hline 42 & 13ZM1 & 2,22 & 0,72 & 5,57 & 88 & $6 \mathrm{F2}$ & 3,29 & 0,80 & 16,58 \\
\hline 43 & 12ZM1 & & & & 89 & $5 \mathrm{F2}$ & 3,46 & 0,83 & 22,84 \\
\hline 44 & 11ZM1 & & & & 90 & 4F2 & 2,70 & 0,75 & 10,65 \\
\hline 45 & 10ZM1 & 3,05 & 0,77 & 14,49 & 91 & $3 F 2$ & 2,86 & 0,79 & 13,58 \\
\hline 46 & 9ZM1 & & & & 92 & $2 \mathrm{~F} 2$ & 3,40 & 0,84 & 22,05 \\
\hline & & & & & 93 & $1 \mathrm{~F} 2$ & 3,23 & 0,80 & 15,34 \\
\hline
\end{tabular}


7.3.3 Profil Witznitz 11

\begin{tabular}{|c|c|c|c|c|c|c|c|c|c|}
\hline 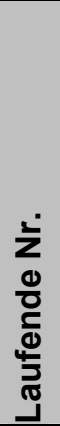 & 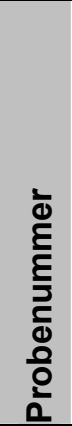 & 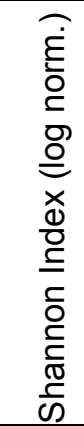 & 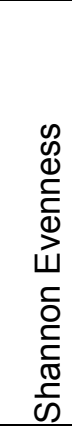 & 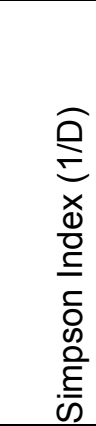 & 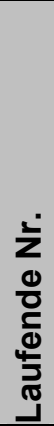 & 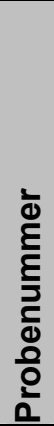 & 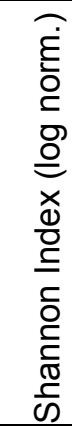 & 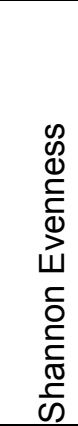 & 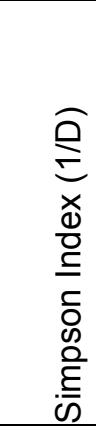 \\
\hline 1 & 21 & & & & 14 & $9 c$ & & & \\
\hline 2 & 20 & 4,05 & 0,98 & 69,47 & 15 & $9 b$ & 3,81 & 0,97 & 52,97 \\
\hline 3 & 19 & 4,1 & 0,98 & 71,79 & 16 & $9 a$ & 3,94 & 0,97 & 61,49 \\
\hline 4 & 18 & 3,86 & 0,98 & 55,55 & 17 & $8 b$ & & & \\
\hline 5 & 17 & & & & 18 & $8 a$ & 3,65 & 0,98 & 45,25 \\
\hline 6 & 16 & 4,03 & 0,98 & 66,78 & 19 & 7 & 3,79 & 0,97 & 52,47 \\
\hline 7 & 15 & 4,01 & 0,98 & 64,88 & 20 & 6 & 3,61 & 0,97 & 43,23 \\
\hline 8 & $14 b$ & 4,1 & 0,98 & 73,41 & 21 & $5 b$ & & & \\
\hline 9 & $14 a$ & 3,89 & 0,98 & 58,92 & 22 & $5 a$ & 3,95 & 0,98 & 63,43 \\
\hline 10 & 13 & & & & 23 & 4 & 3,6 & 0,97 & 42,54 \\
\hline 11 & 12 & 3,92 & 0,98 & 61,55 & 24 & 3 & 3,76 & 0,97 & 50,11 \\
\hline 12 & 11 & 3,9 & 0,98 & 59,94 & 25 & 2 & 3,39 & 0,97 & 33,94 \\
\hline 13 & 10 & 3,74 & 0,98 & 51,08 & 26 & 1 & 3,84 & 0,98 & 56,78 \\
\hline
\end{tabular}



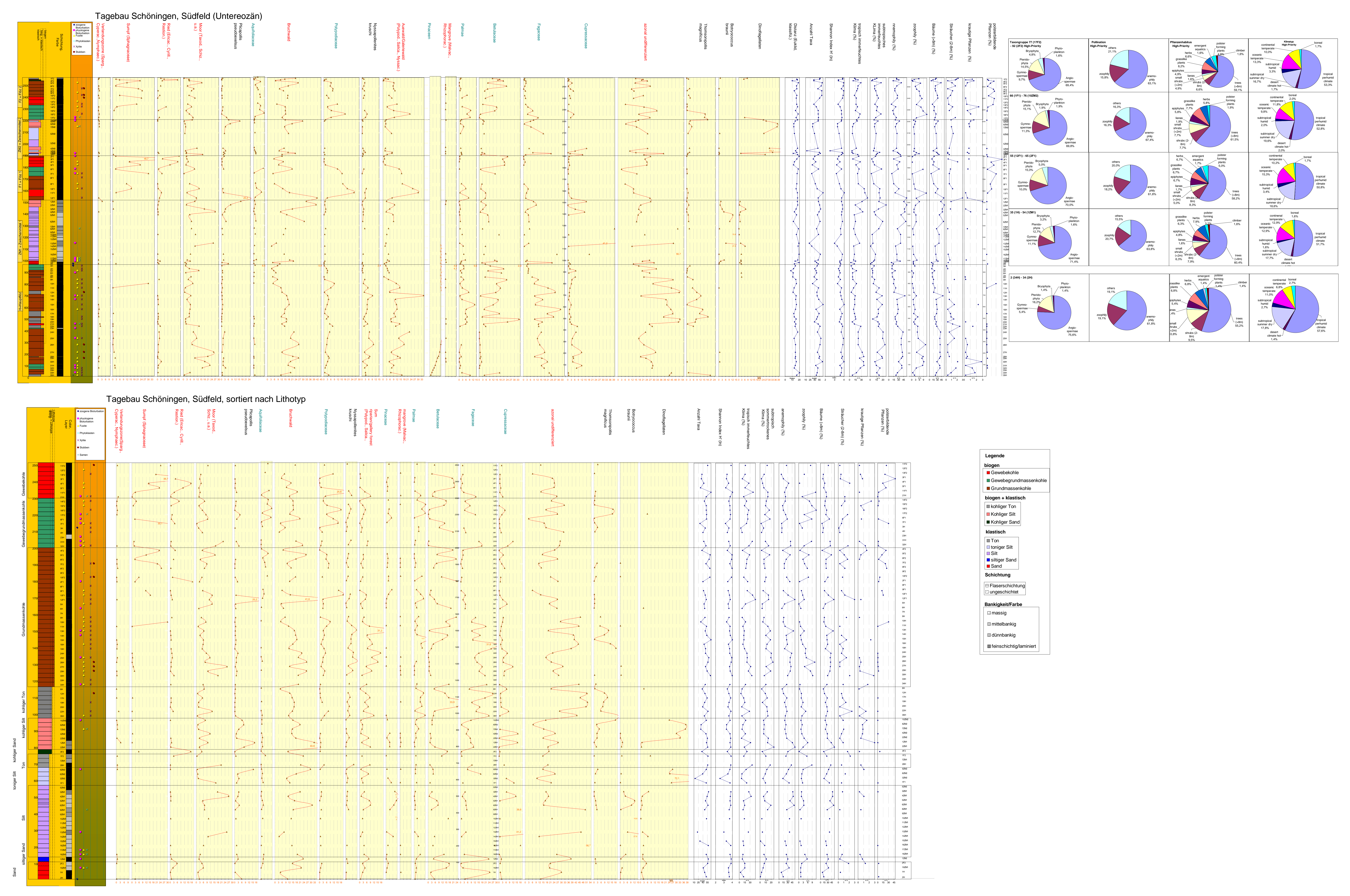
Tagebau Alversdorf (Mititeloozãn)
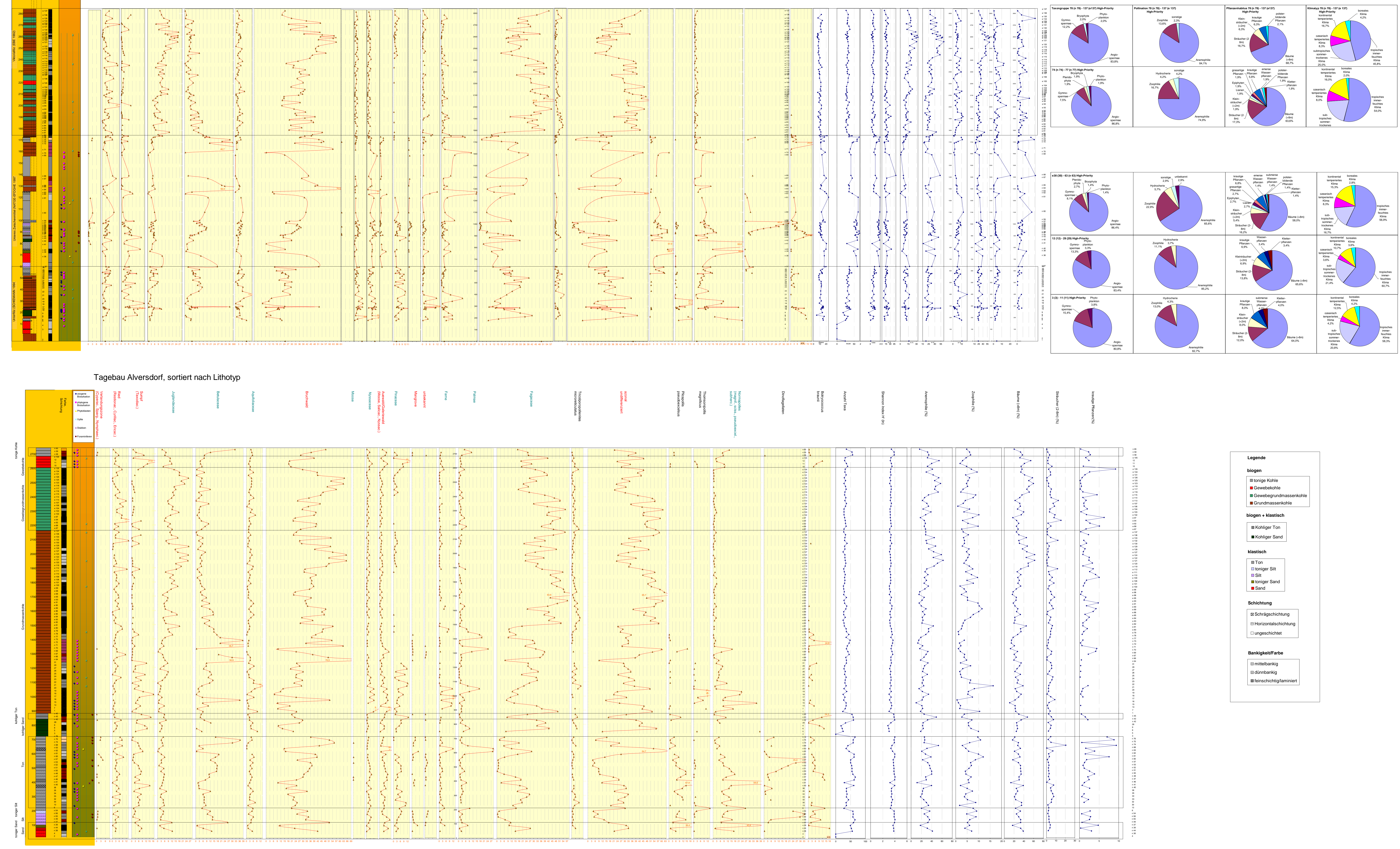


\section{Lebenslauf}

\section{Thomas Bode}

Ludwig-Prandtl Str. 48

37077 Göttingen

0551-39 7962 (dienstl.) 0551-37 2194 (priv.)

e-mail: tbode@gwdg.de

wWw:

www.imgp.gwdg.de/paleobot/paleobot.htm

\section{Persönliche Angaben}

Familienstand: verheiratet, seit 02.03.01

Staatsangehörigkeit: deutsch

Alter: 34

Geburtsort: Göttingen

Eltern: Wolfgang Bode, verstorben am 27.11.91

Sigrid Bode, Angestellte

Geschwister: ein Bruder, 38 Jahre

Kinder: Anna-Carina, geboren am 26.07.01

\section{Ausbildung}

$1973-1977$

Grundschule

Göttingen/Herberhausen

$1977-1983$

Realschule

Göttingen/Weende

$1983-1986$

Fachgymnasium Technik an der BBS II

Göttingen

$1988-1994$

Studium im Fach Geologie

Göttingen

$1995-12 / 2001$

Dissertation mit dem Thema: Ein Datenbanksystem (P.A.S.T) zur Verarbeitung und Interpretation von palynologischen Daten aus dem Paläogen Mitteleuropas mit

Diversitätsbetrachtungen,

Göttingen, im Fach Geologie, Paläontologie

Finanzierung durch folgende Tätigkeiten:

\section{Berufserfahrung}

$1995-1996$

Desktop-Publishing bei Prof. Dr. Hönig

Göttingen

Kieferchirurgie

$1995-1996$

Hilfswissenschaftler bei Prof. Dr. Riegel

Göttingen

Schwerpunkte:Geologie, Betreuung der Abteilungrechner 
$1996-1999$

$1998-2001$

$1986-1987$

01.03 .1995

15.02.1995 (2 Tage) 25.09.1995 (4 Tage) 09.09.1996 (6 Tage)

$1994-2001$

12.10.1992 -

12.02 .1993

01.03.1993 -

31.03 .1993
Wissenschaftliche Tätigkeit im Rechenzentrum der GWDG, Göttingen. Schwerpunkte: Betreuung und Beratung bei Hard- und Softwareproblemen, Netzwerkmonitoring

Betreuung des Netzwerkes der geologischen Institute (GZG, Abteilung Geobiologie), z.T: parallel zur obengenannten Tätigkeit

Schwerpunkte: Installation eines Client-Server-Netzwerkes unter Windows NT (WWW, PDC, BDC, RAID) und Betreuung der Benutzer

Gewerbetätigkeit: Computerhandel, Beratung bei Computerproblemen

\section{Wehr-/Ersatzdienst}

Göttingen

Grundwehrdienst

\section{Qualifikationen}

\section{Kurse GWDG}

Informationsdienste II - NetNews

HTML und Mosaik

Das Datenbanksystem MS-Access 2.0

Autocad

\section{Selbststudium}

Sehr gute Kenntnisse in ACCESS 2.0 - 2000

Visual Basic

Netwerke unter Windows NT

Officeanwendungen (u.a. Word, Excel, Powerpoint)

Grafikanwendungen (Coreldraw, Framemaker, Photoshop)

Grundkenntnisse in Linux

\section{Praktika}

Wille Geotechnik

Erdbaulabor Göttingen

\section{Hobbys}

Computertechnik, Lesen, Musikinstrumente 